6-2019

\title{
Evaluation of Student Perceptions of Introductory Pharmacy Practice Experiences
}

\author{
Jaclyn Boyle \\ Zach Jenkins \\ Cedarville University, zjenkins@cedarville.edu \\ Thad Franz \\ Cedarville University, tfranz@cedarville.edu \\ Charles Cather \\ Nicole K. Stute \\ Cedarville University, nstute@cedarville.edu
}

Follow this and additional works at: https://digitalcommons.cedarville.edu/ See next page for additionalauthors

Part of the Higher Education Commons, Medical Education Commons, and the Pharmacy and Pharmaceutical Sciences Commons

\section{Recommended Citation}

Boyle, Jaclyn; Jenkins, Zach; Franz, Thad; Cather, Charles; Stute, Nicole K.; Harper, Nicole G.; Dittmer, Alison; and Hartline, Christy, "Evaluation of Student Perceptions of Introductory Pharmacy Practice Experiences" (2019). Pharmacy Practice Faculty Publications. 387.

https://digitalcommons.cedarville.edu/pharmacy_practice_publications/387

This Abstract is brought to you for free and open access by DigitalCommons@Cedarville, a service of the Centennial Library. It has been accepted for inclusion in Pharmacy Practice Faculty Publications by an authorized administrator of DigitalCommons@Cedarville. For more information, please contact digitalcommons@cedarville.edu. 


\section{Authors}

Jaclyn Boyle, Zach Jenkins, Thad Franz, Charles Cather, Nicole K. Stute, Nicole G. Harper, Alison Dittmer, and Christy Hartline 
American Journal of Pharmaceutical Education 2019; 83 (5) Article 7654.

\section{AACP REPORT}

\section{Abstracts Presented at the 120th Annual Meeting of the American Association of Colleges of Pharmacy, Chicago, Illinois, July 13-17, 2019}

\section{ADMINISTRATIVE SERVICES \\ Completed Research}

Admission Scores and Characteristics in Relation to Performance Within PharmD Curriculum and on Licensure Examinations. Andrea J. Cameron, University of Toronto, Robert P. Bonin, University of Toronto, Sara J.T. Guilcher, University of Toronto, John A. Pugsley, Pharmacy Examining Board of Canada. Objective: To describe admission scores, including multiple miniinterview (MMI), pre-pharmacy average (PPA) and Pharmacy College Admission Test (PCAT), and covariates (age, prior degree, re-application, gender), in cohorts admitted in 2011 and 2012 to the PharmD program at University of Toronto. To determine predictive validity of admission scores and covariates, with respect to year 3 grade point average (GPA), year 4 advanced pharmacy practice experience (APPE) rotations, and scores on Pharmacy Examining Board of Canada (PEBC) Qualifying Examinations: multiple choice question (MCQ) and objective structured clinical examination (OSCE). Methods: Descriptive statistics and correlations were determined for admission scores and covariates. Multiple linear regression analyses were conducted with PPA, PCAT composite, and MMI scores as predictor variables. Dependent variables were Year 3 GPA, APPE scores (community and institutional), PEBC-MCQ and OSCE scores. Results: Consenting students admitted in 2011 and 2012 with matched PEBC scores totaled 229 (56.8\% female) and 219 (64.8\% female), respectively. Average age $(22.0,21.6)$, PPA $(79.8,80.3)$, PCAT (421.7, 422.5) and MMI \% $(62.5,63.0)$ were similar. In both cohorts, PPA and MMI were significant predictors for annual Year 3 GPA. MMI was a significant predictor for community and institutional APPE (2011); model not significant with 2012. For PEBC exam, PPA, PCAT and MMI were significantly predictive for MCQ; only MMI was significant for OSCE. Implications: MMI was only admissions predictor for PEBC OSCE, supporting MMI continued use. PPA, PCAT, age, prior degree and gender were also significant in some models. Further analysis of in-program course performance is occurring.

A PCOA "Gated Stakes" Model to Ensure Proficiency Prior to Progression. Patrick J. Davis, The University of Texas at Austin, Rochelle M. Roberts, The
University of Texas at Austin, W. Renee Acosta, The University of Texas at Austin. Objective: Develop an administrative process to take the PCOA from a "high stakes" (pass/no pass) model to a "gated stakes" exam in which the level of performance dictated differential levels of remediation prior to progression. The lack of subjectlevel granularity in the PCOA mandated the identification of a more diagnostic process that could (a) identify and (b) remediate deficiencies, (c) confirm proficiency, and (d) if unsuccessful remediation, identify which course(s) need repeating. Methods: Class results were normalized by setting the score for the highest performing student as $100 \%$ (range $=100 \%$ to $55 \%$ ). Students $1-$ StdDev below the mean were designated "grey zone" and those 2StdDev below the mean were designated "red zone." Access Pharmacy's "McGraw-Hill NAPLEX Review Guide" was identified as the diagnostic/remediation/ assessment tool. A validation study conducted with PCOA-successful students showed a direct correlation with overall PCOA scores vs NAPLEX review scores $(\mathrm{R} 2=0.7965)$ and set an acceptable cut score. Results: "Grey-zone" students were "gated" to identifying areas of deficiency, complete a narrative reflection, and meet with their faculty mentor to address how those areas would be addressed prior to and during the P4 year. "Red-zone" students were "gated" to identify areas of deficiency, remediate, and achieve the cut score set by the pilot; all successfully remediated and were then assigned faculty mentors for the P4 year. Implications: The Program Assessment Team has developed a process to extend the non-granular PCOA results to a "gated stakes" process allowing for identification of deficiencies, remediation, and demonstration of proficiency prior to progression.

A Story of 11 Rubrics. Suzanne Carbonaro, University of the Sciences, Lisa Charneski, University of the Sciences. Objective: To describe a process of faculty engagement in program-wide rubric creation Methods: To support assessment within the competency-driven curriculum (fall 2018 start) development of program-wide rubrics become a priority. Three faculty-centered workshops followed by three surveys set the stage for collaboration. Using tenets of Expectancy-value theory, faculty identified key performance indicators of value to our 


\section{American Journal of Pharmaceutical Education 2019; 83 (5) Article 7654.}

college in workshop 1. Informed by survey results, workshop 2 focused on rubrics. In the next survey, faculty identified rubrics of highest priority for development aligned to best rubric type (single point vs analytic). As a result, small working groups were assigned to create rubrics for presentation at the final faculty workshop. Faculty input for needed revisions was elicited in the final survey. Revised rubrics were then mapped to programmatic outcomes in our cloud-based assessment platform. Results: Eleven programmatic rubrics were created between January 2018 through December 2018. Those identified as highest priority in the second survey (response rate 59\%) included: (1) blood pressure; (2) communication; (3) empathy; (4) patient care writing; (5) patient counseling; (6) presentation; (7) reflective writing; (8) prescription dispensing; (9) participation (10) medication history; and (11) device. The third survey (56\% response rate) asked faculty to indicate whether the specific rubric was "Ready for Use" (33-61\%), "Minor Edits Needed" (39-67\%), or "Major Revision" (6\% of responses; 1/11 rubrics). Implications: This process provided transparency and faculty autonomy. The result is a facultyreviewed bank of rubrics, applicable to various contexts, and accessible to stakeholders. Student performance can be aggregated and tracked longitudinally.

Becoming Pharmacists: Exploring the Professional Identity Formation of Student Pharmacists Through Self-Authorship. Curtis G. Jefferson, University of Washington. Objective: To explore how student pharmacists conceive and experience the concept and process of professional identity formation (PIF) using the theoretical framework of self-authorship. Methods: Fourth-year student pharmacists participated in semistructured interviews focusing on their expectations and experiences over the course of pharmacy school. Transcripts of these in-depth interviews were reviewed using an interpretative phenomenological analysis (IPA) approach. Through this iterative analysis process, themes and superordinate themes were identified that capture participants' experiences with PIF and the conditions that appeared most conducive to supporting this type of development. Results: Three superordinate themes emerged as PIF domains: Defining the Profession, Building Confidence \& Competence, and Discerning Values. Development within each domain occurred across three distinct stages that align with the self-authorship framework, which emphasizes the transition between defining one's experiences based on external factors to relying on an internalized sense of understanding. A fourth superordinate theme, Significant Experiences, illustrated factors that helped to support participants in the PIF process: Early \& Sustained Practice Opportunities, Exposure to
Multiple Practice Models/Settings, and a Supportive Learning Environment. Implications: Programs should consider the PIF of student pharmacists in addition to helping them acquire the knowledge and skills necessary for practice. It is through PIF that students begin to understand and internalize the broader context of the pharmacy profession. Earlier placement of curricular practice opportunities, sustained engagement with practice throughout the program, varied practice experiences across settings and practice sites, and an open and welcome environment created by the faculty are potential considerations for fostering student development in this area.

Common Practices and Decision-Making Factors Faculty Use in Accommodating Pregnant Students. Katherine S. Wadas-Thalken, Creighton University, Leah Georges, Creighton University. Objective: To identify common practices and decision-making factors faculty used in supporting and accommodating pregnant students. Methods: An online survey was sent to faculty in one pharmacy program asking about their experiences working with pregnant students. Participants were also asked about their knowledge of Title IX and how it applies to pregnant students. Results: One of the key findings of the study was that school policies and procedures were a strong decision-making factor for participants who have worked with pregnant students in the past three years. Additionally, the findings revealed that faculty preferred accommodations for pregnant students be centralized with the pharmacy school's dean's office. The findings of this study suggest that university-level policies are less influential on faculty decision making than school policies and procedures. Lastly, only half of participants knew that Title IX protected pregnant students from discrimination. Implications: Nearly $75 \%$ of participants had worked with a pregnant student in the past three years and most participants relied on guidance from school policies and procedures for how to work with these students. While participants were aware of legal implications when working with this population, only half of them knew that Title IX was a key law protecting pregnant students from discrimination. Findings from the study suggested that pharmacy schools should have clear policies and procedures for working with pregnant students and that centralizing decision-making with a dean may be more helpful and effective.

Correlating Big 5 Personality Dimensions with Academic Performance. Adam C. Welch, East Tennessee State University, Debbie C. Byrd, East Tennessee State University. Objective: To explore correlations between Big Five Personality Dimensions (Big 5) and academic performance among PharmD students. Methods: 


\section{American Journal of Pharmaceutical Education 2019; 83 (5) Article 7654.}

A 77-question electronic survey was administered to the classes of 2017 and 2018 at one college. It was administered in the spring semester of the P4 year for the class of 2017 and P3 year for the class of 2018. Mean scores were calculated from responses to a five-point Likert scale for questions related to the five personality dimensions (openness, conscientiousness, extraversion, agreeableness, and neuroticism).Mean scores were then correlated (Pearson) with grade point averages (GPAs) during the P1, P2, P3 years, and with scaled score on the NAPLEX. Results: There were 95 of 165 students $(56.2 \%)$ in the classes of 2017 and 2018 that completed the personality assessment. The mean personality scores for all respondents were openness 3.99 , conscientiousness 3.75 , extraversion 3.44, agreeableness 3.59, and neuroticism 2.57. Sixty-six of the students had both personality scores and released NAPLEX scores. The personality dimension with the strongest correlation with NAPLEX was agreeableness $(r=-0.224)$, followed by openness $(r=-0.185)$, extraversion $(\mathrm{r}=-0.121)$, conscientiousness $(\mathrm{r}=-0.118)$, and neuroticism $(\mathrm{r}=0.030)$. Conscientiousness had the strongest correlation with the cumulative GPA for both the P2 $(r=0.151)$ and P3 $(r=0.125)$ year. Extraversion correlated the strongest with P1 GPA ( $\mathrm{r}=-0.184)$. Implications: Conscientiousness had a positive correlation with grades during the didactic curriculum but a negative correlation with scores on the NAPLEX. With further exploration, correlations with personality dimensions and indicators of success in pharmacy school could lead to novel uses of Big 5 Personality with admissions decisions.

Correlation of Student Performance on PCOA Areas to Corresponding GPA. Pamela E. Fernainy, Lebanese American University, Roy Kanbar, Lebanese American University, Lamis R. Karaoui, Lebanese American University, Nibal Chamoun, Lebanese American University, Ghada Khoury, Lebanese American University, Yara Kuyumjian, Lebanese American University, Hanine Mansour, Lebanese American University, Aline Saad, Lebanese American University. Objective: This study examined the relationship between the performance of pharmacy students in the Pharmacy Curriculum Outcomes Assessment (PCOA standard score) areas and the corresponding Grade Point Average (GPA) in the School of Pharmacy program. Methods: For that purpose, the four PCOA areas were mapped to relevant courses in the curriculum. The corresponding weighted GPA for each of the 4 groups of courses was calculated. Pearson correlation coefficient was computed between the PCOA area standard score and the corresponding GPA. Analysis was performed utilizing data collected over the past 4 years, separately and collectively. When available, the overall and per area PCOA standard score of P3 and P4 students was compared. Results: When comparing performance, the overall PCOA standard score is significantly and positively correlated with the cumulative GPA $(r=0.59, p<0.0001)$. When examining this relationship per year, the correlation coefficient remained significant (highest $\mathrm{r}=0.74, \mathrm{p}<0.0001$ and lowest $\mathrm{r}=0.36, \mathrm{p}=0.046)$. Furthermore, the correlations between PCOA area score and corresponding area GPA, computed utilizing cumulative 4-year data, were also significant (area $1 \mathrm{r}=0.43, \mathrm{p}<0.0001$; area $2 \mathrm{r}=0.47, \mathrm{p}<0.0001$; area $3 \mathrm{r}=0.35, \mathrm{p}<0.0001$; area $4 \mathrm{r}=0.40, \mathrm{p}<0.0001)$. However, when computing this correlation per year, it was significant 3 out of 4 years in area 1,2 and 4, and only once in area 3. Finally, when comparing overall PCOA standard score between $\mathrm{P} 3$ and P4, no significant difference was noted, except for area 4 (ANOVA $p=0.01$ ). Implications: The PCOA performance per area is moderately correlated with GPA in corresponding pharmacy courses.

Crossing the Technological Chasm: Implementation of New Online Testing Tool in a Doctor of Pharmacy Program. Amy L. McLaughlin, University of the Sciences, Lisa Charneski, University of the Sciences, Cathy Y. Poon, University of the Sciences, Robert McCunney, University of the Sciences. Objective: To describe a cultural shift during implementation of a new assessment software and the process of faculty development leading to college-wide acceptance Methods: In 2016-2017, implementation began with faculty "innovators" willing to pilot the technology for low-stakes assessments. Pilot results were shared along with a call for volunteers to become "early adopters" for the following year. Coinciding with the launch of our competency driven curriculum in 2018-2019, we entered the "early majority" phase with all required courses using the application. Faculty development was critical to this implementation cycle. Initially, trainings were web-based and focused on key administrators. To support the pilot and "early adopters" focused face-to-face sessions were offered. In the summer 2018, these trainings were expanded for all faculty and administrative staff. The evolution of question tagging was supported by faculty development targeted at the college's assessment committee who were charged with recommending a structure that best supported the curricular assessment plan. Student trainings were also provided. Results: From the pilot year to now, participating courses grew from 3 to 18 and faculty adopters from 3 to 32 (an increase from $6 \%$ to $63 \%$ of all board appointed faculty). Question tagging evolved from tagging to Bloom's taxonomy only, to the addition of programmatic outcomes (measurable abilities), PCOA 


\section{American Journal of Pharmaceutical Education 2019; 83 (5) Article 7654.}

sub-topics, and a few additional key performance indicators (including calculation domains). Implications: Faculty development throughout the implementation of an on-line testing tool lead to widespread acceptance. The data provided by this technology supports the robust assessment plan within our competency-driven curriculum.

Dual-Degree Opportunities in Pharmacy Programs. Dawn E. Havrda, The University of Tennessee. Objective: With the changing job market, student pharmacists want to differentiate themselves and participating in dual-degree programs is an option. The objective of this study was to assess the prevalence and type of dual-degree opportunities in colleges/schools of pharmacy. Methods: Information on dual-degree programs was obtained from pharmacy program websites and cross-referenced with the listed dual-degree offerings in PharmCAS profiles. The number and type of dual-degree programs were correlated to pharmacy program characteristics, including public/private university, presence of an academic health center, when the program was established, and the presence of a branch campus. Results: Out of 141 pharmacy schools, $101(71.6 \%)$ had at least one dual-degree program. Two or more dual-degree options were offered by $62(61.4 \%)$ programs. Dual-MBA was the most frequent $(76.2 \%, n=77)$ followed by a dual-MPH $(41.6 \%, n=42)$. Greater options of dual-degree programs were significantly associated with public universities $(\mathrm{P}=0.002)$, programs established prior to $1995(\mathrm{P}<0.0001)$, an academic health center $(\mathrm{P}<0.0001)$, and the presence of a branch campus $(\mathrm{P}=0.011)$.Public universities were more likely to have the dual-PhD $(\mathrm{P}=0.004)$, and older programs were more likely to have the dual- $\mathrm{PhD}(\mathrm{P}=0.008)$, MBA $(\mathrm{P}=0.004)$, and MPH $(\mathrm{P}=0.038)$. Implications: Almost three-fourths of pharmacy programs have at least one dual-degree program for their student pharmacists. Addition of dual-degree opportunities affords students opportunities to diversity their career path in a changing job market.

Environmental Scan of Opioid-Related Activities in Academic Pharmacy. Dorothy F. Farrell, American Association of Colleges of Pharmacy, Jeffrey O. Ekoma, American Association of Colleges of Pharmacy, Lynette R. Bradley-Baker, American Association of Colleges of Pharmacy, Thomas Maggio, American Association of Colleges of Pharmacy, Jeffrey P. Bratberg, The University of Rhode Island, Andria F. Church, Palm Beach Atlantic University, Sara E. Dugan, Northeast Ohio Medical University, Thomas S. Franko, Wilkes University, Daniel J. Ventricelli, University of the Sciences, Matt Cipriani, American Association of Colleges of Pharmacy, Cynthia P. Koh-Knox Sharp, Purdue University, Jilla Sabeti, Western New England University, Otito F. Iwuchukwu,
Fairleigh Dickinson University, Talia Puzantian, Keck Graduate Institute. Objective: To draw a cohesive picture of the efforts of colleges and schools of pharmacy to address the opioid overdose crisis. Methods: The American Association of Colleges of Pharmacy (AACP) created and disseminated a survey to its institutional members to obtain a detailed report of their opioid overdose crisis-related activities. The initial call for responses was made in July 2018 through the AACP Connect member portal and periodic reminders were sent through September 2018. AACP staff members reviewed submissions and continuously developed and refined overarching categories and descriptive tags to code the submitted activities. A volunteer committee of content experts comprised of members of the AACP Substance Use Disorder Special Interest Group (SIG) helped develop the coding methodology, define categories and tags and followed-up with non-responding colleges and schools. Each activity was independently coded by two separate AACP staff members. Duplicate activities were removed and discrepancies in the coding process were resolved by consensus among AACP staff members and SIG volunteers. Results: Survey responses represented 104 schools and amounted to a total of 462 activities, which reduced to 398 following removal of duplicate and non-opioidfocused submissions. Each activity was categorized as Advocacy (4\%), Education (65\%), Service (23\%), Practice $(8 \%)$ or Research $(22 \%)$ with $20 \%$ of activities assigned to more than one category. A total of 59 tags were defined to capture essential features of each activity. Implications: Academic pharmacy is broadly addressing the opioid overdose crisis in their communities. AACP can support schools by increasing awareness of and support for their efforts among external stakeholders.

Helping Students Manage Stress During Final Exams. Daniel J. Hansen, South Dakota State University, Asha Hertler, South Dakota State University, Nathan Bylander, South Dakota State University. Objective: Evaluate the impact of a series of activities designed to help pharmacy students manage their stress during final exams. Methods: In Fall 2018, student services personnel designed a series of activities centered around helping students manage their stress during final exams. Activities included a midweek mindful study hall, yoga, a Friday finals refresh, and canine comforters. During the midweek mindful study hall and Friday finals refresh, students were given access to additional study space within the pharmacy building, where mindfulness activities (e.g. board games, arts and crafts, coloring) were set-up and snacks were provided. A self-administered questionnaire survey with a 7-point Likert scale was developed to examine student perceptions after participating in the activities. 


\section{American Journal of Pharmaceutical Education 2019; 83 (5) Article 7654.}

Results: Twenty-six P1 and P2 students completed the questionnaire. All of the students (100\%) agreed or strongly agreed with the statements: "I am glad that the College offered this program as part of finals week", "Attending this program helped improve my overall wellbeing", and "If offered, I would attend a similar program in the future." The majority of students agreed or strongly agreed with the statements: "This program will help me manage the stress associated with finals week" and "This program helped me feel better prepared for finals week", $96 \%$ and $65 \%$ respectively. Implications: Student response to this program was positive, and results indicate that it should be continued, and possibly even expanded, in future semesters. This program could be used as a model for other schools of pharmacy looking to provide similar opportunities to their students.

Inclusive Excellence Through a Structured CoCurriculum at Texas A\&M University Rangel College of Pharmacy. Simi Gunaseelan, Texas A\&M University, Asim Abu-Baker, Texas A\&M University, Debra L. Wood, Texas A\&M University, Maria Benavides, Texas A\&M University, Juan J. Bustamante, Texas A\&M University, Indra $\mathrm{K}$. Reddy, Texas A\&M University. Objective: To promote inclusive excellence through a structured co-curriculum. Methods: In accordance with ACPE's guidance on developing a purposeful co-curriculum in PharmD programs, and congruent with the Rangel College of Pharmacy's mission to serve rural health and underserved areas of South and Central Texas, the college has transitioned from a service learning IPPE model to a structured co-curricular program. The goal of this program is to create a culture of excellence towards diversity and inclusion while developing altruistic attitudes and values amongst students. This design entails collegeapproved clinical, interprofessional, non-clinical, and simulation activities. As a measure of excellence in diversity and inclusion, the college has developed extensive community outreach opportunities for targeting the rural, underserved and indigent populations of South and Central Texas. Students are required to complete three co-curricular activities during an academic year, including a required clinical activity. Learning experiences are mapped to CAPE educational outcomes and students' complete reflections at the conclusion of their activities. Results: Since 2017, students have provided health services to approximately 5,760 members of the local and rural communities in the form of health and wellness screenings (4,009 hours), immunization clinics (741 hours), patient education sessions (1,025 hours), and camps for special needs (286 hours). Students have also participated in universitywide annual student-led interprofessional Disaster Day simulations for 538 hours. Implications: Through intentional co-curricular initiatives targeting diversity and inclusion, the college has defined excellence by providing ongoing services in concert with other health professions to underserved, diverse populations in multiple regions of Texas while enhancing students' competencies and professional growth.

Measuring the Overall Wellbeing of Pharmacy Students. Daniel J. Hansen, South Dakota State University, James R. Clem, South Dakota State University, Omathanu P. Perumal, South Dakota State University, Jeremy Daniel, South Dakota State University, Asha Hertler, South Dakota State University, Teresa M. Seefeldt, South Dakota State University. Objective: To measure the overall wellbeing of students in a Pharm.D. program. Methods: As part of the design process of a wellness plan for students enrolled in a Pharm.D. program, college personnel designed a survey aimed to obtain a baseline assessment of their overall wellbeing. The survey asked a variety of quantitative and qualitative questions and was sent via email to all students. A portion of the quantitative questions measured the degree to which students agreed with a series of statements (7-point Likert scale). A chisquare test was used to evaluate differences between the number students who selected an agree-based response versus non-agree amongst the classes (P1-P4). Results: The response rate was relatively strong (Overall $-50 \%$, P1 - 59.8\%, P2 - 47.4\%, P3 - 42.9\%, P4 - 49.4\%). Amongst the fourteen questions analyzed, there was a significant difference in the number of students who agreed with the statement "I feel comfortable approaching college faculty or staff if I am concerned about a classmate's wellbeing" and "I feel comfortable approaching college faculty and staff if I am concerned about a classmate's mental health" (P-Value 0.048 and 0.029 , respectively). In addition, there was a significant difference in how many $\mathrm{P} 1$ and $\mathrm{P} 2$ students agreed with the statement "I would attend counseling myself" versus P3 and P4 students (P-Value 0.006), with P3 and P4 students being more likely to agree with the statement. Implications: The results of this baseline survey will help inform college personnel as they develop an overall wellness program for students in the Pharm.D. program.

Medina's Key Elements of Active Learning as Framework for Faculty Self and Needs Assessment of Preparedness. Elaine L. Demps, Texas A\&M University, Charlotte A. Farris, Texas A\&M University, Merlyn Joseph, Texas A\&M University, Mardoqueo Martinez, Texas A\&M University, Jose Hernandez, Texas A\&M University. Objective: Active learning (AL) is continually being emphasized and implemented in the pharmacy curriculum. The objective of this study was to conduct a faculty self and needs assessment to determine the extent 


\section{American Journal of Pharmaceutical Education 2019; 83 (5) Article 7654.}

to which our faculty perceived they already carried out $\mathrm{AL}$ and to gather their perceptions on how prepared they felt to implement effective AL across the P1 curriculum. Methods: A 19-question survey was administered in July 2017 to all 50 college faculty. For validity, when designing the survey, Medina's set of four key elements of effective AL was adopted as the theoretical framework. For reliability, the draft survey questions were reviewed multiple times and revised accordingly. Texas A\&M University Institutional Review Board determined this study did not involve human subjects. Results: Thirty-five faculty members, $70 \%$, completed the survey in full. Majority of the faculty perceived that they carried out three of the four key elements during lectures: activate prior knowledge (74\%), involve majority of students $(89 \%)$, and provide feedback (91\%). However, only $37 \%$ stated they promoted metacognition, another key element. Moreover, based on the analyses of the qualitative responses, faculty appeared to have misconceptions about what activities constitute the key AL elements. Barriers to incorporating AL that emerged as themes include: lack of student buy-in and lack of faculty know-how. Implications: Although most faculty indicated they integrated AL in their teaching, misconceptions identified indicates the need for extensive faculty development to define and model key elements of AL. Student development on the benefits of $\mathrm{AL}$ is also needed.

Novel Design of an Efficient Process for Meaningful Student Reflections and Feedback. Lisa Finn, University of Maryland, Lisa Lebovitz, University of Maryland. Objective: To devise an efficient and effective process to develop students' affective domains that meets accreditation standards and elicits impactful data, without overburdening students or faculty. Methods: Pharmacy students are required to submit a Plan of Study (PoS) each semester. A faculty advisor then reviews the PoS and provides feedback and approval. The PoS includes quantitative and qualitative questions on career preparedness, involvement and leadership in organizations, stress level and workload. Students are also prompted to briefly reflect on meaningful recent experiences related to problem solving, communication, patient advocacy, interprofessional teamwork, leadership and professionalism. Finally, students develop future goals and action steps. Results: Students develop self-awareness and habits of lifelong learning. Faculty advisors meet regularly in small groups and individually to review their advisees' experiences, stress, workload, career prep, and involvement; any student in crisis can be referred for intervention and support. Administrators monitor these class cohort data, and use it to demonstrate compliance with ACPE Standards 3 and 4 . Findings include: $88 \%$ of P1-P3 students belong to at least one organization and almost half belong to 3 or more; one-third hold leadership positions. Over $50 \%$ of $\mathrm{P} 3 \mathrm{~s}$ attended career planning events last spring; $25 \%$ of P1s reported unmanageably high stress levels. Implications: This approach is an efficient use of time for both students and faculty compared to requiring formal reflective assignments, which can be a disincentive to student participation. Faculty are able to focus on engaging in their advisees' progress and personal growth instead of grading them.

Participant Perceptions of the Aggie Student Pharmacists Initiative for Recruitment|Retention and Education (ASPIR2E) Pre-Matriculation Program. Elaine L. Demps, Texas A\&M University, Amanda Galindo, Texas A\&M University, Amanda Galvan, Texas A\&M University, Simi Gunaseelan, Texas A\&M University. Objective: Once recruited, retaining a diverse student body is instrumental in enhancing the learning experience for all students as well as achieving racial/ ethnic concordance between the future pharmacist and his/her patients for improved patient care outcomes. The ASPIR2E Pre-Matriculation Program (PMP) in summer 2018 was a 6-week, face-to-face and online, grantfunded initiative to retain underrepresented minority (URM) students. The objectives of this study were to understand the student perceptions of the PMP: motivations for applying; the program's effectiveness in enhancing the participants' transition to the P1 year; and areas of improvement. A separate study for determining the academic effectiveness of the PMP was conducted. Methods: Twenty-four Class of 2022 members were selected to participate. Week 1 was on campus with housing and food. Weeks 2-5 were online. Week 6 was on campus but without housing. Participants were guided by-and interacted with-faculty, staff, and peer mentors. Student perceptions were collected through pre/post PMP surveys and pre/post PMP focus groups. Texas A\&M Institutional Review Board determined this study was not human subjects research. Results: Twenty-three participants completed the program. The most common theme for applying was to be successful in the PharmD curriculum; the PMP was viewed as an effective vehicle. Peer relationships formed was the most important factor in transitioning well to the first year. Suggested areas of improvement included content scope/emphases and instructional strategies employed. Implications: The PMP participants matriculated with a strong sense of belonging and an established network of peers, peer mentors, and facultysocial factors that enhance retention.

Prevalence of Depression and Anxiety Symptoms Among Student Pharmacists. Andrew Smith, Samford University, Renee M. DeHart, Samford University, 


\section{American Journal of Pharmaceutical Education 2019; 83 (5) Article 7654.}

Rachel M. Slaton, Samford University, Katherine Smith, Samford University. Objective: The primary objective of this study was to quantify the presence of depression and anxiety symptoms among first through fourth year student pharmacists. Secondary objectives were to identify student subsets who reported these symptoms most and quantify use of university counseling services. Methods: This was a single center cross-sectional study among 474 students. After obtaining Institutional Review Board approval, the Patient Health Questionnaire-9 (PHQ-9, for depression) and the General Anxiety Disorder-7 scale were administered via an anonymous electronic survey over a three-week period in fall 2018. Results: Two hundred fifty-five students completed the study $(53.8 \%$ response rate). The majority were white females between 22 and 25 years of age. Of responders, $40 \%$ had symptoms of at least moderate depression and $41 \%$ had symptoms of at least moderate anxiety. Female students reported statistically more anxiety than males. Other demographics examined (age, race, having children) were not associated with differences in depression or anxiety symptoms. Overall use of university counseling services was very low among these students. Implications: Whether it be due to counselor availability, campus geography, or general reluctance to seek help, use of our university counseling center was infrequent relative to student reported symptoms. Further steps need to be taken to ensure the mental health of our future pharmacists. As a result of this study, several new mental health initiatives have been undertaken within the program, including suicide awareness training for faculty and optional weekly small group discussions for students within the school, among others.

Student Professional Development Program's Effects on Student Self-Efficacy in Learning. Russell $\mathrm{T}$. Attridge, University of the Incarnate Word, Bethany Kalich, University of the Incarnate Word. Objective: Evaluate a professional development program's effect on student self-efficacy in learning. Methods: A structured educational session on evidence-based strategies for effective learning (i.e., spaced practice, interleaving, retrieval practice, elaboration, concrete examples, dual coding) was provided to second-year student pharmacists as a part of a professional development program in fall 2018. The Student Self-Efficacy (SSE) Scale, a validated, 10item, 4-point Likert scale survey, was used to assess SSE pre- and post-intervention in four domains: academic performance, skill and knowledge development, social interaction with faculty, and coping with academic stress. Matched responses were assessed using two-sided paired t-tests. Results: Among 71 student pharmacists surveyed, $65(91.5 \%)$ completed the survey and consented to study inclusion. Post-semester (vs. pre-) surveys demonstrated numerical declines in SSE scores for all 10 items, indicating a decline in learning confidence. Statistically significant declines were noted for learning capability over time (3.67 vs 3.31, p $<0.001$ ), efforts to achieve academic goals ( 3.69 vs $3.49, \mathrm{p}=0.008)$, staying motivated ( $3.28 \mathrm{vs}$ $3.06, p=0.047)$ and ability to be successful despite doubts by others (3.62 vs 3.40, p=0.034). Implications: Pharmacy programs are expected to facilitate student personal and professional growth. An educational session on cognitive learning strategies did not appear to facilitate improvements in student self-efficacy in learning. Unaccounted confounders may have affected our results, including not accounting for the natural progression in course difficulty and not evaluating student application of learning strategies. Continued efforts to evaluate and improve student self-efficacy in learning are being developed as a result of these data.

Student Professional Development Program's Effects on Student Self-Efficacy in Receiving Feedback. Russell T. Attridge, University of the Incarnate Word, Bethany Kalich, University of the Incarnate Word. Objective: Evaluate a professional development program's effect on student self-efficacy in receiving feedback. Methods: A structured educational session on feedback, followed by increased efforts to strengthen feedback quality, was provided to second-year student pharmacists as a part of a professional development program in fall 2018. The Feedback Orientation Scale (FOS), a validated, 20-item, 5-point Likert scale was administered pre- and post-semester to assess self-efficacy in feedback among four domains: utility, accountability, social awareness and feedback self-efficacy. Matched responses were assessed using two-sided paired t-tests. Results: Among 68 student pharmacists surveyed, 65 (95.6\%) completed the survey and consented to study inclusion. Post-semester (vs. pre-) surveys demonstrated numeric improvement in 15 of $20(75 \%)$ items related to feedback self-efficacy in the FOS. Statistically significant improvements were noted in the utility and accountability domains, including increased agreement with "feedback contributes to my success" (4.48 vs $4.28, p=0.047)$ and "it is my responsibility to apply feedback to improve my performance" ( 4.55 vs 4.32 , $\mathrm{p}=0.006$ ). No responses on the FOS postsurvey had statistically-significant decreases in student self-efficacy. Implications: Pharmacy programs are expected to facilitate student personal and professional growth necessary to demonstrate self-awareness, leadership, innovation, entrepreneurship and professionalism. This professional development activity led to modest improvements in self-efficacy related to receiving feedback in some aspects of the utility and accountability domains. We believe the faculty and student development efforts 


\section{American Journal of Pharmaceutical Education 2019; 83 (5) Article 7654.}

related to this may improve student outcomes beyond the scope of this project, and additional efforts deserve exploration to facilitate widespread development among each feedback domain.

The Impact of an Individualized Early Intervention System on Student Learning in a CompetencyDriven Curriculum. Laura A. Mandos, University of the Sciences, Suzanne Carbonaro, University of the Sciences, Karen J. Tietze, University of the Sciences, Lisa Charneski, University of the Sciences. Objective: To describe the implementation of an early intervention system and its impact on student achievement and progression in a competency-driven curriculum Methods: In conjunction with a new, competency-driven doctor of pharmacy curriculum implemented in Fall 2018, the Philadelphia College of Pharmacy at University of the Sciences designed a Learning Support program to provide a structured process for student referrals and intervention. Based on research of existing medical school programs, Learning Support is designed to develop competencies and promote student success. Relying on early intervention and individualized assessments, the program utilizes actionable data, academic coaching, and faculty mentor teams to identify and address the needs of students in, or trending toward, academic difficulty. Learning Support triggers when students receive $<70 \%$ on major assessments within a module. Additionally, a formal process was designed to support students who failed to meet progression standards at the conclusion of a module. The faculty/ student referral process supports students with non-grade related issues, such as professionalism. Results: Fall 2018 data reveal (1) an increase in the proportion of P1 students on the Dean's List; (2) decrease in the proportion of $\mathrm{P} 1$ students failing to progress to the spring semester; and (3) decrease in the proportion of $\mathrm{P} 1$ students entering program probation when compared to cohorts from the previous 5 years. Implications: Learning Support advantages include assisting students with issues early and in real-time. More students are able to stay with their cohort and progress to the next semester. However, the program does require designated resources, coordination with stakeholders, and specific timelines.

Understanding the Motivators of Pharmacy Students Pursuing a Pathway to Rural Pharmacy Practice. Stephanie N. Kiser, University of North Carolina at Chapel Hill, Antonio Bush, University of North Carolina at Chapel Hill, Minnie McMillian, University of North Carolina at Chapel Hill, Anna Gross, University of North Carolina at Chapel Hill, Dzidzai Muyengwa, University of North Carolina at Chapel Hill. Objective: It is well understood that rural communities experience health provider shortages, and most literature addressing this workforce need focuses on physicians. To broaden understanding of recruitment and retention efforts for future rural pharmacists, this study explores perceptions and key motivators of pharmacy students who have chosen to participate in a Rural Pharmacy Health Certificate (RPHC) program and pursue pathways to rural practice. Methods: Nineteen newly admitted RPHC students participated in 30-minute research interviews structured with key questions concerning motivation to pursue the program, RPHC's impact on personal and professional goals, health disparities knowledge in rural settings, and perceived qualities needed to be a successful pharmacist in a rural setting. Interviews were transcribed verbatim. Multiple coders used several rounds of open coding to develop a codebook and identify themes. Trustworthiness procedures included consensus building and analytic memos to promote reflexivity. Results: Several themes were identified: Students' growing up and volunteering and/or working in a rural community led them to a "heart for service" and pursuing the RPHC. Students expressed knowledge of two distinct disparities in rural areas: patients' access to care and health literacy. Students perceived key qualities of a successful rural pharmacist were being a learner of and invested in the community. Implications: Many schools of pharmacy are making efforts to address workforce needs in rural settings and improve the health of rural communities. The data collected in this study could influence the design of recruitment and admission efforts to align with student motivators and be incorporated into curricular considerations for rural practice.

Using a Modified Delphi Technique to Determine Faculty Priorities and Facilitate Faculty Satisfaction. Catherine J. Cone, Roseman University of Health Sciences, Elizabeth J. Unni, Roseman University of Health Sciences. Objective: The objective of this study is to describe a qualitative, iterative approach using a modified Delphi technique to achieve consensus on faculty priorities that facilitate faculty satisfaction and uncover areas of pride that recognize achievement and promote faculty morale. Methods: The Roseman University College of Pharmacy (RUCOP) Assessment Committee used a modified Delphi technique to gather information and form consensus regarding facilitators of faculty satisfaction. Round 1 of the Delphi technique used an open-ended questionnaire asking faculty to list three priorities to improve faculty satisfaction and one source of pride. The results from this round were analyzed qualitatively to generate themes. Round 2 of the Delphi technique required faculty to rank order the themes to establish priorities. Round 3 was an open forum for faculty discussion to achieve final consensus. Results: Seventy-eight percent 


\section{American Journal of Pharmaceutical Education 2019; 83 (5) Article 7654.}

of the faculty participated in Round 1 and $83 \%$ participated in Round 2. By Round 3, faculty were able to reach a consensus for three priorities for RUCOP and three for the University. Priorities identified for RUCOP included workload, recruitment and professional development. Priorities for the University included salary, benefits and money for professional development. Implications: The Delphi Technique promoted proactive engagement from the faculty to determine their priorities for job satisfaction and establish goals for pharmacy administration. While providing anonymity, the holistic process allowed faculty to rank priorities from all respondents to achieve final consensus, in addition to giving them an opportunity to self-reflect on their work environment.

Using Signature Projects, Co-Curricular Transcripts, and Reflective Journal Writing to Track Progress to Programmatic Outcomes. Jeremy A. Hughes, California Health Sciences University, David G. Fuentes, California Health Sciences University, Ahmd Azab, California Health Sciences University, Will Ofstad, California Health Sciences University, Miriam Ansong, California Health Sciences University, Macie Luallen, California Health Sciences University. Objective: To develop and implement a centralized method of documenting and assessing student participation in co-curricular activities, interprofessional education (IPE), as well as to track student development in professionalism and affective domains. Methods: Development of a portfolio system was initiated in response to a need for improved documentation of student activities and progress towards meeting global learning outcomes (GLOs). Project aims were to intentionally document IPE and co-curricular activities and to assess student development in GLOs and affective domains across the program. Students, faculty mentors, and other stakeholders were identified, and desired outcomes were determined for each group. A literature search was done to aid in developing a program definition of co-curriculum and in developing a categorical system of activity types, as well as to identify systems for reflective journal writing (RJW).Platforms, program operations, and assessment methods evaluated. Results: Student portfolios were developed and implemented that include signature projects, discussion boards, studentlogged co-curricular events, and RJW. The PIE-RECAP model was utilized to capture student self-reflections, which were linked to ACPE Standards 1 through 4. Information is housed in Learning Management Software, and available to students and faculty for mentorship discussions. The process was implemented simultaneously across cohorts with trainings given during Orientation and mid-semester and a debriefing after assignment completion. Implications: Professionalism blogs, RJW, co- curricular transcripts, discussion boards, and signature projects can be used to assess student growth in GLOs and CAPEDomains, and can be used by to measure student participation in IPE and co-curricular activities.

Will Increased Co-Curricular Activity Lead to Stronger Professional Engagement? Benjamin D. Aronson, Ohio Northern University, Kelly M. Shields, Ohio Northern University, Kristin K. Janke, University of Minnesota. Objective: To explore the relationship between co-curricular experiences and professional engagement. Methods: Students enrolled in a 0-6 direct-entry PharmD program completed a survey assessing cocurricular experiences and professional engagement. Co-curricular experiences included student organizations (school-related and professional), leadership experiences, "high impact practices" (i.e., study abroad, research), and pharmacy employment. Professional engagement was measured using a modified Student Pharmacist Inventory of Professional Engagement (S-PIPE). Mann-Whitney U tests were used to compare engagement across experiences. Results: A total of 669 students participated (92.7\% response rate). Students with pharmacy work experience had significantly higher professional engagement than those without $(\mathrm{p}<.001)$. No differences in engagement were detected across those with leadership experience or participating in high impact practices. Out of 31 possible types of organizations, differences in engagement were only found for five. Students participating in university-wide service organizations $(p=.017)$, college committees $(p=.029)$, pharmacy student governance $(\mathrm{p}=.005)$, Phi Lambda Sigma $(\mathrm{p}=.016)$, and the National Community Pharmacists Association $(\mathrm{p}<.001)$ had higher engagement scores compared to those who were not. Implications: In this study, organizational membership and specific activities were not broadly associated with higher professional engagement. Consistent with prior literature, these findings indicate that not all cocurricular experiences will automatically lead to engagement. However, experiences focused on serving others (e.g. community, college, profession) and connecting with the profession (i.e., pharmacy employment) were associated with higher engagement. In creating engagement, the characteristics of the co-curricular activities appear to matter.

\section{Theoretical Models}

After the Crisis is Managed: Exploring the Wellbeing of Student and Academic Affairs Leadership. Cherokee Layson-Wolf, University of Maryland, Shannon R. Tucker, University of Maryland, Andrew Coop, University of Maryland. Objective: Be it academic performance, allegations of cheating, professionalism, mental health, Title IX, probation, or dismissal, there is always 


\section{American Journal of Pharmaceutical Education 2019; 83 (5) Article 7654.}

the prospect of a difficult discussion in the life of student and academic affairs leadership. But how do we support new and existing leadership responsible for these difficult discussions with sometimes long-lasting consequences? This case study examines the informal and formal organizational structures and wellness practices that support new and experienced leaders who are responsible for handling crisis on a day-to-day basis. Methods: This case study explores and contrasts the experience of Student Affairs and Academic Affairs Associate Deans at the University of Maryland identifying practices and support structures that support wellbeing and prevent burnout in critical leadership positions. This retrospective exploration of the leadership transition process includes key factors that would have improved onboarding was reviewed. Results: The major theme of dealing with difficult student issues in student and academic affairs is open communication. The associate deans of both offices are able to accomplish this due to their physical location and regular check-ins. Prior working experience as faculty assisted with transitions of new leadership, and onboarding training during transitions was key in readiness for responsibilities. Implications: We believe that the close working relationship between the leaders in the academic affairs and student affairs office has been key in handling student issues efficiently. An evaluation of how well these two offices work together in other institutions may be warranted to determine areas of strengths and improvement.

Creating a Legacy of Student Success in a Team Based Learning Curriculum. David B. Romerill, The University of Texas at Tyler, Bradley J. Brazill, The University of Texas at Tyler, Rahmat M. Talukder, The University of Texas at Tyler, Kathleen A. Snella, The University of Texas at Tyler, Lane J. Brunner, The University of Texas at Tyler. Objective: The program was developed to promote student retention through academic success and professional engagement. Methods: The Offices of Student Affairs and Academic Affairs collaborated with the Departments of Pharmaceutical Sciences and Clinical Sciences to each target one of 4 key domains across the curriculum in order to promote student success: study and communications skills (Student Affairs), self-assessment of progress in knowledge acquisition (Academic Affairs), and development of writing (Clinical Sciences) and math skills (Pharmaceutical Sciences) to improve academic performance within the PharmD curriculum. The domains were chosen to foster individual student confidence functioning within a team to solve problems, to resolve interpersonal conflicts, and to take responsibility for personal development through self-assessment and reflection. Results: The class of 2023 will be the first to participate in pre-matriculation programming (establishing baseline knowledge deficits, developing study and communications skills). Self-assessment of knowledge acquisition and development of writing and math skills were introduced to current students during the Spring 2019 semester. Progressive communication skill development is planned for successive semesters of the didactic curriculum. Comparisons of student retention, academic performance indicators, with student, faculty and preceptor surveys will be used to report effects. Implications: Providing appropriate tools from the outset to promote success in a team-based learning environment will build student confidence that, with time and reinforcement, will increase engagement and retention.

Describing an Admissions Session Model and Activity that Sets a Psychological Contract. David G. Fuentes, California Health Sciences University, Jeremy Hughes, California Health Sciences University, Yuqin $\mathrm{Hu}$, California Health Sciences University, Lianjie Xiong, California Health Sciences University, Macie Luallen, California Health Sciences University, Leslie Williams, California Health Sciences University, Hope Lemons, California Health Sciences University, Jeanette Munn, California Health Sciences University. Objective: Students applying to pharmacy school need to start thinking about joining an organization for the next few years of their lives. Students need to know their own values and consider their educational environment and specific wants and needs as a learner. Herein, we describe a model and activity that primes students to experience our curriculum, and how they will be learning in our program, all on the day of their admissions interview. Methods: Faculty members facilitate sessions on headache and acute coronary syndrome pharmacotherapy and walk students through the Michaelson Team-Based Learning (TBL) model during admissions days. Activities include an individual and team readiness assessment, and various examples of the active learning strategies incorporated in our teaching across the curriculum. Results: PharmD Candidate applicants $(n=93)$ were all able to engage in a team-based learning activity and it helped them understand and experience the program in its most authentic form to ensure that students' expectations are aligned with TBL methodology and that a psychological contract is made more explicit to our pharmacy school applicants. Implications: Having an authentic approach to admissions and sharing with students the reality of our program's structure can help students prepare for, and transition into, our curriculum. Applicant to deposit conversion rates have increased to $77 \%$ from $56 \%$ before the TBL session was added. Other programs may be inspired by this model to highlight their unique differentiators and 


\section{American Journal of Pharmaceutical Education 2019; 83 (5) Article 7654.}

present them to students as they seek to draw in students who fit most in their organizational culture.

Development, Implementation and Assessment of a Co-Curriculum in a New Accelerated Pharmacy Program. Sandra B. Caballero, Larkin University, Lynne Arric, Larkin University, Kathleen Jodoin, Larkin University, Kenneth C. Jackson, Larkin University. Objective: Larkin University College of Pharmacy developed and implemented a comprehensive co-curriculum program to promote the development of a more holistic pharmacist practitioner. The development, implementation, and assessment of the co-curriculum is described. Methods: The co-curriculum is monitored through Professional Development Units (PDUs), which are housed in an electronic portfolio. Students are first introduced to the PDUs during their first course in the program. Students complete a career pathway assessment, 3-5 life-long SMART goals, and a leadership inventory. With that information, students develop 3-5 life-long professional goals, and an action plan for areas of professional development. In accordance with the action plan, students selfidentify PDU activities and program learning outcomes (PLOs) that support personal and professional development. Upon completion of the PDU, students complete a reflection to identify how the activity relates to professional goals. This reflection is reviewed by their faculty advisor. Results: The co-curriculum was completed by the inaugural class during the first two years of the program. Assessment of the co-curriculum identified the need to create activities related to interprofessional collaboration and innovation/entrepreneurship. The assessment of the co-curriculum also identified the need for improved documentation and training of faculty and students on mapping to appropriate PLOs. Implications: The co-curriculum established at Larkin provides strategies for consideration by other programs related to implementation including approaches for assessment to identify areas for improvement.

Early Identification and Intervention of Students Who Struggle with Verbal and Written Literacy. Merideth Hoagland, Cedarville University, Jeffrey A. Bates, Cedarville University, Nathanael Smith, Cedarville University, Aleda M. Chen, Cedarville University, Rachel Stevenson, Cedarville University. Objective: Some students may face challenges with graduate-level reading comprehension and writing skills, particularly in certain active learning pedagogies such as team-based learning or peer instruction that require significant prereading. The objective of this study is to determine the perceived utility of a reading/writing test and subsequent intervention for first professional year student pharmacists (P1s). Methods: An academic literacy test (speaking/ writing) was developed using fall P1 materials with a linguistics faculty member with expertise in literacy assessment. After pilot testing and adjustments with non-P1 students, the final test was administered to all P1 students by trained facilitators. Students at risk were identified, met with individually to debrief on the assessment, and were offered a year-long, one-on-one tutoring program with a trained staff member. P1 faculty participated in an end-of-semester focus group session with pre-specified guiding questions on whether the assessment correctly identified students with literacy issues and the impact of the intervention. Content analysis was performed on the data. Results: A total of 13 students were identified as atrisk through the assessment. Since tutoring was optional, 8 students met at least once and 2 students met weekly. Faculty from the focus group 1) stated that the assessment accurately identified students who struggled with literacy components and 2) expressed a wish for earlier identification of students with required instead of optional tutoring. Implications: Faculty perceived the tool accurately identified students, but the timing and the volunteer nature of the tutoring limited the success of the intervention.

Structured Lunchtime Co-Curricular Programming to Improve Student Progress Towards Affective Domains and Global Learning Outcomes. Jeremy A. Hughes, California Health Sciences University, Macie Luallen, California Health Sciences University, David G. Fuentes, California Health Sciences University, Ahmd Azab, California Health Sciences University, Will Ofstad, California Health Sciences University, Miriam Ansong, California Health Sciences University, Jonathan Velez, California Health Sciences University, Wendy Duncan, California Health Sciences University. Objective: To develop and implement a structured program that allows students to participate in a robust and varied cocurriculum during the traditional school day of 9-5. Methods: Development of a lunch time co-curriculum was initiated in response to the need for additional structured and unstructured co-curricular activities in concert with existing course schedules. Project aims were to schedule and structure programming to enhance student progress towards global learning outcomes and to kindle growth in students' affective domains. Students, faculty, the Registrar, and other stakeholders were identified, and desired outcomes were determined for each group. A literature search was done to aid in developing core cocurricular elements that should be included. Platforms, program operations, and assessment methods were evaluated. Results: A lunch time co-curriculum program was developed and implemented by the office of student affairs, across all cohorts simultaneously. Overviews of the co-curriculum were presented during Orientation and 


\section{American Journal of Pharmaceutical Education 2019; 83 (5) Article 7654.}

program updates. Required elements included workshops on professionalism, ethical decision making, moral agency, APPE readiness, and cultural competency training. Optional sessions included a weekly speaker series, dedicated time for student organizations, and career development programming. Structure and content were provided by creating co-curricular portals within a learning management system and an e-portfolio was developed to capture student self-reflections, which were linked to ACPE Standards 1 through 4. Implications: A structured lunch time co-curriculum can be utilized to enhance students' development in CAPE Outcomes, affective domains, and growth towards Global Learning Outcomes.

\section{BIOLOGICAL SCIENCES \\ Completed Research}

A New HPLC Method for the Determination of Levodopa in Human Plasma; a Human Pharmacokinetics Study. Fawzy A. Elbarbry, Pacific University Oregon, Van Nguyen, Pacific University, Alan Mirka, Pacific University, Heather Zwickey, National University of Natural Medicine, Richard Rosenbaum, Legacy Health. Objective: The purpose of this study is to develop and validatea simple, inexpensive, sensitive, and accurate HPLC-UV method for the quantification of L-Dopa in low volumes of human plasma. Moreover, the method is applied to study the pharmacokinetics of L-Dopa in Parkinson's patients and to investigate the potential effect of ketogenic diet on L-Dopa's pharmacokinetics. Methods: The method involves a simple protein precipitation procedure to extract both L-Dopa and methyldopa, the internal standard. The chromatographic analysis was achieved on a Shimadzu LC 20A HPLC system equipped with a Zorbax Eclipse XDB C18 column and an isocratic mobile phase consisted of $20 \mathrm{mM} \mathrm{KH} 2 \mathrm{PO} 4(\mathrm{pH} 2.5)$ and methanol HPLC grade $(95: 5, \mathrm{v} / \mathrm{v})$ run at a flow rate of 1 $\mathrm{ml} / \mathrm{min}$ for 10 minutes. The UV detection wavelength was set at $30 \mathrm{~nm}$. Results: The method exhibited good linearity $(\mathrm{R} 2>0.999)$ over the assayed concentration range $(0.1-10 \mu \mathrm{g} / \mathrm{mL})$ and demonstrated good intra-day and inter-day precision and accuracy (relative standard deviations and the deviation from predicted values were less than $15 \%$ ). This method was also successfully applied for studying the potential effect of ketogenic diet on the pharmacokinetics of L-Dopa in Parkinson's patients. Implications: Our data analysis indicates that ketogenic diet does not significantly affect the pharmacokinetics of L-Dopa

A Qualitative Study of Evaluating Pharmacy Faculty Perceptions on Empathy in Education. Yuan Zhao, Sullivan University, Lynn Z. Fuller, Sullivan University, Kimberly K. Daugherty, Sullivan University. Objective: Faculty attitude and demeanor in the classroom could have a profound effect on students. Currently, there have been no studies undertaken to evaluate the empathetic capacity of faculty, especially those who teach in health care professional programs. Our study aimed to evaluate pharmacy faculty perceptions on empathy in an educational setting. Methods: A qualitative research approach was utilized for this study. Semi-structured interviews were conducted for ten faculty members randomly selected from the Pharmaceutical Sciences Department and the Clinical and Administrative Science Department. All interviews were transcribed, and an interpretive phenomenological approach was used to describe, code, and analyze the experiences. Emergent themes from all data sources informed the findings about the faculty perceptions on the role of empathy in education. Results: Faculty had a consensus on the role of empathy in education and believed it is important for building relationships with students and can enhance student learning. Most faculty believed that while empathy is an innate ability, it can, however, be developed through life experiences and training. Major challenges of being empathetic in an educational environment include knowing when to draw a line between empathy and fairness to all students, holding all students to the same educational standards, and dealing with time constraints. Implications: Empathy is a critical skill and characteristic for health care profession educators to have. Based on the results, empathy training modules may be helpful to improve faculty's empathy level.

Biological Therapy in Immune-Mediated Inflammatory Diseases: A Comparative Analysis and Market Trend Forecast. Chi Phan, Roseman University of Health Sciences, Manas Mandal, Roseman University of Health Sciences. Objective: To determine the current market value and projected potential market share of TNF- $\alpha$-targeting biologicals in autoimmune disease(s) affecting US and global population. Methods: Immunemediated inflammatory diseases (IMIDs) are characterized by over-expressed inflammatory cytokines. Incidences of rheumatoid arthritis (RA) in US and globally, total market and comparative value of Tumor necrosis factor alpha (TNF- $\alpha$ )-targeting biologicals' with predictive future market trends were analyzed. Patients, cost of treatment and market value/share of each biological were calculated using available/ published data. Regression analysis and time series forecasting methods projected the potential and continuous market value based on Python Platform. Compound annual growth rate (CAGR) with future projection was calculated based on market reports. Results: RA affects $\sim 1 \%$ of the global and $\sim 0.6 \%$ of the US adult population with a predictive increase in patients globally and in US. TNF- $\alpha$-targeting biologicals Humira ${ }^{\circledR}$, Enbrel $^{\circledR}$ and Remicade ${ }^{\circledR}$ are the 


\section{American Journal of Pharmaceutical Education 2019; 83 (5) Article 7654.}

major drugs with Humira ${ }^{\circledR}$ enjoying the largest market share (45.6\% in 2017). Globally, total anti-TNF- $\alpha$ drug sales for all inflammatory diseases increased consistently between years 2013-2017 with continued predictive increase thru 2025. CAGR of global and US TNF- $\alpha$ inhibitor market was $\sim 6.4 \%$ (years 2013-2017), with a predictive CAGR of 7.1\% globally (years 2018-2023). Implications: Biological drug treatment for IMIDs are increasing with TNF- $\alpha$ targeting Humira ${ }^{\circledR}$ capturing the largest market share with predicted further future growth. A comprehensive analysis of patient information, cost of treatment, current market share and future trend of the TNF- $\alpha$-targeting biologicals may help formulate policies and also design a biological therapeutics course for interdisciplinary education.

Bridging the Great Divide: Survey of Activities That Orient Non-Pharmacist Faculty to the Pharmacy Profession. Sigrid C. Roberts, Pacific University Oregon, Kimberly K. Daugherty, Sullivan University, Margarita V. DiVall, Northeastern University, Jennifer Lamberts, Ferris State University, Jeremy W. Vandiver, University of Wyoming, Lisa Lebovitz, University of Maryland. Objective: To collect information on activities geared to orient non-practice faculty to the pharmacy profession, and to determine the outcomes of such activities and individual perceptions of their importance. Methods: We deployed an IRB-exempt survey through the AACP Connect Council of Deans and Council of Faculty communities. Respondents were asked to describe the importance and primary purpose of activities, identify outcomes, rank effectiveness, and provide examples. Rankings, selections from drop-down menus, and comment-based questions were administered. Results: Total respondents were 158 individuals from 90 colleges and schools of pharmacy; $61 \%$ represented the pharmacy practice discipline and 54\% held no administrative role. The majority ( $82 \%)$ reported that it was extremely or very important to orient non-practice faculty to the profession. More than half $(52 \%)$ reported that the primary reason to support this orientation was "to improve effectiveness of PharmD students' ability to apply central principles of science to practice," and 92\% felt that fostering collegial relationships within and across academic units, disciplines, and professions was at least moderately important. Various intentional activities were reported as fostering this orientation process, but only $17 \%$ rated these collective activities as extremely or very effective. Many $(35 \%)$ noted lack of time as the biggest barrier, while smaller numbers (15\% each) noted perceptions of silos/adversarial relationships or lack of personal interest. Respondents suggested potential solutions including toolkits, integrated team-teaching efforts, and shadowing. Implications:
Formalized activities, collegial attitudes, and leadership support are necessary to instill a spirit of collaboration amongst faculty from diverse educational backgrounds.

Chemoprevention of Prostate Cancer in TRAMP Mice by Alpha-Santalol. Ajay K. Bommareddy, Wilkes University, John Oberlin, Wilkes University, Kaitlyn Blankenhorn, Wilkes University, Aaron Knopp, Wilkes University, Sarah Hughes, Wilkes University, Erica Mabry, Wilkes University, Isiah Pinkerton, Wilkes University, Linda Gutierrez, Wilkes University, Adam VanWert, Wilkes University, Chandradhar Dwivedi, South Dakota State University. Objective: Investigate the in vivo efficacy of $\alpha$-santalol using TRAMP (Transgenic Adenocarcinoma of Mouse Prostate) mice as a model. Methods: Animal study approved by institutional IACUC committee was carried out according to standards set by the committee. Two groups of 9 mice/group were injected i.p either $100 \mathrm{mg} / \mathrm{kg}$ of alpha-santalol in $5 \%$ Tween-80 saline solution (vehicle) or vehicle (control group). Starting at 6 weeks, mice were injected for 20 weeks (26 weeks final age) and sacrificed under $\mathrm{CO} 2$ asphyxiation. Tumor/prostate samples collected were analyzed employing Immunohistochemical and Western blotting analysis. Statistical analysis was performed using Graph Pad Prizm software. Results: Analysis of tumor/ prostate samples revealed a statistically significant decrease in tumor incidence $(11.1 \%$ vs $55.5 \%)$, average wet weights of urogenital organs $(0.31 \pm 0.05$ vs. $1.28 \pm 0.42 \mathrm{~g})$ and prostate weight $(0.13 \pm 0.03 \mathrm{vs}$ $0.28 \pm 0.10 \mathrm{~g})$ in alpha-santalol-treated mice compared to control mice. The dorsolateral sections of prostate from $\alpha$-santalol-treated mice exhibited decreased cell proliferation (Ki-67 staining) in association with induction of apoptosis (TUNEL-positive cells). Western blotting analysis of prostate/tumor samples from $\alpha$-santalol-treated group revealed a decrease in surviving, XIAP, PCNA, cyclin D and CDC2 levels compared to control samples. c-RAF, a proto-oncogene was also down regulated in alpha-santalol treated group compared to the control group. Implications: The study revealed chemopreventive potential of $\alpha$-santalol against development of prostate cancer in TRAMP mice by decreasing cell proliferation, and inducing apoptosis and warrants future studies for its clinical development.

Comparing Student and Preceptor Perceptions of Advanced Pharmacy Practice Experience Readiness and Performance. K. Paige D. Brown, Campbell University, Myrah Stockdale, Campbell University, Timothy J. Bloom, Shenandoah University. Objective: A sequential mixed methods design (QUAN $>$ qual) was used to explore differences in student and preceptor perceptions of expectations and performance on first 


\section{American Journal of Pharmaceutical Education 2019; 83 (5) Article 7654.}

Advanced Pharmacy Practice Experience (APPE) rotations. Methods: Quantitative data were collected using parallel anonymous surveys to preceptors and students following the first APPE rotation in 2017 and 2018. Each survey had Likert-type items centered on the themes of patient care, professionalism, communication, cultural diversity, and foundational knowledge. Student and preceptor survey responses from both years were pooled and analyzed using a comparative analytical approach. To boost credibility, confirmability, and dependability (Lincoln and Guba. Naturalistic inquiry. Sage; 1985), this analysis was done independently by two team members followed by discussions to find consensus. The results of the survey were used to develop interview questions for one-on-one semi-structured interviews with students and preceptors to explore the quantitative data. Results: There was limited variability between student and preceptor ratings when analyzing the five themes. For 26 of 33 paired survey questions, students and preceptors had the same most frequent response. Students were more likely than preceptors to rate their professionalism as proficient and strongly agree they were able to interact with and counsel patients of diverse backgrounds. Preceptors were less likely than students to agree that students had difficulty with efficient searching of medical literature. Implications: When evaluating APPE preparation and performance, students tended to rate themselves more highly than preceptors in areas difficult to address in typical didactic courses, supporting the importance of cocurricular and experiential activities.

Comparing Students and Faculty Perceptions at 0-6, 3- and 4-year Schools/Colleges of Pharmacy. Nina Pavuluri, Lake Erie College of Osteopathic Medicine, Timothy J. Bloom, Shenandoah University, Lisa Lebovitz, University of Maryland. Objective: This retrospective study analyzed the summarized aggregate results of AACP curriculum quality surveys to study the perceptions of faculty and graduating students at colleges/ schools of pharmacy (C/SOPs) with three different program formats: 0-6 year, 3-year and 4-year. Methods: Peer schools were determined by program type, and results of the 2016 - 2018 curriculum quality surveys for faculty and graduating students were extracted from the AACP survey system. The total percentage of respondents who selected "Strongly Agree" and "Agree" on the Likert scale for each item were calculated. Chi-square comparison of proportions was performed to compare perceptions in each population and to determine the statistical significance of differing perceptions between the three types of C/SOPs. Results: Of the 141 programs included in this study, eight were categorized as 0-6 year; sixteen as 3year and 117 as 4-year programs. Students from C/SOPs with 3-year programs indicated significantly $(\mathrm{p}<0.05)$ lower satisfaction with their preparation in "Approaches to Practice and Care", but higher satisfaction with their general "Student Experience" and "Introductory Pharmacy Practice Experiences" when compared with 0-6 year and 4-year programs. Faculty from C/SOPs with 3year programs indicated significantly $(\mathrm{p}<0.05)$ higher satisfaction than those with 4-year and 0-6 year programs with "Administration"; "Infrastructure" and "Curriculum, Teaching and Assessment". Implications: We hope to share the information from this study to promote insight into pathway comparability and potential drivers and strategies to improve perceptions and satisfaction for students and faculty at different models of pharmacy schools.

Design, Oversight, and Assessment of a Strategic Planning Initiative. Craig A.H. Richard, Shenandoah University, Teresa A. Schweiger, Shenandoah University, Robert DiCenzo, Shenandoah University. Objective: Design a new strategic plan with the input of appropriate stakeholders, create an oversight process for the progressive achievement of goals, and assess faculty perceptions regarding strategic planning. Methods: The design of a new plan was initiated in 2017 , overseen by a committee, guided by a management consultant, and incorporated input from appropriate stakeholders. A strategy map and work plan were created to communicate, guide, track, and document the goals and achievements of the strategic plan. A voting process was created for the faculty to select the top annual priorities in the strategic plan to receive increased focus and resources. Data from the 2016, 2017, and 2018 ACPE faculty surveys were reviewed for changes in faculty perceptions about strategic planning. Results: Survey results show that $92.3 \%$ of faculty agree or strongly agree that their input was requested during the development of the current strategic plan, and $88.5 \%$ of faculty agree or strongly agree that the school effectively employs strategic planning. The data demonstrates a significant improvement $(\mathrm{p}<.05)$ over prior years and are similar to, or greater than, national averages. Implications: The initiatives used to develop and oversee the strategic plan are associated with improved perceptions from the faculty compared to prior years. The positive perceptions of the faculty are likely the result of their valued involvement into the development of the strategic plan and their continued involvement into the steady achievements of the strategic plan.

Difference in Education Level Impacts Student Perceptions of an Interprofessional Simulation. Teresa M. Seefeldt, South Dakota State University, Brittney A. Meyer, South Dakota State University. Objective: Interprofessional education (IPE) is an essential component of 


\section{American Journal of Pharmaceutical Education 2019; 83 (5) Article 7654.}

health professions education. Accounting for differing levels of education among student participants is a challenge in IPE design. The objective of this study was to evaluate the impact of education level on student perceptions of an interprofessional simulation. Methods: An interprofessional simulation involving pharmacy and nursing students was conducted in two different semesters. In the first semester, the simulation involved second year pharmacy students $(\mathrm{n}=32)$ and final (fifth) semester nursing students $(n=41)$. Second year pharmacy students $(n=38)$ participated with fourth semester nursing students $(n=56)$ in the second semester. All students completed a post-activity survey on student perceptions of the simulation. Results: The survey response rate was $95 \%$. A lower percentage of nursing students in the first semester indicated the activity was excellent or very good compared to pharmacy students (39\% vs 50\%). Comments from the nursing students on the open-ended questions indicated frustration with the difference in education level between the nursing and pharmacy students. After moving the IPE simulation up one semester in the nursing curriculum, the percentage of nursing students indicating the simulation was excellent or very good increased $(39 \%$ to $59 \% ; p=0.05$ ); however, no change was observed in the pharmacy students' perceptions of the simulation. Overall, the students felt their interprofessional teams functioned better in semester 2 compared to semester 1 $(p=0.001)$. Implications: Health professional students' educational level should be considered when designing IPE activities. Further research should determine how educational level impacts student learning in interprofessional simulations.

Effects of Brominated Flame Retardants on Cytokine Production by Mast Cells. Ethan D. King, Western New England University, Jonathan M. Mahoney, Western New England University, Dylan T. Krajewski, Western New England University, Warren M. Smith, Western New England University, Clinton Mathias, Western New England University, Diptiman Bose, Western New England University. Objective: Polybrominated diphenyl ethers (PBDEs), extensively used as flameretardants, have been shown to cause neurotoxicity, immunotoxicity and endocrine disruption. However, the exact mechanisms are largely unknown. In this study, we examined the effects of PBDE-mediated immunotoxicity in MC/9 mast cells. Methods: We assessed the effects of two of the most commonly occurring brominated diphenyl ether (BDE) congeners, BDE-47 and -99, and a metabolite of BDE-47, 6-OH-BDE-47 on MC/9 mast cells. BDE-mediated cytotoxicity and mast cell degranulation was measured by MTS assay and $\beta$ hexosaminidase assay, respectively. Results: Our results demonstrate that tetra-brominated BDE-47 and its 6-OH metabolite can suppress cytokine release with increasing concentrations, while penta-brominated BDE-99 decreases IL-6 release only at higher concentrations $(>20 \mu \mathrm{M})$. Mast cell degranulation measured by $\beta$-hexoaminidase also showed that BDE congeners decreased degranulation in a concentration-dependent manner. The $\mathrm{MC} / 9$ cells were exposed to increasing concentrations of the BDE congeners and cytotoxicity was measured at $24 \mathrm{~h}$. The tetra-brominated BDE-47 exhibited more potent cytotoxicity (EC50 $\mu \mathrm{M}=42.38,24 \mathrm{~h})$ in comparison to the penta-brominated BDE-99 (EC50 $\mu \mathrm{M}=48.41)$ in the $\mathrm{MC} / 9$ cells. However, the 6-OH metabolite of BDE-47 was more cytotoxic in comparison to its parent compound BDE-47, as well as penta-brominated BDE-99 (EC50 $\mu \mathrm{M}=25.82,24 \mathrm{~h}$ ). Implications: Our results suggest that PBDE congeners can modulate cytokine release in mast cells and the extent of inhibition is dependent on the bromination of PBDEs. These results address the need to better understand the molecular and cellular mechanisms by which PBDE exposure can mediate immunotoxicity.

Effects of Implementation of Mini-Application Exercises on Individual Student Performance in a Team-Based Learning Setting. Eugene Kreys, California Northstate University, Ruth Vinall, California Northstate University. Objective: The objective of this study was to determine whether implementation of individual applications (iBATs) improves student performance. Methods: Ninety-eight first year pharmacy students taking the Cell and Molecular Biology \& Biochemistry course participated in a randomized, cross-over study. The course consisted of three blocks: block 1 served as a mechanism to identify of high and low-preforming students, iBATs were administered for two randomized groups in a cross-over manner for only blocks 2 and 3. Separately among high and low-performing students, a paired t-test was used to compare average $\mathrm{z}$-scores between student performance on exams covering the material of blocks corresponding with and without iBAT implementation. A subgroup analysis based on the Bloom's taxonomy of exam questions was conducted. A questionnaire assessed students' impression of iBATs impact after each block exam. Results: Low-performing students participating in iBATs scored on average $4.7 \%$ and $2 \%$ higher for block 2 or 3 exams, respectively; resulting in an overall increase of $0.293 \pm 0.900 \mathrm{z}$-scores (pvalue $=0.029$ ). A subgroup analysis limited to critical thinking questions resulted in an overall increase of $0.404 \pm 0.977 \mathrm{z}$-scores ( $\mathrm{p}$-value $=0.006$ ). Majority of students reported that iBATs increased their ability to identify knowledge gaps (88\%), determine their proficiency of 


\section{American Journal of Pharmaceutical Education 2019; 83 (5) Article 7654.}

critical thinking questions (91\%), and increased studying following poor iBAT performance $(83 \%)$, while few felt that iBATs increased stress (30\%), or reduced enjoyment of the course $(46 \%)$. Implications: Implementation of iBATs significantly improved exam performance of low performing students, especially on critical thinking exam questions. Generally, students felt that iBATs had a positive impact on the course.

Evaluation of the Efficiency of Mixed SynchronousAsynchronous Teaching of Biochemical Pharmacology in the PharmD Program. Ahmad Hanif, West Virginia University, Werner J. Geldenhuys, West Virginia University, Julie Lockman, West Virginia University, Mohammed Nayeem, West Virginia University, Paul R. Lockman, West Virginia University, Marina Galvez Peralta, West Virginia University. Objective: To identify best practices for a mixed synchronous-asynchronous teaching on Biochemical Pharmacology. Methods: Ninety-one P1 PharmD students were enrolled in this course. Instructional sessions were offered in 15 min recordings vs. $50 \mathrm{~min}$, intercalating assessment questions that were discussed during the review in-class weekly. Students' preconceptions of asynchronous teaching prior to the course and their perceptions on best practices' after the course were collected on two independent surveys. (Response rate 93\%). Pre-post survey data were compared using Chi-square analysis $(\mathrm{p}<0.05)$. Results: Seventy-nine percent of students were exposed for first time to asynchronous teaching. Students' responses preferred $15 \mathrm{~min}$ over $50 \mathrm{~min}$ ( $84 \%$ vs $15 \%$ ), learned more (48\% vs. $35 \%)$, convenience for saving time ( $69 \%$ vs. $26 \%)$, and preferred choice ( $58 \%$ vs. $35 \%$ ) on asynchronous vs. synchronous, whereas in-class format was better for their interaction with their classmates $(18 \%$ vs. $78 \%)$. Students reported re-watching sessions more frequently when 15 min. videos were offered. Students' expectations matched with post-course survey except for time saving. While in the pre-course survey $91 \%$ of students anticipated that asynchronous teaching will be more convenient to save time, only $69 \%$ kept that perception by the end of the course (Chi-square, $\mathrm{p}<0.0004)$. Implications: The mixed asynchronous-synchronous format is an attractive approach for students to learn and best practices should be investigated for different audiences. An emphasis on time needed to dedicate to asynchronous teaching should be reinforced when introducing this teaching tool to students.

Incidence and Impact of Hypnotic Medication Use on Sleep Quality Among Young Collegiate Adults. Emily Cramer, Western New England University, Sarah R. Lander, Western New England University, Wenola L. Tauro, Western New England University, Jilla Sabeti,
Western New England University. Objective: Objectives: Studies suggest an alarming percentage $(>60 \%)$ of university students self-identify as "poor sleepers" with complaints of difficulties falling asleep and problematic daytime sleepiness. Factors underlying poor sleep quality among students remain unexplored. This study investigated the association between hypnotic medication use and objective measures of sleep quality in young collegiate adults using ambulatory ECG recordings during sleep at home. Methods: Methods: Participants $(\mathrm{N}=$ 79 students) were instructed on a procedure for wearing a sleep data recorder device (SleepImage Technology) that collected autonomic markers of NREM/REM sleep stability. Hypnotic medication use frequency was queried using the standardized Pittsburg Sleep Quality Index. Statistical differences in sleep disturbance rates between hypnotic users and naïve subjects were tested by chisquared analysis of proportions. Results: Results: ECG data were successfully obtained in 77 (97\%) participants. Data revealed elevated markers of disturbed sleep in $40 \%$ of cases. Twenty-four (30\%) students reported pastmonth use of sleep medication to varying extents (less than once a week: $16 \%, \mathrm{~N}=13$; once or twice a week: $11 \%, N=9$; three or more times a week: $3 \%, N=2$ ). Selfdeclared 'poor' sleepers were evenly divided between those reporting sleep medication use (47\%) and medication-naïve individuals (57\%). However, ECG-markers of disturbed NREM sleep were robustly and positively correlated with student's hypnotic-medication use $\left(\chi^{2}=\right.$ 11.24, $\mathrm{p}=0.001)$. Implications: Conclusions: Results revealed disturbed sleep patterns in a substantial number $(40 \%)$ of students overall, but disproportionately higher rates of disturbed sleep in students using hypnotic medications to varying extents.

Integration of ACPE Appendix 1 and PCOA Prescribed Toxicology Content Areas in PharmD Curriculum. Vishakha Bhave, Philadelphia College of Osteopathic Medicine, Anuj A. Patel, Philadelphia College of Osteopathic Medicine, Christopher J. Moses, Philadelphia College of Osteopathic Medicine, Avadhesh C. Sharma, Philadelphia College of Osteopathic Medicine, Naushad K. Ghilzai, Philadelphia College of Osteopathic Medicine. Objective: Toxicology is an important content area in a pharmacist's knowledge arsenal, which is reflected in it being part of the 2016 ACPE standards and in the NABP's PCOA guidelines. However, the extent to which these topics are covered in pharmacy curricula across the nation is not clear. Methods: This project describes how changes were made in the toxicology course offered at Philadelphia College of Osteopathic Medicine PharmD program to comply with the ACPE and PCOA content areas. With the aim of graduating well rounded 


\section{American Journal of Pharmaceutical Education 2019; 83 (5) Article 7654.}

pharmacists, PCOM School of Pharmacy revamped its toxicology course to include topics such as bioterrorism, antidotes, drug overdoses, and non-drug toxicities (pesticides, natural toxins, household products, and substances of abuse). Additionally, a nationwide review of the extent to which toxicology has been incorporated into pharmacy programs across the country was conducted. Results: From 142 ACPE accredited pharmacy programs in the United States, 63 programs offer toxicology in some capacity. Out of these, 38 schools offer toxicology as a required course in their didactic curriculum and 25 as an elective. Ten programs do not have any information about their course requirements and the remaining 69 programs do not offer toxicology as a course. Each of the syllabi were reviewed for coverage of toxicology topics.13 programs include drug overdose, antidotes and non-drug toxicity in their syllabi. Only 8 programs cover some form of bioterrorism. Implications: Our results highlight a potential deficit in coverage of Toxicology content areas in PharmD curriculum, especially topics such as non-drug toxicities and bioterrorism.

Lipocalin 2 Deficiency Protects Against Acetaminophen (APAP)-induced Acute Liver Failure in a Murine Model. Vishakha Bhave, Philadelphia College of Osteopathic Medicine, Christine Okaro, Philadelphia College of Osteopathic Medicine, Christopher J. Moses, Philadelphia College of Osteopathic Medicine, Harish S. Parihar, Philadelphia College of Osteopathic Medicine, Rangaiah Shashidharamurthy, Philadelphia College of Osteopathic Medicine. Objective: APAP overdose is the most common cause of acute liver failure (ALF) in the US. APAP overdose leads to centrilobular necrosis and sterile inflammation. Lipocalin-2 (LCN2), an acutephase innate immune protein, is upregulated during liver injury. However, its role in acute liver failure is not well understood and is the objective of this study. Necrotic hepatocytes release among other things, Damage Associated Molecular Patterns such as High Mobility Group Box 1 (HMGB1), which can mediate progression of liver injury. Cytoplasmic HMGB1 when bound to Beclin-1, can promote autophagy. LCN2 can negatively regulate this binding. We hypothesize that LCN2 mediates progression of liver injury by modulating the interplay between IL-6, HMGB1, and Beclin-1. Methods: WT and LCN2 KO mice were subjected to APAP overdose $(400 \mathrm{mg} / \mathrm{kg})$. Serum and tissue samples were collected over a time course and subjected to ELISA, Western blots, and coimmunoprecipitation. Results: LCN2 KO mice exhibit protection against APAP overdose as assessed by survival studies, significantly less hepatocellular damage (ALT and AST), and higher liver regeneration (PCNA). Proand anti-inflammatory cytokine expression analysis ne- gated the role of IL-10 in protection of LCN2 KO mice. In corroboration with higher PCNA activity in LCN2 KO mice, significantly higher IL-6 expression suggests its role in promoting liver regeneration. There was greater cytoplasmic HMGB1-Beclin-1 binding in LCN2 KO mice at $24 \mathrm{hr}$ compared to WT mice suggesting that the absence of LCN2 promotes autophagy post APAP overdose and thus protects against ALF. Implications: These findings identify a novel mechanism for LCN2 mediated progression of APAP-induced ALF.

Machine Learning Based Method for Accurate Prediction of Breast Cancer. Tuan Tran, California Northstate University, Uyen M. Le, California Northstate University, Hieu T. Tran, California Northstate University. Objective: Main objective of this study is to utilize advanced computing methods for accurate prediction of breast cancer based on preliminary clinical data. Methods: We used a well-known dataset provided by the Wisconsin Breast Cancer Database. First, we performed data preprocessing to identify missing values, outliers and potential predictors. Next, we used the advanced machine learning method, Random Forest, for modeling. To construct our model, data is randomly divided into $70 \%$ for training and 30\% testing. Performance is measured by accuracy, sensitivity (true positive) and specificity (true negative). Results: The dataset included 698 cases with 11 attributes. We identified 16 missing values and removed these associated observations. Analysis revealed that $64.96 \%$ and $35.04 \%$ of cases were benign and malignant, respectively. In addition, correlation analysis showed that CellSize and CellShape are highly correlated with coefficient of $0.91(\mathrm{p}$-value $=0.001)$. This finding helps to eliminate one of the attributes in our model to avoid multicollinearity. Our constructed model achieved very high prediction accuracy of $97.63 \%$ with $95 \%$ confidence interval of $(94.56 \%-99.23 \%)$. The result is higher than our previously published accuracy of $96.63 \%$. Additionally, the model also achieved very high sensitivity and specificity of $98.55 \%$ and $95.89 \%$, respectively. Finally, CellShape and ClumpThickness were identified as the two most important predictors for detecting breast cancer. Implications: The study provided an accurate and effective method for predicting breast cancer. It opened up new avenue for early breast cancer detection and treatments. Our future work will perform parameter fine-tuning to achieve higher accuracy.

Oxidant Production as a Possible Predictor of Hepatotoxicity of Herbal Supplements. Danielle L. Cruthirds, Samford University, Alexandria Lappe, Samford University, Gregory S. Gorman, Samford University, Marshall E. Cates, Samford University. Objective: The incidence of herbal supplement-associated hepatotoxicity 


\section{American Journal of Pharmaceutical Education 2019; 83 (5) Article 7654.}

has increased steadily as the popularity of these products has skyrocketed. Despite widespread use, many consumers are unaware of the safety and efficacy of these products. For these reasons, additional research into potential mechanisms of toxicity is needed in this area. Oxidant production as a mediator of hepatotoxicity has been well established in the literature. Methods: For this study, four herbal products and several combination products were selected for analysis of their potential to induce oxidant production. Herbal products tested included black cohosh, dong quai, Ginkgo biloba, and valerian. A superoxide dismutase (SOD) assay, used as a marker for oxidant production, was performed on each product. Additionally, high performance liquid chromatography was performed on each product to identify differences in compositions that may exist among different manufacturers. Results: Results of the superoxide dismutase assay showed considerable induction of activity by black cohosh and dong quai of approximately $27 \%$ and $35 \%$ respectively. Minimal induction was seen with Ginkgo biloba. In addition, Ginkgo biloba exhibited protective effects against black cohosh-induced increases in SOD activity. In general, all combination products showed less severe effects of increased activity compared to their single-agent controls. Induction of SOD activity correlated with supplement potential to cause hepatotoxicity; black cohosh exhibits high potential for hepatotoxicity, Gingko biloba, very little. Implications: The results of this study reveal that oxidant production may be a possible mechanism of hepatoxicity of herbal supplements and may therefore serve as a marker for evaluation of new product safety.

Predictors of Substance Use Attitudes in Health Profession Students: Interactions of Gender, Education and Self-Esteem. Wenola L. Tauro, Western New England University, Emily Cramer, Western New England University, Sarah R. Lander, Western New England University, Jilla Sabeti, Western New England University. Objective: This study investigated factors predicting permissive attitudes towards social drug use among health professional students. The main questions investigated were: (1) does self-esteem and/or prior substance abuse education impact student drug use attitudes; and (2) do gender differences modulate the magnitude of these effects. Methods: Participants were pharmacy student volunteers $(\mathrm{N}=122 ; 92 \%$ completion rate) completing two validated instruments (SAAS and the Rosenberg Self Esteem Scale), administered online at two times during semester. Participants had either completed or not completed at least one elective course on substance abuse education. No incentives were provided for completing the survey. ANOVAs and Tukey t-tests were run to de- termine main effects of substance abuse education and self-esteem on permissive drug use attitudes. Hierarchical multiple regression was conducted to test if any main effects were modulated by gender. Results: Overall, $35 \%$ of students sampled held highly permissive attitudes towards social use of alcohol, marijuana and other drugs, with female students tending towards the non-permissive spectrum. Logistic regression revealed that both negative self-esteem $(\mathrm{T}=2.5, \mathrm{p}=0.013)$ and lack of substance abuse education $(\mathrm{T}=2.2, \mathrm{p}=0.03)$ facilitated increased permissiveness attitudes toward alcohol and marijuana use, once gender differences were accounted for. The impact of substance abuse education in reducing drug use permissiveness was evident in only the female but not male participants. Implications: To foster positive change in pharmacy student's attitudes towards reduced drug use permissiveness, educational interventions must address both the gender-specific needs and self-esteem issues of students.

Receptor Expressed in Lymphoid Tissues (RELT) Immunostaining is Enhanced in Mice with Innate Immune Colitis. Leo R. Fitzpatrick, California Northstate University, Pachai Moua, California Northstate University, George Talbott, California Northstate University, Robert O'Connell, Bolder BioPATH, Inc., Jim Zapf, Visionary Pharmaceuticals, John K. Cusick, California University of Science and Medicine. Objective: Receptor Expressed in Lymphoid Tissues (RELT) is a human Tumor Necrosis Factor (TNF) receptor that is expressed most prominently in cells and tissues of the hematopoietic system. The role of RELT in intestinal inflammation is unknown. Therefore, we evaluated immunostaining of RELT in the colonic tissue of mice with innate immune colitis. Methods: Female CB-17 SCID mice $(n=9)$ were injected by the intraperitoneal route with $12 \mathrm{mg} / \mathrm{kg}$ anti-CD40 monoclonal antibody to induce colitis. A control group of mice $(n=4)$ were not injected with this antibody. Seven days later, mice were euthanized and colonic tissue collected for histology and immunohistochemistry (IHC) evaluations. Histological pathology was evaluated using a twelve-point severity scale. Colonic IHC ( $n=3$ to 4 per group) was performed with a specific antibody for RELT. Using captured IHC pictures, percent areas of immunostaining were analyzed with an online grid software program. Results: Significant evidence of histological pathology was present in the anti-CD40 injected mice (mean score $=9.0 \pm 0.5$ ), as compared to naïve mice (mean score $=2.6 \pm 0.2$ ). IHC staining was sporadic within the colons of naïve mice. The mean percent area of RELT staining was $35.1 \pm 3.7$. More prominent RELT immunostaining (mainly in the lamina propria) was evident in mice with innate colitis (mean 


\section{American Journal of Pharmaceutical Education 2019; 83 (5) Article 7654.}

percent area $=54.5 \pm 3.4)$. Implications: These results demonstrate that RELT immunostaining is enhanced within the colonic mucosa of mice with innate immune colitis. Moreover, there is an apparent association between colonic histological damage and RELT immunostaining.

Relationship Between Empathy and Other Affective Domains Among Student Pharmacists. Anna Ratka, St. John Fisher College, Sean T. Leonard, St. John Fisher College, Chris Noel, St. John Fisher College. Objective: Student pharmacists must develop proficiency in affective competencies that are essential in delivery of patient-centered care. In our program, we explored the degree to which empathy may be an overarching feature for development in other affective areas. Objectives: (1) Explore how empathy changes longitudinally throughout training. (2) Evaluate relationships between students' level of empathy and their development within other affective competencies. Methods: Student development in affective domains was tracked for several years. Students were assessed annually using a battery of psychometrically-supported measures that evaluate different components of affective development. Each measure was locally-normed and scores were reported on a standard scale. Empathy was measured using the Kiersma-Chen Empathy Scale, alongside measures for professionalism, communication, leadership, moral reasoning, and emotional intelligence. Scores were evaluated using regression analyses, stratified by academic year. Effect sizes were calculated to gauge the degree of empathic development. Results: Measurements from 471 students were included in this project. Regression models for empathy were statistically significant for each year, with R-squared values of 0.303 for $\mathrm{P} 1 \mathrm{~s}, 0.341$ for $\mathrm{P} 2 \mathrm{~s}, 0.348$ for $\mathrm{P} 3 \mathrm{~s}$, and 0.350 for $\mathrm{P} 4 \mathrm{~s}$. The affective domains most strongly associated with empathy were emotional intelligence and moral reasoning. Empathy scores increased with each year of training. The overall effect size for empathic development was positive but small $(\mathrm{d}=0.33)$. Implications: The understanding of relationships between students' empathy and affective competencies may motivate pharmacy educators to inculcate empathy-enhancing interventions into educational methods to help student pharmacists develop and reinforce affective behaviors and skills.

Science-Practice-Connect: Effective Activity to Enhance Student Life-Long Learning. Fawzy A. Elbarbry, Pacific University Oregon. Objective: The Science-Practice-Connect (SPC) activity was implemented to integrate basic science knowledge into pharmacy practice courses, promote application and critical-thinking skills, and help students to be life-long learners.
Methods: Following first-year science or second-year therapeutics lectures, students were divided into groups with an assigned drug. Each group received 50-minutes to review the drug monograph and propose scientific reasoning behind three practice-related recommendations (e.g. drug interactions, dose adjustment, and contraindications). The groups presented their findings to the rest of the class. An anonymous survey assessed the effectiveness of the SPC. Final exam questions were tagged to different learning domains, including "knowledge", "application", "analysis", and "synthesis" as defined by Bloom's Taxonomy. These exam scores were compared to those of similar questions in the previous 2 years to assess improvement in students' learning. Results: Our quantitative assessments compiled after 4 courses provided compelling evidence that students agreed that the SPC activity is an effective tool in helping them integrate foundational science concepts into pharmacy practice (97\%), apply science to patient care $(95 \%)$, improve knowledge base of drug information (96\%), and promote team-based active learning and communications skills (95\%).Although students significantly $(\mathrm{P}<0.05)$ score better in questions tagged to "application", "analysis", and "synthesis" domains, no significant improvement was detected for questions tagged to the "knowledge" domain when compared to the last 2 years. Implications: The SPC is an easy activity to implement and is effective in promoting students' learning and integrating curricular content in a learner-centered educational environment.

Two Years of Data on a Pedagogical Opioid Abuse and Overdose Activity for Student Pharmacists. Kevin S. Murnane, Mercer University, Jill M. Augustine, Mercer University, Leisa L. Marshall, Mercer University, J. Grady Strom, Mercer University. Objective: Pharmacists are at the front-line of combating the opioid crisis, yet the speed of development of this crisis has created a void in the training offered to student pharmacists. Our objective was to implement and assess a "hybrid" flipped-classroom activity designed to increase the motivation and confidence of student pharmacists to address this crisis. Methods: Third-professional year Doctor of Pharmacy students voluntarily completed pre- and post-activity attitude surveys regarding opioid abuse and opioidoverdose prevention. Students were assessed in two different cohorts. The activity included pre-class reading material developed by federal agencies and professional pharmacy organizations, as well as Georgia-specific information covering medical amnesty and local resources for opioid-overdose prevention. The classroom session consisted of a didactic lecture on opioid pharmacology and opioid overdose, viewing of training videos, and engagement in extensive discussion. Results: Based on the 


\section{American Journal of Pharmaceutical Education 2019; 83 (5) Article 7654.}

Wilcoxon Signed Rank test, there were significant changes in the survey areas related to student confidence; fewer changes were seen in the survey areas related to student motivation. Implications: The student pharmacists in this study exhibited a high baseline motivation to assist in combating the opioid crises but lower confidence in the ability to intervene. A "hybrid" flipped classroom activity increased the confidence of student pharmacists in their understanding of the physical and adverse effects of opioids and the application of reversal agents. Increased confidence may support increased intervention.

U50488, a Kappa-Opioid Agonist, Attenuates Alcohol Preference in Drosophila Melanogaster. Megan Longley, Xavier University of Louisiana, Krishna Shah, Xavier University of Louisiana, Starr Villavasso, Xavier University of Louisiana, Sunil Sirohi, Xavier University of Louisiana. Objective: Alcohol Use Disorder (AUD) is the fifth leading risk factor for premature death and disability worldwide, affecting $6.5 \%$ of the United States population. Based on studies utilizing vertebrate animal models, several neurotransmitters receptor systems, including opioid receptor system, have been implicated in mediating the effects of both acute and chronic alcohol exposure. However, it is unclear if similar mechanisms are intact and functionally regulate alcohol drinking behavior in small invertebrate animal models (e.g., Drosophila melanogaster). Methods: The present study investigated the effects of U-50488, a highly selective k-opioid agonist, on alcohol preference in male Drosophila melanogaster Canton S. (4-9 flies per vial; 10 vials total). First, initial baseline food (5\% yeast, $5 \%$ sucrose and dye) intake was assessed using a capillary feeder assay (CAFE). Following a stable food intake, flies were given capillaries containing either normal food with or without ethanol (15\% ethanol) for 24 hrs. Following stability, all flies received water (controls) or U50488 (treatment) mixed in food for $24 \mathrm{hrs}$ and its effect on alcohol preference was assessed on the following days in a withinsubject design. Results: A preference for food containing alcohol was developed over a period of three days. Importantly, this preference remained unaffected under control (water) conditions, whereas U50488 pretreatment abolished this effect. Implications: Previous studies have shown that U50488 negatively regulate alcohol drinking behavior in rodents. The present study suggests the possibility of a functionally similar system in the Drosophila which warrants further investigation to facilitate the drug development efforts for the treatment of alcoholism.

Validation of an Internal "Pre-Assessment for Learning and Mastery" Test as a Predictor of Academic Performance. Ghous M. Khan, Appalachian College of Pharmacy, Craig R. Mullins, Appalachian College of Pharmacy. Objective: This study was done to assess the validity of the "Pre-Assessment for Learning and Mastery" (PALM) test with regard to selected preadmission criteria, including Pharmacy College admission Test (PCAT) score, that are often used to predict students' academic performance. Methods: The PALM test is an internal 50-question test derived from topics that are mostly covered in students' prerequisite science courses. The test is administered to all students at the entry point so that it can be used as a tool to assess students' early academic performance at the ACP, a three-year accelerated Doctor of Pharmacy (PharmD) program. Students from the class of 2014, 2015 and 2016 were selected for this study $(n=194)$. Pre-admission data were obtained from ACP admission office. PALM test scores of individual students were assessed by linear regression analyses for any correlation with students PCAT scores, Science GPA (SGPA) and Math GPA Results: Significant positive correlation was found between the PALM test and PCAT scores $(\mathrm{r} 2=0.19, \mathrm{p}<.001)$. Weak positive correlation was also seen between the PALM test score and MGPA $(\mathrm{r} 2=0.03, \mathrm{p}=.04)$. No correlation, however, could be demonstrated between PALM scores and SGPA. Implications: The PALM test as an internal instrument for assessment could be validated against PCAT score and MGPA, but not against SGPA. Given that the PCAT score is known for its predictable value in assessing students' academic performance, the PALM test could be used as a potential, internal assessment tool for students' early academic performance. This is important for an accelerated program.

\section{Theoretical Models}

Training and Engaging Pharmacy Residents to Lead High Fidelity Simulation-Based Interprofessional Education Cases. Ashim Malhotra, California Northstate University, Welly Mente, California Northstate University, Victor Phan, California Northstate University, Hieu T. Tran, California Northstate University. Objective: ACPE mandates interprofessional education (IPE) for inclusion in pharmacy programs. We created an innovative program to incentivize and engage pharmacy residents by participation in high-fidelity simulation IPE cases for P2 and P3 students. Methods: The Post Graduate Year 1 (PGY1) Pharmacy Residency at the California Northstate University College of Pharmacy (CNUCOP) and the Adventist Health Lodi Memorial (AHLM), and affiliated residency programs, offers a comprehensive experience in advanced clinical practice and academic teaching. The CNUCOP Residency program offered our residents opportunities to develop their teaching skills, facilitate team-based learning (TBL), and earn a Teaching and Learning Certificate. Residents interested in IPE 


\section{American Journal of Pharmaceutical Education 2019; 83 (5) Article 7654.}

assisted with high fidelity simulation IPE cases between the CNUCOP and the California State University Sacramento School of Nursing for P2 and N1 and P3 and N1 students. The PGY1 residents were trained to develop scripts for unfolding IPE cases, supervise student performance, assist with running the manikins, and debriefing after the case. Results: Six pharmacy PGY1 residents contributed to IPE events $(\mathrm{N}=2)$ engaging large aggregate cohorts of students $(\mathrm{N}=200)$. The IPE cases focused on Acute Pancreatitis and Congestive Heart Failure. Residents were trained in IPE principles in a formal training by the IPE Coordinator, who also provided annotated IPEcase scripts for residents in the role of the consulting pharmacist. Residents also received training by the Residency Program Director and CNUCOP faculty. Implications: Our formal program for training PGY1 residents in IPE incentivized and engaged the next generation of the Pharm.D. workforce in recognizing the significance of interprofessional education and practice.

\section{CHEMISTRY}

\section{Completed Research}

$6 \mathrm{~Hz}$ Active Anticonvulsant Fluorinated NBenzamide Enaminones and Their Inhibitory Neuronal Activity. Patrice L. Jackson-Ayotunde, University of Maryland Eastern Shore, Isis J. Amaye, University of Maryland Eastern Shore, Miguel Martin, University of Maryland Eastern Shore, Thomas Heinbockel, Howard University, Zejun Wang, Howard University, Julia Woods, Howard University. Objective: A library of fourteen novel fluorinated N-benzamide enaminones were synthesized and evaluated in a battery of acute preclinical seizure models. Three compounds (GSA 62, TTA 35, and WWB 67) were found to have good anticonvulsant activity in the 6-Hz 'psychomotor' $44 \mathrm{~mA}$ rodent model. The focus of this study was to elucidate the active analogs' mode of action on seizure-related molecular targets. Methods: The N-benzamide compounds were synthesized by amination of the respective $\beta$-diketones, followed by $\mathrm{N}$-acylation of the enaminone intermediates with corresponding aromatic acyl chlorides in a base-catalyzed reaction. The analogs were screened in the acute rodent seizure models at the NINDS, NIH Epilepsy Therapy Screening Program. Electrophysiology studies were employed to evaluate the compounds' ability to inhibit 1) neuronal activity in central olfactory neurons, mitral cells, and 2) sensory-like ND7/23 cells, which express an assortment of voltage and ligand-gated ion channels. Whole-cell patch-clamp recordings were obtained from the mitral cells of the mouse main olfactory bulb and whole-cell recordings from the ND7/23 cells. Results: We did not find any significant effects of the three com- pounds on action potential generation in mitral cells. The treatment of ND7/23 cells with $50 \mu \mathrm{M}$ of GSA 62 , TTA 35 , and WWB 67 generated a significant reduction in the amplitude of whole-cell sodium currents. Similar treatment of ND7/23 cells with these compounds had no effect on T-type calcium currents. Implications: The findings in the study indicate that the fluorinated N-benzamide enaminone analogs tested may have a selective effect on voltage-gated sodium channels, but not calcium channels.

Analyzing Learning Within Medicinal Chemistry and Pharmacology. Adil K. Shivji, Lake Erie College of Osteopathic Medicine, Daniel A. Offei, Lake Erie College of Osteopathic Medicine, Daniel L. Austin, Lake Erie College of Osteopathic Medicine. Objective: This study featured structured enrichment sessions designed to provide students opportunities to develop life-long learning skills through the context of medicinal chemistry and pharmacology content. Methods: Five enrichment activities were developed each containing a self-study and live session featuring unit-specific content (problem solving, metacognition, reading comprehension, case-based problem solving, and structure-based therapeutic evaluation). The voluntary sessions were administered longitudinally to 139 second professional year pharmacy students throughout an academic quarter at the end of each unit during the first course of an integrated pharmacology and medicinal chemistry sequence. Students provided fivepoint Likert survey feedback at the beginning of the course, after the first activity, and at course conclusion. Survey questions were linked to self-assessment domains of metacognition, content relevance, confidence, affective response to content, and session specific themes. Results: Survey responses indicated significant improvement in confidence (3.68 - 4.15) and metacognition (3.20 - 3.82) domains at the conclusion of the course, and significant improvement in affective domain following the first sessions that was maintained at the conclusion of the course (3.52 - 4.10 - 4.20). Perceived relevance of content did not change significantly throughout the study. Implications: These observations are consistent with the notion that interventions have a significant impact on content perceptions, which may be especially important for challenging disciplines. Concurrent introduction of learning topics and application may positively influence emotional response to learning, which may play a role in latent confidence and self-awareness benefits. Further study is necessary to optimize these activities in order to maximize impacts on learning outcomes.

A Novel Bridging Experience to "Close the Gap" in PharmD Student Readiness Prior to Matriculation. Bryson M. Duhon, The University of Texas at Austin, Walter L. Fast, The University of Texas at Austin, Korbin 


\section{American Journal of Pharmaceutical Education 2019; 83 (5) Article 7654.}

Evans, The University of Texas at Austin. Objective: The objectives of this study were to develop a bridging experience with pre- and post-matriculation content that also incorporates active learning strategies and develop assessment methods to evaluate the impact of these experiences on student success. Methods: One difficulty often encountered while teaching first-year pharmacy students is the wide range of student proficiency in STEM subjects. First, an incoming class of 123 PharmD students were encouraged to complete online quizzes prior to the start of the fall semester. Student scores were released and links provided to on-line tutorial materials, allowing students to 'diagnose' areas where review might be helpful. Second, during on-campus student orientation, small group active learning sessions were added to reinforce the same concepts and were followed by a request to repeat the same quiz taken earlier in the summer. Third, students were offered supplementary student-led tutorial sessions during the first few weeks of class. Assessments included scores from the summer quiz, scores from the same quiz repeated after orientation activities, and matched questions on the final exam. Students were also asked to provide narrative feedback. Results: Preliminary analysis indicates that more than half of the students felt the program helped prepare them for the start of the pharmacy curriculum. Preliminary analysis of the chemistryrelated experiences indicates that quiz scores before and after the orientation experience increased 15-20 percentage points. Implications: Early self 'diagnosis' of academic deficiencies, active-learning exercises, and assisted review sessions may be methods helpful to 'leveling' firstyear pharmacy student STEM preparedness and increasing student success.

A Sports Pharmacy APPE: Utilizing Medicinal Chemistry as a Foundational Approach. Matthew J. DellaVecchia, Palm Beach Atlantic University, Richard J. Marrero, Palm Beach Atlantic University, Jenna L. James, Walgreens Pharmacy, Trevor D. Vanscoy, CVS Pharmacy, Jessica Lendoiro, Palm Beach Atlantic University. Objective: To pilot a Sports Pharmacy Advanced Pharmacy Practice Experience (APPE) utilizing medicinal chemistry as a foundational approach. Methods: Three fourth-year student-pharmacists at Palm Beach Atlantic University (PBA), guided by a medicinal chemistry faculty preceptor, researched known and emerging performance enhancing drugs (PEDs) during APPE rotations. General knowledge of PEDs and the perceived value of pharmacists in sports was gauged among PBA student-athletes, athletic staff, and pre-APPE pharmacy students via interviews, surveys, and small focus groups. Results: Survey participants $(\sim 75 \%, \mathrm{n}=134)$ perceived pharmacists as being a valuable resource to student- athletes seeking information on medications and overthe-counter (OTC) supplements. During the APPE, studentpharmacists demonstrated how legitimate medications were chemically similar to substances banned by sports governing agencies. Upon comparing chemical structures/pharmacophores of PEDs to those of various prescription drug classes, drugs and supplements with known therapeutic function were reviewed to discern why athletes misuse these substances for athletic gain. Furthermore, student-pharmacists educated student-athletes and athletic personnel on pertinent topics like marijuana in sports, the importance of avoiding medication sharing, and deciphering an OTC supplement label. Detection risk-level by various analytical methods was discussed. Counseling points on potential adverse health risks posed by PED usage were outlined. Implications: Opportunity exists for expansion of this Sports Pharmacy APPE model here at PBA as well as potential for implementation elsewhere. Pharmacists rely upon pharmaceutical science and pharmacy practice expertise to counsel patients on the utilization of medications and OTC supplements. Using medicinal chemistry as a foundational approach, pharmacists can readily translate this expertise to benefit the "patient-athlete".

Dopamine D4 Receptor-Selective Compounds Reveal Structure-Activity Relationships that Engender Agonist Efficacy. Comfort A. Boateng, High Point University, Sonvia L. Brown, High Point University, Michele S. Maddaluna, High Point University, R. Benjamin Free, National Institute of Neurological Disorders and Stroke, Alessandro Bonifazi, National Institute on Drug Abuse, Michael P. Ellenberger, National Institute on Drug Abuse, Amy H. Newman, National Institute on Drug Abuse, David R. Sibley, National Institute of Neurological Disorders and Stroke, Chun Wu, Rowan University, Thomas Keck, Rowan University. Objective: To understand the receptor-ligand interactions that control efficacy at Dopamine D4 Receptor (D4R) Subtype Methods: A series of novel full-length N-(m-tolyl) acetamide derivatives ligands were synthesized by using standard $\mathrm{N}$-alkylation reactions. The compound $\mathrm{m}$-toluidine was converted to intermediate 2-chloro- $\mathrm{N}$-(m-tolyl) acetamide by reacting with 2-chloroacetyl chloride. The compound 2-chloro-N-(m-tolyl) acetamide was then used to alkylate different arylpiperazine or arylpiperidine amines in the presence of $\mathrm{K} 2 \mathrm{CO} 3$ in $\mathrm{CH} 3 \mathrm{CN}$ to yield the desired target compounds. Their binding affinities were determined using $[3 \mathrm{H}] \mathrm{N}-$ methylspiperone and $[3 \mathrm{H}] 7-\mathrm{OH}-$ DPAT radioligand binding in HEK293 cells expressing dopamine D2-like receptors (D2R, D3R, D4R). These binding studies were coupled with functional studies using radioligand binding $\beta$-arrestin recruitment and cAMP 


\section{American Journal of Pharmaceutical Education 2019; 83 (5) Article 7654.}

inhibition displacement assays. We further performed comparative molecular dynamics simulations of D4R in complex with selected compounds. Results: To identify new D4R-selective ligands, and to understand the molecular determinants of agonist efficacy at D4R, we synthesized and characterized a series of eighteen novel ligands based on the classical D4R agonist A-412997. Compounds were profiled using radioligand binding displacement assays, $\beta$-arrestin recruitment assays, cAMP inhibition assays, and molecular dynamic computational modeling. We identified several novel D4R-selective (Ki $\leq 4.3 \mathrm{nM}$ and $>100$-fold vs. other D2-like receptors) compounds with diverse partial agonist and antagonist profiles, falling into three structural groups. Implications: These studies have further extended our understanding of receptor-ligand interactions that control efficacy at D4R. The results provide insights to targeted drug discovery leading to a better understanding of the role of D4Rs in neuropsychiatric conditions, including Alzheimer's disease and substance use disorders.

Introduction of Augmented Reality Modules into Pharmacy Medicinal Chemistry Lectures. Chase Smith, MCPHS University-Worcester/Manchester, Matthew Metcalf, MCPHS University-Worcester/ Manchester, Carolyn Friel, MCPHS University-Worcester/ Manchester. Objective: Augmented Reality (AR) can be exploited to provide a novel way for student visualization and understanding of complex three dimensional (3D) molecular interactions as compared to more traditional methods. The work details the development of molecular AR content into Medicinal Chemistry lectures. Methods: Molecular models were first obtained from the Protein Data Bank (PDB) (http://www.rcsb.org/) in PDB format. The files were first processed using the University of California San Francisco (UCSF) molecular modeling software package Chimera (https://www.cgl. ucsf.edu/chimera/) and then exported as 3D scenes for secondary processing in the 3D software Blender (https://www.blender.org/). The final 3D content was ultimately exported to be hosted online at Sketchfab (https://sketchfab.com/). The AR content was presented during Medicinal Chemistry lectures and visualized using molecular viewer's on student mobile devices. Students were surveyed following their encounter with the AR content (approval protocol IRB100118S). Results: 51 $(19 \%)$ of the enrolled students responded to the post-lecture educational survey. Of the responder's that either Strongly Agreed or Agreed: a) 88\% thought the AR application was easy to use; b) $95 \%$ were able to visualize the molecular interactions; c) $83 \%$ were able to manipulate the visualization; d) $67 \%$ believed that the AR visualization helped them better understand the concepts being taught and; e) $83 \%$ would like to see additional AR content in the future. Implications: The positive response to the AR material indicates its potential to become a useful classroom tool for the active learning of complex 3D concepts. Further refinement to enhance student interest in AR learning is also possible.

Mixed Cohort Biochemistry Retention Evaluation in a College of Pharmacy Curriculum Compared to Other Professional Programs. David B. Cleary, Sullivan University. Objective: It is important for educational institutions to evaluate programmatic outcomes. Very little data is published concerning long-term biochemistry retention in pharmacy schools. This research quantifies the retention of biochemical knowledge following a ten-week biochemistry course in a three-year college of pharmacy. The hypothesis for this research is: a biochemistry course taught using standard methodologies in an accelerated three-year college of pharmacy program produces the same outcome as a biochemistry course in other professional programs. Objective one is to quantify biochemical retention one year after a pharmacy biochemistry course. Objective two is to compare the current teaching effectiveness versus the effectiveness of other professional programs. Methods: The teaching method utilized was lecture format with questions to actively engage the audience. The course culminated with a cumulative one hundred question multiple choice examination. One year following the examination, all members of the cohort were invited to participate in a retention study consisting of retaking the same examination. Each participant's change in score from initial examination to retake was calculated to determine each participant's retention score. Results: Although teaching methodologies vary across professions, universities, and time, these limitations do not seem to greatly impact the average retention outcome. Data has been collected and pooled for two cohorts $(n=9$ and $n=37)$ revealing an average decrease of $28 \%$ knowledge. Implications: This result is in good agreement with published data from other professional programs implicating a true hypothesis. Continued collection of data is warranted to increase the number of participants and significance of this research.

Phytotoxic Effects of NSAIDs on Pisum sativum Lead to Animal Alternative Drug Assay. Tyler M. Holmes, MCPHS University-Worcester/Manchester, Gabriel Gabro, MCPHS University-Worcester/Manchester, Palak Bhambri, MCPHS University-Worcester/ Manchester, Crystal Ng, MCPHS University-Worcester/ Manchester, Matthew Metcalf, MCPHS UniversityWorcester/Manchester. Objective: Animal drug assays are the gold standard proof of clinical safety and efficacy 


\section{American Journal of Pharmaceutical Education 2019; 83 (5) Article 7654.}

prior to human trials. However, ethical concerns and IACUC protocols state attempts should be made to develop non-animal-based assays. This work describes the development of a plant based alternative assay for the acetic acid antiwrithing assay. NSAIDS were hypothesized to act as auxins in plants and an assay was developed to detect the phytotoxic effects of NSAIDs in Pisum sativum. Methods: Pisum sativum seeds were sprouted in sphagnum moss and initial growth of root and shoot length were measured. Seedlings were then exposed to varying concentrations of drug in a liquid growth solution for 72 hours when growth characteristics were remeasured. Dose response curves were generated using percent change in growth. Results: NSAIDs produced phytotoxicological effects on root growth in a dose dependent manner in Pisum sativum sprouts. NSAID root growth effects [example - naproxen LD50 23.1 $\mu \mathrm{M}$ (95\% CI 16.6-32.2, $\mathrm{p}<0.0001)]$ were similar to auxin effects [example - indole-3-butyric acid (IBA) LD50 $1.7 \mu \mathrm{M}$ $(95 \%$ CI 1.1-2.8, $\mathrm{p}<0.0001)]$ but significantly different than gibberellic acid (GA3), and acetaminophen (APAP) both no effect. For shoots all NSAIDS and auxins inhibited growth $(\mathrm{p}<0.0001)$; GA3 enhanced growth $(p=0.0002)$; and APAP had no effect on growth. Implications: In root and shoot growth, the NSAIDs produced similar effects to the auxins dissimilar to controls GA3 and APAP. This data supports the hypothesis of auxinlike phytotoxic effects on Pisum sativum growth characteristics and the assay can serve as an animal replacement assay.

SAR Maps: A Student Friendly Approach to Teach Medicinal Chemistry in Integrated Courses. Kathleen M. Frey, Fairleigh Dickinson University. Objective: Courses that integrate pharmacology, medicinal chemistry, and pharmacotherapy are widely implemented in pharmacy curriculums. The integration of medicinal chemistry is often challenging given the material and time constraints. The objective of this educational study is to utilize structure activity relationship (SAR) maps as visual aids to teach students medicinal chemistry in an integrated course. Methods: Structure-activity relationship (SAR) maps were designed and implemented within an integrated course focusing on cardiopulmonary diseases. Specific SAR maps used in lecture and class activities included the following: phenylethylamines (adrenergic agonists i.e. bronchodilators); arylimidazoines (antihypertensives i.e. clonidine); and aryloxypropanolamines (beta blockers). Students were assessed in class activities (formative), take home quizzes (low stakes), and exams (high stakes) for specific information surrounding drug structure and the SAR map. Drug properties assessed included essential pharmacophores, pharmacodynamics, physiochemical properties, metabolism, duration of action, and decision-making (Appendix 1, ACPE Standards). These properties were highlighted in the provided SAR maps. Results: Results from assessment item analysis reveal that students performed well on medicinal chemistry questions related to the SAR maps (i.e. $89.8 \%$ correct on first exam). Students revealed in a survey that they used the SAR maps to study medicinal chemistry for the exams. Implications: SAR maps are effective tools that visually teach students key concepts in medicinal chemistry. This millennial student-friendly tool is time-effective and promotes learning as opposed to drug structure memorization. The SAR map can be easily implemented in other integrated courses focused on various disease states.

Synthesis of Potential Medicinal Enaminone Compounds. Ivan O. Edafiogho, University of Saint Joseph, Yatish Parmar, University of Saint Joseph, Ashley A. Bill, University of Saint Joseph, Ola A. Ghoneim, University of Saint Joseph, Doreen E. Szollosi, University of Saint Joseph. Objective: The objective of this study was to synthesize cyclohexenone enaminones and evaluate them for potential medicinal properties. Enaminones are chemical compounds consisting of an amino group linked through a carbon-carbon double bond to a keto group. Methods: Different structural analogs of cyclohexenone enaminones were synthesized. We obtained ten new enaminone compounds (YP1, YP2, YP3, YP4, YP9, YP10, YP13, YP16, YP17, YP19) and three known enaminone compounds (E121, JODI 19 and YP14). Results: We had previously reported E121 as being anticonvulsant, anti-inflammatory, analgesic, anti-tussive, anti-asthmatic, bronchodilatory, and immunosuppressant. Therefore, we expected the new enaminone compounds from this project to have a variety of pharmacological activities. Of significant importance is the synthesis of YP9 which was confirmed by H-NMR spectroscopy. It is expected to be anti-inflammatory by reducing the production ofcytokines such as tumor necrosis factor-alpha (TNF-alpha) and Interleukin-6 (IL-6) in a J774 macrophage cell line stimulated with lipopolysaccharide (LPS). JODI 19 was anti-inflammatory at $0.5 \mathrm{mM}$. Implications: Our data indicate that the enaminone pharmacophore in our novel enaminone compounds is very important in confering desirable pharmacological activity on the compounds.

The "First-Generation Effect" on Perceptions and Academic Performance of Pharmacy Students. Donald Sikazwe, University of the Incarnate Word, Richard Haan, University of the Incarnate Word, Sinin A. Allahalih, University of the Incarnate Word, Matthew C. Garber, University of the Incarnate Word, Russell T. 


\section{American Journal of Pharmaceutical Education 2019; 83 (5) Article 7654.}

Attridge, University of the Incarnate Word, Anita T. Mosley, University of the Incarnate Word, Tanja Stampfl, University of the Incarnate Word, Amy Diepenbrock, University of the Incarnate Word, Raghunandan Yendapally, University of the Incarnate Word. Objective: To evaluate perceptions and P1 academic performance of first-generation students compared to their non-first-generation peers at the Feik School of Pharmacy. Methods: Perceptions related to academic and personal support were assessed through a 49-question survey at the beginning of $\mathrm{P} 2$ or $\mathrm{P} 3$ years. Academic performance was assessed using measures of student success (course grades, GPA, supplemental instruction enrollments, academic infractions) from P1 year. Students were stratified by first-generation student status (defined as neither parent with a bachelor's degree), and comparative statistics were performed to analyze differences in perceptions and academic performance between the two cohorts (firstgeneration students and non-first-generation students). Results: One-hundred twenty-eight (128) of 132 eligible students (97\% response rate) completed our survey, of which $58(45 \%)$ were first-generation students. In 47 of the 49 survey questions, there were no statistically-significant differences between groups; however, first-generation students had a lower perception of their academic success $(p=0.009)$ and were more likely to cite finances as one of their greatest barriers in school ( $86 \%$ vs. $64 \%$; $p=0.005)$. From the fifteen P1 courses reviewed for academic performance, first-generation students had lower final course grades in two foundational science courses (A\&P I, $p=0.006$; Immunology, $p=0.037)$. Otherwise, no significant differences were noted in measures of academic success. Implications: Finances and a lower perception of academic success may be barriers for first-generation students. First-generation status may not hinder academic performance at the graduate level. Programmatic, proactive interventions in identifiable challenging courses may improve perceptions and performance of first-generation students.

Utilizing Medicinal Chemistry to Solve a Clinical Clozapine and Sucralfate Interaction. Susan L. Mercer, Lipscomb University, Lindsey Miller, Lipscomb University. Objective: ACPE Standard 1 - Key Element 1.1 requires that pharmacy graduates be able to develop, integrate, and apply knowledge from the foundational sciences to evaluate the scientific literature, explain drug action, solve therapeutic problems, and advance population health and patient-centered care. However, students often struggle to see the correlation between science and practice providing an opportunity for interdisciplinary collaboration. Herein, we describe a clinical drug-drug interaction that was solved utilizing medicinal chemistry principles. This case was presented after PY1 students completed a unit on acid/base, $\mathrm{pKa}$ and ionization. Methods: A patient was prescribed $300 \mathrm{mg}$ of clozapine; however, upon admission clozapine levels were undetectable. During a medication review, the patient noted taking sucralfate $2-4$ times daily. A structural evaluation of clozapine indicated multiple basic functional groups with three $\mathrm{pKa}$ values of 3.92, 7.75 and 15.90. The amines are fully ionized $(\mathrm{NH}+)$ in a stomach $\mathrm{pH}$ of $1-2$. These ions interact directly with sucralfate; therefore, no free drug is available to elicit the desired CNS effects. Results: The recommended therapeutic treatment options were to either eliminate sucralfate from the patient drug regimen or to administer clozapine via a route that avoids first pass metabolism. Ranitidine was substituted for sucralfate and clozapine levels increased appropriately to 206 $\mathrm{ng} / \mathrm{mL}$. Implications: Evaluating drugs utilizing structure, $\mathrm{pKa}$ and ionization principles directly impacted patient outcomes. Integrating relevant clinical examples to medicinal chemistry coursework highlights the application of foundational science to the clinical decision process.

\section{Theoretical Models}

A Model for Assessing Professional Association Engagement. Emily F. Gorman, University of Maryland, Klarissa D. Jackson, Lipscomb University, Marc W. Harrold, Duquesne University, Susan L. Mercer, Lipscomb University, Matthew Metcalf, MCPHS UniversityWorcester/Manchester, Lisa Lebovitz, University of Maryland, Shannon R. Tucker, University of Maryland, Kirsten F. Block, American Association of Colleges of Pharmacy, Raphael Franzini, The University of Utah, Andrew Coop, University of Maryland. Objective: To develop a model for quantifying engagement with the American Association of Colleges of Pharmacy (AACP) for recipients of the New Investigator Award (NIA) in the Chemistry section Methods: Assessing member engagement with a professional association is critical in determining the success of programs such as the AACP NIA, because engagement leads to organizational growth and is key to long term sustainability. The authors searched peer-reviewed and organizational literature for published models evaluating organizational engagement to create a modified scale to quantify engagement following the NIA.The model was then assessed using archetype personas designed to mimic involvement patterns represented in the Chemistry section. Results: The American Bar Association published an article assessing engagement of its members with the following five tiers, explaining the tiers as a continuum: consuming, promoting, creating, serving, governing. The authors placed section 


\section{American Journal of Pharmaceutical Education 2019; 83 (5) Article 7654.}

and organizational contributions under each category and proposed numerical scores for each (Consuming 1; Promoting 2; Creating 4; Serving 5 or 6; Governing 10). The "height" and "score" of the 6 archetype personas tested through the model increased with the number of years passed since receiving an NIA. Dividing the total score by the number of years after receipt of the NIA resulted in a normalized, comparable score. Implications: This approach allows a comparable score for engagement with an organization and has the potential to allow for longitudinal analysis. Plans for a future study include an analysis of data of actual NIA recipients and the development of a national model for member engagement with organizations.

Mr. Chemistry Goes to Washington: A Case Study on Congressional Testimony. Andrew Coop, University of Maryland, Shannon R. Tucker, University of Maryland, Lisa Lebovitz, University of Maryland, Sandra N. Harriman, University of Maryland, Rebecca J. Ceraul, University of Maryland. Objective: To describe the experiences of a chemistry faculty member testifying on the opioid crisis for the US Senate Health, Education, Labor, and Pensions (HELP) Committee. Methods: The author is a medicinal chemist with expertise in the design and synthesis of new opioid analgesics with reduced abuse liability. In addition to publishing in high impact scientific journals, the author's specialty attracted public attention related to the current opioid crisis. He has given interviews for newspapers, national periodicals, television, and also panel discussions with the public regarding the science of drugs of abuse. This public exposure led to the author being invited to testify in front of the US Senate HELP committee, chaired by Senator Lamar Alexander. Written testimony was required to be submitted prior to the hearing. Results: Preparation for the testimony included discussions with the University's Government Affairs staff, the School of Pharmacy's Office of Communications and Marketing, the American Pharmacists Association (APhA), and other professional organizations. Testimony focused on the mechanisms of action of opioids, current development of effective non-addictive pain compounds, the need for more research into alternative pain management treatments, and the role of pharmacists in helping to ensure that patients who use opioids do so safely and effectively. It was a challenging and stressful experience to explain the science of drug dependency to the audience of laypeople. Implications: While chemistry expertise was essential in testimony, understanding the context of science to the profession of pharmacy, patients, and the public was important to provide context to laypeople influencing public policy.

\section{CONTINUING PROFESSIONAL DEVELOP- MENT}

Completed Research

An Analysis of Learning Styles of the 21st Century Pharmacy Student. Emily V. Olivier, The University of Tennessee. Objective: Recognition and alignment of learning styles for both students and faculty in classrooms has shown positive effects on learning outcomes. This study assesses for significant trends of learning styles among students transitioning from undergraduate studies into a Doctor of Pharmacy program. Methods: Individual, de-identified data on student learning styles was collected from the university's Office of Student Academic Support Services and Inclusion (SASSI), which administers the Inventory of Learning Styles Questionnaire (ILS-Q) to each first-year pharmacy student at the beginning of the PharmD program. Results of those students entering pharmacy school in Fall of 2017 and Fall of 2018 were analyzed for statistically significant differences across four categories of learning styles: active/ reflective, sensing/intuitive, visual/verbal, and sequential/ global. Results: Independent t-test data analysis for equality of means showed a significant difference in the first-year classes between Fall 2018 and Fall 2017 for the learning style categories of sensing vs intuitive (2-tailed significance of 0.022). These results suggest that the Fall 2017 group of students are more sensing learners, that is, they have a preference towards learning facts and being given information versus intuitive learners who prefer discovering information and relationships. No statistical differences were noted between classes for the remaining three categories. Implications: Results from this study suggest that a larger retrospective analysis of ILS-Q surveys of first-year pharmacy students may provide evidence for enhancing teaching and learning methods to adapt to an array of learning styles that provide an effective learning environment for current pharmacy students.

Application of Innovation and Entrepreneurship Competencies: A Capstone Project in Transition to the Profession Course. Hoai-An Truong, University of Maryland Eastern Shore, Marie-Therese Oyalowo, University of Maryland Eastern Shore. Objective: The 2013 Center for the Advancement of Pharmacy Education outcomes and 2016 Accreditation Council for Pharmacy Education standards include Innovation and Entrepreneurship as domain and competency for pharmacy graduates. The objective of this project is to describe the implementation of a capstone project on the application of innovation and entrepreneurship competencies in a Transition to the Profession required course for final professional year student pharmacists. Methods: A capstone group project was developed by incorporating outcomes 


\section{American Journal of Pharmaceutical Education 2019; 83 (5) Article 7654.}

on innovation and entrepreneurship, population-based care, problem-solving, pharmacoeconomics and practice management. Students were required to identify a pharmacy practice issue for intervention and narrow to a specific population based on literature search and health statistics. They had to conduct a Strengths, Weaknesses, Opportunities and Threats (SWOT) analysis, develop a specific, comprehensive plan for intervention, incorporate Pharmacist's Patient Care Process, propose a business plan, including a budget and funding sources. Written reports and presentations to faculty and students were also required. Results: Eighteen groups of 3 students developed and presented innovation and entrepreneurship projects with interventions on topics ranging from vaccination clinics to home visit, contraceptive clinic, cancer prevention program, medication reconciliation tool, phone apps for smoking cessation and medication adherence, foot care clinic and travel clinic. Proposed business plan and budget ranged from $\$ 200$ to $\$ 980,000$, with a total of $\$ 1.8$ million for all 18 groups of 54 students. Implications: Results suggested that students were innovative and entrepreneurial in developing capstone projects on pharmacy-based services for patient care with appropriate consideration of clinical, humanistic and economic outcomes.

CPD for CPD: Lessons Learned in Implementing a Professional Development Course Series. Kathryn A. Schott, Drake University, Cheryl L. Clarke, Drake University. Objective: 1) To describe lessons learned after the first year of implementation of a six-semester continuing professional development (CPD) course series designed to meet Standards 2016 related to personal and professional development; and 2) To determine the impact of course modifications in pedagogical approach and design on student-reported course effectiveness and confidence on CPD skills. Methods: In response to student feedback from IDEA Center course evaluations after the first year of implementation, course modifications were made including 1) fewer written reflection assignments; 2) integration of journaling into classroom activities; 3 ) increased small and large group discussion; 3 ) enhanced classroom culture of well-being; and 4) removal of technology from the classroom. IDEA Center course evaluations and a student self-assessment of confidence related to CPD skills were compared using independent-sample ttests for the first course in the series for the 2017 and 2018 fall semesters. Results: Significant improvements occurred for 32 of 40 evaluation elements, including factors measuring course and instructor excellence. Significant improvements in learning objective achievement were noted, including the development of teamwork skills and ability to express oneself. Mean confidence scores were significantly improved for 5 of 15 student selfassessment items including Writing SMART Goals, Receiving Feedback, Applying CPD, Working on an Interprofessional Team, and Developing your Personal Brand. Implications: Course modifications improved the majority of student-reported course effectiveness measurements and increased confidence in some skills. Responsiveness to student feedback is critical for improving learning outcomes and establishing best practices for the personal and professional development of students.

Describing an Immersive Leadership Elective Engaging Students in Self-Awareness and Unique Leadership Mindset Development. David G. Fuentes, California Health Sciences University, Miriam Ansong, California Health Sciences University, Ahmd Azab, California Health Sciences University, Wendy Duncan, California Health Sciences University, Yuqin Hu, California Health Sciences University, Jeremy Hughes, California Health Sciences University, Farid Khalafalla, California Health Sciences University, Lorilee Perry, California Health Sciences University, Shaowei Wan, California Health Sciences University, Yuwei Wang, California Health Sciences University. Objective: Students are expected to develop leadership skills in areas related to selfawareness as aligned with ACPE Standards and CAPE Outcomes. The opportunity to develop core leadership knowledge and familiarity with theoretical models behind leadership can be enriched with focused electives in this area. Herein, we describe an elective in leadership that walks through self-awareness exercises, organizational health aspects related to teams and teaming, and asks students to focus on the theme of developing a leadership mindset. Methods: Weekly sessions for P1-P3 students with pre-readings, in-class activities, and application of the PIE-RECAP reflection model are used to document student development and progress in their leadership mindset. Pre- and post-surveys are used in various sessions to provide students with additional time for reflection. Results: Student engagement and satisfaction results, as well as themes from student reflections on their most meaningful learning experiences will be shared. PIE-RECAP reflection themes will be connected to selfawareness and student reflection. Faculty and peerfeedback on reflections will be highlighted to show growth in the area of self-awareness, specifically highlighting how students reflected highly in the CAPE Outcome domain areas of self-awareness (4.1), communication (3.6), and leadership development (4.4). Implications: Programs reviewing this work will be able to take our elective and apply it within their elective or required curriculum, based on their programmatic objectives. 


\section{American Journal of Pharmaceutical Education 2019; 83 (5) Article 7654.}

Design of a Continuous Professional Development Course Series. Leigh A. Hylton-Gravatt, Virginia Commonwealth University, Emily P. Peron, Virginia Commonwealth University, Nicole E. Omecene, Virginia Commonwealth University, Jean-Venable R. Goode, Virginia Commonwealth University, Laura M. Frankart, Virginia Commonwealth University, Cynthia K. Kirkwood, Virginia Commonwealth University, Krista L. Donohoe, Virginia Commonwealth University. Objective: To describe the creation of a four-part longitudinal course series, Continuous Professional Development (CPD), that spans the Doctor of Pharmacy curriculum. Methods: Faculty recognized a need to systematically assess the affective domain elements of the Accreditation Council for Pharmacy Education Standards 2016. To accomplish this goal, the current Professionalism course was combined with the Mentoring and Advising Program for Students to track individual student professional growth throughout the Doctor of Pharmacy curriculum at Virginia Commonwealth University School of Pharmacy. The result is a four-part series of longitudinal courses that provide training in professionalism, career planning, ethical decisionmaking, and cultural sensitivity under the guidance of a faculty mentor. In addition, the course series documents student co-curricular experiences. Results: The CPD course series was implemented in Fall semester of 2018 for first, second, and third-year pharmacy students. Student curricular focus groups provided feedback which reflect a positive experience with the CPD courses after the first semester. Select student comments focused on improved faculty guidance and enhanced career development through the APhA Career Pathway Evaluation Program for Pharmacy Professionals. Students also endorsed the value of learning sessions about mindfulness, cultural sensitivity, and completing the CliftonStrengths ${ }^{\circledR}$ assessment. Reporting of required co-curricular experiences is ongoing with students submitting their activities via Google Forms. Implications: The CPD course series represents a successful framework for assessing the affective domains of Standards 2016 combined with co-curricular activity tracking. Results will need to be compared to methods used by other schools of pharmacy to determine best practices.

Effectiveness of an Interagency Collaboration on an Opioid Elective Course Offering. Jaqueline Y. Zavala, California Health Sciences University, Mandeep Rajpal, California Health Sciences University, Lina S. Vang, California Health Sciences University, Macie Luallen, California Health Sciences University, Michael Prichard, Fresno County Department of Behavior Health, Patty Havard, California Health Sciences University. Objective: Opioid overdose deaths in the United States have increased dramatically over the past decade. The epidemic has encouraged health professional programs to assess pain management curricula within their school. An opioid elective course (OEC) was offered at the California Health Sciences University (CHSU) in collaboration with the Department of Behavioral Health, Substance Abuse Disorders, and Aegis Treatment Center. The purpose of this study is to report the effectiveness of an interagency collaboration on an OEC. Methods: The contents focused on the epidemics of opioid use disorders (OUD) and deaths, harm reduction with naloxone and CURES, role of pharmacists in the epidemic, reducing the stigma associated with drug addiction, and medicationassisted therapy (MAT). A 5-Likert Scale survey was developed to assess knowledge and attitude on OUD and stigma. Results: A total of 32 students enrolled in the OEC, with 5/32 (16\%) P1, 18/32 (46\%) P2, and 9/32 (28\%) P3 students, and age ranging from 21 to 44 years. Of the "strongly agree" respondents, a total of 15/21 (71.4\%) of students rated that the OEC positively changed their viewpoint regarding patients suffering addiction; 25/ $32(78.1 \%)$ of students stated enhanced awareness on the role of pharmacists in minimizing abuse risks in patients; and, 24/32 (75\%) stated enhanced their knowledge on harm reduction benefit of naloxone in overdose reversal. Implications: Our data suggest that interagency collaboration for an OEC is effective in increasing pharmacy students' awareness to the opioid epidemics, role of pharmacists in reducing opioid misuse, and harm reduction.

Game-Based Interprofessional Education Event. Janna Roitman, Long Island University, Anna Nogid, Long Island University, Elizabeth Horsley, Brooklyn Hospital Center, Jane Shtaynberg, Long Island University, Joseph P. Nathan, Long Island University, Dominick Fortugno, Long Island University, Julius Johnson, Long Island University, Marion Masterson, Long Island University, Bojana Beric, Long Island University, Lisa Gordon, Long Island University. Objective: To actively engage LIU students in the health professions in a virtual game-based interprofessional educational (IPE) simulation event and assess the event's impact on students' attitudes toward working as an interprofessional healthcare team. Methods: In November 2018, over 500 students from LIU School of Health Professions, LIU School of Nursing, and LIU College of Pharmacy participated in a large-scale IPE event. A virtual game was developed by an interprofessional team of healthcare educators and reflective of patient population that students may come across. The game presented a virtual scenario of a homeless man found on the street who was encountered by the students. Interprofessional teams of students worked collaboratively to decide on care and treatment plans. 


\section{American Journal of Pharmaceutical Education 2019; 83 (5) Article 7654.}

Students actively engaged with each other through discussion while debating the benefits and limitations of each option. Students' attitudes toward IPE were measured using Interprofessional Attitude Scale (IPAS). The survey was administered pre- and post-activity. IRB approval was obtained. Results: Preliminary IPAS results indicate that after this event more than $90 \%$ of students felt well prepared to define teamwork and be an integral part of the interprofessional team. They expressed their confidence in explaining the roles and responsibilities of their own profession and other health professionals (more than $90 \%$ of students agreed or strongly agreed). Majority of students also agreed that this game taught them importance of patient-and community-centeredness and cultural diversity. Implications: An IPE game strategy was an effective modality of IPE. Based on the success of this program LIU is exploring further IPE initiatives.

Impact of Formative (Early) Assessment on Student Success. Henry North, Harding University, James M. Nesbit, Harding University, Melissa Max, Harding University, Shawn Turner, Harding University, Lana Gettman, Harding University, George Kwame Yeboah, Harding University, MaRanda K. Herring, Harding University, Landry Kamdem, Harding University. Objective: The goal of this study was to evaluate the correlation between personalized formative (early) assessment of student academic performance and successful progression in select courses of the pharmacy curriculum. Methods: Coordinators of didactic courses and faculty teaching first-, second- and third-year pharmacy students at Harding University College of Pharmacy were asked to administer a quiz or an exam during the first two weeks of the semester. Courses in the areas of basic biomedical and pharmaceutical sciences, social and administrative sciences, and clinical sciences were included in this study. The initial student academic performance was analyzed in relationship to pre-defined course level outcomes and instructional methods. Teaching and learning were modified and personalized based on the initial assessment data. Student academic performance was reevaluated on future quizzes and exams. Student success was defined as an improvement in quiz or exam scores and successful completion of a course. Results: The data demonstrates that students at academic risk can be identified very early on in the semester and proactive student remediation can begin. Also, based on students' strengths and weaknesses, teaching methods can be modified to help improve student performance and promote successful completion of the course. Implications: Our data suggests that the use of personalized formative (early) assessment in didactic courses leads to student success. Whether it has a similar impact on overall student progression in a pharmacy curriculum and/or on first attempt NAPLEX passing rates, remains to be elucidated.

Individual Development Plans for Tracking Longitudinal Progression of Personal and Professional Development and 5-Year Professional Goals. Margaret Schulte, Touro University California, Vista Khosraviani, Touro University California, Rae R. Matsumoto, Touro University California. Objective: To track longitudinal progression of pharmacy students' 5-year professional goals, knowledge and achievement of key elements of Personal and Professional Development using Individual Development Plans (IDPs). Methods: The Doctor of Pharmacy Class of $2020(\mathrm{~N}=84)$ completed IDPs during the first- (P1), second- (P2), and third-professional-year (P3) to identify career goals, evaluate skills, and formulate action plans to achieve goals. Four key elements of Accreditation Standards for Personal and Professional Development (Self-Awareness, Leadership, Innovation/ Entrepreneurship, and Professionalism) were scored, and student 5-year professional goals statements were categorized by study investigators, with data summarized using descriptive statistics. Results: Analysis of the IDP as a cumulative assessment tool showed growth in all elements; notably Leadership development (78.6\%) and attainment (61.9\%), and Innovation/Entrepreneurship development $(69.0 \%)$ and attainment $(23.8 \%)$ over the first three years of pharmacy school. In addition, SelfAwareness and Professionalism were addressed annually in nearly $100 \%$ of IDPs. The 5 -year professional goals showed a large proportion expressing plans for residency at least once $(87.0 \%)$, with $56 \%$ of students reporting the same goal, $52.4 \%$ adding specific details or expanding an original goal, $6.0 \%$ adding a back-up goal, and $31.0 \%$ switching goals over the three reporting years. Implications: IDPs can be used to track progression of personal and professional development in pharmacy students. Entering their final year, students recognize the need for Self-Awareness and Professionalism, but require further growth in attaining Leadership and Innovation/Entrepreneurship skills and finalizing career goals.

Needs Assessment for PGY1 Preceptors' Professional Development: A Cross-Sectional Study. Sara Mahmoud, Hamad Medical Corporation, Sara Hayder, Hamad Medical Corporation, Dania Alkhiyami, Hamad Medical Corporation, Hani Abdelaziz, Hamad Medical Corporation, Wessam Al Kassem, Hamad Medical Corporation, Palli Abdelrouf, Hamad Medical Corporation, Rasha AlAnany, Hamad Medical Corporation, Moza AlHail, Hamad Medical Corporation. Objective: The aim of this study is to assess the competency and professional development needs of preceptors for the PGY1 


\section{American Journal of Pharmaceutical Education 2019; 83 (5) Article 7654.}

pharmacy residency program (ASHP Accredited) at Hamad Medical Corporation, Qatar. Methods: Before the survey: All preceptors attended the Residency Program Design and Conduct (RPDC) PGY1 workshop conducted by the ASHP \& received orientation. Preceptor development assessment rubric was created based on ASHP criteria and literature review. The rubric was transformed to a self-assessment survey using online survey application which was sent to all PGY1 preceptors. Results: The survey included 16 questions for selfassessment. Emails were sent to 18 preceptors. Response rate was $94 \%(n=17)$ Being a pharmacy role model: On average, preceptors rated themselves highly at $4.29 / 5$ Preceptors showed deficiency in being able to provide written feedback. Teaching skills: All preceptors reported that they are efficient. However, lacked proficiency in changing preceptor roles. Preceptors responses varied in being able to provide tailored teaching strategies. Only 36\% rated themselves as excellent. A portion of preceptors $(17 \%)$ reported that they require improvement in being able to teach management skills. Other required topics included: critical conversation and management skills. Implications: On a global level, preceptor development requires significant improvement especially for pharmacy residency programs. In HMC, there are multiple areas for improvement such as: teaching strategies, writing feedback and presentation skills which will be addressed by designing a structured development program. There is a plan to create a certificate program specific to PGY1 preceptors

SMART Pharmacy Program: Changing Practice by Changing Education. Michael J. Rouse, Accreditation Councilfor Pharmacy Education, Arijana Mestrovic, Pharma Expert. Objective: Originally developed for the Turkish Pharmacists Association, the SMART Pharmacy Program has now been implemented or introduced in 12 countries. The Program is designed to be a sustainable, evidence-based initiative based on the Continuing Professional Development (CPD) Model. Its objectives are to enhance practitioners' competence, expand their scope of practice, improve the quality of clinical services, and positively impact patient and population outcomes. Beyond building knowledge and skills, the Program addresses motivation and commitment to change. Methods: Before implementation in a country, key stakeholders are invited to an initial workshop that includes a SWOT Analysis. National tools and frameworks are developed/ adapted and trainers trained. Pharmacists are trained in $\mathrm{CPD}$, other key principles and concepts, and a clinical module. Pharmacists self-assess their competence and quality of services, apply their learning to the care of patients, measure the results in a standardized way, and document everything in a portfolio. Results: Results have been achieved at the level of the individual pharmacist (improved competence; self-reported); the pharmacist's work setting (improved quality of service; self-reported using standardized indicators of quality); and the patient level (several measures in asthma/COPD and drug related problem identification, intervention, and resolution). In addition, positive impact has been observed at the national (profession-wide) level. Implications: Since its launch in Turkey (2015), over 5000 Turkish pharmacists have been trained in Asthma, Diabetes and Hypertension. In Oman, excellent results have been achieved in Asthma/ COPD and the Pharmacists Patient Care Process/Drug Related Problems. Further expansion of the Program is expected in 2019.

Student Self-Reported Co-Curriculars Prior to Implementation of a Formalized Co-Curricular Process. Leigh A. Hylton-Gravatt, Virginia Commonwealth University, Cynthia K. Kirkwood, Virginia Commonwealth University, Krista L. Donohoe, Virginia Commonwealth University. Objective: To assess baseline cocurricular data of students prior to implementation of a formalized co-curricular process at Virginia Commonwealth University (VCU). Methods: A Google Form was emailed out to all students in the P1-P3 year (Class of 2019-2021) in April of 2018 to assess participation in co-curricular activities. Students self-reported on the following information: professional organization membership(s), professional fraternity/sorority membership(s), current job status (\# of hours worked/month), and types of co-curriculars completed (education, communication, cultural sensitivity, self-awareness, leadership, innovation/entrepreneurship, professionalism) Results: All students $(n=370)$ completed the co-curricular Google Form (response rate: 100\%).During the $2017-2018$ year, $87 \%$ of students $(n=322)$ were a part of a professional organization. Students on average were a member of 2.65 professional organizations (range: 0-7). Sixty-eight percent of students $(n=252)$ worked in a pharmacy, and on average worked 28.7 hours/month (range: 5-96 hours/month). Almost all students (93\%) completed at least one cocurricular activity. Based upon class, $94 \%$ of $\mathrm{P} 1 \mathrm{~s}, 86 \%$ of $\mathrm{P} 2 \mathrm{~s}$, and $98 \%$ of $\mathrm{P} 3 \mathrm{~s}$ participated in at least one cocurricular activity. On average of those who completed co-curricular activities, students were involved in 5.8 co-curriculars/year (range: 0-20). The co-curricular activities that had the highest participation included self-awareness, cultural sensitivity, and professionalism activities. Implications: This baseline co-curricular data will be used to compare participation in co-curriculars after implementing a formalized co-curricular process 


\section{American Journal of Pharmaceutical Education 2019; 83 (5) Article 7654.}

requiring participation in designated types and number of co-curriculars throughout the year.

Use of Student Leaders in Implementation of CoCurricular Activities. Meri D. Hix, Southwestern Oklahoma State University, Sarah J. Ramsey, Southwestern Oklahoma State University. Objective: To describe student-led development and execution of large-scale, professional development events Methods: Since 2014, students from Southwestern Oklahoma State University (SWOSU) College of Pharmacy's Phi Lambda Sigma (PLS) chapter have chaired events to aid in improved chemical dependency awareness and leadership skills development. The intent of the selected topics is to be specific to the interests of the students themselves. Not only are the events used for professional development of the attendees, the chairs also practice and develop skills that may be useful in future endeavors. Student chairs are expected to procure speakers, reserve venues, determine formats, communicate with stakeholders, advertise, and host the events. Results: Now in their fifth year, these events have become large-scale, entirely organized by the student chairs. The chemical dependency seminar has evolved into community-wide event and requires additional attention due to the varied audience. The leadership seminar continues to be fully attended and has included topics such as personality assessments, discussion with pharmacy leaders, and effective communication. Both events count toward co-curricular participation and have varied in topic, format, and speakers to stay relevant to the current interests of our students. Student chairs have practiced leadership skills, overcome personal obstacles, revised their anticipated tasks. Implications: Student leaders can be used in unique channels to further the mission of a college of pharmacy. In so doing they have an opportunity to practice personal leadership and organizational skills while providing a service to their co-students and the community.

\section{EXPERIENTIAL EDUCATION \\ Completed Research}

Addition of a Hospital Unit-Based Experience to an Internal Medicine 1 Advanced Pharmacy Practice Experience. Janet R. Fischer, South Dakota State University, Patrick E.R. Watchorn, South Dakota State University. Objective: The primary objective of this study was to evaluate perceptions of fourth year pharmacy students regarding the inclusion of 2 to 3 weeks of a hospital unit-based experience (UBE) during their Internal Medicine (IM) 1 Advanced Pharmacy Practice Experience (APPE). The UBE was designed to provide students with more hands-on practice with pharmacokinetics, note writing, and medication dose adjustments in renal dysfunc- tion. Methods: Typical IM students round with a team of providers for five weeks. This model divided the rotation into rounds with providers and assisting a pharmacist on a decentralized unit, each for 2-3 weeks. Both activities were supervised by faculty. At the end of the five-week rotation, students were invited to complete a survey. Results: Sixteen of 24 students involved completed the survey. Students agreed each experience was valuable for learning: $93.8 \%$ for rounding, and $87.5 \%$ for UBE. Each experience taught them skills and knowledge they would not have learned in the other experience, with $81.3 \%$ in agreement for rounding and $68.8 \%$ in agreement for UBE.UBE specifically improved their skills in pharmacokinetics, evaluating dosages, and antibiotic stewardship. Seventy five percent of students felt that splitting the rotation between the two experiences would be most beneficial for future IM rotations. Implications: Adding UBE to traditional rounding on IM APPEs results in a high level of satisfaction from pharmacy students and allows them to gain additional skills compared to rounding. Future rotations should be designed in a similar manner to improve satisfaction and increase learning experiences for students.

A New Rubric: Using Entrustable Professional Activities (EPA) to Track Student Progression in Experiential Rotations. Elizabeth Trolli, The Ohio State University, Donald Sullivan, The Ohio State University. Objective: Experiential activities bridge student learning from didactic coursework to clinical practice. The goal of this project was to analyze a newly updated evaluation providing clinical preceptors the ability to access PharmD students' progression toward attaining set EPA goals. Methods: The pilot was completed in 2018 with 105 second year PharmD students. The experiential team mapped clinical activities completed on rotation to specific EPAs resulting in twenty-three questions focused on clinical practice. Eight questions were selected specifically for this study as they correlated directly to the Pharmacists' Patient Care Process (PPCP). Preceptors were trained on EPAs and the PPCP. The evaluation rubric was divided into five levels allowing the preceptor and learner to categorize the amount of supervision the student needed. The tiers of the possible responses started at one (student observe action only) up to a maximum of five (student could supervise junior colleagues). Results: Upon review of the data for the eight targeted questions, the average preceptor score for the level of current student progress towards the EPA was a 2.50/5.0. In comparison, the student self-evaluation response for the same eight items scored an average of 2.49/5.0. The score averages supported the projection score average of 2.5 for the pilot, confirming that EPA focused evaluations can be used to follow progression of students. Implications: Colleges 


\section{American Journal of Pharmaceutical Education 2019; 83 (5) Article 7654.}

and schools of pharmacy can use this method to track student movement towards confident performance of EPAs. Specifically, in experiential, educators can use this method to determine and identify areas of growth and where digress is occurring.

An Interprofessional Simulation: Reducing Medical Errors in Transitions of Care. Crystal Burkhardt, The University of Kansas, Candice Coffey, The University of Kansas, Stephen Jernigan, Sarah Shrader, The University of Kansas, Ashley Crowl, The University of Kansas, Dory Sabata, The University of Kansas, Jessica Kalender-Rich, The University of Kansas. Objective: Develop an interprofessional (IP) simulation that allows pharmacy and medical students to increase their confidence in medical error disclosure. Methods: A simulated educational experience for pharmacy and medical students to emphasize the importance of IP collaboration in error disclosure was developed and implemented using standardized patient actors (SPA). All students completed pre-learning regarding medical error disclosure. Medical students also completed a hospital discharge simulation while pharmacy students completed a medication reconciliation based on a recorded community pharmacist medication therapy management (MTM) encounter and provided patient's discharge medical information. Pharmacy and medical students communicated via a telehealth conference encounter to correct errors and optimize medications during the care transition. Faculty debriefed students regarding their IP experience following the simulation. Quantitative data from a student completed pre-/post-simulation 5-point Likert scale survey were evaluated. Qualitative data from the debriefs were evaluated for thematic findings. Results: Pharmacy and medicine students completed the pre-simulation survey $(\mathrm{N}=173)$ which was compared to a pilot group of students who completed the post-simulation survey $(\mathrm{N}=27)$. Students reported statistically significant improvements in their confidence with disclosing medical errors and in use of telehealth technology to communicate with other professionals $(\mathrm{p}<0.005)$, and improved understanding of MTM as performed by the pharmacist $(p<0.005)$. Themes of team structure, leadership, and communication emerged in the qualitative review. Implications: This simulation afforded pharmacy and medical students the opportunity to increase their confidence in communicating an error, gain insight into telehealth as an alternative form of IP team communication, and increase their understanding of MTM provided by community pharmacists.

Assessing Patient Advocacy Through Advanced Pharmacy Practice Experience (APPE) Student Interventions. Gretchen Jehle, MCPHS University-Worcester/ Manchester, Kara Bonaceto, MCPHS University-
Worcester/Manchester, Nicole Carace, MCPHS University-Worcester/Manchester, Brianne Morin, MCPHS University-Worcester/Manchester. Objective: Review opportunities students engage in during APPE rotations to advocate for patient's health and wellness. Methods: While on APPE rotations, from August 2017 through May 2018, students documented interventions using a field encounter in CORE ELMS. Preceptors were able to review and confirm the student's field encounter and provide feedback. A summary report was created by The Office of Experiential Education to review the categories of interventions related to patient advocacy. Following review, interventions related to patient advocacy were grouped as health and wellness, financial, or medication management. Results: Four hundred twelve intervention field encounters were completed by students. Of the documented interventions, $61.3 \%$ were categorized as health and wellness, $1.6 \%$ were financial, and $28.5 \%$ were medication management. The remaining interventions, $8.6 \%$, were not related to patient advocacy. Implications: Center for the Advancement of Pharmacy Education (CAPE) Outcomes have been adopted as our curricular outcomes for the School of Pharmacy. CAPE 3.3 states that students advocate for the patient's best interests. APPE rotations provide opportunities for students to advocate for patients and completion of interventions allow for review of outcome assessment data to support compliance with Accreditation Council for Pharmacy Education (ACPE) Standard 3.Future implications include an opportunity for students to recognize and reflect on their role in patient advocacy as it relates to Standard 4.1 (Self-awareness).

Assessment of APPE Grading Schemes Used by ACPE Accredited Pharmacy Schools and Colleges. Jane Shtaynberg, Long Island University, Maryann Z. Skrabal, Creighton University, C. Leiana Oswald, Roseman University of Health Sciences, Cheryl L. Clarke, Drake University, Angela S. Clauson, Belmont University, Eric H. Gilliam, University of Colorado, Jennie B. Jarrett, University of Illinois at Chicago, Tina Kanmaz, St. John's University, Jennifer Prisco, MCPHS UniversityBoston, Valerie L. Ruehter, University of MissouriKansas City. Objective: To determine APPE grading schemes across ACPE accredited programs, their perceived attributes, and correlation to residency placements. Methods: A 12-question survey was developed and disseminated to schools/colleges of pharmacy through the Experiential Education Section (EES) discussion board on AACP Connect in October 2018.Nonresponders were contacted via email and phone with at least three attempts made to reach each non-responding program. Information provided by the American Society of Health Systems Pharmacists (ASHP) regarding PGY1 


\section{American Journal of Pharmaceutical Education 2019; 83 (5) Article 7654.}

and PGY2 residency match rates for 2016-2018 was correlated to survey responses. De-identified data was analyzed using descriptive statistics, logistic regression models, and the FREQ procedure. Perceptions of scheme attributes were analyzed via thematic analysis. Study was determined by IRB as non-human research. Results: Responses were received from 122/140 (87\%) pharmacy programs. Forty-nine percent of respondents represented public institutions, sixty percent indicated they use letter grades compared to forty percent who use a pass/fail grading scheme or "other." The use of either grading method did not differ statistically when comparing public to private institutions, with roughly thirty percent of each using a pass/fail scheme. Programs employing pass/fail grading were more likely to have students matched to PGY1 residencies (p-value $<0.001$ ) than programs using letter grading. There was no correlation between APPE grading and PGY2 placement during the same time period. Implications: This data represents APPE grading schemes across the country and supports that Pass/Fail grading does not hinder residency placements. Data may be used by pharmacy and residency programs to determine optimal assessment and candidate considerations.

Assessment of Volunteer Preceptors at Colleges and Schools of Pharmacy in the US. Jane Shtaynberg, Long Island University, Tina Zerilli, Long Island University, Harold L. Kirschenbaum, Long Island University. Objective: To identify the manner in which colleges and schools of pharmacy in the US assess the performance of volunteer preceptors who precept students in advanced pharmacy practice experiences (APPEs). Methods: In summer/fall 2018, directors of experiential education (or equivalent title) at 140 colleges/schools were invited to complete an online, self-administered questionnaire. Several attempts were made via email and phone to encourage participation. Data were analyzed using SPSS (version 25). IRB exempt status was received. Results: Eighty-one (61\%) of 132 fully accredited programs responded ( 8 programs in candidate status were excluded); all but one evaluate the performance of APPE volunteer preceptors. $49 \%$ of respondents reported that preceptors can be promoted in rank, while $59 \%$ noted there is a process to re-appoint preceptors. $99 \%$ of respondents use the same volunteer preceptor evaluation criteria regardless of practice location. The most common attributes assessed by respondents were the preceptors' accessibility, ability to provide feedback to students, communication skills and professional demeanor. The most commonly reported assessment strategies were summative student evaluations (100\%), on-site evaluation of the preceptor by college/school personnel (86\%), formative student evaluations (40\%), student focus groups (37\%) and assessment of attendance/participation in required preceptor development programs (37\%). Implications: Virtually all colleges and schools of pharmacy assess volunteer APPE preceptors. Given the important role of volunteer preceptors in ensuring students are prepared for pharmacy practice, the information provided here will allow colleges/schools to review their assessment strategies keeping in mind the methodologies used by other institutions.

A Sustainable Group Mentoring Program for Professional Student Career Planning. Daniel Kudo, Keck Graduate Institute, Gregory Reardon, Keck Graduate Institute. Objective: Formalized didactic and experiential courses help students to develop knowledge and skills needed to matriculate as practice-ready pharmacists. However, feedback from senior students and alumni suggests that the professional curriculum does not suffice for adequate career exploration. To address CAPE Outcome 4 (personal and professional development), this longitudinal intervention uses a faculty mentor as coach for a stable group of students to identify areas of key interest and potential career paths that align with the fouryear professional program. Methods: This co-curricular group mentoring process begins in the first semester of the P1 year with opportunities for drop-in during later years. Key elements of this group mentoring process include open voluntary participation, expressed commitment to continue active involvement, weekly-scheduled lunchtime group sessions, investigation of postgraduate and career choices with presentation of findings by individual mentees to the full cohort, opportunities for leadership, and direct involvement in advocacy legislative initiatives and with professional associations. Results: This group mentoring program has completed the first three years of operation. Student feedback is favorable, particularly regarding opportunities to examine careers with initial perceived affinity, contrasted with those initially considered to be "undesirable." This process has challenged preconceived assumptions of students regarding career choices, with several students changing their initially-selected career path prior to graduation. Formalized assessment of success of this mentoring program continues. Implications: An individualized approach like this one permits pharmacy schools to achieve effective career mentoring with economies of scale, when compared to traditional one-on-one mentoring.

A Three-Year Analysis of Clinical Track Participation and PGY1 Residency Pursuit and Match Success. Emily Witcraft, University of Maryland, Patricia A. Ross, The Johns Hopkins Hospital, Hyunuk Seung, University of Maryland, Agnes A. Feemster, University of Maryland. Objective: The objective was to examine the 


\section{American Journal of Pharmaceutical Education 2019; 83 (5) Article 7654.}

influence of clinical track participation on Post-Graduate Year (PGY)-1 ASHP Residency pursuit and Match success. Methods: Students graduating between 2016-2018 were included. Residency match information was obtained from ASHP. Students were categorized as participants in the Match if they ranked programs prior to the submission deadline. The Fisher's exact test was performed, and logistic regression analysis was applied for the overall study period adjusting for age, gender and grade point average (GPA). Odds ratio (OR) and 95\% confidence interval $(95 \% \mathrm{CI})$ were calculated. Results: A total of 164 students participated in the ASHP Residency Match during the study period. $72.5 \%$ were female and the mean student age was $22.6 \pm 3.0$. The mean student GPA was $3.53 \pm 0.26 .91$ (19.9\%) students participated in the clinical track program of whom 78 $(85.7 \%)$ pursued residency and $64(82.1 \%)$ successfully matched. Clinical track participants were more likely to pursue a residency than non- participants $(\mathrm{OR}=19.6,95 \%$ $\mathrm{CI}=(10.3,37.0))$. In multivariate analysis, the most significant predictor of match success was clinical track participation $(\mathrm{OR}=6.6,95 \% \mathrm{CI}=(2.9,14.6))$. Age and gender did not influence match success $(\mathrm{OR}=0.9,95 \%$ $\mathrm{CI}=(0.8,1.1))$ and $\mathrm{OR}=1.7,95 \% \mathrm{CI}=(0.7-4.0))$; however, GPA may be associated with match success $(\mathrm{OR}=1.3,95 \% \mathrm{CI}=(1.1-1.6))$. Implications: Clinical track students were highly motivated to pursue a PGY1 residency. While other studies have explored the impact of non-modifiable characteristics on match success, we associate clinical track participation and higher GPA, modifiable factors, with increased success in the PGY1 residency match.

Case Study for Addressing Leadership and Innovator ACPE and CAPE Outcomes in an Independent Study. Ian Watson, Drake University, Erin Ulrich, Drake University, Pramod B. Mahajan, Drake University. Objective: To develop a research leadership educational opportunity and determine what Accreditation Council for Pharmacy Education (ACPE) and Center for Advancement of Pharmacy Education (CAPE) standards are achieved. Methods: A novel organizational structure was created within the context of completing an entrepreneurial grant. In an independent study course, the pharmacy student managed two undergraduate health science research assistants and reported to two faculty investigators. Pharmacy student tasks included: scheduling weekly meetings, identifying tasks to be completed, delegating tasks, setting deadlines, managing undergraduate assistants through the entrepreneurial process, and sending update emails to investigators. At the end of this educational activity, the pharmacy student was asked to measure their perceived growth in terms of ACPE and CAPE standards with Likert response options. The student was asked to provide further detail on what areas they valued and how this opportunity aligns with the brand they want to create for post-graduate job interviews. Results: The student survey demonstrated a positive attitude toward their role on the research team and reflected personal growth. On a Likert scale the student indicated perceived growth in ten objectives under CAPE Outcomes 4.2, Leadership, and 4.3, Innovation and Entrepreneurship, and two elements under ACPE accreditation Standard 4. Student reported ability to develop personal brand for post-graduate job applications. Implications: Integrating students into unique co-curricular activities addresses building skills in the standards, allows them to develop their brand, and assists faculty in research. Pharmacy schools can follow a similar organizational structure in independent studies, co-curricular activities, or capstones.

Characterization or Preceptor-Assessed APPE Competency Deficiencies to Inform Pre-APPE Curricular Revision. Sandra W. Rosa, Albany College of Pharmacy and Health Sciences, Laurie L. Briceland, Albany College of Pharmacy and Health Sciences, Megan Veselov, Albany College of Pharmacy and Health Sciences. Objective: To Characterize APPE competency deficiency such that processes can be standardized to promote competency on rotation as well as drive didactic (Pre-APPE rotation) curriculum changes. Methods: For the 2018-19 APPE cycle, preceptor-assessed competency deficiencies of "Needs Improvement" (NI) and "Significant Deficiency" (SD) were collated under primary competency domain (Pharmacist Patient Care Process (PPCP), Communications, Professionalism, and Self-Directed Lifelong Learning (SDLLL)) and rotation type (Community, Ambulatory Care, Institutional, Inpatient and Elective) to characterizes areas in most need of improvement. Results: For 224 students on 1568 APPEs, competency deficiencies were noted under PPCP (175); SDLLL (110): Communications (52); and Professionalism (23). Deficiencies noted per rotation type follows: Inpatient (119); Institutional (58); Ambulatory Care (65); Community (65); and Elective (53). Implications: APPE competency deficiencies are evidenced across all rotation types and assessment domains, with Inpatient APPEs and PPCP being the most prevalent. Review of didactic curriculum focused on application of knowledge (i.e. Integrated Problem-Solving and Pharmacy Skills courses), specifically in the Inpatient setting, may identify opportunities for improvement. While the domains SDLLL, Communication, and Professionalism may be better remedied on an individualized student reflection basis, focused teaching of the PPCP during didactic 


\section{American Journal of Pharmaceutical Education 2019; 83 (5) Article 7654.}

curriculum could result in a $50 \%$ decrease in rotational deficiencies. Additional breakdown of deficiencies (i.e. number of competency domain deficiencies for each rotation type) should also be characterized for more specific curricular revision implementation. Pre-APPE readiness efforts can be streamlined to meet the demonstrated APPE deficiencies.

Comparison of Full-Time Faculty Preceptor and Volunteer Preceptor Evaluations of APPE Students. Ryan S. Ades, Manchester University, Ashley Cross, Manchester University, Sara N. Trovinger, Manchester University. Objective: To determine if students with volunteer preceptors get a different evaluation experience than students with faculty preceptors. Methods: A retrospective analysis of preceptor evaluations of student pharmacists enrolled acute care or ambulatory care APPEs from June 2016 to May 2018 was completed to determine if faculty preceptors were more likely to give non-passing scores on evaluation questions as compared to volunteer preceptors. Results were analyzed using a chi-square test. Results: 353 acute care and 303 ambulatory care rotations were included in the evaluation. $80 \%$ of the 20 preceptor evaluation questions of student pharmacists did not show a statistically significant difference when comparing full time faculty and volunteer preceptors. "No Pass" grades were assigned at the same rate for volunteer and faculty preceptors in ambulatory care rotations. Faculty preceptors were more likely to assign a student a grade of "No Pass" in acute care settings than volunteer preceptors. Implications: These results are mixed concerning the difference in rigor between faculty and volunteer precepted rotations. While individual questions are evaluated mainly the same across both groups, there was a difference found in the final grade assignment for students in the acute care setting. This difference was not noted in the ambulatory care setting. More research is needed to determine the need for faculty preceptors as quality assurance for APPE students.

Critical Thinking: Exploring Student and Preceptor Perceptions and Expectations. Teresa A. O'Sullivan, University of Washington, Ali Lois Icenogle, University of Washington, Alexander Tu, University of Washington, Rachel A. Allen, University of Washington. Objective: Pharmacy preceptors are frequently asked to assess student critical thinking skills, yet evidence is lacking about how preceptors define critical thinking or how pharmacy students' and preceptors' definitions of critical thinking align. A qualitative study was conducted to better understand elements of critical thinking identified by preceptors and students with the objective of improving how this skill is developed and assessed across the curriculum. Methods: Students and preceptors were surveyed about how they define critical thinking. Replies to these questions were thematically analyzed. Data analysis continued until saturation, where no new themes emerged from additional responses. Investigators independently conducted initial analyses, resolving discrepancies in theme identification until consensus was reached on the final themes. Results: Preceptor and student data yielded three themes: collecting and assimilating data, assessing data, and decision-making. Student data yielded a fourth theme of needing extensive practice in critical thinking. In collecting data, students noted using multiple information sources, while preceptors focused on asking additional clarifying questions. In the assessment theme, both students and preceptors identified deep analysis as an important element of critical thinking. Deep analysis included considering multiple perspectives when problem-solving; anticipating outcomes and implications of decisions; and weighing the appropriateness and acceptability of various solutions. When decision-making, students and preceptors emphasized the importance of a well-reasoned thought process, even in the absence of complete data. Implications: These data, although from a single institution, will better define how students articulate their clinical reasoning and decision-making process and preceptors' model and assess critical thinking.

Designing Scholarship Opportunity for Students Participating in International Advanced Pharmacy Practice Rotations. Stephanie Lukas, St. Louis College of Pharmacy. Objective: Design an opportunity to showcase and expand student learning on international Advanced Pharmacy Practice (APPE) rotations through a mentored scholarship opportunity. Methods: Four to five weeks prior to departing for their international APPE rotation, one-on-one meetings were set with students to describe the optional scholarly poster activity in detail. Those interested in participating were given further instructions, tips and templates and had regular communication with a mentor. All international preceptors were invited to participate, and students could be mentored by a preceptor or an Office of International Programs (OIP) faculty member. Posters were usually completed by the end of their five-week rotation. Results: Between May 2018 and January 2019, 14 of the 34 international APPE students participated $(41.2 \%)$, creating 7 posters. Two of the ten preceptors participated and took on the role of mentor; the remaining posters were developed with the support of OIP. Most of the students who participated were considering a residency or a research-related career. All posters were (or will be depending on the month of the APPE) presented at St. Louis College of Pharmacy's International Experiences Symposium. Three posters were also presented at a regional global health conference. 


\section{American Journal of Pharmaceutical Education 2019; 83 (5) Article 7654.}

Implications: International APPE rotations are a unique opportunity for students to learn not only about culture but to compare and contrast healthcare systems and in some cases be involved in research. If done with enough forward planning, it can also be an opportunity for students to expand their scholarly knowledge and experience and to create a CV-worthy product.

Development and Assessment of a Standardized Preceptor Encounter in an Introductory Practice Experience Course. Anna Kabakov, Midwestern University/Downers Grove, Kelly Lempicki, Midwestern University/Downers Grove, Jill S. Borchert, Midwestern University/Downers Grove. Objective: Simulations assist in preparation for experiential rotations. While standardized patient-based simulations are well described, a standardized preceptor encounter modeling the day-today presentation of a patient to a preceptor has not been described. Our objective was to develop and assess a standardized preceptor encounter in a clinical Introductory Practice Experience (IPPE), the third course in a 3-course sequence offered to a portion of the class each quarter from Spring of the second professional year through Winter of the third year. Methods: During a mid-quarter and final standardized preceptor encounter, students reviewed an electronic medical record, then presented a patient to a preceptor. The preceptor assessed content, style, professionalism, and answers to on-the-spot questions. Students were then given additional questions to research and document. Performance was summarized from mean scores from Spring 2018 through Winter 2019. Student perceptions of preparedness were summarized from post-event surveys. Results: Mean scores on the patient presentation to the preceptor were $93.8 \%(\mathrm{n}=176, \mathrm{SD} 6.3 \%)$ for the mid-quarter event and $92.0 \%(n=178, \mathrm{SD} 9.4 \%)$ for the final event. Feedback from students indicated that the mid-quarter encounter prepared them for their IPPE rotation $(n=30,77 \%)$ and the majority $(n=37,94.9 \%)$ reported presenting patients during their IPPE rotation. Overall, students felt more prepared for APPE rotations after performing the simulations $(n=29,74 \%)$. Implications: Overall, students performed well on the standardized preceptor encounter and felt prepared for rotations through classroom and simulation activities. Other institutions may explore standardized preceptor encounters in order to prepare students for this important component of experiential education.

Effect of Elective Pharmacy Courses on Student Performance During Advanced Pharmacy Practice Experiences. Matthew A. Wanat, University of Houston, Kimberly A. Nguyen, University of Houston, Nancy D. Ordonez, University of Houston, J. Douglas Thornton, University of Houston. Objective: It is unknown whether taking an elective didactic course improves performance on related Advanced Pharmacy Practice Experiences (APPEs). The objective of this study was to compare performance on select elective APPEs based on whether students completed related elective didactic coursework. Methods: P4 students at University of Houston College of Pharmacy who completed a Critical Care or Pediatrics APPE from 2014-2018 were included. Students were grouped based on whether they completed either the Critical Care (offered to P2) or Pediatrics (P1/P2) elective course. Overall student performance on the APPE rotation, and seven performance variables were recorded (Likert scale 1-5). Scores were compared using an unpaired t-test (alpha $=0.05$ ). Results: A total of 151 students completed an elective APPE in Critical Care or Pediatrics, with 56 (37\%) completing the related didactic course. There was no difference in total rotation grade in students completing the didactic elective course versus those that did not $(88.3 \%$ vs. $89.8 \%, p=0.24)$. Students performed similar in their ability to communicate with healthcare providers $(4.4$ vs. $4.4, \mathrm{p}=0.88)$, knowledge of guidelines ( 4.3 vs. $4.3, \mathrm{p}=0.51)$, and disease state knowledge base ( 4.2 vs. $4.3, \mathrm{p}=0.28$ ). Students who did not take an elective course performed better in their ability to prioritize patient problems (4.3 vs. $4.5, \mathrm{p}=0.036)$. Implications: There was no difference in overall APPE performance and most individual outcomes in students who took a didactic elective course during their P1/P2 year versus those that did not. This data supports our curriculum redesign decision to move elective courses to the P3 year, closer to APPEs.

Evaluating Student Perceptions Using Two Interprofessional Assessment Instruments Following Didactic and Clinical Experiences. Vincent C. Dennis, The University of Oklahoma, Melissa A. Craft, The University of Oklahoma, Dale W. Bratzler, The University of Oklahoma, Denise G. Bender, The University of Oklahoma, Christi Barbee, The University of Oklahoma, Melody J. Yozzo, The University of Oklahoma, Stephen Neely, The University of Oklahoma, Margaret Robinson, The University of Oklahoma. Objective: To assess and compare changes in student perceptions through administration of two validated interprofessional survey instruments before and after didactic vs. clinical cohorts. Methods: Students from seven colleges/schools participated in didactic and clinical cohorts during the 20172018 year. Didactic cohorts experienced two interactive sessions six months apart, while clinical cohorts experienced four outpatient clinical sessions once monthly. Baseline and post assessments included random assignment of 865 students to complete either the 14 item Collaborative Healthcare Interdisciplinary Relationship 


\section{American Journal of Pharmaceutical Education 2019; 83 (5) Article 7654.}

Planning (CHIRP) instrument or the 27 item Interprofessional Assessment Scale (IPAS). Pittman's test using permutations of linear ranks was used to determine differences in distribution of scores between baseline and post assessments. Comparisons of pooled results were made for the CHIRP total score, and IPAS total and subdomain scores. For each score, three comparisons were made simultaneously: 1) overall baseline vs. post-didactic cohort; 2) overall baseline vs. post-clinical cohort; and 3) post-didactic cohort vs. post-clinical cohort. Alpha was adjusted to 0.0167 to account for simultaneous comparisons. Results: Baseline and post-cohort survey responses were 62.4 and $65.9 \%$ vs. 58.7 and $58.1 \%$ for CHIRP and IPAS, respectively. Students completing the clinical cohort had significantly higher scores for the IPAS subdomain of teamwork, roles and responsibilities in relation to baseline and post-didactic cohort scores. No differences were seen for the remaining IPAS subdomain scores or CHIRP instrument total score. Implications: The IPAS instrument may discern changes in student perceptions in the subdomain of teamwork, roles and responsibilities following short-term clinical experiences involving diverse interprofessional team members.

Factors that Influence Advanced Pharmacy Practice Experience (APPE) Selection - 1 Year Later. Vicky Shah, Wilkes University, Kristina Powers, Wilkes University. Objective: To assess student perception of factors potentially influencing Advanced Pharmacy Practice Experience (APPE) selections. Methods: Wilkes University Pharmacy students are required to complete 1440 hours of experiential education during their final year of pharmacy school. Students must choose from seven rotations which include ambulatory, community, inpatient general medicine, hospital/health system and three electives. Third (P3) year professional students completed an anonymous survey to determine what factors impact their decision process when selecting rotations. Factors include area of interest, size of institution, location, future employment, preceptor reputation, rotation hours, faculty rotation, non-faculty rotation, peer recommendation, cost/housing, level of difficulty, size of institution, and if a residency is offered. The students ranked up to five top factors which influenced their required and elective APPE selection. Results: 93\% of P3 (68) students responded to the survey. The class selected location as the number one factor which influenced their decision for selecting required rotations. The second most important factors were Area of Interest and Cost/Housing for Ambulatory Care and Community rotations respectively. Peer recommendation was second most important for P3's for Internal Med and Institutional rotations. Areas of interest in specialty was selected as the most important factor for selecting elective APPEs. Implications: Location appears to be the driving factor for selecting required APPE rotations. It would be beneficial to create a map of rotation sites showing the location of the sites in relation to campus, along with the travel time. Further research into the factors that influence ranking on APPE electives is warranted.

Faculty Perceptions of a Town Hall Model for Engagement in Data-Driven Decision Making. Lisa M. Meny, Ferris State University, Mandy R. Seiferlein, Ferris State University, Aleda M. Chen, Cedarville University, Elizabeth Wandling, Cederville University. Objective: The AACP Curriculum Quality Surveys (CQS) are useful tools for gathering data for programmatic assessment and accreditation. Involving faculty in the analysis process can be beneficial for interpretation and continuous quality improvement (CQI). One method to engage faculty is the use of a town hall model. The objective of this project was to determine faculty perceptions of participating in an AACP Faculty CQS Town Hall. Methods: At two institutions, a standard town hall structure was implemented. Visual CQS data reports were created and distributed to faculty prior to the town hall. Items were flagged with star ratings (red: high, yellow: moderate, green: low) based on percent faculty agreement utilizing pre-determined benchmarks. A Town Hall session was held to gain faculty feedback regarding what it would take to "agree" with flagged statements, thereby engaging them in the interpretation of the data for decision making. Pre- and post-surveys (5-point Likert-type) were completed anonymously by faculty and assessed using a Mann-Whitney U test. Results: Faculty $(\mathrm{N}=48$ pre-survey, 26 post-survey) had significant improvement $(p<0.05)$ in their awareness of the types of data used for CQI, how data are used, and their opportunity to participate in the data use process. Faculty agreed they had opportunities to provide feedback and felt comfortable engaging in the data use process $(\mathrm{p}>0.05)$. Faculty strongly agreed (median $=5)$ the Town Hall model was a good use of their time. Implications: Faculty perceived the town hall model as a positive approach to CQI, and discussions provided assessment personnel with valuable information for data interpretation.

Global Health Learning Outcomes by Country Location and Duration for International Experiences. David R. Steeb, University of North Carolina at Chapel Hill, Monica L. Miller, Purdue University, Ellen M. Schellhase, Purdue University, Jodie V. Malhotra, University of Colorado, Jacqueline McLaughlin, University of North Carolina at Chapel Hill, Sarah A. Dascanio, University of North Carolina at Chapel Hill, Stuart T. Haines, The University of Mississippi. Objective: To 


\section{American Journal of Pharmaceutical Education 2019; 83 (5) Article 7654.}

analyze student learning outcome differences by country location and duration for international Advanced Pharmacy Practice Experiences (APPE). Methods: A mixed-methods study evaluated fourth-year pharmacy students who participated in an international APPE at the University of North Carolina at Chapel Hill, Purdue University, and University of Colorado. A pre-post survey was administered to evaluate self-perceived growth across 13 Consortium of Universities for Global Health (CUGH) competencies with additional open-ended questions and focus groups. Qualitative survey and focus group data went through a two-cycle open coding process using a conventional content analysis approach. Quantitative data were analyzed using independent and paired t-tests and multiple linear regression. Results: Eighty-one students completed the survey with 22 opting to participate in the additional focus group. Students who went to a low-to-middle income country (LMIC) $(n=48)$ had significantly greater growth in all CUGH statements compared to those who went to a high-income country $(n=33)$. Rotation location in a LMIC and prior nonvacation travel were significant predictors of growth in the regression model. Qualitative themes for students going to a LMIC suggested cultural progression, skill development, and altered patient care perspective while students going to a high-income country showed increased knowledge of healthcare system differences, pharmacy practice and education, and an appreciation for alternative patient care approaches. There were no differences for rotation duration. Implications: While learning outcomes differed between high-income and LMIC APPE locations, both provided valuable learning opportunities that contributed to personal and professional development with greater growth among students in LMIC experiences.

How May We Help You? Experiential Education Administrators' Solutions to Common Preceptor and Student Issues. Lana M. Minshew, University of North Carolina at Chapel Hill, Charlene Williams, University of North Carolina at Chapel Hill, Lisa M. Dinkins, Wingate University, Wesley R. Haltom, Wingate University, Kathryn Paige D. Brown, Campbell University, Jacqueline McLaughlin, University of North Carolina at Chapel Hill. Objective: The purpose of this study was to identify the specific issues that arise in pharmacy experiential education settings and the solutions Experiential Education (EE) administrators deploy to resolve the issues. Methods: Data were collected using a standardized form by four EE Administrators from three different schools of pharmacy over a two-year period. Data were compiled from the three study sites, de-identified, and descriptive statistics and content analysis were conducted. Results: A total of 221 issues were reported. Most issues were pre- ceptor reported $(n=156,69 \%)$. The most frequent issues identified by preceptors were Student reliability/responsibility $(n=22,14 \%)$, Student knowledge deficits $(n=20$, $13 \%)$, Student professionalism ( $\mathrm{n}=19,12 \%)$, and Student communication $(15,10 \%)$. The top three solutions applied by EE administrators were Coach preceptor $(n=51,24 \%)$, Coach student $(n=42,20 \%)$, Meet with student $(\mathrm{n}=40,19 \%)$. The most frequent issues identified by students were Preceptor communication ( $\mathrm{n}=13,20 \%)$, Preceptor relationships/respect to others $(n=8,12 \%)$, Preceptor professionalism $(n=7,11 \%)$ and Preceptor care/compassion $(n=7,11 \%)$. The top solutions applied to issues identified by students were Meet with student $(n=20,24 \%)$, Coach preceptor $(n=17,21 \%)$, and Coach student $(n=15,18 \%)$. Implications: The goal of the study was to learn more about the issues and solutions that arise in pharmacy experiential education settings. The results are now being used to develop a model for preceptor training, create student support systems, generate effective quality improvement interventions, and enhance policy and procedures framework for managing student and preceptor issues in the experiential setting.

Identifying Interprofessional Patient Care in Ambulatory Care Advanced Pharmacy Practice Experiences (APPEs). Mara A. Kieser, University of WisconsinMadison, Amanda Margolis, University of WisconsinMadison. Objective: To determine the extent of exposure to interprofessional and team-based patient care during required ambulatory care Advanced Pharmacy Practice Experience (APPE) pre and post implementation of Standards 2016. Methods: New APPE courses were implemented to meet Standards 2016 during the 2017-2018 experiential year. To assure quality and determine interprofessional practice opportunities in the new ambulatory care course, students were surveyed regarding the frequency of their interprofessional interactions. Surveys were completed during the 2016-2017 clerkship year (pre Standards 2016) and the 2017-2018 clerkship year (post Standards 2016). Students reported the frequency they participated in or observed 19 interprofessional activities on a scale ranging from daily to none at all. The Chi-squared test was used to determine statistically significant differences. Results: There were 126 and 121 students in the classes of 2017 and 2018 respectively who completed the survey. Students participating in the following interprofessional activities at least 3-4 times weekly are reported for the 2017 and 2018 graduates respectively: participated on an interprofessional team (36\% vs $57 \%, \mathrm{p}=0.0014)$, made recommendations for patient care on an interprofessional team (39\% vs $67 \%$, $\mathrm{p}<0.001$ ), participated in an interprofessional clinic ( $48 \%$ vs $60 \%, p=0.075)$, offered recommendations 


\section{American Journal of Pharmaceutical Education 2019; 83 (5) Article 7654.}

face-to-face $(16 \%$ vs $42 \%, \mathrm{p}<0.001)$, participated in patient interviews with other providers $(15 \%$ vs $30 \%$, $\mathrm{p}=0.006$ ), and responded to drug information questions from providers $(52 \%$ vs $63 \%, p=0.094)$. Implications: Students experienced greater interprofessional experiences during the 2017-2018 year. Several factors may have contributed to this change including the progression of ambulatory care and availability of electronic medical records.

Impact of a Co-Curricular Program on Students' Perceptions of Personal and Professional Growth. Brenda Pahl, Cedarville University, Jeffrey A. Bates, Cedarville University, Zachary N. Jenkins, Cedarville University, Aleda M. Chen, Cedarville University. Objective: With the ACPE Standards 2016, pharmacy schools/colleges must create co-curricular programs to link to the curriculum, but an ideal approach is unknown. Thus, the objective was to describe co-curricular activities and examine changes in student learning outcomes after the first year of a co-curricular program. Methods: The pilot co-curriculum plan included 7 domains (represented ACPE Standards 3/4 and program outcomes) with minimum expectations. Each student created a personal development plan and discussed it individually with the co-curriculum director. After students attended co-curricular events, they completed a post-event form (event details; mini-reflection; 5-point quality rating) to receive credit. The co-curriculum director reviewed all mini-reflections and scored them with a 4-point, Likert-type scale. Prepost first professional (P1) year, students completed a survey on their perceived development in the domains' outcomes. Results: Students (N=45) completed 1004 cocurricular hours in 2017-2018, with service as highest hours (mean \pm SD: $6.7 \pm 9.8$ ). Over $90 \%$ of the reflections were at/above expectations. Students had significant positive change $(\mathrm{p}<0.05)$ in all service, interprofessional collaboration, and spiritual growth outcomes. Changes in other domains were not across all outcomes (leadership: $3 / 5$, professionalism: $1 / 4$, personal/professional development:1/3). There were no changes in innovation/ entrepreneurship. Implications: Student awareness of and participation in co-curricular activities increased, and they expressed personal growth in several domains. These corresponded to topics that received greater focus in our pilot year. In addition, improved collaboration between student organizations and student understanding of the importance of personal and professional development was noted.

Impact of a Collaborative Advanced Pharmacy Practice Experience Curriculum on Student-Perceived Ability and Confidence. Elizabeth Covington, Samford University, Jeffrey A. Kyle, Samford University, Valerie
T. Prince, Samford University, Megan Z. Roberts, Samford University, Mary A. Worthington, Samford University. Objective: This study evaluated the impact of a collaborative Advanced Pharmacy Practice Experience (APPE) model on student-perceived ability and confidence related to care of adult and pediatric patients. Secondary endpoints included impact on career plans and value of activities. Methods: Anonymous, voluntary pre-post surveys were completed by a student pharmacist cohort in a collaborative adult and pediatric hospital medicine APPE model. The model included group discussions, video modules, patient cases, journal scans and case presentations among student pharmacists and medical trainees. Likert scale responses assessed student-perceived ability/confidence related to interprofessional relationships and decision-making skills for adult and pediatric patients. Post-graduate plans and student value of model activities were also assessed. Fisher's exact test was performed to compare percentage of students selecting a " 4 " or " 5 " on Likert scale questions, with " 5 " indicating highest confidence/ability. Results: From the cohort, 79 student responses $(n=44$ pre-rotation, $n=35$ post-rotation) were obtained from May to December 2018. Post-rotation, students showed an increase in confidence to practice pharmacy on an interprofessional team (36\% vs. $100 \%, p<0.0001)$. Significant increases were also noted for therapeutic decision-making regarding antibiotics, anticoagulants, and pharmacokinetics for both adult and pediatric patients. Among students completing the post-survey, $43 \%$ stated the APPE changed his/her career plans. Video modules were the most valued component of the APPE model. Implications: A collaborative APPE model resulted in consistent increases in student-perceived ability and confidence related to care of adult and pediatric patients. This APPE model could be adapted within different care settings and pharmacy curricula.

Impact of an Interdisciplinary Acute Patient Stabilization Simulation on Attitudes Toward Interprofessional Collaborative Practice. Lisa F. Brennan, Wingate University, Samantha Ogle, Wake Forest University, Deb Harding, Wake Forest University, Kimberly Stanbery, Wake Forest University, Allison McBride, Wake Forest University, Modupe Akinola, Wake Forest University, Paula Correa, Wake Forest University, Jon Goforth, Wake Forest University, Roy Strowd, Wake Forest University. Objective: To assess the effect of a standardized-patient simulation embedded within a 2-week medical intern training course on physician, pharmacy and nursing students' perceptions of interprofessional education (IPE). Methods: Fourth-year medical students $(n=41)$, fourth-year pharmacy students $(n=17)$, and 


\section{American Journal of Pharmaceutical Education 2019; 83 (5) Article 7654.}

nursing trainees $(n=22)$ worked in interprofessional teams to simulate stabilization of an acutely ill standardized patient. Teams had physician, pharmacy, and nurse preceptors. Perceptions of IPE were assessed using the validated Student Perceptions of Interprofessional Clinical Education-Revised Instrument, version 2 (SPICE-R2, 5-point Likert). Participants completed the SPICE-R2 survey pre/post-simulation, with medical students also completing on day 1 and day 14 of their course. Data analysis was performed using Fisher's exact test, Chi-square, student's t-test, and ANOVA when appropriate. Results: All learners showed a statistically significant increase in perceptions of roles/ responsibilities (3.75 to 4.35, $\mathrm{p}<0.0001$ ), patient outcomes from collaborative practice $(4.29$ to $4.63 p<0.0001)$ and interprofessional teamwork and team-based practice ( 4.39 to $4.73, \mathrm{p}<0.0001$ ). No significant difference in mean change between disciplines was found. Pharmacy student perceptions improved across multiple simulations, although improvement beyond the first simulation was not statistically significant. Perceptions toward IPE throughout their course improved for MD students participating in the simulation $(n=41)$ compared to those who did not $(n=65)$ although the difference was not significant (mean change 0.12 vs -0.03 , $\mathrm{p}=0.1011$ ). Implications: Interprofessional collaboration during a standardized patient simulation improved learners' perceptions of teamwork, roles and responsibilities, and effect on patient outcomes. These findings support the incorporation of standardized patient-based IPE events even in late-stage education of health professionals.

Impact of Introductory Pharmacy Practice Experiences (IPPE) Remediation on Advanced Pharmacy Practice Experiences (APPE) Performance. Courtney R. Caimano, Albany College of Pharmacy and Health Sciences, Laurie L. Briceland, Albany College of Pharmacy and Health Sciences, Megan Veselov, Albany College of Pharmacy and Health Sciences, Sandra W. Rosa, Albany College of Pharmacy and Health Sciences. Objective: To assess the impact that student specific remediation of deficiencies identified during IPPEs has on APPE performance. Methods: At the completion of all IPPE and APPE rotations, preceptors evaluated students on several competencies. Students receiving at least one Needs Improvement (NI) or Significant Deficiency (SD), while still possibly receiving a passing grade for IPPEs, were identified as needing remediation. Students receiving two or more NI or SD on APPEs were identified to complete an Experiential Success Plan (ESP). APPE data from the Class of 2019 was reviewed with a focus on students who completed IPPE remediation, and compared to the Class of 2018, who was not required to complete remediation. Results: In the Class of 2018, 15 of 33 students $(45.5 \%)$ who would have completed IPPE remediation, if initiative existed, completed 27 APPE ESPs across all rotation types (community, institutional, ambulatory care, inpatient, elective). In the Class of 2019, 9 of 28 students (32.1\%) who completed IPPE remediation resulted in 13 APPE ESPs across four of five rotation types (none in institutional). The total number of ESPs completed by students identified as needing IPPE remediation decreased by 48\% from the Class of 2018 to 2019. Implications: Implementation of remediation activities following IPPE rotations decreases the number of performance deficiencies identified on APPE rotations; remediated students may be more aware of improvement strategies to employ on APPEs. Further review of data may elucidate predictors of APPE performance deficiencies for students not required to complete IPPE remediation such that corrective strategies may be implemented.

Impact of Student Pharmacists on Patient Care During a 5-Week Community Pharmacy APPE Rotation. Nicole M. Gattas, St. Louis College of Pharmacy, Roxane L. Took, St. Louis College of Pharmacy, Michelle Jeon, St. Louis College of Pharmacy, George Vineyard, St. Louis College of Pharmacy. Objective: To describe the impact Advanced Pharmacy Practice Experience (APPE) students have on patient care during their fiveweek Community Care rotation at St. Louis College of Pharmacy. Secondly, to describe student or site characteristics that affect the number or time spent on interventions. Methods: Primary interventions and time spent reported by students on an online system were retrospectively assessed. Data from successful rotations during the 2016-2018 academic years was collected along with student characteristics. Site types were categorized as chain retailer, regional grocery chain, independent, or other. Multiple descriptive and inferential analyses were performed. Data analyses are reported in aggregate and using correlation analysis. Results: A total of 444 students documented 29,565 interventions over 620 hours. On average over the rotation, students educated patients or healthcare professionals 33 times, administered 12 vaccines, provided 5 comprehensive medication reviews, monitored 5 medications, and clarified 3 medication orders. Students answered drug information questions, provided access to medications, and altered prescription therapy twice. Gender, ethnicity, rotation grade, and ontime graduation did not correlate to more interventions. Higher grade point average positively correlated with an increase in time for interventions, but not number of interventions. On average per student rotation, more interventions were reported in the chain retailer setting (75) vs. 


\section{American Journal of Pharmaceutical Education 2019; 83 (5) Article 7654.}

regional grocery chain (70) or independent (51). Implications: This is the first study to explore predictive factors related to amount of APPE interventions in community pharmacy. Students spend significant time providing a variety of care to patients, likely increasing amount and level of patient care provided at the site.

In House Geo-Optimization: A Unique Approach for Nearby Introductory Pharmacy Practice Experience (IPPE) Site Placements. Anita J. Cleven, Pacific University Oregon, Jackson Ross, Pacific University Oregon, Huy T. Hoang, Pacific University Oregon, Melanie J. Belles, Pacific University Oregon, Fallon J. Hughes, $\mathrm{Pa}$ cific University Oregon. Objective: In order to maximize student satisfaction and minimize travel time to sites, the Office of Experiential Education (OEE) devised an inhouse geo-optimization. A geo-optimization is an arrangement of students to sites such that the total distance between where students live and where they complete their IPPE community rotations is as small as possible. Methods: We first determined the distance between every student's address and every potential site they could be placed. This was accomplished by running a script in Google Sheets that converts an address to latitude and longitude, and then using a formula that calculates the distance between two points on the surface of the Earth based on their latitude and longitude. We then input this data into the optimizer. The optimizer configures our site availability to produce a result that minimizes the total sum of distances between sites, student addresses and is feasible with site availability. Results: The smallest sum of total distances between sites and student addresses tends to result in a large number of students being placed very close to their sites, and a small but not insignificant number of students being placed much further away. This propelled us to set rules for the optimizer such that no student could be placed further than 15 miles away from the site. This resulted in the elimination of far-out site placements. Implications: Providing an in-house optimization allows the OEE to enhance overall student satisfaction with site placements. Students are placed within a reasonable distance from their residence.

Interprofessional Experiences Documented by Introductory Pharmacy Practice Experience Students. Jennifer D. Arnoldi, Southern Illinois University Edwardsville, Kate Newman, Southern Illinois University Edwardsville. Objective: To describe interprofessional interactions documented by students completing introductory pharmacy practice experiences (IPPE) at a public university not affiliated with an academic health center. Methods: Students in their first and second professional years are assigned to complete three IPPE rotations at community and institutional sites. Students document the frequency and nature of their interactions with other healthcare students and professionals. This study evaluated the documentation provided by students to determine the extent and nature of interprofessional interactions occurring in IPPE settings. Results: This study analyzed 429 evaluations from 148 students. Students indicated the frequency of their interprofessional interactions as Frequently ( $>3$ times weekly), Periodically ( $\geq$ weekly but $<3$ times weekly), Seldom ( $<$ weekly), or Never. In the community setting, 264 students reported minimal interactions with other students $[$ Never $=208(78.8 \%)$, Seldom $=21(8.1 \%)]$ but more frequent interactions with other healthcare professionals $\quad[$ Frequently $=92 \quad(36.4 \%), \quad$ Periodically $=54$ $(21.3 \%)]$. Telephone interactions with nurses $(n=121)$, physicians $(\mathrm{n}=51)$, and physician's assistants or nurse practitioners $(n=9)$ were most frequently described. Students completing institutional rotations $(n=165)$ also reported infrequent interactions with other students $[$ Never $=85(51.5 \%)$, Seldom $=26(15.8 \%)]$ but high levels of interaction with other professionals $[$ Frequently $=116(71.2 \%)$, Periodically $=35(21.5 \%)]$, describing interactions with nurses $(\mathrm{n}=97)$, physicians $(n=78)$, dietitians $(n=22)$, and physical/occupational therapists $(n=21)$. Implications: This study describes the nature and extent of interprofessional interactions during community and health-system IPPE rotations, highlighting room for expansion in interprofessional collaboration and education efforts. This data may provide useful for faculty preparing students for IPPE rotations and in developing and refining IPPE documentation tools.

Introductory Pharmacy Practice Experience (IPPE) SMART Goals: A Qualitative Analysis. Kate Newman, Southern Illinois University Edwardsville, Jennifer D. Arnoldi, Southern Illinois University Edwardsville, Maggie Clark, Southern Illinois University Edwardsville. Objective: Standards 2016 emphasize the importance of patient care skills and direct patient care experiences in Introductory Pharmacy Practice Experience (IPPE) rotations. Additionally, we strive to model and enhance self-awareness and professionalism through the practice of specific, measurable, attainable, relevant and timely (SMART) goal setting. The goal of this project was to analyze and describe the skills and activities IPPE students identify during a required goal setting exercise. Methods: Two investigators independently analyzed SMART goal reflection papers written by pharmacy students after the completion of IPPE rotations during their first and second professional years. Skills and activities described by students were categorized using Entrustable Professional Activities (EPAs) and the Pharmacists' Patient Care Process (PPCP) as a guide. Reviewers met to 


\section{American Journal of Pharmaceutical Education 2019; 83 (5) Article 7654.}

compare coding results and resolve any differences. Results: A total of 231 reflections were analyzed from 77 students. A total of 788 goals were categorized into 22 different task types. The most common tasks included: Observing the Function of a Health Care System (15.9\%), Educating Patients and Professional Colleagues (13.6\%), Preparing Medications (8.6\%), Analyzing Information (8.1\%) and Basic Medication Knowledge (8.1\%). It was noticeable that many tasks were not represented in the SMART goals as frequently as expected: Collecting Information (2.4\%), Establishing Patient-Centered Goals (1.9\%), Medication Reconciliation (0.6\%) and Documentation $(0.3 \%)$. Implications: This study highlighted a disconnect between expectations of experiential faculty and student goals. This will inform changes to rotation preparation for students. EPA language may be a useful tool when preparing students to set SMART goals for future IPPE rotations.

Preceptor Evaluation of Student Performance on Required APPEs Using EPA Trust Statements. Erin L. Johanson, Roseman University of Health Sciences, C. Leiana Oswald, Roseman University of Health Sciences, Phillip Lawrence, Roseman University of Health Sciences. Objective: The purpose of this educational research was to measure preceptor evaluation of student performance on EPA mapped learning outcomes during core required APPE (Adult Acute Care, Ambulatory Care, Institutional, and Advanced Community) rotations utilizing a trust statement rubric with a 5-point scale. Methods: Preceptors evaluated student performance completing online evaluations at the mid-point and final of all APPEs. The rubric utilized by preceptors to assess student achievement of 10 professionalism and 19 general learning outcomes incorporated EPA trust statements in the 5-point scale, wherein students were required to achieve a rating of $3 / 5$ on each outcome to pass the rotation. Results: Evaluation data from May 2017-January 2019 , reported average scores of $4.48-4.73$ on a 5.00 point scale for the 10 professionalism outcomes and 4.18-4.46 on the 19 general learning outcomes on APPE rotations. Overall, the highest level of trust achieved was reported in professionalism outcome "Student is respectful" (4,46/5.00). The lowest overall scores were reported in learning outcome "Demonstrate the knowledge and abilities to oversee pharmacy operations, including resource management, personnel, and technology of the practice site" (4.18/5.00). Variations existed in rotation types with highest instances of individual scores of 5 on Advanced Community and lowest instances on Adult Acute Care APPEs. Implications: Preceptor evaluation of student progression using EPA trust statements across required APPE rotations provides evidence of student demonstration of activities required for entry-level practice. Limitations include factors affecting student performance including timing of the rotation, site and preceptor variation, and student preAPPE readiness (including IPPE performance) is worthy of future study.

Prescriber-Led APPE Rotations Improve Entrustable Professional Activities Assessment in an Interprofessional Environment. Charles Douglas, California Health Sciences University, Miriam Ansong, California Health Sciences University, Ahmd Azab, California Health Sciences University, Jeremy Hughes, California Health Sciences University, Will Ofstad, California Health Sciences University, Mary E. Solis, Adriana Sanchez, California Health Sciences University, Monica Singh, California Health Sciences University, David G. Fuentes, California Health Sciences University. Objective: Interprofessional Advanced Pharmacy Experiences (APPE) mandated by the 2016 ACPE standards require students to interact with other providers during rotations. Pharmacy programs are challenged to assess student progress towards Entrustable Professional Activities (EPAs) in this new environment. Our objective was to investigate student reflections for improving EPA assessment in prescriber-led rotations. Methods: CHSU offers "prescriber-led" rotations in which students are actively involved in a practice setting with a physician. This rotation type is required for all students in our program. Thirteen physician practices, including GI, psychiatry, oncology, pediatric and internal medicine were established. Forty students in 2018-2019 cohort were assigned to 6-week rotations. Thematic analysis was performed. Data were extracted from a modified Kirkpatrick assessment form and a focused IPE reflection essay completed by every student. Each investigator coded data separately. On completion, codes were compared, discussed, and codes modified, or new codes generated. Results: Forty paired Kirkpatrick and IPE essays were completed. Ninety-five percent of students documented direct patient interactions, $90 \%$ of students reported patient counseling encounters, and $80 \%$ of students described close collaboration with healthcare professionals. Thematic analysis revealed three overarching themes. Topics included interprofessional communications, patient communication, and consultation techniques, and medication therapy in medical practice. Implications: Analysis informed faculty on revisions to current data interpretation to better assess EPAs in this new environment. Investigators also gained insight on barriers to rotation objectives, rotation design limitations, preceptor training, and opportunities to enhance experiential learning in prescriber-led APPE rotations. 


\section{American Journal of Pharmaceutical Education 2019; 83 (5) Article 7654.}

Quality Assurance of APPE Assessment Tools. Sarah R. Peppard, Concordia University Wisconsin, Melissa L. Theesfeld, Concordia University Wisconsin. Objective: Developing assessment tools that reflect student performance during experiential rotations can be challenging given the subjective nature of preceptor evaluations and variability in practice at experiential sites. This analysis looked at the correlation between letter grades derived from our APPE rubric and perceived letter grades from preceptors. Methods: Final letter grades are calculated by the Office of Experiential Education from ratings assigned by preceptors. A question on the final evaluation for all APPE rotations asked preceptors to assign a letter grade based on the students' overall performance. Rubric-derived letter grades were compared to preceptor-perceived grades for 4 consecutive academic years (2014-2018). During the 2017-2018 academic year, preceptors were asked to provide comments on the final evaluation if they believed that students earned less than an "A" for their final grade. When grade discrepancies existed, preceptor comments were categorized. Results: A total of 2367 grades were evaluated.The rubric-derived letter grade agreed with the preceptor's perceived letter grade in 1937 cases $(81.89 \%)$. When grades disagreed and the actual grade was higher than the perceived $(n=413)$, preceptors didn't indicate students were below expectations on the rubric in in 290 cases (70\%).Attitude and motivation, knowledge application, and critical thinking were noted most frequently by preceptors as reasons they perceived students did not earn an "A". Implications: The majority of final letter grades received by students during APPE rotations reflect the perceived grade from preceptors. There is an opportunity to provide preceptor education to optimize the use of APPE assessment tools.

Residency Application Information Regarding Advanced Pharmacy Practice Experiences Considered Desirable or Necessary by Program Directors. Cheryl L. Clarke, Drake University, Robyn Cooper, Drake University. Objective: To determine how postgraduate year one (PGY1) residency program directors perceive factors related to advanced pharmacy practice experiences (APPEs) when selecting candidates for residency interviews. Factors examined included the 1) overall influence of five categories of APPE-related factors including location, structure, elective types, timing, and preceptor references; 2) desirability of 75 APPE-related variables; and 3) necessity of 75 APPE-related variables. Methods: An online cross-sectional nationwide survey of 1,280 PGY1 residency program directors was conducted. Program demographics and survey data were collected from 370 participants (29\% response rate). Results: APPE preceptor reference letters were the most influen- tial category while APPE timing factors were least influential. Influence of APPE location and APPE structure were second and third ranked, while APPE elective types were fourth ranked. Person-environment fit influenced variables perceived as desirable, with respondents desiring factors similar to their own environment as an indicator of fit. Specific desired variables were submission of two APPE preceptor reference letters and letter grades for each APPE. Inpatient electives were considered highly desirable as compared to a balanced elective mix. Necessity of APPE variables was rarely identified. Implications: APPE preceptor reference letters were the most influential APPE-related factor when selecting candidates for interviews. Two references from APPE preceptors were desirable and necessary. Person-environment fit should be considered by applicants when selecting APPEs and preparing residency applications as program directors desire factors that are similar to their organization. However, absence of desired APPE-related variables does not exclude an applicant, as few variables were considered necessary.

Stress and Its Association with Advanced Pharmacy Practice Experiences (APPE) Among Pharmacy Students. Yinan Huang, University of Houston, Shrey Gohil, University of Houston, Sujit S. Sansgiry, University of Houston. Objective: To assess 4th year pharmacy students' perceived stress scale (PSS) score after their 6week rotation and determine its association with students' satisfaction toward APPE and to explore factors associated with perceived stress. Methods: Perceived stress was measured using 10 question Perceived Stress Scale (PSS10). Satisfaction toward APPE was measured based on self-administered questionnaires. Independent sample t-test, ANOVA, Pearson's r correlations and multivariate regression analysis were conducted. Results: There were 122 students (93.9\%) participated in the survey. The majority were female $(60.3 \%)$, single $(83.3 \%)$, and living with family (67.5\%). Mean PSS score was 22.1 (SD 4.8). Mean APPE satisfaction score was 24.7 (SD 4.8). No significant differences were noted on stress based on varying level of students' satisfaction toward APPE. Female students and students' offsite working hour during their APPE were significantly associated with higher PSS scores. Ambulatory care course was significantly associated with lower PSS scores. Implications: Further research could address causes of observed differences in stress among rotation course types. Stress management and work load workshops before APPE rotations may be considered by college administrators.

Student Perceptions Regarding an Interprofessional International Medical Mission APPE. Craig A. Kimble, Marshall University, Robert B. Stanton, 


\section{American Journal of Pharmaceutical Education 2019; 83 (5) Article 7654.}

Marshall University, Amber Payne, Marshall University, Tiffany V. Oldaker, Marshall University. Objective: Assess student perspectives before and after an interprofessional international healthcare medical mission APPE course to Honduras. The goal is to understand how the experience met student expectations, improved their ability to work with other healthcare team members and changed their approach to empathy and care delivery. Methods: Surveys were developed to assess student perceptions and experiences before and after the course using the Qualtrics survey tool. Student participation was anonymous. There were 22 questions pre- and 26 questions on the post-survey. The syllabus for this course was previously designed for student safety and achievement of foundational objectives regarding clinical responsibility and the culture of Honduras. Survey results were compared to identify changes in perspectives, such as, empathy, interprofessional learning opportunities and to ensure students met course objectives. Students were also required to complete a structured reflection paper detailing their experience and a presentation on the Honduran healthcare system or a disease state encountered. Results: APPE students completing the course were surveyed. Number of students is limited due to group lodging space in Honduras.Response rate of surveyed students completing both the pre- and post- surveys was $100 \%$. Students with no exposure to third world healthcare had marked changes in perceptions and empathy. One student participant was from a third world country and had no change in perceptions in empathy based on student reflection. Implications: Results support the value of exposing students to third world healthcare. Most students indicated this was a life-changing experience, which will affect them in their approach to providing care.

Students Provide Interprofessional Public Health Services During Introductory Practice Experiences. Patricia L. Darbishire, Purdue University, Marija Stefanoska, Purdue University. Objective: The purpose of this research was to study the impact of interprofessional teamwork on health professional students, including their perspectives on the benefits and challenges of working with each other. A secondary objective was to highlight the impact of their collective work toward public health. Methods: Groups of three students in pharmacy, nursing, public health, dietetics, or health/kinesiology $(n=801)$ at researched, designed and delivered an interdisciplinary health promotions project targeted toward a select community-based population. Students reflected on their experience and evaluated their peers' contributions to the project. Results: The researchers categorized the students' group projects into 20 health-related topics, with the most common being bone health, mental health, sub- stance abuse, nutrition, and cold/flu prevention. The researchers analyzed quantitative and qualitative student feedback, noting that $92 \%$ of students found value in the collaboration, while $90 \%$ believed they taught useful, discipline-specific information to their group members. The most common benefit described by students was the ability to present their project or service to the audience in a well-rounded matter, with expertise coming from each of the different disciplines. The most commonly noted challenges were logistical issues, such as arranging planning meetings. Implications: This research provides insight into successes and challenges when students from multiple disciplines work together toward a common goal. It also highlights the significance of each student's individual contributions, and the collective magnitude that interdisciplinary, student-driven projects can make toward public health.

The Impact of Exercise-Associated Learning on Experiential Student Perceptions Regarding Stress and Knowledge Retention. Melissa Ruble, University of South Florida, Jaclyn Cole, University of South Florida. Objective: Physical activity is associated with many physical and mental health benefits including improvements in student learning and retention. The objective of this program evaluation was to determine the impact of exercise-associated learning on student perceptions regarding stress and knowledge retention. Methods: A weekly exercise learning program was created by faculty for Adult Medicine APPE rotation students. Students alternated answering questions ranging in complexity on pre-assigned topics while the rest of the group completed an exercise. Students were emailed an anonymous Qualtrics(R) survey at the end of their rotation block to obtain perception data in the form of 5-point Likert Scale and free response. Results: Thirteen students were surveyed after three 6-week rotations. Students provided positive feedback, with all questions scoring an average of 3.5 or higher. The exception was student motivation to complete additional physical activity independently (mean 2.9). Perceptions suggested that this activity decreased daily stress (mean 3.69, SD 0.947). Students strongly agreed that repetition was helpful for learning (mean 5, SD 0), and retention of topics increased as a result of this activity (mean 4.69, SD 0.48). This activity was highly recommended for future rotations (mean 4.769). Free responses identified benefits of teamwork, accountability for answers, and was supported by a judgement-free environment. Implications: Students had positive perceptions regarding stress levels and knowledge retention. Results are limited by data from a small cohort who completed this specific rotation. However, the results suggest that this activity is well- 


\section{American Journal of Pharmaceutical Education 2019; 83 (5) Article 7654.}

received by students on rotations and may be beneficial in additional practice settings.

The Interprofessional Learning Landscape of Preceptors Supporting Advanced Pharmacy Practice Experiences. Teresa H. Truong, The University of Oklahoma, Melissa S. Medina, The University of Oklahoma, Stephen Neely, The University of Oklahoma, Vincent C. Dennis, The University of Oklahoma. Objective: Evaluate how preceptors teach, assess, and/or expose pharmacy students to interprofessional education (IPE) during advanced pharmacy practice experiences (APPE). Methods: Active APPE preceptors from hospital, community, and clinical settings on required and elective APPEs were surveyed using a locally developed 17-item electronic survey. The survey included questions about which professions (healthcare students and providers) pharmacy students directly interacted with during APPE rotations. The survey also assessed IPE concepts taught, what IPE activities were provided, and whether the IPE concepts and activities were assessed. Results: The survey response rate was 38\% (181/477) with $161(90 \%)$ of the responses coming from preceptors in required rotations. Nurses $(83 \%)$ were the most common healthcare provider pharmacy students interacted with, followed by advanced registered nurse practitioners (69\%), physician associates (68\%), and medical attendings (49\%).Pharmacy students most frequently interacted with medical (23\%) and nursing students (21\%).IPE concepts were taught and practiced through observation (67\%), discussion (64\%), and direct interaction with other healthcare providers (64\%).Students are most commonly practicing IPE in the live clinical setting (84\%), followed by patient cases $(27 \%)$, standardized patients $(24 \%)$ and presentations $(13 \%)$ ).Assessment of IPE knowledge, skills, and attitudes occurred in $44 \%(n=79)$ of responses. Implications: These results provide insight into the interprofessional learning environment supported for students by preceptors during APPE rotations, which allows the College to determine whether additional exposure, activities, and assessments are needed to achieve desired outcomes.

The Use of a Three-Bucket Preferencing System to Familiarize Students With Health System Sites. Anita J. Cleven, Pacific University Oregon, Huy T. Hoang, Pacific University Oregon, Jackson Ross, Pacific University Oregon, Melanie J. Belles, Pacific University Oregon, Fallon J. Hughes, Pacific University Oregon. Objective: Rank-choice preferencing results in a forced mental framing in which a 1 st choice is better than a 2 nd choice, a 2nd choice is better than a 3rd choice and so on. This also results in a situation in which a student who received first preference is perceived to have been given a "better" schedule than a student who received their 2 nd choice. We developed a three-bucket preferencing system to shift students focus away from the location and rank choice. Methods: The three-bucket system consists of students ranking health-system introductory pharmacy practice experience sites as either, "highly interested." "interested", or "not interested" in Qualtrics. Students are provided with a site description document to help facilitate their choice ranking into the buckets. Results: The three-bucket system was rolled out for the first time to our P1 class in the Spring of $2018.77 \%$ of students got placed at sites they ranked highly interested and $22 \%$ of students were placed at sites they ranked as interested. None of the students received sites placements in areas they were not interested in. Students were able to familiarize themselves with health systems by reading the site description document prior to ranking the health systems. Implications: We decided to use the same system to preference core experiences for advanced pharmacy practice experiences. For all optimizations, we have been able to provide over $90 \%$ of our students with a site assignment they indicated as "highly interested."

Transitions of Care Education in Doctor of Pharmacy Curricula in the United States. Lisa A. Salvati, Ferris State University, Sophia A Dietrich, Ferris State University. Objective: Gather information on how Schools/Colleges of Pharmacy (S/COP) in the United States are embedding transitions of care (TOC) education into their curricula. Methods: A survey was developed and e-mailed to at least one faculty member or administrator at each S/COP who was involved in curriculum development or experiential education. The survey gathered quantitative information on TOC education methods. Implementation barriers, current approaches to incorporating $\mathrm{TOC}$, and benefits to incorporating TOC into pharmacy education were also evaluated. Results: At least one individual from 22 of the $143 \mathrm{~S} / \mathrm{COP}$ completed the survey $(15.4 \%)$. TOC learning opportunities are established in $70.8 \%$ of the S/COPs. TOC education in didactic curricula occurs most commonly through required courses embedded with TOC topics $(45.5 \%)$, simulations $(27.3 \%)$, elective TOC courses $(22.7 \%)$, and elective courses with embedded TOC topics (22.7\%). Within the advanced pharmacy practice experience (APPE) curriculum, rotations with TOC opportunities most commonly include inpatient (63.6\%), ambulatory (45.5\%), TOC electives (45.5\%), electives embedded with TOC topics (45.5\%), and community (22.7\%). Respondents believed the most common barriers for TOC education implementation include limited faculty experience, restricted curriculum time, and experiential site limitations. The most frequent ways $\mathrm{S} / \mathrm{COPs}$ are educating on TOC are through didactic lectures, 


\section{American Journal of Pharmaceutical Education 2019; 83 (5) Article 7654.}

APPEs, and didactic labs. The greatest benefits of incorporating TOC education include improving patient outcomes, preparing students for patient care, and training students for team-based care. Implications: This survey provided information on current structure of TOC education in S/COP in the United States. Future research could gather information on structure of TOC education within other healthcare professions.

Using Key Indicators in Pre-APPE Performance to Predict At-Risk Students on APPEs. Carinda Feild, University of Florida, Karen Whalen, University of Flor$i d a$. Objective: To evaluate the process for identifying students who may be at risk for substandard performance on advanced pharmacy practice experiences (APPEs) Methods: Prior to the 2018-2019 APPE year, experiential faculty and administrators identified students potentially at risk of poor performance on APPEs. Students were classified as at-risk if they had at least one key indicator in the areas of knowledge (PCOA 50th percentile or lower, GPA less than 2.5, Top 200 drug score below $80 \%$, revised plan of study), skills (OSCE remediation, low teamwork [CATME] scores), and attitudes/values (professionalism infractions). Student performance during APPEs was monitored, with substandard performance defined as rotation grade $\mathrm{C}$ or lower, unacceptable professionalism assessment, or overall performance below level of expectations. Using descriptive statistics, the investigators assessed the accuracy rate of key indicators in predicting substandard performance on APPEs. Results: The panel of key indicators had a 52.9\% accuracy rate of identifying students with an overall performance assessment below the level of expectations for at least one rotation (10 predicted/17 actual), and a $46.7 \%$ accuracy rate of identifying students with an unacceptable level of professionalism ( 7 predicted/15 actual). The panel correctly predicted 11 of 20 students (55\%) who received a grade of $\mathrm{C}$ or lower. Of the 36 students predicted to be at risk of substandard performance, 15 underperformed in any category. Implications: The panel of key indicators performed modestly in identifying students at risk of substandard performance on APPEs. Future steps include examining students with substandard performance not identified by the panel to find other potential predictors.

Using Modified Trust Statements to Evaluate EPA-Based Learning Outcomes in Experiential Education. C. Leiana Oswald, Roseman University of Health Sciences, Phillip Lawrence, Roseman University of Health Sciences, Erin L. Johanson, Roseman University of Health Sciences. Objective: Report aggregate scores provided by preceptors to evaluate Entrustable Professional Activities (EPA)-based learning outcomes for experiential rotations using modified trust statements.
Methods: In May 2018, evaluations forms incorporating the EPAs for pharmacists were implemented across IPPE and APPE rotations. A 5-point grading scheme modified from the trust statements published by Pittenger et al. in AJPE (2016) was simultaneously implemented. A minimum score of $3 / 5$ was required for all learning outcomes to pass each rotation. The evaluation forms and grading scale were reviewed by an Advisory Committee and approved unanimously by faculty prior to implementation. Preceptor evaluation of student performance for each rotation was analyzed. Results: De-identified data was collected for 1147 rotations for 589 students (231 IPPE, 358 APPE) across 2 campuses. Four IPPE rotations (community-based and institutional-based), and 4 APPE rotations (Adult Acute Care, Advanced Community, Ambulatory Care, and Institutional) were analyzed. Average student scores on learning outcomes ranged from 3.90 to 4.22 for IPPE rotations and 4.15 to 4.43 for APPE rotations. The distribution of scores was $1=0.64 \%, 2=0.00 \%, 3=$ $28.13 \%, 4=38.94 \%, 5=32.29 \%$ for IPPE rotations, and $1=0.02 \%, 2=0.30 \%, 3=14.02 \%, 4=45.21 \%, 5=$ $40.45 \%$ for APPE rotations. Implications: Implementing EPA-based learning outcomes into experiential rotations is a novel concept. The formation of an AACP EPA Taskforce in 2018 shows the importance to pharmacy academia. The data presented provides information related to trust statements and EPA-based evaluation forms as utilized by one experiential program.

Utilizing a Mentored Teaching Experience to Improve Preceptor Feedback Skills. Kathryn A. Schott, Drake University, Cheryl L. Clarke, Drake University, Kristin S. Meyer, Drake University. Objective: 1) To determine the extent to which a 75-minute on-site feedback workshop strengthens preceptors' confidence in giving quality feedback; and 2) To determine the perceived value of the format and content of the workshop. Methods: Fifteen pharmacist participants completed a pre-survey to self-assess their confidence level in giving feedback to students across eight competencies. During the workshop, participants engaged in three case discussions through role-play with partners. Each participant received peer feedback using a standard rubric assessing eight competencies addressing feedback skills. Participants completed the same survey assessing their confidence levels immediately following the workshop and again six months later. Results: Descriptive statistics indicated a high degree of satisfaction with the workshop among participants (mean scores for program effectiveness and perceived program value ranged from $4.40-4.75$ on a 5-point scale). Paired samples t-tests revealed a significant difference across all 8 competencies immediately following the workshop. After the six-month period, all 8 


\section{American Journal of Pharmaceutical Education 2019; 83 (5) Article 7654.}

competencies remained significantly improved over the pre-workshop survey. No significant change occurred when compared to the post-workshop survey, indicating retention of knowledge over time. Implications: A 75minute on-site workshop format utilizing case studies and role-play is an effective means to assist preceptors in developing confidence in giving quality feedback to students, residents, and peers/colleagues. This format may be a useful method for delivering preceptor development in other topic areas.

Validation of an Interprofessional Socialization Values Scale for Student Pharmacists. Jennifer Danielson, University of Washington, Curtis G. Jefferson, University of Washington. Objective: The objective was to develop and validate a tool for assessing student pharmacist values and self-efficacy in interprofessional teamwork at the University of Washington School of Pharmacy. Methods: A 12-item tool was developed based on a review of existing tools in the literature and interprofessional education learning goals at the University of Washington School of Pharmacy. Inspiration for our tool was taken from a previously published tool, the Interprofessional Socialization and Values Scale (ISVS). Our tool was tested with a subset of students and refined, then completed by all second- and third-year students in a retrospective pre-post format as part of their interprofessional education curriculum following involvement in intentional interprofessional activities. Results: Analysis of data from two classes of student pharmacists $(\mathrm{n}=$ 251) showed a statistically significant difference between the pre- and post-scores for each of the twelve items ( $\mathrm{p}<$ 0.001). A principal component analysis found that the 12 items loaded on a single factor that met the criteria for unidimensionality. Internal consistency was excellent $(\alpha>0.9)$, and no items had a low item-total correlation. Change between pre- and post- scores were greater for third students than for second year students. Implications: The tool and scale developed is a valid and reliable tool for assessing student pharmacist values and selfefficacy toward interprofessional education and practice. Continued use of the tool as part of longitudinal assessment is planned. Further work will also be done to assess the applicability of the scale to other health professions and institutions.

Variation by PGY1 Residency Type in Desirability and Necessity of APPE-Related Application Information. Cheryl L. Clarke, Drake University, Robyn Cooper, Drake University. Objective: To determine the extent there is variation in postgraduate year one (PGY1) residency program directors' perceptions of the desirability and the necessity of factors related to advanced pharmacy practice experiences (APPEs) when selecting candidates for residency interviews based on PGY1 residency type. APPE-related factors examined included location, structure, elective types, timing, and preceptor references. Methods: An online cross-sectional nationwide survey of PGY1 residency program directors was conducted, resulting in a $29 \%$ response rate. Program demographics and survey data were collected. Results: $50 \%$ of one-way ANOVA tests were statistically significant. $21 \%$ of all pairwise comparisons for desirability were significant, with $74 \%$ of those demonstrating large effect size. $10 \%$ of all pairwise comparisons for necessity were significant, with $62 \%$ demonstrating large effect size. Examples of preferences by residency type were PGY1 Pharmacy for more inpatient than outpatient electives including critical care, emergency medicine, and infectious disease; PGY1 Pharmacy with an ambulatory care focus for anticoagulation and diabetes/endocrinology electives; PGY1 Community-based Pharmacy for independent and chain community pharmacies, and more outpatient than inpatient electives; PGY1 Managed Care Pharmacy placements at locations such as pharmacy benefit management organizations, health plans, and managed care organizations; and PGY1/PGY2 combination residency placements at university-based hospital locations, more inpatient than outpatient electives, and a hospital administration elective. Implications: Differences in perceptions are explained by the concept of personenvironment fit. Residency applicants should consider this concept when selecting APPEs as APPE-related factors demonstrating fit to the desired residency type are considered by directors as desirable and necessary.

\section{Theoretical Models}

A Consortium Approach to Incorporating Entrustable Professional Activities into the APPE Curriculum. Lindsey H. Welch, The University of Georgia, Melody C. Sheffield, The University of Georgia, April Staton, Auburn University, T. Lynn Stevenson, Auburn University, Lori H. Syed, Mercer University, Patricia B. Naro, Samford University, Lena McDowell, Auburn University, James W. Fetterman, South University, Lori J. Duke, The University of Georgia. Objective: To describe a novel process of incorporating Entrustable Professional Activities (EPAs) into the APPE curricula of a six member-school consortium. Methods: The Southeastern Pharmacy Experiential Education Consortium (SPEEC) is comprised of schools in Georgia and Alabama. In 2017, SPEEC began a process to incorporate EPAs into APPEs and to standardize the syllabi of the four required APPEs. Individual EPAs were categorized ("required", "if applicable", "not required") for each rotation type by subgroups tailored to member practice 


\section{American Journal of Pharmaceutical Education 2019; 83 (5) Article 7654.}

experience. A consensus-based process was used to create a final map to APPE types. Since letter grades were assigned for APPEs, a Likert scale was developed to assess student performance on individual EPAs and calculate rotation grades. Assessment of entrustability was integrated for EPA domains only to decrease survey fatigue and did not contribute to grades. Results: Seven new syllabi and evaluation tools were created, including tools for outpatient and acute medicine specialties and non-patient-care experiences. Evaluation tools were beta-tested by faculty preceptors and revised. Final review and approval was obtained from the schools' curriculum and assessment committees. The new syllabi and evaluation tools will be utilized during 2019-2020. Implications: While incorporating EPAs in pharmacy curricula is encouraged, literature is limited describing options for integrating them into various APPE curricula. As more schools incorporate EPAs, the SPEEC process highlights the benefits, for schools and preceptors, of consortium-based approaches despite the challenges of individual school differences. Outside of consortia, this process also presents an option for schools with non-pass/fail APPE curricula.

Final Push: Implementation of a Comprehensive Intermittent Study Design for NAPLEX and MPJE During APPEs. Tonya Dauterman, University of Findlay, Deborah Berlekamp, University of Findlay, Timothy M. Burkart, University of Findlay. Objective: To develop and assess the benefits of a required study program for last year pharmacy students to enhance preparedness for pharmacy licensure Methods: Current literature describes boot camps and review material for NAPLEX preparation but limit comprehensive plans were identified. Survey data has been complied in various studies to identify common NAPLEX preparation material and resources vary among schools. The new approach is implementation of a multifunctional intermittent NAPLEX study plan using a theoretical model observation. Each student's knowledge base was assessed after two APPE rotations using the PreNAPLEX scores. Students identified their weaknesses after the test and developed a study plan. Students were assigned topic modules and assignments/quizzes, 3-4 per month, based on their scores. An assessment was created for each topic requiring topic competency. Law was reviewed over a 6-week period with final assessment required to achieve $>75 \%$. A second Pre-NAPLEX was administered and competency required. Achieving competency at each phase was incorporated into the rotation grades. Students not meeting competency were required to attend a 2.5-day review prior to graduation. Results: Each student took both Pre-NAPLEX exams, wrote a focused study plan, completed assigned monthly topic reviews and assessments, and completed law practice as- sessment. Eight students were determined at risk perPreNAPLEX scores and required to attend boot camp. Seven of the eight passed the NAPLEX at first attempt. Implications: Implementing a comprehensive study plan into student rotation grades during APPEs can help prepare for pharmacy licensure and provide advantages over other review models.

Putting the "IPE" in "IPPE": A Framework for Intentional IPE-IPPE Models within PharmD Curricula. Brooklyn Cobb, University of the Sciences, Jennifer N. Smith, University of the Sciences, Karleen Melody, University of the Sciences, Jason F. Zupec, University of the Sciences, Angela Bingham, University of the Sciences, Diane Hadley, University of the Sciences, Cathy Y. Poon, University of the Sciences. Objective: To describe the framework of two intentional interprofessional education (IPE) introductory pharmacy practice experience (IPPE) models compared to a traditional IPPE model within a Doctor of Pharmacy (PharmD) curriculum. Methods: A comprehensive literature search was conducted to review IPE standards within PharmD experiential education and existing models within professional curricula. Two intentional IPE-IPPE models were developed, alongside a traditional IPPE, to introduce learners to foundational IPE concepts and allow them to develop key skills necessary to succeed in IPE during their advanced pharmacy practice experiences. The IPE-IPPE models were compared and contrasted to a traditional IPPE model. Results: A schematic was created to showcase the framework for each intentional IPE-IPPE model compared to a traditional IPPE model and demonstrate how each align with the Accreditation Council for Pharmacy Education (ACPE) "Standards 2016" and American Association of Colleges of Pharmacy's Center for the Advancement of Pharmacy Education (CAPE) 2013 Educational Outcomes. Additionally, the IPE-IPPE models were compared and aligned to the Interprofessional Education Collaborative (IPEC) Core Competencies. The models were then evaluated for structure/ design, setting, and degree of interaction with other health professional students and/or providers. Implications: These models offer insight in considering an intentional IPE-IPPE framework within professional curricula as compared to a traditional IPPE only. After demonstrating alignment with various standards and competencies, future research regarding the two structured, intentional IPE-IPPE models will assess the development of learner IPE competencies and readiness for interprofessional practice.

Reaching Progressive Curricular Objectives Through an Older Adult Focused Longitudinal IPPE. Susie J. Crowe, East Tennessee State University, Jeffrey 


\section{American Journal of Pharmaceutical Education 2019; 83 (5) Article 7654.}

A. Gray, East Tennessee State University, Katelyn M. Alexander, East Tennessee State University, Bruce Large, East Tennessee State University. Objective: To engage learners in a longitudinal patient care experience spanning the IPPE curriculum Methods: Through a partnership between the college of pharmacy and a large assisted living facility, IPPE student groups were paired with senior residents beginning in their P1 year. Meetings continued each semester between students and residents in the resident's home environment. All students were required to participate while residents participated on a volunteer basis. General discussion topics were provided in advance and included: life story work (P1), health history story work (P2), and medication history work (P3). Students were encouraged to monitor resident's personal, physical, and medical needs. The intention was for student groups to follow the same resident throughout. Each student was required to attend social activities and present a group capstone reflection. Results: Over 240 students have aligned with one of 48 volunteer senior residents each semester. General feedback from student evaluations has been positive. Specifically, students report that interacting with their resident has enhanced their empathy towards older adult patients. Students also reported an improved awareness of potential barriers to health and wellness faced by seniors. Anecdotally, interactions have been well-received by older adult volunteers, facility staff, and faculty. Implications: The learning experience was designed to enhance critical interpersonal skills, including rapport building, empathy, and improved awareness of factors facing older adult patients. Development of foundational communication skills occurred over three years in scaled fashion. We believe ACPE Standards 2.1, $2.4,3.5,3.6$, and 4.4 can be addressed through implementation of an older adult longitudinal IPPE experience.

Redesign of a Community Pharmacy Advanced Practice Experience to Incorporate Entrustable Professional Activities (EPAs). Maria M. Mantione, St. John's University, Carmela Avena-Woods, St. John's University, Emily M. Ambizas, St. John's University, Laura Gianni, St. John's University, Tina Kanmaz, St. John's University, John M. Conry, St. John's University. Objective: To redesign the syllabus for the required Advanced Community Pharmacy Practice Experience to incorporate the EPAs, map the learning experiences and assessments to reflect the EPA requirements, and design a performance assessment rubric. Methods: In December 2017, the College's administration began a redesign of all APPEs to incorporate the EPAs. Individual work groups were appointed for each required APPE. The community pharmacy workgroup was comprised of 3 community pharmacy faculty members and 2 affiliate preceptors.
The workgroup met consistently over the course of the spring semester to develop the new syllabus. Results: Several modifications to the APPE curriculum were proposed before the syllabus was redesigned. A pass/fail system with a professionalism pre-requisite was adopted, and levels of entrustability were defined on a scale of 1-3. Twenty-three EPAs were chosen as learning outcomes and mapped to the Pharmacists Patient Care Process, CAPE 2013 outcomes, NAPLEX, MPJE and IPEC competencies. Five of the required learning outcomes were identified to be exclusive to the community pharmacy APPE. A performance rubric was developed. For successful completion of the experience, students must meet the professionalism pre-requisite; achieve a "Level 3" on the 5 required community specific activities, and a "Level 3" on at least $70 \%$ of the remaining tasks. The syllabus was approved by the faculty for implementation in May 2019. Implications: The redesigned advanced community pharmacy experience will better assess student pharmacists for practice readiness in the community setting.

\section{LIBRARY AND INFORMATION SCIENCE Completed Research}

Drug Information Redesign; Student Practice of Evidence-Based Medicine. Morgan E. Ostinowsky, University of Pittsburgh, Jordan M. Serio, University of Pittsburgh, Lorin B. Grieve, University of Pittsburgh, Colleen M. Culley, University of Pittsburgh. Objective: To evaluate the redesign of the drug information (DI) section in a first professional year (P1) course from a traditional structure (lectures, focused practica, and high-stakes assignments) to an active learning structure of case-based group-learning sessions with low-stakes assignments and follow-up opportunity. The redesign supports skill development through deliberate practice of discovery of DI resources and information retrieval techniques with faculty guidance. In class, groups use these skills to create evidence-based responses to assigned questions. Methods: Performance on twelve cases used with the $\mathrm{P} 1$ students were given to $\mathrm{P} 3$ students in one class session. These students experienced the traditional P1 DI structure. Evidence-based responses were assessed for accuracy, credibility and relevance of resources, format, and efficiency. A survey was administered in 2017 to solicit feedback on the DI experience. Results: Mean case score for P1 students was 26.2 (2017) and 26.5 (2018) and P3 students 26.4 and 25.4, respectively. On a case level, scores were equal between classes for 3 cases (2017 and 2018), P1 scored higher for 4 cases (2017) and 7 cases (2018), and P3 scored higher for 5 cases (2017) and 2 cases (2018). Survey responses were positive for the DI experience. Implications: Results imply that the active- 


\section{American Journal of Pharmaceutical Education 2019; 83 (5) Article 7654.}

learning DI experience prepares students for the rigor of practicing evidence-based medicine. Omission of detailed lectures regarding selection and use of available resources poses initial challenges until students practice. This redesigned DI section format advances students beyond prior surface learning and helps to get them to expert faster.

Evaluating the Impact of Health Literacy Education on High School Students at Pharmacy Camp. MaRanda K. Herring, Harding University, Karlee Kopp, Harding University, Lisa Acuff, Harding University. Objective: The purpose of this study was to evaluate the effectiveness of a Pharmacy Camp lecture and activelearning workshop at improving high school students' understanding of health literacy and confidence in the use of health literature. Methods: This project was granted an IRB exemption. Informed consent was acquired from all participants before the study began. Participants were kept anonymous and answers were recorded for study purposes only. The eHealth Literacy Scale, a validated, 8-item Likert-scale measure of eHealth literacy, was administered to Pharmacy Camp students before and after the completion of the health literacy workshop. A Wilcoxon Signed Rank-Test was utilized to evaluate data. Results: Fifteen students participated in the activity, and fourteen completed the survey in its entirety. In general, students showed the most improvement in identification of health sources (3.4 vs. $4.5, \mathrm{p}=0.002)$ and validity of health sources ( 3.0 vs. $4.5, p=0.001)$. Students showed improvement in confidence in utilizing health literature to make personal health-related decisions (3.0 vs. $4.5, \mathrm{p}=0.002)$. Implications: If repeated and with a larger scale of students, this workshop can give students who are already interested in healthcare the ability to cultivate their health literacy skills earlier. This approach could easily be integrated into a high school health course and pre-pharmacy programs or presented at public libraries or public health outreach events. However, future study is needed to determine if students not interested in a career in healthcare would be as significantly impacted as the study group.

Evaluation of Student Pharmacists' Ability Utilizing Drug Information Resources to Identify DrugDrug Interactions When Unprompted. Ashley Montgomery, Manchester University, Robert D. Beckett, Manchester University. Objective: To evaluate students' ability utilizing drug information resources to identify and manage drug-drug interactions (DDI) using drug information resources when unprompted. Methods: A skills assessment was distributed to pharmacy students in their first- through third-year (P1-P3) of didactic study. Students were presented with three patient cases, each with a new prescription; one contained a contraindicated DDI, one a DDI requiring an intervention, and one with no DDI as a control. Students evaluated each prescription and determined whether to fill it. Each prescribed drug was previously unencountered in the curriculum. Results: Of 215 eligible students, 168 completed the assessment (78.1\% response rate). Of these, $26.2 \%$ were P1 $(\mathrm{n}=75,58.7 \%$ response rate), $33.9 \%$ were $\mathrm{P} 2(\mathrm{n}=72$, $79.2 \%)$, and $39.9 \%$ were P3 $(n=68,98.5 \%)$ students. Based on year of study, $20.5 \%$ of $\mathrm{P} 1 \mathrm{~s}, 87.7 \%$ of $\mathrm{P} 2 \mathrm{~s}$, and $91.0 \%$ of $\mathrm{P} 3 \mathrm{~s}$ appropriately identified and managed the contraindicated DDI $(\mathrm{p}<0.001)$ compared to $38.6 \%, 70.2 \%$, and $62.7 \%$, respectively, for the DDI requiring an intervention $(\mathrm{p}=0.004)$. Appropriate management of the control prescription did not differ by year $(84.1 \%$ vs. $87.7 \%$ vs. $94.1 \%$, respectively, $\mathrm{p}=0.227$ ). Performance did not differ by gender, GPA, work experience, prior degree, resource, or previous experience with the prescribed drug. Implications: Student performance identifying and managing DDIs when unprompted mostly improved with each professional year. Continued practice is needed to help enhance pharmacy students' ability to manage DDIs, particularly those that are not contraindicated but require an intervention. A study limitation was despite incentives for participation there was a low participation rate by $\mathrm{P} 1$ pharmacy students.

"Flipping" Drug Information Responses: Use of Video Responses to Help Students Write Drug Information Responses. Sherrill J. Brown, University of Montana. Objective: Assist students with revision of DI response papers to a shorter, focused response through use of video response activity. Methods: Third-year pharmacy students are required to write a response to a drug information question. To help the students revise the paper, a video response activity was added to help the student focus on the answer to the question and see how the detailed information of the original paper can be summarized more succinctly. Each student recorded their verbal response to the question in a short video. At the end of the semester, students answered reflection questions on their writing process and their impression of the value of the video response assignment. Results: Most (74\%) of the students felt that the video response activity helped them focus their response. Many of these students mentioned that they used the video content to revise their paper. The video assignment did not alter the approach to the revision for the rest of the students, even if the students liked the assignment. In some cases, the students did not like the assignment due to an aversion to watching themselves in a video, and some felt that more guidance was needed for the assignment. Implications: The video 


\section{American Journal of Pharmaceutical Education 2019; 83 (5) Article 7654.}

verbal response assignment helped most students produce concise written DI responses. Based on student comments, the activity instructions will be revised to help students select the video content and understand the link between the verbal response and the revised paper.

Impact of Quizzes Embedded in Recorded Lectures on Student Performance and Engagement. Christopher S. Wisniewski, Medical University of South Carolina, Emily P. Jones, Medical University of South Carolina, Amy Wahlquist, Medical University of South Carolina, Melissa Hortman, Medical University of South Carolina. Objective: To compare student grades and viewing habits when taught via traditional or embedded-quiz pre-class videos. Methods: In a flipped course using videos for pre-class education, willing students completed a demographic survey and baseline assessment. For 3 consecutive weeks mid-semester, students were randomized to complete pre-class quizzes via learning management system (LMS) or embedded-quiz video (EQV). Unique views and total minutes viewed of preclass videos were captured. All students took the same post-class quiz via LMS. Perceptions of students in the EQV group were collected via survey. To test retention, student retook the baseline assessment at the end of the semester. Average grades and viewing behavior were compared between groups descriptively and statistically. Results: Student performance was comparable ( $\mathrm{n}=27$ per group) at baseline (37.04\% vs. 36.42\%). Aggregate grade averages on pre- (LMS: $89.76 \%$ vs. EQV: $90.25 \%$ ) and post-class assignments (LMS: $90.36 \%$ vs. EQV: $88.27 \%$ ) were also similar. Cumulatively, EQVs had more unique views (252 vs. 183) and minutes viewed (1887.65 vs. 1395.15 minutes). Knowledge retention was similar in the LMS $(\mathrm{n}=25)$ and EQV $(\mathrm{n}=19)$ groups $(71.33 \%$ vs. $71.93 \%)$. The only statistically significant difference between groups was total minutes viewed ( $\mathrm{P}$ $=.03)$. EQV-group survey responses $(n=20 / 27)$ showed higher engagement with EQVs $(n=14 / 20)$. Those with partiality preferred EQV quizzes overall $(\mathrm{n}=11 / 19)$ and for class preparation $(n=9 / 12)$. Implications: Student performance was not affected by pre-class video methodology, though students taught with EQV were more engaged with videos and preferred this educational methodology.

Incorporating Entrustable Professional Activities with the Integration of Drug Information and Basic Science. Cambrey Nguyen, The University of Kansas, Judy Q. Wu, The University of Kansas. Objective: The blending of drug information with basic science in a lab setting has not been described in the literature. The purpose of this project was to implement two learning opportunities to integrate drug information (DI) with basic science (translational science) and incorporate Entrustable Professional Activities (EPA) in a pharmacy skills lab. Methods: The PHAR 512 lab consists of introductory DI and translational science topics taught in the first-year fall semester. Core EPAs $(2,10,11,12)$ were mapped out to all activities in the course. A lab focused on drug development was created to integrate both areas taught in the first half of the semester. The students completed an assignment on pre-clinical concepts then answered drug availability questions using DI references. The second learning opportunity required students to present a preclinical, clinical, or practice-based research article in groups of five at the end of the semester. Student learning was assessed through grades and perception was obtained through a Likert based survey. Results: The mean scores for the drug development lab and presentations were 98\% and $95 \%$, respectively, and core EPAs were met through completion of the activities. A total of 66/146 students filled out the survey. The students agreed $(50 / 66)$ the focused learning opportunities helped to connect concepts obtained throughout the course. Implications: The students' responses indicated the content and assignments in PHAR 512 helped integrate drug information with an area of basic science. Future plans are to expand integration of DI with other basic science courses while documenting progression of EPAs.

Longitudinal Assessment of Pharmacy Students' Knowledge, Confidence and Skill in Interpretation of Evidence-Based Medicine. Shweta R. Shah, University of Wisconsin-Madison, Amanda Margolis, University of Wisconsin-Madison, Connie K. Kraus, University of Wisconsin-Madison, Denise L. Walbrandt Pigarelli, University of Wisconsin-Madison. Objective: To assess a longitudinal, evidence-based medicine (EBM) curriculum through pharmacy students' knowledge, confidence, and understanding of EBM Methods: A series of four surveys were administered to the graduating classes of 2017 and 2018: 1) beginning of the P2 drug literature evaluation course, 2) beginning of $\mathrm{P} 3$ year when students write their first EBM paper, 3) beginning of the P4 year, 4) and prior to graduation. The surveys included 5 EBM selfevaluations questions using a 5 -point scale $(1=$ poor to $5=$ excellent $), 9$ self-confidence questions $(1=$ none to $5=$ complete confidence), and 13 knowledge questions. EBM paper scores in the P3 and $\mathrm{P} 4$ years were an instructor-assessed skill evaluation. Intention-to-treat (ITT) analysis was used for students who completed baseline and at least 1 other survey. Confidence and skills were assessed using repeated ANOVA. Descriptive statistics were used to characterize knowledge and EBM paper scores. Results: Out of 264 students, 224 were included in the ITT analysis (85\%). Average correct response to 


\section{American Journal of Pharmaceutical Education 2019; 83 (5) Article 7654.}

knowledge questions improved from $35 \%$ at baseline to $69 \%$ at graduation ( $\mathrm{p}<0.05$ for all questions). Mean confidence scores improved significantly from 2.6 at baseline to 4.0 at graduation $(\mathrm{p}<0.001)$. Mean self-assessed skills improved from 2.6 at baseline to 3.7 at graduation $(\mathrm{p}<0.001)$. The mean overall EBM paper scores increased by $1.1 \%$ from P3 (89.4) to P4 (90.4) year and the proportion of students scoring $>77 \%$ increased by 2.6\% from P3 (94.9) to P4 (97.4) year. Implications: This study provides strong evidence for the effectiveness of teaching EBM using a longitudinal curriculum design.

Pharmacy and Medical Students' Perception of the Utility of Evidence-Based Medicine in Practice. Jennifer M. Toth, The University of Mississippi, Laura J. Nice, University of Louisville Hospital, Harry Schneider, Northeast Ohio Medical University, Grace Grzybowski, Northeast Ohio Medical University, Patrick J. Gallegos, Northeast Ohio Medical University, Mate Soric, Northeast Ohio Medical University. Objective: To compare pharmacy and medical students' perceptions of evidence-based medicine (EBM) and how student perceptions of EBM differs depending on year in the program. Methods: Pharmacy and medical students were invited to participate in an anonymous survey between May 2017 and October 2018. The survey contained questions regarding confidence in EBM skills, utility of EBM in practice, knowledge of EBM concepts, and demographic information. Survey results were analyzed using a chi-squared test. A logistic regression model was created to identify predictors of valuing clinical judgement over EBM. Results: Response rate was 28.9\% (142 pharmacy and 150 medical students). Students valued EBM with $89.8 \%$ agreeing that they will use EBM in clinical practice. Students that completed EBM coursework had significantly lower rates of agreement that clinical judgement was more important than EBM when compared to students that had not taken the EBM course or those that had started experiential rotations $(21.3 \%$ vs $33.9 \%$ and $41.4 \%$, respectively, $\mathrm{p}=0.004$ ), regardless of student gender, college, previous research experience, or EBM knowledge. The groups had similar rates of agreement that EBM is an important tool for practicing medicine, but medical students were significantly less likely to agree that EBM is an important tool for practicing pharmacy $(90.8 \%$ vs $73.3 \%, p<0.001)$. Implications: Most students agreed EBM is useful in clinical practice, emphasizing the importance of EBM in pharmacy and medical curriculum. This research suggests that more interprofessional EBM activities are needed to increase medical students' understanding of the role of EBM for pharmacy practice.

\section{PHARMACEUTICS}

\section{Completed Research}

A Longitudinal Assessment of Compounding Skills in a Pharmacy Laboratory Course. Deborah L. Elder, The University of Georgia. Objective: Determine if the compounding skills of pharmacy students (P2) improve after 10 weeks of hands-on compounding lab activities. Methods: On week one, second year pharmacy students (137) compounded $1 \mathrm{mg} / \mathrm{mL}$ salicylic acid (SA) solution using SA, USP powder utilizing compounding skill learned in P1 exercises. The same product was prepared after 10 consecutive weeks of compounding. An aliquot was removed from each preparation and stored at $-20^{\circ} \mathrm{C}$.Samples were thawed at room temperature and samples were prepared for analysis using visible light spectrophotometry (540nm). A standard curve was constructed to determine the concentration in. Percent accuracy in each sample was determined. Results: Product analysis at week one indicated $12.8 \%$ of all were below $70 \% ; 19.5 \%$ were between 70 to $89 \%$; and $39.8 \%$ were within the desired 90 to $110 \%$ range $(0.0095$ to $0.011 \mathrm{mg} /$ $\mathrm{mL}$ ) of accuracy; with approximately $27.8 \%$ above 110 . After week 11, the percent of student products containing the appropriate amount of SA (90-110\%) was equivalent to week one. Implications: Compounding skills assessment provides feedback to both student compounders and instructors. Analysis of active ingredients will be incorporated into the course and account for $20 \%$ of the student's grant. Instructor will use the data to counsel students to improve their compounding skills throughout the lab course.

Content Analysis of Three Graduate Curricula in the Pharmaceutical Sciences. Amanda Olsen, University of North Carolina at Chapel Hill, Jacqueline McLaughlin, University of North Carolina at Chapel Hill, Kim R. Brouwer, University of North Carolina at Chapel Hill, Joseph Nicolazzo, Monash University, Philip Thompson, Monash University, Chris Porter, Monash University, Stephanie Schorge, University College London, Adam Phillips, University College London. Objective: The purpose of this study was to crosswalk or map pharmaceutical sciences graduate degree programs across three schools of pharmacy to identify similarities and differences in the content of graduate school curricula. Methods: Lists and descriptions of the courses from each degree program were obtained. The title, description, and content of all courses, as well as the expertise of the instructors, were systematically complied into one dataset. Content analysis was used, and all course topics were mapped onto a Venn diagram representing each of the three schools. Results: Each of the three pharmacy schools specialized in their own specific areas, while also 


\section{American Journal of Pharmaceutical Education 2019; 83 (5) Article 7654.}

overlapping in others. For example, areas of individual specialization included reproducibility, G protein receptors, pharmaceutical outcomes, health systems, and thermal analysis, while areas of overlap included drug delivery, lab safety, and stem cell research. In addition, two areas for collaboration across the three schools were also identified. Implications: Across the United States there are approximately 80 universities with graduate degree programs in the pharmaceutical sciences. However, as evidenced by this study, graduate students may obtain very different training depending on the school they attend, as graduate school curricula vary widely. By understanding what is being taught within and across programs, the academy can work toward improving efficiencies by sharing or creating content that strengthens our programs and, subsequently, the training of our graduate students.

Development and Validation of Improved Reversed-Phase HPLC Stability-Indicating Method and Forced Degradation Studies of Curcuminoids. Asish K. Dutta, Notre Dame of Maryland University, Ellison Park, Notre Dame of Maryland University. Objective: Curcumin is found to have multiple therapeutic properties including anticancer, anti-inflammatory, and anti-bacterial effects. It is obtained from turmeric root, and widely used as a common spice and taken as dietary supplement. The objectives of the current study include development and validation of an improved stabilityindicating Reversed-Phase HPLC method to detect and quantitate curcumin, its impurities, and degradants. Methods: Chromatographic system included the Hitachi Elite LaChrom Organizer with PDA detector. Isocratic separation was achieved with $40 \%(\mathrm{v} / \mathrm{v})$ Acetonitrile$60 \%(\mathrm{v} / \mathrm{v})$ water- $0.1 \%(\mathrm{v} / \mathrm{v})$ TFA using Phenomenex Synergy Max-RP column, 20 min run time and column temperature of $45^{\circ} \mathrm{C}$. Forced degradation studies included acid and base reflux; dry heat, oxidation and reduction. Analytical method validation parameters included lower limit of detection (LLOD), lower limit of quantitation (LLOQ), linearity, and range of detection. Results: Curcumin and its impurities, demethoxycurcumin and bisdemethoxycurcumin were detected and resolved at 13.65 $\mathrm{min}, 11.53 \mathrm{~min}$, and $12.25 \mathrm{~min}$, respectively. The degradation products, ferulic acid and vanillin, were found through acid reflux, base reflux and oxidation, at 1.88 min and 2.08 min respectively. Results from heat degradation and reduction were inconclusive. The LLOD and LLOQ was found to be 0.390 and $1.56 \mathrm{mcg} / \mathrm{mL}$, respectively. The range of detection was found to be from $0.390-250 \mathrm{mcg} / \mathrm{mL}$. The linearity was determined to be 0.390-250 mcg/mL. Implications: Curcumin, its impurities and degradation products were successfully detected using the improved and partially validated HPLC method. Future studies include full validation of the analytical method and its application to formulation development and characterization.

Engineering the Immune System: Implications of Nanoparticle Designs on Modulating Inflammation. Ryan M. Pearson, University of Maryland, Jackline Joy Martin Lasola, University of Maryland. Objective: Sepsis remains a common clinical condition resulting in thousands of deaths and costing billions of healthcare dollars annually in the United States alone. Moreover, treatment strategies to limit the overexaggerated immune response in sepsis has thus far failed to successfully limit morbidity and mortality. Here, we test a new approach to treat severe inflammation using immunomodulatory cargo-less nanoparticles. Methods: Polymeric nanoparticles were prepared using a single-emulsion method from poly(lactic acid) (PLA) or poly(lactic-co-glycolic acid) (PLGA) and either neutral or anionic stabilizing surfactants. Cell uptake was assessed using confocal microscopy and flow cytometry. Inflammatory cytokine secretions influenced by particle treatment under lipopolysaccharide (LPS) stimulation were measured using ELISA. LPS-induced endotoxemia studies were carried out in vivo by administering a single dose of nanoparticles prior to a lethal challenge of LPS intraperitoneally (i.p) to mice. Results: Nanoparticle formulations were prepared with similar sizes (400 to $600 \mathrm{~nm}$ ) and different charges depending on the type of surfactant utilized (-50 to $-20 \mathrm{mV}$ ). Nanoparticles prepared from PLA were more effective at inhibiting in vitro inflammatory cytokine secretions by macrophages than those prepared from PLGA. LPSinduced endotoxemia studies showed that i.p. administration of anionic PLA particles prior to a lethal challenge of LPS dramatically improved the survival of mice. Implications: This work contributes to the fundamental and functional understanding of nanoparticle properties and surface chemistry on modulating inflammatory responses. We believe that nanoparticles are a promising treatment strategy to treat a variety of inflammationassociated conditions.

Enhancement of Student Knowledge Beyond Lecture Using Play-Doh ${ }^{\circledR}$ and Beads to Construct Concentration-Response Curves. Forrest L. Smith, Harding University, Ashley Malkowski, Harding University, Jim Tarrant, Harding University. Objective: Maximum Effect-(Emax), Effective Concentration-(EC50), and potency-ratio values are difficult for P1 students to learn during lecture. Knowledge- and skills-based classroom activities promote learning. The hypothesis tested that student creation of concentration-response curves, and associated calculations, result in higher post-activity 


\section{American Journal of Pharmaceutical Education 2019; 83 (5) Article 7654.}

test scores than same day pre-test scores after lecture. Methods: A repurposed ashtray receptacle served as the extracellular fluid containing the beads "drug", while the Play-Doh ${ }^{\circledR}$ surface in a container was the receptor field. Large and small beads constituted different drugs. Student groups measured \%binding (as E/Emax) at increasing concentrations of beads until saturation at the Emax. EC50 and potency-ratio was calculated. Questions answered after lecture (post-lecture), and the same questions answered after the activity (post-activity) assessed the activity's effectiveness to improve knowledge on Emax, ED50, and potency-ratio. A survey at the end measured for affective skills in Domains 3 and 4 of CAPE 2013. Results: Large and small bead values differed significantly: EC50: 21-beads (95\%CL 29 to 24) and 57-beads (95\%CL 54 to 60), Emax values of 57 and 123 beads, respectively, and 2.7-fold potency-ratio. Post-activity test scores were significantly higher than post-lecture scores. Students agreed or strongly-agreed (3.5 of 4.0 scale) with Domains 3 and 4 of CAPE 2013, indicating significant engagement in problem solving, communication, self-awareness and motivation in the group activity (goodness of fit $\chi 2$ ). Implications: Classroom modeling of laboratory-based concentrationresponse curves increased learning, and is ideal for large student classes. The affective behaviors engaged may indicate that certain traits are necessary for group success on activities.

Evaluation of a Compressed Pharmaceutical Calculations Course in First-Year Pharmacy Students. David Mastropietro, Nova Southeastern University, Devada Singh-Franco, Nova Southeastern University, Sean T. Leonard, St. John Fisher College, Michael D. Dressler, Nova Southeastern University. Objective: To evaluate students' performance on summative assessments administered during a compressed-format course and again nine weeks later in an end-of-semester "Bring Back" course. Methods: The College of Pharmacy implemented a new curriculum where a Pharmaceutical Calculations course was compressed from a traditional 15-week semester course to a 4-day immersive course delivered over two weeks. During the compressed course, 203 students completed three individual and team readiness assurance tests (iRAT/ tRAT) administered via Examsoft ${ }^{\circledR}$. From these assessments, six questions, each with a difficulty index $<0.80$ were selected, revised (values and drug names) and re-administered during the "Bring-Back" course. Each question was assigned a single point for analysis. Students' scores during the compressed course (pre) and the "Bring-Back" course (post) were analyzed using a within-subjects ANOVA in SPSS (version 25). Results: Pre- and post-assessment scores were calculated from 203 students. Compared with pre-assessment scores, there was a statistically significant improvement in mean scores post-assessment ( 3.68 vs. $4.16, \mathrm{p}<0.001)$. A limitation was the small number of questions allowed to be administered post-assessment. Implications: Implications: The compressed calculations course incorporated team-based learning activities to increase student engagement and peer-to-peer teaching during didactic sessions. The purpose of the "Bring Back" course was to evaluate retention of key concepts from previouslytaught courses within the semester. Overall, there was an improvement in students' performance post-assessment, utilizing questions that were considered "difficult" based on item analysis of each pre-assessment question. However, further research with a larger number of questions administered post-assessment will improve reliability of the assessment.

Impact of an External One-Day Workshop on Pharmacy Students' Confidence in Their Compounding Skills. David Mastropietro, Nova Southeastern University, Devada Singh-Franco, Nova Southeastern University, William R. Wolowich, Nova Southeastern University. Objective: To evaluate impact of an external one-day compounding workshop on the confidence of pharmacy students using advanced technologies in preparing nonsterile preparations and of procedural processes for sterile and hazardous compounding. Methods: An interactive compounding workshop was embedded within a 7-week summer elective course. Students were exposed to the following activities: interactive lectures, team-based exercises, and hands-on preparation of dosage forms. LP3 Network ${ }^{\mathrm{TM}}$ provided the workshop at their state-of-the-art facility in Aventura, FL. This facility has additional resources and technologies which broadens students' experiences beyond the classroom setting. In the sixth week of the course, students who voluntarily attended the workshop were invited to complete a pre-/post-workshop questionnaire that assessed their confidence using a 5-point Likert scale (not at all confidentvery confident). Eight questions assessed confidence using advanced technologies and determined understanding of protocols for sterile and hazardous compounding. Data were analyzed using NCSS 12.0.8 Statistical Software. The Friedman test compared pre-/post-intervention results. Results: Fifteen students completed both the workshop and the pre-/post-questionnaire. Self-reported confidence was significantly improved among students in using advanced technologies: semi-automatic capsule filling machine, electronic mortar and pestle, ointment mill ( $\mathrm{p} \leq 0.05$ for all). Students' confidence of the functions and operations performed within various environment (e.g., nonhazardous, hazardous) were significantly 


\section{American Journal of Pharmaceutical Education 2019; 83 (5) Article 7654.}

improved after exposure to certified cleanrooms ( $\mathrm{p} \leq 0.05$ for all). Implications: Limited exposure to compounding environments may result in unawareness of the provision of personalized medicine through compounding. Exposure to up-to-date compounding equipment and hands-on laboratory experiences can help improve students' confidence in compounding skills.

Increased Student Knowledge of Differences in IR- and ER-Metoprolol Formulation Dissolution in a Stomach-Duodenum Model. Rana A. Abdulamir, Harding University, Kofi L. Sefah, Harding University, Jacob L. Hatvany, Harding University, Jim Tarrant, Harding University, George Kwame Yeboah, Harding University, Forrest L. Smith, Harding University. Objective: Immediate Release-(IR) and Extended Release-(ER) differences between drugs are often difficult for students to conceptualize. Knowledge- and skills-based activities promote learning. The hypothesis tested that student learning and skills occur by observation of significant differences in the rate of IR- and ER-metoprolol release, and by significant improvement in post-test scores on properties of extended release drugs. Methods: $\mathrm{P} 1$ groups added 50-mg IR- and ER-metoprolol tablets into 50-ml 0.01 N-HCL, 37-C, stir-paddle 150-rpm, using published stomach-duodenum methods measuring drug-disolution. Timed stomach samples were: 10-40-min, followed by duodenal neutralization at 40-min with $100-\mathrm{ml} 0.5 \mathrm{M}$ Na-phosphate buffer with samples at 50-min, 1-h, 2 h 4h, 6-h, 10-h, 18-h. Serial 1:1 dilutions and UV-fluorescence detection brought emission spectra within range of metoprolol standard curves for quantitative values. Pre-/ posttest assessed for improved knowledge on extended release drugs. A survey at the end measured for engagement of affective skills in Domains 3 and 4 CAPE 2013. Results: IR metoprolol achieved maximal concentrations within 10-min and remained so for 18-h. ER metoprolol concentrations were significantly lower, and slowly increased to IR concentrations at 18-h. Post-test scores were significantly higher than pre-test scores. Students agreed or strongly-agreed ( 3.5 of 4.0 scale) with Domains 3 and 4 of CAPE 2013, indicating significant engagement of problem solving, communication, self-awareness and motivation in the group activity (goodness of fit $\chi 2$ ). Implications: Skills-based activity in a laboratory setting comparing IR to ER drug release increased student learning. The affective behaviors elicited may indicate certain traits are necessary for groups to succeed on activities.

Influence of an Algorithm on the Therapeutic Drug Monitoring Decisions of Pharmacy Students. Laura M. Fox, Presbyterian College. Objective: Pharmacy students often struggle with the conceptual problem-solving process required for therapeutic drug monitoring (TDM) calculations and decisions. Algorithms have been used successfully in chemistry, engineering, law, nursing, and medicine education; thus, a TDM Algorithm was implemented in a pharmacokinetics course to enhance student success in TDM decision making. Methods: Scores from TDM cases completed during pharmacokinetics coursework over four years were compared between years prior to the implementation of the TDM algorithm and after implementation. To further test the impact of the algorithm, a TDM assessment was administered to pharmacy student volunteers who were randomly assigned to the experimental group (algorithm provided) or control group (algorithm withheld); the scores between groups were compared. Student opinions of learning resources that influenced their success in designing and adjusting dosage regimens were gathered via a survey. Results: Scores on TDM cases were significantly higher in years when the TDM algorithm was used compared to years prior to implementation. There was no significant difference in P4 scores; however, P3 students in the algorithm group scored significantly higher on the TDM assessment than did those in the control group. Most students rated the TDM algorithm as very to extremely helpful. Implications: Students find algorithms helpful in making decisions during the problem-solving process and the implementation of a TDM algorithm successfully impacted student performance in designing and adjusting dosage regimens. Algorithms can be used to aid students in solving difficult, or multi-step problems to aid them in clinical decision making.

In Vitro Analysis of Embelin in Combination with Afatinib for Treating Breast Cancer. Michael Danquah, Chicago State University. Objective: To examine the anticancer effect of afatinib and embelin alone or in combination on proliferation, apoptosis and migration of MDA-MB-231 breast cancer cells. Methods: Antiproliferative effect was assessed using MTT assay, apoptosis determined using flow cytometry and XIAP expression determined using ELISA. Results: The antiproliferative effect of afatinib and embelin was found to be dose and time-dependent in MDA-MB-231 cells. Afatinib was more potent that embelin regardless of time or concentration. IC50 values for afatinib were $5.65 \mu \mathrm{M}$ and $0.75 \mu \mathrm{M}$ at $24 \mathrm{~h}$ and $48 \mathrm{~h}$, respectively. In contrast, IC50 values for embelin were $7.31 \mu \mathrm{M}$ and $1.74 \mu \mathrm{M}$ at $24 \mathrm{~h}$ and $48 \mathrm{~h}$, respectively. As determined using combination index analysis, simultaneous combination of afatinib and embelin was synergistic $(\mathrm{CI}=0.18)$ at ED75 following treatment for 24 hours. Also, sequential treatment of afatinib followed by embelin exhibited a synergistic effect (CI = 0.15). Combination of afatinib and embelin was more potent in inducing apoptosis compared to monotherapy. 


\section{American Journal of Pharmaceutical Education 2019; 83 (5) Article 7654.}

Additionally, combination of afatinib $(5 \mu \mathrm{M})$ and embelin $(5 \mu \mathrm{M})$ more effectively downregulated XIAP expression in MDA-MB-231 breast cancer cells compared to monotherapy. XIAP expression decreased by $\sim 27 \%$ for combination therapy compared to $\sim 10 \%$ and $\sim 13 \%$ for embelin and afatinib, respectively. Implications: Anticancer effect of afatinib and embelin in MDA-MB-231 breast cancer cells was found to be dose and time-dependent. Combination of afatinib and embelin is more potent in inhibiting MDA-MB-231 breast cancer cell proliferation compared to monotherapy and the anticancer effect is schedule dependent.

Longitudinal Analysis of Student Attainment of Key Proficiencies in Integrated Pharmacotherapeutic (IPT) Courses. Ajoy Koomer, Marshall B. Ketchum University, Rajesh Vadlapatla, Marshall B. Ketchum University, Ashleigh-Anne Au, Marshall B. Ketchum University, Edward Fisher, Marshall B. Ketchum University. Objective: To analyze trends in student performance in common categories that transcends across Integrated Pharmacotherapeutic (IPT) courses. Methods: The College of Pharmacy has implemented ExamSoft ${ }^{\circledR}$ in the Fall Quarter of 2016 to administer didactic assessments to its inaugural class of 2020 cohorts. It has offered eight IPT (six in P2 and two in P3) courses to date accounting for nearly thirty percent of the didactic curriculum. The test items have been categorized to key standards such as ACPE Appendix 1 content areas, CAPE 2013 outcomes/COP PLEO(s) and Bloom's Taxonomy domains. In order to perform this analysis, the student scores are classified into four segments: lowest, class average, standard and highest. Results: Trend analysis using Microsoft Excel 2016 indicated that class average scores for student performance in Bloom's categories showed a linear decreasing trend, comparing six P2 IPT courses with all required courses in $\mathrm{P} 2$ curriculum. Class average student scores for eight IPT courses increased for Patient Assessment, Patient Safety, Pharmacotherapeutics, Clinical Pharmacokinetics and Pharmacology when compared to student performance in P2 IPT courses. Lowest scores for student performance in eight IPT courses in ACPE 1 Biomedical Sciences emphasis area showed an increasing trend that fit to a linear regression equation. Implications: This study throws light on student performance at micro level in IPT courses that evaluate students on challenging cognitive concepts. In addition, the analysis reveals that probable student adaption to this course series that may contribute to increased categorized scores for the entire IPT sequence.

Male Enhancement Nutraceuticals Sold Online in the USA: Claim, Pharmaceutical Quality, and Safety Assessments. Mark Mikhail, University of Saint Joseph,
Harshvir Kaur, University of Saint Joseph, Jessica Sweeney, University of Saint Joseph, Nadine Amine, University of Saint Joseph, Cindy Zheng, University of Saint Joseph, Nada Ahmed, Alexandria University, Heba Eassa, University of Saint Joseph, Doreen E. Szollosi, University of Saint Joseph, Mohamed I. Nounou, University of Saint Joseph. Objective: The American market is invaded with male enhancement nutraceuticals, which claim to be of natural origin and may represent a great health safety threat to consumers. They are sold claiming major therapeutic uses on online retail stores, such as Amazon and eBay. This project objective is to investigate the safety, efficacy and quality parameters of products declaring therapeutic treatment of erectile dysfunction as an alternative to phosphodiesterase-5 inhibitors (PDE5Is). Methods: The analyzed products, purchased from Amazon, were AlphaManXL, Leyzene $^{\mathrm{TM}}$, Golden $\mathrm{X}^{\mathrm{TM}}$, Organic Maca ${ }^{\mathrm{TM}}$, BigRize ${ }^{\mathrm{TM}}$, Men's Testosterone Booster ${ }^{\mathrm{TM}}$, and Horny Goat Weed ${ }^{\mathrm{TM}}$. High- performance liquid chromatography (HPLC) and near infra-red (NIR) were used to determine the content of the nutraceuticals under investigation. Physical properties were also assessed using content uniformity and weight variation test together with friability testing. SeDeM analysis was used to investigate the pharmaceutical quality and inter and intra batch variability within products1. Results: Different products contained undeclared PDE5Is with variable heterogeneous amounts per single dose. They were of poor pharmaceutical quality and consistency; confirmed by HPLC, NIR, content uniformity, weight variation, dissolution, friability, and SeDeM diagram analysis as a quality control and assurance tool. Implications: The male enhancement nutraceuticals sold online in the American market claimed to be $100 \%$ natural and safe, targeting men who cannot safely use PDE5Is. These products are illegally marketed with therapeutic claims without any physician supervision or regulation. Our results corroborate that these products pose a major threat to customers' lives due to the presence of adulterated pharmaceuticals and questionable quality profiles.

Pharmacy, Nursing, and Physician Assistant Studies Student Self-Report and Perceptions Regarding Classroom Etiquette. Eytan A. Klausner, South College, Kandi D. Pitchford, South College, James L. Schmidhammer, University of Tennessee at Knoxville, Billie R. Phillips, South College. Objective: To investigate pharmacy, physician assistant studies, and nursing students' self-report regarding their classroom etiquette and perceptions regarding classroom etiquette. Methods: A survey regarding classroom etiquette was administered to first year, pharmacy, physician assistant studies, and nursing students (156 responders). Statistical analysis was performed to determine differences among various 


\section{American Journal of Pharmaceutical Education 2019; 83 (5) Article 7654.}

student behaviors and perceptions, and among students of the three academic programs. Results: Most students $(>84 \%)$ indicated that arriving late to class, leaving the classroom during lecture without returning, initiating a conversation with a peer during lecture, and conducting non-class-related activities with electronic devices during lecture are unprofessional. Forty percent of students indicated that leaving and returning to the classroom is professional. Students self-reported that tardiness and leaving and returning to the classroom during lecture are not rare. Most students (52\%) indicated that it is acceptable to have a conversation with a peer while the professor is teaching if the conversation is directly related to the lecture material. Most students (68\%) indicated that a conversation between two or more students that occurs in close proximity interferes with their learning process. More students reported that their academic performance is adversely affected by non-academic use of laptop or tablet devices compared to the use of cell phones. Implications: This study suggests that there is room to improve student classroom etiquette in various academic settings. Moreover, this study may bring a greater awareness to the issue of classroom etiquette, thereby encouraging faculty and administration to evaluate, and/or better enforce policies related to classroom etiquette.

Predicting Student Performance in HSRT-N Using Admission Requirements and Academic Performance. Ajoy Koomer, Marshall B. Ketchum University, Rajesh Vadlapatla, Marshall B. Ketchum University, Edward Fisher, Marshall B. Ketchum University. Objective: To demonstrate correlation between student attainment of "critical thinking and quantitative reasoning skills" in a standardized assessment and COP required evaluation tools. Methods: "Health Sciences Reasoning Test- Numeracy (HSRT-N)", developed by Insight Assessment, San Jose, CA, has been administered to the class of 2020 for three consecutive years starting at the beginning of Fall quarter, 2016. Student performance in "overall" and eight sub-categories of HSRT-N for each administration has been correlated with PCAT scores, Pre-Pharmacy overall GPA, Pre-Pharmacy science GPA, admissions interview scores, P1, P2 didactic GPAs and IPPE I, II scores. IBM SPSS 25.0 has been used for statistical analysis. Results: Results indicated that student scaled scores in "Overall" section of HSRT-N correlated strongly ( $\mathrm{r}=0.62-0.68, \mathrm{p}<0.05$ ) with PCAT performance, for all three administrations of the critical thinking assessment. For first administration of HSRT-N, "Deduction", "Inference" and "Numeracy" sub-categories of HSRT-N showed strongly correlations with PCAT scores $(\mathrm{r}=0.68-0.74, \mathrm{p}<0.05)$. Different sub-categories of HSRT-N showed strong statistically significant correla- tions with PCAT for different administrations. Linear regression models generated by stepwise procedure in SPSS indicate that PCAT scores account for 38 to $46 \%$ variation $(p<0.05)$ in HSRT-N “Overall” score, regardless of the timing of administration. Similar trends were reported for various sub-categories of the critical thinking assessment. Implications: Our limited study indicates the usefulness of PCAT as a predictor for student performance in a standardized assessment that measures critical thinking and numeracy skills.

Pre-Formulation Study of Atenolol, Enalapril Maleate and Hydrochlorothiazide Mixtures. Amusa S. Adebayo, Sullivan University. Objective: Conduct pre-formulation drug-drug and drug-excipients interactions study on a triple drug combination of atenolol, enalapril and hydrochlorothiazide (AEH). Develop HPLC analytical method for combination therapy of AEH Ascertain the feasibility of co-formulating atenolol, Enalapril and hydrochlorothiazide for patients with compelling indications, whose goal for the systolic BP and diastolic BP are above $20 \mathrm{~mm} \mathrm{Hg}$ or $10 \mathrm{~mm} \mathrm{Hg}$ respectively Methods: Single, binary and ternary mixtures of atenolol, enalapril, hydrochlorothiazide and some common tablet excipients were subjected to differential thermal analysis. Also, samples stored under temperature stability programs were analyzed at regular intervals using validated HPLC method. Results: Binary mixtures of atenolol and enalapril maleate formed a eutectic mixture at $97.4^{\circ} \mathrm{C}$ and at binary composition of $40 \%(\mathrm{w} / \mathrm{w})$ atenolol and $60 \%(\mathrm{w} /$ w) enalapril maleate. Intimate contact in the solid state of atenolol and enalapril maleate will give rise to eutectic formation due to depression of the melting points of individual components, which may complicate downstream processing during granulation drying and tableting. It may also induce some physical changes in dosage form during storage, shipping and handling. No sign of chemical interactions was observed in HPLC study of mixtures that were stored under temperature programs. Implications: There is need for an alternative formulation approach that would prevent physical mixing of atenolol and enalapril during blending and co-compression with hydrochlorothiazide. Results have implications for cocompounding of other drugs with potential application as combination therapy as this type of hidden physical interaction may impact product stability and drug-release characteristics.

Stability of Dexamethasone Sodium Phosphate in Intravenous Admixtures. Fang Zhao, St. John Fisher College, Ina Buga, St. John Fisher College, Joy Uzoma, St. John Fisher College, Kristin Reindel, St. John Fisher College, Kateryna Rashid, St. John Fisher College, Tuong Diep, Rochester Regional Health, Pamela McCartan, 


\section{American Journal of Pharmaceutical Education 2019; 83 (5) Article 7654.}

Rochester Regional Health. Objective: Diluted IV solutions of dexamethasone sodium phosphate (DSP) are often used in prophylactic treatment of chemotherapyinduced nausea and vomiting. However, there is limited data to support the selection of diluents, storage conditions, and beyond-use dates. This study was designed to investigate these parameters. Methods: DSP injection $100 \mathrm{mg} / 10 \mathrm{~mL}$ vials were used to prepare the IV admixtures in two diluents and at two drug concentrations $(0.08$ or $0.4 \mathrm{mg} / \mathrm{mL}$ ). The admixtures were packaged in PVC bags and stored at room temperature or under refrigeration for up to 14 days. On day-0, 1, 3, 7, and 14, each IV bag was inspected visually, and a sample was withdrawn for $\mathrm{pH}$ and HPLC analysis. Results: The freshly prepared admixtures of DSP appeared clear, colorless, and free of particulates. The initial $\mathrm{pH}$ values were 6.4-6.8 and 7.07.8 for bags in $0.9 \%$ sodium chloride and $5 \%$ dextrose, respectively. The initial DSP concentrations of all samples were within $96-100 \%$ of the expected values. Over the 14 days of storage at room temperature or refrigeration, no significant change was observed for the visual appearance of any IV bags. The $\mathrm{pH}$ of all samples remained within one $\mathrm{pH}$ unit from the initial values. The HPLC results confirmed that all samples retained $94-100 \%$ of original drug concentrations. Implications: IV admixtures of $0.08-0.4 \mathrm{mg} / \mathrm{mL}$ DSP are compatible with $0.9 \%$ sodium chloride and $5 \%$ dextrose in PVC bags. These admixtures are also chemically and physically stable for up to 14 days when stored at room temperature or under refrigeration.

Using Student-Generated Questions With an Audience Response System for Student-Centered Assessment. Lloyd F. Alfonso, D'Youville College. Objective: To assess the use of student-generated questions as a test preparation activity in a pharmaceutical sciences course. Methods: First year pharmacy students in a pharmaceutical sciences course worked in teams of 7 to 8 to write multiple-choice test questions based on previously taught content. The questions were then tested and graded using an audience response system and a rubric. Accuracy, usability, difficulty index, adherence to instructions and mapping to learning objectives were the criteria considered in the grading rubric. The entire question bank was made available to the students as a study aid for the test. The students were also administered a post-activity survey. Results: Based on the survey responses students felt the activity greatly improved their understanding of the content and performance on the test. Students also indicated an appreciation for higher-level question writing. The audience response system component of the activity promoted in-class discussions about the material. Test scores indicated better student performance compared to previous years. Implications: Using student-generated questions coupled with the audience response system increased student engagement and independent research into the content. This activity complemented the didactic component of the course by promoting collaborative learning and improved student performance on the test.

\section{PHARMACY PRACTICE}

\section{Completed Research}

AACP Research and Graduate Affairs Committee: Needs for Research Leadership Development Among U.S. Pharmacy Schools. Marina KawaguchiSuzuki, Pacific University Oregon, Stacy D. Brown, East Tennessee State University, Kathryn E. Meier, Washington State University, Dorothy F. Farrell, American Association of Colleges of Pharmacy, Kirsten F. Block, American Association of Colleges of Pharmacy, Kevin T. Fuji, Creighton University, R. Kiplin Guy, University of Kentucky, Cassandra E. Nelson, University of Maryland, Sridhar Anand, St. John Fisher College, Ami M. Vyas, The University of Rhode Island, James M. O'Donnell, University at Buffalo, The State University of New York. Objective: To investigate current training needs for research leadership among U.S. pharmacy schools Methods: Faculty members serving on the AACP Research and Graduate Affairs (RGA) Committee developed two surveys: one designed for administrators with oversight of faculty research and the other for faculty. Both surveys were reviewed by the RGA committee and AACP staff, including the AACP Institutional Research team. The administrator questionnaire was disseminated by sending a request to Deans to forward to relevant respondents. The AACP mailing list was used to reach faculty members. Responses were anonymously recorded with Qualtrics from November 2018 to January 2019. Results: Fifty-seven and 540 responses were collected for the administrator and faculty questionnaires respectively. Institutions designated as " $\mathrm{R} 1$ : Doctoral Universities - Highest research activity" $(\mathrm{N}=17)$ or "R3: Doctoral Universities - Moderate research activity" $(\mathrm{N}=13)$, according to Carnegie classifications, dominated the administrative responses. Most institutions required or recommended research activity regardless of different types of their faculty. The largest number of faculty responses were submitted by $\mathrm{PhD}$ scientists $(\mathrm{N}=189)$, followed by residency-trained pharmacists $(\mathrm{N}=188)$. According to the faculty questionnaire, $56.1 \%$ disagreed with the statement, "My university provides sufficient training for me to lead a research group." Both faculty members and administrators listed "how to lead a project by collaborating with other researchers" as the most preferred webinar topic, suggesting a need for faculty development in this 


\section{American Journal of Pharmaceutical Education 2019; 83 (5) Article 7654.}

area. Implications: Research is designated as an important part of faculty responsibility. However, there are unmet needs for faculty research leadership development among U.S. pharmacy schools.

A Case-Based, Interprofessional Activity to Enhance Student Understanding of Professional Roles and Clinical Knowledge. Sara K. Richter, St. Louis College of Pharmacy, Justinne Guyton, St. Louis College of Pharmacy, Christina Angel, Washington University in St. Louis, Megan Wren, Washington University in St. Louis. Objective: To evaluate the change in asthma and inhalerrelated knowledge, in addition to perceptions of physicianpharmacist interprofessional collaboration, following an interprofessional education activity. Methods: This study enrolled second-year medical and pharmacy students following completion of pulmonology-related coursework. Students worked in groups of six (two medical students with four pharmacy students) to complete a patient case focused on asthma management. Each profession was instructed to provide education within their group; medical students provided instruction on physical examination, while pharmacy students provided instruction about inhaler devices and administration technique. Asthma and inhaler-related knowledge was assessed pre- and post-activity with a quiz developed by study investigators. Student perceptions about roles and responsibilities for collaborative practice were assessed pre- and post-activity with portions of the Student Perceptions of Interprofessional Clinical Education (SPICE-R2) instrument. Results: 335 students participated in the activity (111 medical, 224 pharmacy). Scores on the knowledge-based quiz increased from pre- to post-activity in both medical $(65 \%$ vs. $85 \%$, $\mathrm{p}<.001)$ and pharmacy $(82 \%$ vs. $91 \%, \mathrm{p}<.001)$ students. The post-activity SPICE-R2 instrument showed that perceptions improved in $68 \%$ and $48 \%$ of medical and pharmacy students, respectively. Perceptions decreased in $5 \%$ of both medical and pharmacy students. Ninetyone $(82 \%)$ medical students and $196(88 \%)$ pharmacy students found this activity to be useful in their learning. Implications: Utilization of a case-based, interprofessional activity improved student knowledge and understanding of roles within a healthcare team. This activity can be adapted to incorporate other disease states and should be further investigated to determine impact on long-term knowledge retention and perceptions.

ACLS Training in the PharmD Curriculum Reveals Need for Reinforcement and Repetition of SkillBased Competencies. Susan E. Smith, The University of Georgia, Andrea Sikora Newsome, The University of Georgia, W. Anthony Hawkins, The University of Georgia, Michael J. Fulford, The University of Georgia, Beth
Phillips, The University of Georgia. Objective: To investigate an association between students' perceptions and actual performance after completing advanced cardiovascular life support (ACLS) training in the Doctor of Pharmacy curriculum. Methods: ACLS training was incorporated into the third-year pharmacy curriculum through a two-hour didactic lecture followed by a twohour simulation-based experience. Students were administered a pre- and post-instruction assessment consisting of 18 questions evaluating perceptions and 10 questions evaluating knowledge. Perceptions were evaluated using the Dreyfus model and were subsequently dichotomized as not competent (novice, advanced beginner) or competent (competent, proficient, expert). Knowledge questions were mapped to perception questions to identify any associations. Results: All students $(n=133)$ completed the assessment. In the post-instruction assessment, student perceptions were generally consistent with knowledge. Knowledge demonstrated on the pre-instruction assessment often exceeded perceptions, representing a lack of confidence related to ACLS skills (e.g. ability to recognize cardiac arrhythmias: $16 \%$ perceived competent; $67 \%$ demonstrated knowledge). In contrast, student perceptions exceeded knowledge in two areas: ability to perform chest compressions (79\% perceived competent; 59\% demonstrated knowledge); and the ability to deliver breaths (57\% perceived competent; $31 \%$ demonstrated knowledge). Implications: Student knowledge and confidence improved significantly after receiving in-class instruction. Student confidence was elevated in the preinstruction assessment on skills that are taught in the Basic Life Support (BLS) course and expanded upon in ACLS. High ratings of competence are likely a result of BLS training, which students receive during the P1 year. The lower than expected performance in these areas potentially highlight that skills-related competencies require repetition and reinforcement.

Advancing Pharmacist Medication Management Through Academic Public/Private Partnerships. Thomas E. Buckley, University of Connecticut, Marissa C. Salvo, University of Connecticut, Nathaniel M. Rickles, University of Connecticut. Objective: To demonstrate the implementation and outcomes of two pharmacist-based Medication Therapy Management (MTM) programs through a School of Pharmacy and Department of Public Health (DPH) partnership. Methods: Through CDC grants, the pharmacy school and DPH developed and implemented two unique MTM programs targeting underserved populations: (1) the SHAPE program through community pharmacies, and (2) the WISEWOMAN program through a community health worker team in ambulatory clinics. Pharmacists obtained MTM 


\section{American Journal of Pharmaceutical Education 2019; 83 (5) Article 7654.}

certification. Faculty and students facilitated: project methodology, database management, MTM implementation with structured assessment of drug therapy problems, MTM documentation, and evaluation of clinical outcomes. Using the Plan-Do-Act framework, the partnership also engaged in frequent quality improvements to enhance the programs. Reports were prepared and findings disseminated at professional meetings and to policymakers. Results: In 5 years of SHAPE, 354 patients with hypertension and/or diabetes were seen in 30 pharmacies for an average of 3 visits. In 2 years of WISEWOMAN, 43 patients with hypertension were seen for an average of 2.1 visits. Both programs identified nearly 5.5 drug therapy problems per patient with $80 \%$ partially or fully resolved. The programs resulted in substantial improvement of clinical outcome measures. Implications: Partnering a state agency, school of pharmacy, and the community can optimize pharmacists' accessibility and improve patient outcomes. Pharmacy schools can play key roles in the feasible incorporation of MTM into public health programs. By understanding community barriers and providing critical MTM support services, pharmacy schools can improve the likelihood MTM programs are effective and sustainable.

Advancing the Practice of Pharmacy in Kentucky Through Education of Protocol-Driven Care. Misty M. Stutz, Sullivan University, Mary K. Probst, Sullivan University. Objective: As a result of newly approved regulation (201 KAR 2:380), pharmacists in Kentucky can now initiate the dispensing of non-controlled medications and other professional services via a prescriber-approved board-authorized protocol. In an effort to transform and advance pharmacy within our state, our goal was to create and deliver interactive training modules to prepare students for this next step of practice. Methods: This education was piloted in a didactic elective entitled Advanced Community-Based Practice. Students completed selfpaced, interactive modules specific to protocol authorized conditions, including tools for implementation. Students then met as a class to apply their knowledge and skills in a case-based format. Results: Results were collected both on the pedagogy of the course through survey, as well as the increase in student knowledge based on pre and post assessments. Our survey response rate was $67 \%$ and students either agreed or strongly agreed that the course construction and delivery was an effective way to learn the material. Knowledge of content also increased from an average score on the pre-test of $50.89 \%$ to $73.11 \%$ on the post-test. After controlling for the fact that we had a small sample size, we still observed an increase of $22.2 \%$, which was statistically significant. Implications: Based on positive student feedback, these educational modules will be modified to meet the ACPE standards for continuing education and the required training for pharmacists to begin initiating protocol driven care. Research will continue on this project to determine the overall impact on the pharmacist's delivery of protocol-driven care.

A Multi-Faceted Approach to Prepare Student Pharmacists for Residency Placement Success. Megan Willson, Washington State University, Cheyenne C. Newsome, Washington State University. Objective: To examine how a multi-faceted approach affected student's ability to secure a pharmacy residency position postgraduation. Methods: WSU Office of Research Assurances has found that the project is exempt from the need for IRB review. Two optional programs were developed to prepare student pharmacists for the residency process. One opportunity was an elective residency preparation course in which students learned and applied skills to be successful in the ASHP residency application process. Students also were provided an opportunity to apply for a residency modeled schedule APPE rotation sequence, APPE residency block schedule. The APPE residency block schedule includes 4-6 rotations at a single site to foster students' clinical and leadership skills. Residency placement data from the National Matching Service was compared for students who participated in one or both of these elective opportunities to students who did not. Results: Sixty-seven students registered for the National Matching Service. Students who participated in the residency preparation elective course, APPE residency block schedule, or both had a 50\% (16/32) acceptance into a residency program. Students who completed neither the course or APPE residency block schedule had a $28.6 \%(10 / 35)$ acceptance rate into a residency position. Implications: Incorporating a residency preparation course and or residency modeled block APPE scheduling could increase rates of securing an ASHP residency placement. This information should encourage other programs to consider implementing targeted approaches to enhance students' success in securing a residency.

A Multi-Pronged Approach to Teaching Palliative and End-of-Life Care to Student Pharmacists. Meredith L. Howard, University of North Texas Health Science Center, Marian Gaviola, University of North Texas Health Science Center, Adenike Atanda, University of North Texas Health Science Center. Objective: To evaluate the impact of a coordinated multi-pronged approach to teaching end-of-life care on student pharmacist perceptions, knowledge, and confidence. Methods: Within our curriculum, topics surrounding end-of-life care have historically focused on pharmacotherapy with minimal time spent on conversation practice and empathy 


\section{American Journal of Pharmaceutical Education 2019; 83 (5) Article 7654.}

development. In spring 2019, we conducted an all-day session incorporating various teaching approaches (i.e. didactic lecture, Konstanz Method of Dilemma Discussion, Death over Dinner workshop) to prepare students to engage in these difficult discussions. A 21-item survey, administered at three time points (pre, mid, post), assessed student pharmacists across three domains: perceptions, confidence, and knowledge. Additionally, students completed post-session reflections. Results: Ninety-six students participated in the session, with $91 \%$ completing the pre-survey, $69 \%$ completing the midpoint, and 59\% completing the post-survey. Forty-nine (51\%) completed matched survey responses across all timepoints. There was significant improvement in 13 $(62 \%)$ of the survey items across three domains: $43 \%$ of items related to perception, $43 \%$ knowledge, and 100\% confidence. Post-hoc analysis showed significant improvements in 12/13 survey items between overall pre-session and post-session survey results, with most improvements occurring in the confidence domain. Implications: A multi-pronged approach to teaching end-oflife care to facilitate difficult discussions surrounding death improved confidence, perceptions, and knowledge among student pharmacists. Given its success, this multipronged, day-long approach including didactic sessions, large group discussions, and the Death over Dinner workshop will continue as a required activity within the curriculum to prepare students for conversations surrounding death and dying within their professional and personal lives.

Analysis of an Interprofessional Education Acute Care Simulation Activity. Valerie L. Ruehter, University of Missouri-Kansas City, Bradley J. Erich, University of Missouri-Kansas City, Michael Scott, University of Missouri-Kansas City, Maqual R. Graham, University of Missouri-Kansas City, Stefanie Ellison, University of Missouri-Kansas City, Emily Hillman, University of Missouri-Kansas City. Objective: Accreditation standards for schools of pharmacy and medicine require interprofessional, interactive learning to train students to effectively function within a healthcare team. We hypothesized that teams would collaborate to provide high quality patient care, with positive characteristics for interprofessional collaboration. Methods: An acute care simulated activity was designed and piloted for all pharmacy students (P3) in the third year of the professional program. Third (M3), fourth M4) and sixth (M6) year medical students were invited to participate. Teams of P3, M3, and M4 students assessed a standardized patient presenting with an acute illness, developed a plan, and presented treatment recommendations to an M6 student serving as the attending physician/evaluator. Student teams were assessed using an analytical checklist and Jefferson Teamwork Observation Guide (JTOG). Students completed a post-simulation survey to determine achievement of learning objectives, growth regarding teamwork, and overall value. Study was determined by IRB as non-human research. Results: All 48 teams achieved passing scores on the analytical checklist. Average score for the JTOG was 49 with a range of 31 to 56. Survey results show 95\% of students Agree to Strongly Agree that simulation learning objectives were met. Team strengths included building rapport, understanding/capitalizing on profession-specific roles, and respect. Overall, 91\% of students Agree to Strongly Agree that the simulation was a valuable learning experience. Implications: Collaboration during an interprofessional simulation activity resulted in effective team behavior and helped students identify components of team approaches to patient care. Similar simulations can be replicated to prepare team-ready health professions students.

A Nationwide Survey of Pharmacy Students' Knowledge and Perceptions Regarding Medical Marijuana. Karen E. Moeller, The University of Kansas, J.M. McGuire, Belmont University, Brittany L. Melton, The University of Kansas. Objective: The goal of the study was to measure pharmacy students' knowledge and attitudes towards medical marijuana and assess educational needs. Methods: A nationwide anonymous survey was distributed to pharmacy students. Equal numbers of schools were randomly selected based on geographical regions, private versus public universities, and the state's legal status of medical marijuana. The survey consisted of the following sections: (1) demographics and personal factors (2) knowledge assessment of indications and adverse effects of medicinal marijuana and (3) attitudes assessment (5-point Likert scale questions). Data was analyzed using descriptive statistics and chi-square for differences between groups. Results: Data from 629 students were analyzed. The majority of students who completed the survey lived in states where medical marijuana was legalized (61\%), attended public colleges $(53 \%)$, were female $(68 \%)$ and had a median age of 23 years. Overall, $91 \%$ of students believed that medical marijuana should be legalized nationally and $62 \%$ believed in legalization of recreational marijuana. A low number of students could correctly identify indications $(14.9 \%)$ and side effects $(7.0 \%)$ of marijuana using a $70 \%$ passing score. Students who had an in-depth discussion about medical marijuana in pharmacy school were more likely to be knowledgeable about marijuana indications than those who did not have a discussion, $\mathrm{p}=0.04$. Only $28 \%$ of students indicated they felt knowledge regarding medical marijuana. Implications: This survey 


\section{American Journal of Pharmaceutical Education 2019; 83 (5) Article 7654.}

showed a knowledge gap among pharmacy students regarding medical marijuana; however, students who received instruction on the topic were more knowledgeable. With increasing legalization of marijuana, this curricular gap needs to be addressed.

An Evaluation of an Escape Room Activity in a Pharmacy Capstone Course. Bethany Sibbitt, Cedarville University, Myriam Shaw-Ojeda, Cedarville University, Katherine Perry, Cedarville University, Kathrine Distel, Cedarville University. Objective: Pharmacy capstone courses serve dual purposes: reviewing overarching principles necessary for entry-level practitioners and addressing new developments prior to advanced practice pharmacy experiences (APPEs). Meeting both objectives often proves challenging for faculty and learners alike. This study evaluated student perceptions of an "escape room" activity in an oncology-focused capstone session. Methods: A multi-challenge escape room activity was developed to review basic science, clinical, and patient care concepts pertaining to breast cancer. Third-year student pharmacists $(n=48)$ enrolled in the capstone course worked in teams to complete five complex challenges addressing facets of pharmacotherapy and patient care. After completion, students provided feedback via anonymous survey regarding their perception of this novel activity. Results: All teams completed the activity with completion time ranging from $60-80$ minutes. The post-event survey, completed by $30 \%$ of the class, provided insight into logistical and educational aspects of the activity. Approximately 50\% of respondents felt this was a good review activity and $80 \%$ said it was more useful than an individual review activity. The majority of respondents affirmed the ability to practice skills and apply knowledge throughout the game. Implications: Overall, a majority of respondents said they enjoyed this activity and would recommend it be used again for future cohorts. In the future, challenges should be varied to leverage the strengths of many team members, allowing higher engagement. A goal of completing the activity rather than racing other teams to finish first may reduce stress and allow focus to remain on academics rather than on the clock.

A Novel Approach to Teaching Medication Reconciliation in a Skills-Based Laboratory Course. David E. Matthews, The Ohio State University, John D. Bickel, The Ohio State University, Joshua W. Gaborcik, The Ohio State University, Anna C. Haas-Gehres, The Ohio State University, Kristy M. Jackson, The Ohio State University. Objective: To develop a novel learning activity to simulate realistic medication reconciliation in the hospital setting. The activity required students to use multiple sources in order to collect, assess, and document informa- tion obtained during a complex patient encounter. This simulation expands upon activities described in existing pharmacy education literature by incorporating greater complexity and requiring use of practical information sources. Methods: Laboratory faculty and hospital preceptors collaborated to develop a live, interactive simulation of medication reconciliation. The simulation was conducted in the second professional year ( $\mathrm{P} 2$ year) of the PharmD program during Autumn 2017 and 2018 semesters. The activity required students to consult five sources of information: (1) A patient; (2) the patient's caregiver; (3) a community pharmacist; (4) a simulated prescription drug monitoring program (PDMP) report; and (5) a simulated EMR. Each source contributed information that was necessary to obtain and document an accurate medication list. Students were invited to complete a survey at the end of the course to gauge their perceived confidence in completing medication reconciliation. This study was determined to be exempt from review by The Ohio State University IRB. Results: A total of 178 out of 254 students across both semesters responded to the survey. Of the respondents, $75.3 \%$ "strongly agreed" that they felt more confident in their ability to complete a medication reconciliation, and $96.1 \%$ either "strongly agreed" or "agreed." Implications: A complex, multicomponent simulation effectively increased students' confidence in their ability to complete medication reconciliation.

A Picture's Worth a Thousand Words: An Infographic Assignment to Visually Transform Clinical Trial Information. Stephanie L. Sibicky, Northeastern University, Alexa A. Carlson, Northeastern University. Objective: Infographics present information in a visually striking way. Compared to written text alone, visuals increase readability $80 \%$ and content including images receives $90 \%$ more views than those without. Despite the increasing use of infographics in fields like marketing and sales, few organizations utilize infographics or have published about using infographics as a teaching and learning tool. Methods: During an Advanced Pharmacy Practice Experience (APPE), we created an assignment for students to create an infographic about a clinical trial to foster creativity, communication, and visual literacy skills to post to our APPE rotation blog. Utilizing different infographic creation websites (e.g., Piktochart, Canva), students transformed pertinent trial information into an infographic. The Infographic Design Rubric (IDR) published by Kibar and Akkoyunlu was used for assessment (1 - Unacceptable, 2 - Needs Work, 3 - Competent, 4 Exemplary). Results: Eleven infographics were graded using the IDR by two faculty members. Median score ranges for content generation (3-4), visual design/big picture (3), text (2.5-4), and colors/visual (2-4). Based on 


\section{American Journal of Pharmaceutical Education 2019; 83 (5) Article 7654.}

informal feedback, students enjoyed creating infographics, were able to better understand pertinent clinical trial information, transformed information in a meaningful way, and saw value in sharing infographics to the public. We are interested in further dissemination of infographics to the public by linking through other social media platforms (e.g., Twitter). Implications: There is increasing potential for the use of infographics as a means for students to transform health information for other health care professionals and educating the public.

Applications for Pharmacy Practice: A Novel Longitudinal Integrated Course. Miranda G. Law, Howard University, Imbi Drame, Howard University, Yolanda McKoy-Beach, Howard University, Simeon Adesina, Howard University. Objective: To develop and implement a novel integrated didactic course that advances practical clinical skills and holistic understanding of semester course content. Methods: The Application for Pharmacy Practice (APP) course series was launched in Fall 2018 for students in their first didactic year and co-coordinated by both clinical faculty and pharmaceutical sciences faculty. The course convened once every three weeks for three hours (for 5 total sessions) and was designed to reinforce semester material through integrated activities that encouraged holistic understanding without introducing new content. Each session included an individual closed-book clinical case (graded with points), a team open-book activity (graded utilizing an Entrustable Professional Activitiesbased rubric), and a debrief session. Students were expected to achieve EPA's at level one for their P1 year (observation). All activities were developed through integration of course material submitted regularly by each of the semester's course coordinators, reflecting content from six courses. Results: Students passed the course with an average course grade of $80.3 \%$ (StDev 3.2\%). Majority of student feedback on the course was positive and students believed the course helped them to: 1) understand real-life applications for semester material, 2) think critically, 3) hone time management skills, and 4) review all semester course material consistently. The course also enhanced their ability to work in teams. Implications: A fully integrated course can prepare student pharmacists, beginning their first year, to apply course content to pharmacy practice activities in an integrative manner, thus preparing students to better achieve high levels across Entrustable Professional Activities upon graduation.

Applying Educational Gaming Success to a Nonsterile Compounding Escape Room. Lauren M. Caldas, Virginia Commonwealth University, Heidi Eukel, North Dakota State University, Abigale T. Matulewicz, Virginia Commonwealth University, Elena V. Fernández, Virginia Commonwealth University, Krista L. Donohoe, Virginia
Commonwealth University. Objective: To design and expand on serious educational gaming techniques by creating an escape room to increase third-year professional pharmacy students' knowledge of nonsterile compounding. Methods: Faculty adapted the design and methods of a diabetes escape room to create a nonsterile compounding escape room. The escape room gaming environment used a simulated locked room, time limit, and rules in which student teams completed multiple puzzles focused on advanced topics of nonsterile compounding. All participating students completed a pre and post knowledge assessment. A previously validated 12-item survey on student perceptions of educational escape rooms was modified and administered at the end of the activity. Results: All students $(n=30)$ completed the knowledge pre- and post-assessment and perception survey (100\% response rate). Three out of four student teams successfully escaped the room. Students' performance on the knowledge assessment scores significantly improved from a median of $50 \%$ (IQR: $33.3 \%, 66.7 \%$ ) for pre-assessment score to a median of $83.3 \%$ (IQR: $66.7 \%, 83.3 \%$ ) for postassessment score, $\mathrm{p}$-value $<0.0001$. Students agreed or strongly agreed that the escape room activity was helpful to their learning. Implications: A nonsterile compounding escape room increased student knowledge and resulted in positive student perceptions of the teaching innovation. Escape room educational games may be successfully translated from diabetes topics to nonsterile compounding. Other content areas in pharmacy education may be explored in the future.

\section{A Simple Evaluation Tool for Pharmacy Student} Co-Curricular Service Learning Experiences. Michelle R. Musser, Ohio Northern University. Objective: Service learning provides a valuable co-curricular experience for students and augments health care in local communities. An evaluation tool is necessary to assess student performance and reflection, track co-curricular learning outcomes, and document service learning impact. A simple, online tool is described that meets these programmatic needs. Methods: Pharmacy students at a rural, private college of pharmacy are required to complete service learning experiences during their professional program. Following each service learning event, each student completes an evaluation and documentation of the experience, triggering a preceptor evaluation of the student using an online survey tool. Data was collected from 1924 student responses and their respective preceptors for evaluation over an academic year. Results: The online tool allowed documentation of 6509 hours of service and care for over 39,000 patients throughout the academic year. Common event locations, patient interactions, and services were identified. Opportunities to 


\section{American Journal of Pharmaceutical Education 2019; 83 (5) Article 7654.}

improve student exposure to interprofessional practice, innovation, and mentorship during service learning were noted. Preceptor evaluations were completed for $78 \%$ of student experiences. Preceptor evaluations were overwhelmingly positive; common feedback included a need for students to increase clinical confidence, but praised students' professionalism. Student self-assessment was supported by preceptor feedback. Implications: A simple online tool allowed data on a large service learning program to be evaluated and documentation. Tracking of service learning opportunities allows program improvement and expansion, while student self-assessment and preceptor feedback allows evaluation of student performance and learning outcome documentation.

Assessing Pharmacy Students' Knowledge and Attitudes About Naloxone. Annette T. McFarland, Butler University, Prachi Arora, Butler University, Erin Hays, Butler University, Lauren Porter, Butler University. Objective: Over 72,000 Americans died from an opioid overdose in 2017. Despite the availability of naloxone, a safe and effective reversal agent, distribution of the opioid antagonist is controversial and stigmatized. This study assessed pharmacy students' knowledge and attitudes about naloxone. Methods: Second year pharmacy students were surveyed pre-post opioid epidemic lectures to assess their knowledge about naloxone and attitudes toward dispensing it in a community pharmacy setting. Attitude questions were adapted from a past, validated survey instrument. Participants ranked the following naloxone dispensing scenarios on a 5-point Likert scale: heroin user, person taking prescription opioids for chronic pain, mother wanting naloxone for her daughter with opioid use disorder (OUD), patient in recovery for OUD, and a college student wanting to carry naloxone. Chi-square and Mann-Whitney two sample tests were conducted for pre-post survey comparisons. Statistical significance was determined at $\mathrm{p}<0.05$. Results: The majority of students completed the pre $(n=104)$ and post $(n=107)$ survey. Postintervention, students' knowledge regarding naloxone increased significantly $(p<0.05)$. Stigma towards 'likelihood of getting in trouble with the law' decreased significantly for 3 out of the 5 scenarios $(p<0.05)$ as did students' attitude towards 'inviting a heroin user to a dinner party' and 'being his/her colleague at work' $(p<0.05)$. Implications: After the intervention, students' knowledge and attitudes about naloxone changed significantly, especially towards heroin users. Study results suggest the need for continued efforts to reduce stigma surrounding OUD as pharmacists play a vital role in combating the opioid epidemic.

Assessing Student Knowledge and Confidence Following a Prescription Review Module. Alex J. Luli, University of California, San Diego, Renu F. Singh, Uni- versity of California, San Diego, Jennifer Namba, University of California, San Diego, Christina Mnatzaganian, University of California, San Diego, Nathan A. Painter, University of California, San Diego, Panteha Kelly, University of California San Diego, Brookie M. Best, University of California, San Diego, David Bao, University of California, San Diego, Eduardo S. Fricovsky, University of California, San Diego. Objective: To assess student knowledge and confidence with performing commonlyencountered community pharmacy related tasks following completion of a prescription review module. Methods: First-year student pharmacists completed a seven-hour, faculty-developed prescription review module within a Pharmacy Practice course. Prior to the module, students watched videos introducing them to various aspects of community pharmacy operations. Module learning objectives included: orientation to a community pharmacy setting and workflow; utilizing online pharmacy resources, supplies, and tools needed to dispense prescriptions; reviewing prescription orders for errors; verifying filled prescriptions; transcribing verbal orders; and dispensing medications. A ten-question knowledge assessment and ten-question confidence survey were administered prior to and after completion of the module. Two-sided Wilcoxon signed rank tests $(\alpha=0.05)$ were used for comparisons. Results: Seventy-two students $(100 \%)$ completed the knowledge assessment. Knowledge scores significantly improved after completion of the module, with a median pre-assessment score of $40 \%$ and post-assessment score of $70 \%(\mathrm{p}<0.001)$. Seventyone students $(98.6 \%)$ completed the confidence survey. The prescription review module significantly improved student confidence on all ten pre-post survey questions $(\mathrm{p}<0.001)$. Implications: A faculty-developed prescription review module within a first-year Pharmacy Practice course significantly increased first-year student pharmacists' knowledge of common community pharmacy dispensing-related tasks and improved self-confidence in their skills.

Assessing Student Pharmacists' Perceptions of Prescription Adjudications in a Pharmacy Practice Lab. Anthony L. Walker, The University of Louisiana at Monroe, Raghda K. Elsayed, The University of Louisiana at Monroe, Stacy Starks, The University of Louisiana at Monroe. Objective: To assess student pharmacists' knowledge of prescription adjudications in a pharmacy practice lab setting. Methods: Eighty-four student pharmacists enrolled in PHRD4049 lab course completed two prescription adjudication labs two weeks apart. Five core Entrustable Professional Activity (EPA) domains were incorporated throughout each laboratory exercise. A voluntary, post-prescription adjudication survey was 


\section{American Journal of Pharmaceutical Education 2019; 83 (5) Article 7654.}

administered after each lab. This study received IRB approval. Results: The survey response rates were $98 \%$ $(n=82)$ and $87 \%(n=73)$, respectively. While approximately $57 \%$ of students worked in a community pharmacy at the time of this activity, only $37 \%$ indicated previous exposure to insurance and/or prescription adjudication, and of those, half were not confident taking care of insurance issues. After the second lab, $84 \%$ were confident. Students' perceived inability to complete a new insurance entry decreased from $34 \%$ to $11 \%$, and their anxiety levels about prescription adjudication also decreased from $20 \%$ to $10 \%$ after completion of the second lab. Completion of the two prescription adjudication labs exposed each student to planned activities within Core EPA Domains 1-5. Implications: Introducing prescription adjudication and insurance in a pharmacy practice lab setting have a positive impact on students' confidence in solving prescription adjudication issues. Embedding Core EPA Domains into pharmacy practice lab exercises assist in educating future pharmacy graduates. Further studies may be warranted due to a small sample size.

Assessing Students' Knowledge and Perceptions of Urine Drug Testing Following a Pharmacy Skills Laboratory. Jessica M. Bates, The University of Kansas, Brittany L. Melton, The University of Kansas, Karen E. Moeller, The University of Kansas. Objective: This study aimed to evaluate changes in pharmacy students' knowledge of urine drug testing (UDT) interpretation, confidence and attitudes regarding use of UDT before and after a skills laboratory Methods: Third-year pharmacy students completed a self-assessment survey before and after a skills laboratory that included instruction on UDT. The 42-item survey consistent of 3 domains. The first domain collected demographic and prior experience with UDT. The second domain consisted of seven multiple choice knowledge questions on UDT. Lastly, the students were asked a series of Likert scale questions on confidence in UDT interpretation and attitudes regarding the practice of UDT. Demographics were assessed using descriptive statistics, and changes in perception of urine drug screens were evaluated using Mann-Whitney U with an a-priori alpha of 0.05 . Results: The majority of students who completed the survey were female $(62.9 \%)$, white $(85.4 \%)$, and non-Hispanic $(97.7 \%)$, with a median age of 23 years. Before the laboratory, only $38.6 \%$ were able to answer $70 \%$ of the knowledge questions correctly. However, after instruction this changed to $63.9 \%$, $\mathrm{p}=0.001$.After the laboratory, statistically significant increases in student confidence interpreting UDT $(\mathrm{p}<0.001)$, confidence interpreting if a sample is adulterated $(\mathrm{p}<0.001)$, knowledge of what drugs can be detected $(p<0.001)$, and comfort answering questions about urine drug testing $(\mathrm{p}<0.001)$ were found. Implications: Providing students with education and training on UDT improved students' knowledge and confidence in interpreting UDT. To our knowledge there is no literature to describe instructional methods on UDT in pharmacy schools' curriculum.

Assessing the Ability of Student Pharmacists to Facilitate Human Immunodeficiency Virus Pointof-Care Testing. Anna Staudt, Cedarville University, Juanita A. Draime, Cedarville University, Nicole K. Stute, Cedarville University, MeiLing G. Norfolk, Cedarville University, Zachary N. Jenkins, Cedarville University. Objective: The advent of HIV point of care testing (PoCT) has helped increase the ease of access to HIV testing in the outpatient setting. As community pharmacy continues to expand, it is crucial student pharmacists are exposed to PoCT in the classroom setting for proper instruction. Otherwise, the first exposure may occur when working as a pharmacist in a community pharmacy. Our objective was to train student pharmacists to perform HIV PoCT and educate patients on the results and implications of testing. Methods: Following a didactic HIV lecture, second-year student pharmacists learned to perform the OraQuick ADVANCE® Rapid HIV-1/2 Antibody Test in a one-hour workshop. Participants were then assessed during a skills lab on their ability to provide PoCT, interpret results, and counsel patients with a 4-point Likert scale. Sample kits were used to perform testing and simulated results were provided. Data were analyzed using descriptive statistics. Results: Students showed competency in their patient interaction, knowledge, and skills when performing the HIV PoCT. In total, 92.7\% of students were able to describe the purpose of the test, and $97.6 \%$ of students were able to interpret the results correctly. Students scored a mean of 41.68 points, with a maximum score of 50. Implications: Results of this study suggest that training student pharmacists to perform HIV PoCT may allow them to administer the test and counsel patients on results in an outpatient setting. Future studies should examine the likelihood that patients will seek training from PoCT-trained pharmacists.

Assessment of a Lecture Series to Prepare Doctor of Pharmacy Candidates for Postgraduate Training. Abir Kanaan, MCPHS University-Worcester/Manchester, Katherine Carey, MCPHS University-Worcester/ Manchester, Cheryl R. Durand, MCPHS UniversityWorcester/Manchester, Aimee D. Dawson, MCPHS University-Worcester/Manchester, Adriana Cabrera, MCPHS University-Worcester/Manchester, Kristine Willett, MCPHS University-Worcester/Manchester. Objective: A Postgraduate Readiness Education Program (PREP) was developed to promote and help students 


\section{American Journal of Pharmaceutical Education 2019; 83 (5) Article 7654.}

prepare for postgraduate (PG) training. Student feedback was obtained to assess the usefulness of the program. Methods: The PREP included a series of 5 sessions targeting various topics relevant to PG training. The sessions were presented by faculty, current PG education trainees, or representatives from career services. Student attendees were asked to evaluate each session by using a rubric that ranked on a 1-5 Likert scale the content importance and presentation, as well as the usefulness of content on career planning and knowledge development. At the end of the series, a focus group was held to obtain additional student feedback. Results: Approximately, 108 students attended each session, and 44\% completed each session's evaluation rubric. Overall, the series received positive feedback with 4.72 point average on all questions for all sessions. The session on interviewing skills had the highest ratings for importance of topic (4.97 points), usefulness (4.91 points) and personal knowledge gain (4.88 points). Feedback from written comments and focus group suggested increasing the time for each session, particularly for the question-and-answer period, providing more information on the matching process, navigating various $\mathrm{PG}$ education resources, and clarifying timelines for research involvement. Students also suggested recording these sessions for later viewing and incorporating information regarding graduate study programs. Implications: The PREP series aimed to prepare PharmD students for postgraduate training was successful based on student feedback. Modifications will be made on future offerings to incorporate student suggestions.

Assessment of Antimicrobial Pharmacokinetics Curriculum in Schools and Colleges of Pharmacy Across the United States. Gary Fong, Chapman University, Elias B. Chahine, Palm Beach Atlantic University, Julie Ann Justo, University of South Carolina, Navaneeth Narayanan, Rutgers, The State University of New Jersey, Emily Heil, University of Maryland, Kayla R. Stover, The University of Mississippi, Jonathan C. Cho, The University of Texas at Tyler, Zachary N. Jenkins, Cedarville University, Conan MacDougall, University of California, San Francisco. Objective: To identify when and how antimicrobial pharmacokinetics is taught in pharmacy curriculums. Methods: An online survey was distributed to 118 schools and colleges of pharmacy across the United States in 2018. Participants were asked to complete a 30minute questionnaire that covered when and how antimicrobial pharmacokinetics is taught and assessed at their institution. Results: The survey response rate was $46 \%$ (54/118). Of the 35 programs with a traditional progressive curriculum, 34 (97\%) had a basic science pharmacokinetics course, $16(46 \%)$ had a clinical pharmacokinetics course and $28(80 \%)$ had a therapeutics course that cov- ered clinical pharmacokinetics. Of the 19 programs with an integrated block curriculum, $17(89 \%)$ had a principles/foundations block and $13(68 \%)$ had an organs/systems block that covered clinical pharmacokinetics. Vancomycin and aminoglycoside were the most common antimicrobials taught. The predominant andragogy was lecture, which accounted for $64 \%$ of didactic time in programs with a traditional progressive curriculum and 51\% in those with an integrated block curriculum. Most courses used summative assessment with numerical responses and offered partial credit. More than two thirds of participants felt that they needed more time to teach clinical pharmacokinetics and about half felt that students were adequately prepared at beginning of clinical rotations. Approximately one third of participants stated that they were undergoing significant changes in their pharmacokinetics curriculum. Implications: Schools and colleges of pharmacy in the United States demonstrated inconsistency in teaching and assessing pharmacokinetics. This study may help understand gaps, establish best practices and create standardized materials in teaching antimicrobial pharmacokinetics.

Assessment of Factors Related to Pharmacy Student Success in Completing Objective Structured Clinical Exams (OSCEs). Lena M. Maynor, West Virginia University, Ashleigh L. Barrickman, West Virginia University, Marina Galvez Peralta, West Virginia University. Objective: To determine predictive factors for OSCE performance in third year (P3) pharmacy students. Methods: Students completing OSCEs in the P3 Spring 2018 semester were eligible for study inclusion. Data were collected, including age, previous degree, institution for pre-pharmacy coursework, cumulative grade point average (GPA), grades in Systems-Based Therapy (SBT) courses, Pharmacy Curriculum Outcomes Assessment (PCOA) exam score, and formative and summative OSCE scores. Additionally, students were surveyed regarding total intern hours completed prior to the start of the P3 Spring semester. Correlation analyses were performed using Spearman R test, double-tailed, $\mathrm{p}<0.05$. Results: Seventy-one of 72 (98.6\%) eligible students participated in the study.There was a moderate correlation between performance in formative OSCEs and summative OSCEs ( $\mathrm{r}$ 0.45).Moderate correlations were found between summative OSCE scores and cumulative GPA and average grade for SBT courses ( $\mathrm{r} 0.43$ and 0.41 , respectively).Weak correlations were found between summative OSCE scores and completion of community intern hours and PCOA score (r 0.32 and 0.20, respectively).A moderate correlation was found between formative OSCE scores and average grade for SBT courses ( $\mathrm{r}$ 0.40).Weak correlations were also found between 


\section{American Journal of Pharmaceutical Education 2019; 83 (5) Article 7654.}

formative OSCE scores and cumulative GPA and completion of community intern hours (r 0.34 and 0.24 , respectively). Implications: In our cohort, we found moderate correlations between OSCE scores and both performance in SBT courses and cumulative GPA.Understanding predictors of performance on OSCEs will be valuable as we integrate additional OSCEs throughout our curriculum and guide students in their preparations for courses with a significant OSCE component.

Assessment of Patient Goals in PharmacistOperated Workplace Wellness Program. Alison Walton, Butler University, Prachi Arora, Butler University, Samantha Stephens, Butler University, Michelina DiFatta, Butler University. Objective: To determine the rate SMART goals were achieved in pharmacist-operated workplace wellness program. Methods: A retrospective chart review of patients who completed at least one annual wellness consult (AWC) with the pharmacist between April 1, 2015 and April 1, 2017. Patients with identified health concerns (body mass index and hemoglobin A1c) during AWC were placed in health coaching (HC) Track 1. Patients set two wellness-related SMART goals during AWC or HC appointments, regarding sleep, weight, dietary, preventative health, healthcare provider establishment, physical activity, mental health, and other. Goal progress was classified as goal obtained, progress made, no progress made, or forgot goals. Percentages of patients obtaining their goal(s) were determined within each HC track. Results: Sample constituted 335 patients (mean age 44; 65\% females): 147 in Track 1 and 188 in Track 2 (classified at last visit). The most common goals set by patients were physical activity (47\%), dietary (28\%), and weight (14\%). Sixty percent of patients in Track 1 and $63 \%$ in Track 2 obtained at least one goal with $22 \%$ in Track 1 and 25\% in Track 2 obtaining both goals. Patients achieving the first goal under Track 1 (37\%) were significantly different than Track 2 (44\%); however, no such differences were found for the second goal. Implications: Pharmacists in a health coaching role could facilitate positive behavior change through achievement of wellnessrelated goals. In order to succeed, workplace programs need to expand pharmacists' role to help employees, specifically with health concerns, achieve their personalized goals.

Assessment of Pharmacists' Patient Care Process Model in Smoking Cessation. Dhara D. Shah, Atlantic Health System, Elly S. Oh, Atlantic Health System, Anasemon Aioub, Atlantic Health System, Anita Siu, Rutgers, The State University of New Jersey, Lucio Volino, Rutgers, The State University of New Jersey, Rupal Mansukhani, Rutgers, The State University of New Jersey. Objective: To assess the confidence and retention of the Pharmacists' Patient Care Process Model (PPCP) and its application to smoking cessation in a teaching environment. Methods: Pharmacy students in their third professional year of pharmacy school attended two 80minute smoking cessation lectures and two interactive counseling sessions. Pre- and post-surveys were administered in order to assess the students' retention of the PPCP Model and their confidence in utilizing the PPCP Model when counseling patients on a newly introduced topic. The outcomes of this study included the incidence of self-identified confidence in the students knowledge in understanding the PPCP Model; comfortability in applying the PPCP Model to any disease state; and the percent of students able to retain and conduct each step within the PPCP Model. Results: A total of 163 students were included in the study; a majority of the participants were of female gender, age 20 to 24 years old, and Asian ethnicity. There was a significant increase in students' knowledge of and confidence in understanding the PPCP Model and their comfort in using this model while counseling in smoking cessation after attending the targeted lecture $(p<0.001)$. Additionally, there was a significant increase in students' ability to collect, assess, plan, implement, and follow-up when counseling a patient on smoking cessation $(\mathrm{p}<0.001)$. Implications: This study demonstrates an effective approach and method to teaching and implementing the PPCP Model to pharmacy students.

Assessment of Student Knowledge, Awareness, and Perceptions of Women's Health Issues in Pharmacy Practice. Courtney A. Robertson, The University of Louisiana at Monroe. Objective: To evaluate students' knowledge of women's health, assess attitudes toward its importance in practice, and observe perceptions of issues affecting healthcare in women. Methods: Nineteen students enrolled in a 15-week elective course focusing on women's healthcare, related disease states, social issues, and health disparities through open topic discussions, case studies, individual reflections, and final presentations. Students completed IRB-approved pre- and postsurveys regarding their knowledge of women's health topics and a final survey of attitudes and perceptions related to the course, importance of women's health in practice, desired changes in topics, and advice to future students. Statistics were calculated for pre- and post-survey items and compared using the Wilcoxon Signed-Rank Test with $\alpha=0.05$. Results: All responses indicated statistically significant increases in knowledge of women's health issues with mean scale changes $p<0.00097$. Students strongly agreed their understanding of roles in providing care (89.47\%), empathy toward women with medical issues $(89.47 \%)$, and confidence in their ability to respond to needs of female patients in pharmacy 


\section{American Journal of Pharmaceutical Education 2019; 83 (5) Article 7654.}

settings $(94.74 \%)$ had increased, and strongly agreed the course was beneficial to their education experience (100\%). Implications: Pharmacists can provide optimal care and education to women through identification, discussion, and active engagement in related health and social issues. Throughout this elective, students were able to increase their knowledge and awareness of women's healthcare needs. Hopes for future women's health courses include class expansion, additional active learning activities, and implementation of second elective course featuring suggested topics into the didactic curriculum.

Assessment of Student Learning Through Multisite Topic Discussion Series Using Web-Based Conferencing Technology. Sweta M. Patel, Mercer University, Lydia C. Newsom, Mercer University, Maria M. Thurston, Mercer University, Jennifer B. Elliott, Mercer University, Kendra R. Manigault. Objective: Incorporation of technology into the didactic curriculum is required by the Accreditation Council for Pharmacy Education. However, the use of technology during Advanced Pharmacy Practice Experiences (APPE) is less well defined. The primary objective of this study was to assess students' knowledge in clinical decision-making through a multisite topic discussion (TD) series using web-based conferencing during ambulatory care APPEs. Methods: A five session faculty-led TD series was conducted during each five-week ambulatory care APPE at five distinct clinical sites June 2016 through October 2018. The TD series utilized $\mathrm{WebEx}^{\circledR}$ that provided audio and visual feeds for all attendees. Students were assigned required readings, guided questions, and patient cases to complete prior to each TD. A student knowledge assessment was administered before and after TD series. Results: During the study period, 144 students participated in the study. A total of $143(99.3 \%)$ students took the pre-assessment and $116(80.6 \%)$ took the post-assessment. Overall student mean scores on knowledge-based and clinical decision-making questions improved from $55.2 \%$ at baseline to $65.6 \%$ at the end of the 5 -week experience. Post-assessment scores increased for all seven disease states covered in the TD series. Implications: Participation in faculty-led web-based TD series during ambulatory care APPEs was associated with an increase in students' knowledge assessment scores. This type of TD series could potentially be applied to other specialties of pharmacy practice, in which multiple preceptors with different geographical practice sites could collaborate to provide a more beneficial and enriching experience for student pharmacists.

Assessment of the Impact of a Leadership Development Workshop on Perceived Leadership Ability. Lisa M. Richter, North Dakota State University, Allison
Hursman, North Dakota State University, Elizabeth R. Tupper, North Dakota State University. Objective: To design, deliver and assess the impact of a leadership development workshop on pharmacists' and student pharmacists' perceived leadership ability. Methods: Pharmacists and student pharmacists completed an optional six-hour leadership development workshop focused on personal and professional growth. Topics included: ability to change practice; financial analysis and pitching an idea; goal setting and time management. Participants completed pre- and post-workshop questionnaires with fifteen 4-point Likert scale questions ( 1 =strongly disagree; $4=$ strongly agree $)$. Cronbach's alpha was used to test internal reliability and a paired sample t-test was used to compare differences in pre- and post-workshop perception. Results: Thirty-four participants completed both questionnaires (pharmacists, $\mathrm{N}=17$; student pharmacists, $\mathrm{N}=17$ ). Three conceptual constructs from the questionnaires were identified and all of them had an alpha $>0.7$ indicating a strong internal reliability. The data also revealed a statistically significant increase among participants in perceived ability to change practice $(3.46 \pm 0.42$ vs $2.95 \pm 0.42, \mathrm{p}<0.001)$, perceived financial analysis skills and ability to pitch an idea (3.10 \pm 0.34 vs $2.18 \pm 0.70, p<0.001)$, and perceived skills in goal setting and time management $(3.44 \pm 0.39 \mathrm{vs}$ $2.99 \pm 0.36, \mathrm{p}<0.001)$ after the workshop completion. Implications: An intensive leadership development workshop has value in changing perceptions of leadership ability of pharmacists and student pharmacists. Additional studies are needed to determine if these improvements in perceptions translate into improved leadership skills.

Change in Pharmacy Students' Attitudes of Pediatric Topics After Participation in Pediatric Pharmacy-Focused Skills Lab. Jordan Ballou, The University of Mississippi, Amanda Capino, The University of Mississippi. Objective: School of Pharmacy curricula vary in incorporation of pediatric-related topics. Specifically, there are differences in learning experiences available, when courses are offered, and contact hours. The purpose of this study was to determine how the implementation of a pediatric-focused skills laboratory experience would influence student's comfort level and confidence regarding pediatric topics. Methods: The pediatric pharmacy activity was incorporated in the required skills laboratory sequence for second-year students. The activity included pre-readings, a pre-lab lecture, and a 2hour laboratory session. Three pediatric topics were covered: measuring devices and dosing errors, children and caregiver communication, and nonprescription recommendations. All students $(n=102)$ received the 


\section{American Journal of Pharmaceutical Education 2019; 83 (5) Article 7654.}

pre-questionnaire one week before the lab, and the postquestionnaire after completion of the lab via Qualtrics. Both questionnaires assessed perceptions of counseling pediatric patients, confidence in communication with children and caregivers, and confidence in making clinical recommendations. Results: A total of 81 students completed the pre-lab questionnaire and 49 students completed the post-lab questionnaire. In the pre-questionnaire, $29.49 \%$ of students did not feel comfortable counseling pediatric patients. This decreased to $18.37 \%$ in the postquestionnaire. Level of complete confidence in appropriate dose recommendations for children increased from $2.7 \%$ to $26.53 \%$ after the session. Additionally, after the lab session, $28.57 \%$ of students reported being completely confident in calculating correct $\mathrm{mL}$ of medications, compared with $5.4 \%$ on the pre-questionnaire. Implications: After the skills lab, some students' level of comfort and confidence regarding pediatric related topics improved.However, not all students were comfortable/completely confident which suggests the need for more pediatric-focused activities throughout the curriculum.

\section{Change in Student Preparation for and Perceived Value of thePharmacy Curriculum Outcomes Assess- ment (PCOA). Michael G. Kendrach, Samford Univer-} sity, Elizabeth A. Sheaffer, Samford University. Objective: Compare survey scores of students' PCOA preparation, anticipated results, and perceived value of the PCOA when they were second-year versus third-year students. Methods: The Class of 2020 students at one school completed an internet-based survey immediately after finishing each PCOA (taken Jan 2018 and Jan 2019). The students were informed of the survey purpose and that results would remain confidential. The PCOA is currently a low-stakes assessment at this school. University IRB approval was obtained for this survey. Results: A total of 94 students completed the survey each year. The majority of students still devoted no preparation time prior to and during Christmas break (91-95\%) but the response to "no time devoted to prepare after Christmas break" increased from $64 \%$ to $78 \%$. Significantly fewer completed the NABP practice PCOA as $\mathrm{P} 3 \mathrm{~s}$ (35\% vs $67 \%$ ). The mean score for students' perceived value of the PCOA decreased from 5.31 to 4.84 (10-point scale). Mean self-assessment scores (5-point scale) decreased for self-preparation (1.84 to 1.49) but increased for attempt to answer all questions correctly (3.67 to 3.82 ) and anticipated results (2.39 to 2.76). The two most common responses for using the PCOA results remained the same: "personal awareness of my strengths and weaknesses" and "prepare for next year's PCOA or NAPLEX". Implications: Survey results from this class of students indicate they prepared less for the PCOA and they perceived the PCOA value less as $\mathrm{P} 3 \mathrm{~s}$ versus $\mathrm{P} 2 \mathrm{~s}$. Besides developing SMART goals, additional uses of the PCOA results for individual students are being developed.

Changes in Stress Level and Stress-Related Factors in First-year Pharmacy Students. Christina A. Spivey, The University of Tennessee, Sara Stallworth, The University of Tennessee, Marie A. Chisholm-Burns, The University of Tennessee. Objective: Previous studies noted that stress levels change over time as students progress through healthcare curricula. The objective was to longitudinally assess stress among first-year student pharmacists and determine how levels and sources ('stressors') of stress change during the first semester of the PharmD program. Methods: Surveys were conducted during orientation and late in the first semester of pharmacy school. Data were collected using the Perceived Stress Scale (PSS) and Pharmacy Environmental Survey (PES), modified from the Dental Environmental Survey. Paired-sample t-tests were conducted to compare mean scale scores from the first and second survey administrations. Results: Participation was 99.5\% (201 of 202) and $78.2 \%$ (158 of 202), respectively, on the first and second administrations. Significant increases in mean PSS and PES summary scores were found between the first and second survey administrations. Significant increases were also noted on individual school-related stressors from the PES: quantity of classwork, grades and exams, feedback, peer competition, cheating, completing graduation requirements, rules, attendance requirements, academic expectations, response to student needs, atmosphere, lack of input in school decision-making, lack of confidence, fear of failing, and insecurity about professional future. Personal stressor scores (eg, lack of relaxation time, physical/ mental health, home atmosphere, roommate relations) also increased significantly. Implications: Significant increases in stress were experienced by first-year pharmacy students during their first year of pharmacy school. This study has important implications for understanding how stress levels and individual stressors change over time, which can lead to development of improved strategies to address student pharmacist stress.

Collaborative Use of a Mock-Trial as TeachingAssessment Activity in Required Courses at Two Institutions. Ettie Rosenberg, West Coast University, Hoai-An Truong, University of Maryland Eastern Shore, Shih-Ying Hsu, West Coast University, Reza Taheri, RxPrep. Objective: To describe cross-institutional collaborative implementation of a mock-trial in first-year (P1) required courses at two pharmacy programs. Methods: After proving mock-trial is an effective selfdirected learning strategy at one institution, faculty 


\section{American Journal of Pharmaceutical Education 2019; 83 (5) Article 7654.}

collaboration across two institutions implemented parallel mock-trials at their respective programs for two subsequent years. Collaboration included learning objectives, standardized procedures, debate topics, and use of identical assessment tools. P1 cohorts at each program were divided into teams to research, prepare, and debate "for" and "against" controversial pharmacy practice topics in courtroom format. Descriptive statistics were used to analyze faculty-judge scores, student-juror scores, student-peer evaluations, and overall scores. Each program obtained Institutional Review Board exempt approval for this study. Results: A total of 222 P1 student-pharmacists in parallel mock-trials (two institutions; different courses) debated identical topics (2017: $\mathrm{N}=117$; 2018: $\mathrm{N}=105$ ).Faculty-judge scores, studentjuror scores, and student-peer evaluations all showed comparable means (difference $<1$ point) and comparable range between two institutions in 2017 and 2018. The only exception found in 2018 faculty-judge scores reported a 1.16-point difference in means between programs. Mean overall scores (across institutions/trials) fell between 55-57/60 points. Implications: The results for student assessment by different evaluators demonstrate consistent student performance in mock-trials across two institutions and courses, irrespective of other variations. Considering these mock-trials were implemented at two institutions with varied features (traditional 4-year versus concentrated 3-year program; public versus private program; east versus west coast demographic), the described mock-trial project can potentially be replicated and adopted as an innovative approach for collaboration and/or competition between other institutions.

Comparing Different Standard-Setting Methodologies for a High-Stakes OSCE. David J. Caldwell, University of Arkansas for Medical Sciences, Ashley N. Castleberry, The University of Texas at Austin, Benjamin S. Teeter, University of Arkansas for Medical Sciences. Objective: To describe the effects of different scoring and standard-setting methods for a high-stakes objective structured clinical examination (OSCE) Methods: Data from a high-stakes, 10-station OSCE were used to model the effects of two variations in item scoring and three methods of standard setting. Two standard-setting approaches, the borderline group (BG) and borderline regression (BR) methods, used standardized participant global ratings to calculate case- and exam-level passing scores, each with two versions of checklist scoring: weighted and unweighted skills items. The third approach, the Ebel method, used expert judgments of individual checklist items to calculate passing thresholds based on the construct of a borderline student. This mod- eling yielded five versions of exam results for comparison. Results: The Ebel method yielded the lowest passing score $(65.8 \%)$ with no student failures, while the BR method with weighted checklist items resulted in the highest passing score $(77.0 \%)$ with 14 failures. The weighted and unweighted BG methods gave similar passing thresholds to the Ebel method $(67.7 \%, 66.3 \%$, respectively) and neither resulted in failures. The unweighted BR method was similar to the weighted version $(75.3 \%$; 11 failures). Implications: Approaches to calculating passing standards for performance-based exams like OSCEs may result in different rates of student failures. Some methods rely on global performance ratings assigned by standardized participants, and others rely on judgements by content experts. The validity of each approach is supported by existing literature, so careful consideration must be given to the exam purpose when selecting standard-setting methodology.

Comparison of Performance During Advanced Pharmacy Practice Experiences (APPEs) After Online and On-Campus Curricular Pathways. Paul Reynolds, University of Colorado, Jason Brunner, University of Colorado, Megan E. Thompson, University of Colorado, Kari L. Franson, University of Colorado, Monika M. Nuffer, University of Colorado, Erica J. Rhein, University of Colorado, Rachel Wagmaister, University of Colorado, Shaun E. Gleason, University of Colorado. Objective: The University of Colorado offers three Doctor of Pharmacy (PharmD) pathways, including an online entry-level International-Trained PharmD (ITPD), an online post-baccalaureate North American-Trained PharmD (NTPD), and an on-campus Entry-Level PharmD (ELPD). Differences between APPE preceptor assessment of clinical domains (communication, professionalism, practitioner skills) for online and on-campus pathways have not been previously described. Methods: Preceptor evaluations of APPE performance were retrospectively collected across a three-year period for all pathways. Clinical domains were evaluated on a 5-point scale $(5=$ beyond entry-level performance, $4=$ entry-level performance, $3=$ entry-level performance with limitations, 2 =beginning performance, $1=$ not ready for advancement). Overall performance was categorized by letter grade. ANOVA or Kruskal-Wallis were used for clinical domains and Chi-squared analysis for letter grades. Results: 2811 preceptor evaluations were recorded $(\mathrm{ELDP}=2696, \mathrm{NTPD}=69$, ITPD $=46)$. Although ELPD students scored significantly $(\mathrm{P}=0.01)$ higher in overall performance (91\% vs $88 \% \mathrm{~A} ; 6.8 \%$ vs $7.4 \% \mathrm{~B}, 0.09 \%$ vs $1.8 \% \mathrm{C} ; 0.04 \%$ vs $2.8 \% \mathrm{~F}$ ), both online programs scored significantly higher $(\mathrm{P}<0.05)$ than ELPD on preceptor evaluations of every clinical domain. 


\section{American Journal of Pharmaceutical Education 2019; 83 (5) Article 7654.}

Median communication scores were 4.9 for ITPD, 4.8 for NTPD, and 4.4 for ELPD. Median professionalism scores were 5.0 for ITPD, 5.0 for NTPD, and 4.5 for ELPD. Median practitioner skills scores were 4.7 for ITPD, 4.7 for NTPD, and 4.3 for ELPD. Implications: Preceptor evaluations of APPE clinical performance remain similar across online and on-campus programs. Although grades were statistically different, this difference was unlikely to be academically meaningful. Limitations include retrospective nature of the data, subjective preceptor evaluations, heterogeneity of rotations, and large differences in sample size between pathways.

Coordinated Student Success Initiative for P1 and Beyond: Do Academic Success Plans Improve Student Retention? Laurie L. Briceland, Albany College of Pharmacy and Health Sciences, Kimberly Keyes, Albany College of Pharmacy and Health Sciences, Jeffrey M. Brewer, Albany College of Pharmacy and Health Sciences, Luk Schmonsky, Albany College of Pharmacy and Health Sciences, Cindy Jablanski, Albany College of Pharmacy and Health Sciences. Objective: To describe our 2018-19 Student Success Initiative and impact of implemented Academic Success Plans (ASPs) on student progression/retention. Methods: A three-pronged coordinated student success initiative, shared by Center for Student Success (CSS) and School of Pharmacy and Pharmaceutical Sciences Academic Affairs (SoPPS-AA), was developed to 1) provide foundational student success support via CSS P1 Orientation Student Success Workshop plus optional CSS workshops semester-long; 2) implement a process for mid-semester identification of students struggling academically via CSS gradebook tracking, with follow-up via CSS and faculty advisor meetings; and 3) enroll students entering the semester on academic probation into a newly developed SoPPSAA/CSS initiative, the Academic Success Plan (ASP), in which the student works with CSS to complete an Academic Probation Self-Assessment and Probation Improvement Worksheet, and then develops a formal plan for improvement, with critical reflection exercise. Results: 34 P1 to P3 students entered the fall 2018 on academic probation and were asked to complete an ASP; 26 (76\%) completed the ASP. At semester end, of the 34 probationary students, 27 (79\%) progressed; 6 of these students had not completed the ASP. The remaining 7 students were either dismissed (3) or assigned new probation; of these 7 students, 2 had not completed the ASP. Implications: Initial results of student success initiatives including ASP have resulted in positive progression/ retention. Our next steps are to compare the progression/ retention data to years prior when ASP was not in place, and to survey students/advisors on merits of ASP.
Correlation Between Types of Advanced Pharmacy Practice Experiences (APPEs) and NAPLEX Pass Rates. Tibb F. Jacobs, The University of Louisiana at Monroe, Jamie M. Terrell, The University of Louisiana at Monroe. Objective: At our institution, $\mathrm{P} 4$ pharmacy students are required to complete seven APPEs, with a minimum of two APPEs with a faculty preceptor and the rest with non-faculty preceptors. The purpose of this project was to determine if the number of APPEs with faculty preceptors influences NAPLEX pass rates and test scores. A secondary objective was to determine if there was a correlation between number faculty APPE preceptors and individual NAPLEX test scores. Methods: The number of APPE faculty preceptors and NAPLEX pass rates for 179 pharmacy graduates from 2016 and 2017 were included in this project for statistical analyses. Chi-square and linear regression were used to calculate results. Results: Analyses revealed that the 179 pharmacy graduates had anywhere between one and five faculty APPEs. The average number of faculty APPE preceptors among students was 2.4. Of the 179 pharmacy graduates, $80 \%$ passed the NAPLEX on the first attempt. Chi-square test revealed no significant difference for the number of faculty APPE preceptors and NAPLEX pass rates $(p=0.234)$ There was no correlation between number of faculty preceptors and NAPLEX scores $(\mathrm{r}=0.0181)$. Implications: There was no significant difference or correlation found for number of faculty preceptors and NAPLEX pass rates or test scores. Our results are limited by a small sample size from only one pharmacy school. Further research, including additional classes, will be conducted to determine if increasing faculty preceptors influences NAPLEX pass rates and test scores.

Correlation of Emotional Intelligence with Academic Success in the First Professional Year. Elizabeth M. Lafitte, The University of Louisiana at Monroe, Laurel A. Sampognaro, The University of Louisiana at Monroe, Gina C. Craft, The University of Louisiana at Monroe. Objective: This research aims to investigate the relationship between self-reported emotional intelligence (EI) using the EQ-i 2.0, pre-pharmacy academic achievement, and success in the first year of the professional pharmacy program. Specifically, the correlations between EI and first semester grade point average (GPA) and qualification for early intervention will be reported. Methods: All students matriculating in the Fall of 2018 completed the EQ-i 2.0, a validated self-report of emotional intelligence, which provides normed competency scores in fifteen areas of emotional intelligence and overall emotional intelligence score. Correlations statistics for total EQ-i scores, each of the fifteen domains, pre-pharmacy academic variables, and first semester GPA were calculated. 


\section{American Journal of Pharmaceutical Education 2019; 83 (5) Article 7654.}

Results: There was no correlation between total EQ-i score and first semester GPA $(r=-0.023)$. None of the 15 areas on the assessment had strong correlation with first semester GPA, however self-expression, independence, and assertiveness had weak negative correlations $(\mathrm{r}=-0.216,-0.186,-0.179)$. Among the positive correlations, while still weak, self-actualization and self-perception had the strongest correlations with first semester GPA $(\mathrm{r}=0.182,0.161)$. The cohort's pre-pharmacy academic achievement as measured by their pre-pharmacy GPA, math and science GPA, and composite score on the Pharmacy College Admission Test strongly correlated with their first semester GPA. Implications: While strong correlations among many variables were not established in this cohort of students, continued study of correlations between emotional intelligence and individual course performance may reveal insights about which students are likely to struggle or succeed in our professional pharmacy program.

Cultural Readiness and Impact: Perspectives of Interprofessional Student/Faculty Medical Missionaries and Natives They Serve. Lisa Hong, Loma Linda University, Jennie B. Jarrett, University of Illinois at Chicago. Objective: Cultural competence is critical for effective integration of a medical mission team. There is limited information regarding the interaction of missionary and native perspectives of cultural competency and medical mission value. Our study aimed to determine whether student/faculty missionaries have baseline cultural competence to understand the needs of their underserved community and in turn, whether natives are culturally prepared for missionaries. Methods: This IRB approved research was a prospective, mixed methods design. Missionaries completed Qualtrics ${ }^{\circledR}$ surveys regarding their service along with the Intracultural Effectiveness Scale (IES), which assesses skill for interaction with natives on three dimensions: continuous learning, interpersonal engagement, and hardiness. Each dimensional component is scored on a scale of 1 to 7 ( 7 being highest/culturally competent). Natives were interviewed or completed a written survey describing their perception of mission service and cultural competency. Results: Missionaries $(n=6)$ from various departments and levels (pharmacy, physician assistant, and speech pathology) averaged an overall IES score of $3.78 \pm 0.34$ and most believed education was more valuable than service. Natives $(n=8)$ averaged $3.88 \pm 0.99$ for missionary cultural awareness and most found equal value in the education and service provided by missionaries. Administrative natives $(\mathrm{n}=3)$ found development of relationships of highest value. Implications: Overall, student/faculty missionaries and natives are only moderately culturally competent.
The IES may identify focus areas for incorporating cultural competency into school curriculum and faculty development programs. Given differences in what is perceived as valuable, communication and a needs assessment may optimize relationship building and the education or services provided.

Current Status of College-Sponsored Postgraduate Teaching and Learning Curriculum (TLC) Programs. Velliyur Viswesh, Roseman University of Health Sciences, Amy H. Sheehan, Purdue University, Tracy Sprunger, Butler University, Jacob P. Gettig, Midwestern University/Downers Grove, Jaclyn Boyle, Northeast Ohio Medical University. Objective: To describe the characteristics of college-sponsored teaching and learning curriculum (TLC) programs, or teaching certificate programs, and evaluate alignment with best practice recommendations. Methods: A 28-item electronic survey instrument was developed based largely on best practice recommendations from AACP/ASHP and ACCP. The survey was designed using Qualtrics software and distributed via email to the 137 accredited colleges of pharmacy in the United States. Items focused on demographics and content, delivery, evaluation of, and resources related to TLC programs. Participation was incentivized by a nominally priced gift card, and data were analyzed descriptively. Results: Response rate was 63\% (86/137), from which 61 institutions had a TLC program, completed the survey, and were included in the analysis. Public institutions comprised $51 \%$ of respondents, and TLC programs had been offered for an average of 8.6 years. Most TLC programs utilized a variety of teaching modalities to deliver content (e.g. lectures, small-group) and taught residents a variety of educationally relevant topics (e.g. active learning, classroom teaching). About $61 \%$ required residents to precept APPE students and 49\% had residents' teaching formally evaluated by students. Notably, $28 \%$ of TLC programs did not undertake any programmatic assessment or continuous quality improvement on an at least semi-regular basis. Approximately $90 \%$ of TLC programs were aware of published best practice recommendations, with about $75 \%$ of colleges reporting having used these recommendations in planning TLC programs. Implications: Despite high awareness and reported use of best practice recommendations, components, delivery and assessment of college-sponsored TLC programs appears to be inconsistent.

Curricular Integration of Population Health and the IHI Model of Improvement through Interprofessional Community Engagement. Veronica S. Young, The University of Texas at Austin, Jennifer K. Seltzer, The University of Texas at Austin, Lydia Aguilera, The University of Texas at Austin, Lucas G. Hill, The 


\section{American Journal of Pharmaceutical Education 2019; 83 (5) Article 7654.}

University of Texas at Austin, W. Renee Acosta, The University of Texas at Austin. Objective: ACPE standard 11 mandates the integration of interprofessional education (IPE) into pharmacy curricula. The Institute for Healthcare Improvement (IHI) triple aim provides a framework for developing IPE experiences that seek to address population health. This is a programmatic description of a required third-year course that engages student pharmacists with their local community to address social determinants of health (SDOH) in the continuum of care. Methods: Initial planning required agreement between three diverse regional campuses, programmatic approval, and support from community stakeholders. The course blends project-based learning and the IHI improvement model as the teaching pedagogy. Community-identified projects align with Healthy People 2020.Students, faculty, and near-peer mentors are matched with projects based on interests or expertise to form diverse teams. Interprofessional collaborations are integrated. Program outcomes include assessing types of interprofessional interactions, project deliverables, and value to the community. Results: There were 22 projects completed over two semesters. All projects aligned with Healthy People 2020 and addressed SDOH. Examples of project categories included health promotion, health empowerment, access to health and social services, program evaluation, and quality improvement. Targeted populations included underserved communities, youths, seniors, college students, teachers, community health workers, and health professionals. Project deliverables varied substantially. Implications: Curricular integration of population health through interprofessional community engagement required extensive planning and coordination, sufficient faculty support, and community ownership. Students developed skills in real-world project management, problem solving, critical thinking, entrepreneurship, and leadership. Projects allowed students to develop an appreciation of SDOH and the significance of interprofessional collaboration in the continuum of care.

Description of a Transitions of Care and Telemedicine Simulation Lab Activity. Danielle M. Candelario, Rosalind Franklin University of Medicine and Science, Kathleen Cunningham, Rosalind Franklin University of Medicine and Science, Sneha Srivastava, Rosalind Franklin University of Medicine and Science. Objective: Transitions of Care (TOC) has become a unique clinical practice area requiring trained health care professionals however there is limited literature describing TOC education in the didactic curriculum. The purpose of this study is to describe and evaluate a TOC simulation activity incorporated into the second year of a Doctor of Pharmacy curriculum. Methods: Student pharmacists received a TOC lecture, followed by a onehour session for simulation case work-up. Students then participated in two distinct simulation encounters: a discharge encounter utilizing automated mannequins and a telemedicine follow-up encounter with standardized patients. Six medication-related problems (MRPs) within the spectrum of the activity were incorporated. Activity documents were collected to identify student competency in TOC. Study was approved by the Institutional Review Board. Results: 59 student pharmacists in 16 teams completed the activity. Twelve (75\%) and 14 teams (87.5\%) accurately identified the discharge and telemedicine encounter MRP respectively. All teams provided medication education and $94 \%$ of teams identified Primary Care Physician follow-up was needed post discharge. Ten teams $(62.5 \%)$ utilized effective interview sequence and structure during both encounters. Implications: This simulation activity was strategically designed to expose student pharmacists to core concepts of TOC. Students successfully completed tasks associated with TOC, however, effective communication and patient interviewing skills were not effectively incorporated. Activity challenges included resources, financial support and SP training. Utilizing case-based learning and simulation to reinforce and master TOC concepts may be a beneficial way for students to be more effectively trained to deliver impactful TOC services.

Design and Validation of Structured Incremental Measurement of Deliberate and Objective Simulation Experiences-Pilot (SIM DOSE-P). Michael Thomas, Samford University, Georges Adunlin, Samford University, Mary A. Worthington, Samford University, Elizabeth Covington, Samford University, Megan Z. Roberts, Samford University, Jennifer W. Beall, Samford University, Jeffrey A. Kyle, Samford University. Objective: To validate and describe methods, instruments, and rubrics used for a simulation-based activity. Methods: Fourth year pharmacy students were recruited to complete a one-day simulation experience. Participants were briefed on the expectations which included completing two anonymous surveys, writing a SOAP note, and watching a video-recorded debrief. Two trained simulation staff served as the voices for the high-fidelity mannequins. Two licensed pharmacists assessed the SOAP notes using a rubric developed based on pharmacy's Entrustable Professional Activities (EPA). Kappa statistics and simple percentage agreement were calculated to test interrater reliability. At conclusion, participants were led through a focus group relating to overall experience, and to solicit feedback on face and content validity of the survey instruments. Results: Student participants' feedback of the 


\section{American Journal of Pharmaceutical Education 2019; 83 (5) Article 7654.}

survey instruments was positive. There was general agreement that the high-fidelity simulation-based activity was well accepted, and challenged them to develop cognitive, technical, and behavioral skills through the use of mannequins, and working medical equipment. Participants experienced some difficulty using unfamiliar computers to upload SOAP notes. There was a low level of agreement $(41.67 \%, \mathrm{k}=0.19)$ between the raters for SOAP notes, which highlights the need to provide rater training. Implications: The overall design is feasible on a larger scale with some modifications such as allowing personal computer use and further training on the assessment rubric. Based on these results, the full study will commence using a larger participant pool and additional cases.

Designing a Comprehensive Curriculum Map to Assess Educational Outcomes in an Accelerated PharmD Program. Terrick A. Andey, MCPHS University-Worcester/Manchester, Kaelen C. Dunican, MCPHS University-Worcester/Manchester, Abir Kanaan, MCPHS University-Worcester/Manchester, Linda M. Spooner, MCPHS University-Worcester/ Manchester, Gretchen Jehle, MCPHS University-Worcester/ Manchester, Carroll-Ann W. Goldsmith, MCPHS University-Worcester/Manchester. Objective: The Accreditation Council for Pharmacy Education (ACPE) Standard 10.7 requires pharmacy programs to map their curriculum to provide continuous quality improvement and assessment of educational outcomes. Pharmacy administrators and faculty are often challenged with identifying a platform that allows a systematic and thoughtful approach to mapping, to inform assessment and curricular revisions. The objective of this project is to describe a faculty-designed and led process for continuously assessing an accelerated PharmD curriculum. Methods: A Qualtrics-based survey was designed to assess a 34month accelerated PharmD curriculum by mapping all core courses to educational outcomes and learning objectives adopted from ACPE Standards 1-4/CAPE Educational Outcomes 2013. The survey design also evaluated the depth and breadth of the curriculum by assessing pedagogical and student self-assessment methods, as well as levels of learning and performance (Zelenitsky et al., 2014). Additionally, professional outcomes relating to interprofessional education, and required portfolios activities were captured. Results: After the survey was designed, workshops were scheduled to orient faculty on the mapping process and to solicit feedback on the usability of the survey. Course coordinators provided feedback on how levels of learning should be captured. The survey was modified accordingly and administered to course coordinators after each semester's offering. Data were then analyzed to identify gaps, minimize redundancies, and ensure increased rigor in skillsbased activities. Implications: The process was thoughtfully designed to address ACPE accreditation requirements and allow faculty to identify and address curricular gaps. Future plans are to incorporate the Pharmacists' Patient Care Process and link outcomes to Entrustable Professional Activities.

Determining the Impact of Self-Assessment and Peer Review in a Simulated Telepharmacy Laboratory Experience. Kimberley J. Begley, Creighton University, Karen O'Brien, Creighton University, Jennifer A. Tilleman, Creighton University, Eric B. Hoie, Creighton University. Objective: To examine the effect of selfassessment and peer review in a simulated telepharmacy experience with first-year pharmacy students. Methods: First-year students enrolled in a pharmacy laboratory course completed a simulated telepharmacy consultation with faculty portraying patients. In this one-on-one video recorded activity, faculty assessed students' ability to obtain a comprehensive medication history. Students analyzed their case and submitted an assessment and recommendation for their patient. Students were then paired with a classmate and scored their partner's recorded consultation using a faculty-designed rubric and reflected on their own performance. Results: Twohundred-ninety-nine students submitted completed data activity sets for analysis. Scores ranged from 16 to 30 with an average of $93 \%$. Student peer review scores were compared to faculty assigned scores. The percent absolute agreement was $60 \%$. Most scoring disagreement occurred on the subjective communication rubric components, versus the objective content/process elements. Students wrote a one-page guided reflection on their perception of the activity. Pharmacy faculty collected qualitative data and conducted theme analysis. Most prominent thematically, students believed this exercise provided a realworld experience that allowed them to consider their roles as practicing pharmacists. A second recurring theme was students' increased confidence in their skills and abilities. A final theme identified students developing a patientcentered attitude, recognizing they could affect quality of care. Ninety-eight percent $(n=294)$ of students found this activity beneficial and worth repeating. Implications: Reflecting on their role as a pharmacist in a simulated environment allows students the opportunity to analyze their performance, identify areas for growth, and cultivate self-awareness.

Developing Empathy in First-Year Pharmacy Students Utilizing an In-Class Video Conference with a Parkinson's Patient. Stephanie Enz, Butler University, Marcos A. Oliveira, Butler University, Prachi Arora, 


\section{American Journal of Pharmaceutical Education 2019; 83 (5) Article 7654.}

Butler University. Objective: To determine if pharmacy students' empathy improved through an in-class video conference focused on a Parkinson patient's medication and illness experience. Methods: Through a lecture in a course module, first-year pharmacy students were introduced to the concepts of empathy, clinical empathy, and patients' medication/illness experience. A Parkinson's patient case, discussing patient's experiences including fear and anxiety related to his illness/medications, was presented. After the lecture, students underwent an intervention, which comprised of a live, video-conference and interactive in-class interview of the Parkinson's patient's illness/medication experiences. Pre-post survey design was used to determine the change in empathy before and after the intervention, with a ten-question survey instrument. All the responses were recorded using a 5-point Likert scale. Pre-post comparisons were conducted using Mann-Whitney-U-test. Statistical significance was determined at $\mathrm{p}<0.05$. Two open-ended questions were also included to capture students' reflections. Results: Sample comprised of 130 and 127 students pre and post the survey, respectively. Statistically significant improvement in empathy was observed for all the questions $(p<0.05)$. Likelihood of achieving an empathic response in the post-survey (vs. pre) was $>70 \%$ for 4 of the 10 questions. These four questions measured students' empathy towards patient's chronic illness, medication experience, side effects and patient's non-compliance with daily medication. Implications: Learning theoretical concepts of empathy are important, however, incorporating live sessions of patients with chronic/end-stage illness could significantly improve students' empathy. Empathy is an essential construct of patient-care; therefore, pharmacy students need to undergo effective interventions to hone their empathic skills/responses early in their education.

Development of a Leadership Elective for Student Pharmacists. Lindsey E. Dayer, University of Arkansas for Medical Sciences, Seth D. Heldenbrand, University of Arkansas for Medical Sciences, Schwanda K. Flowers, University of Arkansas for Medical Sciences. Objective: ACPE Standard \#4 relays the importance for colleges to provide a holistic approach to teaching. A Leadership Elective was developed in 2016 for students to improve their leadership skills with a focus on all-around growth of leadership and self-awareness. Methods: Three faculty members participated in the design. A maximum of 12 students per class is accepted. Classroom and off-campus activities were designed to cultivate student sociobehavioral skills related to core strengths, emotional intelligence, leadership styles, leading change and developing mindfulness, as well as networking. The class culminated with students participating in a leadership related debate.
Results: 33 students have participated over the previous 3 years.2018 participants evaluated their leadership skills in a pre/post evaluation. Twelve students took the presurvey and nine took the post survey. On the pre-survey, students scored themselves the lowest on "I consider myself a leader among my classmates" and "I am good at networking with others in my profession" with a 3.4 and 3.3 average out of a 5-point Likert scale, respectively $(1=$ strongly disagree and $5=$ strongly agree). On the postelective survey, students' average for "I can better identify leadership skills within myself" and "This elective has taught me strategies to build collaborative relationships" were a 4.4. Students also reported benefits from the team-building activities. Implications: It's important for future pharmacists to develop leadership skills. They should be self-aware as well as able to effectively manage relationships. This elective is important for students to gain a better understanding of these "difficult to teach" topics.

Development of an Ambulatory Care Experience Course to Advance Patient Care Skills for the Outpatient Setting. Ashleigh L. Barrickman, West Virginia University, Megan M. Elavsky, West Virginia University, Gretchen M. Garofoli, West Virginia University. Objective: To describe the development and implementation of a new ambulatory care experience course focused on preparing learners to practice in the outpatient care setting. Methods: An updated Ambulatory Care Experience course was re-developed and implemented in fall 2018 that focused on patient care skills and preparing students to appropriately manage chronic conditions in an outpatient setting. This course included interprofessional education, comprehensive medication review simulations, discharge counseling, student-led topic discussions, and patient care activities. The students similarly completed a medication therapy management certificate through a national program. Students were surveyed at the conclusion of the course to provide feedback on the individual course components and overall course content. Results: Twentynine (29) students $(90 \%)$ completed the course survey. The majority of students (96.5\%) agreed or strongly agreed that the activities achieved the goals and purpose of the course. Student comments indicate that the most impactful component of the course was the opportunity to develop and apply the skills necessary to provide medication therapy management to patients. Implications: Overall, this innovative Ambulatory Care Experience course was well-received by students and has helped students apply and advance the knowledge and skills introduced in the didactic curriculum. Courses that provide these opportunities aid in preparation for advanced pharmacy practice experiences (APPEs) and are integral to 


\section{American Journal of Pharmaceutical Education 2019; 83 (5) Article 7654.}

pharmacy curricula. Coursework that advocates for advanced skills and teamwork involvement, especially in ambulatory care, is instrumental when educating the next generation of pharmacists.

Does a Pharmacy Readiness Education Program (PREP) Support Academic Progression? Van T. Hellerslia, Temple University, Michael Borenstein, Temple University, Jennifer L. Andres, Temple University, Justina Frimpong, Temple University, Anisha Grover, Temple University, Margaret A. Miklich, Temple University, Ina Lee S. Calligaro, Temple University. Objective: A one-week preparatory program called Pharmacy Readiness Education Program (PREP) was implemented out of a concern for a reduced national applicant pool potentially resulting in acceptance of less academically competitive students. This study seeks to evaluate the impact of PREP on academic progression. Methods: Students with lower entering grade point averages (GPA) were required to attend PREP as a condition of admission. This retrospective study compared students in the REQUIRED cohort to students who were not required but chose to attend (OPTIONAL) and to students who were not required and did not attend (CONTROL). The primary endpoint was the proportion of students with academic progression into the second semester of Professional Year (PY)-1. Secondary endpoints included GPA for Infectious Disease, Medicinal Chemistry and Immunology, and academic progression to PY-2. Results: Of the 140 registered students invited to PREP, 24 students were in the REQUIRED cohort.Sixty-one students and 50 students were in the OPTIONAL and CONTROL cohorts, respectively. Five student records were excluded due to medical leave or failure to matriculate. The primary endpoint of academic progression to second semester was $100 \%$ in the REQUIRED cohort, $96.7 \%$ in the OPTIONAL cohort and $94 \%$ in CONTROL cohort $(\mathrm{p}=0.37$ between REQUIRED versus OPTIONAL; $p=0.22$ between REQUIRED versus CONTROL). Similar results were found for all secondary endpoints. Implications: Students required to attend PREP had a lower entering GPA but were able to progress comparable to students with higher entrance GPAs. Components of the program that drive success are being explored.

Does a Redesigned Introductory Pharmacy Practice Experience Better Prepare Students for Advanced Pharmacy Practice Experience? Van T. Hellerslia, Temple University, Pete Watkins, Temple University, Leigh A. Godfrey Webber, Temple University, Jennifer L. Andres, Temple University. Objective: This study evaluated through focus groups and surveys whether a more rigorous Introductory Pharmacy Practice Experience (IPPE) helped students develop the necessary skills and confidence for Advanced Pharmacy Practice Experience (APPEs). Methods: An innovative Neurology IPPE offered to pharmacy year 3 (PY-3) students provided direct patient care and interprofessional education (IPE) experiences reflective of an inpatient clinical APPE rotation.PY-3s collaborated with medical students to provide individualized secondary stroke prevention education and optimize stroke pharmacotherapy. Focus group interviews and survey data were collected from PY-3s and reflective written statements were obtained from faculty. Data was analyzed, and themes were derived inductively. Results: Of the 19 PY-3s who completed the Neurology IPPE in 2017-2018, 14 completed an online survey and 10 participated in a focus group Themes derived from student interviews and survey data revealed that students found the experience to be both rewarding and challenging. In particular, students felt they had a positive impact on patients, applied their classroom learning to the realworld, and while they were sometimes intimidated during the IPPE they left feeling more prepared for APPE. Themes derived from faculty reflective statements were achievement of student APPE readiness, student success at higher-order thinking and complex problem solving, and exposure to IPE. Implications: IPPEs that require higher order thinking and complex problem solving can help students gain the confidence and skills to successfully complete APPEs.

Drivers of Technology Enhanced Learning Adoption Within Colleges of Pharmacy. Reid C. Proctor, Mercer University, J. Grady Strom, Mercer University. Objective: To identify institutional drivers of technology enhanced learning (TEL) adoption by faculty at colleges of pharmacy and to recommend steps for institutions to take to support TEL adoption. Methods: TEL was defined as the application of digital technologies to support teaching and learning. Faculty members of the American Association of Colleges of Pharmacy were asked to complete a survey that would gather their perceptions as to which institutional drivers have the greatest influence on their decisions to adopt TEL. Survey responses were stratified by demographics and analyzed. Results: Five hundred and four usable survey responses were collected. Faculty members indicated levels of significance for 19 items identified in the literature that may influence their decisions to adopt TEL. The highest rated item was "reliability of various systems (i.e. campus internet access and online learning management system)". Ninety-one percent rated this item as being of moderate or significant influence. Colleagues sharing their success with TEL to support teaching was also highly rated by a majority of respondents (80\%). Implications: The survey results from the current study may provide guidance to leaders 


\section{American Journal of Pharmaceutical Education 2019; 83 (5) Article 7654.}

at colleges of pharmacy who seek to promote the adoption of TEL among their faculty. The reliability and availability of technical systems used to deliver TEL are a top priority. Opportunities for faculty colleagues to share their successes with TEL should be facilitated.

Drug Information Questions Answered Three Different Ways in a General Medicine Elective. Stephanie L. Sibicky, Northeastern University, Jason W. Lancaster, Northeastern University, Adam B. Woolley, Northeastern University, Michael J. Gonyeau, Northeastern University, Mark Douglass, Northeastern University, Alexa A. Carlson, Northeastern University. Objective: To describe the use of three different methods to answer drug information questions (DIQs) in a general medicine elective. Methods: In 2018, faculty changed DIQs from written responses only to three types: written, videorecorded (vlog), and live-response. Vlogged DIQs were added as students felt less prepared to communicate with healthcare providers. Students post written responses to the course discussion board with references and search strategies. Vlogs are recorded answering the DIQ speaking to a provider. Written and vlogged DIQs are posted within 24-hours. Live-response DIQs are answered inperson during a mock-rounding experience within 15 minutes. Rubrics utilized for assessment have been mapped to Ability Based Outcomes, CAPE Outcomes and EPAs. Student perceptions were gathered via Qualtrics ${ }^{\circledR}$ survey to 2015-2018 cohorts for feedback. Results: Students strongly agree (4) that DIQs are valuable $(2015,4 ; 20163.89 ; 2017,4 ; 2018,4)$ and agreed/strongly agreed (3-4) that they were well prepared to communicate with the medical team $(2015,3.4 ; 2016,3.1,2017,3.28$; 2018, 3.29). Students felt that vlogged DIQs helped them communicate with other providers (59.14), organizing thoughts (58.57), and making persuasive arguments (59.29), compared to written DIQs (scale from written [0] to vlogged [100]). The average scores in 2018 on written DIQs was $24.9 / 27$ (92\%), vlogged 36.75/39 (94\%), and live 49.2/52 (95\%) and data is being analyzed for the 2019 cohort. Implications: Utilizing three different approaches to answering DIQs offered students the opportunity to strengthen written and verbal communication skills as well as respond in a live, real-world situation.

Drug Monograph Creation and Presentation to a Simulated Pharmacy and Therapeutics (P\&T) Committee. Puja Patel, Marshall B. Ketchum University, Azita Alipour, Marshall B. Ketchum University, Joycelyn M. Yamzon, Marshall B. Ketchum University, Elvin Hernandez, Marshall B. Ketchum University. Objective: Measure student pharmacists' perceptions about their ability to create a drug monograph and present findings and recommendations to a simulated P\&T Committee. Methods: A 13-item survey, based on a five-point Likert scale of agreement, was administered to second-year student pharmacists enrolled in a Drug Information, Informatics and Literature Evaluation course before and after drug monograph preparation and presentation to a simulated P\&T Committee. Descriptive statistics such as measures of central tendency were used to analyze continuous and ratio level data. For categorical variables, we conducted chi-square tests to compare statistical differences of student perceptions before and after the intervention. Levels of significance were set to alpha $=0.05$. Results: Student pharmacists' perceptions of their ability to critically evaluate the utility of adding the drug under review to an organization's current formulary were statistically significant and improved between pre- and post-study intervention $(p=0.011)$. There was a statistically significant improvement $(\mathrm{p}=0.018)$ in student perceptions of their ability to critically evaluate other medications available in the current formulary and identify if there is value added to including the drug under review to formulary. Additionally, there was a statistically significant improvement $(p=0.007)$ in student perceptions of their confidence in preparing a drug monograph on APPE rotations. Implications: As student pharmacists perceived themselves as more confident in preparing a drug monograph and making recommendations to a simulated P\&T Committee after this activity, we find promise in implementing similar activities in drug information courses.

Effect of Being in the Same vs Different Teams Across Required Courses: Students' Performance and Satisfaction. Yulia A. Murray, MCPHS UniversityBoston, Lana Dvorkin Camiel, MCPHS University-Boston, Michele L. Matthews, MCPHS University-Boston, Maria D. Kostka-Rokosz, MCPHS University-Boston, Lisa Padgett, MCPHS University-Boston, Jennifer Goldman, MCPHS University-Boston, Joseph Ferullo, MCPHS University-Boston, Paul J. Kiritsy, MCPHS UniversityBoston, Catherine Taglieri, MCPHS University-Boston, Gary Tataronis, MCPHS University-Boston. Objective: To examine if there is a correlation between academic performance and satisfaction for students working in the same vs different teams in two required third year courses (Over-the-Counter Drugs and Pharmacotherapeutic Seminar). Methods: Students were divided into two sections. Section A had the same teams of students across both courses, while section B had different teams in the OTC and Seminar courses. At the end of the semester, students were electronically surveyed to assess their satisfaction and academic performance. Results: Out of 280 enrolled in the courses, 275 students consented to participate. The 


\section{American Journal of Pharmaceutical Education 2019; 83 (5) Article 7654.}

results presented are based on strongly agreed/agreed students' responses of students in the section A (OTC and Seminar) versus section B (OTC) versus section B (Seminar). Students reported that teamwork: 1) contributed to earning a better grade on team assignments $-92 \%$ vs $86 \%$ vs $86 \%$; 2) helped to develop a better overall learning strategy $-75 \%$ vs $77 \%$ vs $83 \%$ : 3) made it easier to understand the content of courses better - $73 \%$ vs $79 \%$ vs $82 \%$. Students were satisfied working in their teams $94 \%$ vs $91 \%$ vs $82 \%$. Students in section A felt teamwork helped to establish deeper supportive relationships with teammates versus those in section B - $82 \%$ vs $68 \%$ and had preference for being assigned to work in the same teams in the future - $69 \%$ vs. 36\%. Implications: Familiarity with the environment appears to be predictive of students' future preferences for assigned teamwork placement.

Effect of Observation Versus Active Participation on Self-Reported Interprofessional Collaborative Behaviors. Nicholas M. Fusco, University at Buffalo, The State University of New York, Kelly Foltz-Ramos, University at Buffalo, The State University of New York. Objective: The purpose of this study was to compare the change in pharmacy students' self-reported competence toward interprofessional collaboration between those students that were active participants and those that were observers of an interprofessional simulation-based learning (SBL) experience. Methods: Second- and third-year pharmacy and senior nursing students participated in an interprofessional SBL experience. Third year pharmacy students and senior nursing students were divided into teams of four (two from each profession) and were the active participants. Second year pharmacy students were observers. All pharmacy students were asked to complete a brief demographic survey and the Interprofessional Collaborative Competencies Attainment Survey (ICCAS) after the SBL experience. Paired sample t-tests were conducted to compare differences in mean change in ICCAS scores. Differences in pre and post ICCAS scores between observers and active participants were analyzed using analysis of variance (ANOVA) methods. Results: A total of 130 (95\%) second and 121 (92\%) third year pharmacy students participated in the interprofessional SBL experience and completed the survey for an overall response rate of $94 \%$. Compared to second year pharmacy students, both the mean pre $[4.94(\mathrm{SD}=0.97)$ vs. 5.23 $(\mathrm{SD}=0.93)]$ and mean post $[5.94(\mathrm{SD}=0.69)$ vs. 6.23 $(\mathrm{SD}=0.68)$ ] total ICCAS scores were higher for third year pharmacy students; this was statistically significant. There was no difference in the mean change from pre to post between second- and third-year pharmacy students $[1.00(\mathrm{SD}=0.89)$ vs. $0.99(\mathrm{SD}=0.81)]$. Implications:
Both observation and active participation in an interprofessional SBL experience may increase pharmacy students' self-reported competence toward interprofessional collaboration.

Effects of Mindfulness Meditation on Mindfulness, Mental Well-Being and Perceived Stress. Therese I. Poirier, Southern Illinois University Edwardsville, Irene Zollars, Southern Illinois University Edwardsville, Junvie Pailden, Southern Illinois University Edwardsville. Objective: The purpose of this study was to investigate the effects of mindfulness meditation using the Headspace $^{\mathrm{TM}}$ app on mindfulness, mental well-being and perceived stress in pharmacy and nursing students. A secondary objective was to evaluate the health promoting lifestyle behaviors of these students and its relationship to mindfulness, mental well-being, and perceived stress. Methods: P1, P2, and P3 pharmacy students and sophomore and junior undergraduate nursing students were recruited to participate. Students were instructed to meditate using the Headspace ${ }^{\mathrm{TM}}$ app for at least 10 minutes per day for four weeks. Students at baseline completed the health promoting lifestyle profile (HPLP II). Data was collected from the pre/post surveys using the Five Facet Mindfulness Questionnaire (FFMQ), Warwick-Edinburgh Mental Well-Being Scale (WEMWBS) and Cohen Perceived Stress Scale (PSS). Results: One hundred students enrolled in the study including 92 pharmacy and 8 nursing students. Seventypercent of the participants completed the study. Only one participant was excluded in the post data analysis due to non-adherence with the protocol. The data reveals that for all scales the intervention was associated with enhanced mindfulness and mental well-being and decreased perceived stress. Further analysis indicated that controlling for various health promoting lifestyle behaviors preserves the positive impact of mindfulness meditation as demonstrated Implications: Mindfulness meditation uniformly and independently improved the participants overall mental health. The data supports a feasible option for minimizing stress and maintaining mental well-being in demanding professional programs. This study encourages students and professional schools to adopt these practices in their curriculum.

Engaging Health Profession Students of a Large University to Address the Opioid Crisis. James W. McAuley, The Ohio State University, Nicole Kwiek, The Ohio State University, Jacqueline Loversidge, The Ohio State University, Gregory Nixon, The Ohio State University, Monica Robinson, The Ohio State University, Kelly Speirs, The Ohio State University, Kelsey K. Schmuhl, The Ohio State University. Objective: To describe the planning, implementation, assessment, and 


\section{American Journal of Pharmaceutical Education 2019; 83 (5) Article 7654.}

continuing efforts of a large Interprofessional Education (IPE) session focused on the opioid crisis. Methods: Addressing the opioid crisis requires the skills and leadership of all health professions disciplines. Thus, an interprofessional steering team of faculty and students created a large IPE event focused on mitigating the opioid crisis. Second-year students from Dentistry, Medical Dietetics, Medicine, Nursing, Occupational Therapy, Optometry, Pharmacy and Physical Therapy were required to complete pre-work before they attended a 2.5-hour IPE session. After a brief keynote address and small group discussions, attendees listened to short talks by trainees on innovative opioid-focused projects. The active phase of the session had each small group of attendees develop an action plan to address the crisis. Then, interested attendees had the opportunity to form IP teams to further develop and implement selected action plans. Results: A large cohort $(n=832)$ of health professional students participated in this student-led, engaged IPE session. A total of 84 action plans were generated by the small group student teams. Student feedback showed that the session objectives were met. A small portion of students $(n=36)$ expressed interest in the implementation phase and have created interprofessional teams in 4 opioid-crisis areas: naloxone, community education, 1 st opioid prescription, and developing a certificate for health profession students. Implications: This active IPE session facilitated a large cohort of students to learn from, with and about other professions regarding the opioid crisis and supported self-selected students to take next steps to combat this contemporary issue.

Enhancing a Pharmacotherapy Course With a Flipped Classroom Approach Based on Cognitive Science of Learning Principles. Tiffany R. Shin, The University of Kansas, Crystal Burkhardt, The University of Kansas, Robyn E. Kelton, The University of Kansas, Ashley Crowl, The University of Kansas, Ryan Funk, The University of Kansas, Sarah Shrader, The University of Kansas. Objective: Determine the impact of a flipped classroom approach based on cognitive science of learning principles on student performance and experience in a pharmacotherapy course. Methods: The flipped classroom method in this study utilized cognitive science of learning principles including pre-class learning (preview), in-class application to cases (retrieval and elaboration), and after-class learning (spaced retrieval) in a required pharmacotherapy course. During 2017, one instructor utilized this flipped classroom method for a module. During 2018, this method expanded to four instructors covering four modules and two-thirds of the course. All other modules used traditional lecture-based methods. The same multiple-choice exam questions were used both years. An ANOVA test compared the deidentified exam performance between teaching methods in both years. A voluntary anonymous student survey used a 5point Likert scale $(1=$ strongly agree, $5=$ strongly disagree) to evaluate the students' experience. Comparisons were analyzed with the independent t-test. Results: The 2017 and 2018 classes included 132 and 137 students, respectively. During the 2 years, exam performance was significantly better with the flipped classroom method ( $n=146$ questions) compared to the traditional method $(\mathrm{n}=262$ questions $) \quad(89 \%, 85 \%$, respectively; $\mathrm{p}=0.001)$.Students in 2017 and 2018 agreed that "the flipped classroom helped me learn," though the 2017 class had a more favorable survey rating $(1.43,2.18$, respectively, $\mathrm{p}<0.001)$. Students felt podcast-based pre-work helped them learn better than reading-based pre-work $(2.18,3.39$, respectively, $\mathrm{p}<0.001)$. Implications: Implementing a flipped classroom approach based on cognitive science of learning principles positively impacted student performance and experience in a pharmacotherapy course.

Enhancing Application and Long-Term Retention of Clinical Knowledge Using an Extracurricular Non-Credit Course. Kimberley J. Begley, Creighton University, Kevin T. Fuji, Creighton University. Objective: To assess a semester-long extracurricular, noncredit course for third-year Doctor of Pharmacy students designed to reinforce foundational clinical concepts and apply them to patient care-oriented activities. Methods: The course used a case-based instructional design with integrated repetitive rules and patterns illustrated by the cases, and teaching techniques such as "talk out loud" strategies. Learning was assessed by an end-of-course focus group and open-ended survey after the third Advanced Pharmacy Practice Experience (APPE) in their fourth year. A qualitative content analysis approach was used to analyze responses, with two investigators independently coding all data, followed by meetings to discuss codes and categories, and identify emerging themes. Results: A total of 24 students completed the course and all assessments. Focus group themes were: a) preparation for APPEs; b) benefit from a low-stakes approach; c) small group discussions enhanced learning; d) repetition of material; and e) connected different courses together. Open-ended survey results revealed that students directly connected course learning with 162 clinical interventions (drug-drug interactions, therapeutic duplication, inappropriate duration of therapy, drug-disease interaction, drugallergy interaction, other) made across APPEs in 16 different practice settings. Survey themes were: a) reemphasized benefit of repetition; b) long-term confidence; and c) looking at the big picture to solve clinical problems. 


\section{American Journal of Pharmaceutical Education 2019; 83 (5) Article 7654.}

Implications: Student learning can be facilitated through extracurricular efforts to complement required didactic courses. Providing students with opportunities for applying clinical decision-making in the context of patient cases is important for reinforcing core clinical knowledge and can be done in a low-stakes environment.

Escape Room Activity and Impact on Student Perceived Team Dynamics. Emily Eddy, Ohio Northern University, Kelly M. Shields, Ohio Northern University, Michelle R. Musser, Ohio Northern University, Jessica L. Hinson, Ohio Northern University. Objective: To design and assess a small-group escape room activity impact on perceived student team dynamics. Methods: Students $(n=141)$ in a required self-care course were assigned into groups for group projects throughout the semester. Prior to starting their academic group work, all groups participated in an "escape room" activity consisting of sequential puzzles that revealed patient case information to allow for students to develop a patient treatment recommendation. After the activity, student perception of the activity as well as group dynamics were assessed using numerical peer rating and free response questions. Results: Prior year peer evaluations of group members were compared to current year evaluations. The average peer group member rating in the year prior was $92.7 \%$ compared to $93.8 \%$; showing no significant difference in scores. Common themes regarding positive aspects of the activity included serving as a good ice breaker and opportunity to learn/ work as a team. Students also learned about team members' problem-solving abilities and personalities. Noted challenges involved logistics including, space, ability to solve problems and familiarity with the activity. Implications: An escape room activity was well received by students and allowed students to get to know their group members and their strengths prior to engaging in future academic projects, despite limited impact on team dynamics. Limitations of the activity included time to complete the activity and uncertainty in directions. While it didn't improve student reported team dynamics, the activity was well received and offered an alternative team building activity.

"Escape the OTC Aisle": An Evaluation of a SelfCare Focused Escape Room. Kate Newman, Southern Illinois University Edwardsville, Miranda J. Wilhelm, Southern Illinois University Edwardsville. Objective: To develop and assess an escape room activity utilizing time-sensitive, team-based puzzles, over-the-counter (OTC) counseling information and patient cases for an advanced self-care elective course. Methods: "Escape the OTC Aisle" was developed to help students practice case-based OTC recommendations, have hands-on counseling experience and review self-care clinical pearls in addition to applying communication and teamwork skills as encouraged by AACP's 2014 recommendation of serious gaming in pharmacy education. The room has been used for 2 years with some modifications after the first offering to enhance learning opportunities and the difficulty of the room. A 9-question anonymous survey of Likert-scale questions was created and distributed at the completion of the escape room to gauge student perception of learning. Results: Over 2 years, 90\% (50/55) of students who completed the escape room completed the survey. Ninety-eight percent of students reported they enjoyed the activity and $96 \%$ would like to have more of these types of activities in the curriculum. All respondents agreed the escape room allowed them to gauge their knowledge of self-care topics (46\% agree, 54\% strongly agree) and $94 \%$ stated the activity enhanced their learning of self-care content (32\% agree, 62\% strongly agree). Additionally, $82 \%$ of students agreed or strongly agreed that this activity made them feel more comfortable in recommending OTC products. Implications: "Escape the OTC Aisle" was perceived to be an enjoyable and useful tool to help students gauge their understanding and enhance their learning of self-care content. The room will continue to be utilized in the advanced self-care course.

Evaluating an Education Specialization for PharmD Graduates. Therese I. Poirier, Southern Illinois University Edwardsville, Katelyn M. Fryman, Southern Illinois University Edwardsville. Objective: The purpose of this study was to evaluate graduates' perceptions of the impact of an education specialization and aspects of the specialization that could be enhanced. Methods: An online survey was emailed to PharmD graduates of the education specialization program. The survey consisted of 12 demographic questions, 18 confidence statements, 14 motivation statements and 3 open-ended questions. Data was analyzed using descriptive statistics. Regression analysis was used to examine: 1) association between overall self-rated competence on abilities and skills as a teacher and the number of types of teaching experiences, and 2) associations between overall self-rated competence as a teacher and history of holding a faculty position. Results: Fifty-six out of 69 alumni (81.2\%) responded to the survey. Graduates reported high levels of confidence in most teaching abilities. There was a high level of agreement that specialization enhanced motivation to participate in teaching. Regression analysis indicated significant association between self-rated competence and participation in variety of teaching experiences and history of holding a faculty position. Enhanced skills in experiential education and teaching outside of academia were identified as further areas for development Implications: The 


\section{American Journal of Pharmaceutical Education 2019; 83 (5) Article 7654.}

specialization can serve as an alternative entry-level approach to increasing student interest in academia and increasing confidence levels in various teaching skills. The majority of graduates were able to apply the knowledge and skills to their current careers.

Evaluation and Analysis of Student Characteristics as a Predictor of Success in Pharmaceutical Calculations. Ashlee Mattingly, University of Maryland, Cynthia J. Boyle, University of Maryland, Simon Keegan, University of Maryland. Objective: Pharmaceutical calculations are an integral part of pharmacy practice and are heavily weighted in the NAPLEX exam. Therefore, calculations are an important component of pharmacy education. Students should master pharmaceutical calculations to become competent practitioners, and yet some students struggle with this important component of the curriculum. This study aims to identify factors that may be predictive of success on a high-stakes calculation competency examination so that appropriate interventions can be made to ensure students' success. Methods: Students were given an algebra assessment at the start of the semester to establish baseline calculation competency. Additionally, demographic information, college GPAs, PCAT scores, and student pharmacy score application ratings were compiled. At the end of the semester, students' scores were analyzed according to whether or not they passed the calculations final exam, and the various elements were analyzed using descriptive statistics. A T-test was performed for continuous variables and a chi-square test or Fischer's exact test were performed for categorical variables in order to compare means and frequency distribution between passed and unpassed students. A multivariate regression model was built using stepwise selection. Results: After bivariate analysis, associations between performance and undergraduate science GPA, PCAT chemistry percentile, verbal ability percentile, quantitative ability percentile, admissions interview score, and algebra assessment score were significant $(\mathrm{p}<0.05)$. With stepwise multiple regression, science GPA, PCAT chemistry percentile, and mean of 4 quizzes were the strongest predictors of success. Implications: This data suggest that certain parameters may be useful in identifying students who are at risk of failing high-stakes pharmaceutical calculations.

Evaluation of a Capstone Course Developing Patient Work-Up Skills Using the Pharmacists' Patient Care Process (PPCP). Beth Phillips, The University of Georgia, Andrea Sikora Newsome, The University of Georgia, Russell Palmer, The University of Georgia, Katie Smith, The University of Georgia, Stephanie Phan, The University of Georgia, Daniel B. Chastain, The University of Georgia, Christopher Bland, The University of
Georgia. Objective: The 2016 Accreditation Standards place emphasis on incorporating the PPCP into the PharmD curriculum. The purpose of this study was to evaluate student perceptions and skill development in patient work-up for a course developed in a new curriculum and identify areas for improvement in future course offerings. Methods: Using a simulated electronic health record, students were expected to work-up complex patient cases and identify drug-related problems utilizing the PPCP individually, prior to class discussion. Students were assessed on these skills through weekly quizzes and two practical examinations, involving timed patient case work-ups with subsequent presentation of drug-related problems and treatment plans in order of priority to faculty in a controlled environment. Student feedback was gathered through interview and end of course evaluations. Results: Student performance $(n=134)$ significantly improved from the first to second practical examinations ( $\mathrm{p}<0.05$, paired student's t-test). A significant correlation between overall quiz average and performance on the final practical examination (Pearson's Correlation, $\mathrm{p}<0.05$ ) was identified. Student confidence in ability to perform skills listed in the course using an adapted Dreyfus Model significantly improved from the beginning to the end of the semester $(\mathrm{p}<0.05$, Wilcoxon Signed Ranks). Students performing well on the practical examinations consistently used a systematic approach for patient work-up. Feedback for course improvement included minimizing variability in weekly case format and quizzes, modeling presentations, and opportunity for small group practice and feedback. Implications: The course improved student skill and confidence in patient work-up utilizing the PPCP. Valuable feedback was obtained to guide course updates.

Evaluation of a Formative Assessment Tool in a Pharmacy Calculations Course. Dana A. Brown, Palm Beach Atlantic University, Catherine D. Harrington, Palm Beach Atlantic University. Objective: To evaluate the impact of an assessment tool, the Critical Question Assessment (CQA), on improvement in student performance on the subsequent exam (following implementation of the CQA) and final course score in a pharmacy calculations course. Methods: As a means to increase chances for student success, a CQA was implemented in a required, first year pharmacy calculations course. This tool is given to students who perform less than $70 \%$ (i.e. passing grade) on any intra-semester exam. The student must address each question that was missed, classify the rationale of why it was missed, and provide a corrected answer. Deidentified data regarding subsequent examination and course performance was analyzed from students enrolled in the class from Fall 2013 through Fall 2018. Results: A 


\section{American Journal of Pharmaceutical Education 2019; 83 (5) Article 7654.}

total of 172 students completing a total of 266 CQAs were included in this analysis. Seventy-one percent of students who completed a CQA passed the next exam, while $29 \%$ of students did not. Students needing 1 or 2 CQAs $(\mathrm{N}=$ 148 ) were highly likely to pass the course $(95 \%)$, those needing more than $2(\mathrm{~N}=24)$ were not as successful $(29 \%)$ (Chi Square 75.1, p < 0.05). Implications: This analysis demonstrates the potential value of a formative assessment tool on student performance and overall success in a pharmacy calculations course. Simply "viewing" a grade in a learning management system does not close the loop on reflective assessment. Thus, this type of assessment tool could be beneficial in courses in which math is a significant component.

Evaluation of an Elective Course to Enhance Well-Being and Self-Awareness. Katherine S. WadasThalken, Creighton University, Kevin Powers, Creighton University, Amy Pick, Creighton University. Objective: To develop and implement an elective course focused on improving skills for well-being and self-awareness and how both impact student success. Methods: Twentythree students participated in a new elective course, PHA 348 Well-being and Student Success, in Fall 2018. The course was taught online, utilizing videos, readings, discussion boards, and assignments. Students completed the Grit scale the second week of the course and again in the final week. In a final reflection essay, students discussed what they learned and how they will implement the lessons in the future. Finally, students completed a survey about how effective the course was in improving their well-being. Results: Twenty-two students completed the pre and post tests of the Grit scale. The average increase in Grit was significant at $.36(\mathrm{p}<.001)$. Class participants $(n=23)$ reported a mean of 4.74 (high of 5) indicating they had a better understanding of how wellbeing impacted their student success. Students rated the usefulness of the course at 4.7 (out of 5). Themes from the final essay revealed that students gained insight for identifying when their well-being decreased and strategies for improving healthy well-being. Topics regarded useful to students' learning were spirituality, emotional health, and physical health. Implications: The initial offering of this course was successful in improving students' well-being and self-awareness. This elective course will continue to be offered. Certain elements of the course may be integrated into the required Professional Development course series.

Evaluation of an Instrument to Students' Personal and Professional Development During the Faculty Advising Process. Justine S. Gortney, Wayne State University, Minakshi Lahiri, Wayne State University. Objective: To evaluate performance of a novel Faculty Ad- visor Assessment of the Advisee (FAAA) tool within a didactic curriculum in a two-year pilot. Methods: The FAAA is a 5-item tool we developed for longitudinal assessment of students' personal and professional development. Using the FAAA, faculty advisors assess their student advisees' values, engagement, self-awareness, professionalism, and leadership yearly. Question anchors describe characteristics exhibited in the student that match categories of beginning, emerging or engaged. De-identified aggregate cohort reports were evaluated to determine consistency of the FAAA's performance. Descriptive statistics, student's t-tests and Cronbach's alpha were used for data evaluation. The hypothesis was that no differences would exist within the individual class cohort comparisons and more student behavior would be assessed as "engaged" as they progressed through the curriculum designated as exempt. Results: 563 students (P1 $n=203, P 2 n=177, P 3 n=184$ ) were evaluated for winters 2017 and ' 18 . The FAA showed high internal consistency with Cronbach's alpha of $0.875(n=258)$ and $0.887(\mathrm{n}=305)$. Comparing mean instrument scores via t-test, no differences were seen comparing P1 or P2 classes of 2017 and 2018; 13.63 vs $14.1(\mathrm{p}=0.31) ; 16.13$ vs $16.75(p=0.0) 3$. P3 cohorts were 17.09 vs. 17.95, $\mathrm{p}=0.03$. Stepwise increases aligning to "engaged" were observed comparing class years in most characteristics (e.g., P1-12, P2-38, P3-51\% were engaged respectively). Implications: A simple to use 5-item tool showed cohort growth in personal and professional development and could be used to provide objective assessment of students in longitudinal advisor-advisee relationships.

Evaluation of an Objective Structured Clinical Examination (OSCE) to Assess Student Interprofessional Skills and Behaviors. Fred Doloresco, University at Buffalo, The State University of New York, Jaime L. Maerten-Rivera, University at Buffalo, The State University of New York, Yichen Zhao, University at Buffalo, The State University of New York, Kelly Foltz-Ramos, University at Buffalo, The State University of New York, Nicholas M. Fusco, University at Buffalo, The State University of New York. Objective: To examine an objective structured clinical exam (OSCE) evaluating interprofessional collaborative practice. Methods: Third-year pharmacy students completed three interprofessional OSCE cases. Students then completed a survey that included seven questions on the quality of the cases and the Interprofessional Collaborative Competency Attainment Survey (ICCAS), which is a retrospective pre and post instrument. Student performance on each case was assessed using the Global Rating Scale (GRS). Paired sample t-tests were performed to compare differences in mean change in ICCAS scores. Correlations between the 


\section{American Journal of Pharmaceutical Education 2019; 83 (5) Article 7654.}

GRS ratings and ICCAS pre, post and change scores were examined. Results: A total of 124 students participated in the study. Overall, the students reported that the OSCE cases were realistic and of high-quality. The average total ICCAS scale (out of seven) score at pre- was 5.07 $(\mathrm{SD}=0.78)$ and at post- was $5.87(\mathrm{SD}=0.58)$; this difference was statistically significant. The mean (SD) score (out of five) for the three cases were $4.17(\mathrm{SD}=0.45)$, $4.54(\mathrm{SD}=0.56)$ and $4.58(\mathrm{SD}=0.46)$; and the mean for the three cases combined was $4.43(\mathrm{SD}=0.31)$. Implications: The interprofessional OSCE developed was effective at providing realistic scenarios that both assessed and improved students' interprofessional collaborative behaviors.

Evaluation of a Pharmacists' Patient Care ProcessBased Patient Presentation Rubric in a General Medicine Elective Course. Jason W. Lancaster, Northeastern University, Alexa A. Carlson, Northeastern University, Stephanie L. Sibicky, Northeastern University, Adam B. Woolley, Northeastern University, Mark Douglass, Northeastern University, Michael J. Gonyeau, Northeastern University, Margarita V. DiVall, Northeastern University. Objective: Describe development, validation, and the inter-rater reliability a newly created rubric. Methods: Between 2015 and 2017, 36 students enrolled in a 16-week general medicine elective course. Students completed three case presentations, graded independently by faculty using a standardized, nine-item, rubric. Faculty created the rubric with dimensions and criteria developed by consensus with iterative refinements. Dimensions were mapped and validated to the Pharmacists' Patient Care Process and the Center for the Advancement of Pharmacy Education Educational Outcomes. Rubric dimensions evaluated competencies related to students' ability to provide a concise summary, patient work-up related to the Collect and Assess steps and competencies related to the Plan, Implement, and Follow-up steps, oral presentation and organizational skills, and their ability to respond to questions. Inter-rater reliability was determined via intra-class correlation coefficient via two-way model using absolute agreement. Statistical analysis was conducted with SPSS Statistics, Version 25 for Windows. Results: 216 rubric evaluations were analyzed from 108 unique student presentations. The median score was $85 \%$ (range $65-100$ ). The ICC coefficient ranged from 0.4 to $0.949,95 \%$ CI $0.51-0.993$; and $85 \%(\mathrm{~N}=184)$ of rubric evaluations demonstrated an ICC coefficient $\geq 0.7$. Implications: Our rubric demonstrated strong inter-rater reliability and allows for generation of learning outcomes data and has broad applicability across a variety of setting including didactic courses, skills labs, and experiential training. Addition- ally, the findings demonstrate that faculty should feel empowered to develop, and evaluate, assessment tools to evaluate student performance, especially as curricular changes are implemented such as introduction of the PPCP.

Evaluation of In Room Versus Live Stream Evaluation Methods During a P1 Practical Assessment. Sneha Srivastava, Rosalind Franklin University of Medicine and Science, Danielle M. Candelario, Rosalind Franklin University of Medicine and Science, Viktorija Barr, Rosalind Franklin University of Medicine and Science. Objective: This study assesses student performance and perception of in-room evaluation versus live-stream remote evaluation for a pharmacy skills practical counseling assessment. Methods: A counseling practical is required of first-year students enrolled in a Skills Lab course they were given 20 minutes to counsel a patient on two different insulin delivery systems. Students performed one counseling session with a faculty evaluator in the assessment room and then to an adjacent room, where they completed a second counseling session regarding insulin pen use while a faculty member utilized SimCapture $^{\odot}$ evaluate via live-stream. A validated evaluation form was utilized. Students and evaluators were sent an anonymous, voluntary survey to address knowledge, confidence and perceptions of both evaluation methods. Results: 50 out of 61 students that participated in the practical completed the survey. Mean score on the practical assessment was $91.8 \% .45 .7 \%$ felt having an in-room evaluator made them nervous but only $28.3 \%$ felt it negatively impacted their performance. Students did not believe a live-stream evaluator made them nervous $(69.6 \%)$ and most did not feel it negatively impacted performance $(73.9 \%)$. Students preferred live-stream to in-room evaluation (56.5\% versus $32.6 \%$ ). $80 \%$ of evaluators preferred live-stream evaluation. Implications: Practical assessments serve to evaluate effective and appropriate practice-based skills. To ensure faculty are providing optimal evaluation methods, this study provides the foundation for further studies that evaluate best practices for assessing practical skills which can greatly influence the pharmacy curriculum.

Evaluation of Patient Counseling Rubrics Utilized in Schools and Colleges of Pharmacy. Jennifer L. Mazan, Midwestern University/Downers Grove, Vasudha Gupta, Roseman University of Health Sciences, Kristen M. Cook, University of Nebraska Medical Center, Courtney L. Bradley, High Point University, Rashi C. Waghel, Wingate University. Objective: The Accreditation Council for Pharmaceutical Education (ACPE) Standards and Core Entrustable Professional Activities (EPAs) list patient education and effective 


\section{American Journal of Pharmaceutical Education 2019; 83 (5) Article 7654.}

communication as essential competencies in pharmacy education. A universal tool to assess student proficiency in patient education is essential but has not been developed. The objective of this project is to describe current practices in evaluating patient counseling within the didactic curriculum at colleges of pharmacy. Methods: A cover letter and questionnaire was sent to all members of the AACP Laboratory Instructors SIG (Lab SIG) with an invitation to submit a patient counseling rubric. Descriptive statistics were used to analyze the questionnaire items. An inductive approach was used for data analysis, which was completed independently by two members of the Lab SIG and then compared to build consensus to establish common themes. Results: Five major themes emerged but with considerable variability in content. More than $76.4 \%$ of tools included an introduction. More than $58.9 \%$ of tools collected subjective information and assessed patient's prior medication knowledge. More than $93.8 \%$ included correct drug information and counseling points. The majority $(93.8 \%)$ included verification of understanding, but only $41.2 \%$ of tools included a closing statement. Both verbal and non-verbal was assessed in 93.8\% of the evaluation tools. Implications: The common themes of introduction, collecting information, counseling session, follow-up, and communication skills should be incorporated when developing a standardized patient counseling rubric. A limitation is that data may be subject to non-response bias as the number of respondents represents $12.7 \%$ of the colleges of pharmacy across the country.

Evaluation of Student Perceptions of Introductory Pharmacy Practice Experiences. Jaclyn Boyle, Northeast Ohio Medical University, Zachary N. Jenkins, Cedarville University, Thad Franz, Cedarville University, Charles Cather, Northeast Ohio Medical University, Nicole K. Stute, Cedarville University, Nicole G. Harper, Cedarville University, Alison Dittmer, Northeast Ohio Medical University, Christy Hartline, Northeast Ohio Medical University. Objective: Introductory Pharmacy Practice Experiences (IPPEs) are focused on sharpening the student's knowledge, skills, abilities, and attitudes related to becoming an effective member of the healthcare team. However, there is no evidence to describe student perceptions surrounding IPPE curricula across colleges of pharmacy. This study gathered perceptions of IPPE rotations from pharmacy students in their first through third year professional years. Methods: A pre- and post-survey instrument was administered to 367 first through third year professional students from two colleges of pharmacy at the beginning and end of the 2017-2018 academic year. Each survey utilized questions with Likert scales designed to detect differences in student expectations and experiences related to IPPE training. Questions primarily focused on demographics, objectives, goals, interprofessional teamwork, application of knowledge, and preceptor engagement. Students ages 18 and older who were enrolled at the participating institutions were eligible for inclusion in this study. Survey completion was voluntary, and students were not incentivized to participate. Results: Sixty-seven students completed the preand post- surveys with matching, identifiable data for analysis. Twenty-nine items demonstrated a more positive perception of IPPEs between the pre- and postsurveys $(\mathrm{p}<0.05)$. Eight items demonstrated a difference by institution $(p<0.05)$, while three items demonstrated a difference between professional years $(p<0.05)$. Implications: Student expectations of IPPEs were significantly less positive in the pre-survey than in the post-survey. Future studies should examine the implications of these results on student IPPE performance and methods to impact student perception of the value of IPPE training.

Evidence of Learning: What Diabetes Camp does for Pharmacy Learners. Nancy E. Kawahara, Loma Linda University, C.J. Jacobson, Loma Linda University, Heather P. Whitley, Auburn University. Objective: To determine the learning that occurs when pharmacy learners volunteer as medical staff at diabetes camp. Methods: National list-serves were used to identify pharmacist who precept pharmacy learners at diabetes camps. Pharmacists were asked to share a pre-camp (completed prior to arriving at camp) and a post-camp (completed within one week following camp) electronic survey with their pharmacy learners. The survey was comprehensive and produced too much data to present in a single poster. Therefore, this poster will focus on how learners shifted up the Bloom's taxonomy continuum for the terms carbohydrate ratio, insulin sensitivity factor, ketones, hypoglycemia, hyperglycemia, glucagon, glucometer, continuous glucose monitor, and insulin pump. The levels of blooms taxonomy were converted to a Likert scale with 1 corresponding to the lowest and 5 corresponding to the highest level of understanding in order to facilitate data analysis. Results: For all terms assessed, except glucometer, the pre-camp mean compared to the post-camp mean were statistically significant with a P-value of $<0.001$. Within most pharmacy curriculums students are trained to perform blood glucose finger sticks so it was not surprising that the pre and post-camp values for glucometer, while still statistically significant (P-value 0.03), were not as dramatic as the gains seen on the other items. Implications: The opportunity to participate in a diabetes camp experience allows pharmacy learners to gain practical application exposure that gives them the ability to cement the learning that has been started in the classroom. 


\section{American Journal of Pharmaceutical Education 2019; 83 (5) Article 7654.}

Extrinsic Assessments in a Competency-Driven Curriculum: Implementation and Impact on Student Confidence. Jane Bowen, University of the Sciences, Tyan F. Thomas, University of the Sciences, Suzanne Carbonaro, University of the Sciences, Islam M. Ghazi, University of the Sciences, Peter J. Harvison, University of the Sciences, Lisa Charneski, University of the Sciences. Objective: To describe the implementation of extrinsic assessments and their impact on student confidence in a competency-driven curriculum. Methods: Philadelphia College of Pharmacy implemented an integrated, modular, competency-driven curriculum, which includes extrinsic summative assessment reassessments (ESARs). ESARs are pass/fail, occur twice per semester, and are structured periods where the curriculum pauses for programmatic evaluation of students' retention and integration of prior knowledge/skills. Students unsuccessful on any ESAR component are referred to learning support and complete an individualized reassessment. A pre/post ESAR self-efficacy survey was administered to students to assess their confidence in knowledge/skills from prior modules, using a five-point Likert scale. Results: Eightyone P1 students completed 2 ESARs in fall 2018. For both ESARs, $87.7 \%-100 \%$ of students passed knowledge assessments, including general knowledge and commonly used drugs, and 50.6\%-99\% passed skills assessments, including calculations, blood pressure, patient counseling, communication with a provider, compounding, and self-care triage. All but 2 students successfully passed the individualized reassessments on their first attempt. Overall, students' confidence in knowledge/skills increased after ESARs. The largest increase in student confidence was in calculations (doses and dilutions) $(p<0.05)$ and blood pressure. Students felt least confident in calculations (creatinine clearance) and molecular biology. Implications: Although time and resource intensive, ESARs are valuable assessments of student retention and integration of previously learned material. ESARs increase students' confidence in knowledge/skills and trigger early identification of students who are at risk and need additional learning support.

Factors Associated with Perceived Stress in First Through Third Year Pharmacy Students: A Pilot Study. Sarah Holman, Notre Dame of Maryland University, Andrea R. Gauld, Notre Dame of Maryland University, Jennifer L. Bailey, Notre Dame of Maryland University, Heather Folz, Notre Dame of Maryland University. Objective: The purpose of this study is to assess the current state of student wellness in a pharmacy program and factors associated with perceived stress. Methods: First through third year pharmacy students completed a baseline survey which included demograph- ics, current personal wellness practices, and the validated Perceived Stress Scale (PSS). Data were analyzed using descriptive statistics, analysis of variance (ANOVA) and analysis of covariance (ANCOVA). Results: One hundred fifty-three students completed the survey. The most common wellness resource used on campus was the fitness center (27\%) followed by group fitness classes (4\%). No students reported sleeping greater than eight hours per night with $83 \%$ reporting six hours or less. The majority of students denied misuse of alcohol (63.7\%) and illicit drugs (96.7\%). Two-thirds of students reported exercising less than one hour per week. The average PSS score was 20.2 (range 0-40) indicating moderate stress. After covariate adjustment, factors with significant differences in mean PSS scores were class year $(p=0.007)$, hours of sleep $(p=0.005)$, and school life balance $(p<0.001)$. Notable factors not associated with differences in mean PSS scores were commute time, number of dependents, exercise, and work hours. Implications: With $85 \%$ of students reporting moderate to high stress there is a clear impetus for attention to student wellness and stress reduction in didactic pharmacy school curricula. These results may help to guide future targeted interventions by pharmacy programs and provide opportunity to address ACPE Standard 4.1 regarding self-awareness.

Factors Influencing the Involvement of Doctor of Pharmacy Students in Research/Scholarship Projects. Tracey L. Mersfelder, Ferris State University, Minji Sohn, Ferris State University, Heather L. Girand, Ferris State University. Objective: To determine factors that stimulate or hinder student pharmacists to participate in research/scholarship during a Doctor of Pharmacy program. We hypothesize that participation is associated with student-specific and academic environment characteristics. Methods: An electronic questionnaire was developed to assess factors that motivate or hinder student pharmacists to engage in research/scholarship with faculty. The survey was emailed to all students enrolled in the program and all faculty. To increase response rates, students were provided class time to take the survey. Responses were collected anonymously and used to perform qualitative analysis. Results: A total of 426 students (73\% response rate) and 35 faculty (78\% response rate) participated. Motivational factors that were rated high by both students and faculty were interest in the topic, comfort level in working with faculty, energetic quality of faculty, and becoming more competitive for post-graduate training. Students indicated that research/ scholarship benefiting the profession/medical community was important to them, while faculty believed that pursuing a position that requires research/scholarship was a key motivational factor for students. The most highly rated 


\section{American Journal of Pharmaceutical Education 2019; 83 (5) Article 7654.}

barrier was lack of time. Implications: Prior to the survey, student participation in research/scholarship with faculty was inconsistent, and little was known about factors that lead student pharmacists to engage in research/ scholarship. There was agreement regarding some motivating factors; however, differences did exist between student and faculty beliefs. Addressing these differences may help increase student involvement in research/scholarship in the future. The study is subject to potential nonresponse bias, and the findings may not correctly reflect non-respondents' perception.

Faculty Development Book Club Series - A Phenomenographic Assessment of Perceptions and an Evaluation of Impact. Carinda Feild, University of Florida, Karen Whalen, University of Florida. Objective: This study evaluated a live book club series faculty development program through a phenomenographic based description, analysis, and understanding of the faculty's perceptions of the book club experience and its impact. Methods: Ten experiential program faculty working across the state participated in a book club series. The books were discussed at live meetings and debriefing sessions were held to capture the faculty experience. A phenomenographic analysis was conducted to examine the dimensions of variation among the faculty with the goal of describing the relationship between faculty and participation in the book club. Results: Most faculty felt the process helped build a collaborative teaching/practice community. Some cited the in-person meetings as validating their professional experiences while others felt sharing expanded their knowledge and skills. Several noted that the live interaction would be most remembered. Others stated the process made them feel less alone/isolated and some felt more likely to reach out to the community for assistance. Some noted the book club process would change how they worked with students and preceptors, while others cited it would impact how they worked with those outside the workplace. Based on faculty experience, the process has developed a rhizome in another teaching community within the college. Implications: A live book club process may help develop collaborative teaching/practice communities by through varied mechanisms decreasing isolation and creating shared knowledge. This may be particularly impactful where faculty are physically or intellectually working separately and may give rise to other communities with the work place.

Faculty Perceptions of Simulation Training Materials and Sessions: Evaluating the Implementation of a Simulated Case. Nicole R. Winston, Augusta University, Matthew Tews, Augusta University, Amanda Kleinheksel, Augusta University. Objective: To assess faculty perspectives of training for, and the subsequent execution of a simulated case for third-year students. Methods: Faculty volunteers $(n=22)$ were provided with online training materials and attended at least one in-person group training sessions. The individual simulations presented a myocardial infarction utilizing high-fidelity mannequins. Faculty were tasked with providing the voice of the patient, evaluating each student's performance, and debriefing the student after their session. Following the simulations, an online survey was administered to the faculty including both quantitative and qualitative items. Descriptive statistics were used to summarize quantitative items. Themes from open-ended questions were developed using the Glaser's constant comparative method. Results: Twenty-one survey responses indicated $76 \%$ worked in a clinical setting and $52 \%$ had previously participated simulations. Respondents reported spending an average of 38 minutes $( \pm 18$ minutes) with online material. The majority agreed that the online $(86 \%)$ and face-to-face $(100 \%)$ training sessions were useful. In addition, 20 respondents indicated that they plan on using simulation to teach students based on this simulation experience. Open-ended question analyses supported Likert-style item results. Additional themes that emerged included smooth workflow $(n=13)$, student performance variability $(n=8)$, the ability to evaluate students' clinical reasoning $(n=7)$, and the utility of simulation $(n=5)$. In terms of improvement, participants suggested prioritizing online training material $(n=3)$ and providing a patient script to respond to common questions $(n=3)$. Implications: Results suggest that faculty felt well-prepared to facilitate the simulations, which they perceived positively. The training materials and survey may serve as a resource for other simulation programs.

Feasibility of Longitudinal Assessment of the Pharmacist Patient Care Process from Didactic Curriculum to APPE Performance. Khaled A. Elsaid, Chapman University, Siu-Fun Wong, Chapman University. Objective: To evaluate the feasibility of evaluating student performance related to the pharmacist patient care process (PPCP) in the didactic curriculum and examine whether it predicts student performance on APPEs. Methods: We utilize a composite electronic examination approach during the didactic curriculum. Across six trimesters, 30 composite exams are administered. Items are mapped to our institutional student learning outcomes (SLOs) statements. Domain 2 of our SLOs reflects the essential components of the PCPP. We also evaluate student performance on domain 2 in APPEs. We used linear regression and computed Pearson's correlation coefficient (r). Other statistical analyses included reporting 


\section{American Journal of Pharmaceutical Education 2019; 83 (5) Article 7654.}

mean performance and $95 \%$ confidence interval (CI) and Student's t-test for two group comparisons using $\mathrm{p}<0.05$. Results: A total of 1,920 items were mapped to domain 2 SLO over 6 trimesters of study. Students performed significantly better $(\mathrm{p}<0.05)$ on items mapped to gathering information (77.5\%; CI: 75.7 to 79.1$)$ and evaluating current therapies (77.5\%; CI: 75.8 to 79.1$)$ compared to items mapped to developing treatment plans $(72.9 \%$; CI: 71.0 to $74.9)$ and managing therapy $(72.9 \%$; CI: 71.0 to 74.9).There was a significant, albeit weak, correlation between domain 2 performance and cumulative APPE performance $(\mathrm{r}=0.32 ; \mathrm{p}<0.01)$. Mean cumulative APPE performance of our top $25 \%$ domain 2 performers was significantly higher than corresponding performance of the bottom $25 \%$ domain 2 performers $(p=0.02)$. Implications: Evaluation of student competency related to the PCPP in the didactic curriculum can be a valuable tool to identify areas of improvement and assess student preparedness for advanced experiential experiences.

Financial Literacy in Pharmacy Students. Adam B. Woolley, Northeastern University. Objective: The average educational debt incurred by Doctor of Pharmacy students continues to rise. Money management skills are not formally taught within our curriculum. The goal of this research is to assess the financial literacy of pharmacy students at baseline, identify gaps in knowledge, and implement an educational intervention that may help improve their comfort level with money management strategies Methods: An IRB approved pre-post survey based, prospective study including a sample of P1-P4 pharmacy students. Students were invited to attend a voluntary one-hour lecture about financial literacy. A pre-survey was administered to assess baseline financial literacy pertaining to credit, money management, income, savings and investment. A lecture was delivered that emphasized key points within each domain. Afterwards, a post survey was administered, on a Likert scale of 1 (strongly disagree)-5(strongly agree), to assess student's satisfaction with the presentation and further interest in financial literacy resources. Results: The pre-survey was completed by 58 participants with an average correct response rate of $61.4 \%$. The correct response rates varied across the domains of spending and credit $(65.8 \%)$, income (65\%). savings and investment (57.2\%), and money management (51.9\%). Mean post survey responses indicated that students enjoyed the activity (4.5), were confident in applying lessons from the activity to their own finances (4.4) and were interested in learning more about finances (4.7). All participants demonstrated considerable interest in additional resources and course offerings to further increase financial literacy. Implications: Pharmacy students demonstrated average financial literacy.
Educational interventions may improve student confidence and warrant further investigation.

Focusing in on Training Upcoming Academicians: Interviews of Pharmacy Resident Teaching Certificate Leaders in Ohio. Emily Eddy, Ohio Northern University, Brittany L. Long, Ohio Northern University, Lindsey Peters, Ohio Northern University. Objective: Though residency teaching certificate programs (RTCP) are common, there is a lack of standardization and published research on what content and requirements should be included within the programs to best prepare residents for a career in academia. The objective of this project is to better identify and compare how residency teaching certificate programs are structured in Ohio. Methods: Ohio RTCP coordinators were contacted via email and asked to participate in an IRBapproved interview, either in-person or telephonically. Standardized questions were developed focusing on six categories: demographics/background, administration/ logistics, content, resident assessment, financing, and continuous quality improvement. Interviews were recorded, documented, and coded. Data was analyzed and reported in aggregate form. Results: All seven colleges were interviewed, representing 186 RTCP participants this year. Logistics including scheduled meetings, content covered, required activities, and faculty involvement were not consistent. One consistency is all programs require a teaching portfolio and completion, rather than demonstrated competency, of required activities. Throughout all the programs existence, only 2 participants did not earn the certificate. Program finances varied; $29 \%$ of RTCP pay the residency programs and $14 \%$ get paid by the residency programs. Program assessment ranged from no routine assessment to grant-funded quality improvement, with most programs lacking annual programmatic review. Six programs (86\%) expressed interest in developing a standard set of requirements and in increasing collaboration across RTCPs. Implications: This research is the second step in a program review and inquiry into the most effective content, delivery methods, resident assessment, and program evaluation for a residency teaching certificate program.

Focusing Student Academic Support on Self-Regulated Learning Strategies: Where to Begin? Shauna M. Buring, University of Florida, Aaron O. Thomas, University of Florida. Objective: Objective: To identify the relationship between self-regulated learning strategies and academic performance in pharmacy students. Methods: Methods: An inventory of self-regulated learning strategies was delivered to 3PD students via an online survey. Students were asked to respond to each statement using a 7-item ordinal scale of describing how often they 


\section{American Journal of Pharmaceutical Education 2019; 83 (5) Article 7654.}

used the SRL strategy (never to always). A multiple linear regression model was used to identify key self-regulatory learning strategies that significantly impacted academic performance in an integrated series of "therapeutics" courses in (Patient Care 6,7 and 8) in the third professional year. Results: Results: 212 students completed the inventory. A significant proportion of the total variation $(28.7 \%)$ in Patient Care grades was predicted by six key self-regulated learning strategies $(\mathrm{F}(6,205)=13.762, \mathrm{p}<$ $0.001)$. These included watching videos faster than $1 X$, not attending help sessions, waiting until the last minute to study, asking the professor questions to clarify understanding, watching the instructional videos before coming to class, finishing all studying before playing video games or socializing. The majority of students $(89.7 \%)$ indicate the frequently/very frequently/always watch the instructional videos prior to class. However, only $40.6 \%$ frequently/very frequently/always ask questions of the instructor when they do not understand a concept. Implications: Implications: We identified self-regulatory learning strategies most impactful upon student academic performance in didactic, integrated pharmacotherapy courses. Recognition of these strategies will allow us to coach students on the self-regulated learning strategies that are most likely to support individuals to improve their own academic performance.

Foundations of Pharmacy Course Hybridization to Improve Student Engagement. Laurie L. Briceland, Albany College of Pharmacy and Health Sciences, Courtney R. Caimano, Albany College of Pharmacy and Health Sciences, Megan Veselov, Albany College of Pharmacy and Health Sciences, Jeffrey M. Brewer, Albany College of Pharmacy and Health Sciences, Sandra W. Rosa, Albany College of Pharmacy and Health Sciences, Cindy Jablanski, Albany College of Pharmacy and Health Sciences. Objective: To describe the conversion of the P1 Foundations of Pharmacy (FoP) face-to-face (F2F) course to an online hybrid as an attempt to improve student engagement with course materials, faculty, and peers. Methods: FoP is a 1-credit required course for P1 students across two campuses. Prior student course evaluations indicated minimal student engagement with the course material, faculty, and peers; some suggested format conversion to online. Faculty received detailed instruction in online course development through enrollment in an instructional online development course, and personalized instruction from a college instructional designer. Seven of 14 F2F sessions were converted to online. Online activities aimed to increase students' engagement with course faculty, material, and peers and included two discussion boards, Pharmacy Career Exploration employing VoiceThread and cultural competency incorporating interprofessional nursing students; and Top 100 Drugs U Should Know (DUSK) quizzes employing gamification via Quizlet and Kahoot. Writing assignments and openbook examination were retained. Course assessment included student evaluations and focus group discussion with instructional designer. Results: The two most favorable activities within the course noted by student evaluations was the inclusion of DUSK gamification/quizzes and the career exploration discussion board; the hybrid nature was in general favorably received. Areas in need of improvement were overload of content/work for a 1 credit course, too many faculty involved, and a disconnect amongst course content. Implications: The discussion board and gamification were the most favorable aspects of the course. Based on student feedback, the hybrid nature will continue, and course content/assignments will be reduced.

Grit in Pharmacy Faculty: An Analysis of Two Universities. Taylor D. Steuber, Auburn University, Sarah A. Nisly, Wingate University, Christopher Gillette, Wake Forest University. Objective: To assess Grit-S scores among pharmacy faculty across two universities and to evaluate if there was a correlation between Grit-S scores and productivity in the domains of teaching, scholarship, and service. Methods: A 36-item electronic survey was administered to pharmacy faculty members at two universities. Data collected included demographics, Grit-S scores, and measures of quantitative measures of productivity in the areas of teaching, scholarship and service. Non-parametric and correlational analyses were conducted to test if higher Grit-S scores correlated with other variables. Results: Faculty had an average Grit-S score of 3.68. Higher Grit-S scores had a moderate positive correlation with the number of peer-reviewed publications in 2017. However, there was no significant correlation between Grit-S scores and components of faculty productivity in teaching, service, and remainder of scholarship productivity measures for the previous academic year or career. Implications: Grit-S scores were not correlated with higher productivity in most components of faculty workload. Pharmacy faculty have high Grit-S scores; however, the implications of this are unknown.

Identification of Physical Examination Skills to be Taught and Performed in a Skills Laboratory. Sara K. Richter, St. Louis College of Pharmacy, Justinne Guyton, St. Louis College of Pharmacy, Patrick M. Finnegan, St. Louis College of Pharmacy. Objective: To identify which physical exams students should be expected to perform upon graduation in order to prioritize learning opportunities and teaching in a pharmacy practice skills lab setting. Methods: All members of the American 


\section{American Journal of Pharmaceutical Education 2019; 83 (5) Article 7654.}

Association of Colleges of Pharmacy (AACP) Pharmacy Practice Section were invited to participate in an anonymous survey in which they were asked to identify at what level a graduating pharmacy student would need to know or perform/teach various physical exams. A list of thirteen physical exams was compiled by study investigators. Participants were asked to characterize pharmacy graduate level of skill as: 1) do not need to know about or perform/ teach, 2) may know about but do not need to perform/ teach, 3) must know about but do not need to perform/ teach, 4) must know about and may perform/teach, or 5) must know about and be able to perform/teach. Additionally, participants were asked to identify physical exams they have performed on patients in the past 12 months. Data were analyzed using descriptive statistics. Results: A total of 450 unique surveys were analyzed. Physical exams scoring the highest on the rating scale included taking vital signs $($ mean $=4.73)$, basic life support $($ mean $=4.70)$, and monofilament exam (mean=4.08). The most commonly performed physical exams in the past 12 months included: taking vital signs ( $73 \%$ performed), monofilament exam (38\% performed), and neurological/ mental status exam (23\% performed). Implications: Results of this survey may be utilized to guide faculty on which physical examination skills should be taught and practiced in a pharmacy skills lab curriculum.

Identification of Top Drug Information Assessment Within United States PharmD Curricula. Michelle M. Bottenberg, Drake University, Carrie Koenigsfeld, Drake University, Nicholas Lehman, Drake University, Erin Engbring, Drake University, Amy Kukacka, CVS/Target. Objective: To identify current processes and procedures utilized in teaching and assessing pharmacy student knowledge of Top Drug (Top 200/ 300) Information. Methods: Following a literature review, an 18-item questionnaire was sent via Qualtrics to department heads/chairs at US colleges of pharmacy utilizing the AACP mailing list for department heads $(\mathrm{n}=$ 117). Demographics and information regarding current assessment practices were collected (courses taught in, years in curriculum, sources utilized, assessment strategies, mandatory pass score, etc.) This study was classified exempt by the institution IRB. Results: Fifty-six surveys were collected (response rate $48 \%$ ). There was a higher private institution response ( $57 \%$ vs $43 \%$ ) and even distribution for schools accredited before and after 1980 (48\% and 52\% respectively). Only 5 institutions reported assessing Top Drug Information during P4 year. Top Drug Information is mainly taught/assessed in skills lab $(63 \%)$ while a specific stand-alone course was offered at 13 institutions. The most common learning sources identified were Access Pharmacy (43\%) and institution-cre- ated lists (43\%). Many institution representatives (57\%) selected they would not change Top Drug Information teaching/assessment at their institution. Implications: While Top Drug Information is not a part of the 2016 ACPE standards, teaching/assessing it is suggested by ACPE guidance documents. Guidance is lacking on source of Top Drug Information leading to variance in schools' teaching approach. Further research into standardization of Top Drug Information would help insure streamlining of baseline knowledge for future pharmacists.

Impact of a Hallucination Simulation and Schizophrenia Speaker on Mental Health Stigma in Pharmacy Students. Stephanie L. Hsia, University of California, San Francisco, Jordan Brooks, University of California, San Francisco, Emily Yao, University of California, San Francisco, Katherine Gruenberg, University of California, San Francisco, Patrick R. Finley, University of California, San Francisco. Objective: The goal was to evaluate effects of a schizophrenia speaker coupled with a hallucination simulation on mental health stigma in pharmacy students. Methods: Hallucination simulations improve empathy, but produce mixed effects on social distance and attitudes, whereas direct contact with mental health patients improves social distance and attitudes. We therefore hypothesized that combining these interventions would decrease mental health stigma without worsening social distance and attitudes. 121 pharmacy students listened to a schizophrenia speaker and then completed tasks while listening to an auditory hallucination simulation. Students completed the Opening Minds Stigma Scale for Health Care Providers (OMS-HC) prior to the speaker and after the simulation. Students were also asked to describe how their perception changed. Results: $63(52 \%)$ of the students completed both surveys. Wilcoxon-signed rank tests and thematic qualitative analysis of comments were conducted. There was a decrease in overall OMS-HC score $(\mathrm{p}=0.005)$, specifically in the attitudes $(p=0.004)$ and disclosure/help-seeking $(p=0.005)$ subscales. There was no change in the social distance subscale $(p=0.182)$. Students described new awareness, reduced stigma, sympathy/empathy, inspiration/admiration, discomfort, and a changed approach to patient care. Sympathy/empathy was the most common theme and commonly co-occurred with patient care. Implications: Combining a schizophrenia speaker with a hallucination simulation decreased mental health stigma among student pharmacists across domains of attitudes and disclosure/help-seeking, with no effect on social distance. Students voiced feelings of sympathy/empathy when describing their changed perception, another important domain of stigma. Coupling a hallucination simulation with a schizophrenia speaker may potentially 


\section{American Journal of Pharmaceutical Education 2019; 83 (5) Article 7654.}

mitigate negative effects of the simulation on social distance.

Impact of an Advanced Cardiovascular Pharmacotherapy Elective on APPE Preparedness. Meredith L. Howard, University of North Texas Health Science Center, Caitlin Gibson, University of North Texas Health Science Center. Objective: During the Advanced Pharmacy Practice Experience (APPE) year, students must demonstrate competency on skills and assignments that may not be emphasized during the didactic curriculum which may limit APPE preparedness. The objective of this study is to determine if an elective course comprised of common APPE activities improves APPE readiness. Methods: Third-year students (P3s) enrolled in two consecutive years of an Advanced Cardiovascular Pharmacotherapy elective completed activities including a journal club, drug monograph, topic discussion, oral case presentation, and clinical controversy presentation. Student perceptions of APPE readiness were assessed via pre- and post-course surveys and a follow-up post-survey during the P4 year. Results: Twenty-five students were enrolled over two years; 18 completed the survey, and nine completed the P4 survey. Students reported increased confidence in analyzing patient data, creating and implementing individualized care plans, applying biomedical literature to patient care and to formulary decisions, and delivering evidence-based presentations, $(\mathrm{p}<0.05)$. Students identified journal club and topic discussions as the most valuable course activities after the course. $\mathrm{P} 4 \mathrm{~s}$ reported the elective prepared them for APPE rotations at a 7.6 on a scale of $1-10 . \mathrm{P} 4 \mathrm{~s}$ reported that the patient care activities the elective most prepared them for were creating patient presentations (56\%), obtaining subjective and objective information for a patient case (44\%), and applying biomedical literature to patient care (44\%). Implications: Integrating APPE activities into a didactic elective improves student-perceived readiness for rotations during the third year and helped students prepare for several key patient care activities on APPE rotations.

Impact of a Personal Lifestyle Goals Assignment on Pharmacy Student Empathy. Heather Folz, Notre Dame of Maryland University, Jonathan Thigpen, Notre Dame of Maryland University. Objective: To assess the impact of a lifestyle goal assignment on student empathy Methods: As an assignment in a complementary medicine course, third year pharmacy students created two personal lifestyle goals and a journal regarding their progress over a five-week period. To assess changes in empathy, students voluntarily completed the 15-item KiersmaChen Empathy Scale (KCES) at three distinct times: A) baseline - prior to assignment, B) "pseudo-baseline" - at week 5 based on how they would now rank themselves at baseline, and C) final - at week 5 based on how they currently felt. Analysis was conducted using paired ttests. Results: Twenty-nine students $(56 \%)$ completed all three surveys. Mean KCES scores (range 15-105) for A vs $C$ increased 1.800 points ( 85.80 vs 87.60 , respectively; $p=0.201)$. Statistically significant increases were found for three questions regarding 1) the necessity for healthcare practitioners to be able to comprehend someone else's experiences $(p=0.021), 2)$ the ability to express understanding of someone's feelings $(\mathrm{p}=0.023)$, and 3$)$ comprehending someone else's experiences $(p=0.004)$. Mean scores between B vs C showed a non-statistically significant increase of 1.674 (86.63 vs 88.30 , respectively; $p=0.078$ ). The two "baseline" surveys results A vs B had similar mean scores (86.14 vs 86.97 ; p-value $=0.580$ ). Implications: Results support that practical assignments like this may help students better relate to patients' experiences when making lifestyle changes. This study provides an easily adaptable model for other courses seeking to promote empathy while also supporting the idea of self-awareness as outlined in ACPE Standard 4.1.

Impact of Applied Improvisation on Student Pharmacists' Patient Communication Skills. Anne LaDisa, Concordia University Wisconsin, Sarah Ray, Concordia University Wisconsin. Objective: Excellent communication skills are essential in clinical pharmacy practice. Applied improvisation (AI) offers an innovative strategy to strengthen students' communication skills. Studies have reported AI use in medical education and when incorporated into a single required pharmacy student course. There is little data on the effectiveness of AI to enhance communication skills when a coordinated AI curriculum is incorporated throughout several pharmacy student courses. Our research aims to discover if students who participated in AI exercises demonstrate superior communication skills during a simulated patient encounter (SPE) compared to students who did not participate in AI exercises. Methods: Beginning with the graduating class of 2020, AI exercises were incorporated into 5 courses between the first-professional year and thirdprofessional year. In the third-professional year, after completing the AI exercise series, students participated in an SPE in an Applied Patient Care course which required skilled communication use. Students' communication skills during the encounter were evaluated by an investigator using an internally validated rubric. Students' ability to listen well, use empathetic responses, and display confidence and spontaneity were evaluated. Rubric ratings for the Class of 2020 (AI participants) were compared to the Class of 2018 (non-AI participants). Results: Sixty-seven AI participants and 75 non-AI 


\section{American Journal of Pharmaceutical Education 2019; 83 (5) Article 7654.}

participants were evaluated in the SPE. AI participants performed better at listening/responding and spontaneity/confidence, $\mathrm{p}=.024$ and $\mathrm{p}=.016$ respectively. Implications: AI participants demonstrated superior communication during the SPE compared to non-AI participants. The AI curriculum is a valuable strategy for strengthening students' communication skills.

Impact of a Strength Finders and Conflict Management Workshop on Growth Mindset, Communication, and Teamwork. Courtney L. Bradley, High Point University, Julie B. Cooper, High Point University, Elizabeth Jeter, High Point University. Objective: Health professions focus on interprofessional education (IPE) with a goal of health system improvement. Many early IPE interventions lack direct instruction on teamwork skills and measures of mindset shown to impact team functioning. The purpose of this study was to determine impact of a teamwork skills workshop on self-views of growth mindset, communication and teamwork. Methods: A multi-week workshop was developed for first-year pharmacy students. The workshop included completion of StrengthFinders2.0, a classroom session on identifying individual and team member strengths, a classroom session on situational communication tools and group conflict resolution models, and two simulated pharmacy situations requiring group conflict resolution in the laboratory setting. An IRB approved pre-post study measured change in growth mindset, teamwork, and communication between beginning and end of one semester using validated survey instruments. Change over semester in which the workshop was delivered to the intervention group (Fall 2018) was compared to a control group that did not receive the workshop (Fall 2017) applying the Wilcoxon Signed Rank Test. Results: Over the semester the control group growth mindset decreased, 3.25to3.10. The intervention group demonstrated an increase in growth mindset, 3.12to3.17, communication, 2.92to3.14, and teamwork, 3.22to3.30. Change over semester for control and intervention groups were statistically different for all three categories, $>0.01,>0.01$, and 0.04 respectively. Implications: The teamwork skills workshop improved self-views. An early workshop may provide necessary teamwork skills for healthcare improvement. Limitations include size and unprofessional design. Future work will focus on longitudinal measurement of self-views to determine the long-term impact of teamwork training interventions.

Impact of Curricular Change on Student Confidence and Potency Accuracy in a Required Compounding Course. Robert P. Shrewsbury, University of North Carolina at Chapel Hill, Sarah Mislan, University of North Carolina at Chapel Hill. Objective: Three in- dicators were evaluated to determine if students performed better and felt more confident about their performance in a required compounding course that was re-designed due to curricular transformation. Methods: Self-efficacy surveys were given to students in both the legacy program (LP; longer, fewer preparations/lab) and the transformed curriculum (TC; shorter, more preparations/lab) at the start and end of a semester to assess their confidence in compounding skills and determined if the self-analysis of their preparations improved their confidence. Students in both groups compounded the same three preparations and potency (percent of label) was determined. Results: Students in both groups reported increased confidence at the semester's end, with TC students showing more dramatic increases. TC students reported a significant decrease in their perception that self-analysis would affect their compounding ability $(9.38 \pm 1.12$ to $8.98 \pm 1.18)$ while LP students had a significant increase $(8.09 \pm 2.18$ to $8.68 \pm 1.82)$. The mean preparation potencies were statistically different. However, the mean of the TC hydrocortisone medication stick was outside the acceptable range of the course's criteria. Implications: Overall confidence in compounding skills was found to be $70 \%$, and the addition of the self-analysis of preparations increased confidence to $85 \%$. The potency results suggested that equivalent competency in the two groups was possible for preparations that involved simple solutions or filled fixed volume molds. The hydrocortisone medication stick data indicated that understanding the science of compounding may require more knowledge or time that provided in the transformed curriculum.

Impact of Disabling Item Review on Computerized Fixed-Item Tests. Michelle L. Caetano, The University of Rhode Island, Jayne Pawasauskas, The University of Rhode Island. Objective: The decision to disable item review, or 'backward navigation,' during computerizedfixed item tests proved controversial among faculty. Investigators sought to determine the effect of disabling backward navigation on performance of exam items. Methods: Exam items that were administered unchanged and without error or adjustment of scoring between 2016 and 2017 were eligible for inclusion in the analysis. Included items were evaluated for change in difficulty index, point biserial, and discrimination index from one year when backward navigation was enabled to the one year after the function was disabled. The study was deemed exempt by the Institutional Review Board. Results: Investigators screened 2033 exam items and identified 576 items that were administered which met inclusion criteria. There were no statistically significant differences in the point biserial or discrimination index. There was a small, but statistically significant decrease in 


\section{American Journal of Pharmaceutical Education 2019; 83 (5) Article 7654.}

item difficulty index (mean change $-0.74 \%$, p value 0.047 , 95\% CI $0.01 \%-1.47 \%$ ) indicating that fewer students selected the correct answer when item review was disabled. This change in the item difficulty index appeared to be driven primarily by a statistically significant decrease for the highest-scoring students (mean change $-0.95 \%, p$ value $0.008,95 \%$ CI $0.25 \%-1.65 \%$ ); and a smaller, non-significant decrease for the lowest-scoring students. Implications: Although there was a statistically significant decrease in item performance, given the small magnitude of the effect, it is believed that disabling backward navigation is unlikely to significantly impact student performance on computerized-fixed item tests.

Impact of MiniMedTM 670G Hybrid ClosedLoop (HCL) System on Glycemic Control; A Retrospective Single Center Study. Maryam T. Fazel, The University of Arizona, Saleh Alqifari, The University of Arizona, Ahmad Alamer, The University of Arizona, Merri Lou Pendergrass, The University of Arizona. Objective: MiniMedTM 670G hybrid closed-loop (HCL) insulin pump system with SmartGaurdTM technology offers Auto Mode basal insulin delivery based on patient's sensor glucose readings. The primary outcome is to determine the correlation between Time in Auto Mode and Sensor Glucose Time in Range (SG-TIR) of 70-180 g/ dL. Secondary outcomes include correlation of SG-TIR to $\mathrm{HbA1c}$, correlation of Time in Auto-Mode to HbA1c, and change in average $\mathrm{HbA} 1 \mathrm{c}$ from baseline. Methods: A retrospective cohort study via chart review was conducted. Adult patients with type 1 diabetes mellitus at an academic medical center endocrinology clinic who were switched to the MiniMedTM 670G HCL System either from multi-dose insulin injections or another insulin pump with or without a continuous glucose monitor were included. Pearson's Correlation Coefficient (r) was used to assess the correlation between variables in question within 9 months of switching. The a priori $\alpha$ level was 0.05. Results: Twelve patients with average baseline HbAlc of $8.2 \%(+/-1.6)$ included. Time in Auto Mode correlated positively with SG-TIR $(\mathrm{r}=0.8 ; \mathrm{P}=0.002)$. There was no correlation between SG-TIR and HbA1c $(\mathrm{r}=-0.18 ; \mathrm{p}=0.576)$. There was an inverse correlation between Time in Auto-Mode and HbAlc $(r=-0.65$; $\mathrm{p}=0.023)$. There was a significant reduction in $\mathrm{HbA1c}$ from baseline (-0.61, CI 0.25-0.97) $(\mathrm{p}=0.003)$. Implications: MiniMedTM 670G HCL system Auto Mode basal delivery appears to be effective in reducing $\mathrm{HbAlc}$ in patients with type 1 diabetes mellitus.

Impact of Pharmacists' Patient Care Process (PPCP) Activity on Student Perception of APPE Readiness. Ayse Elif Ozdener, Fairleigh Dickinson University. Objective: To assess student perceptions of
APPE readiness after the completion of a PPCP activity in a didactic course Methods: A five-point Likert scale questionnaire assessing perception of APPE readiness was disseminated using SurveyMonkey to 76 students after the completion of a 2-week PPCP activity in a P3 didactic course. As part of the PPCP activity, students used a simulated electronic medical record (EMR), talked to a simulated patient to collect information and implemented their recommendation to a simulated provider. Participants' responses were anonymous. Results: Thirty-three students completed the post-PPCP activity questionnaire $(43.4 \%)$. Sixty-three percent of students strongly agreed or agreed the PPCP activity prepared them for APPE rotations. Sixty-seven percent strongly agreed or agreed that they feel more confident in identifying the patient's most acute problem, conducting a patient interview, and assessing the patient's subjective and objective information. Seventy-three percent strongly agreed or agreed they are more confident in collecting pertinent patient information from an EMR. Fifty-five percent strongly agreed or agreed that they feel more confident writing a SBAR note and fifty-eight percent strongly agreed or agreed they are more comfortable communicating their recommendation to another healthcare provider. Sixty-one percent strongly agreed or agreed they are more confident in monitoring drug therapy and following-up with a patient. Implications: This 2-week PPCP activity had positive outcomes on student perception of APPE readiness. Based on these results, more of these activities will be added to didactic curriculum to boost student pharmacists' confidence with the patient care process.

Impact of Student-Driven Educational Model for Reinforcing Clinical Knowledge of Common Medications. Kim Vo, Marshall B. Ketchum University, Tiffany Tsai, Oregon State University, Jessina C. McGregor, Oregon State University, Tanya L. Ostrogorsky, Oregon State University, Harleen Singh, Oregon State University. Objective: The objective of this study was to measure changes in student knowledge and confidence after completion of a student-driven peer teaching course on commonly prescribed medications designed to prepare students for pre-APPE readiness assessments. Methods: All students $(n=98)$ enrolled in P3 Pharmacy Practice "RxReady" course were assigned a specific medication at the beginning of the term. Twenty-four students were randomly selected to teach their peers, with faculty oversight, about the assigned medication. Assessment methods included weekly quizzes, pre- and post-test, and anonymous surveys about the teaching modality. Pre- and post-tests items were analyzed using a paired-t test. The study was deemed exempt by the Oregon State 


\section{American Journal of Pharmaceutical Education 2019; 83 (5) Article 7654.}

University Institutional Review Board. Results: Ninetytwo $(95.8 \%)$ students showed improvement between the pre- and post-test, with a mean percentage increase of $15 \%(\mathrm{SD}=10.7 \% ; \mathrm{p}<0.001)$. There was no difference in mean percent change in pre- and post-quiz scores for students who presented in class compared to those who $\operatorname{did} \operatorname{not}(16.9 \%[\mathrm{SD}=10.2 \%]$ versus $14.5 \%[\mathrm{SD}=10.9 \%]$, $\mathrm{p}=0.333)$. The difference in the mean change between the pre- and post-quiz was higher in students who passed the pre-APPE exam on their first attempt compared to those who had to retake it $(16.2 \%$ [SD $=9.8 \%]$ vs $9.1 \%$ $[\mathrm{SD}=9.1 \%], \mathrm{p}=0.017)$. Implications: This pedagogical approach targets gaps in knowledge in pharmacotherapy based on previous year cohorts' performance on preAPPE readiness assessments. The results indicate that the RxReady course was an innovative active learning pedagogy, to enhance pre-APPE preparedness.

Impact of Student Pharmacist Teaching Within a Diabetes Self-Management Education Class. Kendra R. Manigault, Maria M. Thurston, Mercer University, Adam Gates, Jill M. Augustine, Mercer University. Objective: To assess: 1) student knowledge of diabetes before and after teaching a diabetes self-management education (DSME) class and 2) student self-perceptions of aptitude and confidence before and after teaching the class. Methods: Students assigned to the study investigators' ambulatory care advanced pharmacy practice experience (APPE) between June 2016 to April 2018 were included in this IRB approved study. Students randomly assigned to the intervention group taught a DSME class while students in the control group did not teach a DSME class. All students completed a 10-item diabetes knowledge pre/post evaluation to assess student knowledge of diabetes at baseline and during week 3 or 4 of the APPE. Students completed a pre/post 14-item Likert scale evaluation to assess aptitude and confidence. Results were analyzed using t-test to compare the differences in knowledge scores and to compare difference in aptitude and confidence questions. Results: Forty-five students were included: 26 students in the intervention and 19 in the control. Intervention students had a significant improvement in their knowledge scores $(81.7 \%$ post-intervention versus $68.4 \%$ pre-intervention, $\mathrm{p}<0.001)$. Control students did not have a significant change in knowledge scores from pre- to post-evaluation $(68.0 \%$ versus $74.1 \%, \mathrm{p}=0.48$ ).Additionally, intervention students had significantly higher gain in confidence compared to the control group (intervention $=11.9$ improvement versus 6.7 improvement in control, $\mathrm{p}=0.003$ ). Implications: Student pharmacist participation in a DSME class led to significant improvement in diabetes knowledge, aptitude, and confidence. This supports the concept that students learn the material better if they are required to teach others the same material.

Impact of Virtual Patient Cases and Student Performance Within a Disease and Therapeutics Course. Ryan G. D'Angelo, University of the Sciences, Justin J. Delic, University of the Sciences, Jennifer N. Smith, University of the Sciences. Objective: Use of virtual patient cases (VPCs) has increased within healthcare education. This novel technology can utilize branching decision points to simulate clinical decision-making for a virtual patient. VPCs encourage critical thinking and provide real-time formative feedback. This study aimed to evaluate the impact of VPCs on student exam performance within a single course. Methods: Four VPCs (two required and two optional) were developed for content on exams during the 2017-2018 academic year. Student exam question performance for topics with an associated VPC was compared between students who accessed VPCs more than once vs. only once for required VPCs and between students who accessed VPC one or more times vs. not at all for optional VPCs. Results: For topics with required VPCs, there was no significant difference in mean exam performance for students accessing the VPC more than once compared to students accessing the VPC once $(75 \%$ vs $73 \%, p=N S ; 71 \%$ vs $66 \%, p=N S)$. For topics with optional VPCs, there was a significant difference in mean exam performance for students accessing the VPC one or more times compared to students not accessing the VPC $(76 \%$ vs $69 \%, p=<0.01 ; 83 \%$ vs $68 \%, p=<0.01)$. Implications: Although the use of VPCs did not universally enhance performance on exams, this study shows that VPCs can be a useful tool for additional self-study. Different content, varied levels of exam question difficulty, and differences in student motivation are possible confounding variables. Data from this study will assist determining how VPCs are used effectively in the curriculum.

Implementation and Impact of an Academia Learning Experience for PGY2 Residents: A ThreeYear Experience. Jane Bowen, University of the Sciences, Angela Bingham, University of the Sciences, Sanchita Sen, Mercer University. Objective: To describe the implementation and impact of an academia learning experience for postgraduate-year-2 (PGY2) pharmacy residencies in Critical Care and Pharmacotherapy. Methods: Faculty at the Philadelphia College of Pharmacy created a required academia learning experience for PGY2 residents to gain experience in the 3 faculty roles of teaching, service, and scholarship. A pre and post 5-point Likert-type scale survey was administered to residents to determine the impact of the learning experience on their confidence in faculty roles in academia. Survey 


\section{American Journal of Pharmaceutical Education 2019; 83 (5) Article 7654.}

questions captured previous postgraduate teaching, service, and scholarship experiences and 22 questions assessed confidence in these 3 areas pre and post learning experience. Results: This study included 8 residents over 3 years with most having prior small group facilitation and experiential co-precepting experiences, but minimal lecture and primary precepting experiences. Five $(62.5 \%)$ residents completed a prior teaching certificate program and $1(12.5 \%)$ completed an academia rotation. Residents lacked prior academic committee experience and 3 (37.5\%) had peer-reviewed publications. The experience resulted in a mean percent increase in responses of "agree" and "strongly agree" that the residents were confident in academia-related topics, scholarship, and teaching of $93.75 \%, 68.75 \%$, and $59.38 \%$, respectively. No residents felt confidently prepared for a career in academia before the experience, but after $87.5 \%$ "agree" and "strongly agree" that they feel confident. Implications: Implementing a required academia learning experience for PGY2 residents can be time-intensive, but significantly improves their confidence in teaching, service, and scholarship, and overall preparation for academia.

Implementation of a Naloxone Distribution Program at a Large, Public, University Health Center Pharmacy. Kelsey K. Schmuhl, The Ohio State University, Jason Goodman, The Ohio State University, Phillip Anderson, The Ohio State University, D'Arcy Oaks, The Ohio State University, Jennifer L. Rodis, The Ohio State University. Objective: Naloxone is an important harm reduction strategy available to combat the opioid epidemic, however, stigma and lack of access often limit its use. This study collected perceptions of students, faculty and staff about naloxone and overdose prevention education and informed strategies to improve access to naloxone on The Ohio State University's (OSU) campus. Methods: A 10-question, multiple-choice, IRB approved survey was distributed via email through the OSU daily bulletin and on paper at OSU's Student Health Center Pharmacy between July 15, 2016 and September 30, 2016. Data from written and electronic surveys were analyzed in aggregate using descriptive statistics. Results: 736 total surveys were collected.66\% of respondents reported being affected by addiction ( $61.7 \%$ of $18-25$-yearold participants versus $68.6 \% 25$ years and older). $76.6 \%$ of respondents had heard of naloxone $(59.4 \%$ of $18-25$ year-old participant and $87.4 \% 25$ years and older; $\mathrm{p}<0.001$ ).37.5\% of respondents knew naloxone was available without a prescription from a pharmacy $(24 \%$ of 18-25 year old participants versus $44.6 \%$ of respondents 25 years and older; $p<0.001) .16 .9 \%$ of respondents reported interest in getting a naloxone kit if they were available at the pharmacy while $37 \%$ answered 'maybe' to the same question. $52.5 \%$ of respondents stated they would be interested in education about preventing and responding to overdoses while an additional $26.4 \%$ answered 'maybe' to the same question. Implications: Study results informed the development of a naloxone and overdose prevention education program at OSU's Student Health Center.

Implementation of a Pre-Work Requirement in a Pharmacotherapy Series. Stephanie M. Schauner, University of Missouri-Kansas City, Eric A. Wombwell, University of Missouri-Kansas City, Karen L. HardingerBraun, University of Missouri-Kansas City, Kendall D. Guthrie, University of Missouri-Kansas City, Maqual R. Graham, University of Missouri-Kansas City. Objective: To assess student and faculty opinions and perceptions of classroom engagement after initiating structured prelecture assignments and quizzes within the Pharmacotherapy series. Methods: Pre-lecture work was added to the Pharmacotherapy series in fall 2018 to encourage student review of previously taught coursework, enhance engagement in the classroom, and allow instructors to focus on clinical application of concepts. A pre- and post-course survey was developed and administered to students. In addition, a survey was created and administered to faculty at the end of the fall semester. Results: All students $(\mathrm{N}=135)$ completed the surveys and $81 \%$ of faculty $(\mathrm{N}=13)$ responded. The majority of student respondents $(\mathrm{N}=97,70 \%)$ reported completing pre-lecture assignments throughout the semester. Ninety (67\%) students reported a positive degree of perceived enhancement of their in-class learning by completing the pre-lecture assignments, however $60 \%$ of students reported that pre-work did not encourage them to engage in the classroom. Most of the students felt that the prework was a useful learning aid (45\% agreed or strongly agreed), $36 \%$ were neutral, and 19\% disagreed or strongly disagreed. Faculty survey data suggests that instructors value the addition of pre-work with the majority $(53.8 \%)$ reporting the pre-work allowed them to spend less time re-teaching previous coursework content. Implications: Pre-lecture assignments and quizzes added to a Pharmacotherapy course series provided structure to lecture readings and positively enhanced in class learning, however it did not encourage classroom engagement.

Implementation of a Review Process to Assess Content in a Pharmacotherapy Curriculum. Kendall D. Guthrie, University of Missouri-Kansas City, Eric A. Wombwell, University of Missouri-Kansas City, Stephanie M. Schauner, University of Missouri-Kansas City, Maqual R. Graham, University of Missouri-Kansas City, Karen L. Hardinger-Braun, University of Missouri- 


\section{American Journal of Pharmaceutical Education 2019; 83 (5) Article 7654.}

Kansas City. Objective: To evaluate content in a pharmacotherapy course series using the American College of Clinical Pharmacy (ACCP) Pharmacotherapy Didactic Curriculum Toolkit. Methods: Coordinators utilized the ACCP toolkit to map topics across an 18 credit hour course series. Instructors were surveyed to provide additional insights on the lecture topics and time allotment. The percentage of covered tier one and two topics as defined by the toolkit were calculated. Simultaneously, the course structure was assessed using student evaluations and the institutional course review process. Results: Data was generated from the 2017-2018 academic year. The courses covered $79 \%$ of tier one and $49 \%$ of tier two topics. For the 2018-2019 year, the series was increased by one credit hour. Two courses in the series were restructured from two three-hour lectures weekly to three twohour lectures. Realignment of time occurred allowing an average of two hours per tier one topic and one hour per tier two topic. Topic addition and reorganization resulted in $100 \%$ coverage of tier one topics and $68 \%$ of tier two topics. Implications: The ACCP Toolkit was an effective tool to review content in a pharmacotherapy curriculum. By incorporating this review, missing topics were identified and topics requiring practice competency upon graduation were enhanced. Despite falling under the national average for pharmacotherapy credit hours, the course series was still able to incorporate all tier one and the majority of tier two topics.

Implementation of a Suicide Prevent Training Program. Megan Willson, Washington State University, Christina R. Buchman, Washington State University, Jennifer D. Robinson, Washington State University, Julie Akers, Washington State University, Kimberly C. McKeirnan, Washington State University. Objective: To describe the impact of an innovative suicide training activity measured through a pre- and post-training survey. Methods: Our team revised a state-mandated pharmacist suicide prevention training program to fit the format of a laboratory course. A WSPA trainer taught the course to student pharmacists during a 2-hour laboratory session. Pre- and post-training survey questions were administered to gather student pharmacist opinions regarding the importance of this training and comfort engaging in a discussion with a patient about suicide. Results: Onehundred fifty-eight student pharmacists completed the training and survey. Forty-six percent of students (74/ 158) reported having previous personal experience with suicide. Thirty-six percent of students (57/158) reported they know someone who may need help and intend to reach out to them after completing this training. Eighteen percent of students (30/158) said "yes" to the statement, "From what I just learned, I have decided to seek help for myself." Implications: Suicide claims the life of one person every 12 minutes and is the second leading cause of death for individuals aged 15-34. A recent survey of over 67,000 undergraduates from 108 universities indicated that $24 \%$ of college students reported suicidal ideation and $9 \%$ attempted suicide. The research team was astounded at the number of students participating in the training who intended to seek help for themselves or someone else after completing this training program. Utilization of this student pharmacist suicide prevention training model could be implementable in a variety of courses and settings and added to the curriculum at any university in the country.

Implementing an Innovative Assessment Approach in a Pharmacotherapy Course. Eric A. Wombwell, University of Missouri-Kansas City, Stephanie M. Schauner, University of Missouri-Kansas City, Maqual R. Graham, University of Missouri-Kansas City, Karen L. Hardinger-Braun, University of Missouri-Kansas City, Kendall D. Guthrie, University of Missouri-Kansas City. Objective: To implement and evaluate an innovative electronic examination in a pharmacotherapy course intermixing case-based assessment, multiple-choice, and short-answer questions. Methods: Exams within our pharmacotherapy course historically comprised 4to-5 patient cases. Students provided handwritten assessments and plans for 2-to-4 drug-related problems per case. This assessment method resulted in 1) significant time to complete exams, limiting assessable content and 2) instructor dissatisfaction due to substantial time burden for grading. To increase the number of diseases assessed per exam, and consequently strengthen course rigor, multiple-choice and short-answer questions were added to exams. Case-based questions were retained to align assessment with the pharmacist patient care process (PPCP). To improve instructor satisfaction and limit time answering cases, students typed assessment and plan responses for only the highest-priority drug-related problem identified in each case. Exams were administered and graded through an electronic assessment platform (Examsoft $\left.^{\circledR}\right)$. Five exams were administered during the course, each incorporating approximately 30 multiple-choice and/or short-answer questions and 4-to-5 case-based questions. A post-semester survey of student and instructor perceptions was administered. Results: The number of diseases assessed in the course doubled from 24 to 53. Course rigor intensified with $74 \%$ fewer A's achieved. Nearly all students (94\%) reported the course prepared them to carry out the PPCP. Instructors responded approvingly, $100 \%$ desired continuation of the new assessment model. Instructors preferred the new assessment model $(92 \%)$ and reported it better assessed student 


\section{American Journal of Pharmaceutical Education 2019; 83 (5) Article 7654.}

comprehension of material (85\%) versus the previous method. Implications: This innovative examination method increased assessable content, increased course rigor, and improved instructor satisfaction.

Implementing Portfolios with Formative Assessment of Professionalism and EPA Proficiency for an Entire 250+ Cohort. Catherine Taglieri, MCPHS University-Boston, Jennifer Prisco, MCPHS UniversityBoston, Anela Stanic, MCPHS University-Boston, Matthew R. Machado, MCPHS University-Boston, Gerard G. D'Souza, MCPHS University-Boston. Objective: Implement a process for faculty to individually verify student self-assessment of professionalism and Entrustable Professional Activity (EPA) proficiency. Methods: Associated with portfolio requirements, a Qualtrics ${ }^{\circledR}$ survey with 19 professionalism questions and $15 \mathrm{EPA}$ questions was distributed to 269, 286 and 301 PY1, PY2 and PY3 students, respectively. Utilizing the School's One-to-One Student Faculty Engagement (OSFE) Program, faculty met with individual students to discuss their self-evaluation survey responses. Results: Average self-rated professionalism scores on a 6 -point scale $(0=$ not yet aware to $5=$ always) were 4.34(PY1), 4.39(PY2) and 4.5(PY3). Average EPA proficiency scores on a 6-point scale $(0=$ not yet aware to $5=$ does and teaches others $)$ were 0.96(PY1), 1.65(PY2) and 2.38(PY3). Year-to-year changes were statistically significant (ANOVA $\mathrm{p}<0.01$ ) only for EPA proficiency scores. In professionalism, the lowest rated item for all three student cohorts was "I exceed expectations of others." In EPAs, "Educate patients and professional colleagues regarding the appropriate use of medications" and "Identify patients at risk for prevalent diseases in a population" were the lowest rated skills in PY3. Implications: Using the portfolio, year one implementation established the feasibility of collecting actionable data across the 3 cohorts. OSFE meetings are required annually, at a minimum, and will allow for consistent formative feedback on student self-assessments as they progress through the curriculum. The EPA data echo other measures of curricular performance and have implications for curricular revision. Combined with existing mechanisms in the experiential curriculum, this approach is the cornerstone of the School's plan for compliance with ACPE 2016 Standards 4 and 24.

Incorporating a Virtual Patient Case into a Required Course in a Doctor of Pharmacy Program. Brooke D. Fidler, Long Island University, Tamara Goldberg, Long Island University, Roda Plakogiannis, Long Island University, Elaena J. Quattrocchi, Long Island University, Chosang Tendhar, Long Island University. Objective: Investigate the impact of virtual simulation on students' exam scores pertaining to diabetes.
Methods: The data (10 diabetes questions on each test) for this study was collected from second professional year students in $2017(\mathrm{n}=202)$ and $2018(\mathrm{n}=190)$. Students in 2017 utilized a traditional paper case during a hypertension/diabetes pharmacotherapy workshop. Students in 2018 used an interactive virtual patient simulation (IVPS) case during the same hypertension/diabetes pharmacotherapy workshop. IVPS involved students verbally asking questions to the virtual patient to obtain a medical history to determine the best treatment for this patient. We used SPSS to compute descriptive statistics and perform Independent Sample t-test. A six-item survey (fivepoint Likert type scale) was also administered after the completion of a virtual patient workshop to assess students' perceptions of utilizing this teaching technique. Results: The mean scores were 6.74 and 7.27 for 2017 and 2018, respectively. Students exposed to the IVPS performed better. The mean difference of 0.53 was statistically significant, $\mathrm{t}(390)=-2.88, \mathrm{p}<0.05$. The student survey results demonstrated that the mean scores of each item was greater than 3 (out of 5). Our results suggest that students endorsed the usefulness of virtual learning. Implications: The findings of this study support the effectiveness of a virtual patient in enhancing students' diabetes knowledge. Furthermore, it has implications for pedagogy. Specifically, the use of virtual learning into other disease state discussions in pharmacotherapeutic courses could be equally beneficial.

Incorporating Community Engaged Learning in Pharmacy Elective Courses to Enhance Student Learning About Minoritized Populations. Anisa Fornoff, Drake University, Sally L. Haack, Drake University. Objective: Two new pharmacy electives, centered on minoritized populations, incorporated community engaged learning strategies. Faculty engaged with local community partners to intentionally integrate service-learning to meet learning objectives. Courses were designed to address issues identified in a campus climate survey at this institution. Methods: The first course embedded a food insecurities service-learning experience. Students served local community partners while also working virtually alongside student teams from an institution in Guadalajara, Mexico, impacting food insecurities in both local and global contexts. A LGBTQ health course threaded a community partner throughout the term to engage students in policy and advocacy. Also, students created continuing education coursework with a focus on a LGBTQ health issues geared toward public school teachers. Students used critical reflection activities to demonstrate achievement of course learning objectives. Community partner and student feedback was gathered. Results: The faculty and community partners 


\section{American Journal of Pharmaceutical Education 2019; 83 (5) Article 7654.}

successfully created and delivered a mutually beneficial partnership that positively impacted student learning. Course evaluations, partner feedback, and assessment of critical reflection activities indicated that students examined personal cultural assumptions and unconscious bias. Students were able to practice interpersonal communication skills to demonstrate respect for other perspectives and cultures. Implications: Community engaged learning projects can impact student learning in relation to issues experienced by marginalized populations. Through engagement in the community in areas of social justice, students can gain experience outside of the classroom rather than only using didactic and case-based methods of teaching. Lessons learned and future opportunities for assessment will be explored.

Incorporating Leadership Development Planning into Pharmacy Education: An Evaluation of Best Practices. Mary M. Bridgeman, Rutgers, The State University of New Jersey, Jenelle Sobotka, University of Cincinnati. Objective: To identify approaches for personal professional leadership development planning to derive best practices for incorporating leadership development planning in pharmacy curricula. Methods: A literature review was performed to inventory existing leadership development plan templates, processes, and strategies for student pharmacists. A query of Medline databases through June 2018 was conducted utilizing the terms: "Education, Pharmacy" [MeSH] or "Students, Pharmacy" [MeSH]; "pharmacy education" or "pharmacy student" or "pharmacy curriculum" or "pharmacy curricula"; "leadership" or "leadership development". Available English language data from reviews, abstracts, evaluations, and references from literature pertaining to leadership development in pharmacy curricula were extracted. Results: A total of 373 references were identified; upon further evaluation, 58 of these were identified to more completely reflect the scope of leadership development planning in pharmacy education. This review identified a need for a leadership development plan to include aspects of multimodal leadership development for student pharmacists, encompassing formal curricular, co-curricular, and extracurricular activities. Leadership development planning should include competency areas, including self and other focus, knowledge, behavior, cognitive domain, and affective domain. Curricular mapping of leadership opportunities and training can address gaps in the curriculum or in a learner's development. Implications: Use of leadership development planning, at present, is limited and unstructured according to a literature review. Student leadership development planning should be intentional, with a thought to desired competency areas for development and assessment of the desired skills. Fi- nally, leadership development planning must include an opportunity for self-reflection in order to provide deeper assessment and insight into areas of leadership development needed.

Incorporation of Branched-Logic Survey Software for Patient Case Assessments in Pharmacotherapy Courses. Paul Reynolds, University of Colorado, Scott W. Mueller, University of Colorado, Ashley E. Glode, University of Colorado, Meghan N. Jeffres, University of Colorado, Erica J. Rhein, University of Colorado. Objective: Educational pedagogy that addresses higher orders of clinical reasoning in a pharmacy curriculum can be time-intensive to develop, implement, and assess. Digital branched-logic surveys create interactive, simulated patient cases that allow application of therapeutic knowledge in a low risk setting. Methods: Patient cases were created for three oncologic and two infectious disease scenarios utilizing the Qualtrics ${ }^{\circledR}$ survey platform for two curricular programs, distance-based and third year on-campus pharmacy students. Branched-logic provided feedback based on student answers to various discrete questions, which allowed students to explore therapeutic decision-making while witnessing simulated outcomes in real-time. A log of student decisions also allowed instructors to provide class-wide feedback. Student perceptions of the survey were collected via a 5-point Likert scale $(\mathrm{SA}=$ Strongly Agree, $\mathrm{A}=$ Agree, $\mathrm{N}=$ Neutral, $\mathrm{D}=$ Disagree, $\mathrm{SD}=$ Strongly Disagree). The chi-squared test was used to compare perceptions by program and scenario. Results: A total of 496 surveys were completed for the 5 clinical scenarios. Overall, students preferred the surveys to clinical note writing $(\mathrm{SA} / \mathrm{A}=82 \%, \mathrm{SD} /$ $\mathrm{D}=7 \%$ ). Students found the surveys valuable to learning $(\mathrm{SA} / \mathrm{A}=99 \%, \mathrm{SD} / \mathrm{D}=1 \%)$ and that feedback from the surveys was useful to their learning process (SA/ $A=99 \%, S D / D=0 \%)$. No statistically significant differences were observed between pharmacy programs or case type. Implications: Branched-logic surveys were preferred and enjoyed by students. It provides a log of decision-making trends that can allow for targeted feedback by instructors. Initial survey construction is time-intensive, but efficient provision of feedback and modification of existing cases improves overall instructor efficiency. Further research is needed regarding branched-logic surveys and academic performance.

Influence of Program Type, Curricula Delivery, and Demographics on Test Anxiety: A Multi-Site Study. Kimberly K. Daugherty, Sullivan University, Stephen Neely, The University of Oklahoma, Daniel R. Malcom, Sullivan University, Alicia S. Bouldin, The University of Mississippi, Kristopher Harrell, The University of Mississippi, Melissa S. Medina, The University of 


\section{American Journal of Pharmaceutical Education 2019; 83 (5) Article 7654.}

Oklahoma, Kristen Pate, The University of Mississippi, Michelle O. Zagar, The University of Louisiana at Monroe, Adam Pate, The University of Mississippi. Objective: There are limited data available regarding test anxiety in health professions students. The purpose of this study was to determine the prevalence of test anxiety in pharmacy students and to explore possible related factors. Methods: Students from four pharmacy schools in their final year of the curriculum were asked to complete the Cognitive Test Anxiety Scale-2 (CTAS). Comparisons were made to determine differences in gender, race, curricula delivery, and program length. Results: A total of $176 / 361$ students across the four schools completed the survey $($ mean $=48.8 \%$; range 41.1-61.3\%). Respondents were mostly female $(59.0 \%)$ and Caucasian (71.6\%). Most (55.0\%) students were classified as moderate-high anxiety with a median anxiety for the NAPLEX of 75 (scale $0-100$ ). Only $4 \%$ of students reported receiving accommodations for text anxiety, though most (71.0\%) reported seeking help when needed. No difference on CTAS-anxiety level was found between genders but trended towards more males having low anxiety $(54.2 \%$ vs. $38.5 \%$ ). Female students reported a statistically higher level of NAPLEX anxiety ( $80.5 \%$ vs $70 \%)$. Race was not statically different for any variable. CTAS-anxiety level groups differed by type of program: the three-year program had more students in high-anxiety; hybrid/PBL programs had more in moderate-anxiety; and the integrated modular-based program had more in low-anxiety. NAPLEX anxiety did not differ between programs. Implications: There was a high prevalence of test anxiety among students, with many reporting anxieties about taking the NAPLEX. Three-year and hybrid/PBL programs were associated with more anxiety than modular-based programs, but further research is needed.

Instructors' Reported Successes and Barriers with a Teaching Electronic Medical Record for Pharmacy Student Learning. Olga O. Vlashyn, Purdue University, Omolola A. Adeoye, Purdue University, Kimberly S. Illingworth Plake, Purdue University, Jamie L. Woodyard, Purdue University, Zachary A. Weber, Purdue University, Debra K. Litzelman, Regenstrief Institute, Alissa L. Russ, Purdue University. Objective: Some pharmacy programs are implementing teaching Electronic Medical Records (tEMRs), but best practices for incorporating tEMRs into pharmacy practice are unknown. Our objective was to examine instructors' early experience with a tEMR in order to improve future use in pharmacy education. Methods: This mixed methods study was guided by the technology acceptance model (TAM). Instructors from one college of pharmacy were invited to participate in 30 to 60 -minute, semi-structured interview and a web-based survey. Interview transcripts were analyzed with a hybrid, deductive and inductive approach by two independent analysts. Analysts discussed discrepancies until reaching consensus. The 43-item survey collected baseline data on instructors' experiences with the tEMR, and items were measured on a 7-point Likert scale. Quantitative analyses included descriptive statistics. Results: Interviews were conducted with seven pharmacy instructors. Qualitative data analysis revealed several major themes, including benefits and barriers to tEMR use (6 and 8 subthemes, respectively). The survey was completed by ten pharmacy instructors. Most instructors agreed or strongly agreed that the tEMR was useful for teaching (44.4\%). However, most instructors strongly disagreed that they were satisfied with identifying patient cases $(55.6 \%)$, and they found it difficult to get the tEMR to do what they wanted $(40.0 \%)$. Overall, instructors had a generally favorable attitude towards using the tEMR for teaching (50.0\%). Implications: This research provides valuable insights on both benefits and barriers for pharmacy instructors as they utilize a tEMR for student learning. Our research findings may aid other pharmacy programs and promote more effective use of tEMRs in pharmacy education.

Integration of Modified 'Career Pathway Evaluation Program' in a Pharmacy Curriculum. Ann Parks, Concordia University Wisconsin, Emily Bryant, Concordia University Wisconsin, Michael C. Brown, Concordia University Wisconsin. Objective: To describe the 'Career Pathway Evaluation Program' (CPEP) usage in the Introductory Pharmacy Practice Experiences (IPPEs) and to summarize 5 years of career and critical factor assessment tool results for Pharm.D students. Methods: CUWSOP requires IPPE curriculum as a part of a four-course series during the first and second year of the Pharm.D program. IPPE-3 focuses on career exploration, including a modified version of the APhA CPEP. Our course involves components of the original pathways program (some of the exercises and the assessment tool) and formal participation in a Career Pathways Poster Fair. All P2 students were required to participate and to submit their assessment tool results. Data were collected anonymously via a survey to the course management website. Results: There were $326(95.6 \%)$ respondents to the survey. Respondents' most common career matches included ambulatory care $(71.85 \%)$, health system pharmacy outpatient $(65.51 \%)$, office-based medication management $(45.69 \%)$, and independent community pharmacy (43.63). Ambulatory Care and Health System Pharmacy Outpatient were in the top three matches for all five cohort years. Respondents' most common critical factors were 'helping people' $(61.02 \%)$, 'interaction with 


\section{American Journal of Pharmaceutical Education 2019; 83 (5) Article 7654.}

patients' (49.38\%), and 'job security' (41.93\%). Implications: Our course has been successful in helping students identify potential career matches. These assessment tool results describe a predominant interest in ambulatory care roles, which aligns with their interest in helping people and interacting with patients. Future research may involve investigating a possible association between the CPEP results and student selection of APPEs and electives, further training, and/or careers.

International Advancement of Pharmacy Education Through a Global Self-Assessment Tool. Miranda G. Law, Howard University, Hyunuk Seung, University of Maryland, Abby A. Kahaleh, Roosevelt University, Jessica Lyons, Howard University, Isatu Bah, Howard University, See-Won Seo, Albany College of Pharmacy and Health Sciences, Miranda Sertic, University of Zagreb, Toyin S. Tofade, Howard University. Objective: In 2017, the International Pharmaceutical Federation (FIP) published the Nanjing Statements on pharmacy and pharmaceutical sciences education, detailing 67 adopted statements by 37 countries. The research objective is to develop a global quality assurance selfassessment tool (SAT) based on the FIP Nanjing Statements. Methods: All 67 statements were evaluated and assigned one of the following nine categories: 1. Didactic student competencies, 2. Experiential student competencies, 3. Workforce development, 4. Academic development, 5. Quality assurance, 6. Pharmacy advocacy, 7. Organization operation, 8. Continuing professional development, and 9. Inter-professional education. Statements containing constructs in more than one category received a secondary category. Afterwards, Likert-scale and multiple-choice questions were developed. Statements that contained multiple individual concepts were divided into multiple questions. The final survey was reviewed by five U.S. pharmacists, a statistician, and three international pharmacists for face validity. The SAT was tested by four schools of pharmacy, two in the U.S. and two international pharmacy programs. Based on the reviewer's suggestions from the pilot, revisions were made to a few questions and concepts in the self-assessment instrument. Results: The survey takes approximately 25 minutes to complete online via Qualtrics. The SAT includes a total of 35 items, 21 questions reflecting the intent of FIP's Nanjing Statements, and 14 on demographics. The SAT can be completed by faculty or administrators. Implications: The SAT allows schools and colleges of pharmacy worldwide to evaluate their institutions against globally recognized educational standards. It allows for quality assurance assessments for institutions unaccredited by national pharmacy organizations.
International Survey of Interprofessional Education in Pharmacy Programs Outside the United States. Ayse Elif Ozdener, Fairleigh Dickinson University, Malgorzata Slugocki, Fairleigh Dickinson University, Julie Kalabalik, Fairleigh Dickinson University. Objective: To assess attitudes, standards, implementations, and barriers associated with interprofessional education (IPE) in non-US pharmacy programs Methods: One hundred seven non-US pharmacy programs were invited to complete a 14-question survey. Programs were identified using the International Pharmaceutical Federation and American Association of Colleges of Pharmacy websites. The survey was disseminated using QualtricsXM. Results were collected anonymously. Results: Twenty-four pharmacy programs responded to the survey (22.4\%). Most of the respondents (95.7\%) agreed or strongly agreed that IPE is important to teach and interprofessionalism is relevant to pharmacy practice. Eighty-seven percent stated that pharmacy students in their programs expressed interest in IPE. Approximately one-third of respondents stated that an organization that creates IPE standards for pharmacy programs exit in their country. Over half of respondents $(56.5 \%)$ incorporate IPE into their curriculum; $39.1 \%$ plan to integrate it in the future. Two common barriers to IPE implementation included lack of interest from other disciplines and lack of dedicated time and space in the curriculum to build an IPE program. Implications: Although the study is limited by low response rates, the findings reveal the majority of respondents offer IPE in their curriculum or plan to incorporate it in the future. Only one-third have IPE educational standards set within their own country. This study highlights the need for the development of tools designed to support collaboration between disciplines for providing IPE in non-US pharmacy programs.

Interprofessional Collaboration Between Student Pharmacists and Student Psychologists. Linda D. Logan, The University of Georgia, Andrew Darley, The University of Georgia. Objective: To describe and evaluate an interprofessional experience for student pharmacists and student psychologists serving on behavioral health teams. Methods: P2 and P3 student pharmacists provide pharmacotherapy recommendations to graduate psychology students, nurses, and social workers during supervisory staffing rounds at two community mental health centers. Student pharmacists are paired with student psychologists and are responsible for providing drug information, therapy recommendations, and when requested attend client sessions to provide direct patient care. Students completed the Interprofessional Education Collaborative (IPEC) Competency Survey Instrument based on the IPEC Core Competencies and answered 


\section{American Journal of Pharmaceutical Education 2019; 83 (5) Article 7654.}

questions describing their previous experience on interprofessional teams and their opinion of participating these interprofessional teams. Study results were analyzed using descriptive statistics. Results: Forty-one of 42 students (98\%), representing pharmacy (17), psychology (22) and social work (2) returned completed surveys. Survey results showed that $95 \%$ of students' responses agreed or strongly agreed with the statements in the Values and Ethics Domain, $89 \%$ in the Roles and Responsibilities domain, $86.9 \%$ in the Interprofessional Communication Domain and $82.9 \%$ in the Teams and Teamwork Domain. Statements in the Teams and Teamwork domain with the highest number of neutral, disagree, and strongly disagree responses pertain to describing the team work process $(29 \%)$, describing roles and practice of effective health care teams $(26.8 \%)$ and constructively managing disagreements (26.8\%). Implications: This experience provided student pharmacists the opportunity to serve as medication experts on an interprofessional behavioral health team. Findings indicate that both pharmacy and psychology students self-perceived high competence in the attributes required of successful team members.

Interprofessional Debate Activity as a Method of Literature Evaluation: A Two-Year Analysis. Taylor D. Steuber, Auburn University, Haley M. Phillippe, Auburn University, Bradley Wright, Auburn University, Miranda R. Andrus, Auburn University. Objective: To assess the impact of interprofessional debates as an alternative activity to journal club on perceived pharmacyphysician relationships and literature evaluation skills. Methods: Third year family medicine residents and fourth year pharmacy students participated together in an interprofessional debate activity. A pre-survey using the 10-item SPICE-R2 instrument (Likert scale; $1=$ strongly disagree through $5=$ strongly agree) was administered to the participants via Qualtrics at the beginning of the APPE rotation year to gauge interprofessional teamwork perceptions. Post-surveys were administered directly after the debate throughout the year and matched to assess changes in perceived pharmacist-physician relationships and included questions to gauge perceptions of literature analysis and other skills via this method. Matched survey results were analyzed using descriptive statistics. Changes in perceptions of the SPICE-R2 instrument were analyzed using Wilcoxon Signed Rank test. Results: Fifty-five participants (18 medical residents and 37 pharmacy students) have participated in 9 unique interprofessional debates over a two-year time period. Thirty-five participants (64\%) completed both the preand post-surveys. Level of agreement increased on the post-survey for each item on the SPICE-R2 instrument and was statistically significant for 7 out of 10 questions $(\mathrm{p}<0.05)$. Overall, participants agreed or strongly agreed that the debate activity improved their literature evaluation, problem-solving, critical thinking, teamwork and communication skills. Implications: The debate was well received as a method of literature evaluation by both family medicine residents and pharmacy students. Participants reported positive and improved perceptions of working with an interprofessional team, as well as improved literature evaluation skills and other skills.

Interprofessional Training for Health Profession Students on Opioid Use Disorders. Gina M. Baugh, West Virginia University, Mark P. Garofoli, West Virginia University, Erin L. Winstanley, West Virginia University. Objective: The objective of this study is to describe health profession students' experiences and opinions about patients with opioid-use disorder (OUD) and to summarize the evaluation results of an Interprofessional Program Educational (IPE) event on OUD. Methods: An anonymous pre-event survey $(n=138)$ collected information on attendees' experience and opinions about OUD patients. The post-event survey $(n=143)$ collected information on the attendees' evaluation of the event and identified opportunities to improve future sessions. Results: Forty percent of attendees reported having a friend or family member who has/had an OUD. Less than a third reported that they would be uncomfortable working with OUD patients $(29.1 \%)$ or would prefer not to interact with OUD patients $(27.7 \%)$. The overall event evaluation results were very positive and $85.5 \%$ reported that the information would change or influence their clinical practices. Analysis of the open-ended responses found that the content was informative $(n=36)$; the attendees liked the inclusion of statistics $(n=19)$ and that the content was locally-focused $(n=13)$. Attendees wanted to learn more about OUD patient assessment and management $(n=13)$, treatment $(n=12)$ and patient communication $(n=6)$. Implications: Health profession students have more favorable opinions of OUD patients than previous research has found, and this may, in part, be explained by their personal experiences. Overall, health profession students want to learn more about the management of OUD patients.

Justification and Development of a Scholar in Geriatric Pharmacy Certificate Track. Julie A. Testman, University of Charleston, Leah A. Hall, University of Charleston. Objective: The healthcare needs of an aging population present an opportunity for students to gain specialized knowledge and differentiate themselves in an increasingly competitive marketplace. The justification and development of a geriatrics track will be described. Methods: A literature search identified limited emergence of specialty tracks with varying design 


\section{American Journal of Pharmaceutical Education 2019; 83 (5) Article 7654.}

embedded in PharmD programs. Pharmacy students were surveyed using a five-point Likert scale to assess perceived need, benefits, and interest in pursuing a geriatrics track. Track outcomes and activities were developed to optimize geriatric patient care, with emphasis on social determinants of health and age-related changes that influence therapy management. Application requirements were defined with a maximum of four students selected per cohort. Faculty workload considerations and university approval processes were addressed. Results: Survey response rate was $59 \%$ (120 of 203 students). Respondents somewhat or strongly agreed: the curriculum should provide a focused geriatrics track $(84.48 \%)$; the track will assist in student recruitment $(77.58 \%)$; specialized training would be valuable to future employers $(91.38 \%)$; and they would be interested in pursuing the track $(47.42 \%)$. The track consists of 14 didactic and experiential credit hours, two projects embedded in other courses, 100 volunteer hours, community outreach, research, and professional meeting attendance to be completed longitudinally throughout the P2-P4 years. Marketing materials were developed for prospective and current students. Implications: Upon successful track completion, students will be awarded a Scholar in Geriatric Pharmacy certificate recognized on the official transcript. This provides a framework in which schools may develop concentrations in geriatrics or other areas.

Leader Academy: A Layered Approach to Learning Leadership. Kristy L. Brittain, Medical University of South Carolina, Cathy L. Worrall, Medical University of South Carolina, Alan R. Spies, University of South Carolina. Objective: Leader Academy is a team-based cocurricular experience for pharmacy students to builds communication skills and foster leadership. The program couples' team-based leadership development during the first year of the curriculum with course-based team activities. Methods: An emotional intelligence assessment, EQi 2.0 is administered to students during their first professional year. Students are led, by facilitators through a series of programs targeted at improving communication skills through the use of GiANT Worldwide tools. Students participate in monthly sessions and are assigned activities that occur within required curricular courses. Students conduct peer/self-assessments through CATME SMARTER Teamwork. The EQi 2.0 will be administered again at the end of the Leader Academy program. Results: Student emotional intelligence data was analyzed. On average, students scored lower in the areas of Assertiveness (97), Stress Tolerance (100), Emotional Expression (100) and Independence (101). The lowest overall composite scores were Self-Expression (100) and Stress Management (101). An anonymous survey asked students to address the value and relevance of the GiANT Worldwide tools introduced, how often they used the tools and if they had taught a tool to someone else. Implications: Students indicate that they find the tools valuable and relevant to their life. The group facilitator approach has allowed the students to grow throughout the program, thus providing more buy-in by greater numbers of students. There are specific areas of focus within emotional intelligence where we can continue to build skills in self-expression and stress management. Students are also able to utilize their EQi2.0 reports to set SMART goals.

Mapping of a Clinical Skills Course Sequence to the Entrustable Professional Activities. Jill S. Borchert, Midwestern University/Downers Grove, Lea E. dela Pena, Midwestern University/Downers Grove, Jennifer J. D'Souza, Midwestern University/Downers Grove, Denise M. Kolanczyk, Midwestern University/Downers Grove, Kelly Lempicki, Midwestern University/Downers Grove, Jennifer L. Mazan, Midwestern University/ Downers Grove, Jeffrey Wieczorkiewicz, Midwestern University/Downers Grove. Objective: Clinical Skills is a 3-course sequence spanning the second and third professional years designed to develop a student's skills in communication and identifying and assessing drug therapy problems. Throughout the sequence, students complete multiple simulation-based activities. These activities are potential methods in which to provide instruction and evaluation to build upon in experiential training. Entrustable Professional Activities (EPAs) represent a baseline set of specific activities and tasks that should be performed by new pharmacy graduates. EPAs map to the Pharmacists Patient Care Process (PPCP). Our intent is to describe this course sequence and how these simulations map to EPAs and PPCP. Methods: All simulations throughout the sequence were mapped to the EPAs by the course directors. Descriptive statistics were used to summarize the domains covered, potential overlaps and gaps. Results: The 31 events mapped to 5 of the 6 EPA domains (patient provider, interprofessional team member, population health promoter, information master and practice manager), but not self-developer. Activities mapped to 12 of 14 core statements with the most frequently assessed tasks as collecting $(90 \%, \mathrm{n}=28)$ and analyzing information $(89 \%, \mathrm{n}=25)$, and utilizing evidence $(89 \%, \mathrm{n}=25)$. Using the 2016-2017 AACP Academic Affairs Committee crosswalk, all steps of the PPCP were represented. Implications: Mapping confirms the sequence is designed with repetition of activities for skill-building. Existing activities within the sequence may be suitable for milestone assessment. Mapping needs to be expanded to other courses for a more comprehensive 


\section{American Journal of Pharmaceutical Education 2019; 83 (5) Article 7654.}

picture to identify potential gaps in the curriculum as a whole.

Mapping Postgraduate Year-one Pharmacy Residency Graduation Standards to the Core Entrustable Professional Activities. Jean Y. Moon, University of Minnesota, Sarah K. Schweiss, University of Minnesota, Jody L. Lounsbery, University of Minnesota, Amy L. Pittenger, University of Minnesota. Objective: Compare the American Society of Health-system Pharmacists (ASHP) postgraduate year-one (PGY1) pharmacy residency competency areas, educational goals, and objectives to the American Association of Colleges of Pharmacy (AACP) Core Entrustable Professional Activities (EPAs) and corresponding domains. Methods: Using a consensus model, the two guidance documents were compared and mapped. The ASHP required competency areas, goals, and objectives for PGY1 pharmacy residencies were mapped to the AACP Core EPAs for new pharmacy graduates. The criteria for each ASHP objective and the example supporting tasks of the EPA statements were reviewed. Results: ASHP included one additional tier when comparing organizational levels (i.e. goals) compared to AACP. All of the 15 AACP Core EPAs mapped to at least one ASHP education objective. All of the ASHP education objectives mapped to the Core EPAs except for one objective in the Patient Care and four in the Leadership and Management competency areas. Implications: With only a slight difference in organizational structure and a small gap between ASHP education objectives and AACP EPAs, tracking of progression from new pharmacy graduate to postgraduate pharmacy resident may be possible. More consensus around leveling and general use with EPAs will provide further insight.

Mentoring Near-Peers: Understanding the value in a Developing Pharmacist. Priti Patel, University of Florida, Carinda Feild, University of Florida, Lindsey M. Childs-Kean, University of Florida. Objective: "Near-peer" mentoring allows Doctor of Pharmacy students to engage with college or high school students exploring careers in pharmacy. However, the meaning that students draw from such experiences specifically, when these experiences are not required, is relatively unknown. The purpose of this study was to understand PharmD students' expectations when undertaking near-peer mentoring roles and to discover what they learn or experience in these activities. Methods: Second- and third-year pharmacy students were invited to participate in activities which allowed them to mentor near-peers. These included College of Pharmacy recruitment and information sessions, PharmCAS workshops, and PharmCORP presentations. Near-peer mentoring occurred one-on-one or in larger groups, depending on the activity. Pharmacy students were given anonymous reflective writing prompts that were used to understand their goals for participation in near-peer mentoring. Results: Eighty-five percent of participants were female and one hundred percent participated in multiple near-peer mentoring activities. Twenty-nine percent were the first generation in their families to attend college. "Improving public speaking" and "helping others" were common reasons for participating in near-peer mentoring. The pharmacy students were pleased that they were able to share their "journey" to pharmacy school. Other positive aspects were the ability to "advocate," "generate interest" in their profession, and "inspire." Implications: Near-peer mentoring can be a valuable experience for pharmacy students, in addition to peer mentoring. This can help PharmD students acquire valuable skills in communication, service, and advocacy. Reaching out to local community colleges, high schools, and middle schools may provide educational opportunities for both parties.

Motivational Design to Grab Attention, Establish Relevance, Build Confidence, and Achieve Satisfaction About Evidence-Based Medicine. Jody L. Lounsbery, University of Minnesota, Shannon L. Reidt, University of Minnesota, Amy L. Pittenger, University of Minnesota. Objective: To evaluate an online, evidence-based medicine (EBM) course created using motivational design principles. Methods: An online EBM continuing education course was designed and delivered to pharmacy residents. The validated Instructional Materials Motivation Survey (IMMS) survey and an open-ended survey based on Keller's Attention, Relevance, Confidence, Satisfaction (ARCS) components were used to evaluate the design and instructional materials. A mixed-methods approach was used to analyze the quantitative and qualitative data sources. Results: Twenty-six (96\%) participants successfully completed the course. The overall mean score for the course was $87 \%$. The mean IMMS score was 126.2 $(\mathrm{SD}=14.6)$. Mean scores for individual ARCS components were relevance $3.8(\mathrm{SD}=1.0)$, confidence 3.7 $(\mathrm{SD}=1.1)$, attention $3.4(\mathrm{SD}=1.1)$, and satisfaction 3.0 $(\mathrm{SD}=1.0)$. Design principles identified as being positive influencers of the ARCS components included organized and logical flow of activities, realistic examples and personalized application expectations, a manageable amount of effort and enough flexibility to fit into the busy practice position, and reminder prompts regarding upcoming due dates. The use of multiple learning formats (videos, readings, online discussions, etc.) emerged as especially positive influences of confidence and satisfaction. Implications: EBM can be a challenging topic for pharmacy learners. Motivational design was an effective 


\section{American Journal of Pharmaceutical Education 2019; 83 (5) Article 7654.}

strategy to help learners gain knowledge and skills related to EBM.

Multi-Institutional Assessment of the Impact of Two Educational Activities on Student Cultural Competence. Lakesha M. Butler, Southern Illinois University Edwardsville, Aleda M. Chen, Cedarville University, Nancy Borja-Hart, The University of Tennessee, Anastasia L. Armbruster, St. Louis College of Pharmacy, Sally Arif, Midwestern University/Downers Grove, Angela Riley, Binghamton University, The State University of New York, Natasha J. Petry, North Dakota State University. Objective: To appropriately meet the new ACPE Standards 2016, students should be prepared to engage with diverse patient populations. Thus, the objective was to determine the change in student pharmacist cultural competence after engaging in two classroom activities. Methods: Six institutions implemented 2 educational activities in different cohorts of student pharmacists. Students viewed movie clips focused on stereotypes and completed the Social Identity Inventory to gain awareness of self-biases. Before and after the activities, students were asked to anonymously complete a modified version of the Clinical Cultural Competency Questionnaire, which measures skills (4 items), knowledge (3 items), encounters/situations (5 items), and attitudes ( 2 items) related to cultural competency. There also were 2 additional questions post-activity related to the educational activities (5-point Likert-type). Data were analyzed using descriptive statistics and Mann-Whitney U tests. Results: A total of 291 students in their P1, P2, or P3 year completed the activities. All skills and knowledge items significantly improved along with 4 encounter items $(p<0.001)$. One encounter and none of the attitude items significantly improved. Students indicated a median response of 4 ("quite a bit") that the 2 activities made them more aware of their own biases and stereotypes. Implications: Incorporation of unique active learning activities resulted in improved cultural competence skills and knowledge. Although changes in attitudes were not significant, students already had a high baseline median response. Overall, these activities were easily implemented across institutions, required little preparation by faculty members and could be replicated in other institutions to address Standards 2016.

New Curriculum, New Technology: Analysis of an iPad Initiative. Clark Kebodeaux, University of Kentucky, Jeff J. Cain, University of Kentucky, Patrick J. McNamara, University of Kentucky, Frank Romanelli, University of Kentucky. Objective: Technology continues to play an increasing role in student learning and engagement but research regarding the use of devices such as iPads for health professions education is sparse.
Concurrent with a new curriculum, University of Kentucky College of Pharmacy (UKCOP) provided iPads at no cost to incoming pharmacy students in Fall 2016.This study aimed to provide foundational information regarding student iPad use as well as general perceptions of the initiative. Methods: Two qualitative survey tools were developed. The first survey was administered to professional (PY1) students at orientation while the second was administered at the conclusion of the academic year. Surveys were anonymous and included two cohorts from Fall 2016 to Spring 2018.Descriptive statistics were used to analyze the data. Results: 286 students completed the initial survey (99\%RR; 286/288) while 236 students completed the second survey ( $82 \% R R ; 236 / 288) .32 \%(n=90)$ already owned an iPad prior to entering the program. 93\% of students $(n=265)$ felt that the iPad program positively influenced their perception of UKCOP as an innovative educational institution. At the conclusion of the PY1 year, $79 \%(n=187)$ identified the iPad as 'very crucial' or 'crucial' to their educational experiences and beneficial educational applications included note-taking $(77 \%, \mathrm{n}=182)$, delivery of presentations $(5 \%, \mathrm{n}=12)$, and word processing $(5 \%, n=11)$. Implications: Students felt the iPad initiative positively impacted their perception of the educational experience at UKCOP. Student feedback was used to improve implementation of the iPad initiative. Future research will focus on faculty perceptions and administrative burdens associated with the program.

Paging Future APPE Students! A Mock Rounding Simulation in a General Medicine Elective. Alexa A. Carlson, Northeastern University, Stephanie L. Sibicky, Northeastern University, Mark Douglass, Northeastern University, Jason W. Lancaster, Northeastern University, Adam B. Woolley, Northeastern University, Michael J. Gonyeau, Northeastern University. Objective: In a P3 elective general medicine course, students completed an open book, open-note, open-internet mock rounding activity including EMR cases intended to simulate an APPE rounding environment and student responsibilities (e.g., reading eMAR, responding to drug information questions (DIQ), verbally making recommendations). Methods: Pharmacy faculty and APPE students portrayed medical attendings and residents, and 14 P3 students were divided into four medical teams, with one additional APPE student moderating to keep time and grade. Each student was assigned a patient EMR before class, with new information discovered during rounds prompting a timed DIQ assignment. A mixed quantitative-qualitative analysis based on grading rubrics and a survey of students was used to evaluate this simulation. Results: Thirteen students (93\%) completed the activity, with an average grade of $78 \%$ (range 59-97) on their performance with 


\section{American Journal of Pharmaceutical Education 2019; 83 (5) Article 7654.}

the DIQ. Some students struggled with the 15-minute timeframe and re-summarizing the DIQ when presenting. Seven students completed a post-class survey, where $92 \%$ agreed/strongly agreed that the mock-rounding was valuable to the course/professional development. When ranking class activities from most (1) to least (7) helpful, mock-rounds scored a mean 2.7, indicating its utility which correlated with the $85.7 \%$ agreement that students felt well prepared to communicate with the medical team. Implications: A mock-rounding simulation reinforced skill development while preparing students for expectations of APPEs. Greater variety of patient cases with more standardization of DIQs will be implemented.

Patient Safety and Quality Improvement Education in US Professional Pharmacy Programs: Implications for Curricular Development. Jennifer Chang, University of Washington, Brenda S. Bray, Washington State University, Gordon Sproul, University of Washington. Objective: To compare and contrast coverage of recommended patient safety (PS) and quality improvement (QI) competency themes in pharmacy curricula and to identify effective course delivery models. Methods: This cross-sectional study was conducted via an electronic survey using Catalyst Web Tools. The survey included questions about program demographics and coverage of competency themes recommended for health professionals at the competent skill level as summarized in a systematic review by Moran and colleagues department chairs from 117 accredited US pharmacy schools/colleges were contacted to identify an appropriate faculty representative who was invited to complete the survey. Responses were analyzed using descriptive statistics to identify PS and QI coverage, competency gaps and course delivery models. Results: Forty-two faculty representatives were identified from 117 institutions. Twenty-five faculty completed the survey. A combined 91.6 percent of PS and 86.4 percent of QI competency themes were consistently covered across pharmacy curricula. The most commonly missed PS competency theme was Population-Based Care, while Personal and Professional Accountability for Quality was the largest QI gap. A stand-alone (PS \& QI) course model was associated with the most consistent coverage of the recommended competency themes. Implications: An analysis of PS and QI competency themes across a sample of US PharmD program curricula suggest a high degree of inclusion of recommended competency themes. The findings suggest an opportunity to reach consensus on the essential PS and QI competencies in pharmacy education. The recommended competency themes provide a framework for curricular development.
Performance on Objective Structured Clinical Examinations as Predictors for Pharmacy Practice Readiness. Kathryn L. Kiser, South College, Brittany M. Schmidt, South College, Karen S. Mark, South College, Tyler S. Dougherty, South College, Kimberly L. Zitko, South College, Richard A. Breeden, South College. Objective: To determine if performance on objective structured clinical examinations (OSCEs) can predict advanced pharmacy practice experience (APPE) readiness and practice readiness. Methods: Student pharmacists in a three-year accelerated program $(n=88)$ completed five OSCEs during didactic year two. Student performance data collected from each OSCE included analytical checklist scores mapped to the pharmacist patient care process (PPCP) and communication scores on a Global Impression Scale (GIS). APPE readiness data included rotation grades; additional data included required versus elective rotation and if preceptor was a full-time faculty member. Practice readiness data included North American Pharmacist Licensure Examination (NAPLEX) firstattempt exam scores. Data was analyzed using multiple logistic regression to develop models for predictors. Firstattempt NAPLEX pass was set as the dependent variable for practice readiness and earning a score $>85 \%$ on a required APPE was the dependent variable for APPE readiness. Results: The average GIS score in the degree of logic communication domain was the best predictor for APPE readiness $(p=0.034)$, while average analytical checklist scores in the PPCP plan and implement domains were additional variables for predicting APPE readiness. The general medicine APPE rotation grade was the best predictor for practice readiness (firstattempt pass of the NAPLEX; $p=0.026$ ). The average analytical checklist score in the PPCP assess domain was another variable to predict success in passing the NAPLEX. Implications: Utilizing these predictors will allow for identification of students at risk of poor performance on APPEs and the NAPLEX. This will enable implementation of targeted programs to assist student in successful progression.

Pharmaceutical Care of the Transgender Patient: Student Perceptions of Integrated Lecture \& Case Activities. Annie A. Nebergall, The Ohio State University, John D. Bickel, The Ohio State University, Colleen A. Dula, The Ohio State University, Stacy A. King, The Ohio State University, David E. Matthews, The Ohio State University. Objective: Despite well-documented disparities in healthcare for transgender patients, limited literature exists regarding inclusion of transgender health topics in pharmacy education. We developed, and assessed the impact of, transgender health content integrated within skills-based laboratory curriculum. Methods: 


\section{American Journal of Pharmaceutical Education 2019; 83 (5) Article 7654.}

Transgender health content was introduced in the first and third professional years (P1 and P3) of a skills-based laboratory series. Identical didactic content was delivered in both the P1 and P3 classes. Students were asked to complete a pre-class survey assessing knowledge and confidence in providing care to transgender patients. Didactic material introducing definitions and treatment guidelines was delivered, followed by discussion with a transgender patient regarding their healthcare experiences. A postclass survey examined change in students' understanding and perceptions of transgender patient care. First-year students also completed an individual patient counseling exercise in which a standardized patient portrayed a nonbinary person preparing to initiate hormone therapy. Results: $54 \%$ of first-year (P1) students and $31 \%$ of thirdyear (P3) students responded to both surveys $(n=68$ P1 students, $41 \mathrm{P} 3$ students). 95\% of respondents rated the activities as useful in preparation for practice. $97 \%$ of respondents ( $94 \%$ of $\mathrm{P} 1 \mathrm{~s}, 100 \%$ of $\mathrm{P} 3 \mathrm{~s})$ were able to locate standards of care for transgender patients following this content, compared to $12 \%$ and $11 \%$, respectively, who could do so prior to this unit. Implications: Students' preparedness and confidence in their ability to participate in the delivery of healthcare to transgender patients improves with integrated didactic \& active-learning exercises. A majority of respondents viewed this material as a valuable addition to the curriculum.

Pharmacist and Intern Knowledge and Concerns of the Pharmaceutical Use of Cannabinoids Prior to Legalization. Erin L. Johanson, Roseman University of Health Sciences, Rachel Marie Belcher, Roseman University of Health Sciences. Objective: The purpose of this project was to identify active pharmacist and pharmacy intern demographics, knowledge, and concerns regarding the pharmaceutical use of cannabinoids prior to legalization of medicinal use of cannabis (now passed) in November 2018 in Utah. Methods: Voluntary participants included actively licensed pharmacists $(n=3289)$ and interns $(\mathrm{n}=761)$ with email addresses provided through the Utah Division of Occupational and Professional Licensing. Following IRB approval, the 23-item survey, adapted from an instrument utilized in Minnesota prior to implementation of a statewide medical cannabis program, was sent electronically through Qualtrics in October 2018. Results: $35 \%$ of respondents were concerned about how the Utah Medical Cannabis Act will affect their practice. $62 \%$ were concerned. regarding safety of cannabis use (i.e., drug interaction, contraindications and adverse reactions). $75 \%$ were concerned about the consistency in quality of medical cannabis products. 55\% were concerned or extremely concerned about the limited evidence of therapeutic benefits from cannabis. $73 \%$ were unprepared to provide counseling to patients using medical cannabis as a part of their medical regimen. Implications: Implications of this study have the potential to address gaps in preparedness of pharmacy professionals of medical cannabinoid use for patients by providing a baseline of information needed to better understand the existing knowledge and concerns of the medicinal use of cannabinoids as well as identify trends in demographic variables. Future studies are needed to identify provision of education and training for pharmacists and interns in all states who have legalized patient utilization of cannabinoids.

Pharmacy Student Attitudes Toward Developing Curriculum for a Micro-Credential in the Pharmacist's Patient Care Process. Robert G. Wahler, University at Buffalo, The State University of New York, Jaime L. Maerten-Rivera, University at Buffalo, The State University of New York, Erin M. Slazak, University at Buffalo, The State University of New York, Sarah Lynch, Binghamton University, The State University of New York, Ashley Woodruff, University at Buffalo, The State University of New York, Kalpesh J. Desai, University at Buffalo, The State University of New York, Anne E. Reed, University at Buffalo, The State University of New York, John H. Wild, University at Buffalo, The State University of New York. Objective: To examine students' attitudes towards teaching and learning, Pharmacist's Patient Care Process (PPCP), micro-credentials and group work that was derived from participation in a teaching and learning theory elective course. Methods: A Teaching and Learning Theory elective course included a project-based activity where students worked in instructor-assigned groups (3 students) to develop content for a Pharmacists' Patient Care Process (PPCP) micro-credential. Fifteen students participated in the course and all students completed a survey designed to measure attitude toward their group work, and understanding of teaching and learning, the PPCP and micro-credentials. All items used a 7-point Likert rating scale. Survey questions were grouped together to form scales using the mean across all items. Each scale had a minimum of four items. Descriptive statistics were examined. Results: The mean group work scale score (out of 7) was $5.94(\mathrm{SD}=1.57)$; most students rated these items highly, however, a few students disagreed that their group was cohesive and/or developed a closer relationship than other groups. Across the understanding items, students reported that understanding increased the most in the scale on teaching and learning $(\mathrm{M}=5.77$, $\mathrm{SD}=1.46)$, followed by micro-credentials $(\mathrm{M}=5.51$, $\mathrm{SD}=1.78)$, and PPCP $(\mathrm{M}=5.33, \mathrm{SD}=1.74)$. Implications: This study demonstrates that learning and applying new information through project-based group work can 


\section{American Journal of Pharmaceutical Education 2019; 83 (5) Article 7654.}

be beneficial and satisfying for students. Students reported the highest understanding in teaching and learning overall which confirmed this elective's outcomes. While still positive, students' PPCP understanding was lower than other areas likely due to extensive PPCP training in other courses.

Pharmacy Student's Perception on the Curricular Integration of the Pharmacist Patient Care Process Model. Soumana C. Nasser, Lebanese American University, Nibal Chamoun, Lebanese American University, Yara Kuyumjian, Lebanese American University, Hani Dimassi, Lebanese American University. Objective: To describe the introduction of pharmacist patient care process (PPCP) process during the second professional year of a pharmacy program, and to compare students' perception of their preparedness to apply the process in experiential rotations during the third professional year. Methods: In spring of 2017, PPCP was introduced into the Pharmaceutical Care and Dispensing Laboratory course. Students' self-perception of preparedness to apply PPCP was assessed via a survey composed of questions related to PPCP skillsets. The pre-PPCP lab cohort (control group) took the survey after the completion of their experiential rotations. The PPCP lab cohort (intervention group) took the same survey prior to taking the course, at the end of the course and then following the completion of their experiential rotations. The Pearson's and McNemar Chi-square tests were used for statistical analysis. Results: The findings indicate that the intervention group significantly improved students' perception of their preparedness to apply a standardized pharmacist care process to verify a prescription $(\mathrm{p}=0.032)$, to collect relevant patient information $(\mathrm{p}=0.013)$, and to develop an individual patient-centered care plan $(p=0.031)$. The intervention group not only improved students' preparedness after completion of the course, but also maintained a positive impact in the following year when assessed after experiential rotations $(\mathrm{P}<0.001)$. Implications: Introduction of PPCP model within a dispensing laboratory course prior to students' introductory experiential rotations develops students' preparedness and establishes a bridge between theory and practice.

Pharmacy Students' Perceptions of the Effectiveness of a Mock Interview Program for PGY1 Interview Preparation. Jamie M. Terrell, The University of Louisiana at Monroe, Tibb F. Jacobs, The University of Louisiana at Monroe. Objective: As competition for PGY1 positions increases, students must be extremely prepared for their residency interviews. Faculty at our institution offered an opportunity for P4 students to participate and practice their mock interview (MI) skills. The purpose of this project is to assess students' perceptions of the effectiveness of the MIs. Methods: Students participating in each mock interview were surveyed before and after the interviews to determine how comfortable they were with their current interview skills and activities they participated in prior to the interviews, and also how effective they perceived the interviews to be when completed. Results: Twenty-one students consented and completed the surveys. Prior to the mock interviews, $43 \%$ and $19 \%$ felt mildly or moderately comfortable with an interview, respectively. After the interview $30 \%$ and $65 \%$ felt mildly or moderately comfortable with the interview, respectively. $43 \%$ of students felt not confident in distinguishing themselves from other applicants prior to the MI, which decreased to $5 \%$ after the MI.95\% of students had completed other MIs prior to these P4 MIs. During their MI, $85 \%$ of students discussed appropriate interview language, body language, and their CV. Implications: Mock interviews appear to increase comfort and confidence in the interview process for students who participate. This was a small sample at one college. In the future, students will also be surveyed after the match results to determine if they found the P4 mock interviews helpful for their residency interviews. Future classes of students will also be included in results to add robustness to the data.

Pilot Integration of Newly Available High Fidelity Simulation to Establish Best Practices and Support Curricular Expansion. Liza B. Andrews, Rutgers, The State University of New Jersey, Maria B. Cardinale, Rutgers, The State University of New Jersey, Deepali Dixit, Rutgers, The State University of New Jersey, Christopher D. Adams, Rutgers, The State University of New Jersey, Yekaterina Opsha, Rutgers, The State University of New Jersey, Jackie Johnston, Rutgers, The State University of New Jersey. Objective: To describe the pilot integration of high-fidelity simulation (HFS) into an established professional year 3 (P3) clinical elective to institute best practices for HFS in a newly redesigned pharmacotherapy curriculum. Methods: The availability of new high-fidelity manikins and acute care simulation spaces provided a unique opportunity to rapidly evolve the teaching methods in a 2-credit, small group, P3 critical care clinical elective designed to encourage individualized, rapid clinical decision-making. In its thirteen years, the course's classroom-based active learning environment correlated with improved student confidence and performance as students transitioned into APPEs. During course redesign, faculty developed operational procedures and logistics, session development templates, and debriefing and conduct standards to facilitate consistency with HFS activities. Results: Implementing a flippedclassroom allowed the 14-week course to provide seven 


\section{American Journal of Pharmaceutical Education 2019; 83 (5) Article 7654.}

HFS cases, five active-learning, classroom-based, two HFS lab-based instructional sessions plus a HFS final exam assessment. HFS case sessions included student teams of five to six with one faculty member dedicated to operations and another to student flow, confederate activities and debriefing. Session design was standardized using a planning sheet that outlined logistical and equipment needs, educational design and session flow, and debriefing. Standards governing learner roles and team assignments were developed and optimized based on student and faculty feedback. Implications: Pilot integration of HFS established both operational and educational design standards that allowed for the identification of potential HFS opportunities in core courses and proposed standards across clinical electives to pioneer a tiered and progressive exposure throughout the first through third professional year curriculum.

Pre and Post-ExamSoft Use Perceptions of First Year Pharmacy Students to Improve Testing Administration and Learning. Justine $\mathrm{S}$. Gortney, Wayne State University, Stephanie M. Fern, Wayne State University, Minakshi Lahiri, Wayne State University, Benjamin A. August, Wayne State University. Objective: Evaluate P1 student perceptions of online testing before and after implementation of ExamSoft (ES) to improve testing and learning. Methods: Pre and post-use ES surveys were developed to assess P1 student perceptions of training provided, impact on learning, performance, and academic honesty. The post-use survey was given after 1 semester of use. Most questions were statements with 5-point Likert scale; others commentary. Questions included on both surveys were matched by respondent code and analyzed with Wilcoxan Signed-Rank. Other questions on post-use survey were evaluated by descriptive statistics or qualitative analysis. IRB exempted study. Results: A total of 110 pre-surveys and 67 post-surveys were completed with 48 validated pre-post matches. Students felt "able to use ES with training provided" (79\% agreed). Comparing pre and post-use, no difference was observed regarding a "favorable attitude toward ES." Students did not feel ES "improved course performance" $(p=0.05)$ nor that "electronic testing reduced the risk of cheating" (56\% disagreed). Students felt it would "help them prepare for future professional exams" (67\% agreed). As a result, modifications to program processes included (1) adopting a standardized electronic-exam policy to promote course consistency (2) modifying faculty manual to improve use of ES student exam review capabilities, and (3) working with student leadership to develop a class fundraiser selling privacy screens to enhance security. Implications: Student perceptions provided valuable insight into improving online examination practices and resulted in process improvement. Students and faculty can work together to improve assessment practices to promote student success in any PharmD program.

Pre- and Post-Travel Health Recommendations for PharmD Students Engaged in Global Health Experiences. Gina M. Prescott, University at Buffalo, The State University of New York, Nicholas Bosso, University at Buffalo, Tyler Mullen, University at Buffalo, Monica L. Miller, Purdue University, Helen Berlie, Wayne State University, Joshua Sawyer, University at Buffalo, The State University of New York. Objective: To determine the content and extent of travel health recommendations in preparation for global health experiences. Methods: A 44-question online survey was pilot-tested and sent to accredited and candidate status US PharmD programs. The survey instrument assessed: demographics, available experiences (advanced pharmacy practice experiences (APPEs) and/or short-term experiences in global health (STEGHs), geographic location, pre-trip planning content and format. Descriptive statistics were used to analyze the data. Results: A total of $71 / 127$ programs responded to the survey (response rate 54.2\%). Experiences available included: APPE and STEGHs (45\%), APPE only (30\%), no experiences (17\%), and STEGHs only (9\%). Common geographic locations included: Latin America-Caribbean (40\%), East Asia-Pacific (20\%), and Africa (20\%).Pre-travel preparation was required by $80 \%$ of all programs offering either an APPE, STEGH or both. The preparation format typically took place in person $(\mathrm{APPE}=66 \%, \mathrm{STEGH}=71 \%)$ vs. online $(\mathrm{APPE}=36 \%$, STEGH $=29 \%)$. Health education topics included: vaccines $(\mathrm{APPE}=74 \%$, STEGH $=79 \%)$, travel insurance $(\mathrm{APPE}=53 \%$, STEGH $=63 \%)$, and water/food safety $(\mathrm{APPE}=51 \%$, STEGH $=58 \%)$. Common resources utilized for education included: Center for Disease Control website $(\mathrm{APPE}=62 \%$, STEGH $=61 \%)$, and school specific guidelines (APPE $=49 \%$, STEGH $=45 \%$ ). Schools offering both APPEs and STEGH required students to receive prophylaxis/vaccinations based on school polices (21\%), while others required prophylaxis/vaccinations based on university policies (APPE $=30.2 \%$, $\mathrm{STEGH}=26.3 \%$ ) or through partnering organizations (APPE $=15.1 \%, \mathrm{STEGH}=13.2 \%)$. Implications: Most US pharmacy schools offer trip preparation prior to any international travel. These experiences have common educational themes, formats, and resources consistent with travel health resources. However, requirements on vaccinations and prophylaxis is low among all experiences.

Predicting NAPLEX Performance: Statistical Models to Identify Students in Need of Early Intervention. Nalin Payakachat, University of Arkansas for 


\section{American Journal of Pharmaceutical Education 2019; 83 (5) Article 7654.}

Medical Sciences, David J. Caldwell, University of Arkansas for Medical Sciences, Ashley N. Castleberry, The University of Texas at Austin. Objective: This report describes a predictive modeling approach and demonstrates its performance in identifying PharmD students at-risk of NAPLEX failure for early intervention. Usefulness of the approach in programmatic assessment is also presented. Methods: A database of records for the graduating classes of 2012-2017 is used in an at-risk identification process. Ordinary least squares (OLS) and logistic regressions are used to create predictive algorithms for identifying at-risk students after two high-stakes exams, with NAPLEX results serving as the dependent outcome variable. Performance of the OLS approach is reported as model R-squares, correlations between predicted and actual NAPLEX scores, and a Bland-Altman plot. Performance of the logistic approach is reported as an area under the receiver operating characteristic curve (AUC). Cutoff scores are explored using information from quantile plots and a predictive margins approach. Results: Both predictive models perform reasonably well with adequate to excellent performance statistics. OLS models' R-square are 0.4 and correlation coefficients of predicted and actual NAPLEX scores are 0.6. AUCs of logistic models show good or excellent predictive accuracy. Information generated from the predictions is used to inform the intensity of interventions in a remediation process. Implications: The predictive modeling approach has been proven beneficial to the College's assessment of students and has been formally adopted in the College's programmatic assessment plan. Other colleges of pharmacy may be able to use the predictive modeling approach to identify students for early intervention who may be at risk of failing the NAPLEX.

Predictive Utility of Clinical Capstone and Intersession Course Performance on NAPLEX Performance From 2016 to 2018. Joel C. Marrs, University of Colorado, Jason Brunner, University of Colorado, Sarah L. Anderson, University of Colorado, Sunny A. Linnebur, University of Colorado, Scott W. Mueller, University of Colorado, Joseph J. Saseen, University of Colorado. Objective: The University of Colorado curriculum includes a P3 Capstone course for clinical content and a P4 Intersession course to assess near terminal competency. The aim of this study was to examine the relationship between students' performance in the P3 Capstone course, P4 Intersession course, and NAPLEX. Methods: P3 Capstone, P4 Intersession, and NAPLEX scores were collected for graduates from the Doctor of Pharmacy (PharmD) program in 2016, 2017, and $2018(n=449)$. Correlation was used to examine relationships between variables for each graduating class. Results: The correlations of the P3 Cap- stone and P4 Intersession student performance with NAPLEX scores were $r=0.577$ and 0.508 in 2016, $r=$ 0.464 and 0.312 in 2017, and $\mathrm{r}=0.455$ and 0.440 in 2018, respectively (all $\mathrm{p}<0.001$ ). For all three years combined, these correlations were 0.433 and 0.313 , respectively $(\mathrm{p}<0.001)$. These relationships for the P3 Capstone and NAPLEX score, and P4 Intersession and NAPLEX score, were both independent moderate correlations. Hierarchical multiple regression indicates that the P3 Capstone and P4 Intersession each uniquely and incrementally increase the ability to predict NAPLEX scores. Implications: Student performance in two courses at the University of Colorado, our P3 Capstone and P4 Intersession courses, predicted individual student performance on the NAPLEX exam supporting the continued impact that these courses provide for the success of our PharmD graduates.

\section{Predictors of Outcomes in a Pharmacotherapy}

Course. Karen L. Hardinger-Braun, University of Missouri-Kansas City, Maqual R. Graham, University of Missouri-Kansas City, Eric A. Wombwell, University of Missouri-Kansas City, Stephanie M. Schauner, University of Missouri-Kansas City, Kendall D. Guthrie, University of Missouri-Kansas City. Objective: To assess how pre-pharmacy characteristics, prior course performance, and student engagement influence Pharmacotherapy performance. Methods: In the fall of 2018, pre-lecture work was added to the Pharmacotherapy course to engage students, encourage students' review of previous coursework, and allow instructors to focus on clinical application. Pre-lecture, open-book quizzes and case recitation accounted for approximately $20 \%$ of the grade. A survey was administered to students to assess perceptions and engagement in the course (defined as completing prelecture assignments and quizzes, class attendance, and participating in group learning case recitation). Pearson's correlation was used to determine the relationship between predictors and performance. Results: Prepharmacy characteristics including math and science GPA, cumulative GPA, PCAT composite, and PCAT subscores did not correlate with course performance. Cumulative pharmacy school GPA $(r=0.553)$ and Pharmacotherapy I grade $(\mathrm{r}=0.694)$ had a moderate to high correlation with course grade. There was not an association between course grade and student reported time preparing for class, or frequency of completing assigned readings. There was an association between the percent of time that students reported coming to class and course grade $(\mathrm{r}=0.248)$.Pre-lecture quiz scores (range $\mathrm{r}=0.229$ $0.481)$ and missing case recitation $(\mathrm{r}=0.366)$ correlated with exam scores. Implications: Pre-pharmacy characteristics (GPA/PCAT) did not correlate with course 


\section{American Journal of Pharmaceutical Education 2019; 83 (5) Article 7654.}

performance. Engagement predicted course performance. Students that attended class and recitation and performed well on pre-quizzes had higher exams scores. Previous performance in pharmacy school was the strongest predictor of exam score.

Prevalence and Roles of Vice Chairs in Schools and Colleges of Pharmacy. Joycelyn M. Yamzon, Marshall B. Ketchum University, David Pham, Western University of Health Sciences, Karl Hess, Keck Graduate Institute. Objective: To 1) determine the prevalence of vice chairs (VC), 2) describe the VCs roles and responsibilities, and 3) evaluate why some institutions have VCs while others do not. Methods: An electronic survey was developed and sent to all CEO pharmacy deans through Qualtrics ${ }^{\circledR}$ using the listed email addresses on the Accreditation Council for Pharmacy Education's website $(n=143)$. Up to three reminders were sent and subsequently, one final direct email request was sent for survey completion. Results: 72 surveys were received yielding a $50 \%$ response rate. $64 \%$ of respondents indicated that they currently have a VC $(57 \%$ in Pharmacy Practice, $32 \%$ in Basic Sciences, and 11\% in Social and Administrative Sciences). Across departments, the primary reason for having a $\mathrm{VC}$ was to help support the responsibilities of the department chair. In all departments, the principal responsibilities for VCs were faculty mentorship (35\%) and faculty development (36\%). The most common reason for institutions not to have a VC was that the position was not perceived as needed (55\%). However, the majority of respondents $(85 \%)$ agreed or strongly agreed that department chairs need to mentor a future replacement. Implications: VCs appear to support the activities of their department chair and primarily in the areas of faculty mentorship and development. Furthermore, mentorship of VCs may positively affect departmental succession planning efforts by helping to ensure continuity and stability.

Promoting Student Engagement Through Group Presentations and Peer-Evaluation in a Self-Care and Over-the-Counter Products Course. Marie-Therese Oyalowo, University of Maryland Eastern Shore, HoaiAn Truong, University of Maryland Eastern Shore, Latasha Wade, University of Maryland Eastern Shore. Objective: The 2016 Accreditation Council for Pharmacy Education (ACPE) Standard 10 emphasizes the importance of active learning pedagogy as part of the pharmacy curriculum. The objective of this study is to describe the implementation of active learning strategies, specifically students' peer-evaluation and assessment of group presentations in a longitudinal Self-care and Overthe-counter (OTC) Products required course. Methods: Therapeutics of Self-Care and OTC Products is a longi- tudinal required team-taught course offered during the fall semester to second year students and includes lectures, self-directed learning topics, Patient Case Day, Student Directed Teaching Group Presentations and Assessments. During the group presentations conducted in the fall of 2018, students evaluated their peers using a modified rubric. They were also evaluated by faculty members. To assess students' perception of the activity, students were asked to complete a 7-question feedback survey. Results: A total of 45 students, assigned to 9 groups (4-6 students per group) presented on various topics. Each student provided evaluations for 8 groups. While the average scores provided by the students ranged from 40.3 to 44.9 out of 50 , those from faculty ranged from 44 to 48 out of 50 . Forty-four students completed the feedback survey. While $91 \%$ of the students ranked the overall activity as good to excellent, most of them indicated that presentations were very engaging. Implications: Group presentations and peer-review promoted student engagement in a Self-care and Over-the-Counter Products longitudinal required course. Most students found the activity very engaging and would very likely recommend that the activity remain as part of the course.

Prospective Comparison of NAPLEX Model Prediction With the Actual NAPLEX Results. Kalin M. Clifford, Texas Tech University Health Sciences Center, Sachin Shah, Texas Tech University Health Sciences Center. Objective: To validate reliability and applicability of NAPLEX predictive model. Methods: This study consisted of implementing a previously developed NAPLEX prediction model for students who were expected to graduate in 2017 and 2018. Students' NAPLEX results were predicted a year prior to graduation and compared with actual NAPLEX results. Student results were eligible for comparison if they had taken the NAPLEX by the end of 2019 NAPLEX Trimester One. In the model, students were divided into risk categories utilizing multiple factors (including PCOA score, didactic coursework). Primary outcome was to assess that the prediction model classified students' risk appropriately. Descriptive and comparative statistics were utilized to confirm validation. Results: NAPLEX predictive model made prediction of 298 total students based on their risk-group categories. There were 151 students in 2017 group and 147 in 2018 group. To date NAPLEX exam results were available for 253 students. For Class of $2017,14.6 \%$ of students were predicted to be poor performers compared with $15.4 \%$ of actual poor performers. For Class of 2018, $15.65 \%$ of students were predicted to be poor performers with $8.4 \%$ predicted to fail NAPLEX compared with $18.75 \%$ and $7.14 \%$ actual NAPLEX results, respectively. Implications: The predictive model originator created an interactive app that 


\section{American Journal of Pharmaceutical Education 2019; 83 (5) Article 7654.}

is available to all students and faculty nationwide to improve awareness of individual risk for NAPLEX failure. Actual results from both 2017 and 2018 may reaffirm the validity of the prediction model.

Relationship Between Stress and Academic Performance in First-year Student Pharmacists. Christina A. Spivey, The University of Tennessee, Sara Stallworth, The University of Tennessee, Marie A. Chisholm-Burns, The University of Tennessee. Objective: Studies suggest that student stress levels are associated with academic performance during the first year of various healthcare curricula. The objective was to examine the relationship between first-year student pharmacist (P1) stress levels and academic performance in the first semester of the PharmD program. Methods: P1s $(n=202)$ were surveyed using the Perceived Stress Scale (PSS) and Pharmacy Environmental Scale (PES). Associations between the PSS, PES, cumulative first semester GPA and final grades from three first-year courses, Dermatology, Biochemistry, and Pharmacy Math, were analyzed using correlational analysis. Results: Response rate was 78.2\%. Analysis of individual PES items revealed that P1 fall GPA was positively correlated with stress related to competition for grades among peers, but negatively correlated with completing graduation requirements, expectations of pharmacy school versus reality, fear of failing a course or the year, and lack of input in decision-making process of pharmacy school. Grades in Pharmacy Math, Biochemistry, and Dermatology were negatively correlated with expectations of pharmacy school versus reality and fear of failing a course or the year. Pharmacy Math and Biochemistry grades were negatively correlated with stress related to difficult classwork. Pharmacy Math grades were also negatively correlated with lack of input in decisionmaking process of pharmacy school and lack of relaxation time. Implications: Findings suggest P1 academic performance worsens due to specific stressors such as completing graduation requirements and fear of failing, but improves with increased competition for grades. This study highlights targets for development of improved stress coping mechanisms among first-year student pharmacists.

Reliability of Peer Assessment of Patient Education Simulations. Cathy H. Ficzere, Belmont University, Angela S. Clauson, Belmont University, Phillip Lee, Belmont University. Objective: To determine if student performance on patient counseling simulations can be accurately assessed by peers. Methods: Students were required to participate in patient counseling simulations throughout the semester. For each simulation, students were assigned one role: pharmacist, patient, and peerevaluator. Each pharmacist was required to counsel the patient on a specific product while the peer-evaluator assessed the accuracy and completeness of the pharmacist counseling using a detailed checklist. The patient used a checklist to assess the pharmacist's communication skills. Faculty assessed the student evaluators and the patients by counting the number of discrepancies between the student evaluator's checklist and the live faculty checklist. Students were surveyed at the end of the semester regarding their beliefs and perceptions of peer assessment for the communication simulations. Results: Of 65 students enrolled in the course in spring of 2018, complete recordings and checklists were available for 54 simulations (83.1\%). Interrater reliability was high with all correlation coefficients exceeding 0.86. Students agreed that they were comfortable assessing patient education content (82.14\%) and communication skills (82.14\%). Implications: Our results indicate that peer evaluation during patient education simulation is reliable and acceptable to students.

Residency Match Rates and Pharmacy Program Characteristics. Dawn E. Havrda, The University of Tennessee, Catherine M. Crill, The University of Tennessee, Joseph M. Swanson, The University of Tennessee. Objective: With the changing job market and competition for post-graduate opportunities, changes to pharmacy programs should consider the impact on graduate success. The objective of this study was to determine pharmacy program characteristics associated with higher American Society of Health-Systems Pharmacists (ASHP) PGY1 residency match-rates. Methods: Pharmacy programs with at least one graduating class were included. Program characteristics were obtained from program websites and course catalogs/bulletins and from nationally available data. ASHP PGY1 residency match-rates were obtained for years 2018, 2017, and 2016.Program characteristics and match-rates were evaluated by year and for a 3-year aggregate. Multi-variable regression was used to identify significant characteristics associated with higher match rates. Results: The analysis included 138 programs. The mean (standard deviation) PGY1 match-rates for 2016, 2017 and 2018 were $66(15) \%, 65(14) \%$, and $63(17) \%$ respectively, and the 3-year aggregate rate was 65(12)\%. Advanced Pharmacy Practice Experience (APPE) assessments included pass/fail in 49 (35.5\%) programs, graded in 87 (63\%) programs, and a combination of grading modes in $2(1.4 \%)$ programs. Programs with pass/fail or graded APPEs had similar PGY1 match-rates (67(13)\% vs. $64(12) \%, P=0.063$, respectively). Program factors significantly associated with high matchrates included public universities $(\mathrm{P}<0.0001)$, programs established before $1995(\mathrm{P}<0.0001)$, academic health centers $(\mathrm{P}<0.0001)$, and offering dual-degree programs 


\section{American Journal of Pharmaceutical Education 2019; 83 (5) Article 7654.}

( $\mathrm{P}<0.0001)$. Implications: Older, public universities with academic health centers and dual-degree programs were associated with higher ASHP PGY1 residency match-rates. APPE grade mode was not associated with a difference in PGY1 match-rates. For programs considering pass/fail grade modes for APPEs to measure practice-readiness skills, no affect was found on PGY1 match-rates.

Role of Institutional Structures to Promote Faculty Development in Interprofessional Education. Rahmat M. Talukder, The University of Texas at Tyler, Yumi So, The University of Texas at Tyler, Rebecca Presson, The University of Texas at Tyler, Mohammed A. Islam, American University of Health Sciences. Objective: Existing literature on interprofessional education (IPE) suggests a lack of IPE faculty development opportunities in US colleges/universities offering healthcare education. The objective of this study is to explore the existing organizational structures in US higher education institutions that foster IPE faculty development and rewards. Methods: A thorough Google search was conducted using the keywords: "interprofessional education" combined with "center", "institute" and "faculty development". A list of 82 institutions was created. Each institution's website was visited to search pages with information on IPE centers/institute, IPE faculty development, award/scholarship and grants. All the identified pages were retrieved, and relevant texts were saved in a MS word document. Data extraction from text document was performed using a systematic content analysis method. Results: Out of 82 institutions, 30 were identified to have a dedicated IPE center or IPE institute. IPE activities of other institutions are administered by the university administration/provost office. $37 \%$ institutions have institutional IPE committees; while $54 \%$ offer faculty development opportunities in IPE practices. The common faculty development activities include workshops, mini-conferences, forums, and seminars, and simulation training. Only $27 \%$ institutions have identified that they provide faculty awards/grants on IPE scholarships and research. Faculty development and reward structures are available only in the colleges and universities that have institutional structures for IPE. Implications: This study has identified a lack of IPE faculty development and reward structures. Institutional structures such as IPE centers or institutes are necessary to develop and implement of IPE faculty development and reward structures.

RXcape: A Fun, Effective Tool for Teaching Acute Kidney Injury to 2nd Year Pharmacy Students. Rebecca Maxson, Auburn University, Sarah V. Cogle, Auburn University. Objective: The 2013-2014 Academic
Affairs Committee recommended development of serious games to be used in pharmacy education. These serious games result in learning, skill acquisition, and training while "learning by doing". Additionally, learners receive immediate feedback resulting in a "fun failure" in real-life pharmacy scenarios. (AJPE2015;79:47, AJPE 2017;81:6265) Methods: Groups of 5 to 6 students competed in an RXcape game during the Acute Kidney Injury unit. The following three inpatient scenarios were developed: verifying an order for a sodium bicarbonate infusion, verifying an order for a potassium infusion along with checking the final product, and determining which medications to resume for a patient transitioning from the ICU to the medical floor. Qualtrics surveys were used to provide immediate feedback on answers. The group that escaped from all stations in the shortest time period was determined to be the winner. Results: Student performance on assessment questions related to this activity was strong, with $73 \%$ answering multiple choice questions correctly. The average point biserial was 0.36 and the average discrimination index was 0.5 , indicating that the top performers in the class were able to answer the questions correctly. Course evaluations revealed that students felt the activity helped them identify key concepts, but wished the activity covered additional scenarios. Implications: The RXcape game was very effective at allowing students to apply their knowledge in a real-world setting. Future enhancements involve adding a fourth activity and increasing complexity in the current activities.

Screening, Brief Intervention, and Referral to Treatment (SBIRT) Results From Three Years of Training. Daniel J. Ventricelli, University of the Sciences, Heather M. Santa, University of Pittsburgh, Michael Thomson, University of the Sciences, Alexandra A. Nowalk, University of Pittsburgh, Meghana Aruru, University of Pittsburgh, Janice L. Pringle, University of Pittsburgh, Cathy Y. Poon, University of the Sciences, George E. Downs, University of the Sciences. Objective: To present the outcomes of a three-year Screening, Brief Intervention, and Referral to Treatment (SBIRT) training program involving interprofessional healthcare students and practitioners. Methods: The SBIRT training program was integrated into an interprofessional substance use disorder (SUD) course for pharmacy, physician assistant, occupational therapy, kinesiology, physical therapy and psychology students. The curriculum included selfdirected online activities, in-person workshops, and clinical experiences. All participants were administered a Survey of Attitudes and Perceptions pre- and post-training to assess changes in core knowledge, perceived competency, attitudes, and perceptions. Qualitative data was derived from post-training student group discussions and 


\section{American Journal of Pharmaceutical Education 2019; 83 (5) Article 7654.}

interviews with course Champions. A brief Clinical Encounters survey was sent to trainees to gauge effectiveness and adoption of SBIRT in clinical practice. Healthcare practitioners completed the SBIRT curriculum through asynchronous online modules and a oneday live workshop. Results: 528 students and 97 practitioners completed the training program. Results indicated improvement from pre-training to post-training in core knowledge and self-reported attitudes, perceptions and competence in SBIRT. Clinical encounter reports and qualitative data indicated SBIRT to be an effective tool to guide SUD related conversations with patients. Implications: Training healthcare professional students and practitioners to use SBIRT can improve their overall confidence and ability to engage in SUD related communication. Based on the results of this program, students and practitioners are more prepared to address SUD following completion of SBIRT training. Many healthcare professionals will encounter patients with SUD throughout their careers and these results further support the need to incorporate SBIRT training and skill development into required curricula.

Self-Directed Learning in First Professional Year Pharmacy Students in a Pharmacists' Patient Care Process Course. Kristen Pate, The University of Mississippi, Meagen M. Rosenthal, The University of Mississippi, Jennifer M. Toth, The University of Mississippi. Objective: To evaluate changes in self-directed learning (SDL) scores in first professional year (P1) pharmacy students after participation in a Pharmacists' Patient Care Process (PPCP) course. Methods: AACP SoTL grant funding was used to support the use of adaptive learning technology (ALT) software during the spring semester of a PPCP course series. Students in the PPCP course have completed two surveys (August and November) to measure SDL and demographic characteristics. All surveys were voluntary, with class time given for completion. Results were analyzed using SPSS ${ }^{\circledR}$ software. Results: Survey response rate was 99\%, with nearly all 107 enrolled students participating. The majority of respondents identified as state residents $(86 \%)$, were not first-generation college students ( $85 \%)$, and had attended a single college $(62 \%)$. The mean age of respondents was 23 years $(\mathrm{SD}=$ 1.30). Scores on the SDL factors "assignment management", "stress management", "procrastination management", and "time management" suggest confidence in these areas. Statistically significant differences were identified between the August and November SDL scores on the factors "assignment management", "comprehension competence", and "time management". Implications: ACPE "Standards 2016" recommend a curriculum "delivered via teaching/learning methods that: promote student responsibility for self-directed learning", but research is needed to determine the best teaching methods to support SDL. The next phase of this research will involve measurement of students' SDL at the end of the spring semester, wherein ALT is being used. This research will provide insight into which teaching methods, course designs, and delivery modalities are most effective for improving SDL.

Simulation to Practice: Assessment of Pharmacy Students' Medical History-Taking Skills via Unannounced Standardized Patients. Tina Zerilli, Long Island University, Brooke D. Fidler, Long Island University, Chosang Tendhar, Long Island University. Objective: The use of unannounced standardized patients (USPs) in pharmacy education is limited. This approach was used to investigate whether students could transfer their medical history-taking skills from a simulated patient environment to the real world. Methods: In spring 2018, P1 students enrolled in a physical assessment course (PHM 326) used peer role-play and weekly virtual patient activities to practice medical history-taking skills. Students were assessed using a final virtual patient case. In summer 2018, an USP used a 17-item medical history content checklist (range 0-34) and an 8-item communication/interpersonal skills checklist (range 8-32) to assess a cohort of these students in a community introductory pharmacy practice experience. A correlation analysis between scores on the content checklist and the final virtual patient case was conducted. IRB exempt status was received. Results: The USP assessed 40 students at 22 pharmacies; 39 had usable data. The mean (SD) composite score on the content checklist was 18.1 (4.4). Complete patient contact and allergy information was obtained by $92.3 \%$ and $79.5 \%$ of students, respectively. The mean (SD) composite score on the communication/interpersonal skills checklist was 19.4 (3.7). Thirty-two of 39 students had usable scores on the PHM 326 final virtual patient case; the mean (SD) percentile score was 79.2 (15.2). A correlation $(r=0.15 ; \mathrm{p}=0.41)$ existed between this and the medical history-taking composite score. Implications: Performance in practice and the size of the correlation suggest there is ample room for improvement. Changing didactic teaching methods, perhaps by integrating standardized patient encounters, may be warranted.

Skills Laboratory Activity to Improve Pharmacy Students' Ability to Provide Medication Counseling via Translator. Jennifer G. Smith, The University of Louisiana at Monroe, Savannah K. Posey, The University of Louisiana at Monroe. Objective: To evaluate an activity developed to provide students with practical experience working with a translator in a simulated patient counseling interaction. Participant's perceptions of the activity 


\section{American Journal of Pharmaceutical Education 2019; 83 (5) Article 7654.}

will be collected to evaluate student confidence before and after the activity and assess activity efficacy to improve one aspect of students' cultural competency. Methods: Based on a pilot study, the activity was implemented in a required skills lab course for students in their third professional year. Students completed two surveys: one before the activity and one after activity completion. Graduate student volunteers who served as the "translator" and non-English speaking "patient" completed a single survey following the activity. This study was approved by the institution's IRB. Results: Eighty-seven students and six volunteers completed the activity. Eighty-four students completed the pre-survey and 66 completed the postsurvey. Of these, 57 were able to be matched with pre/post data. Before the activity, students' average rating of confidence in providing counseling to a non-English speaking patient using a translator was $2.49(1=$ not at all confident and $5=$ very confident). After the activity, the students' average rating increased to 4.07 . Students felt that the activity improved their confidence and ability to work through a translator (average 4.39, scale: $1=$ strongly disagree to $5=$ strongly agree). Student and volunteer feedback highlighted the utility of this activity in preparation for clinical practice. Implications: The results suggest that the activity was effective in increasing student confidence when counseling a non-English speaking patient via translator and support continued development of this type of activity as part of the core curriculum.

Student Attitudes and Perceptions of Restructured Objective Structured Clinical Exams Program. Chelsea Gresham-Dolby, Marshall University, Christopher J. Booth, Marshall University, Breanna Linville, Marshall University. Objective: The Objective Structured Clinical Exams (OSCE) program underwent revision and restructuring in the 2018-2019 academic year. Goals of this restructuring included ensuring that skills were being assessed objectively during OSCEs, that students were prepared for OSCEs by adding mandatory Objective Structured Learning Experiences (OSLEs), and that students receive feedback on their performance during the OSCE to inform their plans for improvement. This project sought to evaluate student perceptions of the OSCE program as compared to baseline perception of the OSCE program for the purpose of quality control and improvement. Methods: Students were surveyed prior to completion of OSCE assessments in the new sequence to assess their baseline attitudes and perceptions of OSCE administration in the previous sequence. After completion of the new OSCE sequence, students were surveyed with the same set of questions in addition to a few questions geared toward assessing the students' perceptions of the OSLE activities. Results: When pre- and post- sur- veys were compared, several areas were identified as being improved from the students' perspective, including: provision of feedback, understanding of skills assessed, and organization of OSCE structure. Areas for improvement remain in assuring that students perceive alignment with skills taught in curriculum. Implications: Improving communication to students about expectations during the OSCE through the addition of the OSLE activities decreased student apprehension regarding OSCE assessments. Addition of OSLE activities and feedback mechanisms can improve student attitudes and perceptions toward OSCE assessments. This can hopefully serve the goal of improving student understanding of and ability to perform essential skills.

Student Confidence in Applying the PPCP to Patients Seeking Contraception: Results from Two Colleges of Pharmacy. Autumn L. Stewart-Lynch, Duquesne University, Sarah Lynch, Binghamton University, The State University of New York, Brooke Griffin, Midwestern University/Downers Grove, Kathleen Vest, Midwestern University/Downers Grove. Objective: Several states permit pharmacist prescribed hormonal contraception. Practicing pharmacists identify lack of confidence and training as barriers to engaging in this activity. The purpose of this study is to describe student pharmacists' confidence in applying the Pharmacist Patient Care Process (PPCP) to prescribing contraceptives and assess the impact of student behaviors on perceived confidence. Methods: A cross-sectional survey questionnaire was distributed to students enrolled in therapeutics courses at two colleges. The survey instrument assessed confidence in performing PPCP steps and perceived preparedness in prescribing hormonal contraceptives. Results: A total of 216 responses were received $(91.1 \%$ response rate). Students were most confident in their ability to gather information (COLLECT) from a patient seeking contraception $(123,59.1 \%)$ and least confident in the ability to recommend (PLAN) a specific product $(83,40.3 \%)$. Students who spent more time in or out of class learning were significantly more confident than those spending less. Thirty-one (14.98\%) students felt prepared based on training in the curricula, while $52.17 \%$ anticipated that practice during APPEs would provide needed preparation. Implications: States vary in required training and $\mathrm{ACPE}$ does not specify a minimum amount or type of training for curricula. These results suggest that the amount of time spent learning and studying contraception may be an important factor in student confidence and that current time spent in didactic teaching may not be enough to prepare students this advanced role. Further research is needed to examine whether current didactic approaches are adequate, and 


\section{American Journal of Pharmaceutical Education 2019; 83 (5) Article 7654.}

whether simulated experiences influence student perceptions of confidence.

Student Extracurricular Employment and Impact on Student Curricular Outcomes. Chelsea Gresham-Dolby, Marshall University, Christopher J. Booth, Marshall University, Samantha Stacy, Marshall University, Bryanna Roar, Marshall University. Objective: This study was designed to investigate correlation between student employment and curricular outcomes with the ultimate goal being able to establish optimal work recommendations for incoming students in the Doctor of Pharmacy program. Methods: Students enrolled in the Doctor of Pharmacy program were asked to volunteer information regarding their extracurricular employment during the Fall 2018 semester. Survey items assessed included number of hours worked in pharmacy (community/ institutional) and non-pharmacy (healthcare-related/ non-healthcare-related). For each survey respondent, curricular results such as grade point average, PCOA, and number of course failures were analyzed for correlations compared to type of employment and hours worked. Results: Overall survey response rate was $41 \%$ with 116 student responses completed. Approximately $75 \%$ of students in P2-P4 years reported employment while approximately half of P1 students reported employment. Over $90 \%$ of student respondents who are employed report working as a pharmacy intern. Of students who work, $40 \%$ of the respondents reported working within the range of 6-10 hours per week. Student employment among respondents shifted to only pharmacy interning by $\mathrm{P} 4$ year with number of students reporting non-healthcare-related work decreasing steadily from $\mathrm{P} 1$ to $\mathrm{P} 4$ year. For student respondents currently enrolled in the didactic portion of the curriculum who have failed a course, $72 \%$ report current employment, and $67 \%$ report working more than ten hours per week. Implications: Student employment may have an impact on academic performance that is multifaceted through reinforcement of what is learned in the classroom and by reducing amount of time to complete curricular requirements.

Student Knowledge and Confidence in Furnishing Self-Administered Hormonal Contraception Following a Simulated Patient Case Activity. Elizabeth Harris, University of the Sciences, Karleen Melody, University of the Sciences, Lisa Charneski, University of the Sciences. Objective: Assess student knowledge and confidence with self-administered hormonal contraception following a patient case activity. Methods: Students received a 2-hour lecture on hormonal contraceptives in a required therapeutics course P2 fall. A 2-hour case activity was created and delivered in a required case studies course P2 spring. Students read a protocol on pharmacist- administered hormonal contraception and used a risk assessment tool while watching a video-recorded simulated pharmacist-patient encounter prior to class. In class, students documented their plan in groups. Assessments included a 5-question readiness quiz at the beginning of class; documented plan; 8-question quiz post-activity; and an anonymous 9-question confidence survey. Results: There were 129 and 112 students enrolled in the 2017-2018 and 2018-2019 academic years respectively. Average student performance was consistent on the readiness quiz between the two years $(83.9 \%)$. Students scored an average of $84.2 \%$ and $91.6 \%$ on the documented plan and $96.4 \%$ and $91.2 \%$ on the post-activity quiz for 2017-2018 and 2018-2019 respectively. There were 218 responses to the confidence survey. Students felt most confident with their ability to navigate and interpret a prescription drug formulary, provide the patient with written documentation of the product, and communicate with the patient's prescriber. Students were less confident with selecting an appropriate product based on patient-specific factors and providing education on missed doses. Implications: This case-based activity increased student knowledge and confidence with furnishing selfadministered hormonal contraception. Faculty are encouraged to incorporate similar educational strategies into their curriculum to prepare students to practice confidently in this evolving landscape.

Student Perceived Competency in Using an EHR Following Implementation of a Learning-Based EHR Platform. Lisa Goldstone, University of Southern California, Rory O'Callaghan Kim, University of Southern California, Emily E. Han, University of Southern California, Scott Mosley, University of Southern California, Paul J. Wong, University of Southern California, Paula Phongsamran, University of Southern California. Objective: The objective of this study was to assess changes in student perceived competency in using an electronic health record (EHR) following implementation of a learning-based EHR platform in a case conference course in the second professional year. Methods: A learning-based EHR was incorporated into an existing 15-week case conference course. Students were required to use the EHR for three purposes on a weekly basis which included reviewing the patient health record prior to attending the session, referring to the patient health record during the session as needed, and submitting documentation of the patient encounter following the session. Students were asked to rate their EHR skills on a 5-point Likert-like scale in 4 areas on an anonymous survey administered on the first and last days of the course. Perception of competency was assessed in the following areas: collecting information from the EHR, assessing this information to detect 


\section{American Journal of Pharmaceutical Education 2019; 83 (5) Article 7654.}

medication-related problems, utilizing this information in making clinical decisions or recommendations, and using the EHR for documentation purposes. Results: A meaningful change in perceived competency between pre- and post-implementation was found for all four skill areas. Nearly all students rated their skill level in using an EHR as an advanced beginner level or higher following the intervention with the largest percentage of students classifying themselves as proficient in EHR skills. Implications: Incorporation of an EHR platform in a small group discussion setting is effective in increasing student confidence in their ability to use EHR-related skills.

Student Perceptions of Multisite Topic Discussion Series Using Web-Based Conferencing Technology. Lydia C. Newsom, Mercer University, Sweta M. Patel, Mercer University, Maria M. Thurston, Mercer University, Jennifer B. Elliott, Mercer University, Kendra R. Manigault, John D. Bucheit, Virginia Commonwealth University. Objective: Incorporation of technology into the didactic curriculum is required by the Accreditation Council for Pharmacy Education. However, the use of technology during Advanced Pharmacy Practice Experiences (APPE) is less well defined. The primary objective of this study was to assess students' perceptions of learning through a multisite topic discussion (TD) series using web-based conferencing during ambulatory care APPEs. Methods: A five session faculty-led TD series was conducted during each five-week Ambulatory Care APPE at six distinct clinical sites from May 2015 through October 2018. Students were assigned readings and case-based activities to complete prior to each TD. The TD series utilized WebEx ${ }^{\circledR}$ that provided audio and visual feeds for all attendees. At the end of the TD series, each student completed an online survey to assess their perceptions of learning. Results: A total of 95 students completed the survey. The majority of students felt that web-based TD series enhanced their learning (95.8\%). Over $95 \%$ of students stated the TD series enhanced their confidence to provide patient care and think critically $(96.8 \%$ and $97.9 \%$, respectively.) While $75.8 \%$ of students stated they would prefer to learn as a web-based community, $51.6 \%$ of students felt that this format introduced unnecessary technological complications. Implications: The facultyled web-based TD series during ambulatory care APPEs enhanced student perceptions of learning and confidence in providing patient care. To ensure this technology is a viable format, technological issues must be minimized. A study assessing the impact of web-based TDs on student learning is ongoing.

Student Preferences Between Two Active Learning Session Schedules. Priti Patel, University of Florida, Shane M. Ryan, University of Florida, Lindsey M.
Childs-Kean, University of Florida. Objective: To understand students' perceptions of adding a new online class session to live active learning sessions. Methods: At our college, most courses are taught as team-based learning with pre-recorded lectures followed by live, inclass active learning sessions. Many in-class sessions are scheduled for 4 hours. In two courses in fall 2018, we offered a new format for two sessions: a 3 hour in-class session, followed by a 1 -hour online session the following day. The purpose of this new format was to 1) allow students to digest the material overnight before revisiting the material the following day; and 2) break up the 4-hour session and allow the students and faculty to more fully engage in active learning. Students completed an online survey following each online class. Results: 156/270 (58\%) students completed the survey in the first course; $149 / 270$ (49\%) completed the second course's survey. The most common benefits selected were breaking up the 4 hour session $(24.9 \%$ in the first course) and the convenience of being in a remote location $(25.4 \%$ first course; $25.1 \%$ second course).Difficulty interacting with their team was the most common challenge selected in both courses (40.8\% and 35.5\%).Even though benefits were identified, students indicated preference for the 4 hour sessions: $89.7 \%$ first course, $88.6 \%$ second course. Faculty perceptions of the online session were similar with increased convenience but some lack of student engagement. Implications: While benefits of the online session were identified, students indicated preference for the 4 hour in-class sessions.

Student Response to Incorporating Mindfulness in a First Year Patient Care Lab Course. Elizabeth A. Buckley, Concordia University Wisconsin. Objective: Analyze student response to implementation of mindful awareness training into a lab course to improve wellbeing, resilience, and enhancement of patient-centered care practice. Methods: During the fall semester of the P1 year, five minutes of weekly lecture time was dedicated to introduce mindfulness tools for students to use for their own well-being and enhancement of patient centered communication. Tools were introduced via PowerPoint, videos, interactive Padlet activities, live demonstrations and hands on experience. Techniques included gratitude practice, mindful breathing, body scan, conscious observation, mindful movement, imagery, touchstones, empathy, self-compassion and spirituality. At the end of the semester, a toolkit of topics and resources was provided to encourage continued self-practice. Students responded to different reflection questions via anonymous blackboard survey at the end of each week, and at the end of the semester. Results: Student response was overwhelmingly positive throughout the semester. Students 


\section{American Journal of Pharmaceutical Education 2019; 83 (5) Article 7654.}

articulated how mindfulness positively related to enhancing their patient centered care. Students reported continued use of the skills (94\% at week 5, 100\% at week 12). $89 \%$ of students agreed that the activities made a positive impact on their life, $100 \%$ believed the course should continue to incorporate the skills, and $77 \%$ agreed it should be further expanded in the curriculum (22\% neutral, 1\% disagree). Implications: Positive student response supports continued incorporation of mindfulness into the current course. Data was presented as a faculty development seminar to encourage further exploration for curricular integration to continue creating a culture of well-being and resiliency at our college.

Students' Ability to Implement the Pharmacist's Patient Care Process in a Redesigned Case-based Course. Keri L. DePatis, Palm Beach Atlantic University, Jennifer Wick, Palm Beach Atlantic University, Amy Henneman, Palm Beach Atlantic University, Samantha Axtell, Palm Beach Atlantic University. Objective: To determine if a redesigned case studies series impacted students' ability to implement the patient care process. Methods: This was a prospective, cohort study conducted at and IRB approved by Palm Beach Atlantic University. In 2016 the Lloyd L. Gregory School of Pharmacy implemented a redesigned four semester case studies series to foster critical thinking and formally integrate the PPCP. The original case studies series consisted of a two-semester problem-based course. Students completing the original case studies series and students completing the first two semesters of the redesigned course series were given a case-based assessment consisting of four open-ended questions covering subjective and objective data collection, medication-related problem (MRP) identification, and assessment/plan development. Results were analyzed via repeated two sample t-tests, accounting for unequal and equal variances. Analysis was performed using Excel Data Analysis. Results: One hundred twenty-three students completed the assessment, including 60 students (49\%) from the original case studies series and 63 students $(51 \%)$ from the redesigned course. Compared to students who completed the original case series, students who completed two semesters of the redesigned series scored statistically significantly higher on the overall assessment, as well as the subjective and objective portions. There was no difference in students' ability to identify MRPs or develop the assessment and plan. Implications: Students' ability to implement portions of the PPCP was improved after completing two semesters of a redesigned case studies course. Future study is warranted to determine the impact on advanced practice experience (APPE) readiness and implementation of the PPCP in practice.
Student's Reflections Surrounding a Week-long Health Fair Training Workshop Preparing Pharmacy Students for Outreach Opportunities. Trishia E. Shaw, Chicago State University. Objective: Although students receive didactic training with respect to various disease states, they often lack the skills and confidence to perform skills related to disease state education at local health fairs. The lack of skill and/or confidence may be attributed to lack of training or lack of opportunities to perform these skills outside of the curriculum. Chicago State University College of Pharmacy's Health Outreach Task Force conducted its first week-long training workshop, which trained first through third year pharmacy students in various health fair screening techniques as well as educational modules. Methods: After completing the workshop, students were required to complete a "co-curricular reflection" to outline how the workshop enhanced clinical and communication skills as well as enhanced their development as a future pharmacy professional. Co-curricular documentation occurred using the "Google Sites" platform. Results: 65 students in total completed the health fair training workshop. 63 out of 65 students completed the "co-curricular reflection" survey. $73 \%$ of students increased their awareness of pharmacists' roles in health care and approximately $70 \%$ of the students stated that the workshop helped enhance their clinical skills. Several students also documented how the workshop reinforced concepts learned in didactic courses as well increased their confidence in performing the various screenings. Implications: These reflections indicate that the training workshop not only enhanced their skills but was able to reinforce the knowledge learning within the didactic curriculum. As a result, students are better equipped to perform screenings at health fairs and as a result, participate in more screening events.

Study Behaviors of High-Performing Students Within an Active Learning Classroom Environment. Kimberly C. McKeirnan, Washington State University, Karen J. Colorai, Arizona State University, Anne Kim, Washington State University, Myphuong Vu, Washington State University, Connie M. Remsberg, Washington State University, Angela S. Stewart, Washington State University. Objective: To use qualitative methods to determine the study behaviors of high-performing pharmacy students that aid them in achieving academic success in the active learning classroom curricular delivery model utilized at Washington State University. Methods: Highperforming students, defined as the top $20 \%$ of the class by cumulative GPA, from third and fourth years of the program were recruited through email to participate in the study ( $\mathrm{n}=81)$. Key informant interviews were conducted for 13 students by an experienced qualitative interviewer 


\section{American Journal of Pharmaceutical Education 2019; 83 (5) Article 7654.}

from an outside college. Student interviews were recorded, transcribed, and examined using thematic analysis. Results: Five prevailing themes emerged from the student interviews: preparing for class, preparing for testing, knowing thyself, seeking help, and building on strengths. The high-performing students generally reported completing all pre-class work, but attitudes varied about the benefits of attending class. When preparing for testing, they employed a variety of techniques including frequent exposure to the material and self-testing. These students demonstrated heightened awareness of their own habits, noting their ability to help or to hinder their studying. They commonly sought help by either strategically studying in groups or seeking help from instructors. Finally, they built on their own strengths, employing techniques to minimize their weaknesses. Implications: The high-performing students identified a number of study behaviors they have used to be successful in a curriculum that fully uses an active learning model. This insightful feedback is being incorporated into a best practices document for new pharmacy students to help them adapt to an active learning classroom environment.

Summer Preparation Program for Fall Courses: Prep for P2 Success. Reid C. Proctor, Mercer University, Renee L. Hayslett, Mercer University, Katelynn M. Mayberry, Mercer University, Gina J. Ryan, Mercer University. Objective: To implement a summer preparation program for rising P2 students and assess the impact on learning outcomes. Methods: At the end of the spring 2018 term, participating students $(\mathrm{n}=92)$ completed a 15 -question pre-quiz covering basic nervous system anatomy and physiology (NSAP) concepts needed for a fall P2 nervous system module (NSM). Khan Academy (KA) is a free online education platform. Instructors encouraged these rising P2 students to use the summer months to complete designated KA learning modules focused on NSAP (which were created in collaboration with the Association of American Medical Colleges) to improve prerequisite knowledge for the NSM. The NSM contains physiology, pathophysiology, pharmacology, medicinal chemistry, and pharmacotherapy. At the start of the fall 2018 term, participants completed the same quiz. Investigators also assessed exam performance. Results: Eighty-one $(88 \%)$ of the participants completed both quizzes. Students who completed at least half of the assigned KA modules $(n=21)$ reflected a significant $(\mathrm{p}<.005)$ and positive change in mean scores between the pre-quiz $(\mathrm{M}=40 \%)$ and post-quiz $(\mathrm{M}=60 \%)$. The post-quiz scores and two of the course exams reflected a positive and significant $(\mathrm{p}<.05)$ correlation. A majority $(88 \%)$ of participants indicated that the KA material supported their learning. Implications: The findings of the current study support the hypothesis that addressing gaps in prerequisite knowledge with KA helps students prepare for upcoming coursework. The investigators are motivated to further develop this model of student support.

Survey of Student and Faculty Perceptions of Revised Early Intervention and Remediation Policies. Elizabeth M. Lafitte, The University of Louisiana at Monroe, Laurel A. Sampognaro, The University of Louisiana at Monroe, David J. Caldwell, University of Arkansas for Medical Sciences, Gina C. Craft, The University of Louisiana at Monroe, Michelle O. Zagar, The University of Louisiana at Monroe, Kassidy Voinche, The University of Louisiana at Monroe, Victoria Rathcke, The University of Louisiana at Monroe. Objective: The purpose of this research was to involve students and faculty in continuous quality improvement for the early intervention and remediation policies that were revised and implemented in the Fall of 2015. Methods: All students and faculty were offered a survey of perceptions and workload related to the revised policies. Both students and faculty were provided with links to the revised policies and descriptive data that showed the improvement in delayed graduation statistics that occurred since the implementation of the policies. Results: Approximately one fourth of students and one half of the faculty responded to the survey in the spring of 2018. About seventy percent of student and faculty respondents agreed or strongly agreed that the time required to participate in the early intervention and remediation processes was worth the effect achieved. Ninety percent of faculty felt that the improvement in progression occurred as a result of implementing the revised policies. Most faculty and students also agreed that the exam averages that trigger early intervention were appropriate and that the criteria for individual remediation were appropriate. Implications: Many methods for improving student success require a considerable investment of faculty and student time. The implementation of revised early intervention and remediation policies, improvement in delayed graduation, and favorable student and faculty perceptions and workload represent an important model that should be shared with other programs that desire to improve delayed graduation.

Telecounseling in Advanced Over-the-Counter Course. Megan Veselov, Albany College of Pharmacy and Health Sciences, Elizabeth R. Brown-Higdon, Albany College of Pharmacy and Health Sciences. Objective: To describe the addition of telecounseling simulations into the elective Advanced Over-the-Counter (AOTC) course to increase students' exposure to triage, counseling, and adoption of emerging technologies. Methods: Second- and third-year pharmacy students across two campuses (Albany and Vermont) enrolled in 


\section{American Journal of Pharmaceutical Education 2019; 83 (5) Article 7654.}

AOTC received faculty instruction on telecounseling and the use of video conferencing technology (Zoom $(\mathrm{C})$. Students were given three opportunities to practice telecounseling during the semester. Students were matched with a partner on the distance campus to simulate a remote experience and were required to act as both a mock patient and pharmacy intern. Students were surveyed using Likert-scale and short-answer response questions throughout and at the end of the semester on technical aspects of using Zoom and overall experience with telecounseling. Results: Twenty-four of the thirty-six students enrolled in the course completed the end-of-course telecounseling survey. The results included students indicating "agree" or "strongly agree" that telecounseling activities helped to learn appropriate OTC recommendations (95.8\%); helped to learn patient triage (100\%); and were as effective as in-person counseling $(87.5 \%)$. Students reported that they enjoyed interacting with students from "the other campus" and valued the opportunity to gain experience in an emerging field. Implications: The positive results from the end-of-course survey reinforced the importance of exposing students to new counseling methods. Information obtained from students' surveys provided valuable feedback for updates for future course offerings. Based on feedback from students, the potential for OTC telecounseling implementation is being investigated for use in the community pharmacy setting.

The Impact of Instagram ${ }^{\circledR}$ on Student Engagement and Learning of Infectious Diseases. Drew W. Cates, Philadelphia College of Osteopathic Medicine, Jonas Arthur, Philadelphia College of Osteopathic Medicine, Timothy Coyle, Philadelphia College of Osteopathic Medicine, Edo-abasi U. McGee, Philadelphia College of Osteopathic Medicine, Essie Samuel, Philadelphia College of Osteopathic Medicine, Vishakha Bhave, Philadelphia College of Osteopathic Medicine. Objective: To evaluate the impact of Instagram ${ }^{\circledR}$ on Doctor of Pharmacy (Pharm.D) student engagement and learning of infectious diseases in the didactic curriculum. Methods: In the 2018-2019 academic year (AY), second year pharmacy students enrolled in the Infectious Diseases course at Philadelphia College of Osteopathic Medicine School of Pharmacy (PCOM-SOP) were informed of Instagram ${ }^{\circledR}$ utilization to enhance learning. A presurvey and pre-knowledge assessment were administered on day one of the class to assess student perception and knowledge. Uniquely curated content was created and posted daily to Instagram ${ }^{\circledR}$ to increase student engagement and knowledge. Throughout the term, Instagram ${ }^{\circledR}$ posts were scheduled one to three times per day on weekdays. Upon completion of the course, a post-knowledge assessment and post-survey were re-administered to stu- dents. Iconosquare ${ }^{\circledR}$, an analytical platform, was used to determine student impressions of "comments", "likes", "saves", and "reach" on Instagram ${ }^{\circledR}$. Results: A total of 88 students were enrolled in the course. The average preknowledge assessment score was significantly lower compared to the average post-knowledge score. Throughout the term there was a total of 329 "comments", 1,100 "likes", 304 "saves", and 4,400 "reached" on Instagram ${ }^{\circledR}$. Implications: Use of Instagram ${ }^{\circledR}$ is an innovative teaching method, which has had a positive impact on student knowledge and increased engagement beyond the realm of the classroom.

The Use of Flipped Classroom Pedagogy in US Pharmacy Schools. Eugene Kreys, California Northstate University, Tiffany-Jade M. Kreys, California Northstate University, Christina Stephenson, California Northstate University, Janie $\mathrm{Yu}$, California Northstate University, Tiffannie Nguyen, California Northstate University, Shawna Evans, California Northstate University, Luis Tolento, California Northstate University, Harpreet Kaur, California Northstate University, Tiffany Wong, California Northstate University, Ravneet Kaur, California Northstate University. Objective: To evaluate the use of flipped classroom pedagogy in U.S. colleges of pharmacy. Methods: This was a cross-sectional study conducted using an anonymous eight-item multiple choice survey. The survey was administered to the deans of academic affairs of 138 active pharmacy schools in the US. The survey items included questions inquiring information on the characteristics of pharmacy schools, the type of pedagogy used within the curriculum, and to what extent these methods are used based on the general type of courses. Wilcoxon signed-rank test was used to determine the difference in the use of active-learning strategies between general course types, while the association between colleges' general characteristics and pedagogy was evaluated using multivariable logistic regression. Results: Seventy-four (54\%) of the subjects completed the survey. A total of $81 \%$ of schools employed flipped classroom pedagogy, of which $27 \%$ exclusively employed TBL, $2 \%$ exclusively employed PBL, while $72 \%$ employed a combination or another form of flipped classroom pedagogy. The implementation of flipped classroom varied depending on the general course type; with Pharmacy Practice courses demonstrating the most frequent use, followed by SAS courses $(p=0.017)$, and Basic Science courses demonstrating the least frequent use $(p<0.001)$. Smaller rather than larger schools were less likely to employ flipped classroom pedagogy with an OR of 0.15 (95\% CI: 0.02-0.97), as were private rather than public institutions with an OR of 0.16 (95\% CI: 0.03-0.95). Implications: Some use of flipped classroom is common; 


\section{American Journal of Pharmaceutical Education 2019; 83 (5) Article 7654.}

however, the frequency at which this pedagogy is used varies greatly based on the course type.

Toxicology Course Offerings by US Schools/Colleges of Pharmacy. Paniz Hazrati, West Coast University, Fred Farris, West Coast University, Mohammed A. Islam, American University of Health Sciences. Objective: The aim of this study is to assess toxicology contents/course offering in undergraduate, graduate, and professional degree (PharmD) programs in US schools/ colleges of pharmacy. Methods: The websites of 133 ACPE accredited schools/colleges of pharmacy were visited during November-December 2018. Pages with curriculum, toxicology course/content information, course catalog descriptions, were identified and retrieved. Data extraction and analysis were performed by using a systematic content analysis method. All data were entered in Microsoft Excel spreadsheet. Descriptive statistics was used for data analysis. Results: Fifty-nine percent of the US PharmD programs offer toxicology contents in didactic curriculum mainly focusing fundamental concepts of the science and practice of clinical toxicology. Thirtyeight programs $(28 \%)$ offer toxicology elective courses in an average 3.3 semester credit hour with a wider depth and breadth in contents (common toxicants, acute and chronic toxicities of different therapeutic drug classes, chemicals, and household products in humans, and role of pharmacists in poison control centers). A total of 30 programs offer MS degrees in Pharmacology and Toxicology/Toxicology with an average of 6.6 semester credits of dedicated toxicology contents. Eleven programs $(8.3 \%)$ offer toxicology contents/courses in BS programs. Thirty-two programs (24\%) offer PhD in Pharmacology and Toxicology track. Five programs were identified offering graduate certificate in toxicology. Implications: Opportunities exist to expand the offering of toxicology contents in PharmD core curriculum. our study suggests a lack of emphasis of toxicology in graduate programs. The results of the study may serve as a useful guide for pharmacy schools/colleges or other institutions to review toxicology content offerings.

Transferability of an Educational Escape Room: Student Outcomes from Two Institutions. Jeanne E. Frenzel, North Dakota State University, Heidi Eukel, North Dakota State University, Rachel J. Kavanaugh, Medical College of Wisconsin. Objective: To evaluate the impact of an educational escape room on student knowledge and perceptions when transferred between two institutions with major programmatic differences. Methods: Faculty at the Medical College of Wisconsin and North Dakota State University (NDSU) collaboratively transferred and replicated an educational escape room originally developed and assessed at NDSU. Simi- lar to North Dakota students, Wisconsin students completed a knowledge assessment one week prior to, immediately after, and six weeks after participating in the escape room to assess their baseline and retained knowledge of diabetes management, treatment guidelines, goals of therapy, and preventative screenings. Students also completed a set of questions to measure perceived value of the activity on their learning. Results: Student scores for the pre-game and post-game knowledge assessments at North Dakota $(\mathrm{p}<0.001)$ and at Wisconsin $(p<0.05)$ showed a statistically significant difference. Both student groups were administered a perception survey following participation in the educational escape room. Results suggest positive perceived engagement with and usefulness of the educational escape room. Implications: Despite significant programmatic differences including class size, class sequencing, and activity timing, the use of an educational escape room resulted in statistically significant gains in knowledge and students' perceptions at an institution to which the activity was transferred. This suggests that the educational escape room is a transferrable activity that can be used by other colleges or schools of pharmacy.

Update on Research Requirements in PharmD Curriculums - A National Survey. Marion K. Slack, The University of Arizona, Amy H. Schwartz, University of South Florida, Michael P. Dorsch, University of Michigan, Justin Cushing, The University of Arizona, Amber Cox, University of South Florida, Gillian P. Leung, University of Michigan. Objective: To quantify the number of PharmD programs that require completion of a research project. To describe the types of research being undertaken within PharmD programs. To compare current responses with those obtained from the last survey. Methods: Collaborative effort including students and faculty at three pharmacy programs (AZ, FL and MI). Cross-sectional analysis utilizing survey (with modifications) developed in 2007. Peer review of questions (facevalidity); format included dichotomous response, multiple-choice, and open response. Qualtrics ${ }^{\circledR}$ survey was distributed via email to Academic Affairs (or comparative) Deans at AACP affiliate institutions. Descriptive analyses. Project deemed exempt by IRB. Results: Seventy-four programs responded (54\% response rate). A total of $22(30 \%)$ pharmacy programs have research project requirement compared to $25 \%$ in 2007 . Project requirements were similar among programs requiring a project: proposal (95\%), IRB application $(90 \%)$, collect and analyze data (77\%), prepare written report (86\%), and present findings $(82 \%)$. Commonly utilized data collection methods included surveys $(91 \%)$ and chart reviews $(86 \%)$. Research related coursework was similar between 


\section{American Journal of Pharmaceutical Education 2019; 83 (5) Article 7654.}

programs except for Research Methods, which were 36\% for requiring versus $14 \%$ for those not requiring a project. Implications: Most pharmacy curriculums include coursework related to research. The number of programs requiring student research appears to have increased since 2007. Students with an interest in research can expect to receive equivalent experiences attending any of the programs with a research requirement. The value of student participation in research remains uncertain, however in a data-driven society, the skills will be quite useful.

Use of Concept Mapping to Deepen the Understanding of Pharmacists' Roles in Public Health. Dan Cernusca, North Dakota State University, Mark A. Strand, North Dakota State University. Objective: Pharmacists are increasingly expected to analyze new information and use the conclusions drawn to solve complex problems in the public health realm. Toward the goal of achieving ACPE standards 4.3 (Innovation) and 2.4 (Population-based care), and address these learning needs, we integrated concept mapping strategies in lectures and active learning sessions. Methods: A Public Health for Pharmacists course was redesigned from a three-hour lecture format to a one-hour lecture and a two-hour active learning case-driven segments. After the first couple of semesters, the instructor worked with an instructional designer to integrate concept-mapping both in the lecture and active learning segments. For one case study, Hepatitis $\mathrm{C}$, student teams were asked to build a concept map that synthesized the process to improve the Hep C population health. A prior-knowledge and an exit surveys focusing on student perceptions and beliefs were administered during the first semester and with the concept mapping implementation. Results: An independent-samples t-test showed that the concept mapping-cohort had a statistically significant higher mean for perceived engagement $(\mathrm{t}(167)=-2.27, \mathrm{p}<.05)$, perceived positive impact of case studies $(\mathrm{t}(167)=-2.12, \mathrm{p}<.05)$ and self-efficacy a proxy for learning performance $(\mathrm{t}(167)=-4.01$, $\mathrm{p}<.001$ ). For the concept-mapping cohort, the perceived impact of case studies had a strong positive correlation with the perceived impact of concept-mapping on own learning, $r(85)=.72, p<.001$. Implications: The implementation of concept mapping in the Public Health for Pharmacist course showed a significant impact on student engagement with the active learning tasks and on their beliefs about the effectiveness of the learning process in the course.

Using a Heart Failure Themed Escape Room in a Therapeutics Course to Enhance Learning. Roda Plakogiannis, Long Island University, Nubriel Hernandez, Long Island University, Anna Nogid, Long Island University, Abraham Stefanidis, St. John's University. Ob- jective: To assess students' perceptions of the HF Escape Room Activity Methods: Second professional year pharmacy students were taught Heart Failure pharmacology and pharmacotherapeutics prior to the escape room activity. Students were randomized into groups of approximately eight. All students received an email of their group and room assignment, with a reminder to bring one electronic device per group. At the completion of the escape room activity, students were asked to fill out a survey on their perceptions of the escape room activity. A follow-up survey of their perceptions was conducted four weeks after their participation in the activity. Results: 178 and 110 responses were collected during the first and second data collection periods, respectively. Students reported increased levels of satisfaction immediately after the completion of the escape room activity. Students agreed that the escape room activity helped them engage in team-learning, think about the material in a new way, learn from their peers, effectively review the topic of heart failure, learn new information, and that they would recommend this activity to other students. Based on the follow-up survey, conducted four weeks later, students' increased satisfaction levels toward the escape room activity were sustained. The independent samples t-test results revealed no statistically significant difference between students' perceptions reported immediately after the completion and in the follow-up survey. Implications: Students' perceptions of the escape room activity were positive and enhanced students' review of heart failure. The escape room activity is an innovative learning methodology that can be employed to effectively teach pharmacy students.

Validity of a Gaming Perception Tool for Educational Escape Rooms Across Multiple Instructional Contexts. Hannah J. Larson, North Dakota State University, Jeanne E. Frenzel, North Dakota State University, Dan Cernusca, North Dakota State University, Heidi Eukel, North Dakota State University. Objective: To determine the reliability of a gaming perception tool for use with educational escape rooms used across multiple instructional contexts including diabetes, cardiovascular, and interprofessional education topics. Methods: A faculty-developed gaming perception tool (published in AJPE) was administered at 4 institutions. One had a large cohort $(\mathrm{N}=85)$, one a medium size cohort $(\mathrm{N}=42)$ and two had smaller cohorts $(\mathrm{N}=29-30)$. Of these, 3 institutions implemented an educational escape room and used the perception tool in pharmacy courses and one in an interprofessional master-level course. Results: Analysis indicated a very strong internal reliability of all constructs for all participating groups, with Cronbach's Alpha ranging from 0.86 to 0.95 , above 0.70 , the value for strong 


\section{American Journal of Pharmaceutical Education 2019; 83 (5) Article 7654.}

internal reliability. An independent samples t-test indicated that the mean gaming perception values (4.2 to $4.8)$ were statistically significant higher $(\mathrm{p}<0.001)$ than " 3 ", the average perception. A one-way omnibus ANOVA was significant $(\mathrm{p}<0.001)$. Post-hoc analysis showed that the large pharmacy diabetes group had a mean perception statistically significant lower that all other groups $(\mathrm{p}<0.05)$. Implications: Results of the comparisons of the pooled data to individual institution data showed a positive perceived value of educational escape rooms on learning. As the class size of various institutions increased, the perceived value on learning decreased slightly. Overall, students, regardless of educational level or health profession, portrayed positive perceptions of educational escape rooms. The perception gaming tool was proven to be a reliable tool for measuring these domains.

Virtual Patient Simulation to Enhance Physical Assessment and Medical History Taking Skills of Pharmacy Students. Brooke D. Fidler, Long Island University. Objective: If utilizing a virtual simulated patient program improves the health history taking and physical assessment skills of first year professional pharmacy students. Methods: Students were assigned a virtual patient case to complete during week one and week 13 of a 14week physical assessment course. Scores from the pre and post test case were compared focusing on the ability to perform a physical exam, take a medical history and provide education and empathy. Students were also asked to self-assess their skills before and after having been exposed to the virtual program. Results: The total number of paired observations used in the final analysis of the pre and post virtual test case was 171 students. The student performance score, which included the ability to obtain subjective and objective data, provide patient education and display empathy, improved from $52 \%$ at week 1 to $78.42 \%$ at week $13(\mathrm{p}<.0001)$.There was a significant increase in students' perception of their ability to take a patient's medical history from the beginning to the end of the semester $(57.37 \%$ vs. $82.68 \%, \mathrm{p}<.0001)$. Overall, $76.54 \%$ of students strongly agreed or agreed that the virtual patient program made them feel more confident in their medical history skills when they begin their introductory community pharmacy practice experiences. Implications: While the value and best way to implement simulation in pharmacy education continues to be investigated, use of virtual simulation in one of our required courses improved students' ability and confidence in obtaining information during a health history and performing a physical exam.

Virtual Versus Paper-Based Cases to Reinforce the Pharmacists Patient Care Process (PPCP). Jonathan Newsome, The University of Texas at Tyler, Takova
Wallace-Gay, The University of Texas at Tyler, Osama A. Shoair, The University of Texas at Tyler, Nofisat Iginla, The University of Texas at Tyler. Objective: To compare the use of virtual vs. paper patient cases to reinforce the Pharmacists' Patient Care Process (PPCP). Methods: This was a randomized, crossover study that was conducted in an ambulatory care elective course. Students completed both paper- and virtual-based patient cases that emulated the PPCP over 6 weeks. Students were randomly assigned to either complete paper-based cases for weeks 1-3 then virtual-based cases for weeks 4-6 or vice versa. At the conclusion of each 3-week period, students were surveyed to assess their satisfaction with the instructional method they experienced during that 3 -week period in reinforcing the PPCP. Additionally, a focus group was conducted to qualitatively evaluate students' experience with virtual and paper cases. Results: There were 27 students who were recruited and completed the study. There were no statistically significant differences in students' satisfaction with the two instructional methods except that students agreed more that paper cases improved their ability in collecting information about patients. Students' performance on the parts of the cases related to the 'Assess' element of the PPCP was higher using paper cases. However, during the focus groups, students reported that virtual cases promoted their critical thinking and ability to practice collecting information about patients more than paper cases. Furthermore, students stated that virtual cases forced them to recognize and document relevant information. Implications: Though there were mostly no significant differences in students' satisfaction with either instructional method in reinforcing the PPCP, students reported that virtual cases provided a more realistic and engaging experience.

\section{Theoretical Models}

A State-Wide Community Pharmacy Enhanced Services Training Program: Pennsylvania Case Study. Nicholas Leon, Thomas Jefferson University, Nicole Pezzino, Wilkes University, Suzanne Higginbotham, Duquesne University, Elizabeth Harris, University of the Sciences, Anisha Grover, Temple University, Rebecca M. Wise, Lake Erie College of Osteopathic Medicine, Gina Bellottie, Thomas Jefferson University, Melissa A. McGivney, University of Pittsburgh. Objective: To describe how all seven schools of pharmacy in Pennsylvania (PA) collaborated to develop a community pharmacy enhanced services training program for the Pennsylvania Pharmacists Association (PPA) and the CPESN Pennsylvania Pharmacists Care Network (PPCN), and the subsequent incorporation into each school's curriculum. Methods: In 2017, the University of Pittsburgh School 


\section{American Journal of Pharmaceutical Education 2019; 83 (5) Article 7654.}

of Pharmacy and PPA, led a coalition of faculty representatives from all seven PA schools to develop the third edition of the PPCN training that includes a 6-hour on-line home study and 3-hour live training. Faculty collaboratively developed the 6-hour on-line home study materials and serve as trainers for the 3-hour live training for pharmacists and students. In 2017, PPCN launched its first payer contract, and the training continues to be a requirement to patriciate in the provision of billable patient care. Students who are trained are able to support PPCN pharmacists. Results: In 2017, 299 students completed the training from three schools. In 2018, 571 students completed the training program from five schools. In 2019, all seven schools will be incorporating the training program into their curriculum. Six schools or colleges have implemented the training in the required curriculum; one school offers an elective. Implications: The community pharmacy landscape is a rapidly changing environment employing more pharmacists than any other setting. This Pennsylvania case study represents a successful collaboration between a state association and academic pharmacy (entire state), working together, to simultaneously advance current community pharmacy practice while helping to facilitate the training of new graduates for contemporary practice.

Creation of a Human Resource Blueprint: An Important Consideration for a New or Revised Curriculum. Kristin A. Casper, The Ohio State University, Katherine A. Kelley, The Ohio State University, Maggie A. Merry, The Ohio State University, Marcia M. Worley, The Ohio State University. Objective: The concept of faculty workload has been studied in both the pharmacy and educational literature. Understanding how faculty workload, particularly in the area of teaching and learning, can be impacted by curricular revision has not been evaluated. In addition, the curricular change process is complex, with the primary focus often on content delivery instead of resource needs. The objective of this project is to create a curricular resource blueprint to better predict the human resource needs of a new or revised curriculum. Methods: A literature search was conducted to determine best practices for calculating faculty workload around teaching and learning. These concepts were used to generate a model to predict overall human resource needs for a significantly revised curriculum. Student contact hours were used as the common denominator. Additional modifiers of effort included utilization of active learning, introduction of new technology and classroom space, curricular integration, and modular structure of the curriculum. Results: The curricular resource blueprint was applied to the new curriculum following the final year of implementation, with opportunity for faculty input. Additional human resource needs were identified, as was the need to more clearly define the roles of faculty and other specialized support personnel. Implications: The use of a curricular resource blueprint for predicting human resource needs is an important component of curricular revision that can often be overlooked. Utilization of this model in the early stages of curricular revision could prove to be beneficial for implementation and understanding of faculty workload.

Creation of a Mentorship Program in the Pharmacy Practice Section. Allison M. Bell, University of Cincinnati, Jennifer Ball, South Dakota State University, Seena L. Haines, The University of Mississippi, Kristi W. Kelley, Auburn University, Rebecca Schoen, Texas Tech University Health Sciences Center, Susan E. Smith, The University of Georgia. Objective: To describe a conceptual framework for creation of an inaugural mentorship program within the AACP Pharmacy Practice (PP) section. Methods: An 18-member task force was convened within the PP section and charged with developing and implementing a formal mentorship program. Four subteams were devised to apply an evidence-based approach to this charge. Each sub-team had specific objectives: 1) mission/vision, 2) structure/content, 3) assessment/impact, and 4) dissemination/scholarship. Results: The mission of the AACP-PP Mentor Program is to provide a senior faculty support system focused on personal and professional development of more junior faculty and to create networking opportunities, with the goal of career development in teaching, scholarship, service, and best practices for academia. The vision is to establish a leading mentorship program for AACP members to promote the development of junior faculty to their fullest potential. The task force is following a systematic process to determine structure/expectations, content areas, mentee needs, capacity/scalability, and targets/goals of the program. A unique programmatic element is integration and maintenance of the program between the task force, PP section, and Association. Implications: Mentorship programs have been described in other disciplines and are most often implemented within a single institution. This program builds upon exemplars and challenges of existing programs by providing structured framework for developing and implementing a program that meets the diverse needs of a professional association's membership. The program will begin implementation in 2019 and be evaluated for short-term and long-term successes related to recruitment, retention, and mentor/mentee development.

Curation and Praxis of Active Learning: Gagné's Events of Instruction for Teaching Blood Pressure Measurement. Jack J. Chen, Principal Consultant, Herman Johannesmeyer, Marshall B. Ketchum University. Objective: To create a high impact instructional 


\section{American Journal of Pharmaceutical Education 2019; 83 (5) Article 7654.}

framework blending active learning (learnercentered constructivist) with Gagné's learning model (teacher-centered cognitive) in the provision of a curated learning experience for auscultatory blood pressure (BP) measurement. Methods: Lesson planning, instructional flow, and activity sequencing was based on Gagné's cognitive learning theory and nine events of instruction (see supplemental handout). Active learning fulfilled the definition by Bonwell and Eison. Learning outcomes were based on Bloom's 2001 revision and Simpson's taxonomies for cognitive and psychomotor domains, respectively. Rubric-based measurement of learning outcomes was performed by direct observation during an objective structured clinical examination (OSCE). High impact practices for each of nine events was derived by experience and empiric observation. Instructor agency was guided by knowledge building principles. Results: All students $(n=40)$ accessed the pre-session reading and video material. Four instructors served as session facilitators. Pairing students into dyads (20 pairs) facilitated peer teaching and empathy during face-to-face blood pressure measurement simulations. Utilization of rubric-based assessment with directed observation aided by teaching stethoscopes during an OSCE improved fidelity of assessment. Two students (5\%) failed to achieve required proficiency in BP measurement. During and after the lesson, students reported transient performance anxiety but overall enhanced knowledge and skill retention with the given instruction, flow, and formative/summative assessments. Implications: This pedagogic approach embeds Bonwell and Eison's constructivist active learning within Gagné's cognitive learning model and nine steps of instruction to provide curation and design of learning for BP measurement. The model is generalizable to other skills topics.

Describing a Renal Function Assessment Methodology Model and Application in a Patient Care OSCE. Rebecca Leon, California Health Sciences University, Will Ofstad, California Health Sciences University, David G. Fuentes, California Health Sciences University, Yuqin Hu, California Health Sciences University, Alexis Woods, California Health Sciences University, Jeremy A. Hughes, California Health Sciences University, Maya Leiva, California Health Sciences University. Objective: Educators can integrate methodologies and rubrics to help students develop skills to apply what they know to authentic case scenarios and prepare them for practice in ways that can be more easily assessed during an objective structured clinical examination (OSCE). This model offers a stepwise methodology to develop/assess students' performance, identify and manage renal drug problems, recognize risk factors impacting renal function, estimate renal function, and perform renal dosing adjustments. Methods: Faculty teaching our therapeutics course reviewed the process education methodology development literature and created a three-phase model for learners to engage in a step-wise approach to renal function assessment. The methodology and rubric that could be used for any scenario was developed and reviewed by course faculty and used during a class team application on vancomycin dosing. Students were assessed on their ability to apply it during a high-stakes OSCE focused on addressing methotrexate and anti-hypertensive medication problems. Results: P2 students $(n=77)$ applied the renal function assessment methodology to a practice case in class and during the workup of a complex case as part of an OSCE. $83 \%$ of students successfully applied the entire renal methodology on the first attempt, and $100 \%$ of students were successful after one remediation session. The methodology and drug-independent rubric will also be presented. Implications: Use of the renal function assessment methodology was successful in helping students approach making clinical decisions based on a patient's renal function. This model is general and can be applied to other cases and curricula.

Objective Structured Clinical Exam Development and Curricular Mapping for Pre-Advanced Pharmacy Practice Experiences. Jordan Sedlacek, Larkin University, Andria F. Church, Palm Beach Atlantic University, Sandra B. Caballero, Larkin University. Objective: The Pharmacy Outcomes Curriculum Assessment (PCOA) is usually administered immediately prior to students beginning advanced pharmacy practice experiences (APPE). The PCOA measures basic pharmacy knowledge while not completely assessing basic clinical skills, which is critical for success in APPEs. A pre-APPE objective structured clinical examination (OSCE) can help identify the skills in which a student is deficient, particularly in areas such as patient-centered care, communication, education, and professionalism. Despite the implementation of OSCEs in pharmacy curricula, minimal information is known on the relationship between OSCE performance and APPE success. The pre-APPE OSCE developed at our 3-year accelerated program was intended to evaluate students' preparation for the clinical component of APPEs. Methods: Fifteen OSCE stations were developed which varied in length from 5 minutes to 40 minutes each. Five stations required in-person faculty evaluators. All stations were evaluated in ExamSoft ${ }^{\circledR}$. The final station was a clinical note graded by faculty based on a predefined rubric. All station assessments were mapped to program learning outcomes (PLOs). Faculty peer evaluated stations prior to the OSCE administration. Results: This project describes the implementation of a pre-APPE OSCE in an 


\section{American Journal of Pharmaceutical Education 2019; 83 (5) Article 7654.}

accelerated curriculum. Steps required for development of a successful end of didactic curriculum OSCE require planning, peer evaluation, and curricular mapping. The outcomes of the OSCE based on PLOs identifies areas for improvement in the curriculum. Implications: This model for implementation of the OSCE has shown promise regarding assessing students' clinical skills prior to APPEs. The curricular mapping also allows the program to identify areas for didactic improvement.

\section{SOCIAL AND ADMINISTRATIVE SCIENCES Completed Research}

A Mediation Analysis of PCAT, Grades, and PCOA. Aaron O. Thomas, University of Florida, Shauna Buring, University of Florida. Objective: An examination of the direct and indirect effects of the PCAT upon GRADES and PCOA scores. Methods: Using historical PCAT, PCOA, and course data, a mediation analysis was employed to model the relationship between PCAT (prior-knowledge) to both GRADES (curriculum knowledge) and PCOA (competency knowledge) with one cohort of 1PD students $(\mathrm{N}=219)$. This study employed a weighted average for course grades which provided statistical and practical value. Results: PCAT as a predictor of PCOA overall scaled scores accounted for $22.8 \%$ of the variation $\mathrm{F}(1,218)=63.878, \mathrm{p}<.0001$. When PCAT and GRADES were combined into a multiple regression, $47.8 \%$ of the variation in PCOA overall scaled scores was explained $\mathrm{F}(2,217)=98.579, \mathrm{p}<.0001$. For every five points of PCAT composite score increase, PharmD grades improved by one percentage point approximately. A change in PharmD grades by one percentage point was estimated to result in a 3.976 increase in PCOA overall scaled score which translated to a 1 and 4 percentiles change with the greatest impact to lower performing students. Indirect effects of PCAT upon PCOA (.787 [95\% CI: $.5545,1.041])$ were greater than total effects $(\mathrm{c}=.6645)$ supporting the mediation model. Implications: The findings support the predictive power of the PCAT composite score upon grades and PCOA, thus supporting the use of PCAT in admissions. Mediation models can be used to evaluate the impact of PCAT as a measure of priorknowledge that has direct effects upon GRADES and indirect effects upon PCOA.

A New Approach for Increasing PharmacistPatient Communication Effectiveness. Nathaniel M. Rickles, University of Connecticut, Marissa C. Salvo, University of Connecticut, Paul D. Tieger, iConnectHealth. Objective: To demonstrate the implementation of a 3-part communication style profile program to help student pharmacists tailor their patient communications using information about self and patient communication styles. Methods: Eighty-three students in a required first professional year course in pharmacy communications participated in the first part of the communication style profile program: (1) completion of a validated online Preferred Communication Style Questionnaire (PCSQ) that assesses students' communication style; and (2) review of an automatically generated and personalized Communication Success Profile (CSP) that identifies their innate communication strengths and potential blind spots. After completing this first part, a background survey was administered $(n=70)$. Students participated in the program's second part involving a live lecture about communication style profiles. A second survey $(n=74)$ elicited feedback about this lecture. The program's third part involved small group role-playing exercises. A final survey $(n=54)$ elicited feedback on the entire program. Surveys were linked by identification numbers. Data were analyzed using descriptive and bivariate statistics. Results: $89 \%$ of those who completed the PCSQ reported their communication style profiles were "extremely" or "very accurate." Across all surveys, the 3-part program significantly increased respondent awareness of their communication style and its importance. Approximately $70 \%$ reported it would be very or extremely valuable to include this type of training in the pharmacy communication course. Implications: More pilots are needed to generalize findings to other pharmacy education programs and to determine if this communication style profile program impacts communication effectiveness, the pharmacist-patient experience, and patient outcomes.

An Innovative, Active Learning Approach to Teaching Pharmacy Students About Social Determinants of Health. Chamika Hawkins-Taylor, South Dakota State University, Brittney A. Meyer, South Dakota State University, Teresa M. Seefeldt, South Dakota State University, Samuel Joseph Koob, South Dakota State University. Objective: To evaluate the impact of an active learning board game on pharmacy students' social determinants of health $(\mathrm{SDoH})$ empathy and knowledge. Methods: P1 students attending pharmacy skills lab and P3 students in a Public Health course took a 21-question survey before and after playing a SDoH board game. Four instructors were trained and facilitated the game during three, 2-hour labs and one, 3-hour class (split into two 1.5hour sessions).Students paired up within groups of 6-10 players and created a single character profile with race, ethnicity, sex, and sexual orientation. Coins indicated health status. Teams advanced through life stages with the roll of a die and lost or gained coins depending on their SDoH and life-situations encountered. Discussion occurred throughout with debriefing following the game. Paired T-tests assessed the change in scores following 


\section{American Journal of Pharmaceutical Education 2019; 83 (5) Article 7654.}

game participation. Students, presented with a list of CDC-defined SDH, selected those they considered SDoH. Pre- to post- SDoH identified were summarized descriptively. Results: In total, 154 students (81 P1, 73 P3) participated in the game, with $50 \%$ over age 22 . Females outnumbered males $3: 1$. Regardless of class, a statistically significant improvement in mean empathy and knowledge scores were discovered $(\mathrm{p}<0.005)$. Transportation as a SDoH had the largest change (13\%) with $77 \%$ of students recognizing it before and $90 \%$ after. Implications: A SDoH board game provides an innovative, active learning approach to expose pharmacy students to $\mathrm{SDoH}$, which is expected to enhance patient connections and stimulate critical thinking about the impact of SDoH on patient health goals.

A Pre-Matriculation Program (PMP) Improved Baseline Knowledge and Retention Rates for FirstYear Underrepresented Minority Pharmacy Students. Amanda Galvan, Texas A\&M University, Simi Gunaseelan, Texas A\&M University, Elaine L. Demps, Texas A\&M University, Amanda Galindo, Texas A\&M University, Juan J. Bustamante, Texas A\&M University, Lacy Daniels, Texas A\&M University, Hamed I. Ali, Texas A\&M University, Mohammad T. Nutan, Texas A\&M University, Nephy G. Samuel, Texas A\&M University, Steven L. Peterson, Texas A\&M University. Objective: To evaluate the effectiveness of a pre-matriculation program designed to ameliorate disparities of underrepresented minority (URM) students in their baseline knowledge necessary to progress during the first year of pharmacy school. Methods: A six-week, face-to-face and online pre-matriculation program (PMP) was initiated to enhance retention rates of URM students. The PMP was constructed with five subject areas - biochemistry, organic chemistry, pharmaceutical calculations, physiology, and medical terminology; the prime areas determining the readiness of pharmacy students during their P1 year. This PMP was carried out by five pharmacy faculty subject experts who constructed the self-paced modules with built-in assessments. Quantitative preand post-PMP data were collected from these five content-based readiness assessments. The post-PMP readiness assessment results were compared to the remaining incoming first-year students' readiness assessment results. Upon completion of the PMP, the participants' success in the first semester was tracked and compared to the percentage of students who were dismissed at the end of the first semesters in the previous years. Results: The implementation of the PMP resulted in enhanced increase in participants' knowledge in all the five subject areas. Participants' mean percentage score positively shifted at post-PMP (70.32\%) when compared to their pre-PMP performance $(52.03 \%)$ and performance of non-PMP participants $(57.2 \%)$. The number of URM students dismissed at the end of the fall 2018 semester was reduced by $50 \%$ when compared to the previous fall semesters. Implications: Implementation of a pre-matriculation pharmacy program can significantly decrease the number of dismissals of first-year URM students while enhancing their retention rates.

Assessing Preceptor Use of Cognitive Apprenticeship: Is the Maastricht Clinical Teaching Questionnaire Appropriate? Michael D. Wolcott, University of North Carolina at Chapel Hill, Anne M. Rodino, University of North Carolina at Chapel Hill. Objective: To evaluate the utility of the Maastricht Clinical Teaching Questionnaire (MCTQ) to assess preceptor use of cognitive apprenticeship teaching methods in pharmacy practice. Methods: Student pharmacists and pharmacy residents at a large academic medical center completed the MCTQ to evaluate pharmacist preceptors. A confirmatory factor analysis (CFA) was used to determine how consistent the data were with the five-factor structure of the cognitive apprenticeship teaching methods (eg, modeling, coaching, articulation, exploration, and safe learning environment). A structural equation model (SEM) was used to evaluate the relationship between the five factors based on theoretical and previous empirical research. Preceptor and student perceptions were also collected to determine the relevance and utility of the instrument in practice. Results: Thirty-eight learners (second-year and fourth-year student pharmacists and pharmacy residents) submitted 157 evaluations. The CFA showed appreciable fit with the five-factor structure of the cognitive apprenticeship framework after two modifications (removal of one item and moving one item to a different factor). The SEM had poor model fit compared to previous studies and an exploratory analysis suggests there is a complex relationship between the various teaching methods. Preceptors and students highly agreed the instrument was valuable for providing feedback. Implications: The MCTQ is a viable tool to assess how well pharmacy preceptors engage in teaching methods that are consistent with the cognitive apprenticeship framework. Additional research is warranted to further develop and validate tools for reliable preceptor evaluation and to inform future applications of cognitive apprenticeship within pharmacy education.

Assessing Student Characteristics to Identify Predictors of Student Leadership Engagement. Marina Dykhne, West Coast University, Shih-Ying Hsu, West Coast University, Sarah McBane, West Coast University, Ettie Rosenberg, West Coast University, Reza Taheri, RxPrep. Objective: To determine correlation of peer- 


\section{American Journal of Pharmaceutical Education 2019; 83 (5) Article 7654.}

evaluations, learning styles, and critical thinking with student leadership engagement (SLE) in pharmacy students. Methods: This study included four cohorts of pharmacy students. Each student completed the Kolb Learning Style Inventory (LSI) and Health Science Reasoning Test (HSRT) during orientation. Peer evaluation scores were obtained from 3 separate courses throughout P1 to P3 year. Predefined criteria for leadership were determined and correlation between leadership and LSI, HSRT, and peer evaluation scores was assessed. Descriptive analysis was conducted while independent groups t-test was performed to test the group difference on continuous outcomes. Differences in LSI between the two groups were assessed using Fisher exact test. This study is Institutional Review Board approved. Results: This study included 219 pharmacy students from Classes of 2018 to 2022; 62 students (28\%) had SLE. No significant differences were found in the peer evaluation scores between students with and without SLE. Students with SLE showed significantly higher scores in all eight specific domains of the HSRT and its overall score $(p<0.005)$. No significant difference was found between the two groups for the Kolb's learning styles and learning flexibility scores. Implications: Early prediction of SLE in the program provides Schools/Colleges of Pharmacy an opportunity to better equip future leaders of the profession. Of the variables assessed in this study, a test of critical thinking skills (HSRT) was highly correlated with leadership engagement. Other variables, that are broadly used across the academy, need to be investigated as predicators of leadership engagement.

Association of Physical Activity and Academic Performance in an Accelerated Pharmacy Curriculum. Daniel L. Austin, Lake Erie College of Osteopathic Medicine, Janene M. Madras, Lake Erie College of Osteopathic Medicine, Rachel R. Ogden, Lake Erie College of Osteopathic Medicine, Benjamin P. Vroman, Lake Erie College of Osteopathic Medicine, Christina Hanson, Lake Erie College of Osteopathic Medicine, Dave Hopkins, Lake Erie College of Osteopathic Medicine. Objective: The purpose of this study was to determine if there is a relationship between academic performance of professional students enrolled in the Lake Erie College of Osteopathic Medicine (LECOM) School of Pharmacy three-year accelerated pathway and their utilization of the LECOM Wellness Center. Methods: This retrospective study included first- and second-year pharmacy students enrolled in the three-year accelerated program. Total number of Wellness Center visits during the academic year, as well as a voluntary student survey were used to obtain physical activity information. Average grade point averages (GPA) for respective academic years were used as indicators of academic performance. Results: The number of visits was not significantly correlated with student GPA. Of the 268 students included in analysis, 43\% made 10 or fewer visits during the academic year. Of the 118 students who completed the survey, 56\% reported overall decrease in activity as a pharmacy student, $58 \%$ reported fluctuation in activity level throughout the year, $58 \%$ reported decreased activity when experience stress associated with academics, and $47 \%$ reported utilization of the Wellness Center for majority of their physical activity. Implications: This analysis was limited by low student utilization of the Wellness Center. Additionally, the timespan of available visit data may have obscured trends within smaller timeframes, as the majority of survey respondents indicated fluctuations in activity throughout the year. To build upon this exploratory work, further study is necessary to determine if and how behaviors pertaining to physical activity are significantly associated with academic performance of pharmacy students.

Associations Between Food Purchasing Decisions and Weight Status Among College Students. Cassidi N. Crosby, Auburn University, Kimberly B. Garza, Auburn University. Objective: To assess the food purchasing behaviors among underweight/healthy weight students compared to overweight/obese students on a college campus. Methods: An online survey was distributed via email to a purposive sample of 8000 students on a college campus to assess associations between weight status and food purchasing behaviors, including foods away from home, use of nutrition information, and considerations while grocery shopping. Participants were classified as either underweight/healthy weight $(\mathrm{BMI}<25)$ or overweight/obese (BMI $>=25)$ based on self-reported height and weight. Descriptive statistics and Chi-squared were used for analysis. Results: Of 514 students who completed surveys, $65 \%$ were underweight/healthy weight, and $35 \%$ were overweight/obese. Overweight/obese students were more likely to eat at fast food or pizza places than underweight/healthy weight students $(\mathrm{p}=.003)$. Underweight/healthy weight students were more likely to use nutrition information in making purchasing decisions at restaurants with wait staff $(\mathrm{p}=.030)$. Weight status was not associated with reasons for eating at fast food/pizza places, such as lower cost, nutrition, taste, convenience, or socializing. Weight status was not associated with the use of the Nutrition Facts panel or ingredients lists to make food purchasing decisions. There was no association between weight status and consideration of price, nutrition, taste, or ease of preparation while grocery shopping. Significantly more overweight/obese students $(86 \%)$ than underweight/healthy weight students $(78 \%)$ reported shelf life as an important consideration while 


\section{American Journal of Pharmaceutical Education 2019; 83 (5) Article 7654.}

grocery shopping $(\mathrm{p}=.027)$. Implications: These factors related to food purchasing behaviors should be considered when developing interventions to improve diet targeted toward overweight/obese college students.

Can We Find Pharmaceutical Calculations Low Performers Before Class Starts? Identifying Problem Solving Deficiencies. Benjamin D. Aronson, Ohio Northern University, Emily Eddy, Ohio Northern University, Jennifer Grundey, Ohio Northern University, Brittany L. Long, Ohio Northern University, Jessica L. Hinson, Ohio Northern University, Kristen N. Sobota, Ohio Northern University. Objective: To determine the relationship between an algebra-based word problem pretest and pharmaceutical calculations performance to identify those at risk of low performance. Methods: First-year students in a 0-6 direct-entry PharmD program were screened using a pretest for ability to solve algebraic word problems using proportional reasoning, unit analysis, and percent. Pretest scores were compared with scores on 3 summative calculations assessments from the fall of their second year. Linear regression was used to understand the relationship between pretest and calculation assessment scores after controlling for demographic and pre-admission factors. Results: Consent was obtained from 118 students. The mean pretest score was $15 / 18$, ranging from 5 to 18 . The mean total calculations score was $116 / 150$. Pretest scores correlated with subsequent total calculations points. This relationship remained significant after controlling for age, gender, high school grade point average, and ACT math, science, and English sub-scores $(\mathrm{p}=0.008)$. Cutoff scores of the pretest are explored to determine the sensitivity, specificity, and accuracy of the pretest to identify those at risk of failing. Implications: This work demonstrates that scores on a pretest aimed at identifying deficiencies in algebra-based math skills are associated with grades on later summative pharmaceutical calculations assessments, even after controlling for age, gender, earlier grades, and standardized test scores. The next step in this line of inquiry is to determine how to reduce this deficit through deliberate supplementary content and structured problem-solving activities for those in need.

Collaboration Among Ambulatory Care Faculty Results in Multi-Center GLP-1 Impact Study. Judith T. Barr, Northeastern University, Tayla Rose, Northeastern University, Michelle Jacobs, Northeastern University, Debra J. Reid, Northeastern University, Carla Bouwmeester, Northeastern University, Michael Conley, Northeastern University, Borna Fatehi, Northeastern University, Thomas M. Matta, Northeastern University. Objective: Conduct an eight-center, ambulatory care study to evaluate the impact on monthly glucose-lowering medication cost, HbA1c, weight, and polypharmacy after adding a glucagon-like peptide-1 receptor agonist (GLP-1) to medication regimens of patients with uncontrolled diabetes. Methods: Ambulatory pharmacy faculty at Northeastern University practicing at 7 federally qualified community health centers and a Program of All-inclusive Care for the Elderly collaborated to design and conduct a retrospective, pre-post cohort $(n=120)$ study. Agreed upon standards were developed for: baseline and 6month-post-GLP-1 initiation periods, demographic and clinical eligibility criteria, and outcome variables (primary: changes in glucose-lowering drug costs, HbAlc, weight) and (secondary: polytherapy impact -- name, dose, number of daily doses or injections). Electronic medical record data were collected at initiation of GLP1 and after 6-12 months. Bivariate and multivariate analyses were conducted to compare changes. Monthly faculty meetings monitored study progress, data analysis, and manuscript development. Results: Study population was largely female, age $55.8 \pm 11.7$ years, obese, $71 \%$ minority, equal English/non-English speaking, with high pill and injection burden, and 10\% baseline HbAlc. Monthly glucose-lowering medication cost increased $\$ 586.85$ (overall), $\$ 741.68$ (oral-only baseline regimen), and $\$ 530.55$ (insulin + oral baseline regimen) (all $\mathrm{p}<$ 0.001). Overall HbA1c decreased 1.7\% (18 mmol $/ \mathrm{mol})$ $(\mathrm{p}<0.001)$ and was consistent across subgroups. Weight decreased overall $(-1.8 \mathrm{~kg}, \mathrm{p}<0.001)$. A significant shift occurred toward fewer oral agents, insulin, and daily injections. No meaningful outcome differences were associated with age, gender, English-speaking status, nor race. Implications: This multi-site pharmacy faculty collaboration, leveraging intra-institutional faculty partnerships to conduct clinical research, produced data evaluating GLP-1 initiation.

Communicating with Clinicians on Fasting during Ramadan: The Patients' Perspective. Mohamed E. Amin, Beirut Arab University, Ahmed Abdelmageed, Manchester University, Marwa Farhat, Manchester University. Objective: Caring for millions of Muslim patients who decide to fast during Ramadan can be challenging for clinicians. Using a theory guided approach, this study explores Muslim patients' perspectives on factors influencing communication with clinicians in relation to fasting during Ramadan. Methods: Semistructured interviews were conducted with a purposeful sample of patients in Egypt (9) and the US (8). Participants were purposefully sampled to assure variance in age, gender, education, decision to fast and ethnicity. Data emerging from narratives were mapped to constructs within the Linguistic Model of Patient Participation in Care (LMOPPC). Using framework analysis, iterative 


\section{American Journal of Pharmaceutical Education 2019; 83 (5) Article 7654.}

sampling and analysis continued until saturation. Results: Drawing on LMOPPC, participants' narratives were mapped and clustered into predisposing factors (perceptions about fasting and its significance including sense of spiritual benefit when fasting and sense of guilt when not fasting, prior experiences including prior conversations with clinicians on fasting and experience of fasting while sick, patients' personality and locus of control, belief in the legitimacy of participation, motivations and perception of need to communicate with clinicians about fasting, provider verbal and nonverbal responses and provider-patient rapport) and enabling factors (knowledge about the topic and repertoire of communicative skills, presence of companions during appointment and timing of appointments). Implications: LMOPPC framework provided insight into patients' perspectives in barriers and facilitators for communication with clinicians about fating during Ramadan. It is especially important for pharmacists and pharmacy educators to with consider those issues when implementing interventions aiming to provide better care for this group of patients.

Comparing Project Quality of Groups Assigned Using Strengths Quest vs. Self-Selection. MaRanda K. Herring, Harding University, Sarah Steely, Harding University, Kassandra Fetz, Harding University. Objective: Research comparing outcomes of self-selected student groups to strategically created StrengthsQuest groups in pharmacy curricula is lacking. The purpose of this project was to compare the quality of work performed by students assigned to groups using StrengthsQuest with students who self-selected groups for a longitudinal, high stakes business plan project. Methods: This project was approved by the IRB. Informed consent was acquired from all participants. Students enrolled in a core, pharmacy practice management course were asked to self-select working groups. Afterwards, to ensure blinding, a third party used a random number generator to identify half of the groups to be reassigned and utilized the students' Gallop StrengthsQuest strengths to ensure each group included a student with each of the following predominant themes: executing, influencing, relationship building, or strategic thinking. Groups completed a longitudinal, high stakes business plan project, and grades were compared. Results: On average, students in StrengthsQuest groups performed $5.99 \%$ better on the business plan project and $2.07 \%$ better in the course than students who self-selected groups. A statistically significant difference was shown in comparing overall project grades $(\mathrm{p}<0.05)$ but not final course grades. Implications: Best practices suggest heterogeneous groups are best for team-based assignments but offer no guidance on how best to assign such groups. Our study shows observable differences in performance when groups were assigned using Gallop StrengthsQuest compared to self-selected groups. Further study is needed to determine if this strategy would improve student learning in other courses that utilize teamwork-based learning activities, like pharmacotherapy.

Developing Student Empathy in a Personal and Professional Development Course. Rosalyn P. Vellurattil, University of Illinois at Chicago, Nicole KaminskiOzturk, University of Illinois at Chicago. Objective: UIC College of Pharmacy implemented a new core course in personal and professional development (Phar 463) for second year pharmacy students. Teaching and learning centered on social competence (i.e., social skills, empathy) is included. The objective is to assess student learning on empathy and to evaluate their individual growth and self-awareness. Methods: The 15-item Kiersma-Chen Empathy Scale (KCES) was administered pre/post a class session delivered on social competence utilizing clinical role-playing scenarios for active learning. Students indicated their level of agreement or disagreement with each item $(1=$ strongly disagree, $2=$ disagree, $3=$ somewhat disagree, $4=$ neutral, $5=$ somewhat agree, $6=$ agree, and $7=$ strongly agree), with four items coded in reverse. Pre/post-item bias interaction analyses were conducted using FACETS ${ }^{\text {TM }}$. A Multi-Faceted Rasch Measurement model examined the difference between students' pre/ post survey results on a logit scale. Results: The KCES was administered to all students enrolled in Phar 463 in Fall 2018, 141 students provided both pre and post responses ( $72 \%$ of the class). Of the 15 items, 9 were found to have a significant level of bias interaction $(\mathrm{p}<0.05)$ between pre/post administrations, and item responses. The largest difference observed at 0.64 logits $\mathrm{t}(267)=$ $3.90(\mathrm{p}<0.001)$ was found in item 14 addressing practitioner perspective. Item 11 related to having difficulty identifying with someone's feelings exhibited the least difference at 0.18 logits $\mathrm{t}(278)=-2.39(\mathrm{p}<0.05)$. Implications: The class session on social competence supported students' understanding of patient perspectives, and comprehension of their experiences. Students showed least significant growth in identifying someone else's feelings; a skill to be further developed as they progress through the curriculum.

Disability and Accommodations in Pharmacy Practice and Education. Michaela M. Almgren, University of South Carolina, Whitney Maxwell, University of South Carolina, Amy D. Grant, University of South Carolina, Amee Shah, University of South Carolina, Heather Hembree, University of South Carolina. Objective: Colleges of pharmacy are tasked with producing graduates who provide competent pharmacist-delivered care, while Boards of Pharmacy (BOPs) are responsible for 


\section{American Journal of Pharmaceutical Education 2019; 83 (5) Article 7654.}

protecting the health, safety, and well-being of their constituents. As learners with disabilities are now formally identified at colleges of pharmacy, challenges arise of providing equitable, high-quality education for all students, while simultaneously protecting the public. As there is currently little published data related to the process of reasonably accommodating these individuals, we have undertaken a multi-part research study to assess the required capabilities of practicing pharmacists, the prevalence of disabilities being reported by colleges of pharmacy, and the types of accommodations provided for these learners. Methods: The first phase of research was an assessment of the technical standards and physical capabilities required by BOPs in the United States. BOPs in all 50 states were contacted by email and follow-up phone calls to assess technical standard requirements for licensure and allowable accommodations. Results: Only 2 of the 29 responding Boards of Pharmacy (7\%) indicated that pharmacists would need to demonstrate any level of physical capability for licensure. Accommodation options were typically not well defined. Additionally, two states required a practical compounding exam. Implications: Licensure requirements through BOP technical standards do not appear to pose substantial barriers for pharmacy graduates with disabilities. Further study into the prevalence of disabilities and accommodations being provided to learners within colleges of pharmacy is also needed to assess for potential barriers.

Does Your Teaching Count? A Frequency-Based Instrument for Peer Evaluations of Teaching. Jacqueline McLaughlin, University of North Carolina at Chapel Hill, Amanda Olsen, University of North Carolina at Chapel Hill, Skye Zambrano, North Carolina State University, Antonio Bush, University of North Carolina at Chapel Hill, Jacqueline M. Zeeman, University of North Carolina at Chapel Hill. Objective: The purpose of this study was to examine the psychometric properties of the Teaching Practices Inventory (TPI), an instrument designed to measure the frequency of evidence-based practices utilized by a classroom instructor. Methods: Participants classified as experts (e.g., individuals with a doctoral degree, or those who have taught college courses) and novices (e.g., individuals without a doctoral degree, students, and those who have not taught college courses) were recruited to complete a modified version of the TPI while watching a recorded biostatistics classroom lecture within a Doctor of Pharmacy (PharmD) curriculum. The TPI instrument was further modified using Round 1 participant feedback and was administered to a different group of expert and novice teachers for Round 2 . Interrater reliability (IRR), Mann Whitney/independent samples t-tests, and chi-square analysis were used to an- alyze the data. Results: The IRR for the categorical data between the first and second rounds increased from 0.33 to 0.60 . For the continuous data, the IRR increased from 0.61 to 0.73 . There were no differences in the frequencies of the evidence-based practices between rounds reported on the TPI by individuals in the expert and novice groups.

Implications: Teacher experience (i.e., expert, novice) does not appear to influence how observers rate teaching practices as defined by to the TPI. Since the TPI is an evidence-based instrument that records frequency-based measures, the TPI could provide a more objective approach to evaluating teaching practice.

Empowering Student Pharmacists to Manage Vaccine Hesitancy: A Simulation-Based, Assertive Communication Training Program. Justin Gatwood, The University of Tennessee, Kenneth C. Hohmeier, The University of Tennessee, Chelsea Renfro, The University of Tennessee, Dylan C. Knox, The University of Tennessee, Madison Williams, The University of Tennessee, Dviti Mody, The University of Tennessee, Tracy M. Hagemann, The University of Tennessee. Objective: To improve student pharmacists' confidence in providing accurate and assertive pneumococcal vaccine recommendations to high-risk adults. Methods: Following completion of the APhA immunization certification curriculum, a multi-phase vaccine hesitancy and communication workflow training program was implemented in a required first-year course. A five-part online module included content on pneumococcal vaccine recommendations, communication theory, improvisation techniques, and managing hesitancy in adults. Students then participated in live patient simulations at the UTHSC Center for Healthcare Improvement and Patient Simulation. Surveys evaluating hesitancy management were completed before and after the entire program by Mann-Whitney U tests, and focus groups captured direct student feedback. Results: Initial confidence in addressing and overcoming pneumococcal vaccine hesitancy in adults was generally higher among students with community pharmacy work experience ( $p<0.05$ in 4 of 6 items), but this difference was less pronounced after the training ( $<<0.05$ in 2 of 6 items). Five out of six measures of hesitancy management (addressing concerns, overcoming concerns, time to address hesitancy, influencing decisions, and convincing hesitant adults) improved as a result of the training (all $\mathrm{p}<0.01)$. Emerging themes from direct student feedback indicated that time constraints may limit real-world applications of the communication workflow and that application to influenza vaccination may be more critical. Implications: Results suggest that a combination of preparatory online and live simulation training can improve student pharmacists' confidence in addressing 


\section{American Journal of Pharmaceutical Education 2019; 83 (5) Article 7654.}

the pneumococcal vaccine needs of adult patients. Findings will inform an expansion of the training to practicing pharmacists who will undergo more advanced, simulationbased hesitancy and assertive communication training.

Entrepreneurial and Intrapreneurial Intentions: An Exploratory Comparison of Student Pharmacists in the United States and Oman. Nazneen Fatima Shaikh, West Virginia University, Mona Nili, West Virginia University, Nilanjana Dwibedi, West Virginia University, S. Suresh Madhavan, University of North Texas Health Science Center, Jayasekhar P. Nair, National University of Science and Technology, Oman, Alka Ahuja, National University of Science and Technology, Oman. Objective: To identify which personality traits (internal locus of control, innovativeness, autonomy, risk-taking propensity, proactiveness, achievement motivation, people liking, problem solving, and leadership) are associated with future entrepreneurial and intrapreneurial intentions among US and Omani student pharmacists. Methods: An Entrepreneurial-Intrapreneurial Pharmacist Questionnaire developed by the authors was administered to a convenience sample of second year or higher 134 US and 75 Omani student pharmacists. Independent t-test was used to compare differences in means between US and Omani students, and multiple linear regression (MLR) models of entrepreneurial and intrapreneurial intentions were built by entering all the personality traits, sex, age and country (US or Omani) as independent variables. Results: A majority of the study sample (both US and Omani) were females and between 22 and 25 years of age. Omani students had significantly $(\mathrm{p}<.05)$ higher entrepreneurial and intrapreneurial intentions as compared to US students. Compared to US students, Omani students had significantly lower internal locus of control and people liking mean scores, however, they had significantly higher mean scores on innovativeness, autonomy, and risk-taking propensity. In the first MLR model, autonomy $(\beta=.19, p<.01)$, achievement motivation $(\beta=.14, p=.02)$, being Omani $(\beta=.59, \mathrm{p}<.01)$ and being female $(\beta=.15, p<.01)$ were positively associated with higher entrepreneurial intention $(\mathrm{R} 2=0.45, \mathrm{p}<.01)$. In the second MLR model, achievement motivation $(\beta=.17, p=.01)$, leadership $(\beta=.21, p=.02)$, and being Omani $(\beta=.28, p<.01)$ were positively associated with higher intrapreneurial intention $(\mathrm{R} 2=0.30, \mathrm{p}<.01)$. Implications: Healthcare system, social, and cultural differences may explain the significant differences between US and Omani student pharmacists' personality traits and entrepreneurial and intrapreneurial intentions.

Evaluating Pharmacy Student Consultations on Opioid Medication Use and Discussion of OpioidSpecific Risks. Tanvee H. Thakur, University of
Wisconsin-Madison, Meredith L. Frey, University of Wisconsin-Madison, Betty A. Chewning, University of Wisconsin-Madison. Objective: The primary aim was to assess third year pharmacy students' entry level verbal and non-verbal communication skills when addressing sensitive topics during opioid consultations with standardized patients. Sensitive topics included describing the medication as an opioid or narcotic, side effects, potential for opioid dependence, and risk of overdose. This assessment was utilized to identify gaps that skills training programs need to address for students and pharmacists. Methods: Seventy-one students were video-taped consulting with standardized patients receiving a one-month supply of oxycodone for low back pain. The consults were coded quantitatively for what topics students discussed with the patient, terms used, eye contact, and filler words. Coding of video-recording had high inter-rater reliability (kappa = 0.90). Results: The majority of pharmacy students discussed common opioid side effects and severe side effects of opioid use, such as respiratory depression. However, only $30 \%$ explained to patients that the medication was an opioid or narcotic and only $23 \%$ of students initiated a conversation about dependence, addiction, or overdose risk. Students used more filler words when discussing dependence, addiction, or overdose risk as compared to the rest of the consult. Afterwards, students expressed discomfort and the need for additional training and resources for communicating with patients about opioids. Implications: There is a need to expand education within schools and colleges of pharmacy on mechanisms to discuss sensitive information about opioids with patients. This can be achieved by structured lectures and instruction-based labs focusing on communication about pain management with opioid analgesics.

Evaluating Student Competencies and Influences on Behavior Change in a Personal and Professional Development Course. Rosalyn P. Vellurattil, University of Illinois at Chicago, Nicholas G. Popovich, University of Illinois at Chicago. Objective: The affective domain has been identified as an essential component in the education of a pharmacist by both the American Association of Colleges of Pharmacy, and the Accreditation Council for Pharmacy Education. The UIC College of Pharmacy (UIC COP) implemented a new core course (Phar 463) for second year pharmacy students to achieve outcomes in self-awareness, leadership, innovation and entrepreneurship, and professionalism. The objective of this study was to assess the professional development of pharmacy students in a didactic personal and professional development course. Methods: Assessments in Phar 463 included formative and summative reflections, $360^{\circ}$ performance evaluations, creation of a mission/vision statement, and 


\section{American Journal of Pharmaceutical Education 2019; 83 (5) Article 7654.}

conceptualization, design and delivery of a team service learning project to the community. A randomized sample of summative reflections were analyzed. Students' selected competencies from the UIC COP Competencies and Outcomes, and stages of behavior change (i.e., Transtheoretical Model of Behavior Change) were coded. Themes were identified through qualitative analysis. Results: Thirty-two summative reflections were reviewed by researchers. All reflections referenced at least one of the following competencies while enrolled in the course: teamwork $(n=20)$, leadership $(n=15)$ and communication $(n=17)$. Most students $(63.5 \%)$ felt their selected competencies could be attained. The service learning project was the major driver affecting student development and behavior change. Implications: Phar 463 develops and supports students' progression professionally in the PharmD program. Most often, students were able to develop competence in teamwork, leadership and communication. Service learning was found to be most beneficial for student development.

Evaluation of a Substance Use Disorder Educational Event for Doctor of Pharmacy Students. J. Douglas Thornton, University of Houston, Matthew A. Wanat, University of Houston, EeVien Low, University of Houston, Soham Yande, University of Houston, Stephanie Crowley, University of Houston, Elizabeth P. Pitman, University of Houston, Marc L. Fleming, University of North Texas Health Science Center. Objective: A pilot, substance use disorder (SUD) educational event was developed to improve student pharmacist's knowledge and awareness on nonmedical prescription drug use, with the long-term goal of incorporating a similar training event into the Doctor of Pharmacy curriculum. The objectives of this study were to evaluate the effects of this educational event on students' attitudes toward the disease, knowledge of medications used to treat patients with SUD, and ability to assess risk factors of SUD in patients. Methods: First-year through third-year Doctor of Pharmacy students at the University of Houston College of Pharmacy were required to attend a four-hour event on SUD. Faculty, staff, and community members presented material during the event that included didactic information as well as interactive patient cases. Students were given pre/post-test questionnaires to assess attitudes regarding SUD, ability to identify risk factors, and knowledge of treatment options for SUD. Results: A total of 246 students completed a pre-test survey, with 137 of those students $(55.6 \%)$ also completing the post-test survey. Prior to the event, $151(61.5 \%)$ students indicated they had little or no experience with the topic of SUD. After the event, interest in learning more about SUD increased $(75.1 \%$ pre vs. $88.2 \%$ post, $p=0.006)$. Students felt more comfortable in counseling patients with SUDs $(38.7 \%$ pre vs. $51.5 \%$ post, $\mathrm{p}=0.016)$, and were more confident in their ability to identify patients with SUD $(28.8 \%$ pre vs. $59.3 \%$ post, $\mathrm{p}<0.001)$. Implications: The substance abuse education event was effective in improving students' knowledge base and attitudes towards SUD.

Evaluation of the Effectiveness of an ActiveLearning, Simulation-Based Healthcare Communications Course Utilizing the PaCT Assessment. David M. Baker, Western New England University, Izabela A. Collier, Western New England University, Courtney DoyleCampbell, Western New England University, Melissa Mattison, Western New England University, Katelyn Harrison, Western New England University, Quan Wei, Western New England University. Objective: Intent was to determine, by utilizing the validated Patientcentered Communication Tools (PaCT), whether an active-learning, intensely simulation-based healthcare communications course was effective in improving students' communication skills, in particular those involving patient counseling. In the course, each student performed four patient counseling sessions and presented in three group presentations; doing self-evaluations and receiving both peer and instructor evaluations on each one. Methods: Prior to enrollment in Healthcare Communications, students were informed of the study and volunteered, consenting to perform a pre- and post-course video-recorded patient counseling session, and complete pre- and post-course perception surveys.Each recorded counseling session, involving standardized faculty-actors as patients, were evaluated by the faculty-researchers utilizing the $\mathrm{PaCT}$ assessment. The pre- and post- surveys and $\mathrm{PaCT}$ results were compared to determine the course's impact on the students' communication skills. Results: Twenty-nine students participated, completing both the pre- and post-course study requirements. In all areas of the PaCT (i.e., establishing connection, exploring/integrating the patient's perspective, demonstrating interest and empathy, collaborating and educating, and communicating with finesse), the students' post-course counseling session evaluations, as judged by the faculty-researchers, were significantly $(\mathrm{p}<.000)$ improved over their pre-course counseling session evaluations. In addition, the students' own perceptions of their communications skills significantly improved $(\mathrm{p}<.05)$ subsequent to taking the Healthcare Communications course. Implications: Healthcare Communications, required in PY1, was designed to provide students with both didactic knowledge, and experiential and active learning beneficial to being effective pharmacist communicators. Using $\mathrm{PaCT}$ analysis of pre- and post-course counseling 


\section{American Journal of Pharmaceutical Education 2019; 83 (5) Article 7654.}

sessions demonstrated the benefit of an intensely simulation-based communications course.

Expanding the Evidence Base for the KiersmaChen Empathy Scale (KCES) Across Multiple Health Professions. Benjamin D. Aronson, Ohio Northern University, Aleda M. Chen, Cedarville University, Mary E. Kiersma, Accreditation Council for Pharmacy Education. Objective: Accreditation standards promote the importance of student empathy development, bolstering the need for measurement. One popular measure of empathy is the Kiersma-Chen Empathy Scale (KCES). Since its initial validation in 2013, the KCES has been used at multiple institutions, but there is limited new evidence of validity and reliability. The objective of this study was to explore the validity and reliability of the KCES across several administrations in health professionals. Methods: Data from 20 administrations of the KCES $(\mathrm{n}=3,065)$ were compiled. Participants included nursing students, pharmacy students, nurses, and respiratory care. Response patterns within and across cohorts were investigated. A paired-sample t-test and correlation coefficient were computed for pre- and post-activity KCES scores. Cronbach's alpha was calculated as a measure of internal consistency. Confirmatory and exploratory factor analyses were used to test and explore factor structure. Results: Response patterns to most items were consistent across administrations. Negatively-worded items displayed lower mean values and more fluctuation between cohorts, and some response categories were rarely endorsed for positively-worded items. Cronbach's alpha for the entire scale was 0.714 . Pre- and post-scores were moderately correlated $(\mathrm{r}=0.595, \mathrm{p}<0.001)$, with postscores significantly higher than pre-scores $(\mathrm{p}<0.001)$. The data did not fit proposed 1 or 2-factor models. Exploratory factor analysis revealed alternative factor structure. Implications: The KCES appears responsive to change with relatively consistent responses across different cohorts of health professionals and students. Some potential modification to the KCES are proposed to optimize the instrument.

Factors Associated With Attempting to Quit Illicit Substance Use. Fadi M. Alkhateeb, Qatar University, Omar F. Attarabeen, Marshall University, Isha Patel, Marshall University, Charles Babcock, Marshall University, Michael J. Rudolph, University of Kentucky, Rana Moawad, Marshall University, Nile M. Khanfar, Nova Southeastern University, Ahmad Hanif, West Virginia University, Faruk Khan, University of Charleston. Objective: Mortality rate due to illicit substance overdose is the highest in West Virginia among all states in the US. Using Social Cognitive Theory, this study investigated the factors associated with attempting to quit illicit substance use in a sample of users in West Virginia. Methods: After Institutional Review Board approval was obtained, data were collected from February to December 2018 using a 30 -item cross-sectional questionnaire that was administered through face-to-face interviews. Prospective participants were approached at local health departments. Adult men and non-pregnant women who used illicit substances during the past 30 days and were not receiving pharmacologic addiction treatment were eligible for inclusion. Binary logistic regression analysis was utilized to answer the research question. Results: Out of 360 individuals approached, 244 individuals agreed to participate and completed the questionnaire (response rate $=68 \%$ ). Median age was 37 years whereas average history of illicit substance use was 14 years. Heroin was the most commonly used substance (reported by $83 \%$ of participants). The logistic regression model demonstrated that individuals with 1) more positive attitudes regarding quitting illicit substance use and 2) higher self-efficacy regarding their ability to quit illicit substance use were significantly more likely to attempt to quit illicit substance use. Implications: Health behavior interventions that incorporate attitudes towards quitting illicit substance use and selfefficacy regarding ability to quit can be effective in encouraging quitting illicit substance use. Future research may address predictors of successfully quitting illicit substance use in this population.

Faculty Perceptions of Participating in and Goal Attainment During the \#RxWriting Challenge. Juanita A. Draime, Cedarville University, Aleda M. Chen, Cedarville University, Emily C. Wicker, Cedarville University. Objective: The \#RxWriting Challenge is an international, community-building initiative for encouraging faculty to make writing a priority for 14 days. Thus, the objective of this project was to determine faculty perceptions of participating in the \#RxWriting Challenge and goal attainment. Methods: Faculty were encouraged to sign up to participate in the Challenge and were supported through a school-wide initiative, including reserving writing space with snacks, group webinar attendance, goal setting, and prizes for participation and goal achievement. A Qualtrics-distributed survey (categorical and 5-point Likert-type agreement) was created to address key components of the Challenge and local initiative. Descriptive statistics were performed. Results: Of the 14 participants, $12(85.7 \%)$ faculty completed the survey. Ten (83.3\%) stated that they met their goals. The two who were unable to meet their goals stated that they did not set aside time to write and did not have the time and space to write at their practice site. Participants agreed (median $=4$ ) that the Challenge made it easier to write, the local initiative was motivating, and the writing space was beneficial. 


\section{American Journal of Pharmaceutical Education 2019; 83 (5) Article 7654.}

All agreed/ strongly agreed (median=5) that they would participate in the \#RxWriting Challenge again. Implications: Setting aside time to write is can be the toughest part of successful scholarly publication. Participation in the \#RxWriting Challenge that included local support resulted in helping faculty to meet their writing goals and were positively received by faculty. Accountability and friendly competition assisted with fostering a scholarly environment.

Falling In and Standing Out: Exploring Racially Minoritized Women's Path to Become Pharmacy Faculty. Antonio Bush, University of North Carolina at Chapel Hill, Katie Furl, University of North Carolina at Chapel Hill, Blake Martin, University of North Carolina at Chapel Hill, Estella Obi-Tabot, University of North Carolina at Chapel Hill. Objective: Recruiting and retaining diverse faculty members is of critical concern in health professions, and particularly of interest in pharmacy due to faculty shortages in recent years. This study explores the decisions made by self-identifying underrepresented racial minority (URM) women on the path to becoming pharmacy faculty. Methods: This qualitative study utilized 60 to 90 -minute semi-structured interviews with 15 faculty from multiple schools/colleges of pharmacy across the United States. Interview recordings were transcribed and analyzed using constant comparative analysis and thematic coding. To maximize trustworthiness, the research team used multiple coders, regularly engaged in consensus building, and employed a dependability audit. Results: Respondents found themselves "falling in" to a career as pharmacy faculty, through chance, social networks, practical concerns like time and money, and proximity to loved ones. The importance of mentorship, and seeing the possibility of a good fit, emerged as particularly salient themes among respondents. Respondents also expressed how their experiences in pharmacy school impacted their trajectory to initially not consider a faculty role, illustrating the importance of cyclical support. Implications: Results have the potential to guide future diversity initiatives in pharmacy program admissions, and to promote adequate racial, ethnic, and gender diversity along the pathway to a career as pharmacy faculty. This study sheds light on the lived experiences of URM women pharmacy faculty, and how the overlapping oppressions associated with URM and female status operate in the lives of pharmacy faculty.

Fourth Year PharmD Students' Perceived Achievement of CAPE Educational Outcomes. Omar F. Attarabeen, Marshall University, Isha Patel, Marshall University, Craig A. Kimble, Marshall University, Angel M. Kimble, Marshall University, Brian Heng, Marshall University, Kimberly A. Broedel-Zaugg, Marshall Uni- versity. Objective: In order to exhibit the required knowledge, skills, and attitudes, PharmD students are to meet the Center for the Advancement of Pharmacy Education (CAPE) 2013 educational outcomes. This study aimed to explore fourth year students' perceived achievement of these outcomes at a public pharmacy school that employs the flipped classroom active-learning andragogy. Methods: A 64-item cross-sectional online questionnaire was utilized to collect data. The questionnaire assessed students' agreement on the extent to which they met different educational outcomes. The items were measured on a 5-point Likert scale that ranged from "Strongly Disagree" to "Strongly Agree". During data analyses, scales were normalized to percentages. Data collection took place from March to May, 2018. All fourth-year students $(\mathrm{n}=81)$ were invited to participate in the questionnaire. Institutional review board approval was obtained. Results: Data were collected from 80 students (response rate $=99 \%$ ). On average, students' agreement on the extent to which they met CAPE educational outcomes was $84 \%$. Average agreement levels for Domain 1 (Foundational Knowledge), Domain 2 (Essentials for Practice and Care), Domain 3 (Approach to Practice and Care), and Domain 4 (Personal and Professional Development) were $82 \%, 83 \%, 84 \%$, and $85 \%$, respectively. Implications: Overall, fourth year students demonstrated high perceived achievement of CAPE outcomes. This may indicate students' confidence in their educational capabilities, or a perceived view of a robust curriculum at the school. Future research might examine the association between students' perceived achievement of CAPE outcomes and actual competency of CAPE domains and learning objectives.

Hands-on MUE and ABS Activities in Didactic Courses. Staci Hemmer, University of Montana, Jean T. Carter, University of Montana, Jenner Minto, University of Montana, Sherrill J. Brown, University of Montana. Objective: To increase pharmacy student readiness to perform medication use evaluation (MUE) and antimicrobial stewardship (AMS) activities in APPEs through increased practice in didactic courses. Methods: Analyzing data and reporting MUE results were the focus of 3 hours of didactic and 3 hours of hands-on instruction in the final pre-APPE year. Students applied clinical, statistical, and writing skills to prepare a mock MUE report. In another 3-hour class period, students were introduced to antibiotic tracking and reporting and used real-life lab data to construct an antibiogram. The experiences were evaluated by observation of student questions, results of their submitted work, and responses to a short questionnaire. Results: All students enrolled in the courses $(\mathrm{N}=59)$ responded. Overall, students gave positive 


\section{American Journal of Pharmaceutical Education 2019; 83 (5) Article 7654.}

feedback for the MUE and AMS activities on an end-ofcourse survey. Most students agreed or strongly agreed that they better understood the MUE process and AMS activities (83\%) and felt confident in their ability to make an antibiogram (75\%). Fewer students (61\%) were confident in their ability to present written results of an MUE. This last assessment was consistent with student performance on the MUE report assignment in less than half $(45 \%)$ of the submitted papers properly reported the statistically significant results. Implications: Results indicate student understanding of MUEs and AMS was augmented by the expanded hands-on learning activities. However, the ultimate benefit will not be known until students are exposed to MUE and AMS activities in their APPE rotations.

Has the Bubble Burst? Observing Pharmacist Workforce Trends and Current Jobs Data. Lisa Lebovitz, University of Maryland, Michael J. Rudolph, University of Kentucky. Objective: To characterize pharmacist workforce trends and current job postings to demonstrate the urgent need for critical changes that will increase demand, maintain high salaries, and preserve the profession's high regard. Methods: US Bureau of Labor Statistics, Pharmacist Demand Indicator, and other available data were mined for pharmacist workforce trends. Burning Glass Technologies' Labor Insight $₫$ was used to evaluate more granular data on PharmD job postings including salaries, employers, and locations. Results: Forecasts of pharmacist shortages were predicated on the profession moving toward mostly non-distributive functions. PharmD programs opened unabated; student enrollment drastically increased and patient-care learning outcomes were enhanced. Now the job market is flooded with highly trained graduates with large debt burdens. In actuality, the distribution of pharmacist job postings by practice type remained relatively constant from 20102018 (56\% retail, $31 \%$ health care, $13 \%$ other), with no evidence of growth in emerging areas. Average advertised salaries for pharmacists with 0-2 years of experience increased from $\$ 98,900$ in 2010 to $\$ 115,948$ in 2018 , although year to year increases are flattening. Data suggest a shift in geographic demand with the greatest increases in job postings occurring in the Midwest, West, and Pacific regions. Nevertheless, total projected 2019 graduates $(14,564)$ indicate saturation relative to the projected job openings for new pharmacists entering the field $(15,300)$. Implications: Armed with data regarding the pharmacist workforce, professional associations should urgently advocate for and demonstrate positive outcomes of changing the profession. Perhaps the recent pharmacy chain mergers present a collaborative opportunity to catalyze the stalled forecasts.
Health Literacy in College Students. Michael J. Gonyeau, Northeastern University. Objective: Health literacy (HL) is the ability to obtain, communicate, process, and understand basic health information necessary to make medical decisions. Lower levels of HL have been associated with increased hospitalizations, comorbidities, decreased medication adherence and use of preventative health services. To assess HL levels and the perceived importance of HL in college students to determine the need for core curriculum modifications to improve education and health. Methods: A prospective survey utilizing the validated METER HL assessment tool was offered to university students. METER contains a mix of 70 correct and false medical words and requires participants to identify the actual medical words. Two validated scoring methods were utilized; one calculated correct choices from 40 actual health words, the second subtracted for incorrectly selected words. Participant demographics, majors, history of health-related coursework, HL attitudes and frequency of healthcare access were collected. Results: Respondents completed 553 surveys (female $=80 \%$ ) with all colleges, 76 majors, and all years of study represented. Most respondents(54.3\%) had not completed a health-related course (range:0-7), while $82.4 \%$ stated confidence in understanding health-related information and considered HL important (92\%). Most participants $(69.9 \%)$ had functional HL (1st method: mean:37.05 \pm 3.14 ; 2 nd method: mean:35.4 \pm 3.84$)$, with $29.2 \%$ marginal and $1 \%$ low HL. Significant differences between mean scores were not found to exist between different colleges and demographics. Implications: While a majority of college students appear to have functional HL, a population with marginal and low HL exists, highlighting the potential for additional health education within and across all colleges of the university.

Identifying the Challenges in Implementing the Co-Curriculum Accreditation Requirement: A National Survey. Jaime L. Maerten-Rivera, University at Buffalo, The State University of New York, Aleda M. Chen, Cedarville University, Jill M. Augustine, Mercer University, Richard d'Assalenaux, West Coast University, Kelly C. Lee, University of California, San Diego, Cameron C. Lindsey, University of Missouri-Kansas City, Laurie S. Mauro, The University of Toledo, Daniel R. Malcom, Sullivan University, Nina Pavuluri, Lake Erie College of Osteopathic Medicine, Michael J. Rudolph, University of Kentucky, Siu-Fun Wong, Chapman University, Jacqueline M. Zeeman, University of North Carolina at Chapel Hill, Paula Zeszotarski, Albany College of Pharmacy and Health Sciences, Paula Zeszotarski, Albany College of Pharmacy and Health Sciences. Objective: The ACPE 2016 Standards introduced a co- 


\section{American Journal of Pharmaceutical Education 2019; 83 (5) Article 7654.}

curriculum requirement. This study, conducted by a committee within the Assessment SIG, examined challenges, areas of concern, and confidence in the cocurriculum across accredited schools/colleges of pharmacy. Methods: A co-curriculum survey was administered to assessment representatives at all ACPE-accredited pharmacy programs, which collected information on: a) challenges in implementation and evaluation of cocurriculum, b) areas of concern with satisfying the ACPE co-curriculum requirement, and c) confidence in ability to satisfy the requirement $(1=$ unconfident, $10=$ confident $)$. Descriptive statistics were examined, and analysis of variance (ANOVA) was performed to examine differences in confidence based on program characteristics (ie, year founded, cohort size, year of last accreditation review). Results: The survey response rate was $75 \%$. Most programs indicated faculty/staff/committees did not have enough time for co-curriculum $(57.0 \%)$ and there were insufficient personnel to design, implement and evaluate the co-curriculum $(54.4 \%)$. A substantial number of programs reported that a lack of clear understanding of cocurriculum and how to enact it was a challenge (38.3\%). Additional reported areas of concern included closing the loop $(50.5 \%)$, assessment $(50.5 \%)$, and documentation $(49.5 \%)$. Few reported an inadequate number of activities available $(8.4 \%)$ as a concern. The mean confidence score (out of 10) was 7.91 ( $\mathrm{SD}=1.59)$, with no significant differences based on program characteristics. Implications: Most programs report confidence in their ability to satisfy the ACPE 2016 Standard requirements. A common challenge reported was resources to implement the co-curriculum. Additional research is needed to determine ways to efficiently implement co-curriculum models.

Impact of a Mental Health Elective on Reducing Stigma. Jennifer D. Robinson, Washington State University, Nancy N. Johnson, Washington State University, Damianne Brand-Eubanks, Washington State University, Anne Kim, Washington State University, Connie M. Remsberg, Washington State University. Objective: The development and evaluation of a mental health elective to decrease mental illness related stigma. Methods: A two-credit Mental Health First Aid elective was developed where second- and third-year student pharmacists received Mental Health First Aid training and engaged in activities to gain insight about individuals living with a mental illness. Pre- and post-class surveys administered to all enrolled students consisted of the 15 item "Opening Minds to Stigma Scale for Heath Care Providers" including 1) attitudes of health care providers, 2) disclosure and help-seeking behavior, and 3) social distance. Results: The pre- and post-course surveys resulted in 51-paired responses for a $93 \%$ (51/55) response rate. When comparing each individual's pre to post responses, significantly less stigma was reported in 5 of the 15 question items following completion of the elective $(p<0.05)$. The greatest change was observed with the survey item, "Despite my professional beliefs, I have negative reactions towards people who have a mental illness," where 29/51 (56\%) and 44/51 (86\%) answered disagree/strongly-disagree during the pre-course and post-course surveys, respectively. When prompted with "I would be reluctant to seek help if I had a mental illness," 17/51 (33\%) respondents selected agree/strongly agree pre-course while 11/51 $(22 \%)$ selected agree/strongly agree post-course. Statistically significant changes were observed in all domains: attitudes of health care providers $(\mathrm{p}<0.0001)$, disclosure or help-seeking behavior $(\mathrm{p}=0.0008)$, and social distance $(p=0.044)$. Implications: Results indicate respondents' willingness to support and treat individuals living with a mental illness. The same group displays hesitancy when seeking out care for their own mental health.

Incorporating History of Pharmacy Into a Required Pharmaceutical Calculations Course. Michael A. Hegener, University of Cincinnati. Objective: To determine student perceptions of pharmacy history topic inclusion into a required calculations course. Methods: Although pharmacy history is a required element of ACPE standards 2016, no PharmD program currently has a required, standalone history course. Most programs meet this requirement via inclusion in other required courses. Optional clinical and history of pharmacy short answer questions were added to ten weekly, required homework sets in a first professional year pharmaceutical calculations course. These questions all had a connection to a drug mentioned in the homework sets. There was one of each question type per homework set and the number of clinical and history questions answered per student were tracked. A survey, consisting of three 5-point Likert-scale questions $(5=$ strongly agree, $1=$ strongly disagree) was administered to students after the course to determine their perceptions. Results: 86 students were enrolled in the course and $70(81 \%)$ completed the survey. Students answered more of the history questions than the clinical ones (49.7\% and $47.4 \%$ respectively). Ten students did not answer any of the clinical questions while 7 did not answer any history questions. The mean survey response for the questions stimulating interest in pharmacy history was 4.2. The mean responses for both a desire for more courses to incorporate history of pharmacy topics and interest in taking a pharmacy history elective course were 3.8. Implications: Based upon question completion frequency and survey responses, students found the inclusion of pharmacy history topics interesting and would like 


\section{American Journal of Pharmaceutical Education 2019; 83 (5) Article 7654.}

history topics incorporated into additional PharmD courses.

Incorporating Wrappers to Evaluate Pharmacy Students' Self-Assessment Abilities. Norman E. Fenn, The University of Texas at Tyler, David B. Romerill, The University of Texas at Tyler, Jennifer Smith, University of Texas at Tyler, Bradley J. Brazill, The University of Texas at Tyler. Objective: The purpose of this study was to compare student self-perceived versus actual performance outcomes using wrappers as a self-assessment tool. Methods: All third professional year students $(n=101)$ enrolled in the 8-week Oncology/Critical Care Pharmacotherapy course were included in the study and completed the wrappers surveys as a course requirement. Two questionnaires were created to assess five separate time points. The 13-item survey was issued at the end of each major assessment and was completed prior to exiting the exam. This survey was issued three times during the term and assessed student study habits and perceived performance on each assessment. The 7-item survey was completed during each of two exam review periods. It repeated the last three items of the survey for each incorrect response on the exam, which collected rationale for each incorrect response as well as actual assessment performance. Results: Most students (70.2\%) performed at the level they expected for the assessments. Approximately $20 \%$ performed better than expected, while $10 \%$ performed worse than expected. Students described spending most of their study time creating study guides (range: $18.3-22.0 \%, \mathrm{p}=0.78$ ), reviewing class notes (range: $18.3-25.9 \%, \mathrm{p}<0.001$ ), and rereading textbook chapters (range: $14.6-18.8 \%, \mathrm{p}<0.001$ ). Time spent preparing for major assessments varied widely, with the most common ranges being $21-30$ hours per exam (17.2\%) over three days $(24.5 \%)$. Implications: Most third professional year pharmacy students' self-assessed performance was reflective of their actual performance on major assessments. Further studies will involve pharmacy students in their first or second professional year to evaluate variation between years.

Influence of Item Structure Upon Student Performance Using Select all that Apply and True/False Items. Scott A. Baggarly, The University of Louisiana at Monroe, Jeffery D. Evans, The University of Louisiana at Monroe. Objective: To determine if item structure influences student performance on Select all that Apply (SAA) items and true/false (TF) items in first (P1)- and third (P3)-year pharmacy students when identical information is tested in the two different formats. Additionally, we intend to investigate the impact of awarding partial credit for SAA items upon student performance. Methods: The College has a two-semester series of Phar- macy Law and Ethics courses. The instructors developed two instruments, each containing 4 SAA course-related items and 1 SAA control item from a topic that students were unlikely to know. In addition to the 5 SAA questions, Instrument $\mathrm{A}$ contained $15 \mathrm{TF}$ questions derived from SAA items of Instrument B, and Instrument B contained 15 TF questions derived from SAA items of Instrument A. The instruments were randomly assigned and administered to volunteers from the first- and third-year classes. Results: The instruments were completed by 68 P1 and 52 P3 students. Overall, students had lower scores on SAA items vs. TF items $(\mathrm{SAA}=41.9 \%$; $\mathrm{TF}=74.4 \%$; $\mathrm{p}=0.0401)$ and on control items vs. course-related items (Control $=34.8 \%$; Course-related $=66.3 \% ; p=0.0022)$.On course-related material, $\mathrm{P} 1$ and $\mathrm{P} 3$ students performed similarly on SAA items $(\mathrm{P} 1=41.5 \% ; \mathrm{P} 3=42.6 \% ; \mathrm{p}=0.4574)$ and on $\mathrm{TF}$ items $(\mathrm{P} 1=74.8 \% ; \mathrm{P} 3=68.5 \% ; \mathrm{p}=0.3838)$. When partial credit was awarded for SAA items, overall student scores increased significantly (No partial credit $=$ 59.9\%; Partial credit $=67.2 \% ; p=0.0401)$. Implications: These data demonstrate that item structure influences student performance when identical information is tested in two different formats. Further studies are needed to investigate the effects of other item formats.

Innovative Virtual Symposium: Connecting Leaders Across AACP. Kerry K. Fierke, University of Minnesota, Gregory M. Zumach, Oregon State University, Jenelle Sobotka, University of Cincinnati, Andrew S. Bzowyckyj, Pacific University Oregon, Kim M. Jones, Union University, Ashley Crowl, The University of Kansas, Edward C. Portillo, University of WisconsinMadison. Objective: To explore various approaches to implement identified leadership frameworks and assessments that meet the needs of students and post-graduate trainees through a Virtual Symposium (VS) in order to address ACPE Accreditation Standard 4, specifically Key Elements 4.2 (leadership) and 4.4 (professionalism). Methods: The Leadership Development (LD) SIG created a VS designed to provide case study vignettes to AACP members at a variety of stages in LD (beginner, intermediate, advanced). Each video introduced leadership framework(s) used, steps implemented, outcomes observed, and lessons learned, all in five minutes or less. AACP Connect was employed to disseminate the weeklong asynchronous VS. The presenters for the ten case studies were requested to facilitate discussion electronically via Connect that week. Results: A total of 130 views occurred during the VS. A post-evaluation, including four scaled evaluative statements and three open-comment questions, was completed by ten participants. One hundred percent of respondents rated each of the scaled questions 'strongly agree' or 'agree', including the statement, 


\section{American Journal of Pharmaceutical Education 2019; 83 (5) Article 7654.}

"The video presentations were an effective learning tool." Comments indicated that attendees found the VS relevant and several noted takeaways of ideas to incorporate into their curriculum/co-curriculum. Ninety percent noted they would like to see more VS offerings from the LD SIG. Implications: Disseminating case studies through a VS is a practical approach to providing faculty with the knowledge and tools to address LD in a timely, flexible manner. This format may provide a new communication approach to facilitate dissemination and discussion of leadership materials amongst colleagues.

Interest in Lung Screening Among a Sample of Adult Muslims in the United States. Omar F. Attarabeen, Marshall University, Fadi M. Alkhateeb, Qatar University. Objective: Muslims in the United States (US) exhibit high rates of tobacco use, which make them more vulnerable to lung cancer. The current study investigated the associations between Social Cognitive Theory factors and being interested in being screened for lung cancer in a sample of adult Muslims in the US Methods: After Institutional Review Board approval was obtained, we examined a convenience sample of adult ( $\geq 18$ years) Muslims who resided in the US and had no personal history of lung cancer. A cross-sectional on-line survey was used to collect data from November 2016 to March 2017. Binomial logistic regression analyses were conducted to answer the research question Results: Two hundred seventy-one eligible participants from 30 states completed the questionnaire, of which $59.9 \%$ expressed an interest in being screened for lung cancer. Individuals were more likely to express an interest in lung cancer screening if they had 1) more positive views about lung screening, 2) higher perceived value of screening, and 3 ) greater selfefficacy with regard to ability to undergo lung screening Implications: Cognitive factors can influence interest in lung screening. Lack of adherence to lung screening guidelines was apparent in our sample. Improving attitudes to lung screening, increasing perceived value of screening, and boosting self-efficacy regarding ability to complete lung screening could be important factors to consider in devising future interventions aimed at increasing interest in lung screening in adult US Muslims

Inter-Rater Reliability of Four Reflection Rubrics in an Elective Global Health Course. Rebecca Cope, Long Island University, Suzanna Gim, Long Island University. Objective: To establish the assessment rubric which maximizes inter-rater reliability (IRR) when evaluating pharmacy student reflections for an elective course in global health. Methods: A 6-item 4-point scale reflection rubric developed by Tsingos et al and previously validated in pharmacy students was utilized to grade an assignment for the course. The IRR score was compared to two other validated rubrics and one non-validated rubric to determine a preferred assessment tool. Student reflections were blinded and scored by two raters using all four rubrics. IRR for each rubric was determined using a two-way mixed, absolute, average-measured intra-class correlation (ICC). Student participants voluntarily completed an anonymous survey regarding perceptions of the rubrics. Exempt IRB status was obtained. Results: Nineteen reflections were evaluated. The non-validated rubric had the highest ICC of 0.864 and resulted in a mean score of $87 \%$. The rubrics validated by 1) Tsingos et al, 2) medical students, and 3) general students resulted in ICCs of $0.8,0.8$, and 0.76 , respectively, and resulted in mean scores of $89 \%, 81 \%$, and $81 \%$. Of the 10 survey respondents, $70 \%$ indicated a preference for the rubric by Tsingos et al. Implications: Incorporation of reflective practices into pharmacy education is challenging due to a significant gap in the literature regarding the use of reflective rubrics to consistently assess reflective thinking. Students preferred the rubric which would result in the highest score, however, the non-validated rubric outperformed the validated rubrics in regard to IRR. Validation of this rubric in a pharmacy student population will be pursued.

Longitudinal Impact of an Interprofessional Education Session on the Development of Team and Values Competencies. Emily Laswell, Cedarville University, Elizabeth Sled, Cedarville University, Felisha Younkin, Cedarville University, Aleda M. Chen, Cedarville University. Objective: In order to prepare students who are team-ready and practice-ready, schools must incorporate interprofessional education (IPE) experiences and activities that allow health professions students to develop needed competencies. Thus, the objective of this study was to examine the longitudinal impact of an IPE session on student development in defining roles/responsibilities and team values/ethics. Methods: Pharmacy (first professional year), nursing, psychology, pre-med, and allied health students attended an 8-hour training session on providing holistic patient care. Students were placed into small interprofessional groups, and interprofessional faculty teams facilitated the sessions. There were specific discussion questions and activities related to the IPEC 2016 competencies of values/ethics and roles/ responsibilities. Students completed surveys pre-session, post-session, and 3, 6, and 8.5 months post-session. Data were analyzed using a Friedman test for longitudinal changes; descriptive statistics were performed. Results: A total of 169 participants attended (mean age 20.5 \pm 3.1 years; $82.8 \%$ female). At the time of the training, $55.4 \%$ of participants had less than one year of patient experience, while $18.5 \%$ had none. Longitudinally, participants 


\section{American Journal of Pharmaceutical Education 2019; 83 (5) Article 7654.}

felt significantly more competent in defining roles and responsibilities and defining appropriate values and ethics among interprofessional healthcare teams over time $(p<0.05)$. Implications: A training session was beneficial to students from various health professions students in developing IPE competencies. Additional incorporation of IPE activities throughout the curriculum are recommended to further develop the skills needed for team-based care.

Mapping Didactic Courses to the Five Steps in the PPCP Model. Kimberly A. Madson, University of Montana, Jean T. Carter, University of Montana. Objective: Measure faculty and student understanding of how didactic courses are linked to the five principle steps of the JCPP Pharmacist Patient Care Process (PPCP) model. Methods: Faculty and third-year students indicated which of the $38 \mathrm{P} 1-\mathrm{P} 3$ didactic courses covered one or more of the five principle PPCP steps: Collect, assess, plan, implement, follow-up. Information was collected on a paper form displaying the PPCP wheel by individual faculty members and groups of third-year pharmacy students. Descriptive statistics and nonparametric comparisons of faculty $(\mathrm{N}=36)$ and student $(\mathrm{N}=66)$ responses were performed. Results: Most faculty (89\%) and students $(100 \%)$ participated. The number of didactic courses where all five steps were addressed increased steadily from three $(21 \%)$ in the P1 year to $12(100 \%)$ in the P3 year (100\%). Faculty thought all five steps were addressed in half $(50 \%)$ of the courses and one to four steps were covered in the 19 remaining courses. Students estimated $22(57 \%)$ of the courses covered all five steps, $12(32 \%)$ for one to four steps, and $11 \%$ (4) with none of the five principle steps. The four courses without a step were social administrative courses that students indicated fit into other aspects of the PPCP such as communication and documentation. Implications: Mapping the five principle PPCP steps to courses indicated both faculty and students have some recognition of the model and how it is incorporated into the didactic curriculum.

Measuring Depression and Anxiety Prevalence Among Iraqi Healthcare College Students Using Hospital Anxiety and Depression Scale. Ali Azeez AlJumaili, University of Baghdad, Sarmed H. Kathem, University of Baghdad, Malak Noor Aldeen, University of Baghdad, Noor Najah, University of Baghdad. Objective: The study objectives were to 1) measure prevalence of depression and anxiety among Iraqi pharmacy and medical students using Hospital Anxiety and Depression Scale (HADS) and 2) evaluate the association of various sociodemographic factors and students' HADS scores. Methods: Methods: This was a cross-sectional descriptive study in four universities in Baghdad, Iraq. Depres- sion and anxiety were screened using an Arabic version of the HADS. An electronic survey was administered via Facebook groups to convenience samples of students at four colleges of pharmacy and a college of medicine between April and June 2018. Demographic characteristics were collected at the end of the survey. Results: Results: We received 750 usable surveys. The participating students spent more time browsing social media (6.64 hours/ day) than studying (1.92 hours/day) and exercising ( 2.83 hours/week). Around forty-five percent $(45.9 \%)$ of the participants reported depression symptoms and one-quarter $(24.8 \%)$ reported depression borderline symptoms. More than one-half (52.1\%) of the participants reported anxiety symptoms, while $20.1 \%$ reported anxiety borderline symptoms. According to the linear multiple regression analysis, five factors - sleep hours at night, study hours weekly, academic achievement, and colleagues and family social support during exams - were significantly (P-value $<0.05$ ) associated with depression and anxiety symptoms. Implications: The students of healthcare colleges are vulnerable for mental illness and anxiety because high stress due to study and exams. To reduce their levels of anxiety and depression, they need more social support, more exercise, more sleep and a lower academic workload.

Perfectionism, Test Anxiety, and Gender Differences in Student Pharmacists Enrolled in an Accelerated 3 Year Program. Lilia Z. Macias-Moriarity, South University, Starlette M. Sinclair, South University, Erin Dalton, South University, Maha Coucha, South University, Kirsten M. Boggs-Mock, South University, Shaina Varughese, South University. Objective: While often deemed a positive trait, perfectionism can be adaptive (healthy) or maladaptive (unhealthy). Students who are maladaptive perfectionists demonstrate high self-blame, high performance expectations, and are more likely to experience test anxiety as compared to their adaptive perfectionist counterparts. The objectives of this study were to (1) assess perfectionism and test anxiety in student pharmacists (2) determine relationships between perfectionism, test anxiety, conscientiousness, and neuroticism (3) investigate differences between these traits as they relate to gender. Methods: An anonymous online survey was administered to a convenience sample of student pharmacists. Demographic variables and standardized psychological instruments designed to measure perfectionism, conscientiousness, neuroticism, and testing anxiety included the Almost Perfect Scale-Revised, Frost Multidimensional Perfectionism Scale, Big Five Personality Inventory, and Test Anxiety Inventory. T-tests identified group differences. Pearson correlations measured relationships between all variables of interest. Alpha 


\section{American Journal of Pharmaceutical Education 2019; 83 (5) Article 7654.}

was set at 0.05 . Results: 157 student pharmacists completed the survey: $72 \%$ female and $26 \%$ male with the median age of 25 . Females reported more neuroticism than males $(\mathrm{p}<0.05)$. Male perfectionism correlated with greater test anxiety $(r=0.382, p<0.05)$; however, female perfectionism correlated with greater test anxiety $(\mathrm{r}=0.303, \mathrm{p}<0.05)$, neuroticism $(\mathrm{r}=0.535, \mathrm{p}<0.05)$, and conscientiousness $(\mathrm{r}=0.454, \mathrm{p}<0.05)$. Implications: While conscientiousness is desired in healthcare professionals, perfectionism can be crippling in extreme forms. If self-destructive and self-limiting behaviors can be identified early in student pharmacists, interventions may be developed to increase coping strategies, potentially decreasing these behaviors and, ultimately, improving their performance as future pharmacists.

Pharmacy Artifact Sorting Team (PAST): Bringing Pharmacy History Into the Pharm.D. Program. Roberto W. Linares, Oregon State University, Karina Nguyen Destine, Oregon State University, Abigail J. Larson, Oregon State University, Kameron T. Kauffman, Oregon State University, Jessica Foerster, Oregon State University, Eliza Ammon, Oregon State University, Ann Zweber, Oregon State University. Objective: While Pharmacy programs struggle to meet ACPE pharmacy history curriculum requirements, they also often find themselves in possession of historical pharmacy artifacts. Our program has a large collection of pharmacy artifacts. Objectives were to: Use a systematic process to catalog, digitize, identify, and share information about the items; Use the collection to enhance the teaching of pharmacy history in our program. Methods: Two faculty members and several pharmacy students examined a pharmacy artifact collection of over 4000 items dating back to the late 1800's. Steps included: 1) developing cataloging strategies, 2) creating a database using Airtable TM , 3) cataloging and photographing individual items, 4) sharing results. A History of Pharmacy elective was designed to engage students with further research on the collection.

Results: The historical pharmacy items were assigned a number, a brief description, an image, a manufacturer, and a grouping. Electronic ways of sharing items and their history included the state organization newsletter and website, social media, and the College newsletter and website. Displays of items and their stories were created to share at state organization meetings, sites on campus, and other venues. A history of pharmacy elective course syllabus has been developed. Implications: Having a detailed database for the collection facilitates further research into the history of items. A syllabus has been developed, allowing students to further explore the history of pharmacy, with individual investigations into specific artifacts. The database will allow for develop- ment of themed exhibits to share online and at a variety of venues.

Pill Counter, Business Person or Health Care Provider? A Discourse Analysis of Professional Identity. Jamie L. Kellar, University of Toronto, Elise Paradis, University of Toronto, Cees PM van der Vleuten, Maastricht University, Mirjam GA oude Egbrink, Maastricht University, Zubin H. Austin, University of Toronto. Objective: The objectives of this study were to explore (1) what have been the professional identities in pharmacy education over the last century in North America and (2) which one(s) currently dominate the curricula. Methods: A Foucauldian informed historical critical discourse analysis was undertaken to uncover the educational assumptions underpinning pharmacist identity over time. This analysis allowed for the questioning of the roles of the pharmacist that are taken for granted and assumed as rational and inevitable. Results: This study identified five prominent discourses in the literature related to the professional roles of pharmacists over the last century. The identities are the "apothecary", "druggist", "merchandiser", "expert advisor" and "health care provider." Each of these discourses constructs the pharmacist's professional role in different ways and as such makes possible certain language, subjects, and objects. An unexpected finding of this research was that over the years there were no clear discursive shifts in the education literature, but rather discursive "pile-ups" of all identities. Implications: Each identified discourse remained present throughout the entire archive. These "discursive pile-ups" suggest that each discourse continues to have relevance to pharmacy educators, and as such competes for space in the curriculum. This likely contributes to the identity challenges pharmacists currently face. This critical discourse analysis reveals that pharmacist identity constructs are not straightforward, self-evident, or progressive. Pharmacy educators must determine the ultimate goal of pharmacy education and begin a formal process of inculcating the ideal identity discourse in pharmacy curricula.

Prestige \& Versatility: Choosing Pharmacy. Dane D. Osmond, Keck Graduate Institute, Natalia Shcherbakova, Western New England University, Sally A. Huston, Keck Graduate Institute. Objective: With steadily declining applications to pharmacy programs nationwide, there is a need to recruit high quality students to meet population healthcare needs. The objective of this study was to identify factors influencing pharmacy student's choice of pharmacy as a degree. Methods: The institutional review boards at Western New England College of Pharmacy (WNE) and Claremont Graduate University approved this study. Focus groups at WNE (2) and 


\section{American Journal of Pharmaceutical Education 2019; 83 (5) Article 7654.}

Keck Graduate Institute School of Pharmacy and Health Sciences (3) were conducted to elicit factors motivating students to pursue a PharmD. Focus group results were used to develop a survey questionnaire administered to first year WNE students. Participants ranked the importance the factors influencing pharmacy choice on a 5point scale. Results: A total of 36 students participated in the focus groups. Identified themes included prestige of a doctorate degree, patient interaction, career versatility, less intense than medical school, and post-graduation competition. A total of 59 MA students (70\% female; average (SD) age 23 (4)) completed the survey (97\% response rate). The factors ranked by students as important were having financial stability (95\%), having a career in healthcare (94\%), having a good work life balance (87\%), having a flexible work schedule (86\%), and being in a versatile career (84\%). Alternative degrees considered included MD (21\%), nursing (12\%), DPT (9\%), and PA (9\%). Implications: The emerging themes and quantitative findings generated from this mixed methods study may assist recruitment efforts of pharmacy admissions offices nationwide.

Prevalence of Suicide and Substance Abuse Related Mortality Among Healthcare Professionals. Muzammel Rizvi, The University of Tennessee, Marie A. Chisholm-Burns, The University of Tennessee, Christina A. Spivey, The University of Tennessee. Objective: Several factors place healthcare professionals (HCP) at high risk for suicide and substance abuse including access to controlled medications, high-stress job environments, and stigma related to being treated for substance abuse disorders. The objective is to examine mortality rates for suicide and substance abuse disorders among HCPs (physicians, pharmacists, nurses, dentists) using US Census data from 26 states. Methods: The National Occupational Mortality Surveillance (NOMS) database was used to determine proportional mortality ratio (PMR) related to ICD-10 codes for intentional self-harm (X60-X84, Y87) and mental disorders related to substance abuse (F10F19) among selected HCPs. PMR indicates if the proportion of deaths due to a specific exposure is high $(>100)$ or low $(<100)$ in a certain occupation compared to all occupations. Mortality data were matched with US Census occupational codes for HCPs to facilitate a comparison of suicide and substance abuse-related mortality versus allcause mortality across all HCPs using odds ratios. Results: The following PMRs for were found for intentional selfharm and mental disorders related to substance abuse, respectively: Pharmacists (204,117); Physicians $(254,59)$; Dentists $(322,134)$; and Nurses $(96,60)$. Odds ratios, wherein specific HCPs experienced disproportionate mortality due to either cause of death, were also reported
Implications: This study identifies specific HCPs (pharmacists, physicians, and dentists) that are at a higher risk for mortality related to substance abuse and suicide compared to all other occupations. Further studies are needed to determine the potential work-related factors that may have a causal link to these findings.

Promoting Cultural Awareness in a Pharmacy Program Using Student-Led Health Education Workshops for Refugees. Amany K. Hassan, D'Youville College, Vicky Belousova, D'Youville College, Hiba Al-Naji, D'Youville College, Anna Pisano, D'Youville College. Objective: To describe the implementation of a series of student-led educational workshops offered to refugees and evaluate the perceived benefit to improve cultural awareness among student pharmacists. Methods: Students volunteered to participate in health education workshops for refugees as either presenters or interpreters. The students prepared the educational material with the help of faculty members with a focus on overcoming cultural and language barriers. Two orientation sessions were scheduled prior to each workshop to train the students and review the material. Students completed an anonymous survey and submitted a short reflection about their experiences after each workshop. Results: We received 25 responses from students $(83.3 \%)$ who participated in the first two workshops on medication literacy and safe use of complementary medicine. All students $(100 \%)$ agreed that the workshops increased their cultural awareness, improved their skills as healthcare educators, and contributed to their professional development. However, $16 \%$ agreed that the information was not well received due to language barriers. A summary of themes from the students' reflections showed that they valued the experience, thought it was an eye-opener, and inspired them to volunteer in similar events. They also thought the workshops exposed the great need to educate this population on medication use and safety. Responses from refugees showed that $90 \%$ or higher agreed that workshops were useful, easy to understand, and that presenters were helpful. Implications: The workshops exposed students to the culture and health care needs of refugees. Expanding these experiences to the didactic courses can be used to improve cultural awareness.

Providing Validation Evidence for a ClinicalScience Module: Improving Test Reliability with Quizzes. Michael J. Peeters, The University of Toledo, M. Kenneth Cor, University of Alberta, Erik D. Maki, Drake University. Objective: Towards validation of learning assessments, reliability is vital evidence for its generalization inference. In classrooms, a short exam may not be very reliable on its own, and so should be supplemented. Might quizzes help? This study's objective was to 


\section{American Journal of Pharmaceutical Education 2019; 83 (5) Article 7654.}

understand how adding quiz data to a traditional examination during a clinical-science module impacted reliability. Methods: During second-year of a PharmD program, students took a clinical-science course. This 15-week course contained three modules, with one 5-week module focused on cardiovascular therapeutics. This module involved seven quizzes leading to an examination. Generalizability Theory (Gtheory) was used to combine these multiple testing occasions, based on a model where students were crossed with items nested in eight fixed testing occasions ( $\bullet \bullet x$ i ; mGENOVA used). Results: Onehundred students took seven quizzes and one exam. The exam had 32MCQ $($ KR-20 reliability $=0.67)$. Quizzes had a total of 50MCQ (5-9MCQ each), with most KR-20's less than or equal to 0.54 . Using quiz \& exam weightings of $18 \% \& 82 \%$, the reliability of the composite grade was 0.73 (i.e., improved from the exam alone). Doubling the quiz-weight to $36 \%$, increased the reliability of module grade to 0.77 . Reliability of 0.80 was achieved with equalweight for quizzes and exam. Implications: Using quizzes can improve reliability beyond examinations; however, to contribute meaningfully, weighting of quizzes needed to approach weighting of examinations. While one quiz (with KR-20 of 0.00) showed that quizzes do not necessarily improve the reliability, if aligned to general content in the module, quiz data can enhance reliability of composite grades.

RAMCOM: A Tool for Communication with Muslim Patients Considering Fasting During Ramadan. Mohamed E. Amin, Beirut Arab University, Ahmed Abdelmageed, Manchester University. Objective: 1) Explore clinicians' perspectives on factors affecting care provided to Muslim patients considering fasting Ramadan. 2) Propose a communication tool, RAMCOM, that can assist clinicians in caring for such patients and gathers perspectives on its potential to be implemented. Methods: Semi-structured interviews were conducted with a purposeful sample of clinicians in Egypt (11) and the US (10). Clinicians were purposefully sampled to assure variance in age, gender, time in practice, specialty, and religious background. Data emerging from narratives were mapped to constructs within Social Cognitive Theory (SCT). Using framework analysis, iterative sampling and analysis continued until saturation. Results: Twenty physicians and one ambulatory care pharmacist were interviewed. Drawing on SCT, clinicians' beliefs were mapped and clustered into self-efficacy (belief in ability to care for those patients), collective efficacy (belief in medical group's ability to provide care), outcome expectations (anticipated consequences of providing such care), knowledge of Islam, Ramadan, and fasting guidelines, observational learning (learning by observing other clini- cians), normative beliefs (cultural norms and beliefs about acceptability and prevalence of care provision), environmental barriers and opportunities, and behavioral skills (communication approach). Factors contributing to using RAMCOM included perception of tool (need for use, perceived burden of use), perceived norm (perceived patient expectations), and ability to use tool (time, frequency of seeing patients, knowledge of Ramadan and Islam). Implications: Clinicians provided valuable perceptions on caring for Muslim patients and on the potential to be implemented of RAMCOM. To facilitate better care for Muslim patients considering fasting, these perceptions should be considered by different stakeholders including educators.

Resident and Preceptor Development Sessions on Large Group Teaching Using Four-Component Instructional Design. Katherine Gruenberg, University of California, San Francisco, Conan MacDougall, University of California, San Francisco. Objective: To explore the use of Four-Component Instructional Design (4C/ID), a cognitive science-derived instructional design model, to plan and execute a large group teaching development session. Methods: We developed two sessions on large group teaching using the 4C/ID framework. One session was delivered to first- and second-year pharmacy residents as part of a teaching certificate program. The other session was offered to pharmacist preceptors at the University of California San Francisco for preceptor development. Using 4C/ID, we defined learning tasks aligned with session objectives, active learning methods, and informal assessment and feedback. Participant satisfaction was elicited using an electronic survey. Results: Twenty-six residents and fourteen preceptors participated in their respective sessions. Resident (77\% response rate) and preceptor participants ( $42 \%$ response rate) both indicated that presentation content, pace and sequencing of information, and use of active learning were effective to highly effective. Implications: The 4C/ID framework serves as an effective instructional design tool for constructing resident and preceptor development sessions.

Resource Utilization and Clinical Outcomes of Inpatients with Alzheimer's Disease and Related Dementia in the US. Vassiki Sanogo, University of Florida. Objective: Alzheimer's disease and related dementia (ADRD) a leading health problem faced today, with an increase in hospitalized patients. This study compared patients' socio-demographic, clinical characteristics, determined estimates of prevalence, mortality, resource utilization and predictors for hospitalized U.S ADRD patients. Methods: Using the Nationwide Readmissions Database with ICD-9. - 10-CM codes, analyzed retrospective cohorts of aged $>18$ years admitted to the 


\section{American Journal of Pharmaceutical Education 2019; 83 (5) Article 7654.}

U.S. hospitals between 2010 and 2015. Descriptive analyses were conducted for socio-demographic and clinical characteristics. Multivariate analyses were conducted for healthcare resources utilization and predictors. Results: Of $83,243,478$ adults discharged from U.S. hospitals (2010-2015); 529,198 were diagnosed with ADRD. The yearly inpatient of ADRD ranged from 47,569 to 41,154 ( $\mathrm{P}<.0001)$, encompassing $0.65 \%$ of all hospitalizations. Over the study period, hospital mortality decreased from $3.60 \%$ to $1.73 \%(\mathrm{P}<.0001)$. The average LOS increased from 7.35 to 8.89 days $(\mathrm{P}<.0001)$. The average charges increased from $\$ 30,259$ to $\$ 35,896$ case $(\mathrm{P}<.0001)$. Moreover, total national charges related to ADRD decreased from $\$ 3,543$ million to $\$ 2,594$ million. Although $97 \%$ of inpatients were late-onset ADRD ( $>$ mid-60s), $3 \%$ were early-onset (30-mid-60s). mortality predictors included the number diagnoses per admission $(\mathrm{OR}=1.099 ; 95 \% \mathrm{CI}, 1.09$ to 1.11 /diagnosis), number of procedures per admission $(\mathrm{OR}=1.140$ per procedure; $95 \% \mathrm{CI}, 1.12$ to 1.16$)$, and mortality score $(\mathrm{OR}=1.018$; $95 \%$ CI, 0.98 to 1.06). Most important predictors of cost, charge, and LOS were identified $(\mathrm{P}<.05)$. Implications: This study provides evidence on characteristics and healthcare resources utilization associated with ADRD patients from 2010 to 2015 in U.S.

Revealing Trends of Overdose Deaths and Opioid Prescriptions via Google Keyword Search. Tuan Tran, California Northstate University, Erika Titus-Lay, California Northstate University, Uyen M. Le, California Northstate University. Objective: The objective of this study was to reveal trends of overdose deaths and filled opioid prescriptions in the U.S. via Google keyword search. Methods: Data was collected from the Centers for Disease Control and Prevention (CDC), National Institute on Drug Abuse (NIDA), and Google Trends including opioid prescription rates from 2013 - 2017, number of overdose deaths per 100,000 persons in 2016 and frequencies of "opioid" keyword searches associated with geographical locations from years 2013 - 2016. Correlation analysis was used to identify trends of overdose deaths and opioid prescriptions of different states. Keyword search ranking identified the top 10 states with the most overdose deaths. Results: Pearson correlation analysis showed strong correlation of filled prescriptions from 2013 - 2017 with coefficients greater than 0.96, pvalues $<=0.001$. Findings showed consistent prescription filling trends across the states over time. Analysis of Google keyword searches from 2013 - 2016 showed strong correlation with overdose deaths in 2016 with coefficients from $0.56-0.78$, p-values $<=0.01$. Additionally, high correlations of monthly keyword searches and overdose deaths in 2016 were identified, ranging from
$0.61-0.78$, p-values $<=0.01$. Finally, keyword search ranking correctly identified $80 \%$ of the top 10 states having the most overdose deaths in 2016. Implications: The study proposes a novel way for estimating trends of overdose cases and opioid prescriptions across the states in the U.S. Policy makers can use it for public health surveillance. Our future work includes refining keyword combinations and collecting more data for predictive modeling.

Students' Self-Assessment of Global Health Competencies and Perceived Value in the PharmD Curriculum. Prosperity Eneh, University of Minnesota, David R. Steeb, University of North Carolina at Chapel Hill, Rebecca Cope, Long Island University, Suzanna Gim, Long Island University, Olihe N. Okoro, University of Minnesota. Objective: (1) Determine students' selfassessed competency in global health topics and perception of importance to pharmacy practice; (2) identify attributes associated with competency and perception of importance; and (3) explore students' perspectives on how best to incorporate global health content into pharmacy education. Methods: A cross-sectional online survey was administered to students in three Colleges of Pharmacy. Survey items were adapted with permission from the Consortium of Universities for Global Health (CUGH) inter-professional competency statements and the Global Health Competency (GHC) survey for health professionals. Data was analyzed using descriptive statistics, Fischer Exact tests and ANOVA. Results: The survey was completed by 487 students. Students were most confident in their ability to "Acknowledge one's limitations in skills, knowledge, and abilities within a global health team" and least confident to "Describe the major public health efforts to reduce disparities in global health." Exposure to global health content outside the pharmacy curriculum was significantly associated with higher competency scores $(p<0.05)$. Race/Ethnicity and speaking a non-English language were associated with perceived importance of global health topics to a pharmacy career $(\mathrm{p}<0.05)$. Most respondents $(96 \%)$ agreed that relevant global health education should be incorporated into the pharmacy curriculum. Implications: Pharmacy students generally perceive global health competencies to be of great value in practice and as global citizens but acknowledge their deficiencies in this area. Ensuring that pharmacists understand their role in global health teams and achieve the necessary level of competency to function in interdisciplinary initiatives requires more strategic incorporation of relevant content into the curriculum.

Team-Based Learning in a Pharmacy Management Course. Marwa Noureldin, Manchester University, Mohamed E. Amin, Beirut Arab University, Joseph K. 


\section{American Journal of Pharmaceutical Education 2019; 83 (5) Article 7654.}

Bonnarens. Objective: Few published studies have examined the utility of team-based learning (TBL) as a pedagogy for pharmacy management courses. This study evaluates the impact of an innovative pharmacy management course on student perceptions of the value of TBL and confidence in developing a clinical service business plan. Methods: A TBL course is required for third year pharmacy students and includes a business plan project incorporating a clinical pharmacy service and pharmacogenomics. Assessments included Readiness Assurance Tests (RATs); in-class engagement; peer evaluations; and business plan checkpoints, final report, and presentation. Using IASystem ${ }^{\text {TM }}$ (University of Washington), students evaluated the course overall, the level of academic challenge and engagement (CEI score), their perceptions on the value of TBL components, and their confidence in completing several business plan sections. Likert scales were utilized to examine perceptions and confidence. Descriptive statistics were conducted. The institution's IRB deemed the study exempt. Results: Sixty-six students completed the course during Fall 2018. Based on course evaluations, the median summative course rating was 4.4 (out of 5). Students rated the course as 4.9 on the CEI Index (out of 7, higher scores indicate higher challenge and engagement). All students strongly agreed/agreed/ slightly agreed that TBL was the preferred instructional method for the course. Most students ( $>80 \%$ ) strongly agreed/agreed they were confident in their ability to develop a business plan for a new patient service and recognize the potential role of pharmacogenomics within these services. Implications: TBL is an appropriate and valuable approach to enhance students' application of pharmacy management and business planning principles.

The Efficacy of CASPer ${ }^{\circledR}$, an Online Situational Judgement Test (SJT), in Pharmacy School Admissions. Harold I. Reiter, McMaster University, Heather Davidson, Altus Assessments. Objective: A challenge in health professions education admissions is on the assessment of applicants' personal competencies, which have been shown to be stronger predictors of future job performance and lapses in professionalism than traditional cognitive measures (e.g. GPA, PCAT), in a time- and cost-effective manner. In 2018, three pharmacy programs in Canada adopted CASPer ${ }^{\circledR}$, an online SJT, as a way of incorporating non-cognitive characteristics into their screening process. This study explored the validity, feasibility, and acceptability of this addition to the pharmacy school admissions process. Methods: Retrospective data were analyzed, including psychometric data on test internal consistency and inter-rater reliability, and qualitative and quantitative data on perceptions of CASPer ${ }^{\circledR}$ from pharmacy programs and applicants. Results: These analyses suggest that the psychometric evidence and applicant acceptability of CASPer ${ }^{\circledR}$ in pharmacy education is in line with what has been found in the North American medical school context, showing both high reliability $(\alpha=.81, \mathrm{ICC}=.81)$ and generally positive applicant and program perceptions. Results are also presented from case studies on the three programs who have adopted CASPer ${ }^{\circledR}$ thus far. Implications: These preliminary results support the use of CASPer ${ }^{\circledR}$ in pharmacy school admissions for assessing personal competencies. Future directions, including the need for longitudinal data on predictive validity in pharmacy education, are discussed.

The Impact of a Structured In-Depth Academic Instruction Program for PGY-2 Pharmacy Residents. Nicholas G. Popovich, University of Illinois at Chicago, Hannah Dalogdog, University of Illinois at Chicago, Kayeromi Gomez, University of Illinois at Chicago. Objective: Teaching is an essential function of a pharmacy resident. Unfortunately, not all residency programs incorporate in-depth teaching certificates, and the current number of programs that do so is not known. The objective of this study was to determine whether participation in an indepth academic instruction program influenced the confidence of participating PGY-2 residents in their teaching skills. Methods: A voluntary survey was conducted among UIH PGY-2 pharmacy residents who participated in a UIC Academic Instruction Program from August 2017 to May 2018. The survey data was measured in three parts: (1) a retrospective pre-post survey, (2) an agree/ disagree portion, and (3) open-ended questions. Descriptive analysis was used to evaluate the progress in each resident's perception of their attitudes, confidence, and knowledge in participating in an academic-focused program from beginning to end. Results: Eleven residents completed the three-part assessment, with 100 percent completion. The data for Section 1 was on a four-point scale of "Weak" to "Very Good," and examined using the Means Procedure (mean: 1.89, SD: 0.73 [pre-analysis]; mean: 3.20, SD: 0.60 [post-analysis]). Section 2 had fourpoint scale of "Disagree" to "Agree". The information from Section 3 was collated into a separate deidentified report. Implications: Data analysis demonstrated the residents who participated in the program considered themselves overall more confident in their acquired teaching skills and, therefore, better prepared to enter academic pharmacy. This study should be conducted with other pharmacy programs offering teaching certificates for comparison of results and standardization of programs.

Thirty-Day Readmissions Associated with Ischemic Heart Disease, Predictors and Ethnoracial Differences. Arinzechukwu Nkemdirim Okere, Florida A\&M University, Vakaramoko Diaby, University of Florida, 


\section{American Journal of Pharmaceutical Education 2019; 83 (5) Article 7654.}

Vassiki Sanogo, University of Florida. Objective: Hospital readmissions among patients with ischemic heart disease (IHD) is common. As expected, 30-day readmissions associated with IHD places financial burden on society and negatively impact patients reported quality of life. Efforts to minimize preventable IHD readmissions should be based on the understanding of the incidence and predictors. Nonetheless, less is known about the rate of IHD related 30-day readmission, cost and risk factors. In this study, we evaluated the rate of readmission for composite IHD related diseases, costs and underlying predictors. Methods: We conducted a retrospective analysis of all hospitalizations associated with IHD. This was done by using the public dataset from Florida Agency for Health Care Administration from 2006 - 2016. The rate and cost of 30-day readmission were examined, and a generalized linear model was used to identify the predictors of 30-day readmission. This study was approved by the institutional review board. Results: We evaluated 59,589 patients. Based on our analysis, 30-day readmissions for composite IHD related disease was $6.47 \%(\mathrm{p}<$ 0.001 ). The female sex, age, hypertension, congestive heart failure, COPD, atrial fibrillation, and increased length of stay were independent predictors for IHD related readmission $(\mathrm{P}<0.0001)$. Additionally, patients of African American and Hispanic origins had a higher rate of readmission when compared to Caucasian $(\mathrm{P}<0.001)$. Finally, the average total cost per IHD patient was $\$ 105,116$ ( $\pm 2.14 \mathrm{E} 6$ ). Implications: These findings underscore the importance of identifying amenable improvement quality measures to alleviate IHD related conditions that will translate to reduction in preventable readmissions and cost.

Towards Better Assessment of Educational Outcomes Through Mapping of Exam Questions. Monina Lahoz, MCPHS University-Boston, Lana Dvorkin Camiel, MCPHS University-Boston, Gerard G. D'Souza, MCPHS University-Boston, Tewodros Eguale, MCPHS University-Boston, Paul J. Kiritsy, MCPHS UniversityBoston, Matthew R. Machado, MCPHS UniversityBoston, Jennifer Prisco, MCPHS University-Boston, Brian Rittenhouse, MCPHS University-Boston, Anela Stanic, MCPHS University-Boston, Andrew Szumita, MCPHS University-Boston. Objective: The school's Assessment Committee implemented a mapping initiative to: (1) determine the breadth and depth by which written exams assess educational outcomes, and (2) identify gaps in required content in the PharmD curriculum. Methods: In 3-4 member peer-review groups, faculty mapped exam question packets from didactic courses (PY1, PY2, and PY3) during weekly faculty meetings over a 3-month period according to three criteria: (1) ACPE Standards
2016 Appendix 1, (2) CAPE Outcomes 2013, and (3) Bloom's Taxonomy of Learning Objectives. To test interrater reliability, two groups mapped the same packets. Results: A total of 3401 exam questions from 32 courses were mapped. Per Standards 2016, the content areas with the most questions were pharmacotherapy (694), pharmacology (459), and biochemistry (287); those with the least questions were ethics (5), history of pharmacy (2), and cultural awareness (1). Per categories, 33\% of the questions mapped to pharmaceutical sciences, $32 \%$ clinical sciences, $20 \%$ biomedical sciences, and $15 \%$ social sciences. Per CAPE Outcomes domains, $77 \%$ of the questions mapped to "Foundational Knowledge" and 15\% "Essentials for Practice and Care". "Foundational Knowledge" questions decreased progressively from PY1 to PY3 (90\% to 59\%) while questions in the other domains increased. Per Bloom's Taxonomy levels, $68 \%$ of the questions mapped to "knowledge and comprehension", $24 \%$ "application and analysis", and 7\% "synthesis and evaluation". Interrater reliability ranged from $72 \%$ to $80 \%$. Implications: This mapping initiative prepared faculty for the implementation of a computer-based testing application. We acknowledge that comprehensive curriculum assessment requires more than exam question mapping.

Understanding the Learning Styles of P1 Students at One College of Pharmacy. C. Lea Lea Winkles, Mercer University, Jill M. Augustine, Mercer University. Objective: To identify most common learning styles among pharmacy students at one college of pharmacy; and, identify if learning styles impact performance in the firstprofessional year (P1). Methods: This retrospective descriptive project assessed P1 students' learning styles using the Index of Learning Styles (ILS) questionnaire. Questionnaire results indicated students' primary, secondary, tertiary, and quaternary learning style. Results were compared to students' grades in P1 courses, number of $\mathrm{P} 1$ courses failed, gender, and if a previous degree was completed. Differences in learning styles and outcomes were determined using T-tests and chi-square analysis. Results: One-hundred and forty-three students completed the ILS questionnaire. The Sensing learning style was most frequent $(\mathrm{n}=58,40.6 \%)$ followed by Visual $(\mathrm{n}=28,19.6 \%)$, Reflective $(\mathrm{n}=25,17.5 \%)$, and Active $(\mathrm{n}=12,8.4 \%)$. There were no differences in learning styles between genders $(p=0.07)$, previous degree $(p=0.18)$, and number of courses failed $(p=0.06)$.Among the most frequent learning styles (Sensing, Visual, Reflective, and Active which accounts for $86 \%$ of students), students had higher grades in non-science courses in the P1 curriculum $(\mathrm{p}<0.001$ for all styles $)$; in longitudinal courses ( $p<0.001$ for all styles); and in application-based 


\section{American Journal of Pharmaceutical Education 2019; 83 (5) Article 7654.}

courses $(\mathrm{p}<0.001$ for all styles). Implications: Students were most commonly a Sensing, Visual, Reflective of Active learner, regardless of gender. No particular learning style was associated with failure of P1 course. Students with these learning styles had significantly higher grades in certain courses. Understanding of a students' learning style can help faculty when developing lectures and classroom instruction or when assisting students individually who may struggle in their course.

Use of an Annual Performance Evaluation to Assess Professional Growth in Student Pharmacists. Maria G. Shelley, Purdue University, Jennifer Dexter, Purdue University, Kimberly S. Illingworth Plake, Purdue University, Holly L. Mason, Purdue University. Objective: The annual performance evaluation (APE) was developed to address ACPE accreditation standard 4 and provide an opportunity for students to reflect on their growth and receive evaluative feedback on their progress. The objectives of this study were to assess students' professional growth in self-awareness, leadership, professionalism, and career planning, as well as students' satisfaction with the APE process. Methods: First professional year students $(\mathrm{N}=150)$ reported and reflected upon professional growth activities in the areas of selfawareness, leadership, professionalism, and career planning, as well as created an action plan for continued growth. Faculty assessed students' reflections using a standardized rubric (accomplished, developing, novice) and facilitated small group discussions focused on continued professional growth in each of the areas. Students were surveyed regarding their satisfaction with the APE process. Descriptive statistics were performed on measures assessing student performance and satisfaction. Results: Less than $10 \%$ of students were considered "accomplished" in any of the professional growth areas with the majority of students considered as "developing" and a somewhat smaller number classified as "novice". When asked about the most impactful learning experience in the past year, $46 \%$ wrote about their first introductory pharmacy practice experience, which helped in their comfort level when interacting with patients (21\%). Approximately $89 \%$ of the students found it helpful to think about their professional growth and $93.3 \%$ indicated the small group discussion was a good learning experience. Implications: The Annual Performance Evaluation was a unique way to document and encourage students' professional growth. Students viewed the APE as a positive educational experience.

Using Social Cognitive Career Theory to Examine Pharmacy Residents' Research Intentions. Spencer E. Harpe, Midwestern University/Downers Grove. Objective: According to social cognitive career theory
(SCCT), interests, intentions, and actions result from interactions between self-efficacy and outcome expectations, which are influenced by sources of self-efficacy, learning experiences, and personal and environmental factors. The objective of this study was to use SCCT to predict pharmacy residents' research intentions. Methods: An online questionnaire containing the shortened version of the Research Training Environment Survey (RTES), the Mentorship Effectiveness Scale (MES), the revised Research Outcome Expectations Questionnaire (ROEQ), and assessments of practice-related research attitudes, research self-efficacy, and investigative career interests was distributed to a random sample of 600 PGY1 and 600 PGY2 residents. The SCCT framework was examined using structural equation modeling. Model fit was assessed using the comparative fit index (CFI) and root mean square error of approximation (RMSEA). The institutional review board granted this study exempt status. Results: Overall, 470 residents responded (39\% response rate; 44\% PGY1; 74\% female; mean [SD] age: 26.9 [3.2] years). Proposed relationships between environmental factors (e.g., residency setting) and research intentions were not statistically significant and removed. The trimmed model showed acceptable fit (CFI $=0.952$, RMSEA $=0.067$ ). Positive total effects were found for research outcome expectations $(\beta=0.684)$ and research self-efficacy $(\beta=0.025)$. As suggested by SCCT, personal factors, sources of self-efficacy, and learning experiences (e.g., previous research output and positive research experiences) had positive effects mediated by research selfefficacy and outcome expectations. Implications: SCCT may be useful in predicting resident research intentions. Residency research training may benefit from improvements guided by SCCT-based approaches.

Using Theatrics to Design an Escape Room Activity in a Biostatistics Course. Amany K. Hassan, D'Youville College, Vicky Belousova, D'Youville College. Objective: To describe the design of an educational escape room used to assess knowledge of students in biostatistics and evaluate the perceived benefit of the activity. Methods: A combination of online and physical locks and clues hidden in the classroom were used. All clues were part of a script based on Harry Potter books. The facilitators dressed as characters from the book and videos from the movies were played for theatrical effects. Students worked in teams to escape in 50 minutes. An anonymous survey was sent to students for evaluation. Data were analyzed using descriptive statistics and Wilcoxon signed-rank test. Results: Forty-one responses were received $(72 \%)$. Forty students $(97.5 \%)$ agreed that the activity encouraged them to think about the course in a new way, learn from peers, and that it was effective to review 


\section{American Journal of Pharmaceutical Education 2019; 83 (5) Article 7654.}

the material. However, 14 students (34\%) agreed they felt stressed, and $17 \%$ agreed that the non-educational part (cyphers) were distracting. On a scale from one to ten, students' median score for the escape room level of engagement was 9.0 compared to 7.0 for class discussions $(\mathrm{p}=0.001), 8.0$ for online polls, and 8.0 for jeopardy games. The median score for learning effectiveness was 8.0 for escape room compared to 7.0 for class discussions $(p=0.244)$. Comments indicated that the Harry Potter theme was fun, interactive, and engaging. Implications: The study describes the design of a popular educational game that can be used for a large group of students and provides an insight on how it compares to other active learning activities.

Utilizing International Mission Work to Promote Interprofessional Education While Reinforcing Clinical Knowledge and Skills. Sarah E. Raake, Sullivan University, Emily R. Esposito, Sullivan University. Objective: Health professions education is rooted in the translation of novice didactic knowledge into practiceready clinical skills. Logistical and curricular constraints often hinder early integration of clinical experiences in didactic curricula. By providing an opportunity for learners to partake in international mission work, health professions educators can promote interprofessional education (IPE) in an informal, service learning environment while also reinforcing clinical knowledge and skills. Research from recent mission trips at a College of Health Sciences demonstrates the positive impact of allowing trip participants to learn with and from other professions in authentic clinical settings, emphasizing the need for early immersion in clinical care and interprofessional experiences. Methods: Two international short-term medical mission trips were organized in which participants were divided into mixed-ability, interprofessional teams. The Readiness for Interprofessional Learning Scale (RIPLS) survey was administered, measuring learner perceptions in areas such as teamwork/collaboration, professional identity, and roles and responsibilities. Experiences were detailed throughout the trip and mapped to curricular topic (including disease specific care), programmatic outcomes, ACPE standards, and IPEC domains. Results: Trip participants affirmed that working together on a multidisciplinary team advanced their knowledge in their respective professions, noting an improvement in communication after working with other health professions $(\mathrm{p}<0.05)$. While caring for patients, learners relied heavily upon recall and application of an assortment didactic course material, including knowledge of over 25 different disease states. Implications: Utilizing international mission work can be a tool for promoting authentic IPE while reinforcing clinical knowledge and decision-making skills early on in health professions education.

Validation Evidence for PharmD Student Case Presentations: A Pilot Study. Jennifer S. Byrd, Union University, Michael J. Peeters, The University of Toledo. Objective: There is a paucity of validation evidence for assessments of clinical case presentations by PharmD students. In Kane's Framework for Validation of learning assessments, one important aspect is scoring while another is generalization. Towards validation of a case presentation assessment, our objectives were to characterize and improve scoring, as well as build initial generalization evidence. Methods: Third-year PharmD students worked up patient-cases from a local hospital. Students orally presented/defended their therapeutic care-plan to pharmacist preceptors and fellow students. Evaluators scored presentations using an 11-item instrument with a 6-point rating-scale. In addition, evaluators scored one global item on a 4-point rating-scale. Rasch Measurement was used for scoring analysis, while Generalizability Theory was used for generalization analysis. Results: In Fall 2017, 35 students each presented five cases that were evaluated by 15 preceptors. Using Rasch, eight of the 11 items measured students' ability $(73 \% ; 3020$ data-points). The 6-point rating-scale was dysfunctional; functionality improved when collapsed into a 4-point rating-scale - though still showed redundancy (separation 8.62, reliability 0.99 ). Alone, the global item performed reasonably (separation 2.57 reliability 0.87 ). Using Generalizability Theory, composite reliability for the series of case-presentations was 0.74 . A trade-off in reliability estimates showed that one evaluator for each of nine presentations (reliability $=0.81$ ), or two evaluators for five presentations (reliability $=0.83$ ) seemed best. Implications: Our results demonstrate that scoring should be simple (scale \& instrument); a longer 11-item instrument had redundancy whereas one global item provided favorable measurement. Reliability (and generalization) was more dependent on multiple case presentations than on number of evaluators per presentation.

What Differentiates Exceptional New Pharmacy Practitioners? Kristin K. Janke, University of Minnesota, Robert A. Bechtol, University of Minnesota, Eliza Dy-Boarman, Drake University, Michael H. Nelson, Drake University. Objective: To determine: 1) pharmacy employers' perspectives on the variables differentiating exceptional new practitioners (ENPs), and 2) the utility of a Delphi process in collecting external constituents' perspectives to support curriculum advancement. Methods: A modified Delphi process was conducted with employers in inpatient and outpatient pharmacy settings. Possible panelists were nominated by one of six 


\section{American Journal of Pharmaceutical Education 2019; 83 (5) Article 7654.}

partnering Midwest pharmacy schools and interested individuals confirmed eligibility (i.e., significant experience on the job; observation of the new practitioner work; involvement in the hiring process; exposure to new practitioners from multiple schools). Panelists responded to open-ended questions on behaviors of ENPs (i.e., Round 1) followed by rating the themes derived from those responses (i.e., Round 2). Consensus was defined as 90\% of ratings as Agree/Somewhat Agree. The process and data were examined to determine the Delphi's utility for gathering external constituents' input. Results: Nine panelists participated in Round 1. Six panelists returned for Round 2 with five additional panelists joining to provide additional, new perspectives. One hundred sixteen (116) codes were identified and nine (9) themes were developed, including "Speaking up for quality improvement and patient safety" and "Developing self through successes and failures." All themes achieved consensus. The process produced five, single-spaced pages of text $(2,124$ words) for analysis. Implications: The results expand the evidence base supporting the design of professional development curricula. The Delphi process captured panelists' responses to focus group-similar questions with the added benefits of: 1) asynchronous participation without travel, 2) documenting a response from each person, and 3) no hosting or transcription costs.

What Employers Want: Identifying Necessary Skills in Pharmaceutical Sciences Graduates. Lana M. Minshew, University of North Carolina at Chapel Hill, Jacqueline McLaughlin, University of North Carolina at Chapel Hill, Jeffrey Aube, University of North Carolina at Chapel Hill, Wendy C. Cox, University of North Carolina at Chapel Hill, Daniel Gonzalez, University of North Carolina at Chapel Hill, Kelsey Lamb, University of North Carolina at Chapel Hill, Nicholas J. Klus, University of North Carolina at Chapel Hill, Kim R. Brouwer, University of North Carolina at Chapel Hill. Objective: The goal of this study was to identify the core skills that employers look for when hiring doctoral graduates in the pharmaceutical sciences. Methods: Semiscripted interviews were conducted in November and December of 2017, with 12 pharmaceutical scientists representing diverse expertise, career paths and current positions in global pharmaceutical companies, small startups, government, and academia. Transcripts of interviews were coded by two data analysts and codes were synthesized to identify common themes. The themes were reviewed by the research team for consensus building, and an independent qualitative analyst confirmed the findings. Results: Analysis revealed seven broad themes regarding the skills and abilities employers desire when hiring pharmaceutical sciences doctoral graduates. Four of the themes were expressed by all 12 experts: knowledge, communication, collaboration, and motivation/curiosity. Three themes were salient, yet not present in every interview: experiential training, adaptability, and critical thinking/problem solving. The findings suggest that regardless of career path, employers value and desire similar skills, traits, and characteristics of graduates across different areas of the pharmaceutical sciences. Implications: The employability of doctoral students in the pharmaceutical sciences is a key concern for graduate programs. Identifying strategies that optimize student development of the above skills and attributes is imperative for graduate programs aimed at differentiating students and meeting the needs of employers. To attract and support students in today's competitive job market, doctoral programs must create programs that foster the qualities and skills most valued by future employers upon graduation.

What were You Thinking? Student and Pharmacist Situational Judgments About Empathy. Michael D. Wolcott, University of North Carolina at Chapel Hill, Nikki G. Lobczowski, University of North Carolina at Chapel Hill, Jacqueline M. Zeeman, University of North Carolina at Chapel Hill, Jacqueline McLaughlin, University of North Carolina at Chapel Hill. Objective: To describe the features of the response process (i.e., what participants think about) when completing a situational judgment test (SJT) intended to measure empathy. Methods: Thirty participants -15 students and 15 pharmacists - completed a 12-item SJT designed to measure empathy. Each SJT item included a case scenario and participants ranked five responses to the scenario in order of appropriateness. Each participant engaged in a thinkaloud interview during the SJT in which they spoke about their thought process while they completed the test. This was followed by a cognitive interview with specific questions about their decision-making processes. Interviews were audio-recorded and transcribed for qualitative analysis. Salient themes were identified and used to create a model to describe the SJT response process. Results: The overarching SJT response process can be described by a four-component model: comprehension, retrieval, judgments, and response selections. Each component was influenced by multiple factors. For example, comprehension was influenced by the task to be completed. Job-specific knowledge and experiences comprised a substantial portion of the retrieval process. Moreover, there was evidence that SJTs were highly contextual and that item characteristics such as setting, actors, or relationships influenced judgements. Lastly, there was evidence that individuals utilized specific strategies when selecting their final responses, such as an ordered approach and guessing. Implications: This 


\section{American Journal of Pharmaceutical Education 2019; 83 (5) Article 7654.}

study provides a comprehensive evaluation of SJT response processes. Overall, design of these instruments must be attentive to items characteristics and the perspective of the test-taker that may relate to answer selections.

\section{Theoretical Models}

Evaluation of Student's Perceptions of SmartDrugs Use Among College Students: Instrument Development. Fatimah M. Sherbeny, Florida A\&M University, Diena Almasri, King Abdulaziz University, Askal A. Ali, Florida A\&M University, Barry A. Bleidt, Nova Southeastern University. Objective: Non-medical use of Smart-drugs (SDs) among students has been growing to enhance cognitive performance. Among college students, $10 \%$ used SDs, while $11.3 \%$ of health professions students admitted to misusing prescription stimulants, $70.5 \%$ of which were pharmacy students. The objective of this study was to develop an instrument to evaluate students' perception of the need, safety, and fairness of using smart-drugs among college students. Methods: In this study, SDs were defined as prescription stimulants, used to treat ADHD, such as methylphenidate and amphetamine. A survey was developed to measure and evaluate students' perception. It had four components: perception, need, safety, and fairness of using SDs. The instrument included 24 items agree/disagree, 5-point Likert scale, type of questions. Then, the survey was sent to an expert panel for evaluation and feedback. SPSS statistical software was used for factor analysis. Results: With a response rate of $79 \%$, factor analysis showed that the instrument items were loading on 6 factors instead of 4 . Some of the items were loading on more than one factor. Therefore, 4 items were removed, and a second factor analysis was performed. It resulted in 4 factors as the original hypothesis. However, based on the results of the factor analysis, the items needed to be rearranged for better loading. Implications: Research on nonmedical stimulant use has been focused on traditional drug misuse issues. Stimulant use for cognitive enhancement is not well studied. Sociobehavioral analysis of nonmedical stimulant drug use among college students is needed for better prevention and intervention strategies.

Health Systems Science: Should This Proposed "Third Pillar" of Medical Education Be Adopted by Pharmacy? Gregory Reardon, Keck Graduate Institute. Objective: In 2013, the American Medical Association (AMA) funded 11 demonstration projects to address perceived deficits in knowledge, skills and attitudes within the Flexnerian dyad of basic and clinical science education. Several medical schools have since adopted the nascent discipline of Health Systems Science (HSS), based in systems thinking, and proposed as a possible "third pillar" of medicine. This study explores the origins of HSS, summarizing learning frameworks and competencies and evaluates implications for pharmacy education. Methods: Abstracts from PubMed and International Pharmaceutical Abstracts were analyzed using search terms "'systems thinking' and (health or medicine)," "'health systems science,"” "('systems thinking' or 'health systems science' or 'social and administrative sciences') and (pharmacy or pharmacist)." Cited works were further evaluated for consideration. A total of 324 full text articles and one HSS textbook were reviewed. Results: The number of HSS publications appears to have grown exponentially. Most defined HSS competencies are consistent with related systems-based-care/populationhealth/policy/economics ones in ACCP Competencies 2017, ACPE Standards 2016, and CAPE Outcomes 2013. Many HSS competencies are likely currently offered in social/administrative or clinical practice courses of most pharmacy colleges, though HSS emphasizes much more of an integrated systems, rather than compartmental, blending within the didactic portion of clinical sciences (M3 and M4 years in medicine) and extends much further into post-graduate education. Implications: Subject to critical study, HSS offers pharmacy educators an opportunity to achieve health systems and public health competencies and to integrate these more fully within didactic clinical practice teaching, with potential extension to experiential rotations and post-graduate residencies.

Social Capital and the Pharmacy Service Mix. Oscar W. Garza, University of Minnesota, Caroline A. Gaither, University of Minnesota. Objective: To identify a conceptual model for understanding the relationship between organizational social capital and pharmacy service development. Methods: A cross-sectional, descriptive survey design was used for collecting and analyzing data. Data were collected using a self-administered questionnaire that was mailed to a random sample of 5,200 licensed pharmacists in the US. An 11-page questionnaire was developed and each of the items were previously found to be reliable and valid in prior studies and thus included in the instrument. Data were analyzed using descriptive statistics. Results: Approximately 1,389 questionnaires ( $28 \%$ response rate) were returned and included in the analyses. Almost half (50\%) did not feel like they could trust the people they work with, over a third (35\%) did not feel committed to their pharmacy organization, and over a third (36\%) did not perceive normative structures tying them to the organizational purpose. More than half $(55 \%)$ of respondents did not feel like they had any control in their work environment. Approximately half $(50 \%)$ of respondents reported that their pharmacy practice site offered 1 or less pharmacy service of a 


\section{American Journal of Pharmaceutical Education 2019; 83 (5) Article 7654.}

possible 11 pharmacy services. Implications: The movement toward value-based health care has presented a potential boon for pharmacy to survive the ever-shrinking margins in medication dispensing-based business models. Organizational commitment is important for maximizing value of the pharmacy profession. However, largely unaddressed is the basis of the exchange within the organization and its subsequent impact on the ability to meet the needs and opportunities of the healthcare marketplace.

\section{7-2018 NEW INVESTIGATOR AWARD WINNERS}

Addressing Racial Disparity in Heart Failure Outcomes and Beta-Blocker Response. Jasmine A. Luzum, University of Michigan, Solomon K. Musani, University of Mississippi Medical Center, Stanford E. Mwasongwe, University of Mississippi Medical Center, Edward L. Peterson, Henry Ford Health System, David E. Lanfear, Henry Ford Health System. Objectives: Heart failure (HF) is a major public health problem, but it is especially burdensome in African-Americans. The beta- 1 adrenergic receptor is critical to HF pathophysiology and beta-blocker pharmacology. A genetic variant, rs11196454, was recently discovered to increase expression of the beta- 1 adrenergic receptor in hearts from HF patients, and the variant is most frequent in AfricanAmericans (34\%). Therefore, the objective of this study was to determine whether rs 11196454 is significantly associated with all-cause and beta-blocker mortality benefit in African-Americans with HF. Methods: Retrospective analyses of two existing clinical/genomic datasets of African-Americans with HF were performed using Cox proportional hazards regression adjusted for significant clinical risk factors and population stratification. The test dataset was the HF genomic registry at Henry Ford Health System (HFGR; $n=543)$. The validation dataset was HF patients from the Jackson Health Study (JHS; $\mathrm{n}=518$ ). Results: The HFGR sample was $40 \%$ women with (mean \pm sd) age $64 \pm 12$ years, $3 \pm 2$ years follow-up, and $22 \%$ mortality rate. The JHS was $61 \%$ women with age $66 \pm 14$ years, $7 \pm 3$ years follow-up, and $36 \%$ mortality rate. rs 11196454 was not significantly associated with time to death in either dataset: adjusted HR $=1.25(95 \%$ $\mathrm{CI}=0.77-2.01) \quad \mathrm{p}=0.369$ in HFGR and adjusted $\mathrm{HR}=0.97(0.59-1.60) \mathrm{p}=0.916$ in JHS. rs11196454 did not significantly interact with beta-blocker mortality benefit in either cohort: $p=0.934$ and $p=0.906$ in HFGR and JHS, respectively. Implications: A novel regulatory variant for the beta-1 adrenergic receptor in heart tissues (rs11196454) was not significantly associated with mortality nor beta-blocker mortality benefit in AfricanAmericans with HF.
Development of Glutaminase and Isocitrate Dehydrogenase Inhibitors for Cancer. Md. Imran Hossain, The University of Mississippi, Ajit G. Thomas, Johns Hopkins University, Barbara S. Slusher, Johns Hopkins University, Hoang V. Le, The University of Mississippi. Objective: Glutaminase and isocitrate dehydrogenase are two critical enzymes in the enhanced production of $\alpha$-ketoglutarate in cancer cells. Our objective was to develop novel inhibitors for these enzymes to study the effects on cancer cells when either one of the enzymes is inhibited, as well as when both of the enzymes are inhibited. Methods: Substrate-based irreversible inhibitors for glutaminase were designed and synthesized. Docking suggested that $L$ - $\gamma$-methyleneglutamine-based compounds that carry a Michael acceptor could inhibit the activity of glutaminase by forming a covalent bond with Cysteine 418. Mechanism-based inactivators for isocitrate dehydrogenase were designed and synthesized. Docking suggested that isocitrate-based compounds that carry an appropriate leaving group could use the catalytic activity of isocitrate dehydrogenase to activate them into reactive species inside the active site, which then inactivate the enzyme. Results: An efficient synthetic route to the biological relevant $L-\gamma$-methyleneglutamine and its amide analogues was discovered. A small library of these compounds was synthesized and tested for their potential as glutaminase inhibitors for cancer treatment. Unfortunately, none of the compounds showed inhibitory activity against kidney-type glutaminase (GLS1). It is possible that these compounds can inhibit other two isoforms of glutaminase, liver-type (GLS2) and the splice variant of GLS1 (Glutaminase C or GAC), or other glutaminedependent enzymes. The synthesis of our designed isocitrate-based compounds for isocitrate dehydrogenase is being carried out. Implications: This effort provided a much easier access to $L$ - $\gamma$-methyleneglutamine and its amide analogues for further studies on their potential as inhibitors of glutamine-dependent enzymes.

Drug Discovery and Repurposing Against a Key Clostridium sordellii Exotoxin. Dong Xu, Idaho State University, Aoxiang Tao, Idaho State University, Mike Aldape, Sandoz. Objective: The mortality rate of Clostridium sordellii infections in human is $70 \%$. We recently reported the discovery and characterization of a previously unknown metalloproteinase (Mcs1) exotoxin that cleaves human vascular cell adhesion molecule (VCAM)1, directly responsible for the extreme leukemoid reaction and fatality associated with C. sordellii infection (RSC Advances, 2017, 7, 13928-13938). In this project, we aim to identify novel Mcs1 inhibitors for the purpose of mitigating the circulating exotoxin at the critical stage of C. sordellii infection and improving patient survival. 


\section{American Journal of Pharmaceutical Education 2019; 83 (5) Article 7654.}

Methods: We carried out a large-scale pre-clinical drug screening and repurposing campaign using in silico structurebased drug design. The identified hits were then validated using in vitro assays. Results: The in-silico screening of National Cancer Institute compound library yielded seven validated Mcs 1 inhibitors at $\mu \mathrm{M}$ level inhibitory activity. The screening of approved and investigational drug library further yielded ten inhibitors at sub-nM level inhibition, five of which are FDA-approved drugs. Implications: With the discovery of novel Mcs1 inhibitors, particularly new such indications of some FDAapproved drugs, this project represents an important step forward in the direction of anti-microbial toxin development and its results demonstrate the feasibility and validity of the computational and experimental strategies.

Effects of Lead $\left(\mathrm{Pb}^{2+}\right)$ on Oxalate Nephrolithiasis. Greg M. Landry, MCPHS University-Boston, Anthony J. Branco, MCPHS University-Boston, Harleigh B. Becotte, MCPHS University-Boston Objective: An important cause of renal calcium oxalate $(\mathrm{CaOx})$ formation that remains insufficiently studied is environmental exposure to nephrotoxic metals, specifically $\mathrm{Pb}^{2+}$. Epidemiological data indicates that $\mathrm{Pb}^{2+}$ exposure $(\mathrm{BLL}=0.48-3.85 \mu \mathrm{M})$ confers a $35 \%$ increased risk of $\mathrm{CaOx}$ nephrolithiasis development. The purpose of this study was to determine the mechanism(s) that underlie the association between $\mathrm{Pb}^{2+}$ exposure and renal $\mathrm{CaOx}$ crystallization. Methods: Isolated female Drosophila melanogaster anterior Malpighian tubules (MT) (wild-type, WT and inositol $(3,4,5)$-triphosphate receptor, $\mathrm{IP}_{3} \mathrm{R}$ transgenic knockdown), as well as mouse inner medullary collecting duct (mIMCD-3) cells were utilized to determine the effects of $\mathrm{Pb}^{2+}$ on total $\mathrm{CaOx}$ crystallization, $\mathrm{IP}_{3} \mathrm{R}$ mediated $\mathrm{Ca}^{2+}$ mobilization, and epithelial cell damage. $\mathrm{Pb}^{2+}$-induced effects were assessed via $\mathrm{CaOx}$ crystal quantitation ex vivo using differential interference contrast (DIC) microscopy and ImageJ, fumarase and lactate dehydrogenase release, and intracellular $\mathrm{Ca}^{2+}$ concentrations in vitro. Results: $\mathrm{Pb}^{2+}+\mathrm{Ox}^{2-}$ increased mIMCD-3 necrotic cell death in vitro at $48 \mathrm{~h}$ sensitizing collecting duct epithelium to $\mathrm{CaOx}$ crystal nucleation and increasing the likelihood of extracellular $\mathrm{Ca}^{2+}$ release contributing to subsequent $\mathrm{CaOx}$ crystallization. Additionally, $\mathrm{CaOx}$ crystal number and total area were significantly increased ex vivo $(\sim 5$-fold $)$ in $\mathrm{Pb}^{2+}+\mathrm{Ox}^{2-}$-exposed MTs compared to $\mathrm{Ox}^{2-}$ alone controls. Contrastingly, $\mathrm{CaOx}$ crystal number and total area in $\mathrm{Pb}^{2+}+\mathrm{Ox}^{2-}$ exposed $\mathrm{IP}_{3} \mathrm{R}$ knockdown MTs were significantly decreased $(\sim 3$-fold $)$ indicating a role for $\mathrm{IP}_{3} \mathrm{R}$-mediated $\mathrm{Ca}^{2+}$ mobilization as a mechanism for $\mathrm{Pb}^{2+}$-induced increases in $\mathrm{CaOx}$ crystallization. Implications: These findings suggest that
$\mathrm{Pb}^{2+}$ exposure plays a significant role in renal CaOx crystal formation via an $\mathrm{IP}_{3} \mathrm{R}$-mediated mechanism.

Effects of the First US Biosimilar on Patient Clinical and Economic Outcomes. Minghui Sam Li, The University of Tennessee, Junling Wang, The University of Tennessee. Objective: Filgrastim and its biosimilars are commonly prescribed to treat chemotherapy-induced neutropenia in cancer patients. Filgrastim-sndz, the first U.S. biosimilar, was approved in March 2015. The objectives were to examine differences in 1) patient characteristics, 2) economic outcomes, and 3) clinical outcomes between users of filgrastim-sndz and filgrastim. Methods: This retrospective incident user cohort study used South Carolina Medicaid and cancer registry linked database. Incident users of filgrastim/filgrastim-sndz were observed based on Medicaid claims and were identified between July 2015 and June 2017. Outpatient visits, hospitalizations, emergency department visits, and total medical costs were measured during the six-month follow-up period. A generalized linear model, with a log link and a gamma distribution, was used for economic outcomes. A negative binomial regression was used for clinical outcomes. Results: This study identified 40 incident users of filgrastim and 17 incident users of filgrastim-sndz during the study period. Except for race, other patient characteristics were similar between users of filgrastimsndz and filgrastim. Filgrastim-sndz users were more likely to be African Americans (58.82\% vs. 20.51\%, $\mathrm{p}=0.0049)$ compared to filgrastim users. After adjusting for covariates, total medical costs were not significantly associated with filgrastim/filgrastim-sndz use (cost ratio: -0.75 ; 95\% CI: $-1.74 \sim 0.24)$. Similarly, outpatient visits (incidence rate ratio, IRR: $0.89 ; 95 \%$ CI: $0.46 \sim 1.31$ ), hospitalizations (IRR: 0.23 ; 95\% CI: $0.00 \sim 1.20$ ), emergency department visits (IRR: 1.09; 95\% CI: 0.00 4.02) were not significantly associated with filgrastim/filgrastim-sndz use. Implications: Filgrastim-sndz and filgrastim had similar economic/clinical outcomes in the Medicaid population. African Americans were more likely to use filgrastim-sndz.

Enhancing the Delivery of Ibuprofen Through the Skin Using in silico Modeling. Gabriella Baki, The University of Toledo. Objectives: Trial-and-error approach to formulation development is laborious, time-consuming and expensive. With growing time and cost pressures in the pharmaceutical industry, the need for computer modeling is greater than ever. In this project, emulgels were designed using in silico modeling for the topical delivery of ibuprofen. Methods: Formulating for Efficacy ${ }^{\text {TM }}$ (FFE), an in silico program was utilized to select five skin penetration enhancers (SPEs) for ibuprofen, design emulgels and simulate skin penetration studies. Emulgels were 


\section{American Journal of Pharmaceutical Education 2019; 83 (5) Article 7654.}

formulated; $\mathrm{pH}$, viscosity, spreadability, droplet size and stability were evaluated. Franz cell studies were performed to test in vitro drug release on regenerated cellulose membranes, drug permeation in vitro on Strat-M $\mathrm{M}^{\circledR}$ membranes and ex vivo on porcine ear skin, a marketed ibuprofen gel was the control. A validated HPLC method was used to determine drug concentration. Results: Oleyl alcohol (OA), combined with either dimethyl isosorbide (DMI) or diethylene glycol monoethyl ether (DGME) provided the highest permeation in 24 hours via Strat$\mathrm{M}^{\circledR}$ membrane, which was significantly higher than the marketed product $(\mathrm{p}<0.01)$. OA + DGME significantly outperformed OA ex vivo $(\mathrm{p}<0.05)$. FFE ranking of SPEs was in correlation with solubility results; in silico predictions correlated well with in vitro and ex vivo penetration results. Emulgels were opaque with a skin compatible $\mathrm{pH}$, they were stable at $25^{\circ} \mathrm{C}$ for 6 months, had viscosity and spreadability comparable to a marketed emulgel. Implications: This study confirmed that FFE is a fast, useful and reliable tool for aiding formulators in selecting SPEs, designing topical products for ibuprofen, and simulating Franz cells studies.

Evaluation of Cytochrome P450-Mediated Metabolism of the Synthetic Cathinones. Joshua Appel, Manchester University, Ping Lin, Manchester University, Diane M. Calinski, Manchester University. Objective: Synthetic cathinones (a.k.a. "bath salts") have become popular drugs of abuse. Despite the rise in use of synthetic cathinones, a complete and through understanding of synthetic cathinone metabolism has yet to emerge. Here we investigated the phase 1 metabolism of butylone, and analyzed the synthetic cathinones, butylone and MDPPP, as potential mechanism-based inactivators (MBI) of cytochrome P450s. Methods: Butylone was incubated with Supersomes containing P450s of interest (CYP2D6, CYP2B6, CYP2C19, and CYP1A2) and the phase 1 metabolites were rank quantified after LC-MS/MS analysis. In a parallel set of experiments, butylone was incubated with Human Liver Microsomes (HLM) with and without inhibition of the specific P450s noted above and analyzed in a similar manner. To investigate the synthetic cathinone-mediated loss of $\mathrm{P} 450$ catalytic activity, MBI experiments were performed for butylone and MDPPP with CYP2D6. Results: Rank quantification of the phase 1 metabolites of butylone demonstrated that the predominate metabolite is dihydroxybutylone. Depending on the system, Supersome or HLM, N-hydroxybutylone or norbutylone respectively were the next most prominent metabolites. CYP2D6 was identified as the most important P450 for dihydroxybutylone production, and CYP2C19 was the most important for the N-hydroxybutylone and norbutylone metabolites. Butylone was demonstrated to be an MBI for CYP2D6, with $K_{\mathrm{I}}=84.7 \pm 41.7 \mu \mathrm{M}$ and $k_{\text {inact }}=0.047 \pm 0.012 \mathrm{~min}^{-1}$. MDPPP was not an MBI. Implications: A thorough characterization of the metabolism of the synthetic cathinones will assist scientists and clinicians in identifying potentially toxic metabolites and developing treatment regimens for toxicities.

Hyaluronic Acid Surface-Modified Cationic Liposomes for Dual Molecular Targeting of Triple-Negative Breast Cancer. Terrick A. Andey, MCPHS University-Worcester/Manchester. Objective: The basallike/triple-negative breast cancer (TNBC) subtype is very aggressive and difficult to treat due to the lack of specific and selective targets. This project describes the engineering of nanoliposomes, surface-modified with hyaluronic acid (Ha-NoL) for specific trafficking to the TNBC microenvironment; and selective targeting of oncogenic HMGA1 and PPARD. Methods: HMGA1 and PPARD were identified as potential targets by analyzing breast cancer data from The Cancer Genome Atlas. HMGA1 and PPARD expression was confirmed by immunoblotting in TNBC (MDA-MB-231, MDA-MB-468) versus non-TNBC (MCF7, SK-BR-3) cells. Ha-NoL containing either or both siHMGA1 and siPPARD $(60 \mathrm{nmol})$ were prepared using DOTAP, DPPC, DSPE-mPEG [2000], and cholesterol at a mole ratio of 2:3:1:3 (N/P ratio 10:1). The formulations were characterized for size, charge, loading efficiency and cellular uptake. Efficacy of the Ha-NoL-siHMGA1 and Ha-NoL-siPPARD were investigated using resazurin dye assay in MDA-MB-231 co-cultured with adipocyte differentiated 3T3-L1-MBX cells. Results: HMGA1 was significantly upregulated in MDA-MB-231 (100\%) and MDA-MB-468 (30\%) versus MCF7 (7\%) and SK-BR-3 (9\%); PPARD expression was relatively insignificant across cell lines $(100 \%, 96 \%$, $100 \%$ and $76 \%$ respectively). Ha-NoL (110-120 nm) and non-modified NoL (85-92 nm) yielded cationic nanoliposomes (13-18 $\mathrm{mV}$ and $22-25 \mathrm{mV}$ respectively) with high therapeutic loading (90-92\% and 88-93 respectively) and cellular uptake. Ha-NoL-siHMGA1, Ha-NoL-siPPARD, and Ha-NoL-siHMGA1+ siPPARD produced significantly decreased MDA-MB-231 cell viability ( $74 \%, 67 \%$ and $52 \%$ respectively), compared to non-modified NoL batches and control $(\mathrm{P}<0.05-$ $\mathrm{P}<0.0001)$. Implications: HMGA1 and PPARD are important mediators of the aggressive TNBC phenotype, that present with opportunities for therapeutic targeting.

Microparticulate Cancer Vaccines: Interpreting an Innovative Approach to Immunotherapy. Michelle Ubowski, St. John Fisher College, Ryan VanSice, St. John Fisher College, Morgan Marriott, St. John Fisher College, Lipika Chablani, St. John Fisher College. Objective: To formulate a microparticulate murine breast 


\section{American Journal of Pharmaceutical Education 2019; 83 (5) Article 7654.}

cancer vaccine and determine the associated innate and adaptive immune responses. Methods: Murine breast cancer cells, 4T07, were grown to confluence and subjected to lysis to prepare a whole cell lysate (WCL). The WCL was formulated with Eudragit FS 30 D, hydroxyl propyl methylcellulose acetate succinate, and bovine serum albumin. The formulation was spray dried to form vaccine microparticles. Separate formulations devoid of WCL were used to prepare placebo microparticles. Murine dendritic cells, DC2.4, were cultured and exposed to the following microparticle treatments: (a) vaccine, (b) vaccine + Poly (I:C) adjuvant, (c) placebo, (d) placebo + Poly (I:C) adjuvant ( $\mathrm{n}=3$ each). The stimulated cells were co-incubated with EL4.IL2 murine T-lymphocytes. The co-cultured dendritic cells were evaluated for their adaptive immune responses by evaluating the expression of CD40, CD80, CD86, MHC I, and MHC II by flow cytometry. The innate immune responses were evaluated by ELISAs to quantify the expression of IL-2, 10, 12, TNF alpha, and IFN-gamma in the co-culture supernatants. Results: The vaccine + adjuvant Poly (I:C) treatment group was most effective in stimulating the adaptive responses. This group expressed significantly higher levels of CD40, CD80, MHC I, and MHC II when compared to the control $(\mathrm{p}<0.05)$. Additionally, the innate immune responses observed via ELISAs indicated no significant differences in IL-2,10,12, and IFN-gamma levels. However, the vaccine and vaccine + adjuvant groups significantly reduced the TNF-alpha levels $(\mathrm{p}<0.05)$. Implications: The microparticulate breast cancer vaccine is eliciting its immune responses by activating the adaptive immune pathway.

Personalized Erythropoiesis in Hemodialysis Patients: The Novel PREDHICT Tool. Calvin J. Meaney, University at Buffalo, The State University of New York, Wojciech Krzyzanski, University at Buffalo, The State University of New York, Ly Minh Nguyen, University at Buffalo, The State University of New York, Mandip Panesar, Erie County Medical Center, Gauri Rao, University of North Carolina at Chapel Hill. Objectives: Determine the influence of erythrocyte lifespan and erythropoiesisstimulating agent (ESA) exposure on variability of hemoglobin response. Methods: A prospective, observational, 10 -week, clinical pharmacology study was conducted in 28 chronic hemodialysis patients receiving ESA (epoetin alfa). Biweekly blood sampling included hemoglobin, serum erythropoietin concentration and erythrocyte dynamics (production rate, lifespan) determined using flow cytometry. A pharmacokinetic (PK) pharmacodynamic (PD) model was developed for 15 of the subjects which included a sigmoid Emax function for drug effect, precursor and transit compartments for erythrocyte matura- tion, with covariate testing using a Bayesian approach. Results: Age was $56.5 \pm 14.8$ years. Epoetin alfa dose was a $159 \pm 204$ units $/ \mathrm{kg} /$ week. Laboratory parameters included hemoglobin of $10.59 \pm 0.62 \mathrm{~g} / \mathrm{dl}$ (range $6.8-13.9 \mathrm{~g} / \mathrm{dl})$, transferrin saturation of $36.5 \pm 9.5 \%$ and ferritin of $785 \pm 301 \mathrm{ng} / \mathrm{ml}$. Serum erythropoietin concentrations were $19.14 \pm 34.45 \mathrm{mIU} / \mathrm{mL}$ (range $0.36-249.89 \mathrm{mIU} / \mathrm{mL}$ ). Erythrocyte production rate (kRBC) normalized to the number of reticulocytes was $0.928 \pm 0.256$ days $^{-1}$ (range 0.466-1.89 days ${ }^{-1}$ ). The posterior distributions of PD parameters from the model were: maximum drug effect, $\mathrm{E}_{\max }: 1.0-2.2-4.7$ $\left(2.5^{\text {th }}-50^{\text {th }}-97.5^{\text {th }}\right.$ percentile $)$; drug potency, $\mathrm{EC}_{50}: 20-$ 50-128mIU/mL; erythrocyte lifespan, $\mathrm{T}_{\mathrm{RBC}}$ : 67-100150days; population baseline $\mathrm{HGB}, \mathrm{HGB}_{0}$ : 3.9-5.3-6.9 $\mathrm{g} / \mathrm{dL}$. Hemoglobin observations were well predicted for $12 / 15$ subjects 4 weeks in advance (22/28 observations within $95 \%$ credible intervals). Implications: Variability in ESA dose, erythropoietin exposure, and hemoglobin response was high as anticipated. Erythrocyte production rate had less variability and allowed for improved hemoglobin prediction using Bayesian PKPD modeling. ESA dosing algorithms based on these parameters may improve target hemoglobin attainment in dialysis patients.

Prescription Drug Therapy Outcomes Among Older Cancer Survivors in the United States. Traci LeMasters, West Virginia University, Usha Sambamoorthi, West Virginia University, Nazneen Fatima Shaikh, West Virginia University. Objective: The study objectives were to determine: the prevalence and associations with potentially inappropriate medications (PIMs) and adverse drug events (ADEs) among older cancer survivors; and the association of PIMs and ADEs with prescription drug expenditures (PDE). Methods: A retrospective cohort study using SEER-Medicare data (20082014) identified cases of breast, colorectal, prostate, lymphoma, cervical, ovarian and uterine cancers $(\mathrm{n}=$ 286,761). PIMS were identified using the NCQA's list of high-risk medications. ADEs were identified using diagnostic and external cause of injury codes specified by the HCUP. Expenditures were standardized to 2014 dollars. Adjusted odds ratios (AOR) with 95\% confidence intervals (CI) were estimated for the likelihood of PIMs and ADEs. Results: The prevalence of PIMs was $20 \%$ among all survivors, and the highest among cervical $(30.8 \%)$ and lowest among prostate cancer survivors (15.3\%). Almost $5 \%$ of all survivors had an ADE, ranging from $13.2 \%$ (ovarian) to $1.8 \%$ (prostate). Compared to prostate cancer survivors, cervical, lymphoma, uterine and colorectal cancer survivors were 1.66 to 1.24 times more likely to have PIMs. All cancers were more likely to have an $\mathrm{ADE}$, than prostate cancer survivors, especially 


\section{American Journal of Pharmaceutical Education 2019; 83 (5) Article 7654.}

lymphoma $(\mathrm{AOR}=6.22)$, ovarian $(\mathrm{AOR}=5.32)$ and cervical $(\mathrm{AOR}=3.67)$. Excess prescription expenditures associated with PIMs was $\$ 1,963$ and with ADEs was $\$ 1,447$ over two years. Implications: Survivors of cancers that are commonly diagnosed at later stages and/or have worse prognosis are more likely to use PIMs and experience ADEs, resulting in greater prescription drug expenditures.

Production of Predation-Associated Antimicrobials from Cryptic Biosynthetic Pathways. Cole Stevens, The University of Mississippi, Shukria Akbar, The University of Mississippi, Barbara Adaikpoh, The University of Mississippi. Objective: To access metabolites from predatory myxobacteria that are not observed during axenic cultivation conditions by introduction of quorum signals produced by ecological bacterial prey. Methods: Cultivation of Cystobacter ferrugineus, Archangium violaceum, and Archangium sp. strain Cb G35 with and without introduced signaling molecules from prey bacteria provided extracts for comparative, metabolomic analysis using Global Natural Products Social (GNPS) Molecular Networking. Supporting transcriptomics were also obtained via RNAseq to determine biosynthetic gene clusters (BGCs) activated upon signal exposure. Results: Our data suggests that predatory myxobacteria respond to prey quorum signals with dynamic changes in specialized metabolism. We observe activation of metabolism not utilized during axenic conditions as well as deactivation of metabolism utilized during axenic conditions. Implications: This data suggests that predatory myxobacteria have evolved sensory mechanisms to detect and respond to chemical signals present in microbial communities. We suggest that continued natural product discovery from myxobacteria will benefit from utilizing ecologically relevant chemical signals to induce such predatory features.

Strengthening Student Pharmacist Engagement and Teamwork with Productivity Software. Justin Gatwood, The University of Tennessee, Kenneth C. Hohmeier, The University of Tennessee, Mehmet Kocak, The University of Tennessee, Marie A. Chisholm-Burns, The University of Tennessee. Objective: To understand the extent to which productivity software facilitates effective student engagement, course management, and team-based project management. Methods: All secondyear students $(\mathrm{N}=166)$ used Evernote Business as the course management system for Pharmacy Practice Management and Pharmacoeconomics, training for which was led by Evernote personnel and after which students used the software for the course and other functions. Mixed methods, including structural equation modeling, Wilcoxon signed-rank tests, and focus groups, assessed technology acceptance, student comparisons to Blackboard, and the applicability of the software to learning and practice. Results: Technology acceptance was lower for Evernote than Blackboard (all $\mathrm{p}<0.001$ ) including behavioral intention to use (all $\mathrm{p}<0.001$ ); however, effort expectancy and attitudes toward technology improved in 3 of 4 items each for Evernote (all $p<0.001$ ) and anxiety toward using the new software declined from baseline (all $\mathrm{p}<0.001)$. All items loaded significantly on their corresponding factor for Blackboard, but model fit was not ideal. This pattern was also observed for Evernote, suggesting similar conditions for acceptance of either software and in this case performance expectancy may not be a significant predictor of intention to use. Direct student feedback suggested the following emerging themes: facilitating collaboration, comparatively distinct product function, non-technological learning methods, seamless access to information, lack of intuitive product features, product adoption, and future applications. Implications: Introducing productivity software in the middle of the curriculum is not ideal for project management or learning needs, but it could be impactful if provided earlier, offered real-time interactivity, and integrated with existing software systems.

\section{INNOVATION IN TEACHING COMPE- TITION-WINNER}

Master Adaptive Learning: Re-Engineering Core Integrated Pharmacotherapy Curriculum for 21st Century Learning. Denise H. Rhoney, University of North Carolina at Chapel Hill, Amanda H. Corbett, University of North Carolina at Chapel Hill, Sarah M. Anderson, University of North Carolina at Chapel Hill, Tom Angelo, University of North Carolina at Chapel Hill, Ian Hollis, University of North Carolina at Chapel Hill, Kathryn A. Morbitzer, University of North Carolina at Chapel Hill, Phil Rodgers, University of North Carolina at Chapel Hill. Objective: In order to support development of the expertise needed to function efficiently in the complex healthcare environment, we re-engineered how we teach pharmacotherapy using the master adaptive learner (MAL) framework. Methods: The Integrated Pharmacotherapy (iPHTH) series (three courses) bridges foundational learning in the classroom with experiential learning in clinical practice. It is pivotal for ensuring preAPPE readiness. The iPHTH series is innovative in its design, delivery, and assessment. The design innovation applies four learning strategies across the phases of MAL, moving students sequentially from: (1) foundational knowledge to more specialized knowledge; (2) general thinking skills (critical thinking) to more specialized thinking skills (clinical reasoning and decision-making); 


\section{American Journal of Pharmaceutical Education 2019; 83 (5) Article 7654.}

(3) basic principles and guidelines to applications in practice; and (4) simple to complicated to complex problems. The delivery innovation is based upon A) following a non-organ-based approach to reflect the complexity of problems in practice and B) using the Pharmacist Patient Care Process to support skill development in clinical decision-making. The assessment innovation is incorporating the same "entrustment" aspects (EPAs) of the experiential program across the course series. Results: iPHTH course assessments demonstrated that students achieved demonstrably higher levels of knowledge learning and retention along with higher levels of clinical skill development per the assessment of EPAs. Conclusion: The iPHTH course series is the most contemporary example of the belief that we can no longer focus on imparting information to students, but we must embrace developing a set of self-regulated, self-directed learning skills within our student pharmacist with the goal of developing expertise for life-long learning.

\section{INNOVATION IN TEACHING COMPE- TITION-HONORABLE MENTION}

Collaborative Learning Teams to Longitudinally Teach and Assess Teamwork Behaviors and Attitudes. Michelle Z. Farland, University of Florida, Diane E. Beck, University of Florida. Objective: We created an innovative model of continuous development of teamwork skills (CDTS) using a longitudinal peer feedback process across multiple courses with collaborative learning teams. Components of the CDTS model include: 1) team longevity for the academic year; 2) team contracts; 3) reliable peer feedback tool; 4) team debriefings; 5) targeted faculty coaching for individuals and teams. Methods: Students complete 3 formative and 1 summative peer assessment each academic year. Assessments were implemented using the Comprehensive Assessment of Team Member Effectiveness (CATME) instrument to assess individual performance and team dynamics. Individual students and team performing below the performance standard engage in faculty coaching to assist with improving teamwork behaviors, attitudes, and norms. A systematic thematic analysis was conducted on free-text peer evaluation comments. Results: The majority of students were rated highly for teamwork behaviors and attitudes. Across three years, 35 students (4.5\%) and 51 teams $(19.5 \%)$ were identified as requiring faculty coaching related to teamwork. Themes identified include: 1) contributing to the team's work/expecting quality, 2) psychological safety, 3) identification of team member strengths and weaknesses, 4) team development, and 5) adjourning. Conclusion: The CDTS model is an innovative approach to teach and assess teamwork behaviors. It can be used to identify students who require a personalized approach to become collaborative team members.

\section{AWARD FOR EXCELLENCE IN AS- SESSMENT-HONORABLE MENTION}

A Multimodal Real-Time Assessment Process and Its Impact on Student Achievement and Progression in the First Year of a Doctor of Pharmacy Program. Lisa Charneski, University of the Sciences, Suzanne Carbonaro, University of the Sciences, Laura A. Mandos, University of the Sciences, Tyan Thomas, University of the Sciences, Sarah Kleinfeld, University of the Sciences, Jesse Swartz, University of the Sciences, Islam M. Ghazi, University of the Sciences, Karen J. Tietze, University of the Sciences, Jane F. Bowen, University of the Sciences. Objective: To describe the implementation of a multimodal real-time assessment process paired with an early student intervention system and its impact on student achievement and progression in the first professional year of a Doctor of Pharmacy program. Methods: A multimodal real-time assessment process was implemented in fall 2018 to coincide with the transition to a competencydriven curriculum. The availability of actionable data resulted in an enhanced Learning Support system. "Extrinsic summative assessment and reassessment" (ESAR) periods provide curriculum pauses for programmatic evaluation of students' mastery of knowledge and skills expected at specific points within the curriculum. Adoption of ExamSoft ${ }^{\circledR}$ for all required courses with question category tagging to program outcomes and the implementation of an cloud-based assessment system to house common rubrics provide meaningful feedback to all stakeholders in real-time. Sustainability is maintained through intentionally designed faculty teams, an office of assessment and a robust technology infrastructure. Results: Fall 2018 data reveal a significant increase in the proportion of P1 students on the Dean's List; (2) significant decrease in the proportion of $\mathrm{P} 1$ students that failed to progress to the spring semester; and (3) significant decrease in the proportion of $\mathrm{P} 1$ students that entered program probation when compared to each cohort from the previous 5 years. Conclusion: A real-time multimodal assessment system paired with Learning Support results in increased student success. Continued research and tracking of these methods will determine the system's efficiency in maintaining student achievement and consistent progression.

\section{TRAINEE POSTER COMPETITION}

Academic Dishonesty and Students in Pharmacy Education: A Global Review. Minh N. Van, The University of Texas at Tyler. Objective: The objective of this 


\section{American Journal of Pharmaceutical Education 2019; 83 (5) Article 7654.}

study was to evaluate the global literature addressing student academic dishonesty (AD) in pharmacy education. Methods: A comprehensive search was conducted through PubMed, MEDLINE, Academic Search Premier, PsycInfo, CINAHL, and Scopus using the search terms "academic integrity" OR "academic dishonesty" OR "cheating" OR "plagiarism" AND "pharmacy" AND "students" OR "education." A total of 210 articles were retrieved from all databases. Inclusion criteria for this study were articles relating to academic dishonesty and pharmacy education. Demographic information was recorded if available. Results: Twenty-one studies over a sixteen-year period met the inclusion criteria, evaluating 7,607 students across eight countries and three continents. Most respondents were female. The most commonly reported acceptable form of AD was submitting assignments without citation (plagiarism) with as many as $90 \%$ of respondents approving of this action. Eight to seventeen percent of students viewed cheating on an exam as acceptable. The incidence of AD varied considerably between studies, with two authors describing greater than $90 \%$ of student responders admitting to some form of AD behavior in pharmacy school. Identified rationale for $\mathrm{AD}$ conduct included stress, perceived insignificance of the act, lack of understanding, and fear of failure. Six studies evaluated technological implications on $\mathrm{AD}$. Fifteen studies proposed solutions to combat AD, including the use of plagiarism detection software, education, interventions, and family or community engagement. Implications: AD remains a challenge in pharmacy education worldwide. Further research is needed to identify effective methods of reducing or preventing AD behaviors.

Analysis of Compounded Preparations to Evaluate a "Practice Makes Perfect" Aseptic Technique Teaching Method. Sarah Mislan, University of North Carolina at Chapel Hill, Robert P. Shrewsbury, University of North Carolina at Chapel Hill. Objective: This study determined if a new "practice makes perfect" aseptic technique teaching method would result in significant improvements in the potency of student compounded preparations. The potency was compared between two student cohorts that differed by student composition, instructor level, supervision, and the number of practice opportunities. Methods: Cohort 1 TAs received no precourse training, were pharmacy residents naïve to the course, and provided group supervision during two practice opportunities. Cohort 2 TAs received six hours of training, were PharmD students in the program, and conducted six individualized practice opportunities. Students in both cohorts were evaluated transferring drug solution from a vial (Vial), an ampule (Ampule), and a reconstitute powder (Reconstitution) into an IV bag. Potency for each preparation was determined and statistically compared using Wald z-test and $\mathrm{p}=0.05$. Results: Cohort 2 students meeting the goal of $+/-10 \%$ of label was $62.3 \%$ (Vial), 65.1\% (Reconstitution), and 55.1\% (Ampule), compared to $59.3 \%$ (Vial), $50.4 \%$ (Reconstitution), and $80.3 \%$ (Ampule) in Cohort 1. Reconstitution in Cohort 2 was statistically different $(\mathrm{p}=0.039)$. In Cohort 2 , a single TA group used peer video recording and review during practice sessions and had statistically different results for Vial, Ampule, and Reconstitution compared to seven other TA groups. Implications: Increased practice and individualized instruction showed limited improvement in student's ability to compound acceptable preparations. Use of video recording improved student performance. This suggests traditional teaching methodologies commonly used for knowledge-based assessments do not adequately prepare students for skill-based assessments, and that peer-coaching may be beneficial in skill-based teaching methodologies.

An Analysis of Pharmacy Curriculum Coverage Regarding Transgender Patient Care and Gender Affirming Therapy. Jessica L. Robertson, The University of Texas at Austin, Steven D. Swank, The University of Texas at Austin, Michelle Thien-Tam Tran, Justina Lipscomb, The University of Texas at Austin, Ashley S. Oliver. Objective: The purpose of this study is to describe the current state of gender affirming therapy (GAT) education provided at colleges of pharmacy around the nation. Methods: A seven question Likert-scale survey will be distributed to 152 pharmacy programs listed in the Pharmacy College Application Service system to assess curriculum coverage regarding the care of transgender patients. Respondents will be asked questions regarding the duration of required and elective coursework as well as transgender sensitivity training. Participation in the survey was both anonymous and voluntary. No written consent was required. Results from the survey will be analyzed using descriptive statistics and student's t-test as appropriate. Results: We hypothesize that the majority of pharmacy school curriculums in the United States do not offer required or elective education on GAT. We also hypothesize that most of the current required curriculum will primarily consist of cultural competency and sensitivity training rather than pharmacotherapy education. Further results are pending. Implications: Pharmacists are being called upon for their clinical expertise more frequently, which is reflected in the shift of pharmacy school curriculums in the last several years. It is clear that colleges of pharmacy have been and will continue to adapt to changes in the landscape of healthcare and should prepare students to manage diverse populations, including 


\section{American Journal of Pharmaceutical Education 2019; 83 (5) Article 7654.}

transgender individuals. This study has potential to highlight the gaps in education in current curriculums across the nation as it pertains to the management of gender affirming pharmacotherapy in transgender patients.

An Evaluation of a Current Events Self-Directed Learning Activity. Francesca Napolitano, Concordia University Wisconsin, Elizabeth A. Buckley, Concordia University Wisconsin, Andrew Traynor, Concordia University Wisconsin. Objective: Lifelong self-directed learning and the ability to understand emerging issues that may impact pharmaceutical care are core professional attributes of pharmacists. Research on current events activities in schools of pharmacy is limited. This project sought to implement and analyze student perceptions of a current events self-directed learning activity. Methods: This prospective cohort study assigned $75 \mathrm{P} 1$ students in an applied patient care course, to each develop a 10minute presentation and handout on a current event. Students filled out a survey and reflection after completion. Quantitative and qualitative results were analyzed. Results: Of the 73 respondents, all recognized value in self-directed learning and $98.6 \%$ agreed that self-directed learning helped them achieve the lab purpose. Most (97.2\%) indicated completing this activity improved skills including; identifying resources, staying updated on current events, developing and delivering a presentation. All indicated increased current event awareness and the importance of pharmacists understanding healthcarerelated news. Students highlighted the following themes about why it is important for pharmacists to stay up to date: pharmacists are professionals and continuous learners, pharmacy is ever-changing, and it is important for patient care. Qualitative analysis of 75 student reflections indicated positive perceptions of lessons learned, assignment organization, and teaching methods. Most $(89 \%)$ wanted to expand their current events in pharmacy knowledge. Implications: Students found value in this self-directed learning activity and expressed a desire for continued current events education. Positive perceptions of the activity aligned with student performance. Schools of pharmacy should consider incorporating current events opportunities throughout the curriculum.

A New Mechanism of Action of Exemestane (an Aromatase Inhibitor). Allison P. Garcia, Harding University, Jacob L. Hatvany, Harding University, Michael A. Murphy, Harding University, Landry Kamdem, Harding University. Objective: To measure Osteoprotegerin (OPG) levels in postmenopausal women before and after exemestane administration. Methods: A single oral 25 $\mathrm{mg}$ dose of exemestane was given to 14 healthy postmenopausal female volunteers. Study subjects were enrolled with the written informed consent under a protocol that was reviewed and approved by the local IRB. Urine baseline samples were collected prior to dosing. The subjects returned to give urine samples 72 hours after dosing for pharmacokinetic and pharmacodynamic analysis. Urine concentrations of exemestane and its major metabolite 17-hydroexemestane were quantified using LC-MS/ MS. Data collection and processing were performed using the Analyst software package. A human OPG ELISA kit from Sigma-Aldrich was used to quantify OPG urine levels according to manufacturer's guidelines. Results: Our findings showed that urine OPG levels were decreased 72 hours after exemestane administration $(61.37 \pm$ $24.12 \mathrm{pg} / \mathrm{mL}$ vs. $45.74 \pm 22.09 \mathrm{pg} / \mathrm{mL}$ ). We found no statistically significant correlations between fold decrease in urine OPG levels and the pharmacokinetics of exemestane and its major metabolite 17-hydroexemestane. Implications: Our results indicate that one of the pharmacological effects of aromatase inhibitors (e.g. exemestane) involve a decrease in OPG levels, as supported by the evidence of our findings. Urine levels of OPG could be used as a biomarker of toxicities associated with exemestane use.

An Exploration of Student Pharmacist Development on Advanced Practice Experiences. Laura K. Sjoquist, University of North Carolina at Chapel Hill, Brad L. Wingo, University of North Carolina at Chapel Hill, Nicole P. Reitter, University of North Carolina at Chapel Hill. Objective: The purpose of this study is to use an accepted theory for student identity development in order to categorize sources of dissonance that occur during advanced pharmacy practice experiences (APPEs). Methods: Student pharmacists in the fourth professional year enrolled in a capstone course while simultaneously completing practice experiences were included. Qualitative analysis was performed on reflections completed in response to a structured prompt regarding a seminal experience that was difficult, conflicting, or disorienting during APPEs. An initial codebook around Chickering's Seven Vectors of Identity Development framework was developed. Upon consensus by all investigators, all data was categorized, and emerging themes were identified. A dependability audit was implemented to ensure trustworthiness. This study was determined except from review by the Institutional Review Board. Results: A total of 137 reflections were available; of those, $99(72.3 \%)$ were submitted by female student pharmacists. Coding analysis revealed student pharmacist reflection in all seven vectors of Chickering's theory. Most frequent reflection occurred in areas of intellectual, and interpersonal competence as well as recognition, incorporation, and management of emotions. Additional prominent areas of reflection emphasized progression toward interdependence and 


\section{American Journal of Pharmaceutical Education 2019; 83 (5) Article 7654.}

growing ability to establish identity. Some reflections begin to indicate early stages of development around mature interpersonal relationships, purpose, and integrity. Implications: Presence of all seven vectors indicates that Chickering's theory may be a relevant framework for evaluating student identity development during APPEs. Future efforts should be focused on identifying key recommendations for supporting student identity development during practice experiences.

A Novel IPE Collaboration Between a Pharmacy Compounding Club and a Medical Dermatology Club. Emily C. Darst, Washington State University, Damianne Brand-Eubanks, Washington State University, Michael Scott, Pacific Northwest University of Health Sciences. Objective: Create a unique interprofessional education (IPE) experience between pharmacy and medical schools, through which students will tangibly act in their future role within a collaborative healthcare team alongside other disciplines to create individualized treatments for optimal patient care. Methods: An IPE experience involving the pharmacy Compounding Club and the medical Dermatology Club was designed to introduce future prescribers to available compounding pharmacy services and provide future pharmacists insight into the process of diagnosis and treatment determination. Students were briefly presented background information for a dermatological case patient, a review of diagnosing and prescribing information, and an introduction to the compounding process. Pharmacy and medical students worked in groups to assess and diagnose the patient, then prescribe, compound, and dispense a topical preparation. Results: This IPE experience, while not designed to obtain objective data, met the curricular goal of developing a novel, interactive IPE session, and further strengthened the professional relationship between the schools. The experience achieved the designed educational outcomes of collaborative learning across both disciplines, and successful creation of appropriate, personalized compounded products. Implications: Compounding provides unique opportunities to customize medication for optimal patient care, and as such, prescribers should be familiar with the role of compounding in providing personalized treatments. This IPE session is customizable and can be replicated with interchangeable cases to provide differing experiences. The future of pharmacy relies on effective collaboration between various healthcare disciplines; consistent implementation of interactive IPE events such as this within the curricula will foster collaborative behavior in future healthcare practitioners.

A Novel Iron (II) Preferring Dopamine Agonist Chelator Suppresses $\alpha$-Syn- and MPTP-Induced Toxicities In Vivo. Banibrata Das, Wayne State University,
Subramanian Rajagopalan, Buck Institute for Research on Aging, Joshi Gnanada, Wayne State University, Liping $\mathrm{Xu}$, Wayne State University, Sokol Todi, Wayne State University, Julie K. Andersen, Buck Institute for Research on Aging, Aloke K. Dutta, Wayne State University. Objective: Parkinson's disease (PD) is a neurodegenerative disorder affecting $1-2 \%$ of the elderly population causing profound motor impairments along with non-motor symptoms. Although the etiology of PD is still not completely understood, research has provided insights into the basic pathogenic factors of PD such as aggregation of $\alpha$-synuclein ( $\alpha$-Syn) protein, oxidative stress, and nigral iron dysregulation. Current therapies are focused on the symptomatic aspect of PD and consequently the disease progression continues. Thus, there is an unmet need for development of disease modifying drugs which will provide great benefit and a paradigm shift over the current treatment options. Methods: We have designed and synthesized a novel dopamine D2/D3 agonist molecule, D607 . In vitro binding and functional assays at D2/D3 receptors were carried out to investigate receptor affinity and activation. Various in vitro and in vivo assays were conducted to demonstrate the symptomatic and neuroprotective effects of the compound. Results: The molecule displays strong antioxidant and efficient preferential iron (II) chelation properties, accompanied with potent in vivo efficacy in a reserpinized PD animal model. D-607 also rescued neuronal $\mathrm{PC} 12$ cells from toxicity induced by iron and 6-OHDA in a dose-dependent manner. In an in vivo, Drosophila melanogaster model expressing a diseasecausing variant of $\alpha$-Syn protein, the compound suppressed toxicity significantly compared to controls. Finally, D-607 was able to rescue dopaminergic neurons from toxicity of MPTP in mice. Implications: These observations strongly suggest that D-607 is a promising multifunctional lead molecule for a viable symptomatic and disease modifying therapy of PD.

A Proposed Model to Improve Cardiovascular Health Outcomes in Underserved Communities. Kenya E. Covarrubias, California Health Sciences University, Madison N. Fesperman, California Health Sciences University, Patty Havard, California Health Sciences University, Farid Khalafalla, California Health Sciences University. Objective: Cardiovascular diseases [CVD] are a leading cause of death. Hispanic fieldworkers from Calwa neighborhood in California's Central Valley are at a higher risk for CVD due to prevalent obesity, genetic predispositions, socioeconomic status, poor diet, and limited access to health care. Healthy dietary modifications and access to medical care can effectively decrease CVD risks. This project will assess the effects of dietary coaching and accessible clinical services on 


\section{American Journal of Pharmaceutical Education 2019; 83 (5) Article 7654.}

cardiovascular health outcomes among Calwa fieldworkers. Methods: To achieve project objectives, our team will facilitate: (1) Culturally competent dietary coaching sessions and hands-on training focused on behavior modification, setting achievable healthy eating goals, and overcoming potential barriers; (2) A channel for referral of medical services for clients with abnormal blood pressure (BP) and glucose screenings through collaboration with a local Federally Qualified Health Center. Outcomes of these interventions will be evaluated through one-year longitudinal monitoring of body mass index (BMI), BP, blood glucose levels, A1c, and scores on a nutrition perception screening questionnaire (NPSQ9). Results: We expect that health literacy appropriate dietary coaching and access to clinical referral will improve clinical markers and risk factors for CVD including BMI, waist circumference, BP, blood glucose levels, and A1c. Implications: This innovative project, built upon improving health literacy and facilitating clinical service channels, is unprecedented in California's Central Valley. Consistent with our mission, this proposed model will improve health outcomes and quality-of-life for underserved Hispanic Calwa fieldworkers. This model can be expanded to involve other underserved communities in the Central Valley and state of California.

A Randomized Controlled Trial Evaluating the Impact of a Community Pharmacy-Based Naloxone Education Program. Lindsey Hohmann, Auburn University, Brent Fox, Auburn University, Kimberly B. Garza, Auburn University, Chih-Hsuan Wang, Auburn University, Denton Beau Scott, Auburn University, Christopher Correia, Auburn University, Geoff M. Curran, University of Arkansas for Medical Sciences, Salisa C. Westrick, Auburn University. Objective: Community pharmacists lack training and confidence in communicating with patients about receiving naloxone. The purpose of this study was to assess the impact of the Empowering Community Pharmacists program on pharmacists' knowledge, intentions, and confidence to provide naloxone. Methods: A 2-group pragmatic randomized controlled trial was conducted in 2018-2019. Community pharmacists in 20 Alabama counties with the highest opioid overdose mortality rates were randomized to either: 1 ) basic informational flyer (control); or 2) basic flyer + webinar discussing naloxone dispensing, implementation strategies, and communication strategies. Outcomes were measured via online survey pre- and post-intervention. Primary outcomes included changes in intention and confidence to provide naloxone using 7-point Likert-type scales $(1=$ strongly disagree, $7=$ strongly agree $)$. Secondary outcomes included change in knowledge score (percent correct on a 7-item index). Changes were analyzed using 2-sided Wilcoxon Signed-Rank and Mann-Whitney U tests (alpha $=0.05)$. Results: Sixty community pharmacists completed assessments at both time-points $(n=30$ control, $\mathrm{n}=30$ intervention). Most participants were female $(77-80 \%)$, white $(80 \%)$, and employed in independently-owned pharmacies (34-43\%). From pre to post, mean (SD) intervention group intention (5.41(1.48)$6.09(0.97), \mathrm{p}=0.001)$ and confidence (5.56(0.99)$6.14(0.68), \mathrm{p}<0.001)$ increased; increases were statistically significant compared to control (intention $p=0.005$, confidence $\mathrm{p}=0.003)$. Intervention knowledge scores increased pre to post (78.10(14.88)-89.52(11.21), $\mathrm{p}=0.001)$, but the difference was not significant compared to control $(p=0.588)$. Implications: The program was effective at increasing pharmacists' intention and confidence to provide naloxone, potentially offering a means to increase naloxone access. Although the study is limited by the short time-frame, sustained changes in key variables will be measured in future investigations.

Assessing Drug Information Resource Utilization by South Dakota Registered Pharmacists. Miranda P. Boraas, South Dakota State University, Brittney A. Meyer, South Dakota State University. Objective: To examine the utilization of and preference for drug information resources and different influencing factors in drug information resource selection by South Dakota pharmacists. Methods: A 22-question electronic survey was emailed to all pharmacists registered in the state of South Dakota with a valid e-mail address. Results were summarized descriptively. Results: A total of 579 pharmacists out of 1,421 completed the survey (40.8\% response rate). All pharmacists were registered in SD, but $63.7 \%$ primarily practiced in SD. Several practice settings were represented including mail order, ambulatory care, long term care, academia, and nuclear pharmacy, but hospital (37.7\%) and community (29.6\%) were the most common. The following lists the drug information resources utilized by SD pharmacists in the last year: Lexicomp (67.0\%), Pharmacist's Letter $(67.0 \%)$, UpToDate (57.5\%), Micromedex (55.0\%), Google (48.4\%), Sanford Guide (44.0\%), PubMed (42.7\%), Drug Facts and Comparisons $(33.9 \%)$, individual reference textbooks (24.2\%), Clinical Pharmacology (22.3\%), others not listed (12.4\%), and DynaMed (3.1\%). A majority of pharmacists prefer Lexicomp to answer general drug information questions (39.53\%), with Micromedex as the second choice $(18.23 \%)$. The primary influencing factors for preferred drug information resource was validity/accuracy of information and ease of use. Overall, Lexicomp was identified as the leading drug information resource of pharmacists registered in South Dakota, which is comparable to 


\section{American Journal of Pharmaceutical Education 2019; 83 (5) Article 7654.}

published data for other states. Implications: Colleges of pharmacy may focus time in their curricula to train student pharmacists on drug information resources that will most likely be used in the workforce. Continued analysis will compare practice settings.

Assessing Students' Skill Knowledge in a Health Fair Setting. Dakhari J. Williams, Chicago State University, Andre Roberts, Chicago State University, Samuel Babatunde, Chicago State University. Objective: Participation in health fair screenings and providing disease state education to communities are two co-curricular activities performed by student pharmacists. The American Council of Pharmacy Education does not require a formal class for health fairs and many students lack the confidence and the skill to perform required health fair activities. The purpose of this study is to assess self-reported student confidence in performing multiple health fair screenings such body mass index (BMI), diabetes, cholesterol, and hypertension screenings, before and after participating in a workshop geared toward standardizing training to perform such screenings. In addition to the aforementioned screening, training for HIV Pre-Exposure Prophylaxis (PrEP) education and naloxone education was provided. Methods: We administered the surveys to Chicago State University pharmacy students. Students were asked to complete an anonymous survey prior to completing a week-long training workshop geared towards providing screenings and education at health fairs. The post-survey was administered to the students after completing at least one fair subsequent to the training workshop. Results: Post-workshop survey results are not available, but results are available from the pre-workshop survey. Pre-survey results were based on students' self-assessment. Overall, 28 students participated in the survey. After a pre-survey, $57 \%$ of pharmacy students were not confident in their knowledge regarding PrEP and when it is indicated. Pharmacy students were confident in their knowledge regarding BMI screening, diabetes screenings, cholesterol screenings, hypertension screenings, vaccinations, and naloxone education (75\%, $96 \%, 64 \%, 96 \%, 71 \%$, and $71 \%$, respectively). Implications: Student pharmacists were not confident in their knowledge regarding HIV PrEP and when it's indicated.

Assessing the Effectiveness of a Teaching Method for Calculation Skills Within a Pharmacy Laboratory Course. Marwah H. Dabaja, Manchester University, Holly D. Robison, Manchester University. Objective: The primary objective was to study the effects of required calculation worksheets within a pharmacy practice lab (PPL) course on pharmacy student performance within a required calculations course. The secondary objective was to observe the impact of calculation methods on performance within a calculations course and on a highstakes summative examination (OSCE). Methods: Students concurrently enrolled in PPL and calculations courses during fall 2018 were required to complete calculations worksheets. Calculations course scores for these students were compared to students in fall 2017 who were provided optional worksheets. Calculation methods were determined as follows for each student of the fall 2018 cohort: proportions, dimensional analysis, mixed methods. Student OSCE scores and calculation course scores were analyzed for differences among methodology subgroups. Results: Calculation course scores for 74 students from Fall 2017 and 73 students from Fall 2018 were analyzed. No significant differences were found between the two cohorts. No significant differences were found between calculation methodology and calculations course scores or OSCE scores. Descriptive statistics showed a trend toward lower calculation course scores for students who use proportional methods. Implications: Required completion of calculations worksheets within a PPL course did not significantly impact student performance within a calculations course. This study found no significant impact of calculation methods on calculations course scores or OSCE scores. Further research is needed to evaluate the effects of calculation methods on calculation course performance.

Assessing the Frequency of Clinically Relevant CYP2C19 Variants in Over 2.2 Million Direct-to-Consumer Genetics Research Participants. Yelena Ionova, University of California, San Francisco, James Ashenhurst, 23andMe, Inc, Jianan Zhan, 23andMe, Inc, Hoang Nhan, 23andMe, Inc, Cindy Kosinski, 23andMe, Inc, 23 andMe Research Team, Bani Tamraz, University of California, San Francisco, Alison Chubb, 23andMe, Inc. Objective: Cytochrome P450 (CYP) 2C19 metabolizes many medications. This study assessed the frequencies and ethnic distribution of clinically relevant variants $(* 2, * 3$, and $* 17)$ in the CYP2C19 gene and examined the prevalence of patients prescribed medications metabolized by CYP2C19. Methods: This study utilized two separate cohorts. The first consisted of 23 andMe research participants ( $\mathrm{N}=\sim 2.3$ million) who were genotyped on Illumina arrays that included CYP2C19 *2, *3, and *17 alleles. The second was composed of University of California, San Francisco (UCSF) patients $(\mathrm{N}=\sim 880,000)$ whose de-identified medication histories were obtained from UCSF's electronic medical records database and sorted by age, sex, and ethnicity. Results: The overall frequencies of $* 2, * 3$, and $* 17$ were $15.2 \%, 0.3 \%$, and $20.4 \%$, respectively, but varied by ethnicity. The most common genotypes were $* 1 / * 1$ at $41.2 \%, * 1 / * 17$ at $26.0 \%$, and $* 1 / * 2$ at $19.4 \%$. The less common $* 2 / * 17$, 


\section{American Journal of Pharmaceutical Education 2019; 83 (5) Article 7654.}

$* 17 / * 17$ and $* 2 / * 2$ genotypes occurred at $6.0 \%, 4.4 \%$, and $2.5 \%$, respectively. A total of 143,398 UCSF patients (16.3\%) were prescribed one or more high-pharmacogenetic-risk CYP2C19 medications (2012-2019). Implications: Almost $60 \%$ of genotyped individuals had at least one increased activity or loss-of-function CYP2C19 allele, and based on this finding, the potential number of UCSF patients with a clinically relevant variant surpasses the number of those likely to be CYP2C19 normal metabolizers. This insight suggests that pharmacogenetic testing may be warranted more broadly and that directto-consumer testing offers one potential mechanism for widespread preemptive screening.

Assessment of a Student Led Formalized Tutoring Program in a College of Pharmacy. Dylan C. D'Aguanno, The University of Rhode Island, Colin Dimond, The University of Rhode Island, Brian Bishop, The University of Rhode Island. Objective: The purpose of this study is to retrospectively determine if optional tutoring sessions offered by peer mentors within the College of Pharmacy were beneficial to students who attended them. Methods: To increase support of students' academic performance, the College of Pharmacy implemented a student-run tutoring program. For this program, students within the college's honor society offered scheduled tutoring sessions for first-year pharmacy students. The students responsible for conducting the sessions were trained from the University Academic Enhancement Center to determine best practices. Data supporting this study will encompass 15 review sessions in the course of one semester. Objective course grades of students who attended will be compared in aggregate to scores of students who did not attend sessions. Results: Preliminary data found that of the 135 first-year pharmacy students, those who attended sessions throughout the semester expressed they received benefits from these sessions. Seventy-five students provided written feedback. This retrospective study was started as part of a quality review process to determine if a connection exists between attendance at these sessions and academic performance, and analysis on the data is still in progress. Implications: If the data demonstrate a strong correlation between attendance and academic performance, an evidence-based link may be established in the College of Pharmacy and an investment of resources may be more readily allocated to these programs. A limitation of the study is that students who would have otherwise excelled in the class may have attended the review sessions more readily than those who were unlikely to succeed.

Assessment of Empathy Among Pharmacy Students Through a Polypharmacy Medication Regimen Simulation. Katie E. Ronald, Southern Illinois University
Edwardsville, Megan Underwood, Southern Illinois University Edwardsville. Objective: To assess change in empathy among first year pharmacy students through a polypharmacy simulation. Methods: First year pharmacy students enrolled in Personal and Professional Development I were recruited to participate in a volunteer, pilot polypharmacy simulation. A mock medication regimen consisting of eight prescriptions taken for seven days was distributed. Both qualitative and quantitative data were obtained. Baseline characteristics, personal insight, and empathy [Kiersma-Chen Empathy Scale (KCES)] were assessed in pre- and post-surveys. The Wilcoxen signed-rank test was used to determine statistical significance. Results: Eighty-two students were eligible for recruitment. Matched data from thirty-nine student participants was included. Of the 15 questions on the KCES, one question, "I will not allow myself to be influenced by someone's feeling when determining the best treatment," produced a significant change in mean score ( 4.77 vs $4.10, p=0.02)$. The average percentage of late and missed doses, as self-reported by students, was 5.63\% and 5.04\%, respectively. Qualitative responses indicated forgetfulness as the primary cause of non-adherence. When asked how this experience will impact patient counseling on multiple medications, a common theme included: a better understanding of the difficulty of medication self-management. Implications: Limited statistically significant findings could be contributed to high empathy scores at baseline in this population. Based on qualitative data, this simulation should be considered for incorporation into future curriculum plans.

Association of Holistic Student Admissions Criteria and Academic Failure at a Team-Based Learning Institution. Allen Keshishian Namagerdi, California Health Sciences University, Shaowei Wan, California Health Sciences University, Yuqin Hu, California Health Sciences University, Farid Khalafalla, California Health Sciences University, Jeanette Munn, California Health Sciences University, David G. Fuentes, California Health Sciences University, Jeremy Hughes, California Health Sciences University, Will Ofstad, California Health Sciences University, Chandra Kolli, California Health Sciences University, Lianjie Xiong, California Health Sciences University. Objective: There is no evidence on the association between holistic admissions requirement and students' performance at a team-based learning (TBL) Doctor of Pharmacy program. The objectives of this study are to calculate the cumulative incidence of student academic failure and examine if difference exists in the overall admission score between those with and without academic failure. We hypothesize that overall admission score is higher in those without academic 


\section{American Journal of Pharmaceutical Education 2019; 83 (5) Article 7654.}

failure than those with academic failure. Methods: This was a retrospective cohort study that includes 4 classes of students admitted to California Health Sciences University (CHSU) College of Pharmacy, a TBL institution from August 2014 for the inaugural class to August 2017. Data collected includes: student demographics, overall admission scores consisting of scores for cumulative GPA, pre-requisite GPA, personal statement, extracurricular activity, attainment of undergraduate/graduate degree, pharmacy/other work experience, and onsite interview scores (interview and writing essay score), and academic failure data. Academic failure is defined as an inability of the student to progress in the pharmacy program due to academic reason, being on alternative progression plan (APP) or at least one grade of "D" or "F". Descriptive analysis will be conducted for pre- and post-admission characteristics. Cumulative incidence of academic failure will be calculated at each didactic year and overall admission score will be compared in those with and without academic deficit or failure after adjusting other covariates. Results: Results will be available for poster presentation. Implications: Holistic admissions approach can be used to predict students' likelihood of academic success at a TBL pharmacy school.

A Sustainable Business Model for Comprehensive Medication Management in a Patient Centered Medical Home. Patricia H. Fabel, University of South Carolina, Tyler David Wagner, University of South Carolina, Bryan Ziegler, University of South Carolina, Paul Fleming, Robert E. Davis. Objective: The primary objective was to develop a sustainable business model for pharmacist-provided comprehensive medication management (CMM) services in a patient centered medical home (PCMH). The secondary objective was to evaluate the impact the pharmacist had on clinical (A1C, LDL, and blood pressure) and economic (physician productivity and cost avoidance) outcomes. Methods: This pilot project took place at Palmetto Primary Care Physicians (PPCP) Trident office in North Charleston, SC from October 2013 to September 2014. At the time, the practice employed 5 physicians and 2 nurse practitioners and served more than 20,000 patients. We executed an agreement with PPCP to develop a collaborative practice agreement (CPA) and integrate a pharmacist into one of their PCMH locations. Retrospective chart reviews were conducted in October 2014 to gather pre-CMM and postCMM clinical marker data (A1C, LDL, and blood pressure). Results: The pharmacist was able to see an average of 11 patients per day, which was $72 \%$ of his capacity. The practice collected about $\$ 7,400$ per month for services rendered by the pharmacist. The average daily payment for services rendered by the physicians in the practice increased by $20.6 \%$. Over $70 \%$ of uncontrolled patients had an improvement in clinical outcomes, such as A1C, LDL and blood pressure. Implications: This project demonstrates the sustainable business model for imbedding a pharmacist into a PCMH within South Carolina. When pharmacist-provided CMM services are billed using medication therapy management (MTM) or "incident to" physician evaluation and management services $(E / M)$ codes, a PCMH can generate $65 \%$ of the pharmacist expenses.

A Three-Year Assessment of APPE Placement and Schedule Changes: Cause and Effect. Mitchell Robak, The University of Iowa, Christine Lawson, The University of Iowa, Ellen Overholtzer, The University of Iowa, Cassandra Streeter, The University of Iowa, Susan S. Vos, The University of Iowa. Objective: The purpose of this study is to evaluate APPE (Advanced Pharmacy Practice Experiences) schedule changes to determine the primary reasons for placement changes, identify the sites most frequently involved, and measure the overall time required to perform schedule changes. Methods: The Office of Experiential Education documents all APPE schedule changes using a standardized change tracking form. Data were collected from APPE graduating classes of 2016 to 2018. Information collected included: reason for change request, who initiated change, paid or volunteer preceptors, site type, site location, propagation of change (e.g., number of cycles affected, additional paperwork needed, elective category change), number of days between change request and resolution, and number of days until affected cycle. Results: Of the 147 requests analyzed, eight were removed due to insufficient information. Of 139 requests, 73\% (101) were initiated by preceptors and/or sites, while $21.5 \%$ (30) were initiated by students. Reasons for a preceptor or site-initiated change included job change, medical leave, short-staffed, accepted student from another school, and affiliation agreement issue. On average, $71 \%$ of our APPE are offered by volunteer preceptors; however, $86 \%$ of the schedule changes were initiated by volunteer preceptors. Two-thirds of the schedule changes involved Elective APPE, despite students doing an equal number of electives versus required APPE. Schedule changes were resolved within 9 days, on average (range $=0-230$ days, median $=3$ days). Implications: Elective APPE, as well as volunteer preceptors and sites, are at a higher risk for schedule changes. Many changes are resolved quickly.

Attitudes and Perceptions of Poverty: An Interprofessional Poverty Simulation. MaryCatherine E. Heighton, Western New England University, Beth E. Welch, Western New England University, Melissa Mattison, Western New England University. Objective: The objective was to evaluate the impact of poverty 


\section{American Journal of Pharmaceutical Education 2019; 83 (5) Article 7654.}

simulations on student attitudes and perceptions of those living in poverty after taking part in a simulation in which students step into the shoes of someone living in poverty. Methods: Western New England University College of Pharmacy and Health Sciences collaborated with three other health profession programs from multiple institutions to conduct poverty simulations. Two separate poverty simulations were conducted with a total of 160 students from pharmacy, physician assistant studies, social work and nursing. A pre-survey and post-survey were administered (Attitudes Toward Poverty-short form). Overall mean scores and item mean scores of matched responders were compared using a paired t-test from pre- to post- assessing change in attitudes and perceptions. Results: Response rates for the pre- and postsurvey were $70 \%$ and $43 \%$, respectively, with 60 matched surveys that could be utilized for comparisons. No changes were found in overall means scores from preto post- $(\mathrm{p}=0.276)$. However, significant improvements were found in 8 of the 30 items, mostly falling within the area of stigma and structural perspective. Implications: While a significant overall change was not found, there were improvements in several domains. Poverty simulations can provide the opportunity to combine interprofessional education and the need of health profession students to understand social determinants of health, such as poverty.

California Pharmacists' and Pharmacy Technicians' Opinions on Administration of Immunizations in Community Pharmacies by Technicians. Zachary Charles Hackworth, Loma Linda University. Objective: Pharmacy technicians are important health care professionals; however, they are prohibited from administering immunizations in California. Pharmacists in Idaho and Rhode Island can delegate the task of immunization to a duly trained technician. If similarly implemented in California, and throughout the United States, this could reduce the burden on pharmacists, improve workflow, and promote public health through immunization. This study determines the opinions of California pharmacists and pharmacy technicians on technicians administering immunizations in community pharmacies. Methods: A web-based Qualtrics ${ }^{\circledR}$ survey was developed to query pharmacists and pharmacy technicians on different aspects of immunization administration. The survey was distributed in June 2018 electronically to approximately 1,200 pharmacists and technicians throughout California through district managers of CVS, Rite-Aid, Walgreens, and through managers of independently-owned community pharmacies via emails containing the survey link. Results: From the 144 responses, the majority of both parties agreed that pharmacy technicians are capable of administering vaccinations and would benefit the pharmacy's workflow. Although, both parties showed concern of liability with the added responsibility. Pharmacists strongly agreed that technicians should receive additional hours of immunization training. Technicians more strongly supported that California should change the law to allow adequately trained technicians to administer immunizations. Implications: Both pharmacists and technicians were in favor of technicians administering immunizations as a part of their job description. This research is ongoing, and data will be updated throughout the year. Future studies focusing on other regions of California are needed to better understand this topic.

Case Element Analysis for Automated Generation of Cases in Pharmacy Education. Carolyne Ma, University of North Carolina at Chapel Hill, Tazim Kabir, University of North Carolina at Chapel Hill, Robert Hubal, University of North Carolina at Chapel Hill, Jacqueline McLaughlin, University of North Carolina at Chapel Hill. Objective: Current methods for developing vignettes for case-based learning in healthcare education are labor- and time-intensive. We are developing a guided authoring tool to efficiently generate tailored clinical vignettes on demand. In this phase, we analyzed vignettes to identify case elements and evaluate the frequency of these elements as they appear in cases. These elements will serve as the foundation for our case generation tool and additional research in case construction, element analysis and typology. Methods: Around 800 cases were sourced from Objective Structured Clinical Experiences, published pharmacy practice literature, and other training and assessment environments within the School. Sixtytwo cases representing a variety of disease states were selected for initial coding. A consensus building process was used with three coders who independently coded each case and convened to discuss and reconcile these elements. Results: The coding process resulted in 36 case elements including race, medications, and chief complaint. The most common elements were age $(n=57$, $91.9 \%)$, gender $(n=54,87.1 \%)$, and patient identifiers $(\mathrm{n}=48,77.4 \%)$. Case structures and wording were highly variable, with elements presented in different magnitudes (range 4 to 18 elements), using different descriptors and in different sequences. Patterns in the codes indicated rules for case generation, such as: labs are less likely to appear in certain settings (e.g., community pharmacy). Implications: Cases in pharmacy education have a common set of elements and rules that govern their typology. Case construction for pharmacy education could benefit from further understanding of case structures and wording and their influence on level of difficulty. 


\section{American Journal of Pharmaceutical Education 2019; 83 (5) Article 7654.}

Cefazolin versus Anti-Staphylococcal Penicillins for the Treatment of Patients with Staphylococcus aureus Infection: A Meta-Analysis. Lolade S. Bakare, University of Florida, Anthony M. Casapao, University of Florida, Kenneth P. Klinker, University of Florida, Lindsey M. Childs-Kean, University of Florida, Ariel F. Pomputius, University of Florida, John M. Allen, University of Florida. Objective: Staphylococcus aureus (S. aureus) is a gram-positive bacteria and a common cause of infection. S. aureus infections are typically sorted into two categories, methicillin-sensitive (MSSA), or methicillinresistant (MRSA). Among options for MSSA infections (MSSAI), controversy exists on whether anti-staphylococcal penicillins (ASPs) or cefazolin should be the first-line option. The study purpose was to determine which agent is superior at reducing mortality. Secondary outcomes included clinical failure, antibiotic discontinuation due to adverse effects $(\mathrm{D} / \mathrm{C})$, and infection recurrence (IR). The predicted outcome: cefazolin is at least equivalent to ASPs for efficacy (i.e., mortality) and has superior safety outcomes in MSSAI. Methods: The study was conducted with PRISMA guidance. We searched PubMed/Medline, Cochrane Library, and Google Scholar from database inception through August 31, 2018, and performed reviews for unpublished studies. Inclusion criteria included studies evaluating cefazolin to ASPs in patients with MSSAI reporting mortality as an outcome. We conducted a random-effects meta-analysis for studies fitting the inclusion criteria. Two independent investigators conducted study selection, data extraction, risk of bias, and study analysis. Results: Eighteen studies ( $\mathrm{n}=$ 13, 233 patients) met inclusion criteria. Compared to ASPs, cefazolin was associated with a mortality benefit (OR 0.77; 95\% CI: 0.68-0.88). D/C was lower with cefazolin (OR 0.25; 0.16-0.39). However, IR was higher with cefazolin (OR 1.38; 1.02-1.85). Implications: Compared to ASPs, cefazolin was associated with reduced all-cause mortality, reduced clinical failure, improved patient tolerance, but IR was increased. Generally, cefazolin should be the primary option for MSSAI. However, other considerations including treatment indication must be evaluated.

Challenges and Successes of PharmD Students Working Collaboratively Around the World: PharmAlliance Student Domain. Clara H. Kim, University of North Carolina at Chapel Hill, Vraj Patel, University of North Carolina at Chapel Hill, Sarah A. Dascanio, University of North Carolina at Chapel Hill, David R. Steeb, University of North Carolina at Chapel Hill. Objective: The objective is to describe the development of a threeway international pharmacy student partnership and various challenges and strategies for global collaboration.
Methods: PharmAlliance is an international partnership that was started in 2015 between the schools of pharmacy at the University of North Carolina at Chapel Hill, Monash University, and University College London in order to impact health worldwide. A student domain was created to encourage student collaboration. Student leaders from each institution met in person to reflect upon challenges and strategies to address them based on prior projects conducted. Results: Over 90 students have participated in PharmAlliance across the three universities. International collaboration has strengthened through the sharing of resources and joint projects. These projects include an annual international case competition addressing global issues such as medication safety and non-communicable diseases, grant acquisition from community education initiatives, and a student blog. Student projects have contributed to global health initiatives including the World Health Organization's Medication Safety Challenge and the Fight the Fakes Campaign. Challenges encountered include communication barriers, cultural differences, maintaining equal collaboration, and project sustainability. Strategies used to navigate challenges included the utilization of team building events, social media, project management software, transition plans, and establishing group expectations. Implications: The PharmAlliance student domain has given students the opportunity to become globally minded leaders through engaging in collaborative projects with students from international universities. Global student partnerships may enhance personal and professional development in accordance with ACPE 2016 Standards by providing leadership, cultural awareness, communication, and innovation.

Change in Pharmacy Student Empathy After Completion of Book Club Course. Whitney J. Ly, University of the Sciences, Quynh-Nhu Truong, University of the Sciences, Laura Pontiggia, University of the Sciences, Alice L. Scaletta, University of the Sciences. Objective: The Center for Advancement of Pharmacy Education (CAPE) standards state students should be able to demonstrate and listen with empathy when interacting with others. However, studies have shown a decline in empathy in health profession students as they progress through schooling. At the Philadelphia College of Pharmacy, a new elective course entitled "PP324 Pharmacy Book Club" began in Spring 2016. The purpose of this study is to measure change in student empathy after completion of the book club course. Methods: Students enrolled in PP324 read four autobiographical books detailing real experiences with chronic disease. Students completed the Kiersma-Chen Empathy Scale (KCES) questionnaire describing their feelings towards patients before and after 


\section{American Journal of Pharmaceutical Education 2019; 83 (5) Article 7654.}

completion of the course. Scores were collected during the 2016, 2017, and 2018 offerings of the course, and were statistically analyzed using paired t-test. Results: A total of fifty-five students completed the KCES. The mean pretest KCES score was 80 and the mean post-test KCES score was $89(\mathrm{p}=<0.001)$, indicating an increase in empathy. In $2016(n=18), 2017(n=17)$, and $2018(n=20)$, students saw in increase in empathy from a mean of 77.6, 81.4 , and 81.6 to a mean of $85.9,91.7$, and 91 , respectively. Implications: These results illustrate the efficacy of this course in improving empathy skills in pharmacy students, which aligns with CAPE standards for pharmacy students to demonstrate empathy and compassion for patients. Activities from this course will be incorporated into other courses at the College to further enhance empathy skills in students.

Continuous Curricular Improvement: Comparison of Performance in Therapeutics I and II by Matriculating Year. Clayton $\mathrm{H}$. Williams, The University of Louisiana at Monroe, Elizabeth M. Lafitte, The University of Louisiana at Monroe, Jamie M. Terrell, The University of Louisiana at Monroe. Objective: Our college recently made a series of curricular changes to resequencing key therapeutics modules within the curriculum and better prepare students for an integrated learning experience. The purpose of this retrospective review of student performance was to ensure continued success of all students by comparing exam grades, course grades, and number of students qualifying for early intervention under each curricular sequence. Methods: This review assessed student performance in two integrated modules among two cohorts: students matriculating in consecutive years (Fall 2017, 2018). The students matriculating in 2018 are the first group to participate in the revised Doctor of Pharmacy curriculum. The cardiovascular module was moved from the fall of P3 to the P2 year in the new sequence. The endocrinology module remains in the fall of the $\mathrm{P} 2$ year. This review compared mean exam scores and course averages between the two cohorts. Results: Student performance in the endocrine module was the same among each of the cohorts; the average exam grades and course grades were not statistically different. The percent in each matriculating class meeting the criteria for early intervention was the same $(19.5 \%$ and $20 \%)$. Students matriculating in 2017 outperformed the 2018 cohort across all exams in the cardiology module and had a higher course average. Additionally, only $4.6 \%$ of students in the class entering 2017 met the criteria for early intervention compared to $12.6 \%$ in the 2018 cohort. Implications: Careful consideration will be given to ensure successful transition through the resequenced curriculum as result of this review.
Counseling Her, Zir, and Them: Educating Student Pharmacists in the Care of Transgender Patients. Sam J. Miller, Karan N. Dawson, University of Washington. Objective: In the National Transgender Discrimination Survey, 19\% of respondents reported they had been refused care by a medical provider due to their transgender status, and $28 \%$ reported delaying care due to discrimination or fear of discrimination. As one of the most accessible health providers, pharmacists have a unique opportunity to improve the health outcomes of this community, but the majority of pharmacy schools have little or no required education in caring for the transgender community. Our objective in this educational brief is to outline the health and cultural considerations student pharmacists should understand when caring for the transgender community. Methods: Our initial literature review sought resources such as the Center of Excellence for Transgender Health, World Professional Association for Transgender Health, and other available guidelines to identify clinical knowledge pertinent to an introductory pharmacy practice course. After consulting stakeholders from local community organizations as well as transgender healthcare providers, a lesson plan and three case scenarios were developed. Results: The lesson plan addressed a pharmacist's role in providing genderaffirming care, injectable hormone administration, basic terminology used by the transgender community, and markers of an inclusive and welcoming pharmacy environment. The educational patient case studies illustrate common communication barriers for providers and best practices to provide patient-centered care. Implications: With education and practical experience providing care to transgender patients, student pharmacists can help improve access to care and help create a welcoming and more inclusive pharmacy environment.

Critical Moments in Student Learning on International Rotations. Sarah A. Dascanio, University of North Carolina at Chapel Hill, David R. Steeb, University of North Carolina at Chapel Hill, Monica L. Miller, Purdue University, Ellen M. Schellhase, Purdue University, Jodie V. Malhotra, University of Colorado, Stuart T. Haines, The University of Mississippi. Objective: To evaluate self-perceived critical moments that were transformative to learning in students who participated in an international Advanced Pharmacy Practice Experience (APPE). Methods: Fourth-year pharmacy students from University of North Carolina at Chapel Hill, Purdue University, and the University of Colorado who went on an international APPE rotation participated in a post-rotation focus group which evaluated critical moments that impacted their personal and professional development. Focus group recordings were transcribed and coded using a 


\section{American Journal of Pharmaceutical Education 2019; 83 (5) Article 7654.}

conventional content analysis approach. Data went through a two-cycle open coding process and major themes were identified. Results: Twenty-two students participated in the focus group with four students going to a high-income country and 18 students going to a lowto-middle income country (LMIC). The majority of students described their critical moment as a situation which resulted in a new point of view, often in regard to patient care. Students who went to an LMIC reflected on a newfound appreciation for the large impact small changes can produce, usually at the individual level, as well as enhanced empathy for their patients. Students who went to a high-income country commented on an appreciation for how alternative healthcare models can impact patient care. Student comments often indicated self-reflection on their personal values or future professional behavior. Implications: Critical moments in learning for students who went on an international APPE rotation often involved transformation in their attitudes or perspective. Incorporation of reflection during international experiences may help to enhance the transformation of attitudes and student development.

Design and Evaluation of an Interdisciplinary Escape Room-Themed Non-Prescription Medications Lab. Bethannee C. Horn, Union University, Kate Norville, Union University, Ashok E. Philip, Union University, Christopher Stoltz, Union University. Objective: To evaluate the effectiveness of escape room-themed overthe-counter (OTC) medications lab in reinforcing knowledge and interpersonal skills of first-year pharmacy students. Methods: Four escape room-themed OTC lab sessions were developed to cover: 1) Cough, Cold and Allergy (CCA) 2) Ophthalmic Disorders 3) Dermatologic Conditions and 4) Dietary/Herbal Supplements. 53 P1 students were divided into 9 randomized groups for the first CCA lab. Teams completed a series of tasks pertaining to the Pharmacists' Patient Care Process within 35 minutes to successfully escape. Students were challenged to collect patient information, review Beer's criteria and nonpharmacologic therapies, and utilize resources to identify an appropriate OTC medication. To measure the impact on student learning and interpersonal skills, students completed pre/post assessments and a perceptions survey. Comparisons were made using a priori alpha level of 0.05 . Results: 53 students from the P1 cohort completed the pre/post assessments and the perceptions survey. Majority of the students strongly agreed this activity reinforced information learned in the lecture portion of class $(79.3 \%)$, fostered problem-solving skills $(69.8 \%)$ and improved team dynamics (70\%). Additionally, $84.9 \%$ of students felt confident in recommending OTC medications for CCA. A paired sample t-test revealed a statisti- cally significant increase in knowledge post completion of the first escape room $(p=0.000)$. Implications: Students indicated the intentional design of the escape roomthemed CCA lab encouraged them to collaborate and learn from each other, in a simulated environment. Three more escape rooms are in the pipeline to assess the overall benefit of this educational game.

Design and Synthesis of Dopamine D4 Receptor Subtype Agonist Ligands to Treat Neuropsychiatric Disorders. Charity E. Amenya, High Point University, Joseph C. Ezeigwe, High Point University, R. Benjamin Free, National Institute of Neurological Disorders and Stroke, Chun Wu, Rowan University, David R. Sibley, National Institute of Neurological Disorders and Stroke, Thomas Keck, Rowan University, Comfort A. Boateng, High Point University. Objective: To understand the role of Dopamine D4 Receptor (D4R) subtype-mediated signaling in treating D4R-associated disorders Methods: We designed and synthesized a small generation of compounds using computational modelling approach based on the D4R crystal structure with parental compound agonist compound A-412997 (2-(4-(pyridin-2-yl)piperidin-1-yl)-N-(m-tolyl) acetamide). The compounds were synthesized by using substituted or unsubstituted m-toluidine to react with 2chloroacetyl chloride to give substituted or unsubstituted 2-chloro-N-(m-tolyl)acetamide. Followed by alkylation with substituted or unsubstituted piperidine and pyridine moieties as well as modifications to the alkyl chain length. Their binding affinities were determined using both agonist and antagonist by competitive inhibition of $\mathrm{OH}-$ DPAT and $[3 \mathrm{H}] \mathrm{N}-$-methylspiperone radioligand binding assays respectively in HEK293 cells expressing dopamine D2-like receptors (D2R, D3R, D4R). Additionally, functional signaling analysis studies were performed using $\beta$-arrestin recruitment and cAMP inhibition assays Results: We found that substitutions on the piperidine and pyridine moieties, as well as modifications in linker length, led to several novel lead compounds with improved selectivity and potency, and varying degrees of agonist efficacy. Compounds displayed high binding affinity $(<100 \mathrm{nM})$ for the D4R with a $>1000$-fold selectivity comparable to D3R and D2R. Implications: These ligands displayed a range of agonist efficacies. Interestingly, some of the compounds with similar binding affinities showed divergent functional efficacies. These studies have further extended our understanding of ligand-receptor interactions and the relationship between bindings to the two pockets of the receptor: the orthosteric binding pocket and the extended binding pocket. Hence, these studies can potentially lead to the discovery of a possible new pharmacotherapeutic treatment to Neuropsychiatric Disorders. 


\section{American Journal of Pharmaceutical Education 2019; 83 (5) Article 7654.}

Determining the Impact of an Interprofessional Simulation Focused on Social Determinants of Health. Zachary T. Wellner, The University of Kansas, Ashley Crowl, The University of Kansas, Michelle Levy, The University of Kansas, Christina Boyd, The University of Kansas, Jessica M. Bates, The University of Kansas, Jessica R. Barnes, The University of Kansas, Sarah Shrader, The University of Kansas. Objective: The purpose of this study is to determine the impact on pharmacy students' satisfaction, self-confidence, and collaborative behaviors after participating in an interprofessional simulation focused on social determinants of health (SDOH). Methods: Students completed a voluntary pre- and postsurvey after participating in a simulation focused on SDOH and substance abuse. Questions utilizing a Likertscale assessed the students on their understanding of $\mathrm{SDOH}$, knowledge of resources for $\mathrm{SDOH}$, and ability to screen for $\mathrm{SDOH}$. Additional questions assessed the student's confidence in asking patients about their substance abuse and their willingness to refer their patients to a social worker, which were secondary objectives. The data was analyzed using a Wilcoxon Signed-Rank Test via SPSS. Results: Sixty-eight pharmacy students completed both surveys. After participation in the interprofessional simulation, pharmacy students improved their understanding and knowledge of SDOH $(p=0.006$ and $\mathrm{p}<0.000$, respectively); as well as, skills addressing and counseling on SDOH ( $p=.000$ for both). No statistically significant change in students' confidence when asking about or advising a patient on their substance abuse. There was a significant change in how likely pharmacy students would refer patients to social workers $(\mathrm{p}<0.001)$. Implications: This simulation proved to be an effective way to improve pharmacy students' self-reported knowledge of $\mathrm{SDOH}$, as well as, skills for assessing and counseling on resources for SDOH. In addition, the simulation was effective in exposing students to the role of social workers on the team to address $\mathrm{SDOH}$ issues.

Development and Evaluation of an Immunization Information System Training Program Using a Participatory Design Approach. Tessa J. Hastings, Auburn University, David R. Ha, Keck Graduate Institute, Jingjing Qian, Auburn University, Brent Fox, Auburn University, Joni Lakin, Auburn University, Salisa C. Westrick, Auburn University. Objective: As pharmacists have increasingly provided immunization services, there is a growing concern of incomplete immunization records. Only $35 \%$ of pharmacists report vaccination information into their respective Immunization Information System (IIS). This study's objective was to develop and evaluate an IIS Training Program to improve independent community pharmacists' knowledge and intention to enroll in
ImmPRINT, the Alabama IIS. Methods: A participatory design approach, including qualitative interviews and review panel of pharmacists and IIS representatives across five states was used to develop and refine the training program. Twenty-five semi-structured telephonic interviews were conducted identifying potential strategies to improve pharmacy IIS implementation. A panel of six pharmacists and IIS representatives reviewed the program and provided feedback using the Delphi method. Results: An ACPE-accredited two-hour CE course was created as an online article with demonstration videos. Topics include: 1)IIS introduction, 2)IIS policies, 3) Benefits of participation, 4)Enrollment, 5)Historical/administered vaccine documentation, 6)Assessment/recommendation of additional vaccines, 7)Using IIS to provide patient records, 8)Vaccines for children, and 9)Pharmacy recommendations. Panel input was solicited throughout development to ensure that information was relevant and format was acceptable. Preliminary analyses of six pharmacists demonstrate significant increase in mean knowledge $(p=0.009)$ from baseline $(39.6 \%)$ to post-training $(81.2 \%)$ and intention to enroll in ImmPRINT $(\mathrm{p}=0.014)$. Implications: Using a participatory design approach, a pharmacist-centered training program focused on practical strategies to integrate IIS into pharmacy workflow was developed. While this program contains some information specific to ImmPRINT, it could be adapted and disseminated to other states. Further research is needed to evaluate actual IIS enrollment and participation.

Development and Evaluation of a Student-Run Tobacco Cessation Program in an Interprofessional Free Clinic. Elyse A. Keating, University of WisconsinMadison, Rachel Gibbons, University of WisconsinMadison, Molly Lehmann, William S. Middleton Memorial Veteran's Hospital, Ashley Alter, University of WisconsinMadison, Maria Wopat, William S. Middleton Memorial Veteran's Hospital, Andrea L. Porter, University of Wisconsin-Madison. Objective: To describe the studentdriven development of a pharmacy-run tobacco cessation program in an interprofessional free clinic and to assess the implementation and impact of the program. Methods: From October 2017 to February 2019, a pharmacy-run tobacco cessation program in a school-affiliated interprofessional free clinic was designed, developed, and implemented. Student pharmacists and pharmacy preceptors were integrated into the clinic as providers where they led patient visits centered on tobacco cessation and motivational interviewing and collaborated with patients to create comprehensive care plans. Individualized plans were equipped with nicotine replacement therapy and bi-weekly follow-up interactions. Data collected from 


\section{American Journal of Pharmaceutical Education 2019; 83 (5) Article 7654.}

clinic inception (March 2018) until February 2019 included patients reached, nicotine replacement products prescribed, and follow-up interactions completed. Data will continue to be collected through May 2019. Results: Thirty-one patients have been consulted regarding tobacco cessation and nine have enrolled in the program. Fourteen prescriptions for nicotine replacement therapy have been written: 7 for nicotine patches, 1 for nicotine gum, and 6 for nicotine lozenges. Sixteen follow-up calls have been made with two patients successfully reached. Fifteen student pharmacists have volunteered with the program. Implications: This program provides care to underserved patients and introduces other health care professionals to the expanded roles of a pharmacist in the primary care setting. Other student-initiated primary care pharmacy-led initiatives could be implemented in schoolaffiliated free clinics and would increase patient access to healthcare services.

Development of an Interprofessional Military Academic Enrichment Elective. Megan L. Grochowski, Medical College of Wisconsin, Isabelle M. Sviatoslavsky, Medical College of Wisconsin, Sean Blaeser, Medical College of Wisconsin, Michael Nagy, Medical College of Wisconsin. Objective: Collaborate to develop an Interprofessional Military Academic Enrichment Elective (MAEE) course and create an objective examination to measure trainee attainment of knowledge, skills, and attitudes (KSAs) for military and veteran health care. Methods: A MAEE course, for up to 50 students, will adapt materials from Terregino et al. Instructional methods will minimize the use of lecture by leveraging participation from military members and veterans from the community, as well as incorporate an active learning pedagogy. A pre and post examination will consist of 5-10 questions from each topic written by the instructor. Student achievement will be compared before and after the course to determine attainment of KSAs. A paired t-test will be used for the before and after comparison. Results: Pending conclusion of the MAEE course and will be presented at the 2019 AACP annual meeting. Implications: Possible implications include the ability to share this innovative interprofessional course with other pharmacy schools and illuminate the potential need to increase didactic education on military and veteran health care as a social determinate of health.

Development of a Novel MCH1 Receptor Antagonist for the Treatment of Obesity. Sarah C. Steely, Harding University, James Narens, Harding University, Jim Tarrant, Harding University. Objective: Melanin Concentrating Hormone $(\mathrm{MCH})$ is a nineteen amino acid cyclic peptide that is produced primarily by the lateral hypothalamus and interacts with two identified recep- tors, $\mathrm{MCH} 1$ and $\mathrm{MCH} 2$. The $\mathrm{MCH} 1$ receptor is highly expressed in the shell of the nucleus accumbens (NAcSh) and has been tied to feeding and stress behavior in humans. In a prior study, 6-(4-chloro-phenyl)3-[3-methoxy-4-(2-pyrrolidin-1-yl-ethoxy)-phenyl]-3Hthieno[3,2-d]pyrimidin-4-one (GW803430) was shown to act as a potent, orally available, receptor-specific brain penetrant in mice and canines that could be used to selectively antagonize MCH1-R. However, research of GW803430 was discontinued due to cardiac toxicity. The purpose of this project is to develop a novel compound based upon this previous work that is orally bioavailable and can selectively and safely inhibit or downregulate the MCH1-R reward response in the NAcSh to treat obesity. Methods: Using an inexpensive, readily available starter compound, a nine linear step synthesis process (11 total) was developed to produce the MCH1-R antagonist compound, avoiding the need for chromatography purification steps by using a Horner-Wadsworth-Emmons reaction. Results: Yield potentials of approximately $18 \%$ have been achieved, an increase from $9 \%$ yields when compared to the original synthesis methodology. Implications: Studies suggest that selective antagonism of MCH1-R may decrease a patient's craving for and learned response to food. A therapeutic intervention that acts in this manner could reduce the desire for excess food consumption and change the quality of life for those suffering from obesity. Further development and clinical studies will be necessary to support this compound's efficacy for this implication.

Development of a Novel Rural Health Elective Course Integrating Community Engagement and Pharmacist Service Development. Matt J. Huppert, University of Wisconsin-Madison, Andrea Gray, University of Wisconsin-Madison, Kevin Look, University of Wisconsin-Madison, Casey Gallimore, University of Wisconsin-Madison, David A. Mott, University of WisconsinMadison, Robert M. Breslow, University of WisconsinMadison, Mara A. Kieser, University of Wisconsin-Madison, Edward C. Portillo, University of Wisconsin-Madison. Objective: Limited literature exists on the development, structure, and content for pharmacy-specific rural health courses. This project was undertaken to determine core competencies for pharmacy students interested in rural pharmacy practice as well as how core competencies can be incorporated into a semester-long rural pharmacy practice course. Methods: A planning faculty workgroup was formed in October 2016 at the University of Wisconsin-Madison School of Pharmacy. Core rural health competencies were determined through a focus group with 14 Rural Wisconsin Healthcare Cooperative 


\section{American Journal of Pharmaceutical Education 2019; 83 (5) Article 7654.}

members. A faculty representative presented themes from this focus group to the Health-System Pharmacy Advisory Board of the Pharmacy Society of Wisconsin for feedback. After determination of core competencies, students and faculty collaborated to incorporate a longitudinal project. The framework model breaks down the curriculum into six factors: external factors, content, objectives, teaching strategies, learning experiences, and evaluative measures. Results: The topics selected are listed in chronological order as follows: The Agricultural Community in Rural Wisconsin, Epidemiology of Rural Areas, Rural Healthcare Delivery, Interprofessional Care in Rural Communities, Ambulatory Care Leadership, Inpatient Pharmacy Leadership, Community Pharmacy Leadership, Underserved Populations, Critical Access Hospitals, and Health Care Reform and Rural Access. The longitudinal project incorporated three learning components: research on a rural community using population health data, a community site visit, and design an evidence-based pharmacy service. Implications: Determination of core rural health competencies and incorporation of these competencies within a pharmacy practice course is one step to improve student preparation for this important practice setting.

Dietary Supplement and Medication Use and Knowledge in Collegiate Athletes of Rural West Texas. Athena Brindle, Texas Tech University Health Sciences Center, Sivaramakrishna Koganti, Texas Tech University Health Sciences Center, Rubini Pasupathy, Texas Tech University Health Sciences Center. Objective: College athletes' use of dietary supplements and medication is a topic of interest for individuals who work directly with competitive athletes. The controversies of college athletes' use of dietary supplements and medications are primarily focused on the use to gain a competitive edge, i.e. doping. While performance enhancement is one outcome that can result from dietary supplement and medication use in athletes, more concerning outcomes are the side effects and long-term health risks. College athletes' lack of knowledge about the substances they consume increases the risk of harm and testing positive for banned-substances. The purpose of this study is twofold; the first is to evaluate college athlete's prevalence of use of dietary supplements and medications. The second purpose is to investigate college athletes' sources of knowledge of dietary supplements and medications. Methods: A survey study of 755 National Collegiate Athletic Association (NCAA) registered athletes in two institutions of higher education in rural West Texas is being conducted to evaluate their prevalence of use and knowledge of dietary supplements and medications. Results: Expected in 2 months Implications: Pharmacists are actively utilized among healthcare teams as the dietary supplement and medication resource. Previous studies have evaluated the resources athletes use to ensure the safety of the substances they consume, revealing that pharmacists have been underutilized in this area. Pharmacists could play a larger role in ensuring that dietary supplements and medications are appropriate for athletes' medical needs and that the health benefits outweigh the risks that can occur with these substances.

Discussion of Diversity in the Pharmacy Curriculum. Caroline Kruszecki, The University of Rhode Island, Lauren DellaGrotta, The University of Rhode Island, Kendra Walsh, The University of Rhode Island, Angela Dansereau, The University of Rhode Island, Aaron Shaposhnikov, The University of Rhode Island, Megan Gray, The University of Rhode Island, Anne L. Hume, The University of Rhode Island. Objective: Pharmacists serve increasingly diverse patient populations. Student pharmacists should have a solid foundation regarding the factors that may influence patient medication needs, especially before beginning APPEs. Not only to understand the biological differences between patients of diverse backgrounds, but to also be able to establish trust and rapport with patients. The purpose of this project is to review the content of the curriculum related to sex-, race/ ethnicity-, and age-related differences discussed in P1, P2, and P3 courses using student observers during the 2018-2019 academic year. Methods: The review was conducted through the use of two students in each professional year. Each student had access to their own document to record the date, class, and topic associated when a professor addressed either age, gender/sex, race, or ethnicity. To eliminate bias, the project was not discussed with faculty besides those working on the review itself. Students sat in different areas of the auditorium and did not discuss, nor have access to, another's data collection document. Data collected will be analyzed at the end of the academic year in order to allow comparisons between the frequency of topics discussed in a single class and between each class in the curriculum. Results: As this is an ongoing project, final results are not available. Implications: The results of this review can lead to improvements in the curriculum by adding diversity education where it is lacking, thereby ensuring that students leave the institution understanding the impact that patient diversity has on patient care.

Effectiveness of an Advanced Pharmacogenomics Independent Study Course for Doctor of Pharmacy Program. Shannon L. Bailey, Western New England University, Susan Capasso, Bay Path University, Shannon Kinney, Western New England University. Objective: Since ACPE included genetics and pharmacogenomics in 


\section{American Journal of Pharmaceutical Education 2019; 83 (5) Article 7654.}

the recent standards, pharmacy programs are striving to fulfill this requirement. Our objective was to design an independent study that required interpretation of 23 andMe personal genomic testing (PGT) data and development and implementation of a genetic test to enhance abilities in utilizing genetics and pharmacogenomics. Methods: Within the course, the student translated their PGT data into genetic variants that could be used to identify phenotypic medical pre-dispositions and potential response to medications ('dry-lab'). The genetic test was designed to determine blood type using cancer cell line DNA ('wetlab'). We utilized a pre/post-survey to assess perceptions of, and a pre/post-examination to assess skills within, the aforementioned areas. During the pre/post-examination, the student was allowed to utilize Pharmgkb.org and ncbi.nlm.nih.gov databases. Results: The post-survey demonstrated increased confidence in capabilities and understanding of genetics. The pre-examination score was $67.1 \%$, which increased to $94.2 \%$ in the post-examination. When comparing the pre/post exams, areas of growth included medical genetic diagnosing and prescribing, determining phenotype, and identifying gene locations. The student's ability to translate PGT data was validated by their 23andMe report. Implications: This independent study format is unique in that it utilized both 'dry' and 'wet' lab content. Our results suggest the student developed expertise in understanding and implementing pharmacogenomics gaining skills that go beyond an introductory course. We are currently designing a similar advanced experiential rotation. These course formats may be of interest to other pharmacy programs that are incorporating pharmacogenomics education into their curricula.

Effectiveness of Post-Discharge Education on Reducing Proton Pump Inhibitor (PPI) Usage in Conjunction with Stewardship Efforts. Mathew Olean, VA Tennessee Valley Healthcare System, Monica Barrett, VA Tennessee Valley Healthcare System, Michelle Moseley, VA Tennessee Valley Healthcare System, Elizabeth Mitchell, Lipscomb University, Jessica Wallace, Lipscomb University. Objective: The primary objective was to assess the impact of a post-discharge telephone followup call on successful discontinuation of an inappropriate PPI prescription as part of a PPI stewardship program. Successful discontinuation was defined as lack of a refill or new script within four months post-discharge. Secondary outcomes included the number of patients with symptomatic reflux at follow-up, the number of inappropriate PPI prescriptions discontinued as part of the stewardship effort, and the number of patients who tolerated dose deescalation. Methods: A quality improvement study evaluating a PPI stewardship program with patient education was conducted. Patients were included if admitted to the hospital with an active inpatient order for an oral PPI that was deemed to be inappropriate. Patients were excluded if an appropriate indication for long-term PPI therapy was identified (i.e. esophageal strictures, Barrett's esophagus, and Zollinger-Ellison syndrome). Patients who had dual antiplatelet therapy, concomitant anticoagulation and nonsteroidal anti-inflammatory, or chronic steroid use were also excluded. Data collected included demographics, PPI medication including dose and indication, and stewardship-effort outcome at the time of identification, ten-days post-discharge, and four-months post-discharge. Patient education performed included discharge counseling regarding PPI discontinuation and a post-discharge follow-up phone call to provide additional education regarding the reasons for discontinuation, inquiring about pertinent symptoms, and counseling on nonpharmacological interventions. Results: Data collection is underway. Implications: If an association exists between postdischarge patient education and successful PPI discontinuation, this may improve PPI stewardship efforts and subsequent patient outcomes by avoiding unnecessary adverse effects from long term PPI use.

Effect of a Medication Discharge Program on Primary Medication Non-Adherence. Christopher J. Damlos, Purdue University, Julia M. Smith, Purdue University, Heather A. Jaynes, Purdue University, Margie E. Snyder, Purdue University, Alan J. Zillich, Purdue University. Objective: This study aims to describe primary non-adherence among subjects who opted-in to a bedside medication delivery (Meds-to-Beds) program at discharge compared to subjects who opted-out. Methods: This is a retrospective, observational cohort study, which consisted of a pharmacist delivering medications to bedside at discharge. The intervention cohort was defined as all hospitalized patients eligible for and opting into the "Meds-to-Beds" program. Eligibility criteria included all patients discharged to home from a general medical/surgical, progressive care or intensive care unit and prescribed one or more new medications filled within 30 days after discharge. New discharge medications were classified as over-the-counter, as needed, short-term, or chronic by a multidisciplinary research team. Analyses occurred at the prescription level, and differences between groups were compared using a chi-square, student's t-test or Mann-Whitney U-test. Results: The final cohort consisted of 500 subjects in the intervention group, receiving a total of 1,112 new medications at discharge, and 1,306 subjects in the control group, receiving a total of 2,336 new medications. Subjects in the intervention group filled a greater percentage of total first time medications within 30 days following discharge compared to the control group (Intervention $=87.8 \%$ vs Control $=$ 


\section{American Journal of Pharmaceutical Education 2019; 83 (5) Article 7654.}

$38.4 \%, \mathrm{p}<0.00001)$. Intervention group subjects filled new medications in less days following discharge compared to the control group (Intervention $=0.4(2.9)$ vs Control $=2.6(5.6), \mathrm{p}<0.00001)$, and both trends were statistically significant across all categories of medications. Implications: Patients offered a medication discharge program filled more first-time prescriptions and had a shorter time to first fill of medications compared to the control group.

Effect of Competitive Longitudinal Advanced Pharmacy Practice Experience on Residency Pursuit and Match Rates. Parna Haghparast, University of Houston, Julianna Fernandez, University of Houston, Nancy D. Ordonez, University of Houston, Kimberly Nguyen, University of Houston, Elizabeth A. Coyle, University of Houston, Matthew A. Wanat, University of Houston. Objective: Many pharmacy schools offer the option of competitive longitudinal advanced pharmacy practice experience (CLAPPE). These programs allow students to participate in four to five APPEs at a single institution and provide a longitudinal experience similar to a residency with a research project and mentorship program. An additional advantage of these programs is that students will spend less time orienting and acclimating to a new practice site. The purpose of this research project is to look at the residency pursuit and match rates of students participating in a CLAPPE compared to students who participated in traditional advanced pharmacy practice experiences (APPE). Methods: This is a retrospective study being conducted at the University of Houston College of Pharmacy. The residency pursuit and match rate of students that completed a CLAPPE or traditional APPE (from 2014-2018) will be compared using data from the experiential office and ASHP MATCH results. To prevent confounding data, students in each cohort will be matched based on grade point average. Results will be analyzed using a chi-squared analysis. Results: Data collection and analysis of results are pending. Results to be presented include residency pursuit and match rates among both cohorts. Implications: This would be the first study, to our knowledge, to evaluate the effect of CLAPPE programs on residency pursuit and match rates. The results of this study could provide evidence for the benefit of such programs that are available at some colleges of pharmacy.

Effect of Exercise on Perceived Stress in School of Pharmacy Students and Faculty. Jamie Haas, Virginia Commonwealth University, Julie A. Patterson, Virginia Commonwealth University, Lauren Pamulapati, Virginia Commonwealth University, Kelechi C. UnegbuOgbonna, Virginia Commonwealth University, Victoria A. Keel, Virginia Commonwealth University, Lauren M. Caldas, Virginia Commonwealth University. Objective:
(1) Compare School of Pharmacy (SOP) students and faculty exercise levels to their Cohen's Perceived Stress Scale (PSS) and (2) Assess for a relationship between weekly step count, calories burned, minutes of exercise, the frequency of daily exercise, and type of exercise and reduced perceived stress. Methods: This prospective study utilizes monthly surveys of PSS and activity level, reported through self-reported surveys or electronically shared via Apple Watch users. A baseline survey collected demographic data, self-reported activity level, and perceived stress using the PSS. Monthly follow-up surveys collect repeated PSS and activity outcomes. Baseline data will be analyzed with descriptive statistics and PSS repeated measures with ANOVA. Results: The project is in data collection phase, but preliminary data is available. Enrolled participants $(n=54)$ were primarily female (83\%) and students (83\%) with an average age of 26 years (range: 21-63). The mean baseline PSS score was 16.7 (SD: 5.93; range: 6-29). The Apple watch participants $(n=21)$ to date had a daily average of 7011 steps, 435 active calories, 33.6 total activity minutes, and 11 hours of Apple Watch's definition of standing. Implications: While this project is in the data collection phase, if there is a foreseeable difference in perceived stress score and activity level, this may be an area for SOPs to focus support on increasing student activity level. If institutions have the option for students to self-enroll in wellness monitoring programs, then early student interventions could occur.

Empathy and Confidence in Pharmacy Students in a Skills-Based Learning Environment Regarding Opioid Overdose. Joshua Wollen, University of Houston, Alexa L. Vyain, University of Houston, David A. Wallace, University of Houston, Dhara D. Surati, University of Houston. Objective: A skills-based learning activity was designed to determine if a continuing education article, a naloxone training activity, and a drug diversion activity will increase empathy and change attitudes towards patients at high risk for an opioid overdose. Methods: Prior to the skills-based learning activity, students engaged in reading a segment of a continuing education (CE) article that focused on the hardships of patients suffering from opioid use disorder. The skillsbased learning activity was divided into two portions: a naloxone training activity and a drug diversion activity. The naloxone training activity involved a 10-minute lecture followed by a role-playing activity. After the roleplaying activity, the students engaged in a reflection. The drug diversion activity consisted of two 15-minute roleplaying activities; one involved internal drug diversion and one involved external drug diversion. This activity also ended with a reflection focusing on the 


\section{American Journal of Pharmaceutical Education 2019; 83 (5) Article 7654.}

diversion perpetrator in the role-playing scenario. Empathy and understanding were assessed with a pre- and postassessment. The evaluation tools used were the KiersmaChen Empathy Scale (KCES) and the concerns and readiness portions of the Opioid Overdose Attitudes Scale (OOAS). Results: Results will be analyzed by a t-test and to identify the degree of change in empathy and attitudes from the described activity. Implications: Implications will be better assessed upon completion of this project. If the hypothesis is correct, this activity may provide a template for colleges of pharmacy to better prepare the next generation of pharmacists to more effectively care for patients in the wake of the opioid epidemic.

Evaluating Preventable 30-day Readmissions Among Patients Offered a Medication Discharge Program. Julia M. Smith, Purdue University, Christopher J. Damlos, Purdue University, Heather A. Jaynes, Purdue University, Margie E. Snyder, Purdue University, Alan J. Zillich, Purdue University. Objective: Transitions between care settings is a vulnerable period for patients. Therefore, cost-effective programs for transitional care from hospital to home are needed to improve patient outcomes. The purpose of this study was to evaluate preventable readmissions among patients who were offered a medication discharge program. Methods: This is a retrospective, observational cohort study during a 1-year period in a medium-sized suburban health-system. The "Meds-to-Beds" program consisted of a pharmacist delivering patient's medications to bedside at discharge. The intervention cohort was defined as hospitalized patients eligible for and opting-in to the program, and the control cohort were those who opted-out. The primary outcome was defined as any preventable 30 -day readmissions across both study groups using the Agency for Healthcare Research and Quality's (AHRQ) potentially avoidable hospitalization for ambulatory care sensitive conditions classification. Results: Data was collected for 500 intervention and 1591 control patients. The proportion of patients with preventable readmissions within 30 days was $2.8 \%(n=14)$ in the intervention group and $4.4 \%(n=70)$ in the control group. In the multivariate model, patients in the control group were more likely to be readmitted for a preventable cause within 30-days than patients in the intervention group $(\mathrm{OR}=2.03 ; 95 \%$ CI: 1.14-3.86, $\mathrm{p}=0.02)$. Implications: This "Meds-toBeds" program reduced 30-day preventable hospital readmissions. Within health systems, transitions of care interventions are needed and must target reducing avoidable hospitalizations. Future studies should determine the cost-effectiveness of this "Meds-to-Beds" program and evaluate the effect of the program on primary nonadherence.
Evaluating the Impact of Problem Solving and Medication Education on Diabetes Self-Care and Disease Management. Sushma Dey, University of South Florida, Briana Williams, University of South Florida, Deena Albashir, University of South Florida, Nour Salhab, University of South Florida, Rachel Franks, University of South Florida. Objective: To evaluate if patients' knowledge and ability to self-manage diabetes improves after participation in an educational workshop lead by pharmacy students. Methods: Patients 18-79 years old who have diabetes and have not received diabetes education within a year will be recruited from the Family and Internal Medicine Clinic from USF Morsani. A pharmacy-student led diabetes education workshop will be conducted that will focus on diabetes medications and problem-solving related to diabetes medication. A preworkshop and post-workshop survey will be performed to measure the change in patients' knowledge, attitudes and problem-solving skills. Survey responses from patients with diabetes who are consistent with our sample criteria but did not attend the workshop will also be collected as control group data. Results: Results pending workshop in March. Implications: In the future we could do additional studies that follow up with patients to see how our intervention affected their A1C. We could also do a general post survey to see how much information was retained from our educational workshop. We could also provide additional diabetes education workshops to the same treatment group to see if there was any further improvement in patient outcomes.

Evaluating the Quality and Utilization of Multiple-Choice Questions in a NAPLEX Preparation Book. Tina Danh, University of South Florida, Victoria R. Herrmann, University of South Florida, Tamara A. Desiderio, University of South Florida, Heather M. Lyons, University of South Florida, Frankie L. Patrick, University of South Florida, Kamila A. Dell, University of South Florida, Gwendolyn Wantuch, University of South Florida. Objective: To evaluate the quality and utilization of multiple-choice questions (MCQs) by examining the item construction of questions in a commonly used North American Pharmacist Licensure Examination (NAPLEX) preparatory book. Methods: Seven analysts reviewed MCQs, including those in the "select all that apply" format, from the RxPrep 2018 Edition Course Book. The item construction of MCQs and utilization of case-based questions were evaluated using best practices for item construction. Item writing flaws (IWFs) were identified individually by the analysts and later agreed upon through group consensus. Results: A total of 298 questions were reviewed. Thirty-two (10.74\%) questions met all best practices for item construction. Amongst 


\section{American Journal of Pharmaceutical Education 2019; 83 (5) Article 7654.}

questions that did contain flaws, an average of 2.58 IWFs per MCQ were found. The most common best practice violations identified were answer choices with differing verb tense and length (20.99\%) and question stems containing too much or too little information necessary to eliminate distractors (16.62\%). The majority of casebased questions did not require utilization of the case provided (61.98\%). Implications: This study identified IWFs in a majority of the questions from one NAPLEX preparatory source, which may impact the utility of these questions as study material. The results of this analysis align with previous literature, in and outside of pharmacy. Flawed MCQs and underutilization of case-based content may not represent item construction on board exams and, therefore, may advantage or disadvantage certain test takers. Further evaluation of other preparatory materials, including test banks, is needed.

Evaluation of a Game Design Activity as an Active Learning Strategy in a Laboratory Setting. Olivia M. Kim, University of Wisconsin-Madison, Andrea L. Porter, University of Wisconsin-Madison. Objective: To assess the impact on student knowledge and perceptions of a game design activity on anticoagulation in a pharmacotherapy skills laboratory course. Methods: At the beginning of the spring 2019 semester, all P2 students in the course were given a pre-intervention survey gauging their attitudes toward educational game-based learning activities in the classroom. Later in the semester, students will complete a five-question knowledge-based assessment on an anticoagulant. Subsequently, students will work in teams of four or five to design a game focused on educating patients about their respective medication. A week later, students will be given the same knowledge-based assessment they had completed previously. They will also complete a post-intervention survey that will evaluate their perceptions of the activity. A subset of questions on the survey will be identical and compared to those from the pre-intervention survey. Results: A total of 134 students $(93.7 \%)$ completed the pre-intervention survey. Of those students, $84.3 \%$ had played educational games in previous classes and $52.2 \%$ of students had designed a game previously. In addition, $68.7 \%$ of students felt that the use of games would be helpful in achieving a deeper understanding of a concept. The remainder of the data collection and analysis is in progress and will be completed by May 2019. Implications: This study evaluates an innovative game design activity as an active learning strategy for student pharmacists. Student feedback and performance surrounding the game design activity will lead to optimization of game-based learning for future pharmacotherapy laboratory courses.
Evaluation of a Novel Pharmaceutical Industry Track as an APPE for Doctor of Pharmacy Students. Justin G. Moots, Purdue University, Brian M. Shepler, Purdue University, Amy H. Sheehan, Purdue University. Objective: Interest to participate in industry fellowships has grown significantly in recent years, reaching 500 PharmD fellows in 2018, with fellowship positions becoming more competitive. Thus, a pharmaceutical industry track APPE could increase students' probability of successfully obtaining an industry fellowship position. The primary objective of this study is to evaluate the effectiveness of a pharmaceutical industry focused advanced pharmacy practice experience (APPE) designed to prepare students for acquiring a post-doctoral pharmaceutical industry fellowship. Methods: This pharmaceutical industry track program consists of blocked scheduling to include up to three consecutive APPE rotations (four weeks each) at the selected pharmaceutical industry site. An electronic survey instrument was developed to be distributed to 16 fourth-year Doctor of Pharmacy students who participated in the pharmaceutical industry track program and the ASHP fellowship selection process. The guiding framework for the survey instrument used Kirkpatrick's four-level training evaluation model including reaction and results and consisted of 25 items using a 5-point Likert scale. The electronic survey instrument will be distributed on March 11th, 2019 to collect demographics, motivating factors and students' opinions regarding the program, and to assess students' success in obtaining an industry fellowship position. Data will be analyzed by descriptive statistics using the Statistical Package for the Social Sciences (SPSS) version 24. Study procedures were reviewed and determined to be exempt from IRB review. Results: Not Applicable (Research in Progress) Implications: Not Applicable (Research in Progress)

Evaluation of a Rural Health Pharmacy Practice Elective Course Through Student Engagement, Confidence, and Attitudes. Jenna Nehls, University of Wisconsin-Madison, Patrick Holland, University of Wisconsin-Madison, Edward C. Portillo, University of Wisconsin-Madison, Casey Gallimore, University of Wisconsin-Madison, David A. Mott, University of WisconsinMadison, Robert M. Breslow, University of WisconsinMadison, Kevin Look, University of Wisconsin-Madison, Mara A. Kieser, University of Wisconsin-Madison. Objective: This evaluation assesses impact of a new rural health pharmacy elective course on confidence, attitudes, and student engagement towards rural pharmacy practice. Methods: A total of 18 P3 students completed the Topics in Rural Health elective course in Fall 2018. Two independent course observers evaluated student responses to 


\section{American Journal of Pharmaceutical Education 2019; 83 (5) Article 7654.}

(1) a 36-item questionnaire assessing student confidence using a 7-point Likert scale (2) focus groups assessing student attitudes towards class content and (3) student engagement during in-class activities. Questionnaires were completed before and after course completion to evaluate degree of change in confidence and attitudes performing activities rural pharmacy practice activities. Using questionnaire data, two focus groups were evaluated using thematic analysis to better understand course impact. Student engagement was assessed using a validated tool categorizing students as engaged, disengaged, and unknown. Results: Students enrolled in the rural health elective course on average expressed greater intention to practice as rural health pharmacists $(0.66$ point average increase). Students also demonstrated greater confidence performing a community assessment and engaging with local health departments (1.67 point average increase for each). Focus group themes regarding attitudes towards the class included: a strong connection to rural community assessment, applicability to pharmacy practice, and pharmacist opportunity to serve patients through service development. Students experienced the greatest engagement during student and guest speaker presentations. Implications: A rural health elective class can result in greater student intention to enter rural practice and increased confidence engaging with community stakeholders.

Evaluation of a Simulated Professional Conference for Student Pharmacists: Conference on Conferences. Hannah Hecht, University of Wisconsin-Madison, Catherine Kuecker, William S. Middleton Memorial Veterans Hospital, Andrea L. Porter, University of WisconsinMadison. Objective: To describe the development and implementation of a mock professional conference for student pharmacists and evaluate student confidence in activities associated with professional meetings. Methods: In October 2018, students participated in 3 hours of programming including networking bingo, developing an elevator speech, pharmacist roundtables, and poster session etiquette. Student pharmacists from all schools of pharmacy in Wisconsin were invited to attend. Student pharmacists completed a pre- and post-event survey that evaluated confidence in performing various professional meeting activities using a 10-point scale $(1=$ not confident at all, $10=$ very confident). A thematic analysis of short answer questions was conducted to identify strengths and areas for improvement. Results: There were 125 student tickets sold for the event and 24 pharmacists attended to facilitate sessions. The pre- and postsurveys were completed by $76.8 \%$ and $74.4 \%$ of student pharmacists, respectively. An increase in reported confidence was observed for all surveyed measures, with high- est increases for maintaining meaningful connections after meeting someone new at a conference (4.8 to 7.0) and interacting with poster presenters (5.0 to 7.7). Student pharmacists responded they enjoyed developing an effective elevator speech and practicing new networking skills but felt a longer duration for the pharmacist roundtables would be beneficial. Implications: Student pharmacist confidence performing a variety of professional meeting activities increased after a mock professional conference, and feedback indicated the event was successful. A similar event could be implemented at other schools and colleges of pharmacy to introduce students to professional meetings in a safe environment.

Evaluation of Patient Risk Factors in Bortezomib-Induced Peripheral Neuropathy in SWOG Trial S0601. Jayson Yumang de Guzman, University of California, San Francisco, Siu-Fun Wong, Chapman University, Hongli Li, Fred Hutchinson Cancer Research Center, Brian Till, Fred Hutchinson Cancer Research Center. Objective: To evaluate the potential difference in the incidence and severity of peripheral neuropathy in mantle cell lymphoma subjects enrolled in SWOG clinical trial S0601 based on patient specific parameters and demographics (age, gender, ethnicity, BSA) reported in the literature that may influence tolerability to the anticancer drug bortezomib. Methods: Subject information will be obtained from the SWOG statistical center database. The incidence and severity of peripheral neuropathy based on CTCAE criteria will be analyzed by enrolled subjects' risk factors of interest including age ( $>61$ vs. $<61$ ), gender, BSA (total cumulative dose), and ethnicity (Caucasian vs. non-Caucasian). An unpaired t-test with a p-value set at 0.05 will be analyzed for incidence and severity of peripheral neuropathy for each of the above risk factors. A peripheral neuropathy toxicity profile will be formulated based on the patients at risk for peripheral neuropathy when treated with bortezomib. Results: A total of 68 patients were screened and 65 were eligible for evaluation. Patients were aged $36-85$ with a median of $61,13(20 \%)$ were female, bortezomib was dosed at $1.3 \mathrm{mg} / \mathrm{m}^{\wedge} 2$ with a mean dose intensity of $96 \%$, and 61 (94\%) were Caucasian. Data analysis is ongoing. Implications: Currently, no standard of care approach exists to predict for patients who may be at risk for dose limiting peripheral neuropathy toxicity when treated with bortezomib. This project hopes to identify at risk patients to facilitate for early recognition and preventative measures to optimize anticancer treatment outcomes, minimize adverse events, and improve patient's quality of life.

Evaluation of Peer-to-Peer Feedback in a Pharmacotherapy Skills Course. Catherine Kuecker, William S. Middleton Memorial Veterans Hospital, Casey 


\section{American Journal of Pharmaceutical Education 2019; 83 (5) Article 7654.}

Gallimore, University of Wisconsin-Madison, Andrea L. Porter, University of Wisconsin-Madison. Objective: To evaluate the impact of purposeful instruction on students' perceived and actual ability to provide effective peer-topeer feedback within a skills-based laboratory course. Methods: During Fall 2018143 P2 students were taught to provide peer feedback and were given opportunities to practice during four in-lab recorded simulations (1 medication history, 2 consultations, 1 OTC consultation). After the semester, a random, representative sample $(\sim 25 \%$ of enrolled students) was selected. A standardized rubric was used by two evaluators to assess effectiveness, quality, and type of feedback provided. Inter-rater reliability was completed to ensure consistency. All students were invited to complete pre- and post-semester surveys to selfevaluate their ability to provide peer feedback using a 7point scale from strongly disagree to strongly agree. A thematic analysis will be conducted to identify studentperceived strengths and areas for improvement. Results: Recorded simulations of 140 students were assessed (35 per simulation) totaling 861 pieces of feedback. More feedback was provided for medication history or consultation simulations (range 207-238 items) compared to the OTC consultation ( $\mathrm{n}=191$ items). Across the simulations, feedback was tangible (88-92\%), specific (80-89\%), and user-friendly $(100 \%)$. Actionable and constructive feedback throughout the semester (16-26\%) was consistent with highest rates occurring during the second consult lab. Positive feedback statements varied (52-67\%) and negative statements trended downward throughout the semester from $21 \%$ to $15 \%$. Student survey results are pending. Implications: Incorporating opportunities for students to practice providing formative peer feedback had a positive impact and further integration to improve actionable and constructive feedback would be beneficial for peer learning.

Evaluation of Pharmacy Student Burnout and Effectiveness of Intervention Strategies. Jenna Summerlin, Union University, Drew Wells, Union University, Ashok E. Philip, Union University, Kim M. Jones, Union University. Objective: To evaluate impact of stressreduction strategies in reducing burnout among Union University College of Pharmacy (UUCOP) students. Methods: Consenting P1 - P3 students were randomly assigned to control or intervention groups. UUCOP provided stress-reducing activities from October 2018 to April 2019 for the intervention group. The Maslach Burnout Inventory-General Survey for Students (MBI-GS) was used to determine baseline and mid-point scores for all participants. Participants completed a pre-intervention survey, using a scale of $0-5(0=$ no stress, $5=$ highly stressful), which identified stressors and stress-reducing activities. Post-intervention MBI-GS scores and a postintervention survey will be collected and analyzed, at study conclusion in April 2019. Results: The control and intervention groups comprised 40 students, respectively. The mean scores for the top 3 stressors were 4.4 (exams), 4.2 (competencies), and 2.6 (class assignments). The mean scores for top 3 stress-reducers were 4.1 (family time), 3.8 (personal time), and 3.6 (exercise). Control group baseline and mid-point MBI-GS median individual average scores for Emotional Exhaustion (EE) were 4.2/ 4.2, Personal Efficacy (PE) 4.5/4.2, and Cynicism (C) 2.2/ 2.6. Intervention group baseline and mid-point median individual average scores for EE were 4.8/4.2, PE 4.5/ 4.8, and C 2.5/2.0 The intervention group MBI-GS scores improved, but the difference was not statistically significant (p value: EE 0.2, PE 0.6, C 0.07) after ANCOVA analysis. The average attendance rate was 15 students per intervention in fall 2018. Implications: At mid-point, activities provided by UUCOP contributed to stressreduction. Upon study completion, results will be used to guide UUCOP efforts to promote overall health and wellness of students.

Evaluation of the Impact of Prior Pharmacy Experience on Performance in a First-Year Skills Laboratory Course. Leah Allison Selznick, Virginia Commonwealth University, Teresa M. Salgado, Virginia Commonwealth University, Fawaz Alotaibi, Virginia Commonwealth University, Lauren M. Caldas, Virginia Commonwealth University. Objective: (1) Assess the impact of prior pharmacy practice experience on first-year pharmacy students' performance and confidence in a onesemester skills laboratory course on Top 300 Exam, prescription graded activities, and final course grade. (2) Evaluate the potential consideration for students to optout of certain assignments. Methods: First-year pharmacy students $(n=266)$ from two cohorts were surveyed on prior pharmacy experience and confidence on future performance. Success to consider opting-out of components of the course was categorized as a score of $100 \%$ on the Top 300 exam, $100 \%$ on prescription grades, and an A $(93 \%)$ for their final course grade. Frequency and percentage were reported for categorical variables and mean (SD) were calculated for continuous variables. Logistic regression model was performed to assess the association between prior pharmacy experience and the predictors. Results: Students with prior pharmacy experience (75\%) had more perceived confidence (OR: 8.84, 95\% CI: $3.86-20.21)^{*}$ and were more likely to receive a final grade of an A (OR: 1.06, 95\% CI: 1.00-1.13)*. Both Top 300 exam and prescription grades showed an increase however neither were significant. ${ }^{*} \alpha=0.05$. Implications: Pharmacy students with varying levels of practice 


\section{American Journal of Pharmaceutical Education 2019; 83 (5) Article 7654.}

proficiency enter programs where faculty are tasked to create meaningful learning experiences despite students' differences. Students with prior experience demonstrated increased performance in the final course grade but not in the individual course activities. This evaluation did not support opting out of course activities but may be an indication to further evaluate the performance discrepancy.

Examining Pathophysiology Course Placement Within PharmD Curriculum Across the United States. Meagan L. Dias, Western New England University, Kaitlin J. Armstrong, Western New England University, Megan M. Pantos, Western New England University, Joshua J. Spooner, Western New England University, Daniel R. Kennedy, Western New England University. Objective: To examine the amount and placement of pathophysiology, anatomy, and physiology courses within pharmacy curricula, looking specifically at variations in program length, class size, prerequisites, and institution type in order to determine the level of consistency across pharmacy programs. Methods: The websites of 146 pharmacy programs were examined for information related to pathophysiology, anatomy, and physiology courses and instruction. Findings were examined for potential differences based on common program demographics. Categorical and continuous data were analyzed using chi-square and $\mathrm{T}$ tests, respectively. Results: Eight programs listed uninterpretable or incomplete website data and were excluded, producing a final sample size of 138. Most programs ( $n=113,81.9 \%)$ had integrated pathophysiology courses. The majority of these $(\mathrm{n}=95,84.1 \%)$ used solely integrated pathophysiology courses, while some $(n=18,15.9 \%)$ had both stand-alone and integrated pathophysiology courses. The remaining programs $(n=25,18.1 \%)$ used stand-alone pathophysiology courses only. Of those with stand-alone pathophysiology courses, the mean number of credit hours was 5.0 (range: 2-9). A majority of programs (89.8\%) required anatomy and/or physiology, with more public programs than private programs requiring it as a prerequisite (77.9\% vs. $55.7 \%$; $=0.0068)$. Implications: Pathophysiology, anatomy, and physiology are fundamental components of pharmacy education and are integral to pharmacists' knowledge of pharmacotherapy. Within pharmacy curricula, pathophysiology was found in various formats, but most commonly integrated within other courses. A wide variety of credit hours were assigned to stand-alone pathophysiology courses. Anatomy and physiology were prerequisites for most pharmacy curricula, with public programs more likely to require it as a prerequisite.

Expanded Scope of Practice for Pharmacists at a Diabetes Camp and Experiential Education Development. Emma C. Hatfield, St. Elizabeth Healthcare, Leah
E. Fuller, University of Cincinnati, Nicholas Messinger, University of Cincinnati. Objective: To evaluate a new advanced pharmacy practice experience (APPE) at a diabetes camp. Methods: : Camp Korelitz is an American Diabetes Association (ADA) diabetes camp in Ohio for children living with diabetes. Two pharmacists, credentialed in diabetes education, were granted an expanded scope of practice by a consult agreement with the medical director. Under the consult agreement, the pharmacists were responsible for the campers and counselors in their assigned cabins. Roles included, but were not limited to, blood glucose monitoring, carbohydrate counting, adjusting and administering insulin doses, managing insulin pumps, and treating hypo- and hyperglycemic episodes. An APPE was subsequently designed and provided to enhance student learning. To evaluate the APPE, students completed pre- and post-camp assignments. The precamp assignment consisted of a survey and one openended question. The post-camp assignment consisted of a reflection and the survey. Results: Twelve students completed the APPE at Camp Korelitz since its development. For the first cohort of students, improvement was reported for confidence in diabetes management and insulin pump therapy. Utilizing student feedback, an elective course was designed to better prepare students interested in the APPE. Confidence in diabetes management and insulin pump therapy continued to improve for students in following years. Overall, reflections indicated the APPE had a favorable impact on professional development and ability to empathize with patients. The second and third cohorts indicated the elective course enhanced preparation for the APPE. Implications: The expanded scope of practice for pharmacists at a diabetes camp offered a unique, challenging, and rewarding learning experience for students.

Exploiting Exosomes for Targeted Cancer Delivery. Coy A. Fitts, The University of Mississippi, Chalet Tan, The University of Mississippi. Objective: The goal of this study is to establish and optimize the isolation, characterization, and encapsulation of anticancer drugs into exosomes. Methods: Human embryonic kidney cells (HEK293T) were cultured to $80 \%$ confluency and the media was harvested. To isolate the exosomes from cell culture media, a two-step process of ultrafiltration followed by size exclusion was conducted. Exosomes were characterized by the size and protein expression profiling by western blot. Exosomes (60 $\mu \mathrm{g}$ protein) were incubated with paclitaxel $(20 \mu \mathrm{M})$ for 1 hour at $37^{\circ} \mathrm{C}$. The unincorporated paclitaxel was removed from the sample by a $100 \mathrm{kDa}$ membrane, resuspended in PBS, and centrifuged at 14,000 rpm for 10 minutes. The exosomes contained in the supernatant were lysed, and the paclitaxel 


\section{American Journal of Pharmaceutical Education 2019; 83 (5) Article 7654.}

concentration was measured by HPLC. Parallelly, to enable fluorescence labeling, exosomes (300 $\mu \mathrm{g}$ protein) were also incubated in a DiD solution $(15 \mu \mathrm{M})$ for 1 hour and analyzed by near-infrared spectroscopy. Results: Exosomes had an average diameter of $111.86 \mathrm{~nm}$ and highly expressed CD63 and Flotillin 1, without calnexin expression (ER protein). The encapsulation of paclitaxel and DiD were $1.48 \mu \mathrm{M}$ and $0.85 \mu \mathrm{M}$ respectively. Implications: Exosomes are able to be reproducibly isolated via ultrafiltration and size exclusion. Exosomes are promising delivery carriers for imaging-guided therapy and targeted drug delivery.

Exploration of Military and Veteran Health Care Education Within Pharmacy School Didactic Curricula. Isabelle M. Sviatoslavsky, Medical College of Wisconsin, Megan L. Grochowski, Medical College of Wisconsin, Sean Blaeser, Medical College of Wisconsin, Michael Nagy, Medical College of Wisconsin. Objective: Compare the incorporation of military and veteran health care education within pharmacy school didactic curricula nationwide. Methods: Study team members will assess publicly accessible curricular information listed on each pharmacy school website, including class lists and course syllabi. If contact information is available, a direct inquiry through phone or email, will be made to elaborate on the publicly available information. If a school includes military and veteran health care in their curriculum, academic year in school, course type (elective vs required), duration, topic coverage, and instructional methodology will be collected. General information about each pharmacy school, such as private or public, size of school, proximity to a VA, and school ranking will also be collected to help identify any trends in veteran and military health education. We hypothesize proximity to a VA or military base will correlate to the amount of military and veteran health care didactic content within the curriculum. We will use descriptive statistics to assess school factors that are associated with the incorporation of military and veteran health care education. Results: Results pending conclusion of the investigatory stage and will be presented at the 2019 AACP annual meeting. Implications: Possible implications include the identification of appropriate placements within the didactic curricula to teach military and veteran health care. Through analysis of topic coverage and instructional methodology, current practices can be shared nationwide. This investigation could potentially uncover gaps in pharmacy didactic curricula regarding current exposure of military and veteran health care as a social determinate of health.

Exploring Entrustable Professional Activity Milestones in the Curriculum. Kara E. Provence, The University of Tennessee, Christa M. George, The Univer- sity of Tennessee, Chelsea Renfro, The University of Tennessee. Objective: To determine faculty and preceptor perceptions of expected levels of entrustment for student pharmacists' pre-introductory pharmacy practice experiences (IPPE), pre-advanced pharmacy practice experiences (APPE) and upon graduation. Methods: An electronic survey instrument was developed and administered to all University of Tennessee Health Science Center (UTHSC) College of Pharmacy faculty and preceptors via QuestionPro. Questions were developed to gather respondent demographics and perceptions of expected levels of entrustment for the Patient Care Provider and Interprofessional Team Member Domains pre-IPPE, preAPPE, and upon graduation. Questions were structured in Linkert format with level one being the lowest (limited knowledge and insufficient skill) to level five being the highest (supervision of others). The survey instrument was distributed to all faculty members and preceptors utilizing a modified Tailored Design methodology. Descriptive statistics were utilized to analyze data. Results: The survey was completed by 84 faculty and preceptors (response rate $=11 \%$ ) with $76 \%$ preceptors and $24 \%$ faculty responding. Of the preceptors completing the survey, 25\% serve as a preceptor for the APPE Advanced Institutional rotation and $22 \%$ precept the APPE Medicine rotation. Over half the responders $(51 \%)$ believed student pharmacists should perform at level five, upon graduation for each EPA. All survey data have been collected and are being analyzed. Implications: The survey determined faculty and preceptor perceptions of EPA levels of entrustment. Most faculty and preceptors believe student pharmacists should perform at the highest level of entrustment upon graduation. An orientation process to the levels of entrustment may be needed to better define expectations for student assessment.

Exploring Montelukast as a Potential BroadSpectrum Thiol Isomerase Inhibitor. Megan M. Pantos, Western New England University, Christina A. Verbetsky, Western New England University, Sophie Nock, University of Reading, Lisa-Marie Holbrook, Univserity of Reading, Jonathan Gibbins, University of Reading, Daniel R. Kennedy, Western New England University. Objective: Thiol isomerase enzymes have both potential anti-cancer and anti-thrombotic indications. Zafirlukast, a known leukotriene inhibitor, was recently identified as the first FDA approved drug that is a broad-spectrum inhibitor of thiol isomerases. However, zafirlukast is considerably less common than montelukast, an agent which dominates the same class. Therefore, we sought to explore whether montelukast also can inhibit thiol isomerases. Methods: Montelukast was examined for inhibition against PDI, ERp5, ERp57, ERp72, and thioredoxin for broad- 


\section{American Journal of Pharmaceutical Education 2019; 83 (5) Article 7654.}

spectrum thiol isomerase activity using the insulin reductase assay. Inhibition of platelet aggregation was measured by optical densitometry, p-selectin exposure was measured using flow cytometry, while cancer cell growth inhibition was measured by the presto blue assay using A549 lung cancer and HCT colon cancer cell lines. Results: We verified that montelukast was a broadspectrum thiol isomerase inhibitor, blocking the enzymatic activity of all thiol isomerases examined. Montelukast also inhibits platelet aggregation, albeit less potently than zafirlukast. P-selectin exposure, which was inhibited by zafirlukast, was not significantly inhibited by montelukast. Finally, montelukast inhibited cancer cell growth but also less potently than zafirlukast. Implications: Montelukast is a broad-spectrum thiol isomerase inhibitor which has the ability to inhibit platelet aggregation and cancer cell growth. Due to its availability and decreased side effect profile, montelukast is another potential drug that could be used to target aberrant thrombosis and antineoplastic growths. However, its decreased potency for both indications in comparison to zafirlukast, raises questions of whether it can reach similar levels of pre-clinical efficacy.

Exploring the Effect of PDI Inhibition on Cellular Model of Cancer Induced Thrombosis After Chemotherapy. Christina A. Verbetsky, Western New England University, Megan M. Pantos, Western New England University, Lisa-Marie Holbrook, Univserity of Reading, Jonathan Gibbins, University of Reading, Daniel R. Kennedy, Western New England University. Objective: Cancer patients, particularly those receiving systemic chemotherapy, have a significantly increased risk of developing both arterial and venous thrombosis. Thiol isomerase enzymes have both anti-platelet and anti-neoplastic activity, which gives them great potential in targeting cancer induced thrombosis. The goal of this study is to determine whether the PDI inhibitor zafirlukast can decrease activation of the clotting cascade after systemic chemotherapy in various cancer cell lines. Methods: Colon, ovarian, and lung cancer cell lines, all of which are associated with high incidence of cancer induced thrombosis, were selected. Cells were treated with cisplatin and gemcitabine and then tissue factor activity was measured in the presence or absence of zafirlukast by determining the generation of factor Xa. Cancer cell growth after chemotherapeutic treatment with or without zafirlukast was also determined using the presto blue assay. Results: Cell surface tissue factor activity increased after cell lines were treated with chemotherapy. Zafirlukast, as well as a known PDI inhibiting antibody, blocked the production of Factor Xa. Adding zafirlukast to the chemotherapeutic regimen had no detrimental effects to the anti-growth effect of cisplatin and gemcitabine. Implications: In this study, treating cells with cisplatin and gemcitabine lead to increased procoagulant activity, which was attenuated with the PDI inhibitor zafirlukast. Furthermore, zafirlukast had no inhibition or negative effect on chemotherapeutic agents and contributed to decreased factor Xa generation. Taken together, this data indicated that the addition of zafirlukast to a chemotherapeutic regimen may have potential for reducing the incidence of cancer induced thrombosis.

Exploring the Effects of Copper (II) $\alpha$-(N)-Heterocyclic Thiosemicarbazone Complexes on Human DNA Topoisomerase IIß. Jacob M. Keck, Lipscomb University, Jennifer D. Connor, Tennessee Technological University, Xiaohua Jiang, Tennessee Technological University, Edward C. Lisic, Tennessee Technological University, Joseph E. Deweese, Lipscomb University. Objective: Cells utilize type II topoisomerases to alleviate topological strain in DNA associated with replication and transcription and to remove knots and tangles in the DNA. Topoisomerase II is an effective anticancer target, but several clinically used agents display significant off-target toxicities and adverse events. Therefore, it is critical to continue to identify compounds with activity against topoisomerase II. We previously analyzed $\alpha-(\mathrm{N})-$ heterocyclic thiosemicarbazone copper $[\mathrm{Cu}(\mathrm{II})]$ complexes against human topoisomerase II $\alpha$ (Top2A), but humans also express topoisomerase IIß (Top2B), which has distinct functional roles. It is critical to explore whether these compounds also impact Top2B. Methods: We examined two $\alpha-(\mathrm{N})$-heterocyclic thiosemicarbazone copper $[\mathrm{Cu}(\mathrm{II})]$ complexes for activity against Top2B in a purified system. The $\mathrm{Cu}$ (II) complexes, copper(II) acetylpyridine-ethylthiosemicarbazone and copper(II) benzoylpyridine-ethylthiosemicarbazone were examined using plasmid DNA cleavage, relaxation, ligation, enzyme inactivation, and DNA binding assays with Top2B to determine whether these compounds act similarly against both enzymes. Results: The $\mathrm{Cu}$ (II) thiosemicarbazone complexes disrupted the function of Top2B in a way similar to the previously studied Top2A. In particular, Top2B DNA cleavage activity is increased in the presence of these compounds while the relaxation activity is inhibited. Notably, these compounds display the ability to stabilize the N-terminal DNA clamp and to rapidly inactivate the enzyme when the compounds are present before DNA. Implications: Our data show that the $\mathrm{Cu}$ (II) thiosemicarbazone complexes utilize a similar mechanism against both isoforms of the enzyme. Together with our previous results, the evidence supports an interaction with the ATPase domain of Top2A and Top2B outside of the ATP binding pocket. 


\section{American Journal of Pharmaceutical Education 2019; 83 (5) Article 7654.}

Extending the Learning Tree: 4th Year APPE Students Learning Through Teaching During Their Academic Rotation. Andrew A. Yabusaki, Washington State University, Joshua J. Neumiller, Washington State University, Kimberly C. McKeirnan, Washington State University. Objective: To assess whether academic fellow supervised 4th year APPE students can effectively create, lead, and manage a high stakes review session for 2 nd year pharmacotherapy students, and whether the entire experience is perceived beneficially by each tier of the learning tree. Methods: Ten APPE students on their elective academic rotations at Washington State University were assigned to lead biweekly review sessions for competency-based block testing for the PY2 pharmacotherapy course. The APPE students had the same access to material as the PY2 students and were trained using various educational tools. De-identified PY2 student test performance $(n=30)$ from students who attended the review session was tracked, compared to their performance when not attending the review session, the class performance as a whole, and will be analyzed using SPSS to determine significance using a two sample T-test. Additionally, Likert-style surveys will be administered to PY2 students, PY4 APPE students, and the academic fellow to determine perceptions of this activity. Results: Full results are still being gathered, but preliminary results after one semester are promising. 7 out of 10 APPE students have completed their review sessions and have completed their end of rotation surveys. 19 out of 30 PY2 students have completed their surveys on the review session. Every review session group scored higher than the class average with constant turnover in the attendees. Implications: This is a potentially easy to implement intervention that can have broad learning enhancement across multiple branches of the learning tree.

Factors Associated with Social Isolation Among Graduate and Professional Healthcare Students in a Midwestern Public University. Ali Azeez Al-Jumaili, University of Baghdad, Mary E. Ray, The University of Iowa, Jessica M. Coon, The Uiversity of Iowa, Miranda Fullerton, The Uiversity of Iowa. Objective: The study goal was to qualitatively determine factors associated with social isolation in graduate and professional health science students. Methods: The study gathered qualitative data via an online survey including an-open ended question from graduate and professional students in the Colleges of Pharmacy, Dentistry, Medicine, Nursing and Public Health in a Midwestern university during 2017. We examined respondent comments using thematic analysis. The research team cross-checked comments and used an inductive, data-driven analytic methodology following a constructivist paradigm. Themes were con- structed from common trends emerging from student comments. We classified themes according to the source into four levels: Individual, interpersonal, organization (college/program) and community. Results: The survey completion rate by college among potential participants was as follows: Pharmacy (33.7\%), Public Health (17.9\%), Medicine (11\%), Nursing (10.9\%), and Dentistry $(9.8 \%)$. The comments from 115 respondents were reviewed, but 96 (83.5\%) were analyzed (ie, discussed factors relative to social isolation). The student comments revealed seven risk factor themes in four categories: Individual (feeling different from peers), interpersonal (competitive/ exclusionary atmosphere), organization (too busy with coursework/ dual-degree programs/ online program) and community (relocation reduces social support). We noted two protective factors: Organization (activities encouraging socialization) and community (supportive outside group). Implications: This study revealed numerous factors associated with increased risk of social isolation in healthcare students and demonstrates the value of support systems to help students remain engaged with others. Understanding associated factors and designing strategies to reduce student social isolation may enhance the quality and well-being of future health professionals and scientists.

High Risk Opioid Prescribing When Multiple Prescribers Are Involved. James Gregory Berain, Idaho State University, Catherine M. Oliphant, Idaho State University, Thomas G. Wadsworth, Idaho State University, Rylon Hofacer, Idaho State University, Rick Tivis, Idaho State University. Objective: To quantify the number of chronic opioid patients in Idaho who received an overlapping prescription for a benzodiazepine or controlled central nervous system (CNS) depressant. To determine the frequency of these high-risk combinations occurring when multiple prescribers were involved. Methods: This study reviewed all patient profiles reported to the Idaho Prescription Monitoring Program (PMP) from January to December 2017. Patients who received an opioid for $>90$ consecutive days were defined as chronic opioid users. These patients where then reviewed for overlapping benzodiazepine or CNS depressant prescriptions received from multiple prescribers. Results: Of the 301,975 patients in Idaho that filled a controlled substance in 2017, there were 100,916 (33\%) patients that met chronic opioid use criteria. Of the chronic opioid users, 24,520 (24\%) were also prescribed a benzodiazepine or controlled CNS depressant in the same year. Of this population, 10,789 (44\%) had more than one prescriber involved in the high-risk combination(s). Implications: Almost one in four chronic opioid users received a high-risk opioid combination. Of these patients, $44 \%$ received this 


\section{American Journal of Pharmaceutical Education 2019; 83 (5) Article 7654.}

combination when more than one prescriber was involved. The remaining 56\% were written by the same prescriber. It was initially suspected that the large majority of patients would be receiving opioid-benzodiazepine combinations from different prescribers. Although this did occur, a higher percent of patients received these combinations from the same prescriber. These results suggest continued prescriber education on appropriate opioid prescribing is warranted. In addition, it appears pharmacists have many opportunities to facilitate prescriber communication, educate patients on increased overdose risks and offer opioid-antagonists.

Identifying Gaps in Non-Therapeutic Geriatric Pharmacy Competencies in a Pharmacy School Curriculum. Jane Frances Nazareno, University of Washington, Leigh Ann Mike, University of Washington. Objective: The increasing population of older adults emphasizes the need to train pharmacy students in geriatrics to improve patient outcomes. We compared the University of Washington School of Pharmacy's (UWSOP) core didactic curriculum with the American Society of Consultant Pharmacy's (ASCP) Geriatric Curriculum Guide to identify gaps in coverage. Methods: We chose to focus on non-therapeutic topics to be more inclusive of the entire UWSOP curriculum. Non-therapeutic topics defined by the ASCP Curriculum Guide incorporate socioeconomics of aging, geriatric syndromes, and communication and medication-related issues. We audited years 1-3 of UWSOP's didactic core curriculum spanning years 2017-2019. The content reviewed included syllabi, recordings, materials on course websites, personal class notes and handouts, and communication with course instructors. Coverage of each topic was determined by its presence in the course, and the extent or method in which it was included. Results: The majority of non-therapeutic topics included in the UWSOP curriculum are covered but are only mentioned or make up a fraction of an entire lecture - these include deprescribing, continuum of care, weakness/frailty, and advanced directives. Topics not covered include sensory deficits, malnutrition, and elder abuse. Topics such as medication adherence, health literacy, and polypharmacy are covered to a greater extent than the other non-therapeutic topics. Implications: The UWSOP curriculum has gaps in coverage for nontherapeutic geriatric pharmacy competencies. We will work collaboratively with course instructors to identify opportunities to include these topics in the future.

Identifying Pharmacy Students' Career Path Drivers to Better Aid Career Advising and Work Satisfaction. Jennifer Denise Suarez, University of North Texas Health Science Center, Danh Nguyen, University of North Texas Health Science Center, Summer Haj, Uni- versity of North Texas Health Science Center, Fahraj Ahmed, Texas Tech University Health Sciences Center, Krystal L. Edwards, Texas Tech University Health Sciences Center, Randy Martin, University of North Texas Health Science Center. Objective: To identify key drivers that influence pharmacy students in selecting career paths to pursue. Methods: Students from two pharmacy programs completed a voluntary survey in conjunction with a career fair. Survey elements included demographics, career paths being pursued, and career path drivers. Respondents could select multiple career paths and drivers. Career paths included: community, hospital, government, post-graduate education, and other. Drivers included: financial need, pride in work, work/life balance, schedule flexibility, rigor/challenge, location, and prerequisites. Phi coefficient (f) was used to identify and quantify relationships between demographics, drivers, and career paths. Effect size (i.e. relationship strength) was classified as weak $(\mathrm{f}<0.2)$, moderate $(f=0.2-0.5)$, or strong $(f>0.5)$. Results: 156 respondents completed the survey. 13 relationships of moderate effect size and 8 of weak effect size were detected and statistically significant. Moderate drivers for choosing hospital pharmacy included: schedule flexibility $(\mathrm{f}=0.287)$, worklife balance $(f=0.247)$, and rigor/challenge $(f=0.222)$. Moderate drivers of community pharmacy included: financial need/concern $(\mathrm{f}=0.353)$ and $\mathrm{P} 4$ progression $(f=0.207)$. Moderate drivers of post-graduate education included: pride in work $(\mathrm{f}=0.259)$, rigor/challenge $(\mathrm{f}=0.279)$, job outlook $(\mathrm{f}=0.246)$, and self-confidence $(\mathrm{f}=0.249)$. Gender and previous work experience demonstrated weak, but detectable, influence upon select career drivers. Implications: Understanding career path drivers can aid in career advising and placement of pharmacy students, enhancing job satisfaction.

Immunization Training in Pharmacy Schools in the United States. Nicole M. Ondrush, Western New England University, Emma R. Thomas, Western New England University, Sabrina M. Gaffney, Western New England University, Daniel R. Kennedy, Western New England University, Joshua J. Spooner, Western New England University, Courtney Doyle-Campbell, Western New England University. Objective: To determine how immunization training is delivered in pharmacy schools throughout the United States. Methods: An 18-question survey was developed and sent to 145 US pharmacy schools via SurveyMonkey in February 2019. Questions included if immunization training was required within the curriculum, when it occurred, the number of faculty involved, and consequences for not successfully completing training. Immunization coordinator contact information was provided by the American Pharmacists 


\section{American Journal of Pharmaceutical Education 2019; 83 (5) Article 7654.}

Association (APhA); for schools that did not use APhA's training program, their Dean of Academic Affairs (or equivalent) was contacted. Results: The response rate was $43.8 \%$ (64 schools). While all respondents offer immunization training, $3(4.7 \%)$ of these schools do not require it. Nearly all schools (98.4\%) use APhA's materials to train students. The majority of schools $(63.3 \%)$ conduct their training in the first professional year, with fewer schools training students each subsequent academic year (2nd year: $26.7 \%$; 3 rd year: $8.3 \%$; 4 th year: $1.7 \%$ ). Half of respondents indicated that students completed immunization training prior to their first IPPE rotation. Most respondents $(91.8 \%)$ required students to administer 3 simulated immunizations on a partner to complete the training, and $81.7 \%$ indicated that state laws did not limit their ability to conduct training. Implications: This research provides a useful benchmark for pharmacy immunization training in US pharmacy schools. Immunization training is widespread, though how it is operationalized varies. Future research opportunities abound, including student perceptions on training and experience while on IPPE to help identify the most opportune timing for training in relation to IPPE commencement.

Impact of an Elective Mock Exam on First-Year Pharmacy Student Exam Performance and Perceptions of Confidence. Ansley M. Gayle, The University of Georgia, Brittany T. Jackson, The University of Georgia, Michael J. Fulford, The University of Georgia. Objective: To evaluate the impact of an elective mock exam on student performance and perceived confidence prior to the live exam. Methods: Third-year pharmacy students developed a one-hundred question mock exam to assess first-year students' knowledge of the 75 most frequently prescribed medications. The exam measured the ability to match brand or generic name to corresponding dosage forms, doses, contraindications, precautions, pharmacology, adverse effects, and/or patient counseling points. An electronic survey was sent to participants to ascertain their perceived confidence and preparedness at baseline and after completing the mock exam. Deidentified data sets of exam scores and survey responses were obtained. Exam scores between the participants were compared to the scores of students not completing the mock exam. Results: Completion of the mock exam $(n=77)$ was associated with an increased mean score of 5.55 points on the live exam in comparison to students who chose not to participate $(n=69)$. First-year students reported improved self-perceptions of preparedness, confidence, and study strategy after taking the mock exam. Students ranked mock exam format, question content and online administration among the most beneficial in their preparation for the live exam. Implications: An elective mock exam im- proved exam performance and perceptions of confidence for first-year pharmacy students. Hosting a student-led mock exam promoted collaboration between pharmacy students at various points in the curriculum and provided a platform for students to ask questions about study techniques and exam-taking strategies.

Impact of an Interactive Top 300 Drug Game on a Pre-APPE Competency Exam. Amanda A. Clouser, Long Island University, Rebecca Cope, Long Island University, Sara Grossman, Long Island University, Joi YauLin, Long Island University. Objective: To determine the impact of an interactive game modeled after "Who wants to be a millionaire?" on students' Top 300 drug knowledge and performance on a pre-APPE competency exam. Methods: In November 2018 and January 2019, P1-P3 students were invited to participate in an interactive program entitled "Who wants to be a PharmD?" developed by Rho Chi student officers. An anonymous 14 -item survey was distributed to participants following each event to measure the perceived impact of the program. Following administration of a pre-APPE competency exam focused on Top 300 drugs in January 2019, the pass rates of P3 students who did and did not participate in the event were compared using a Chi square test. Mean scores of the groups were compared using a two-tailed t-test. IRB exempt status was received. Results: A total of 154 contestants (69\% P3 students) who participated in one or both of the events completed the survey. Of $\mathrm{P} 3$ respondents, $93 \%$ felt motivated to prepare for the Top 300 drug exam following the activity, and $96 \%$ were able to identify personal areas for improvement. The exam pass rate was greater $(p=0.003)$ for those who participated in the activity (66\%) compared to those who did not participate $(43 \%)$. Participants also had a higher mean score on the exam $(74 \%$ vs. $67 \%$; $<<0.001)$. Implications: P3 students who participated in the interactive program performed better on the Top 300 drug preAPPE competency exam. The data suggest that this activity should be incorporated into co-curricular development planning.

Impact of a Wellness Laboratory on Student Pharmacists' Stress Management. Prajakta H. Waghmare, Purdue University, Chien Yu Huang, Purdue University, Chelsea M. Anderson, Purdue University, Jamie L. Woodyard, Purdue University, Kimberly S. Illingworth Plake, Purdue University. Objective: Nationally, college students are increasingly reporting symptoms of stress, depression and anxiety. The primary objective of this study was to assess the impact of a required wellness laboratory on student pharmacists' ability to cope with stress. Methods: First, second, and third year student pharmacists' $(\mathrm{N}=381)$ participated in a wellness 


\section{American Journal of Pharmaceutical Education 2019; 83 (5) Article 7654.}

laboratory focused on identifying and responding to symptoms of depression and anxiety, as well as specific stressors. A 10-item survey was utilized prior to and after the laboratory to assess students' comfort level in discussing wellness topics as well as addressing symptoms of stress, depression, and anxiety. Eight additional items were included on the post-survey to assess students' intention to adhere to SMART wellness goals and overall feedback on the laboratory. A five-point Likert scale was utilized for both surveys. Descriptive statistics were performed on all items and paired t-tests were used to identify differences in pre- and post-responses. Open-ended items were coded by two researchers independently and themes were generated. Results: There were statistically significant differences in the pre- and post- responses for all items $(p<0.01)$. Students' perceptions of their comfort in managing their stress improved after participating in the laboratory. In qualitative analysis, students indicated that the laboratory assisted in identification of specific stressors, means of dealing with stressors, and methods of helping others with stress. Implications: A wellness laboratory was positively received by students. Laboratory content assisted students in identifying stressors and developing methods to cope with them.

Impact of Class Attendance on Exam Performance in an Active-Learning Classroom Model. Ashley T. Ta, Washington State University, Anne Kim, Washington State University, Joshua J. Neumiller, Washington State University, Connie M. Remsberg, Washington State University, David Gothard. Objective: To examine the relationship between class attendance and exam performance within an active-learning classroom model. Methods: Second-year students enrolled in the pharmacotherapy course series were included in this study $(n=160)$. Class attendance was taken manually by members of the study team over a one-year study period (Fall 2017 and Spring 2018 semesters). Attendance was not required and had no impact on student grades. Scores from the six competency-based exams in each semester and overall course grades were linked to class attendance records. Results: Class attendance was collected over 48 class sessions. Of the six assessments given each semester, students during the fall course required approximately one retake during the semester, with an increase to 1.5 retakes on average in the spring course. There was a significant negative correlation found indicating that missing more classes was related to a lower final course percentage for both courses $(p<0.001)$. Further regression analysis predicted a final course grade of $90.78 \%$ in the fall course and $88.25 \%$ in the spring course if no classes were missed. For each missed class session, there was a reduction in overall course grade by $0.138 \%$ and $0.140 \%$ in the fall and spring courses, respectively. Implications: A positive relationship between class attendance and assessment scores for both semesters indicate attending class in an active-learning curriculum may be essential for students to succeed in learning, developing, and applying pharmacotherapy concepts. The results of this study illustrate the importance of attending active-learning sessions to attain higher assessment scores.

Impact of CliftonStrengths ${ }^{\circledR}$ Assessment on Team Construction and Dynamics in Second-Year Pharmacy Students. Apryl N. Anderson, Virginia Commonwealth University, Julie A. Patterson, Virginia Commonwealth University, Krista L. Donohoe, Virginia Commonwealth University, Laura M. Frankart, Virginia Commonwealth University, Abigale T. Matulewicz, Virginia Commonwealth University, Emily P. Peron, Virginia Commonwealth University, Kacie Powers, Virginia Commonwealth University, Lauren M. Caldas, Virginia Commonwealth University. Objective: This study aims to assess team construction using CliftonStrengths ${ }^{\circledR}$ domains on (1) peer evaluations, (2) team dynamics, and (3) performance on team-based learning (TBL) activities. Methods: Students were randomized into teams $(n=21)$ during the fall semester. Teams were constructed in the spring using CliftonStrengths ${ }^{\circledR}$ Top 5 Strengths, stratifying students to represent at least one of the four domains per group. Peer evaluations were assessed with modified Koles method, student-reported team dynamics with Macke's "Feelings about Teams" survey, and teams' performance via RATs. Changes in overall peer evaluation and RAT scores will be assessed using Wilcoxon signed-rank tests. McNemar's test will be used to analyze changes of positive responses in peer evaluations and Macke's survey items. Results: Top 5 Strengths $(n=610)$ demonstrated 38\% relationship building domain, $36.2 \%$ executing, $19.7 \%$ strategic thinking, and $6.1 \%$ influencing. In the fall, students $(n=122)$ scored 33.6 of 36 points $(93.4 \%+2.24)$ on the peer evaluation. The fall semester Macke's responses $(n=64)$ will be compared to spring semester responses on a per question basis. Fall students' average performance was $86.2 \%$ for iRATs, $98.6 \%$ for tRATs, and 0.87 on iRAT:tRAT ratio. Final data collection and analysis will be completed at the conclusion of the spring semester in April 2019. Implications: Pharmacy education relies heavily on TBL with limited consideration on team construction. Strategic team construction could improve team dynamics and student performance on TBL. Final results and conclusions of this study are expected in April 2019.

Impact of Former College Athletic Participation on Student Pharmacists Wellbeing and Burnout Rates. Chadarryl L. Clay, Virginia Commonwealth 


\section{American Journal of Pharmaceutical Education 2019; 83 (5) Article 7654.}

University, Lauren Pamulapati, Virginia Commonwealth University, Julie A. Patterson, Virginia Commonwealth University, Taryn A. Hayes, Virginia Commonwealth University, Kelechi C. Unegbu-Ogbonna, Virginia Commonwealth University, Benjamin W. Van Tassell, Virginia Commonwealth University, Lauren M. Caldas, Virginia Commonwealth University. Objective: (1) Assess pharmacy student's burnout and wellbeing through the Oldenburg Burnout Inventory (OLBI)-student questionnaire and Warwick-Edinburgh Mental Wellbeing Scale (WEMWBS), respectively, comparing prior student athletes to their peers and (2) determine a difference between length of involvement, level of activity, and type of sport on burnout and wellbeing. Methods: This prospective survey-based study will recruit student pharmacists for 5 weeks via email. Demographic data will be collected, including type of sport, level of activity (i.e., intramural, club, or collegiate), and length of involvement. Students will self-report burnout and wellness ratings. Descriptive statistics will be used to summarize student characteristics and scores on the OLBI exhaustion scale and WEMWBS. Univariate and multiple linear regression will be used to describe associations between student characteristics and exhaustion and mental wellbeing. Results: The project is in the data collection phase with expected results by April 2019. Implications: No current studies examine the relationship between prior participation in athletics with wellness and burnout rates in pharmacy students. However, studies have shown that students who have participated in competitive sports before entering medical school experience lower burn-out rates compared to non-student athletes. Higher levels of involvement in sports resulted in a lower academic burnout. If this study shows that prior student athletes have improved wellness and lower burnout rates, schools of pharmacy may be able to apply this information in admissions efforts by more heavily weighting prior athletic involvement in the application review process.

Impact of Pharmacy Student Teaching Assistant Positions on Career Aspirations and Involvement in Student Learning. Kenny Nguyen, The University of Texas at Austin, Ashley Dinh, The University of Texas at Austin, David V. Giang, The University of Texas at Austin, Victoria Vuong, The University of Texas at Austin, Kristin Marie Janzen, The University of Texas at Austin, Claire Latiolais, The University of Texas at Austin. Objective: Teaching and mentoring is a central motif in the field of pharmacy. Whether in a formal setting like a classroom or assuming a preceptorship or mentorship role for students, preparing pharmacy students for these roles is essential in advancing the profession and next generation of pharmacists. However, there is limited research examining the role of a student academic position and its influence on graduates pursuing academic pharmacy or becoming a certified preceptor. This study analyzes the relationship between previous and current pharmacy students in academic positions and their current or future role in pharmacy. Methods: Qualtrics ${ }^{\circledR}$ surveys were sent to graduates and current pharmacy students, respectively, who served in academic positions from 2013 to 2018 at the University of Texas at Austin College of Pharmacy. Both qualitative and quantitative data were gathered regarding their current or future interests in pursuing an academic-related career before and after being in an academic position. The survey was open for a three-week period and following data collection, we assessed the data and relationship between having teaching experience in pharmacy school and post-graduate involvement as a mentor, preceptor, or in academia. Results: Work in progress. Implications: Depending on obtained results and analysis, it would be advantageous for pharmacy schools to consider implementing academic teaching positions for students. This study aims to show that having these experiences available will help pharmacy students eventually pursue an academic-related career.

Impact of Student- Versus Instructor-Led Learning on Student Pharmacists' Performance in a SelfCare Module. Heather L. Walser, Idaho State University, Kevin W. Cleveland, Idaho State University, Rebekah Dunkley, Idaho State University. Objective: To evaluate the impact of student-led versus instructor-led self-care module facilitation on student pharmacists' ability to identify appropriate over-the-counter recommendations and counseling technique. Methods: A retrospective cohort study of first-year professional student pharmacists who completed the self-care module during an introductory course in the Fall semesters of 2017 and 2018. The 2017 Fall semester involved student-led presentations for 15 common self-care topics such as heartburn, cough/cold remedies, insomnia, etc. The 2018 Fall semester cohort was faculty-led presentations covering the same self-care subjects. Students then completed a case-based patient counseling session by identifying an appropriate overthe-counter recommendation to treat the patients presenting symptom. Evaluations utilized a 10-item rubric on students' ability to identify the appropriate recommendation and counseling technique (discussed in an earlier section of the same course). The impact of the module on student performance was compared between studentled and faculty-led cohorts. Results: 164 pharmacy students completed the self-care module in the Fall 2017 and Fall 2018 semesters. A chi-squared analysis will be utilized to compare evaluation data. Approval granted by the Idaho State University Institutional Review Board. 


\section{American Journal of Pharmaceutical Education 2019; 83 (5) Article 7654.}

Implications: This study will provide insight on the impact of student-led versus instructor-led facilitation of material in the self-care module. Knowledge gained from this study could apply to any course that employs traditional classroom learning modules or an educational program that aims to develop clinical application skills. Limitations of this study include year-to-year variability of students and rubric evaluators.

Implementation of a Grant-Funded Medicare Call Center and a Medicare Elective: Estimated Cost Savings for Patients. Natalie Peng, Roseman University of Health Sciences, C. Leiana Oswald, Roseman University of Health Sciences. Objective: Evaluate the cost savings between the Medicare Call Center (MCC) as a grantfunded program and as a non-funded Medicare elective course. Methods: The on-campus MCC began in July of 2016 through a $\$ 50,000$ grant awarded from Noble Cause. Students volunteered to become certified Medicare counselors through a partnership with the Nevada State Health Insurance Program (SHIP). During the grant funding period, two Nevada SHIP counselors were compensated to oversee day-to-day operations of the MCC. The student volunteer Call Center transitioned to a for-credit elective APPE course in March of 2017 that was overseen by a faculty member receiving no additional compensation. Within the MCC, areas of cost savings were calculated on an annual basis, for example: callers are screened for federal and state assistance programs like Medicaid and upon determination of qualification, the Part B premium of $\$ 134$ a month would yield an annual savings of $\$ 1,608$. A comparison of the cost savings from July 2016-February 2017 to March 2017-February 2019 was completed. Results: Cost savings documented from July of 2016 to February of 2017 (7 months) as a grant-funded volunteer call center estimated to be at $\$ 522,491$ (approximately $\$ 74,641 /$ month). Cost savings from March 2017 to February 2019 (24 months) as an APPE elective course estimated to be $\$ 564,086$ (approximately $\$ 23,503 /$ month). Implications: Student pharmacists who are well educated in Medicare can have a positive economic impact on beneficiaries. Institutions seeking to start an on-campus MCC may see larger reported cost savings when a dedicated individual is compensated to oversee the project.

Implementation of Journal Club Series for Second Year Pharmacy Students. Ashley Perkins, Marshall University, Brittany L. Riley, Marshall University, Angel M. Kimble, Marshall University. Objective: Not much data exists on how to best teach journal club skills. A journal club series was implemented into two professional year 2 courses with the first activity in the Fall semester. The purpose of this study was to determined student confidence after the first journal club activity. Data will be used from this study to develop the second session of the journal club series. Methods: A 13 question survey was administered to $\mathrm{P} 2$ students after completion of the first part of the series. The questions were all Likert Type questions that assessed the students perceived confidence in the journal club process. The questions were a mix of confidence before, during and after the journal club activity. The before questions asked the students to retrospectively rate their confidence. Results: Approximately $42 \%$ (27) of the P2 class completed the survey. Prior to the first session $22 \%$ of students ranked themselves as novice and $65 \%$ of students ranked themselves as developing. After the first session 4\% of students ranked themselves as novice, $22 \%$ as developing and $57 \%$ as skilled. The majority of the respondents strongly agreed or agreed $(91 \%)$ that having the class on the journal club process increased their confidence in performing a journal club. Implications: A basic introduction to journal clubs for second professional year students can improve their confidence in preparing journal clubs. Information from this survey will help refine the second journal club series activity.

Implicit Professional Bias in Interprofessional Peer Evaluations. Karen Juco, Rosalind Franklin University of Medicine and Science, Scott D. Hanes, Rosalind Franklin University of Medicine and Science, Ziemowit Tomasz Mazur, Rosalind Franklin University of Medicine and Science, Steven A. Miller, Rosalind Franklin University of Medicine and Science. Objective: To determine if profession-based bias exists in peer evaluations of an interprofessional (IP) course between pharmacy $(\mathrm{COP}, \mathrm{n}=69)$ and physician assistant $(\mathrm{PA}, \mathrm{n}=63)$ students. Methods: Students enrolled in an IP course completed anonymous Likert-scale peer evaluations for group members, which rated their peers' effectiveness while working on case-based issues throughout the quarter. Multilevel models were used to determine if there were interactions between evaluator and target school or gender. Results: In the evaluation of a PA student, PA evaluators and COP evaluators gave similar scores. In the evaluation of COP students, PA evaluators gave lower scores than COP evaluators $(\mathrm{p}<0.001)$. Gender was not found to be an influence in peer evaluation scores. Implications: From an analysis of peer evaluation scores in an IP course with COP and PA students, PA evaluators scored PA students higher than they scored COP students, while no such difference was apparent in COP evaluators, indicating a potential professional bias in the PA students. This bias may be due to the difference in IP exposure in a first-year PA student compared to a third-year COP student. Future studies should observe the scores of peer 


\section{American Journal of Pharmaceutical Education 2019; 83 (5) Article 7654.}

evaluations over the course of an academic year to determine if how a student's opinion of his or her IP group changes over time. Additionally, other professions should be examined to determine if profession-based biases are present in other healthcare providers or just a select few.

Increasing Diversity: Impact of an Intensive Summer Pipeline Program for Pre-Pharmacy Students. Amanda M. VanInwegen, Virginia Commonwealth University, Rollin L. Ballentine, Virginia Commonwealth University, Krista L. Donohoe, Virginia Commonwealth University, Taryn A. Hayes, Virginia Commonwealth University, Kelechi C. Unegbu-Ogbonna, Virginia Commonwealth University, Lauren Pamulapati, Virginia Commonwealth University, Lauren M. Caldas, Virginia Commonwealth University. Objective: To assess the impact of the Summer Academic Enrichment Program (SAEP) on diversity at Virginia Commonwealth University (VCU) School of Pharmacy. Methods: This is a retrospective analysis of the diversity goal of SAEP, a six-week summer residential program that simulates the first-year of dentistry, medicine, pharmacy, or physical therapy professional school instruction. Matriculation and demographic data of previous SAEP participants (from 2012 to 2018) and pharmacy students was obtained via program and school administration. Results: Of the 68 pharmacy-track participants, $57.4 \%(n=39)$ have enrolled at VCU School of Pharmacy to date. Of those who matriculated, $33.3 \%(n=13)$ represented one or more of the following underrepresented minority (URM) backgrounds: $28 \%$ identified as Black or African American, $5.1 \%$ as Hispanic or Latino, and $2.6 \%$ as American Indian. Comparatively, the same URM classifications for students admitted to VCU School of Pharmacy over the last seven years (Class of 2016 - Class of 2022) ranged from 5-11\% Black or African American, 0-5\% Hispanic or Latino, and $0-0.1 \%$ American Indian with a total URM representation range of $7 \%$ to $13 \%$ over this time period. Implications: Programs focused on increasing students from underrepresented backgrounds, like SAEP, may be used to enhance diversity in pharmacy schools. While there are several efforts at the university focused on increasing and retaining URM populations, SAEP has demonstrated a direct impact on matriculating students from underrepresented backgrounds.

Inhibition of DNA Methyltransferase Activity Enhances IgE-Mediated Mast Cell Functions. Kaitlin J. Armstrong, Western New England University, Edwin B. Kaczenski, Western New England University, Dylan T. Krajewski, Western New England University, Shannon Kinney, Western New England University, Clinton Mathias, Western New England University. Objective: Mast cells play a critical role during allergic responses.
We have previously shown that epigenetic modulation of mast cell function via inhibition of histone deacetylation results in suppression of mast cell-mediated effects during food allergy. We therefore hypothesized that other epigenetic processes such as DNA methylation may have similar effects on mast cells and influence its functions during allergic responses. Methods: 5-Aza-2'-deoxycytidine (DAC) is a DNA methyltransferase inhibitor that induces gene activation through hypomethylation and chromatin remodeling. To examine the effects of DAC on mast cells, IgE-activated bone marrow-derived mast cells (BMMCs) were treated with DAC and their functions were examined. Similarly, the effects of DAC were also examined in vivo using an ovalbumin (OVA)-induced model of food allergy in mice. Results: IgE-induced activation of BMMCs resulted in enhanced expression and secretion of IL-4, IL-6, TNF and IL-13. Interestingly, DAC pretreatment further enhanced the expression and secretion of these cytokines. In contrast, treatment of wild-type mice with DAC in a model of food allergy resulted in a significant attenuation in the development of food allergy symptoms including decreased mast cell activation and allergen-specific IgE. Implications: Our data suggest that inhibition of DNA methylation has the potential to enhance mast cell activation during allergic responses. However, systemic hypomethylation in vivo may turn on gene activation in multiple immune cells including $\mathrm{T}$ cells that can counteract the mast cell-dependent effects. Further studies are needed to examine the effects of DNA hypomethylation on specific cell subtypes and to determine the mast cell-specific effects during allergic responses.

Interprofessional Education with Medical Students: Meeting the ACPE Standards. Victoria L. Freniere, Western New England University, Melissa Mattison, Western New England University, Beth E. Welch, Western New England University. Objective: To determine the amount and extent each school or college of pharmacy was able to include medical students in their interprofessional education (IPE) in the pre-APPE curriculum. A second objective was to identify, barriers in meeting the updated standards. Methods: A survey was sent via email to accredited colleges and schools of pharmacies in the US; Deans or faculty who have a focus on IPE were selected to receive the survey. The survey included 16 questions pertaining to demographics, and the amount and type of interprofessional education offered at each institution with a focus on medical student inclusion. Results: Initial response rates are $21 \%$; however, survey results are still being collected. The demographics showed that schools from all eight NABP districts were represented; $41.38 \%$ public and $58.62 \%$ private 


\section{American Journal of Pharmaceutical Education 2019; 83 (5) Article 7654.}

institutions. Overall, $82.14 \%$ of institutions include medical students in their pre-APPE curriculum, with $48.28 \%$ of institutions having one interaction per year, $75 \%$ utilize simulations, and $6.9 \%$ of institutions had none/unknown interprofessional education with medical students. Implications: Currently, not all schools are meeting the ACPE updated standards in regard to inclusion of IPE with medical students in the pre-APPE curriculum, and of those institutions who did include medical students, many of them only included one interprofessional education session per year. Results also showed more interactions per year with other professions than medical students. Using the comment boxes provided, institutions cited barriers, which included distance, planning, and scheduling.

Interviews to Assess Older Adults' Preferences for Features of Community-Based Fall Prevention Programs. Natalie Hohmann, Auburn University, Jingjing Qian, Auburn University, Salisa C. Westrick, Auburn University, Caralise Hunt, Auburn University, Ana L. Hincapie, University of Cincinnati, Kimberly B. Garza, Auburn University. Objective: Falls in older adults are a major public health problem. Although evidence-based fall prevention programs exist in community settings, their utilization is low. We investigated older adults' preferences for features of community-based fall prevention (CFP) programs. Incorporating these preferences into existing program designs may help improve program engagement and adherence. Methods: We interviewed older adults ( $\geq 65$ years), caregivers, and fall prevention experts to gather their opinions on the most important features of exercise-based CFP programs, and preferences for frequency and cost of program sessions. Older adults/caregivers were recruited at an outpatient cardiac rehabilitation clinic for face-to-face interviews. A fall prevention expert was purposively recruited from a fall prevention clinic for telephonic interview. The investigators took written field notes during the interviews, which were coded by hand by two investigators and assessed using qualitative content analysis. Results: Saturation was reached after six interviews $(n=5$ older adults/caregivers, $n=1$ expert). Key elements for CFP programs included social interaction and improving confidence in self-management skills. Preferred frequency of program sessions ranged from one to five times per week and varied with program location and transportation needs; however, expert views stressed that sessions should ideally be attended three times per week to meet physical activity guidelines. Older adults' willingness to pay for a CFP program ranged from $\$ 0$ to $\$ 150$ per month. Implications: Results were used to develop a survey to investigate preferences for CFP program features in a larger sample. Ultimately, designing CFP programs in a patient-centered manner may improve older adults' program engagement, adherence, and outcomes.

Investigating the Role of Statin Therapy in the Reduction of Gentamicin-Induced Nephrotoxicity. Dan D. Arendt, Northeast Ohio Medical University, Mate Soric, Northeast Ohio Medical University. Objective: The primary objective of this study is to determine the overall impact of statin therapy on the mean change of serum creatinine in adult patients receiving gentamicin for at least 4 days. Methods: This study will compare the mean change in serum creatinine from baseline to peak in patients receiving gentamicin who are treated with concurrent statin therapy to those without statin exposure. This will be a retrospective cohort study including patients 18 years or older who received gentamicin for at least 4 days. Patients will be excluded from the trial due to Stage 4 or higher CKD, pregnancy, baseline hypotension, dehydration or acute kidney injury. This trial will require a sample size of 39 patients in each group to provide $80 \%$ power and an alpha value of 0.05 . Unpaired t-tests will be used for continuous variables and the chi-squared test will be used to evaluate dichotomous variables. Results: The trial is currently in the data collection stage, results will be presented at the AACP conference. Implications: Aminoglycosides have been shown to cause high rates of nephrotoxicity, predominantly related to uptake in the proximal tubule of the nephron via the multi-ligand receptor megalin. Statin therapy has been shown to decrease receptormediated endocytosis by megalin via inhibition of HMGCoA reductase. This nephrotoxicity has been shown to be reduced by statin use in animal models but has yet to be properly evaluated in human subjects. Statins may represent a safe and cost-effective method of preventing the nephrotoxicity of aminoglycosides in at risk patients.

Knowledge, Beliefs, and Attitudes of UMKC School of Pharmacy Students and Faculty Regarding Cannabis/Cannabinoids. Matthew X. Laing, University of Missouri-Kansas City, Cydney E. McQueen, University of Missouri-Kansas City. Objective: To gather and compare student and faculty attitudes and beliefs regarding cannabis and cannabinoid medicines. Knowledge of Missouri laws and regulations regarding cannabis was also assessed. This study also gathered opinions about whether or not to include additional education on cannabis in future curriculum at the UMKC School of Pharmacy. The results gathered were compared to a similar study performed at UMKC in 2016. Methods: Students and faculty were given the opportunity to complete an online survey created via REDCapTM. Topics covered included: general information; law; knowledge, beliefs and attitudes; relative risk; and additional/miscellaneous. Results: Students and faculty were asked about 


\section{American Journal of Pharmaceutical Education 2019; 83 (5) Article 7654.}

increasing academic coverage of cannabis and cannabinoid medicines and 4 out of 5 respondents were in favor of increasing coverage. Regarding the legal status of cannabinoid medicines, one-hundred and fifty students $(77.1 \%)$ answered incorrectly about the legality of cannabis in Missouri. Implications: There is a lack of knowledge regarding cannabis/cannabinoid medicines and an interest in adding additional cannabis/cannabinoid medicines coverage to future curriculum at the UMKC School of Pharmacy.

Knowledge Comparison in a Community Pharmacy Lab Between Students with and Without Prior Technician Experience. Tyler Marie Kiles, University of Houston, Alexa L. Zhao, University of Houston, Matthew A. Wanat, University of Houston, Catherine L. Hatfield, University of Houston. Objective: It is unknown if students with previous pharmacy technician experience benefit from the community pharmacy dispensing lab. Past course evaluations have indicated that students with previous technician experience did not feel a substantial benefit from the course. The purpose of this project was to evaluate perceptions, knowledge, and academic performance of those with and without prior technician experience in a re-designed, integrated community lab course. Methods: Pharm.D. students enrolled in a community pharmacy lab course were included in the study. All students were administered a pre-course survey (20 multiple-choice questions) and knowledge assessment (20 scenario-based questions), to be followed by a postcourse survey and knowledge assessment. Select survey variables to be analyzed include length of experience, confidence with community pharmacy practice, and course expectations. The knowledge assessment evaluates understanding of community pharmacy law, workflow, inventory, insurance, and prescription verification. Results were analyzed using descriptive statistics. Results: A total of 233 students (response rate $90 \%$ ) were included in the analysis. Students with previous technician experience scored higher on the pre-course assessment than students without experience (P1's, $\mathrm{n}=122$ : $53 \%$ vs. $25 \%, \mathrm{p}<0.0001$; P2's, $\mathrm{n}=111: 59 \%$ vs $45 \%$, $\mathrm{p}<0.0001)$. Future analyses will look at student perceptions and knowledge gained after completing the course. Implications: There is a baseline knowledge gap between students with technician experience and those without. Results of this study will identify specific areas that should be emphasized in the new curriculum. This study may provide data for discussion of initiation of separate tracks for experienced and non-experienced students.

Leveraging Student Leader Involvement in Creating and Administering a Co-Curricular Program. Kevin T. Nguyen, University of California, San Diego,
Arianna Choi, University of California, San Diego, Linda Dao, University of California, San Diego, Sarah M. Lorentz, University of California, San Diego, David Bao, University of California, San Diego, Anjan Debnath, University of California, San Diego, Irina Kufareva, University of California, San Diego, Renu F. Singh, University of California, San Diego. Objective: To incorporate student leadership into the implementation of a new co-curricular program Methods: To fulfill 2016 ACPE accreditation requirements, our school developed a cocurricular program that enhances student learning beyond the classroom. The program requires P1-P3 students to participate in and reflect upon professional, community, and networking events. Program administration is overseen by a faculty committee while student leaders assist with defining program requirements and serve as liaisons between faculty and student organizations. Alongside faculty, student leaders established a cloud-based platform, the Student Organization Weekly Newsletter (SOWN) for event sharing and attendance tracking and provided fellow students with programmatic information and updates. In addition, an approval process for new student-initiated events was developed. Results: The co-curricular event list contains approximately 400 events classified into 35 event types, each assigned to one or more of four co-curricular domains: (1) Leadership \& Innovation/Entrepreneurship, (2) Cultural Understanding, Community Outreach, \& Service, (3) Personal Development \& Wellness, and (4) Professionalism \& Career Development. At the initiation of the program, events from previous years were assigned to specific domains. During the first 6 months of the program, 85 new event requests were approved. Twelve student organizations and affiliated student groups documented events on the SOWN. This approach enabled faster buy-in from the student body, took advantage of the students' technical skills, and enhanced the program's efficiency by reducing workload for the faculty. Implications: Student leadership involvement proved a productive strategy in establishing a new co-curricular program and may be adopted by other schools of pharmacy.

Managing Medication Error Disclosures in Community Pharmacy. Ali Lois Icenogle, University of Washington, Karan N. Dawson, University of Washington, Brilliana Hou, University of Washington. Objective: The objectives of this qualitative study are to identify what pharmacists consider effective medication disclosure, learn what resources are available to pharmacists regarding medication error disclosure, and use study results to enhance a current teaching module on medication error. Methods: A purposeful sample of 20 pharmacists with experience working in community practice, as 


\section{American Journal of Pharmaceutical Education 2019; 83 (5) Article 7654.}

educators, and for pharmacy professional organizations are selected for interview. Interviews are conducted using a semi structured interview guide that consists of openended questions relating to how medication errors are disclosed, what training pharmacists receive regarding error disclosure, and how pharmacists can best prepare for medication error disclosure according to the interviewees. Interviews are audio recorded and transcribed via a mobile application and through note taking. The lead investigators will analyze interview transcripts for common themes relating to medication error disclosure and, through an iterative process, devise a set of coding rules to classify the data into these themes. Two auditors will independently apply the coding rules to the transcript sections to check the dependability of the coding system. Recommendations for best practices for medication error disclosure will be reported, and gaps in disclosure that may relate to legal and professional support may be identified. Results: Results pending Implications: Study results will be used to enhance a current teaching module on error disclosure and as a basis for study authors to help train pharmacy students and practicing pharmacists regarding error disclosure. In addition, gaps in medication error disclosure practices and/or training may emerge to stimulate future research and program development.

Mentorship in Pharmacy Education: Student Perceptions of Value, Preferences, and Likelihood to Engage. Ashley S. Crumby, The University of Mississippi, Alicia S. Bouldin, The University of Mississippi, Sujith Ramachandran, The University of Mississippi, Jennifer L. Adams, Idaho State University, Chelsea W. Bennett, The University of Mississippi. Objective: The objective of this study is to measure the value of mentorship among student pharmacists by examining the importance of mentorship functions and preferences for the relationship. The study also assesses how perceived personal relevance and valuation influence the likelihood that students will engage in a mentoring relationship. Methods: Data collection involves self-report via an online survey among student pharmacists (P1-P4) at four participating universities. To measure the value of mentorship, an adapted version of the 29-item Mentoring Functions Scale will be used which evaluates both career and psychosocial functions. Perceived personal relevance will be measured using the Revised Personal Involvement Inventory Scale. Preferences for the structure of the mentoring relationship will be elicited using a discrete choice experiment which includes evaluation of twelve pairs of "promotional program descriptions" for various mentoring programs. For each pair, students will be asked to identify which program they prefer. Results: Work in progress. Implications: Results from this study will help to identify the level of value students attribute to mentorship and the aspects of the mentoring relationship students prefer. This could allow for the creation of mentorship programs, whether formal or informal, that would be valued by students and utilized to their fullest potential, resulting in positive outcomes for all involved. This research could potentially seed targeted mentorship development education, to better train mentors in the initiation and cultivation roles.

Model of Interprofessional Education in Underserved Populations. Patrick Haverty Lee, The University of Rhode Island, Kelly L. Matson, The University of Rhode Island. Objective: To demonstrate the University of Rhode Island Academic Healthcare Collaborative (-URI AHC) Jamaica Mission Trip may serve as a model of interprofessional practice in an international mission trip setting for other colleges of pharmacy Accreditation Council for Pharmacy Education (ACPE) 2016 Standards and Center for the Advancement of Pharmacy Education (CAPE) 2013 Outcomes. Methods: Twenty-two pharmacy, nursing, and physical therapy students who voluntarily participated in the 2019 URI AHC Jamaica Mission Trip were offered an anonymous, voluntary post-trip survey comprised of 5-point Likert-scaled statements. Survey statements were developed to elicit responses from mission experiences based on practice essentials/approach and interprofessional education from current CAPE outcomes and ACPE Standards. Results: Majority of all students surveyed responded favorably that the 2019 Jamaica Mission Trip met CAPE outcomes of health and wellness promotion $(89.5 \%)$, population-based care (94.7\%), education (94.7\%), interprofessional collaboration $(94.7 \%)$, and communication $(87.7 \%)$. Furthermore, URI AHC students perceived the interprofessional global mission met ACPE Standards of health and wellness (84.2\%), interprofessional collaboration $(94.7 \%)$, cultural sensitivity $(100 \%)$, communication $(100 \%)$, interprofessional team dynamics $(94.7 \%)$, interprofessional team education $(94.7 \%)$, and interprofessional team practice (89.5\%). Implications: Students perceived their participation in URI AHC Jamaica Mission Trip met CAPE outcomes and ACPE Standards centered around communication, cultural competency, team-building, and leadership. Study limitations may include selection and observer biases based on survey participants included and specific statements assessed, respectively. This trip may serve as a model of a global interprofessional mission trip for other colleges of pharmacy to meet CAPE outcomes and ACPE Standards.

Observational Review of Leukopenia in PostKidney Transplant Recipients. Ngoc-yen Pham, The University of New Mexico, Amanda J. Condon, The 


\section{American Journal of Pharmaceutical Education 2019; 83 (5) Article 7654.}

University of New Mexico, Melanie A. Dodd, The University of New Mexico. Objective: The University of New Mexico Health System has the longest running kidney transplant program in the state since 1977. As of August 2018 , there are 218 patients awaiting kidney transplant at UNMH, with $77 \%$ are of Native American or Hispanic descent. While our patient population is mainly comprised of Hispanics and Native Americans, this population remains largely underrepresented in transplant literature. Anecdotally, this population appears more sensitive to leukopenia-inducing medications. The purpose of this study is to describe the incidence and complications of leukopenia in kidney transplant recipients of Hispanic, Native American, and non-Hispanic or non-Native American descent. Methods: This will be a single-center, retrospective, study conducted at the UNMH.Patients 18 years or older who received a kidney transplant at UNMH between January 1, 2006 and September 30, 2017 will be included in the study. The UNMH transplant database and electronic medical record will be utilized for data collection. All data will be recorded without patient identifiers and maintained confidentially. The primary outcome will be to describe the incidence of leukopenia among the prespecified populations. Secondary outcomes include evaluation of time to leukopenia, treatment of leukopenia, and one-year graft outcomes. Data will be analyzed using chisquare test and analysis of variance (ANOVA). Results: The results of this study are still in progress. Implications: By exploring the rate of leukopenia in patients of Native American and Hispanic descent, immunosuppressive therapies could be tailored to improve safety and efficacy in this patient population.

Ohio Pharmacists' Knowledge and Utilization of the Patient Care Process. Abagail Agler, University Hospitals Geauga Medical Center, Mate Soric, Northeast Ohio Medical University, Lukas J. Everly, Northeast Ohio Medical University. Objective: The Joint Commission of Pharmacy Practitioners adopted the Pharmacists' Patient Care Process (PPCP) in 2014 to define expectations of pharmacists. It is unknown if pharmacists are aware of these expectations and how it is being used across the profession. The primary objective of this study is to determine the proportion of Ohio pharmacists who are familiar with and utilize the PPCP. Methods: A validated survey was emailed to all licensed pharmacists in Ohio. Pharmacists who spend less than $20 \%$ of time per week in direct patient care were excluded from the study. The survey was designed to assess a pharmacist's usage of the PPCP in a hypothetical patient scenario. Case vignettes assessed a pharmacist's process for care delivery. Data regarding practice setting, precepting roles, and pharmacy training was also collected. Results: Of the
812 responders who met inclusion criteria, 698 completed all components of the survey. The majority held a Doctor of Pharmacy degree (49\%), were out of school five years or longer $(64.9 \%)$, precepted students $(60.9 \%)$, and practiced in a community setting (39.0\%). While $62.8 \%$ selfidentified as having good knowledge of the PPCP, 20.2\% ranked their knowledge as none or poor. Only $17 \%$ ranked their knowledge as excellent. Only $48.2 \%$ scored $4 / 5$ or better when applying the PPCP to the hypothetical case. Implications: As Ohio pharmacists just received provider status, use and evaluation of the PPCP is critical. Due to the rate of self-identified lack of knowledge, education on and further understanding of how best to apply the PPCP is necessary.

Peer Health Educators on Campus: Teaching Safe Medication Practices. Olga O. Vlashyn, Purdue University, Baylee Q. Bryan, Purdue University, Murphy K. O'Toole, Purdue University, Stephanie A. Deremiah, Purdue University, Jane E. Krause, Purdue University. Objective: Objectives included: (1) to assess the impact of a prescription drug abuse/ misuse peer education program ("Safe Medication Practices") on knowledge of University students; (2) to obtain feedback on the presentation; and (3) to obtain reflections from the peer educators on the experience. Methods: A 30-minute educational presentation was developed and offered to student organizations during the 2018 spring semester. Student attendees completed an anonymous knowledge assessment containing ten multiple choice items prior to and immediately following the presentation. The postassessment also included items that gathered feedback on the presentation. The paired t-test was used to compare changes in pre- and post-assessment items and scores. Feedback on the presentation was summarized. Results: In collaboration with a pharmacist faculty mentor, five students from the College of Pharmacy and College of Nursing served as peer educators. The presentation was delivered 13 times to a total of 494 students. Results from the pre- and post-assessments indicated a statistically significant increase in eight of the ten multiple choice items and a mean total score increase of $19.13 \%(\mathrm{p} \leq 0.05)$. Following the presentation, $90.49 \%$ of the attendees agreed they were more knowledgeable about the dangers of misusing prescription drugs and $95.34 \%$ of the attendees agreed the information was presented effectively. The peer educators found impacting the knowledge of fellow students to be rewarding and enjoyed collaborating on this inter-professional initiative. Implications: Results support the notion that peer education is an effective way to raise awareness about prescription drug abuse/ misuse on college campuses. 


\section{American Journal of Pharmaceutical Education 2019; 83 (5) Article 7654.}

Pharmacist and Pharmacy Student Wellness and Mental Health Analysis. Ashley A. Kang, University of North Carolina at Chapel Hill, Abby MacCauley, University of North Carolina at Chapel Hill, Suzanne C. Harris, University of North Carolina at Chapel Hill, Heidi N. Anksorus, University of North Carolina at Chapel Hill. Objective: Survey a large sample of pharmacists and pharmacy students in various practice areas and pharmacy schools, respectively, to assess three areas of wellness: mental, physical, and personal health. Methods: The Qualtrics surveys have two arms with IRB approval: PharmD students and practicing pharmacists. In December 2018, the North Carolina Board of Pharmacy disseminated a survey to all actively licensed pharmacists in North Carolina. In January 2019, representatives, including Directors of the Office of Student Affairs of 10 accredited U.S. pharmacy schools disseminated the student arm of the survey. Surveys will collect pertinent demographics and assess mental (stress, anxiety, depression, substance use), physical (diet, exercise, sleep habits), and personal (school- or work-life balance, hobbies, burnout, career and relationship satisfaction) health, incorporating previously validated tools adapted to create a comprehensive survey. The survey will be open through March 2019 with full analysis anticipated in April. Results: At this point in data collection, the student arm of the survey has received 794 responses from 10 schools of pharmacy across the U.S., which equates to a response rate of roughly $13 \%$. The pharmacist arm has received 1,311 responses, which is a response rate of about $10 \%$. The current completion rate is in line with initial estimations. Implications: When looking at contributors to wellness, it is anticipated that connections will be identified among the three domains assessed, allowing employers and pharmacy schools to better understand the needs of their respective populations, hopefully leading to increased resources and programs to support wellness.

Pharmacist and Physician Engagement in Tertiary Prevention of Opioid Use Disorder. Aaron J. Salwan, East Tennessee State University, Nicholas E. Hagemeier, East Tennessee State University, Kelly Foster, East Tennessee State University, Jesse Arnold, East Tennessee State University, Bill Brooks, East Tennessee State University, Arsham Alamian, East Tennessee State University, Robert Pack, East Tennessee State University. Objective: To compare physician and pharmacist engagement in tertiary prevention behaviors in patients at risk for opioid use disorder and accidental overdose. Methods: Two paper-based survey instruments were developed to assess community pharmacists' $(\mathrm{N}=2206)$ and primary care physicians' $(\mathrm{N}=1890)$ engagement in prevention behaviors for patients at risk of opioid use disor- der (OUD). A profession specific scenario was pretested and thereafter included in each survey instrument to gauge engagement in prevention behavior. Given 10 patients with OUD, pharmacists and physicians were asked how many patients they would dispense/prescribe buprenorphine/naloxone, and for how many patients they would co-prescribe/dispense naloxone. Participants were asked to evaluate perceived prevalence of evidence-based medication assisted treatment (MAT) practices among physicians in Tennessee. SPSS version 25 was used to calculate descriptive statistics. Results: Usable responses were obtained from 433 pharmacists and 296 physicians. Given 10 patients with evidence of poly-substance misuse, $61 \%$ of physicians indicated they would discharge 10 of 10 patients. For a patient with a buprenorphine/naloxone prescription, $35 \%$ of pharmacists indicated they would never $(0 / 10)$ dispense buprenorphine/naloxone. Given 10 patients, pharmacists reported they would discuss co-dispensing naloxone with $3.3 \pm 4.03$ patients, and physicians would co-prescribe naloxone to $2.02 \pm 3.65$ patients. Pharmacists and physicians perceived a minority of MAT providers (30\% and $32 \%$, respectively) to engage in evidence-based MAT. Implications: Opportunities exist for pharmacists and physicians to increase engagement in OUD prevention behaviors. Future research is warranted to understand drivers of behavioral engagement.

Pharmacist Preceptor Perceptions of Experiential Learning: A Phenomenological Study. Shauna Gerwing, University of Saskatchewan. Objective: The purpose of this study is to describe the lived experience of experiential learning by pharmacist preceptors. Questions guiding the research include: what are pharmacy preceptor perceptions of experiential learning at the University of Saskatchewan? And, what enhances or constrains pharmacy preceptors understanding, desire, or ability to engage in experiential learning? Methods: Qualitative methodology, in particular, hermeneutic phenomenology as guided by Max van Manen (Researching Lived Experience, 1990) is used in the study. This method is an iterative process that involves turning to the phenomenon of interest, investigating experience as it is lived rather than conceptualized, reflecting on the essential themes of the phenomenon, describing the phenomenon through writing and rewriting, maintaining a strong and oriented relation to the phenomenon, and balancing the research context by considering the parts and the whole. Semi-structured, one-on-one interviews, with nine preceptors from hospital and community practice were conducted. Themes, anecdotes, and detailed descriptions to gain understanding and insight into the lived experience of experiential learning of preceptors are provided in this interpretive, descriptive, phenomenological analysis. 


\section{American Journal of Pharmaceutical Education 2019; 83 (5) Article 7654.}

Results: Preliminary themes identified include: time for everything, finding a balance, building relationships, learning and teaching, motivation, and difficult situations. Analysis is currently ongoing. Implications: This phenomenological project may allow others, including educational institutions, to appreciate the lived experience of pharmacy preceptors, and may encourage others to act in a tactful, empathetic manner when modifying experiential learning curriculums. It may enhance the quality and quantity of experiential placements to benefit both students and preceptors.

Pharmacist Preparedness and Perceptions Regarding Gender Affirming Therapy. Michelle ThienTam Tran, Steven D. Swank, The University of Texas at Austin, Jessica L. Robertson, The University of Texas at Austin, Justina Lipscomb, The University of Texas at Austin, Ashley S. Oliver. Objective: The purpose of this study is to describe practicing pharmacists' preparedness in caring for transgender patients. Methods: An eleven question Likert-scale survey was distributed to practicing pharmacists to assess foundational knowledge and comfort level regarding gender affirming therapy (GAT). Participants were asked to rate their preparedness on a scale from 1 to 5 ( 1 being "not prepared", 5 being "very prepared"). Participants were also asked to provide demographic information and if they received education regarding GAT during or after graduating from pharmacy school. Participation in the survey was both anonymous and voluntary. No written consent was required. Results from the survey were analyzed using descriptive statistics and student's t-test as appropriate. Results: A total of 92 pharmacists completed the survey. Preliminary results show that $92.4 \%$ did not receive formal GAT education while in pharmacy school. On average, pharmacists rated themselves at 2.13 and 2.20 in providing recommendations for and counseling transgender patients on GAT, respectively. Fifteen pharmacists indicated that they previously received continuing education in GAT. On average, they rated themselves at 2.93 and 3.07 in providing recommendations for and counseling transgender patients on GAT, respectively. Implications: Transgender populations are growing in America, and the likelihood that a pharmacist will care for a transgender person is increasing. The results of this study show the importance of including GAT education in pharmacy curriculums. Moreover, providing continuing education to practicing pharmacist increases their ability and confidence in providing care for transgender patients.

Pharmacist-Prescribed Hormonal Contraception: Gauging Pharmacist Attitudes, Interest, and Knowledge in North Carolina. Gwen J. Seamon, Mountain Area Health Education Center, Allison Burke,
Walgreens, Casey Tak, University of North Carolina at Chapel Hill, Amy Lenell, Walgreens, Macary W. Marciniak, University of North Carolina at Chapel Hill, Mollie A. Scott, University of North Carolina at Chapel Hill. Objective: The objective of this study is to evaluate the attitudes, interest, and knowledge regarding pharmacist-prescribed hormonal contraception in North Carolina. Methods: A web-based survey was distributed via email to all actively licensed pharmacists residing in North Carolina from November 14, 2018 - December 13, 2018. Demographic data and pharmacy practice characteristics were gathered. Respondents in community-based settings were asked their perceptions regarding feasibility of implementation, barriers to implementation, and personal views on prescribing. All respondents were asked regarding support towards pharmacist-prescribed hormonal contraception. Descriptive statistics were used to characterize the responses. Results: This Institutional Review Board-approved study had a $6 \%(713 / 12,001)$ response rate. Of those, $65 \%$ were female, $53 \%$ were less than 40 years old, $71 \%$ received a PharmD degree, and $16 \%$ completed a Postgraduate Year One pharmacy residency. The majority of respondents, $54 \%$, reported their primary practice site as community-based. Community-based pharmacists reported the most common barriers to impact prescribing ability were added responsibility and liability (81\%), time constraints (79\%), and need for additional pharmacist training (77\%). Perceived benefits of prescribing ability were increased patient contact $(91 \%)$, increased use and adherence by patients $(81.02 \%)$, and improved access to hormonal contraception ( $80 \%)$. Overall, $46 \%$ of community-based pharmacists felt that pharmacists are well-trained/educated to prescribe versus $51 \%$ of non-community pharmacists. Implications: Assuming barriers are removed and that sufficient training is provided, most community-based pharmacists in North Carolina are likely to prescribe hormonal contraception. Study limitations include a low response rate and a convenience sample of respondents. This research supports legislation to permit pharmacist-prescribed hormonal contraception.

Pharmacists' Roles When Interacting with Persons Living With HIV of African Origin: A Narrative Inquiry. Alina Cernasev, University of Minnesota, William L. Larson, Allina Health, Todd Rockwood, University of Minnesota, Cynthia Peden-McAlpine, University of Minnesota, Paul L. Ranelli, University of Minnesota, Olihe N. Okoro, University of Minnesota, Jon C. Schommer, University of Minnesota. Objective: Different studies have demonstrated pharmacist's roles in adherence to the Antiretroviral (ART) regimen. To our knowledge, there was no previous research to explore the role of the 


\section{American Journal of Pharmaceutical Education 2019; 83 (5) Article 7654.}

pharmacist in African-origin persons living with HIV when navigating the U.S. healthcare system and their medication regimen. Methods: A qualitative approach was used for this study. Fourteen participants were recruited from Minnesota pharmacies and HIV clinics. One-on-one Narrative Interviews were conducted over five months in 2018. All interviews were audio-recorded and transcribed verbatim. An inductive content analysis was conducted using Dedoose, qualitative software. Codes were inductively derived, and similar codes were grouped into categories that revealed themes. Results: The participants were originally from seven different African countries. Analysis of the interview data resulted in two distinct themes that relate to the study objectives. Theme 1: Accessing the U.S. healthcare system Some of the participants are still learning how to navigate the U.S. healthcare system. Whenever they had questions about ART medications, they would rely on the physician's advice. Participants seemed unaware of the potential role that could be played by the pharmacists in their therapy management. Furthermore, there were situations when participants experienced various insurance issues; they sought help from a case manager. Theme 2: Role of herbal remedies in their treatment Some of the participants have faith in herbal remedies and use them. Implications: Pharmacists have the opportunity to take additional patient care roles in helping these individuals to navigate the unfamiliar U.S. healthcare system and the ART treatment landscape.

"Pharmacy Battle", a Group-Based Study Game Implemented in a Top-200 Drugs Class. Micah E. Miller, Washington State University, Christina R. Buchman, Washington State University. Objective: To create and apply a completely custom-created, group-based study game to improve retention of first-year-level "Top-200 Drugs" content while increasing motivation, enjoyment, and socialization. Methods: Game creation and pre-testing lasted from Fall 2018 into Summer 2019, with final game implementation occurring in Fall semester of 2019 within a cohort of first-year pharmacy students at the Yakima extension of the Washington State University College of Pharmacy and Pharmaceutical Sciences. Game sessions were held outside of class, on a voluntary basis. Each group of 3-5 students could participate in 6 separate game sessions during the semester, with each session held the week before the upcoming exam. Two rounds of surveys gathered subjective motivation- and experience-centric information at the middle and end of semester. Participants' exam performance was gathered and compared to two non-participant cohorts. Results: In total, 952 non-participant and 71 participant exam performance results were gathered. Incidence of retests (scoring
$<80 \%$ on initial assessment) in the participant group was $6(8.5 \%)$, versus $161(16.9 \%)$ in the non-participant group. Additionally, the participant group had higher average tests scores $(9.27 / 10$ versus $8.71 / 10$ average of non-participants). Surveys also revealed several positive outcomes related to motivation, enjoyment, and socialization. Implications: Pharmacy Battle represents a unique product as an educational game, purpose-built from scratch rather than an adaptation of an existing product. This process admittedly requires greater initial effort for the game creator, but the successful outcomes noted in this project suggest there is great potential for such tailormade, game-based solutions in the future of pharmacy education.

Pharmacy Student Perception of School Related Stress and Awareness of Resources. Eric P. Tobin, University of Colorado, Benjamin Chavez, University of Colorado, Megan Leeds, Regis University. Objective: This study aims to assess the impact of stress on pharmacy students. It also aims to increase awareness of student stress to make adjustments in campus resources and better serve students. Methods: A 12-question survey on the perception of school-related stress was sent out electronically to all students enrolled in Colorado entry-level PharmD programs $(n=817)$. Questions used a 4-point Likert scale rating from strongly agree to strongly disagree. The survey measured the perception of stress from topics like financial, academic performance, and future career opportunities. It included questions geared toward how school stress impacted mental and physical health, the ability to enjoy life outside of school, and awareness of resources related to stress management and coping skills. The survey also presented students with campus resources for helping with management of stress. Results: A total of 415 students (51\%) responded, 272 were female (66\%). Results showed $67 \%$ of respondents felt pharmacy school had impacted their physical health and mental well-being. Seventy-three percent stated their life outside of pharmacy school had suffered since starting the program. About a third (30\%) responded that stress from pharmacy school had contributed to regretting entering pharmacy school. Only $45 \%$ of respondents felt their university had enough resources to help them cope with stress. Implications: Stress experienced in pharmacy school impacts mental and physical health of students, as well as their lives outside of school. Methods to increase awareness and improve resources should be explored further in order to preserve student's health and ability to manage stressrelated negative effects.

Pharmacy Student Preferences and Perceptions of In-Person Versus Video Evaluations in a Skills-Based Laboratory Course. Jenny Beal, Purdue University, 


\section{American Journal of Pharmaceutical Education 2019; 83 (5) Article 7654.}

Zachary A. Weber, Purdue University, Alex N. Isaacs, Purdue University, Kimberly S. Illingworth Plake, Purdue University, Alan J. Zillich, Purdue University, Jamie L. Woodyard, Purdue University. Objective: To determine pharmacy students' preferences and perceptions of inperson and video evaluation methods for formative and summative activities in a skills-based laboratory course. The relationship between student evaluation and academic achievement has been established, but little research has been conducted on student perceptions of evaluation methods. Methods: An online survey was administered to 447 first-, second-, and third-professional year pharmacy students. Fifteen items using a 5-point Likert-type scale $(5=$ strongly agree to $1=$ strongly disagree $)$ measured student perceptions and ten items measured preferences ("video", "in-person", or "both are equal"). Paired t-tests compared student perceptions of the two evaluation methods and independent t-tests compared the difference in perceptions between students exposed and not exposed to video evaluations. Results: Pharmacy students (99.3\% response rate) perceived in-person and video evaluations as significantly $(\mathrm{p}<0.05)$ different, with in-person evaluations being perceived more positively on all items except for nervousness. Students exposed to video evaluations felt significantly more positive towards video evaluations than video-naïve students on nine of 11 items but felt significantly less positive towards video evaluations in terms of quality (delta $=1.24$ vs. 0.83 ) and amount (delta $=1.14$ vs 0.77) of written feedback received. Implications: Students view in-person evaluations significantly more positively than video evaluations. This effect is largely mitigated by exposure to video evaluations, suggesting that concerns regarding video evaluations are based on conjecture rather than experience. Adjustments are needed to improve the quality and amount of written feedback provided to students evaluated via video.

Pharmacy Students' Desired Post-Graduation Job and Lifestyle Attributes and Confidence Choosing from Potential Job Offers. Drake J. Reiter, Drake University, Erin Ulrich, Drake University, Kelli Welter, Drake University, Jonathan R. Hurdelbrink, Drake University. Objective: To determine pharmacy student preference in lifestyle/city characteristics, job traits, and confidence in selecting post-graduation jobs with varying job attributes. Methods: Pharmacy students in the NABP/ AACP District 5 completed a one-time, anonymous, online questionnaire. Measures of interest included: subject characteristics (gender, year in pharmacy school, and size of hometown), important lifestyle/job attributes, preference of position when given four rounds of hypothetical job posting, and confidence in their decision after each round (Likert scale response). A summed confidence score was calculated (range score 4-12). Kruskal Wallis and Mann-Whitney $U$ tests analyses were conducted to determine if individual characteristics were related to summed confidence. Results: A total of 283 students completed questionnaires from in Iowa, South Dakota, North Dakota, and Saskatchewan. The majority of respondents were female, P3 students, and from a hometown with a population greater than 2,500 . The top lifestyle characteristics were cost of living (75.4\%) and sense of community (52.7\%). The preferred job attributes were flexible schedule and autonomy (57.1\%) and salary (55.5\%). Overall, when given hypothetical job listings, students were not confident in any of their decisions (means ranged from 9.6-51.1). Out of all locations, confidence was only related to year in school $(p=0.025)$ in Saskatchewan. Implications: Pharmacy students are not confident in the selecting a post-graduate position. Schools of pharmacies should consider implementing resources, support, and co-curricular activities to assist in building confidence during the job search process.

Pharmacy Students' Perceptions of Electronic Peer-Review of Journal Clubs During a Drug Literature Evaluation Course. Kaitlin J. Montagano, Purdue University, Amy H. Sheehan, Purdue University. Objective: Journal clubs are frequently used to teach important literature evaluation skills to pharmacy students. Peer review is one method to improve students' competency regarding journal clubs. The objective of this study is to determine whether pharmacy students' perceptions of participating in electronic peer-review sessions during class time influences their perceived self-competence toward journal clubs. Methods: Second-year professional students participated in three separate in-class, electronic, anonymous peer-review sessions to provide and receive feedback for their three written journal club drafts. Following grading of the third journal club, students were invited to complete a 23-item survey instrument based on the Self-Determination Theory. The Intrinsic Motivation Inventory (IMI) and Perceived Competence Scale, were used to assess students' interest in and perceived value of the electronic peer-review sessions, as well as their perceived self-competence regarding journal clubs. Results: The survey response rate was 98.6 percent, with 146 of 148 students completing the survey. Based on scales of one to seven, students demonstrated moderate interest in the peer-review sessions (mean: 3.86), viewed them as valuable (mean: 5.25), and had a moderate level of perceived self-competence regarding journal clubs (mean: 3.74). Additionally, students' interest and perceived value of the electronic peer-review sessions both significantly influenced their self-competence in completing quality journal clubs. Implications: An in-class 


\section{American Journal of Pharmaceutical Education 2019; 83 (5) Article 7654.}

electronic peer-review system offers an efficient and anonymous method for providing and receiving feedback on journal club assignments. Increasing students' interest in and perceived value of peer-review may improve their self-competence regarding journal clubs.

Pharmacy Students' Use of Social Media for Learning and Communication. Ruth N. Jeminiwa, $A u$ burn University, Fatana Shamsuddin, Nashville General Hospital, Jeff J. Cain, University of Kentucky, Kevin A. Clauson, Lipscomb University, Brent Fox, Auburn University. Objective: The use of social media is highly prevalent with over $80 \%$ of Americans owning a social media profile. Although social media platforms such as Wikipedia and YouTube hold promise in teaching and learning, little is known about students' perceptions of using these platforms for learning and communication. The objective of this study is to determine pharmacy students' perceptions of using social media as tools for learning and communication. Methods: A cross-sectional survey of P1, P2, and P3 pharmacy students in two Universities was performed. In addition to demographic information, survey questions elicited information on students' use of social media for education and patientrelated communication. Descriptive statistics and the Chi square test were computed. Results: A total of 335 students completed the survey (response rate $=51 \%$ ). Most participants were female $(67.2 \%)$, white $(75.5 \%)$, and between 20 - 30 years old (92\%). About $64 \%$ of the students used Wikipedia for health-related information when studying. Conversely, only $21 \%$ of students used Wikipedia for health-related information for patient-related questions. Eighty-seven percent used YouTube for health-related information when studying. Again, there were fewer students utilizing YouTube to visually demonstrate health-related information to patients (33\%). A Chi square post-hoc analysis revealed that P1s were underrepresented among students utilizing YouTube for health-related information when studying. ( $p=0.002)$. Implications: Majority of students utilize social media for learning, however, few utilize this platform for patient-related communication. Future studies should explore factors influencing students' use of social media for patient-related communications.

PharmCasting the Future of Pharmaceutical Sciences and Pharmacy Practice Education. Amanda Olsen, University of North Carolina at Chapel Hill, Lana M. Minshew, University of North Carolina at Chapel Hill, Jacqueline McLaughlin, University of North Carolina at Chapel Hill. Objective: The objective was to build consensus and rank order items regarding the future of pharmacy practice and pharmaceutical science education. Methods: Two separate Delphi studies were conducted, one with leaders who specialized in pharmacy practice education and one with leaders who specialized in pharmaceutical science education. Using the Delphi method, a systematic process where experts generate, reduce, and rank order items to indicate importance, experts from three different institutions identified current/emerging innovations and professional skills that students may need to develop to be successful in the next decade. Results: For current/emerging innovations, pharmacy practice experts identified, 1) digital health, 2) greater incorporation of pharmacists in team-based care, and 3) personalized medicine, while the pharmaceutical science experts identified 1) personalized medicine, 2) immunotherapies, and 3) big data. Regarding professional skills, the pharmacy practice experts identified, 1) clinical decision making, 2) creative problem solving, and 3) collaborating with other professionals, while the pharmaceutical science experts identified, 1) creative problem solving, 2) communication skills, and 3) self-directed learning. Although pharmacy practice and pharmaceutical science students are trained differently, experts in these domains identified areas of overlap such as personalized medicine and creative problem-solving. Implications: When developing curricula for professional (PharmD) and Doctor of Philosophy (Ph.D.) degrees, it is often not known what educational content is needed for continued student success. The results from this study provide insight into different areas that should be integrated into graduate and professional programs so students can remain at the forefront of their respective fields.

PharmD Student Roles in Development and Implementation of an Applied Patient Care Skills Course. Julia S. Lee, University of California San Francisco, Crystal Zhou, University of California, San Francisco. Objective: To describe student contributions to curriculum and implementation of a new skills course for first-year PharmD students. Methods: Students in the Applied Patient Care Skills (APCS) course meet two hours per week to exercise critical thinking and problemsolving skills through complex patient cases; practice patient communication, including motivational interviewing and medication counseling; and receive training in using various devices, such as inhalers and nicotine replacement products. The Office of Education and Instructional Support (OEIS) employs two student interns (second- or third-year students) to work with the APCS course director. Student interns for the APCS course spend approximately six hours weekly working in conjunction with the course director to develop patient cases, facilitate group discussions, provide individualized feedback for weekly reflections, assist during structured office hours, and design the top 200 most prescribed "brand and 


\section{American Journal of Pharmaceutical Education 2019; 83 (5) Article 7654.}

generic drug quizzes" for the first-year PharmD students. Results: Student interns found their role beneficial in that it bolstered their clinical knowledge, allowed insight into course design, and provided opportunities for mentoring and establishing collegial active-learning environments. Faculty found the role of student interns to be instrumental in building a new curriculum through compiling patient cases, reviewing assignments, and assisting with structured office hours. Implications: Students can play a valuable role throughout the design and implementation of new curricula. This model of employing educational student interns is beneficial for both student learning and faculty support that cannot be provided by non-PharmD trainees (e.g. graduate students) or staff.

Phenylalanine Hydroxylase Deficiency: The Pharmabiotic Treatment Approach. Chloe E. LeBegue, University of South Carolina, Jacob C. Massey, University of South Carolina, Michael D. Wyatt, University of South Carolina. Objective: Phenylketonuria, now known as phenylalanine hydroxylase (PAH) deficiency, is a disorder of metabolism affecting approximately one in every 15,000 infants born in the United States. Patients have nonfunctional PAH enzyme secondary to one or more genetic mutations. The enzyme deficit results in destructive supraphysiologic blood phenylalanine levels upon consumption of the essential amino acid phenylalanine. Current standards of care mitigate signs and symptoms of the disorder, but do not approach a cure. The methods for creating a prototype pharmabiotic as an innovative treatment strategy for PAH deficiency are described. Methods: DNA molecular cloning techniques were utilized to engineer a novel plasmid termed LiLi5, which was subsequently transformed into Lactobacillus helveticus. Results: A cDNA for human PAH was amplified and inserted into a gram-neutral shuttle vector to create LiLi5, which expresses human phenylalanine hydroxylase. The LiLi5 plasmid was confirmed by restriction enzyme digest and sequencing of the cDNA insert. Successful transformation of LiLi5 into Lactobacillus helveticus generated a novel strain. PCR-based detection confirmed the presence of the human PAH cDNA in Lactobacillus helveticus. Implications: Phenylalanine hydroxylase deficiency is a debilitating disorder causing somatic, psychosocial, and financial distress for patients and their families. Current treatment strategies do not adequately address the decreased quality of life experienced by patients. Lactobacillus helveticus is a GRAS gram-positive bacterial species commonly formulated in commercially marketed probiotics. Lactobacillus helveticus expressing human phenylalanine hydroxylase will be formulated into a pharmabiotic for testing as a new treatment for phenylalanine hydroxylase deficiency. Patent pending.

Piloting A Branched Narrative Learning Pedagogy to Promote Critical Thinking In Student Pharmacists. MeiLing G. Norfolk, Cedarville University, Bethany Sibbitt, Cedarville University, Zachary N. Jenkins, Cedarville University. Objective: Traditional lectures may not always not mimic the complexity of decision making in patient care. Branched narrative learning (BNL) is a pedagogy that utilizes patient cases that evolve based on student response and may promote critical thinking through the simulation of real-life decisions and their consequences. The objectives of this study were to evaluate the ability of BNL to strengthen student performance on higher Bloom's level questions, as well as addressing the Pharmacist Patient Care Process (PCPP), in a therapeutics course. Methods: Third-year professional pharmacy students completed a series of four assessments throughout the hematology/oncology course, as well as a survey on their perceptions of BNL. The first assessment was administered prior to topic preparation for evaluation of baseline knowledge. The second assessment was administered following lecture-based content delivery, the third was given after BNL content delivery, and the fourth was administered at the culmination of the course to assess extended retention of knowledge. Questions were mapped to Bloom's Level and the PCPP, and scores were analyzed using repeated measures ANOVA. Results: In this 48-student cohort, performance from the first through the fourth assessments for all Bloom's taxonomy levels, PPCP principles, and overall examination scores showed significant improvement from baseline. Students had positive perceptions of this pedagogy. Implications: BNL may improve students' critical thinking and better prepare them for real-life scenarios of navigating through clinical decision making in practice. Future studies will directly compare this pedagogy to other methods.

Positive Deviants for Medication Therapy Management: A Mixed-Methods Evaluation of Community Pharmacy Practices. Omolola A. Adeoye, Purdue University, Leslie M. Lake, Kroger Central Division, Karen S. Hudmon, Purdue University, Alan J. Zillich, Purdue University, Margie E. Snyder, Purdue University. Objective: Applying the Positive Deviance model, generate hypotheses for practices that enable community pharmacies to achieve top performance on widely accepted Medication Therapy Management (MTM) quality measures. Methods: This work incorporates two complementary conceptual frameworks. First, to explain reasons for deviations in MTM performance, the Positive Deviance model was adapted and applied to inform study 


\section{American Journal of Pharmaceutical Education 2019; 83 (5) Article 7654.}

design. Second, because MTM focuses primarily on optimizing health outcomes among the chronically ill, the Chronic Care Model was applied to guide data collection and analysis. Pharmacies within a supermarket-based community pharmacy chain were ranked by composite scores on five MTM quality measures comprising the 2017 Medicare Part D Star Ratings: Adherence Measures for cholesterol, hypertension, and diabetes medications, High-Risk Medication use in the elderly, and Comprehensive Medication Review completion rates. A purposive sample was drawn from the first, third, and fifth quintiles of a ranked list to generate high-, moderate-, and lowperforming categories respectively for recruitment. Pharmacy staff engaged in MTM delivery at selected locations were invited to participate in semi-structured interviews characterizing MTM delivery practices. Using an iterative process throughout data collection, study investigators will conduct an abductive thematic analysis of interview data collected. Qualitatively derived themes will be cross-tabulated with performance categories and demographic data. Primary outcome measures will include key findings pertaining to areas of overlap and variation across the three performance categories. Results: Pending Implications: With a long-term goal of optimizing Medicare Part D MTM policy, the resulting data could inform recommendations for practices consistent with top performance on MTM quality measures.

Price Transparency in Prescription Drug Advertisements on Television/Streaming Devices: Consumer Perspectives. Michael T. England, Shah Afzal, Medical Marketing Economics LLC, Amit S. Patel, Medical Marketing Economics LLC, Mansi Shah-Patel, Medical Marketing Economics LLC, John P. Bentley, The University of Mississippi. Objective: Examine consumers' perspectives on including drug prices in directto-consumer advertising via television/streaming devices (TV-DTCA). Methods: This study employed a crosssectional design using a self-administered web-survey; respondents were recruited via an online consumer panel. Inclusion criteria: prescription drug insurance, taking at least one prescription, and viewed prescription TVDTCA in the last 3 months. Primary outcome variables: opinion of whether price should be included in TVDTCAs and behavioral intentions (e.g., information search, request a prescription) if price were included. Attitude scales, sociodemographics, self-reported pricing knowledge, and awareness of the Trump Administration's proposed policy were used as predictors in multivariable models. Results: Of the 267 respondents, $60 \%$ agreed that manufacturers should show drug prices in TVDTCAs. Significant predictors of this opinion included: negative attitude toward pharmaceutical pricing and pos- itive attitude toward pricing in TV-DTCA. There was a moderate level of intention to engage in behaviors motivated by drug price in advertisements. Significant predictors of intentions were positive attitude toward TV-DTCA in general, toward pricing in TV-DTCA, and past behavior. A majority (54\%) of respondents stated a preference to see out-of-pocket (OOP) costs (average, lowest, highest) for patients with insurance in such advertisements (vs. other options like list price). Implications: Even though a majority of consumers appear to support the inclusion of patient OOP costs in TV-DTCA, price information varies by insurance and plan type and may be more misleading than helpful. It may affect patient requests for prescriptions; however, it is unclear whether the practice will influence how manufacturers price their products.

Primary Care Physician and Community Pharmacist Opioid-Related Communication and Screenings Behaviors. Tyler C. Melton, East Tennessee State University, Nicholas E. Hagemeier, East Tennessee State University, Kelly Foster, East Tennessee State University, Jesse Arnold, East Tennessee State University, Bill Brooks, East Tennessee State University, Arsham Alamian, East Tennessee State University, Robert Pack, East Tennessee State University. Objective: To compare Tennessee primary care physicians' and community pharmacists' primary (risk communication, risk assessment) and secondary (screening) opioid use disorder (OUD) prevention behaviors. Methods: After calling physicians and pharmacists to confirm practice setting, a survey instrument was sent to all Tennessee primary care physicians $(n=1890)$ and community pharmacists $(n=2206)$ using a modified, 4-wave Tailored Design Method approach. Three OUD scenarios (primary, secondary, tertiary) were pretested with providers and thereafter included in the profession-specific survey instruments. Respondents were asked to report, given 10 similar scenarios, the number of times ( 0 to 10 ) they would engage in prevention behaviors. Means and standard deviations were calculated using SPSS version 25. Results: Response rates of $16 \%$ and $20 \%$ were obtained for physicians and pharmacists, respectively. Given 10 similar scenarios, physicians indicated they would assess for risk of opioid abuse 1.66 $+/-3.25$ times and for current opioid abuse $3.08+/-4.31$ times, while pharmacists would assess risk of opioid abuse $2.11+/-3.7$ times and for current opioid abuse $2.08+/-3.7$ times. Pharmacists reported communicating risks associated with prescription opioids to patients 7.6 $+/$ - 3.5 times, while physicians reported doing so 8.86 +/- 2.77 times. Implications: Pharmacists and physicians rarely use tools such as the Opioid Risk Tool and Current Opioid Misuse Measure to assess for risk of opioid abuse or for current opioid abuse. Further 


\section{American Journal of Pharmaceutical Education 2019; 83 (5) Article 7654.}

research is warranted to understand factors influencing pharmacists' and physicians' engagement in OUD prevention behaviors.

Proteomic Evaluation of Cetuximab Susceptible and Resistant Colorectal Cancer Cell Lines. Travis John Holmes, Belmont University, Amy-Joan L. Ham, Belmont University. Objective: Colorectal cancer (CRC) is the 3rd leading cause of cancer-related death in the United States. Resistance to treatment, like the monoclonal antibody cetuximab, occurs in $90 \%$ of patients in 18 months. This research was conducted to identify pathways of resistance in CRC and investigate novel targets for future drug development. Methods: Two cell lines were derived from the same HCA-7 progenitor line; SC which is resistant and CC which is susceptible to cetuximab. Whole cell lysate was subjected to gel electrophoresis, combined with LC-MS/MS analysis, to fractionate and collect peptide mass spectra. Database searching using the Myrimatch algorithm was employed to identify proteins and IDPicker 3.0 was used to reassemble the proteins and filter the data to result confident identifications. Differences in protein expression were determined by spectral counting using the quasi-likelihood modeling for spectral counting. Results: A total of 125,927 spectra were filtered and matched to 1,545 distinct protein groups with a false discovery rate (FDR) of less than $1 \%$. Statistically significant protein downregulation was identified for 53 genes that were mapped to a total of 10 common cell pathways using Web gestalt. The BAD and GNAI2 genes were observed in multiple affected pathways. Implications: BAD is a gene that codes for a protein which normally results in cellular apoptosis, and GNAI2 codes for a protein that normally inhibits the generation of cAMP. With these pathways downregulated, the cell is constituently proliferative and ignores standard signals for termination. Future studies will investigate BAD and GNAI2 pathways as possible targets to reverse cetuximab resistance.

Quality Control and Quality Assurance for Pharmaceutical Botulinum Neurotoxin Type A (BoNT/A). Jennifer Y. Tran, The University of Utah, James N. Herron, The University of Utah, Aiping Wei, Agri-Analysis, Shawn C. Owen, The University of Utah, Andrew S. Dixon, The University of Utah. Objective: Determine whether an in-vitro simultaneous assay system for BoNT/A is more sensitive and faster than the gold standard mouse lethality bioassay for determining the potency of pharmaceutical BoNT/A Methods: Pharmaceutical BoNT/A comprises a $50 \mathrm{kDa}$ light chain and a $100 \mathrm{kDa}$ heavy chain. The heavy chain binds to the neuron, internalizing the neurotoxin. Inside the neuron, the light chain cleaves synaptosomal-associated protein 25 (SNAP25) preventing acetylcholine release. In this study, different light chain expression conditions were optimized by varying vector, bacterial host cell, induction temperature, and induction time. Expressed light chain activity was evaluated via a fluorogenic enzymatic assay using a SNAP25 mimic (SNAPtide). BoNT/A heavy chain expression is being optimized with similar procedures, except that its activity will be validated through an immunoassay. The final in-vitro assay system combines both assays to detect active heavy and light chains simultaneously, producing a calibration curve to determine the unknown potency of a pharmaceutical BoNT/A. Results: Optimal expression of active BoNT/A light chain occurred with pF1K T7 Flexi ${ }^{\circledR}$ vector, SHuffle ${ }^{\circledR}$ T7 Express Competent E. coli, induction temperature of 7.5 degree Celsius, and induction time of 24 hours. Results for the protein expression of BoNT/A heavy chain and the simultaneous assay system are in progress, but an increase in sensitivity by $25 \%$ to $50 \%$ and a reduction in assay time from 48 hours to 5 hours are expected. Implications: As compared to the mouse lethality bioassay, this in-vitro simultaneous assay system for BoNT/A is expected to result in significantly higher sensitivity and shorter assay time.

Rational Design of Isoform-Selective HDAC3 Inhibitors for Reversal of HIV Latency. Sarah Marie Reasoner, Concordia University Wisconsin, Henry North, Harding University, Tzvia I. Springer, Concordia University Wisconsin, Terry-Elinor Reid, Concordia University Wisconsin. Objective: Despite advancement in antiretroviral therapy (ART), complete eradication of HIV has not been achieved. One approach for eliminating HIV latent reservoirs is the "shock and kill" method by latency-reversing agents (LRAs). Histone deacetylase (HDAC) inhibitors are among the most prominent LRAs. HDAC3 isoform activity is reported to be essential for restricting the HIV-latent integrated provirus. Although there are class I HDAC inhibitors currently on the market, most are not isoform selective thereby resulting in reduced potency as LRAs. Hence, it is pertinent to identify isoform-selective HDAC3 inhibitors that can be included in latency eradication strategies. Methods: To achieve our goal of designing isoform-selective HDAC3 inhibitors, we utilized in silico approaches to; (1) identify potential hits, (2) conduct hit-to-lead optimization of hits by capitalizing on unconserved amino acids within the binding site and, (3) to design molecules that target the more recently discovered co-repressor binding site. We experimentally validated hits by conducting in vitro fluorogenic assay screening. Results: Virtual screening of our in-house chemical library lead to the discovery of two promising novel HDAC3 inhibitors that are weakly active. Structure-guided hit-to-lead optimization of our hits 


\section{American Journal of Pharmaceutical Education 2019; 83 (5) Article 7654.}

lead to the generation of a small library of structures for synthesis and SAR studies. In addition, we designed Inositol mimics that may selectively bind the more unconserved co-repressor binding domain of HDAC3. Implications: The exploitation of unconserved regions of HDAC3 is important in the design of isoform-selective LRAs to be used as part of a potential HIV eradication strategy.

Revision and Mapping of Entrustable Professional Activity Supporting Tasks. Bailey K. Buenger, South Dakota State University, Teresa M. Seefeldt, South Dakota State University. Objective: Entrustable Professional Activities (EPAs) are skill-based competencies that students can be entrusted to perform independently once they have received training and demonstrated competence in performing the skills. The Core EPAs for New Pharmacy Graduates were recently developed by the American Association of Colleges of Pharmacy. This document includes 15 core EPAs, and each EPA has a list of exemplary supporting tasks. The objectives of this project were to refine the supporting tasks for each EPA for implementation in a Pharm.D. program and map the EPAs to the pre-APPE competency statements. Methods: The example supporting tasks in the core EPA document were reviewed and compared to the pre-APPE competency statements. Mapping of the supporting tasks to these competency statements was conducted to identify areas of similarity and gaps. The results of the mapping were used to develop a program-specific draft of the EPA supporting tasks. Feedback on the draft was obtained from faculty in the pharmacy practice department, and the supporting tasks were further refined based on this feedback. Results: Following revision, 51 supporting tasks were included in the final document. The EPA with the most revisions to the supporting tasks was "Oversee the pharmacy operations for an assigned work shift." The final EPA supporting task document was approved by the college's curriculum committee in Fall 2018. Implications: The EPAs and associated supporting tasks will be used to guide curriculum development and assessment of APPEreadiness and practice-readiness.

Risk of Heart Failure Associated with Gabapentinoid Use: A Systematic Review of Case Reports. Oliwier Nowak, Philadelphia College of Osteopathic Medicine, Jonathan J. Park, Philadelphia College of Osteopathic Medicine, Jiehyun Lee, Philadelphia College of Osteopathic Medicine. Objective: Pregabalin and gabapentin are widely used in treating neuropathic pain, however there has been suspicion of development and/or worsening of heart failure (HF). The purpose of this systematic review is to evaluate the potential relationship between gabapentinoid use and HF. Methods: A system- atic literature review was performed on August 23rd, 2018 using three electronic databases (Pubmed, Embase, and Cochrane Library). The literature review was restricted to case reports and series written in English. Results: Among 799 search results, 10 case reports were identified from 2007 to 2014. All cases involved the use of pregabalin with no HF cases reported with the use of gabapentin. Out of the 10 cases observed, 2 cases had no prior history of HF, and 8 cases had a history of HF. Out of these 8 cases, 7 were staged as New York Heart Association (NYHA) class II or higher prior to exacerbation, with only 2 being staged as class III or higher. The dosing of pregabalin varied from 50mg twice daily up to $300 \mathrm{mg}$ once daily. All reported cases exhibited ejection fraction of less than $56 \%$ on admission, and nearly all interventions involved diuretic therapy. Implications: Even though both drug package inserts advise the use of caution in patients with NYHA Class III-IV, it is unclear whether gabapentinoid use can be recommended in patients with NYHA Class I-II or with multiple risk factors for HF. Our findings will help bring awareness to healthcare professionals prescribing these medications and trigger further research to assess the association between $\mathrm{HF}$ and gabapentinoids.

Safe Handling and Disposal of Oral Anticancer Chemotherapy - Patient/Provider Survey Studies, an Interim Analysis. Siu-Fun Wong, Chapman University, Sun Yang, Chapman University, Priya S. Patel, Chapman University, Alexandra Corcoran, Chapman University, Samantha Arellano Isidro, Chapman University, Eric Dobberpuhl, Chapman University, Dustin Le, Chapman University, Analise Klassen, Chapman University, Jay Rho, Chapman University, Vi Tran, Chapman University. Objective: Oral anticancer chemotherapy (OC) has become more prevalent since the 1940s. The combination of patients' perceptions of OC as safer than its intravenous counterpart and the lack of standardized guidelines may predispose for increased risk of improper handling and disposal, leading to in-home and environmental contamination. The primary study objective is to evaluate the knowledge/awareness, practice, and attitude of OC handling and disposal in the home setting. Secondary objective aims to identify gaps and barriers to safe handling and disposal of OC. Methods: A 38-item healthcare provider survey and an IRB approved 36-item patients/caregivers survey were created and distributed by the Chapman research team, the California Society of Health-System Pharmacists, and the University of California, San Francisco. Surveys were administered in person or online using Qualtrics. Expansions to national study population are in progress with collaborators such as the Research Advocacy Network (patients/caregivers) 


\section{American Journal of Pharmaceutical Education 2019; 83 (5) Article 7654.}

and SWOG (providers). Statistical and descriptive analyses were used to analyze data collected. Results: Preliminary analysis of 12 patients and 15 providers showed suboptimal knowledge/awareness and practice in both patients and providers. The overall item score for both patients and providers average at $65 \%$ and $63 \%$, respectively. This study is ongoing. Updated, detailed scorings of each section and analyses of potential gaps and barriers will be presented. Implications: Our goals are to use evidence-based survey data to create safe practice model, to partner with boards of pharmacy to advocate for national legislative change, and to engage key stakeholders in this patient safety initiative to optimize population and environmental safety.

Service Learning: A Co-Curricular Program Promoting Public Health, Fostering Community Engagement, and Enhancing Student-Pharmacist Development. Zaynib A. Hassan, University of Colorado, Eric Hartsfield, University of Colorado, Susie Lee, University of Colorado, Maria Pearson, University of Colorado, Samantha Roming, University of Colorado, Patricia Meyer, University of Colorado, Dana P. Hammer, University of Colorado. Objective: Through the CU SSPPS Service Learning Program, first-year student pharmacists provide longitudinal health and nutrition education to underserved 3rd-5th grade students. The objective of this descriptive, exploratory study is to assess the program and its impact on student pharmacists, elementary students, and their teachers. Methods: Lessons based on state-mandated K-12 science learning standards include a variety of interactive and complex topics, permitting each student pharmacist to perform assigned and situational leadership activities and facilitate summative discussions. Five years of data will be analyzed including student pharmacists' reflections, elementary students' knowledge assessments, teacher ratings of student pharmacists' performance, as well as general feedback regarding the program. Quantitative ratings and scores will be aggregated, averaged, and compared over time. Qualitative reflections and teacher feedback will undergo thematic content analysis. Results: Working in a classroom provides real-life experience on how to lead one's peers in dynamic situations, which may be directly transferable to clinical practice. While not hypothesis-driven, analysis of 2014-2019 data based on teacher feedback and selfreflection will provide insight on student pharmacists' communication, leadership, and professionalism. Quantitative results will be presented graphically, while thematic analysis will be represented by excerpts from our findings. Implications: Anecdotally, this innovative cocurricular service learning model seems to have a positive impact on student pharmacists, elementary students, and their teachers, all while promoting public health. The program's success has led to additional partnership events including flu clinics and Pharmacist-for-a-Day, a nationally recognized program. Limitations of this evaluation include its lack of generalizability and the descriptive nature of its data.

Stability of Antibacterial Diterpenes in Dry Andrographis paniculata Stems. Aaron M. Parsons, South College, Truyen Nguyen, South College, Zhihong $\mathrm{Xu}$, South College. Objective: Andrographis paniculata is a medicinal herb that has been used for centuries in Southeastern Asia to treat infections, inflammation, and many other disease states. Two of the main antibacterial compounds found in A. paniculata are the two diterpenes, andrographolide and 14-deoxy-11,12-didehydroandrographolide. Our objective was to determine how much of these two diterpenes is lost from the raw material over time. Methods: Methanol was used to extract various compounds, including the diterpenes, from A. paniculata plant stems of different years. Sonication was used to complete the extraction, and a rotovap was used to evaporate the solvent. Utilizing commercially available andrographolide and 14-deoxy-11,12-didehydroandrographolide as standards, we analyzed the quantity of both antibacterial diterpenes in each dried plant extract using HPLC. Results: The percentages of the two diterpenes in each dried plant extract were obtained by HPLC analysis. Our results showed that over the years the quantity of andrographolide has decreased while the quantity of 14-deoxy-11,12-didehydroandrographolide has increased. However, we observed that there has not been a significant change in the total amount of these main antibacterial diterpenes in A. paniculata stems. Implications: Our results indicated that dry herb A. paniculata stems for antibacterial use may be stored at room temperature.

Student Led Public Health Initiatives: Naloxone. Nicole M. Perea, Washington State University, Kimberly C. McKeirnan, Washington State University, Angela Nguyen, Washington State University, Katherine Dier, Washington State University. Objective: The primary objective of this research is to determine if student pharmacists, in addition to a pharmacist mentor, can make a positive impact on increasing the public's knowledge on naloxone. The secondary objective of this research is to observe the quality and sustainability of a student led public health initiative. Methods: This study utilized a qualitative survey given to a variety of community members at the beginning and end of each presentation as a Pre/ Post format. The questions are to determine the quality of the content, perceived benefit of the training, and the comfort level regarding the use and administration of naloxone. To observe the sustainability of the student 


\section{American Journal of Pharmaceutical Education 2019; 83 (5) Article 7654.}

led initiative, mentor perception and student perception were utilized in an informal discussion to determine preliminary outcomes. Results: As a qualitative survey, the data so far has shown favorable results at this point in time. The data collection will continue through April with data analyzation completed by May. The observations made on quality and sustainability of the student led process will also take place in May. Implications: As accidental overdoses from opioid medications continue to ravage the country, naloxone is a cost-effective way to save lives and to reduce the financial burden placed on the healthcare system. The pharmacy profession has a great depth of knowledge on medication administration and a unique ability to convey this information to nonmedical personnel. Student pharmacists are in the unique position to create community partnerships and to make a positive public health impact within our communities.

Student-Led Quality Engagement Team Supporting a Community Pharmacy Enhanced Services Network: Pennsylvania Case Study. Sophia M. Cothrel, University of Pittsburgh, Joni Carroll, University of Pittsburgh, Katie Doong, University of Pittsburgh, Kim C. Coley, University of Pittsburgh, Stephanie H. McGrath, Pennsylvania Pharmacists Care Network, Brandon Antinopoulos, University of Pittsburgh, Melissa A. McGivney, University of Pittsburgh. Objective: To describe the formation of a student-led Quality Engagement Team (QET) within a Community Pharmacy Enhanced Services Network (CPESN) to support initial implementation of a payer contract. Methods: Student pharmacists from a single school were recruited to form a QET for the Pennsylvania Pharmacists Care Network (PPCN) CPESN. In September of 2017, PPCN launched its first payer contract with a regional Medicaid plan. The goal of the QET was to support successful implementation of comprehensive medication management (CMM) services to patients cared for in 84 independent community pharmacies. Faculty and PPCN leadership provided ongoing coaching to the QET. Results: In 2017, five students supported 68 pharmacies, and in 2018, nine students supported 84 pharmacies as the QET. The QET developed a semi-structured interview guide to assist with information gathering during bi-weekly telephone calls to the pharmacists. Calls were audio recorded and transcribed over four months in 2017, and over 6 months in 2018. Students utilized rapid-cycle problem solving to support the pharmacists. Students met bi-weekly with faculty and were continually coached throughout the time periods on how to address scenarios uncovered during the interviews. Learnings regarding CMM implementation were shared bi-weekly with PPCN to facilitate wrap-around support for the pharmacists. Students elicited nearly real time, qualitative case studies which were shared with the payer. Implications: Utilizing student pharmacists versed in rapid-cycle problem solving is a unique and beneficial approach to support initial implementation of a statewide payer contract for pharmacist-provided patient care services.

Student Perceptions Concerning the Use of Simulated Medications in a Pharmaceutical Care Lab. Maiah D. Hardin, The University of Louisiana at Monroe, Courtney Smart, The University of Louisiana at Monroe, Anthony L. Walker, The University of Louisiana at Monroe, Raghda K. Elsayed, The University of Louisiana at Monroe, Stacy Starks, The University of Louisiana at Monroe. Objective: To assess student perceptions regarding the substitution of in-house prepared, simulated medications for commercial, active medications in pharmacy school practice labs as a follow-up to a pilot study. Methods: A survey was used to assess perceived benefits and challenges of using simulated medications including amoxicillin suspension, ampicillin and ceftazidime powders, and Tamiflu capsules prepared in-house at the College of Pharmacy. The survey included students' opinions of in-house simulated medications compared to commercial active medications used in previous labs. An openended question was included to obtain student perceptions on the overall advantages and disadvantages of using simulated medications. Results: The survey revealed a majority of the students (97\%) understood the simulated products' educational intentions and purpose in their practice labs. There was a diverse response in relation to simulated Tamiflu capsules providing adequate substitution for commercial Tamiflu capsules. Overall, for future labs, simulated lab products were preferred $(43 \%)$ over commercial drugs (23\%) and no opinion (34\%). Safety concerns and cost efficacy were addressed in the open-ended responses given by the students. Implications: This project relates to students' perceptions concerning the use of in-house prepared, simulated medications versus commercial, active medications in pharmacy practice labs and the impact that it has had, if any, during their academic career. Our findings suggest that implementing simulated medications was accepted by students and will help with the advancement of future practice lab courses.

Student Pharmacists Preparedness and Perceptions Regarding Gender Affirming Therapy. Steven D. Swank, The University of Texas at Austin, Michelle Thien-Tam Tran, Jessica L. Robertson, The University of Texas at Austin, Justina Lipscomb, The University of Texas at Austin, Ashley S. Oliver. Objective: The purpose of this study is to assess current student pharmacists' preparation and attitudes in caring for transgender 


\section{American Journal of Pharmaceutical Education 2019; 83 (5) Article 7654.}

patients. Methods: An eight question Likert-scale survey was distributed to student pharmacists to assess their access to education and perceptions about gender affirming therapy (GAT) on a scale from 1 to 5 (1 being "not important", 5 being "very important"). Students on Advanced Pharmacy Practice Experience (APPE) rotations were asked two additional questions to assess comfort level in providing recommendations for and counseling patients on GAT. Participation in the survey was both anonymous and voluntary. No written consent was required. Results were analyzed using descriptive statistics and student's t-test as appropriate. Results: A total of 107 students completed the survey. Preliminary results show that they rated the importance of understanding GAT as 4.52 on average. Despite this, only $16.8 \%$ reported having access to formal GAT education at their institution. Seventeen P4 students on APPE rotations rated their preparedness to provide recommendations on GAT as 2.06 on average and their preparedness to counsel transgender patients initiating GAT as 2.12. Implications: Transgender patients who choose to undergo hormone replacement as part of their GAT require education on their medication regimens and managing side effects associated with these medications. Our assessment suggests that new pharmacy graduates are not prepared by their curriculum to provide recommendations or counsel patients about gender affirming hormone therapy.

Students' Attitude Toward Communication Training and Their Self-assessment of Oral Communication Skills in Three-Year Pharmacy Curriculum. Jean J. Vincent, Larkin University, Cucnhat Walker, Larkin University, Priscilla T. Ryder, Larkin University, Lynne Arric, Larkin University. Objective: To assess how pharmacy students' attitudes and perceptions of their oral communication skills change before and after communication class and at the end of their pharmacy training. Methods: A two-part survey was administered to pharmacy students twice, at the beginning and at the end of the communication class in a voluntary manner. All study investigators were blinded to student identity. Part 1 of the survey measures students' attitudes towards communication skills learning. Part 2 of the survey assesses students' perception of their communication. There were 3 additional demographic questions. This survey will be administered again at the end of these students' advanced pharmacy practice experience (APPE) year. SPSS version 24 was used to analyze the data. Results: Positive attitude subscale for students who English is not their first language was marginally higher than for the English-native speakers (mean 54.7 vs. 53.3, t54.3 = $-2.36, p=0.058$ ) while there was no difference in nega- tive attitude subscale. There was no difference among questions in survey part 2 . The overall positive attitude subscale decreased from pre- to post-communication class $(\mathrm{t} 43=2.780, \mathrm{p}=0.008)$. Score changes did not differ by any demographic factor. Implications: Possible reasons for a decrease in positive attitude before and after communication class need to be further investigated to identify improvement areas for the course faculty. The third survey data will tell us whether this trend will continue or improve after students' progress through their second year of didactic curriculum and third year of APPE rotations.

Students' Perception of Using Criterion-Referenced Assessments to Determine a Passing Score. YeeAnn Chen, University of California, San Francisco, Crystal Zhou, University of California, San Francisco, Andrew L. Leeds, University of California, San Francisco, Jaekyu Shin, University of California, San Francisco. Objective: Educators face the challenge of determining passing scores in a defendable and reliable manner. In preparation for the new competency-based curriculum, we piloted criterion-referenced assessments in a therapeutics course by using the Angoff method to set the passing score before administration of assessments. Understanding students' perception of criterion-referenced assessments may inform course directors on how to help students prepare for them in the new curriculum. Methods: In this cross-sectional study, we surveyed 122 pharmacy students who took the Therapeutics II course in Spring, 2018. The 10-question survey utilized a 5-point Likert scale for students to rate their perception of fairness/satisfaction, stress/test anxiety level, and likelihood to compare test scores with classmates, all regarding criterion-referenced versus norm-referenced methods. Descriptive statistics were used to summarize survey data and univariable analyses were used to determine correlation between survey results and academic performance. Results: The survey response rate was $96 \%$. The majority agreed that criterion-based assessments were fairer $(61.0 \%)$ and reflected competency better (61.9\%) compared to norm-referenced assessments. Conversely, most students felt that criterion-based assessments increased stress levels (43.6\%) and test anxiety (39.8\%). Though not significant, an increased level of test anxiety trended towards correlation with decreased assessment scores (coefficient $=-1.81, p=0.097$ ). Implications: The majority of students perceived the criterion-based assessments favorably. Issues that were identified include increased associated stress and test anxiety. This could possibly be improved over time with familiarity with the grading method and avoiding announcement of passing grade prior to assessments. 


\section{American Journal of Pharmaceutical Education 2019; 83 (5) Article 7654.}

Student Stress Management and Wellness Programs Among Colleges of Pharmacy. Kelly Ann Moran, Midwestern University/Downers Grove, Sally Arif, Midwestern University/Downers Grove, Ana C. Quinones-Boex, Midwestern University/Downers Grove. Objective: The objective of this study is to describe programmatic interventions that colleges of pharmacy have implemented to support student stress and wellness, as well as the barriers that prevent colleges from implementing support measures. Methods: A questionnaire and cover letter were distributed to college of pharmacy faculty teams attending the American Association of Colleges of Pharmacy (AACP) Fall Institute in 2018. Surveys were collected following completion and secured during the duration of the study period. The questionnaire contained 5 parts: (1) how perceived student stress levels are tracked and assessed, (2) the types and formats of stress-coping interventions offered, (3) the measured impacts of any stress-coping interventions, (4) the level of faculty training and involvement in student stress remediation, and (5) institutional demographics. Results: Over half of respondents (59.1\%) reported currently measuring student stress levels. Common student interventions offered were: individual counseling (95.5\%), academic advising (86.4\%), exercise classes (77.3\%), and peer to peer mentoring (68.2\%). The majority $(68.2 \%)$ of schools do not provide formal training in dealing with mental health related issues. The most reported reasons for the absence of training include lack of time $(13.6 \%)$ and it being considered a low priority (13.6\%) for the college. Lastly, a very limited number of schools $(4.5 \%)$ currently assessed the impact of student stress and coping interventions. Implications: Colleges of pharmacy are addressing student stress and wellbeing, yet variability exists and, barriers to improvement remain. Mental health concerns should be continually addressed in order to provide students with an effective learning experience.

Student Training for Community Screening Events: Asynchronous and Live Training Comparison. Haley M. Fox, University of Illinois at Chicago, Ryan Hill, University of Illinois at Chicago, Jacquelyn Punches, University of Illinois at Chicago, Heidi Olson, University of Illinois at Chicago, Alice N. Hemenway, University of Illinois at Chicago. Objective: Pharmacy students participate in community blood pressure and blood glucose screening events to give back to the community and practice interacting with patients. Instruction on proper methods for community screening events ensures that student participants have been properly trained and follow current policies and procedures. At our college of pharmacy, health screening training had been com- pleted via live, in-person training. However, with a curriculum change, we were able to pilot an asynchronous online training program. The purpose of this study was to determine if the online training course provides similar competency when compared to live training. Methods: An anonymous survey was given before and after the live session in 2017, and before and after the asynchronous, online session in 2018. Data from these surveys were compared using descriptive statistics to see if the increase in knowledge and confidence was similar between the live and online programs. Results: The live training event had 69 participants and the online training event had 77 participants. The live and online trainings each had 100\% successful completion. Both trainings saw increases in student-reported confidence, with a slightly greater increase seen for the live training (average increase of $34.7 \%-69.1 \%$ per question live, average increase of $14.4 \%-63.2 \%$ online). Both formats were considered convenient by participants (9.7/10 for live, 9.6/10 for online). Implications: Use of an online, asynchronous course is a viable option for student training to prepare for participation in community screening events.

Synthesis, in-vitro Evaluation, and Physicochemical Properties Enhancement of Modified JOAB Series as Potential Pro-inflammatory Mediator Suppressants. Ashley A. Bill, University of Saint Joseph, Yatish Parmar, University of Saint Joseph, Mohamed I. Nounou, University of Saint Joseph, Doreen E. Szollosi, University of Saint Joseph, Ivan O. Edafiogho, University of Saint Joseph, Ola A. Ghoneim, University of Saint Joseph. Objective: Infection triggers pro-inflammatory cytokine release such as tumor necrosis factor-alpha (TNF-alpha) and interleukin-6 (IL-6) from immune cells. In our lab, E121 suppressed TNF-alpha and IL-6 in lipopolysaccharide (LPS)-stimulated macrophages. E121 performance encouraged us to examine the key structural components of its effect. JOAB series were designed, synthesized, and found to surpass E121 in reducing pro-inflammatory cytokines in vitro. During in vivo evaluation, concerns rose over their physicochemical properties, mainly solubility, partition coefficient and diffusion. Herein, we present synthesis, in vitro evaluation and physicochemical optimization of modified JOAB series. Methods: Synthesis was accomplished by amination of the methyl/ethyl 2,4dioxo-6-methylcyclohexane-carboxylate with the desired $\mathrm{N}$-substituted piperazine. In vitro biological activity was tested in an LPS-stimulated J774 macrophage cell line. Cytokine release was measured after 24 hours using enzyme-linked immunosorbent assay. Solubility parameters were investigated in various solvents and $\mathrm{pH}$ systems with diverse polarity indices. Cyclodextrin complexation, polyethylene glycol solid dispersions and co- 


\section{American Journal of Pharmaceutical Education 2019; 83 (5) Article 7654.}

solvency were also explored. Results: Sixteen original JOAB series plus two modified analogs were synthesized. Their ability to reduce TNF-alpha/IL-6 was tested in a J774 macrophage cell line and exhibited superiority to E121. DMSO, chloroform and ethanol are the best solvents solubilizing the JOAB series at 20,10 , and $5 \mathrm{mg} / \mathrm{ml}$ respectively. Acidified ethanolic aqueous solution showed promising solubility parameter at $7.5 \mathrm{mg} / \mathrm{ml}$. Implications: JOAB series represent novel approach for autoimmunity as pro-inflammatory cytokine suppressants. Improving the physicochemical parameters would help transition JOAB series from bench to bedside, as well as, facilitate its efficient formulation and delivery along with clinical performance.

The Effects of Beta - Caryophyllene in Aged Heterozygous Triple Knock-in Alzheimer's Disease Mice. Melissa Milfort, Mercer University, Kevin S. Murnane, Mercer University, Cedrick M. Daphney, Mercer University. Objective: Since the discovery of Alzheimer's disease (AD) in 1906, AD is the number one cause of dementia among older adults. In the proposed study, we targeted brain CB2 receptors as agonism of these receptors has been shown to yield profound anti-inflammatory effects. We tested the efficacy of the cannabinoid compound (Beta-Caryophyllene) using a cutting-edge genetic (non-transgenic) knock-in mouse model (APPNL-G-F mice) of $\mathrm{AD}$ that recapitulates key aspects of $\mathrm{AD}$-related neuroinflammation. This APPNL-G-F mouse model exhibited phenotype of extensive neuroinflammation, age-related cognitive decline, and cell-type and temporal specificity of alpha-beta expression and plaque deposition. Methods: Baseline cognitive behavioral tests such as the Y-Maze, Novel Object Recognition Test, OpenField locomotion test, and Light-Dark transition test were assessed. The 8 mice were then administered $178 \mathrm{mg} / \mathrm{kg}$ of Beta-Caryophyllene IP to reduce inflammation. The same behavioral tests were re-run under the same conditions post-administration of the intervention, BetaCaryophyllene. Results: Results: Y - Maze: No improvement in female mice. Improvement in cognition seen in male mice, NORT: No improvement in female mice. Improvement in cognition seen in male mice, Open - Field Locomotion: Controlled locomotor test. No difference between both groups, and Light - Dark Transition: Reduction in anxiety observed in Female mice compared to Male mice. Implications: Based on the results from the behavioral tests, the anti-inflammatory compound appeared to positively impact cognition in male mice as compared to female mice. While positively impacting affective behavior in female mice as compared to male mice. Further testing will be conducted to analyze inflammatory markers from blood collected.
The Emotional Intelligence of Academic Pharmacy Administrators. Vonda K. VanDyke, East Tennessee State University. Objective: There is a growing interest in determining if emotional intelligence (EI) is a predictor of leadership effectiveness in pharmacy academia. This study explored differences between the administrative ranks of academic pharmacy administrators and their total emotional quotient (EQ) as measured by the Genos Emotional Intelligence Inventory. A secondary purpose identified differences between the administrative ranks of academic pharmacy administrators and their EQ competencies (Self-Awareness, Awareness of Others, Authenticity, Emotional Reasoning, Self-Management, and Positive Influence). The relationship between demographic characteristics of academic pharmacy administrators and total EQ scores was evaluated. Methods: A quantitative, non-experimental design was employed to measure EI in a stratified random sample of academic pharmacy administrators from ACPE fully accredited schools. Results: The results revealed a significant difference between the demographic characteristics of academic pharmacy administrators by rank. As determined by a MANOVA, significant differences existed among six continuous demographic variables. Results of chi-square probability testing of categorical demographic variables by administrative rank revealed nine significantly different variables. The results showed no significant differences between the total EQ scores of academic pharmacy administrators. The data revealed significant differences between the demographic characteristics of academic pharmacy administrators and EQ competencies. Implications: This study filled a void in academic pharmacy research by assessing the importance of EI to academic pharmacy administrative positions and the demographics of leaders as they related to EI. The results also provided a baseline measurement of EI function as it related to overall pharmacy leadership.

The Evolution of Prepharmacy: How Prerequisites Have Changed Over the Last Decade. Laura J. Bobbitt, University of North Carolina at Chapel Hill, Jacqueline McLaughlin, University of North Carolina at Chapel Hill. Objective: To characterize current prepharmacy curricula and compare them to prepharmacy curricula from 2009. Methods: Prepharmacy course requirements for every Doctor of Pharmacy (PharmD) degree program accredited by the Accreditation Council for Pharmacy Education (ACPE) were collected from each school's website in July 2018. The frequency of prepharmacy course requirements were compared to data from a white paper by Boyce and Lawson, which described pharmacy prerequisites in 2009. Analyses included descriptive statistics and chi-square tests. 


\section{American Journal of Pharmaceutical Education 2019; 83 (5) Article 7654.}

Results: In 2018, schools required $66.1 \pm 8$ (range 41 to 91) semester hours of prepharmacy coursework. Most programs required Biology (93\%), General Chemistry (100\%), Organic Chemistry (100\%), Calculus (99\%), and English (85\%). Courses such as Anatomy/Physiology, Microbiology, Physics, Statistics, Communication/ Public Speaking, and Economics were required by approximately $60 \%$ to $80 \%$ of programs. Biochemistry, Genetics, Immunology, Psychology, Ethics, Other Culture and/or Diversity, and Fine Arts were required less frequently. In comparison to 2009, prepharmacy curricula have remained largely unchanged. All schools required General Chemistry and Organic Chemistry in 2009 and 2018. However, a significant increase was found in the percent of schools requiring Anatomy/Physiology (62\% vs. $77 \%, \mathrm{p}=0.02)$ and Statistics $(56 \%$ vs. $75 \%, \mathrm{p}<0.01)$. Implications: Boyce and Lawson recommended more uniform prepharmacy curricula in 2009, but curricula have remained largely unchanged since then. As curricula and pharmacy practice evolve, schools must revisit their prepharmacy curriculum to ensure it is relevant and contemporary. Reference: Boyce EG, Lawson LA. Preprofessional Curriculum in Preparation for Doctor of Pharmacy Educational Programs. Am J Pharm Educ. 2009;73(8).

The Influence of PCAT Requirements on NAPLEX Pass Rates, On-Time Graduation Rates, and USNWR Ratings. Mallory A. Paszkiewicz, Western New England University, Joseph A. Rubilar, Western New England University, Deana A. Rodriguez, Western New England University, Daniel R. Kennedy, Western New England University, Joshua J. Spooner, Western New England University. Objective: To determine if the requirement for Doctor of Pharmacy program applicants to complete the Pharmacy College Admission Test (PCAT) had any bearing on traditional metrics of program success. Methods: A retrospective data analysis was conducted for the 125 Doctors of Pharmacy programs with full ACPE accreditation by December 2015. The graduating class of 2017 was examined; PCAT requirements were obtained from AACP for the corresponding admission cycle for that class (2012-2013 cycle for four-year programs; 2013-2014 cycle for three-year programs). NAPLEX pass rates were obtained from NABP. On time graduation rates were obtained from program websites; the most recent (2016) USNWR pharmacy program ratings were utilized. Tests of significance ( $\mathrm{T}$-tests and chisquares) were utilized for continuous and categorical data, respectively. Results: During the relevant admissions cycle for the Class of 2017, 101 programs $(80.8 \%)$ required applicants to complete the PCAT. While school characteristics (public vs. private; 3 year vs. 4 year) did influence metrics of program success, requiring applicants to take the PCAT did not influence these metrics. There were no statistically significant relationship between PCAT requirements and program NAPLEX pass rates, on time graduation rates, or USNWR pharmacy program ratings (all $\mathrm{P}>0.1$ ). Implications: While pharmacy program success is the product of many factors, the decision on whether or not to require applicants to take the PCAT does not appear to influence traditional metrics of pharmacy program success at this time.

Transformative Learning: Collaborating Through Interprofessional Simulations. Emily L. Fedor, Western New England University, Beth E. Welch, Western New England University, Melissa Mattison, Western New England University, Courtney Doyle-Campbell, Western New England University. Objective: Interprofessional education is important in preparing future healthcare practitioners to be collaborative practice ready with the goal of improved patient outcomes and greater satisfaction for both patients and providers. The objective was to create transformative learning through an interprofessional simulation day. Methods: Western New England University College of Pharmacy and Health Sciences designed and executed a multisite simulation day which included three institutions and students from four healthcare professions (pharmacy, nursing, physician assistant studies, and occupational therapy). Students were assigned to either ambulatory care simulations using standardized patients or acute care simulations using high-fidelity human simulators. The students worked in interprofessional teams to assess, diagnose, develop, and implement a care plan. To investigate the impact of the simulated experience on the beliefs, behaviors, and attitudes that underlie interprofessional collaboration, preand post-surveys (Interprofessional Socialization and Valuing Scale-24) were administered. Results: A total of 175 students participated, with 71 (41\%) matched survey responses usable for comparisons. Mean scores significantly increased from pre- to post- (114 vs. 124, respectively $(\mathrm{p}<.05))$. Scores significantly increased in 21 out of 24 items and across three factor domains: selfperceived ability to work with others, value in working with others, and comfort in working with others. Implications: The simulation day increased participants' confidence to work on an interprofessional team, created awareness of each profession's role and responsibilities, and strengthened skills needed to effectively contribute to a team. Partnering with other institutions using interprofessional simulations can provide transformative learning experiences and prepare future healthcare practitioners to be collaborative practice ready. 


\section{American Journal of Pharmaceutical Education 2019; 83 (5) Article 7654.}

Using Peer Review to Enforce SOAP Documentation Skills. Antonia Akrap, The University of Kansas, Sarah Shrader, The University of Kansas, Ashley Crowl, The University of Kansas, Tiffany R. Shin, The University of Kansas, Ryan Funk, The University of Kansas, Crystal Burkhardt, The University of Kansas. Objective: To evaluate the impact of a peer review process on the clinical note writing skills of third year pharmacy students. Methods: Students in a required course submitted four individual clinical notes assessed using a standard grading rubric throughout the 2017 and 2018 fall semesters. In 2017 , faculty randomly graded one of four student notes throughout the semester which serves as the historic comparator. In 2018, the first note was graded by faculty and the final three notes were randomly assigned for three anonymous peer reviews. In 2018, the students received additional instruction on the use of the rubric to complete peer reviews. All scores were deidentified and analyzed using descriptive statistics. Performance on note one and four was analyzed using a paired t-test for the 2018 class. Final note performance between 2017 and 2018 was compared using a two-sample t-test. Qualitative data from a focus group of the 2018 class was analyzed using conventional content analysis approaches. Results: Note performance improved significantly $(\mathrm{p}=0.001)$ from note one to four $(79.58 \%+/-12.04 \%, 93.26 \%+/-6.10 \%$ respectively) for the 2018 class $(n=133)$. The 2018 class performed significantly better $(\mathrm{p}=0.001)$ on their final SOAP compared to the 2017 class $(n=124)(93.26 \%+/-6.10 \%$, $90.20 \%+/-9.49 \%$ respectively). The focus session recurrent themes highlighted student's preference to submit individual notes and to have a clear, guided rubric. They also stated the time devoted to the assignments were significant, however they voiced satisfaction with the learning experience. Implications: These findings suggest peer review can advance students' clinical note writing skills.

Utilization of Entrustable Professional Activities to Develop Professional Identity and Reflective Learning Skills. Brianna McQuade, University of Illinois at Chicago, Sheila M. Allen, University of Illinois at Chicago, Margaret A. Choye, University of Illinois at Chicago, Jennie B. Jarrett, University of Illinois at Chicago. Objective: Development of identity and reflective learning skills are critical to the success of a professional, but difficult to teach in the classroom. Entrustable Professional Activities (EPA) described in the Patient Care Provider domain (EPAs 1-5) align with the Pharmacist Patient Care Process (PPCP). The tasks performed in a second-year, skills-based course focus on communicating pharmacotherapeutic recommendations utilizing these EPAs for pharmacy graduates. This study describes the use of pharmacy EPAs to cultivate professional identity and reflective learning techniques in a skills-based course. Methods: After each skills laboratory, students reflected on their activities using EPAs 1-5 to describe the activity performed, their efficiency (in minutes) and level of entrustment (scale 1, thoughtfully observe, to 5, perform and teach) to perform in an actual patient care environment. Paired t-test and descriptive statistics were used to determine improvement in skill across the course (pre/post) and activity efficiency. Results: One hundred eightyseven students completed the course. The average level of entrustment (scale 1-5) increased from baseline to end of course across all EPAs with all p-values $<0.05$ [EPA 1 (3.24 to 3.82), EPA 2 (2.90 to 3.64), EPA 3 (2.74 to 3.5), EPA 4 (2.74 to 3.41), and EPA 5 (3.06 to 3.34)]. The average time to complete all 5 EPAs decreased from their first recitation (mean=163 minutes) to the end of the course (mean $=81$ minutes). Implications: While students' self-reported level of entrustment and efficiency for performing the PPCP EPAs both improved, further student development is required to decrease inflation of their capabilities.

Validation of a Pre/Post Survey to Measure Change in Student Social Justice Attitudes and Empathy. Shannon N. Vaffis, The University of Arizona, Elizabeth J. Anderson, The University of Arizona, Rebekah Jackowski, Midwestern University/Glendale, Terri L. Warholak, The University of Arizona, Elizabeth A. Hall-Lipsy, The University of Arizona. Objective: Despite endorsement by the Center for Advancement of Pharmacy Education (CAPE), Doctor of Pharmacy (PharmD) students are infrequently taught concepts related to social justice and empathy in a formal capacity. No validated measure exists to examine changes in these attitudes. Therefore, we tested the ability of a survey to predict changes in social justice and professional empathy attitudes in PharmD students. Methods: Retrospective pre-post data collected from PharmD students after a population health course at two schools of pharmacy were used to assess validity and reliability of the Social Justice and Empathy Assessment (SJEA) survey. A partial-credit Rasch model was used to test for unidimensionality, person and item fit, and rating scale functioning, as well as construct and content validity. Multiple linear regression was used to predict the effect of demographic covariates on the extent of pre-post change. Results: Of 396 participating students, $60 \%$ were non-Hispanic White and 57\% were male. The scale demonstrated evidence of unidimensionality of a construct designated "social justice self-efficacy". The rating scale functioned best as a three-option scale. Four items were removed to optimize person-item distribution, though few items were difficult 


\section{American Journal of Pharmaceutical Education 2019; 83 (5) Article 7654.}

to endorse. Only attending the private school was significantly associated with increased pre-post change. Implications: The SJEA is functional as a criterion-referenced assessment to determine whether minimum competency in social justice self-efficacy is experienced before or after participation in a CAPE-informed educational program. Further research should explore contextual predictors of change in social justice self-efficacy.

Virtual Pharmacopedia: Analysis of StudentDeveloped Expert-Reviewed Modules for PharmD Curricular Expansion. Amy L. Dorszynski, University of North Carolina at Chapel Hill, Minh-Thi Ton, University of North Carolina at Chapel Hill, Amanda Mintz, University of North Carolina at Chapel Hill, Edward Lee, University of North Carolina at Chapel Hill, Michael B. Jarstfer, University of North Carolina at Chapel Hill, Jacqueline McLaughlin, University of North Carolina at Chapel Hill. Objective: Virtual Pharmacopedia (VP) is an online educational resource that houses studentdeveloped, expert-reviewed modules to supplement the core pharmacy curriculum. As a platform for self-directed learning, VP offers abbreviated NAPLEX review, rotation preparation, and exposure to unfamiliar content. The purpose of this study was to evaluate the perceived need and utility of VP for students. Methods: Students were separated into two groups: those who created module content (creators) and those who utilized the module (consumers). Learning was evaluated before (pre-) and after (post-) module creation via multiple-choice assessments, and perceptions were evaluated afterward via surveys and interviews. Perceived need and utility for the modules were also evaluated by consumers using five-point Likert survey items. Pre- and post-data were evaluated using paired t-tests $(\alpha=0.05)$. All other data were analyzed descriptively. Results: Most consumers $(n=72,47 \%$ response) agreed they would use VP (94.4\%) and that module content enhanced their understanding of the material $(87.5 \%)$. Preliminary creator survey data $(n=5$, $100 \%$ response) revealed increased perception of knowledge $(\mathrm{p}<0.05)$ and application $(\mathrm{p}<0.05)$ from pre- to post-module. Additionally, $100 \%$ strongly agreed that generating module content increased their knowledge. Preliminary consumer assessment scores demonstrated improvements from pre- to post-module for those who reviewed the module ( $\mathrm{p}<0.05)$. Implications: VP modules provide statistically significant increases in learning and application of knowledge for both module creators and consumers. VP has demonstrated to be a useful resource exhibiting potential for practical utility in education.

What Are the Perceptions Of Pharmacists Collaborating On Mental Healthcare Teams? A Systematic Review. Brandy R. Davis, Auburn University,
Kimberly B. Garza, Auburn University. Objective: Pharmacist involvement in mental healthcare teams can positively impact patient care, but acceptance by patients and other providers is unknown. This systematic review synthesized perceptions of pharmacist involvement among healthcare providers and patients. Methods: The search was conducted using Pubmed, PsychInfo, International Pharmaceutical Abstracts and clinicaltrials.gov from inception to November 2018. Search terms included "pharmacist", "mental health", "collaboration", "interprofessional", "patient care team", and "cooperative behavior". Inclusion criteria was: inclusion of pharmacists on mental health teams, perceptions of pharmacists recorded, and written in English. Articles were excluded if perceptions about pharmacist involvement were not assessed or pharmacists were not on collaborative teams. Results: A total of 360 unique articles were identified in the initial search, and 330 were removed after the initial screening. Thirty full text articles were screened; six were included in the review. Perceptions were obtained from pharmacists $(n=5)$, patients $(n=3)$, and other health care providers $(n=4)$, including psychiatrists, psychologists, a mental health team, general practitioners, nurses, and social workers. Pharmacists were seen as valuable by patients $(n=2)$ and other health care providers $(n=4)$. Some patients were not aware of pharmacists collaborating on mental health care teams $(n=1)$, while some healthcare providers thought that collaboration with pharmacists should be expanded further $(n=1)$. In community settings, pharmacists were the initiators in collaboration efforts $(\mathrm{n}=1)$. Implications: Pharmacists are perceived as valuable members to mental health collaborative teams. Further research should be performed to see how collaboration efforts can be expanded and make collaboration a natural part of the healthcare system.

What Motivates Domestic vs. International Students to Select Pharmacy as a Major: Intrinsic, Extrinsic or Both? Stephanie Yang, Northeastern University, Jungeun Lee, Northeastern University, Jenny A. Van Amburgh, Northeastern University. Objective: Intrinsic motivation refers to internal or personal interest versus extrinsic motivations are outside of the individual's desires. Through this study, we will analyze the factors that influence students' decision to pursue pharmacy (pre-pharmacy through P4 year) and the possible correlation these factors have on academic and social involvement outcomes. Methods: A cross-sectional study was conducted using a 14-question online survey through Qualtrics. Participants will be recruited from the School of Pharmacy Classes of 2018/19 through 2023/2024 (P4 to pre-pharmacy students). The survey included three 


\section{American Journal of Pharmaceutical Education 2019; 83 (5) Article 7654.}

major sections: demographics, motivational factors, and social/academic involvement. Descriptive statistics will be used to analyze and report collected data with the $\mathrm{p}$ value set as $\mathrm{p}<0.05$. Results: Survey was sent to 693 students, 303 students responded with 246 (81.2\%) completed the survey. Of those who completed, $57.9 \% \mathrm{P} 1-\mathrm{P} 4$ students, $83.0 \%$ domestic, $65.6 \%$ born in the US, $56.8 \%$ Asian, and $66.8 \%$ of parents born outside the US. In preliminary review, individuals with one or both parents born in the US had more internal motivation to pursue pharmacy, whereas individuals with neither parent from the US had higher external motivation. Further analysis will be completed and reviewed. Implications: The results of the study will provide information to increase awareness of factors that influence students' decisions to selecting pharmacy as their major and how such factors influence academic and social involvement outcomes.

Who Mentors the Mentors? Mohammad I. Ansari, University of Maryland, Shannon R. Tucker, University of Maryland, Andrew Coop, University of Maryland. Objective: The purpose of this program is to prepare chemistry trainees to become educators in academic pharmacy. Pharmacy teaching is not simply instructing, rather than it is a continual learning process of how to effectively disseminate information to students with the goal that the students apply it in a clinical setting. From observation, significant challenges were present for both graduate students and postdocs transitioning from research to professional education. Teaching methods were predominantly focused on basic research, rather than applied science. Methods: We formalized the process with an approach aimed at training trainees to become mentors by: 1 . Delivering monthly talks in group meetings that mimic lectures in the PharmD curriculum, 2. Supporting the involvement of rotating PharmD students in group meetings, 3. Feedback and evaluation by faculty and PharmD students, 4. One on one interaction with PharmD faculty and students. Results: At the conclusion of the pilot program case study, the following improvements were observed: the author grew in confidence in relaying information to students in drug pharmacology, pharmacokinetics, metabolism, excretion, and adverse effects, trainees became more familiar with pharmacy setting, curriculum, and teaching methodologies, and feedback improved the learning outcomes. Implications: By applying these methods there was a significant improvement and confidence in the teaching abilities of the author, who is a trainee. Feedback from faculty and students proved to be invaluable in assisting the future faculty members with their transition to academic pharmacy setting.

\section{RESEARCH/EDUCATION AND TRAINEE POSTER COMPETITION AB- STRACT ROSTER OF REVIEWERS}

AACP would like to acknowledge the following individuals for their contributions to the abstract review process and their service to the Association. Their time and expertise have been instrumental in ensuring the quality of posters presented at Pharmacy Education 2019.

\author{
ADMINISTRATIVE SERVICES SECTION \\ Russell T. Attridge \\ Kenza E. Benzeroual \\ Vishakha Bhave \\ Bernadette K. Brown \\ Jack J. Chen \\ Avani Desai \\ William M. Gentry \\ Daniel J. Hansen \\ Jennifer A. Henriksen \\ Erik Jorvig \\ Paul W. Jungnickel \\ Matthew M. Lacroix \\ Lisa Lebovitz \\ Jill A. Morgan \\ Michael W. Neville \\ Richard O'Brocta \\ Jason T. Range \\ Kelly M. Shields \\ Joel P. Spiess \\ Joshua J. Spooner \\ Kara Sweet
}

\section{BIOLOGICAL SCIENCES SECTION}

Felix Amissah

Teresa A. Beam

Alok Bhushan

Timothy J. Bloom

Ajay K. Bommareddy

Daniel A. Brazeau

Melissa A. Burmeister

Rahul R. Deshmukh

Joseph E. Deweese

Abir T. El-Alfy

Marina Galvez Peralta

Amy-Joan L. Ham

Ayman K. Hamouda

Yagna P.R. Jarajapu

David A. Johnson

Patricia Jumbo-Lucioni

Shannon Kinney

Jennifer Lamberts 
American Journal of Pharmaceutical Education 2019; 83 (5) Article 7654.

Anastasios Lymperopoulos

Mattia Migliore

Islam Mohamed

Shekher Mohan

Fatima Mraiche

Kalyan C. Nagulapalli Venkata

Sreejayan Nair

Henry North

Amy L. Parkhill

Vanishree Rajagopalan

Shantanu Rao

Terry-Elinor Reid

Sigrid C. Roberts

Monzurul A. Roni

Jilla Sabeti

Noha N. Salama

Nathan Shankar

Alok Sharma

Horrick Sharma

Sunil Sirohi

Jozef Stec

Jody K. Takemoto

Marilyn E. Thompson Odom

Yuan Zhao

\section{CHEMISTRY SECTION}

Dennis A. Brown

Daniel J. Canney

Matthew J. DellaVecchia

Rahul R. Deshmukh

Ola A. Ghoneim

Ian S. Haworth

Edward B. Roche

David J. Weldon

Ragunandan Yendapally

\section{CONTINUING PROFESSIONAL} DEVELOPMENT SECTION

Jordan Baye

Paul P. Belliveau

Swati Betharia

Andrea Burns

Peter J. Hughes

Kristin K. Janke

Arinzechukwu Nkemdirim Okere

Fatimah M. Sherbeny

Elizabeth Trolli

Elizabeth J. Unni

James Wheeler

Catherine A. White

EXPERIENTIAL EDUCATION SECTION

Sheila M. Allen
Kelly J. Anderson

Jennifer D. Arnoldi

Gina M. Baugh

Meghan Bodenberg

Laurie L. Briceland

K. Paige D. Brown

Courtney R. Caimano

Angela S. Clauson

Jennifer N. Clements

Erica F. Crannage

Nicole S. Culhane

Andrew Darley

Keith DelMonte

Melissa M. Dinkins

Eliza Dy-Boarman

Carinda Feild

Brett Feret

Caitlin K. Frail

Wesley R. Haltom

Meredith L. Howard

Delaney Ivy

Jennie B. Jarrett

Tina Kanmaz

Suzanne Larson

Jennifer L. Luciano

Tamara McCants

Lena McDowell

Lisa M. Meny

Rita Morelli

Kate Newman

C. Leiana Oswald

Sara Revolinski

Christine M. Ruby-Scelsi

Kathryn A. Schott

Melody C. Sheffield

Jane Shtaynberg

Maryann Z. Skrabal

Mary Douglass Smith

Alison M. Stevens

T. Lynn Stevenson

Diana Tamer

Julie A. Testman

Angela R. Thomason

Misha M. Thomason

Elizabeth Trolli

Sara N. Trovinger

Lindsey H. Welch

\section{LIBRARY AND INFORMATION SCIENCE SECTION}

Sherrill J. Brown

Vern Duba 


\section{American Journal of Pharmaceutical Education 2019; 83 (5) Article 7654.}

Miki Goldwire

Timothy C. Hutcherson

J. Rosa Kim

Scott Perkins

Christina M. Seeger

Christopher S. Wisniewski

\section{PHARMACEUTICS SECTION}

Lloyd F. Alfonso

Charles S. Asbill

Shaifali Bhalla

Kathleen M. Boje

Bill J. Bowman

Lipika Chablani

Maimoona G. Chinwala

Melgardt M. de Villiers

Asish K. Dutta

Deborah L. Elder

Ian S. Haworth

Sunil Jambhekar

Cynthia B. Jones

Medha Joshi

Eytan A. Klausner

Krishna Kumar

Victoria K. Maskiewicz

William C. Mobley

Tommy C. Morris

Anita T. Mosley

Vaibhav Y. Mundra

Karen M. Nagel-Edwards

Indiran Pather

Rodney C. Siwale

Rahmat M. Talukder

Rajesh Vadlapatla

Catherine A. White

Nicole R. Winston

\section{PHARMACY PRACTICE SECTION}

Cheryl Abel

Michaela M. Almgren

Jason Bandy

Tina C. Beck

Kimberley J. Begley

Allison M. Bell

Paul P. Belliveau

Tricia M. Berry

Lauren Bode

Nancy Borja-Hart

Michelle M. Bottenberg

Courtney L. Bradley

Sherrill J. Brown

Elizabeth A. Buckley
Lakesha M. Butler

Danielle M. Candelario

Katherine Carey

Kacey Carroll

Mary L. Chavez

Jack J. Chen

Lindsey Childs-Kean

Angela $\mathrm{Chu}$

Jennifer N. Clements

John M. Conry

Stephanie Conway

Steven J. Crosby

Ashley Crowl

Brian Cryder

Erin Dalton

Kimberly K. Daugherty

Tosin David

Lisa D. Davis

Aimee Dawson

Maithili Deshpande

KariLynn Dowling

Ian C. Doyle

Eliza - Drake Dy-Boarman

Lea S. Eiland

Teresa M. Elsobky

Jeffery D. Evans

Paula J. Evans

Sarah Jane E. Faro

Charlotte A. Farris

Norman E. Fenn

Cathy H. Ficzere

Jeffrey Fong

Janice R. Frueh

Wendy M. Gabriel

Alice M. Gahbauer

Caitlin Gibson

Heather L. Girand

Chelsea Gresham-Dolby

Sally L. Haack

Lauren C. Haggerty

Leslie A. Hamilton

Jennifer Hartman

Karl Hess

Cheryl K. Horlen

Anne L. Hume

Kendrea M. Jones

Abby A. Kahaleh

Nancy E. Kawahara

Kristi W. Kelley

Michael G. Kendrach

Amber King

Justin M. Kinney 
American Journal of Pharmaceutical Education 2019; 83 (5) Article 7654.

Justin Kirby

Jennifer Kirwin

Christine M. Klein

Kristin C. Klein

Laura E. Knockel

Judith L. Kristeller

Yee Ming Lee

Kelly Lempicki

Troy Lynn Lewis

Laura Licari

Hua Ling

Justina Lipscomb

Alicia M. Lopez

Jody L. Lounsbery

Alex J. Luli

Karen F. Marlowe

Joel C. Marrs

Diane M. McClaskey

Tracey L. Mersfelder

Brittney A. Meyer

Margaret A. Miklich

Gary Milavetz

Christina Mnatzaganian

Cortney M. Mospan

Monique L. Mounce

Fatima Mraiche

Kimberly L. Nealy

Lydia C. Newsom

Timothy V. Nguyen

Samar Nicolas

Arinzechukwu Nkemdirim Okere

Anna Nogid

Nkem Nonyel

Heather A. Pace

Kristine Parbuoni

Sharon K. Park

Edna Patatanian

Isha Patel

Erin E. Pauling

Deborah Pestka

Ann M. Philbrick

Linnea A. Polgreen

Andrea L. Porter

Brittany L. Riley

Janna Roitman

Katie E. Ronald

Nephy G. Samuel

Kyle J. Schmidt

Fred N. Schneiweiss

Rebecca Schoen

Sheila Seed

Courtney A.B. Selby
Manvi Sharma

Kayce M. Shealy

Stephanie L. Sibicky

Caroline Sierra

Nicole M. Sifontis

Kathryn J. Smith

Susan E. Smith

Pamela L. Stamm

Catherine Taglieri

Colleen Teevan

Jamie M. Terrell

Jonathan Thigpen

Tyan F. Thomas

Jennifer Towle

Katherine M. Tromp

Kali VanLangen

Stacy A. Voils

Deepti Vyas

Rashi C. Waghel

Matthew A. Wanat

Kenric B. Ware

Kathryn Wdowiarz

Nancy T. Williams

Jennifer A. Wilson

Cheng C. Yuet

Tina Zerilli

\section{SOCIAL AND ADMINISTRATIVE SCIENCES}

SECTION

Joyce Addo-Atuah

Georges Adunlin

Sarah M. Anderson

Omar F. Attarabeen

Jill M. Augustine

David Rhys Axon

David M. Baker

Benita Bamgbade

Marie Barnard

Kimberly A. Broedel-Zaugg

Chenita W. Carter

Aleda M. Chen

Li Chen

Kelly Conn

Jordan R. Covvey

Utkarsh J. Dang

KariLynn Dowling

Akesha Edwards

Duska M. Franic

Rahul Garg

Kimberly B. Garza

Justin Gatwood 
American Journal of Pharmaceutical Education 2019; 83 (5) Article 7654.

Tyler H. Gums

Nicholas E. Hagemeier

Spencer E. Harpe

Tessa J. Hastings

Ana L. Hincapie

Lindsey Hohmann

Natalie Hohmann

Jan Kavookjian

Monina Lahoz

Minghui Sam Li

David J. McCaffrey

Pramit A. Nadpara

Leslie Ochs

Motolani E. Ogunsanya
Justin K. Owensby

Michael J. Peeters

Ana C. Quinones-Boex

Sujith Ramachandran

Nathaniel M. Rickles

Manvi Sharma

Natalia Shcherbakova

Marion K. Slack

Lori H. Syed

Casey R. Tak

J. Douglas Thornton

Ranjani Varadarajan

Daniel J. Ventricelli

Terri L. Warholak 